\title{
Chemical Synthesis and Medicinal Applications of Glycoporphyrins
}

\author{
Claire Moylan ${ }^{1}$, Eoin M. Scanlan ${ }^{2}$, Mathias O. Senge $e^{1,3^{*}}$
}

${ }^{1}$ School of Chemistry, SFI Tetrapyrrole Laboratory, Trinity Biomedical Sciences Institute, 152-160 Pearse Street, Trinity College Dublin, The University of Dublin, Dublin 2, Ireland; ${ }^{2}$ School of Chemistry, Trinity Biomedical Sciences Institute, 152-160 Pearse Street, Trinity College Dublin, The University of Dublin, Dublin 2, Ireland; ${ }^{3}$ Medicinal Chemistry, Institute of Molecular Medicine, Trinity Centre for Health Sciences, Trinity College Dublin, St James's Hospital, Dublin 8, Ireland.

\begin{abstract}
This review presents an in-depth overview of the modification of porphyrins with bioconjugates and their applications in medicine today. Porphyrin bioconjugates ranging from nucleotides to steroids are under active scrutiny. However, carbohydrates have been at the forefront of such research in recent years and offer significant potential. This is attributed to their own selectivity to lectins on the surface of cancer cells and their influence on the amphiphilicity of the porphyrin macrocycle. These characteristics and the tendency of porphyrin photosensitizers to accumulate in tumor tissues make glycoporphyrins promising candidates for use as photosensitizers. Thus, a detailed overview of the synthesis and biological evaluation of glycoporphyrins is given with a particular focus on their applications in photodynamic therapy and their future prospects as drug candidates have been reported.
\end{abstract}

Keywords: Carbohydrates, Glycoporphyrins, Porphyrins, Photodynamic Therapy, Sugars and Tetrapyrroles.

\section{INTRODUCTION}

\subsection{Porphyrins and PDT}

Porphyrins are ubiquitous in nature and are an essential class of pigments in biological systems. They are found in cytochromes, peroxidases and catalases and carry out a variety of functions in respiration, electron transfer, catalysis and photosynthesis ${ }^{[1]}$. Porphyrins are energy excitable chromophores and have many functions in biochemistry and industrial applications ${ }^{[2]}$. They have a wide range of applications in modeling energy transfer, in supramolecular chemistry and in solar cells ${ }^{[3]}$, but specifically in biological system they are involved in oxygen transport, photosynthesis, and catalysis. In clinical terms they are involved in genetic disorders such as porphyrias, play a role in signaling and other areas of photomedicine ${ }^{[4,5]}$.

Due to their biological relevance and involvement in many clinical problems much research has focused on the development of synthetic porphyrin derivatives for medical research. Medical applications of porphyrin derivatives have focused on both photodynamic detection (PDD) and photodynamic therapy (PDT). Photodynamic detection is a diagnostic technique where a specific light activating molecule, i.e. a photosensitizer (PS) agent is administered to the targeted cells. These cells are then exposed to short wavelength light which produces emission of light at a lower energy wavelength. This light is detected and analyzed spectroscopically ${ }^{[6]}$. PDD refers to the diagnosis, while PDT

*To whom correspondence should be addressed Fax: +353-1-896-8536; Email: sengem@tcd.ie. refers to the actual therapy of malignant and nonmalignant diseases ${ }^{[7,8]}$

PDT is a non-invasive cancer therapy which can allow for more selectivity in destroying tumor cells compared to conventional methods with significantly less side effects. It involves the administration of a PS agent either intravenously or topologically to the body which can accumulate in cancer tissue largely due to the enhanced permeability and retention (EPR) effect ${ }^{[9-11]}$. These PS agents can be activated with specific wavelengths to produce reactive oxygen species (ROS), often singlet oxygen, which in turn induces irreversible cancer tissue damage. Accumulation of PS agents in normal tissue and the inability of light to penetrate deeply into the skin are just two of the drawbacks which highlight the need for continuous research in this field ${ }^{[7,12,13]}$.

PDT has shown significant advances in recent years, from the synthesis of first generation PSs, e.g., the haematoporphyrin derivative Photofrin ${ }^{\circledR}$ to second generation systems, such as Temoporfin ${ }^{\circledR}{ }^{[14]}$. Syntheses of new derivatives focusing on eradicating the problems observed in the first generation derivatives have been investigated extensively in recent times ${ }^{[15]}$. Efforts to improve the selectivity of PS agents have led to the incorporation of targeting agents including bioconjugates which have selectivity for receptors over-expressed on tumor tissue, thus, resulting in the study of third generation PSs. Commercially available PS formulations such as Photofrin ${ }^{\circledR}$ and Foscan ${ }^{\circledR}$ exhibit passive selectivity for malignant tissue modulated by increased metabolic activity of cancer cells. 
Therapeutically this lack of selectivity implicates the dosage of PS administered to the patient so as to obtain significant therapeutic effect. The pharmacokinetics and biodistribution of PS must be tackled to overcome these undesirable characteristics with the goal to provide beneficial palliative treatment.

\subsection{Carbohydrates}

Oligosaccharides play an important role in many biological functions such as cell adhesion, metastasis, signaling, communication, and as growth signals ${ }^{[16,17]}$. The interactions between the receptors and the substrates are very specific, indicating their potential as targets for drug delivery. Complex carbohydrates mediate a wide variety of biological effects ranging from basic energy requirements to cell recognition, cell-cell communication, fertilization, hormone activity, viral and bacterial infections and tumor cell metastasis ${ }^{[18]}$. Thus, glycobiology involves a cornucopia of glycoconjugates including mono-, oligo- and polysaccharides attached to proteins and lipids ${ }^{[19]}$.

Most carbohydrates have a corresponding receptor on the cell surface, i.e. a binding protein receptor called a lectin. Lectins are non-enzymatic proteins which carry out cellular functions such as trafficking, adhesion and signaling through deciphering the "glycocode" ${ }^{[17,20]}$. Many changes in cell surface lectins are brought about by oncogenic transformation which provides impetus for future research in cancer detection and treatment. For example, galectin and glucose transporters ${ }^{[21]}$ are found to be over-expressed on tumor cells ${ }^{[22-24]}$. As a result, noteworthy research has been carried out on galectin-3 as its expression has been associated with oncogenic cell formation in the human gastric system ${ }^{[25]}$ and thyroid ${ }^{[26]}$, to name a few.

The diversity and complexity of glycans is an attribute of their significant importance in biological functions. The genome and proteome have provided substantial knowledge about the relationship of coding with cell function. However, as more is discovered about complex glycans and the glycanprocessing enzymes (glycosyltransferases and glycosidases), the inner workings of cells can now be deciphered through analysis of its oligosaccharide components ${ }^{[27]}$.

Carbohydrate chemistry is dominated by the requirement for the synthesis of glycosidic linkage and protecting groups. One of the major challenges in carbohydrate chemistry is the promotion of stereoselectivity and regioselectivity when forming a glycosidic linkage. Glycosyl reactions require a variety of latent leaving groups, activators, protecting groups, temperature and solvent conditions which can encourage the synthesis of isomerically pure saccharides. Different leaving groups can have a profound effect on the glycosyl donors reactivity. Protecting group chemistry is relied on heavily in this field due to the presence of multiple reactive hydroxyl groups. Careful selection of such groups can selectively form $\alpha$ or $\beta$ products by neighboring group participation. Many saccharides differ only by the orientation of a single hydroxyl group but the biochemistry and physical properties of such compounds differ dramatically. With advances in this field access to complex oligosaccharides with different linkages is becoming easier. The importance of carbohydrates in biological systems is slowly emerging in recent times. Hindered by their low permeability through the intestinal wall due to their high polarity and fast excretion from the body, they were thought not to be ideal candidates for pharmaceutics. However, coupling such characteristics with compounds lacking these pharmacokinetics is highly advantageous and has been used in treatments for diabetes, influenza, epilepsy and osteoarthritis ${ }^{[28]}$.

Carbohydrate units in addition to providing polar hydroxyl groups have potential to lead the conjugates to cell surface binding receptor targets. The interaction between carbohydrates and lectins over-expressed on the surface of cancer cells can be taken advantage of in drug development. For the last couple of decades, carbohydrate modified porphyrins have been explored for use as PDT agents. Glycoporphyrins were envisaged to tackle the obstacles of first and second generation PS agents. Even with the many advances in basic porphyrin functionalization, dedicated reactions for bioconjugates with carbohydrate units are only slowly emerging ${ }^{[29]}$. This is despite the excellent water solubility of glycoporphyrins, a significant improvement over established PSs.

Various methods of linking carbohydrates to porphyrin scaffolds, for example by direct glycosylation to the porphyrin ${ }^{[30]}$, amide [31], or triazole linkages ${ }^{[32]}$ are available. The number and regiochemical arrangement of the sugar units gives scope for fine-tuning the hydrophobicity and hydrophilicity. The major problems with these methods are the low yielding synthesis with condensation methods and the use of very strong Lewis acids, bases and coupling reagents which means that the carbohydrate has to be suitably protected. This is evident in the numerous glycoporphyrins substituted with the simple monosaccharide $D$-glucose. When introducing more expensive and synthetically relevant carbohydrates, it is preferably done in the later steps ensuring stability of the glycosidic linkage on large oligosaccharides. For example, our groups have published work using the $\mathrm{CuAAC}$ reaction under microwave and significantly less harsh conditions which are high yielding for the synthesis of homo- and heteroatom substituted tetraphenylporphyrins ${ }^{[32]}$. This allowed the synthesis of an extensive glycoporphyrin library which has been biologically evaluated and used to identify the intracellular localization of glycoporphyrins depending on the carbohydrate unit present ${ }^{[33]}$. For example, these preliminary results in biological testing showed that triglycosubstituted porphyrins lack the ability to be taken up by esophageal cancer cells possibly due to their high water solubility and reinforced that amphiphilicity of such systems is key to their ability to cross the lipid membrane ${ }^{[34-42]}$. 


\subsection{Porphyrin Bioconjugates}

The significant medical potential of porphyrins has raised interest in related bioconjugates. It is hoped to increase targeting to tumor tissue, enhance the solubility of the porphyrin scaffold and to lower the photosensitivity experienced by patients with current clinically approved PSs. In a conceptual sense the general structure of porphyrinbioconjugates consists of the subunits porphyrin-linkerbioconjugate group. The linker between the porphyrin and the bioconjugate can be introduced into either the meso or $\beta$ positions by condensation methods or through substitution of pre-synthesized porphyrin scaffolds (Figure 1). It can range from polyethylene glycol (PEG) chains, amide bonds, triazole rings, ether linkages, etc., all discussed below.

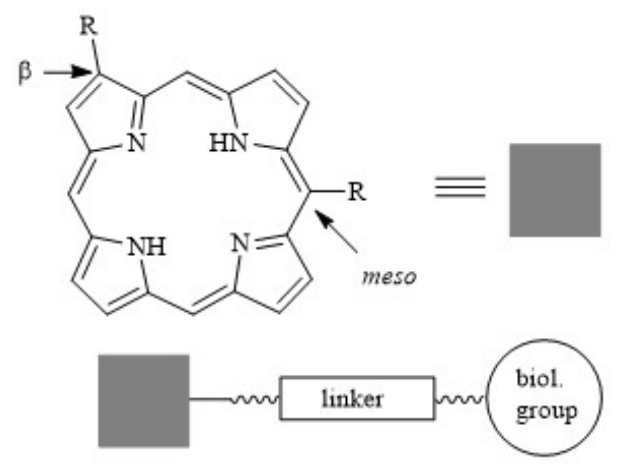

Figure 1. Illustration of porphyrin-bioconjugate systems.

As an established area on its own, there are many examples of such bioconjugates now available ${ }^{[43]}$. These include porphyrins with steroids, lipids, peptides, carbohydrates, lectins and nucleotides. Especially for porphyrins with signaling peptides a significant body of information is now available ${ }^{[44-48]}$. Porphyrins appended with amino acids have been prepared to model haemoproteins ${ }^{[49-51]}$, to investigate ligand binding and oxygenative catalysis and energy transfer in photosynthesis ${ }^{[52]}$. Likewise, the difference in the expression of aminopeptidases in tumor vasculature versus normal vasculature has been identified in some organs ${ }^{[53]}$. Berger et $a l$. synthesized amino acid ALA ( $\delta$-aminolevulinic acid) derivatives which have the potential to act as substrates for aminopeptidase expressed in lung tumor and endothelial cell lines, thus, increasing the accumulation of ALA in malignant tissue and potentially Pp IX (Protoporphyrin IX) ${ }^{[54]}$.

Porphyrin-antibody conjugates have also been studied in depth ${ }^{[55]}$ as well as their non-covalent interactions ${ }^{[56]}$. Levy and coworkers in the 1980's reported the first conjugation of an antibody to a porphyrin macrocycle. The antibodyhaematoporphyrin conjugates showed significant in vitro destruction of cells ${ }^{[57]}$. Lipid-porphyrins have also been synthesized, initially for an investigation of their electron transfer properties ${ }^{[58,59]}$. Current interest in the synthesis of lipid porphyrins aims at the construction of self-organized porphyrin assemblies for modeling electron transfer processes and oxygen transport ${ }^{[60-62]}$. The inclusion of porphyrins into monolayer, bilayer and micellular lipid aggregates forms supramolecular structures with unique physical-chemical properties ${ }^{[63,64]}$ as do nanoformulations [65]. Also, phospholipid vesicles containing porphyrins have been prepared as model systems for haemoproteins for investigating $\mathrm{O}_{2}$ transport systems ${ }^{[66]}$ and electron transfer reactions ${ }^{[67]}$. Such vesicles have been observed under electron microscopes to be $100-150 \mathrm{~nm}$ in diameter ${ }^{[62]}$.

Initial work on porphyrin conjugates focused on steroids [68,69]. For example, the first investigation was aimed at studying the effect of steroid hormones such as estrone and progesterone for targeting an excess of receptors in breast cancer cells ${ }^{[70]}$. A typical example is the synthesis of an estrone derivative $\mathbf{2}$ covalently linked through an alkenyl unit via a Heck type reaction with a meso-iododiphenylporphyrin 1 (Scheme 1) ${ }^{[71]}$. In contrast to these areas, the chemistry of the related carbohydrate conjugates is only slowly emerging in recent years.

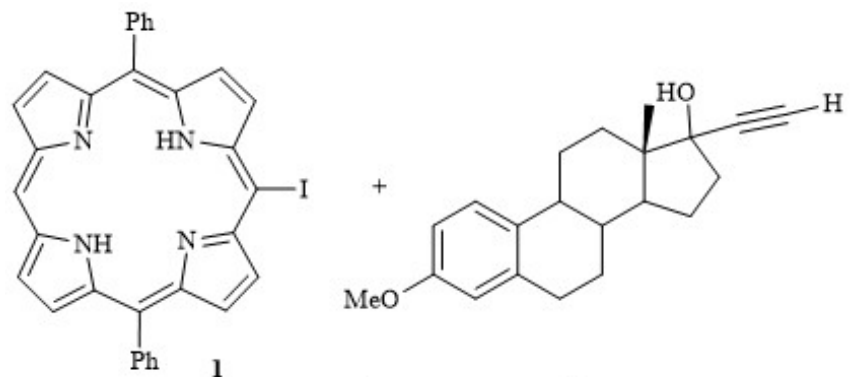

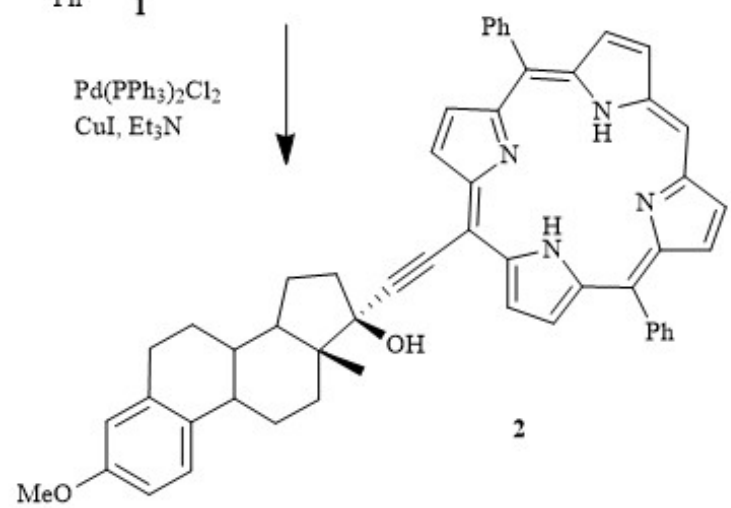

Scheme 1. Synthesis of an estrone-porphyrin conjugate.

Very few examples of naturally occurring glycoporphyrins have been described. One example is a chlorophyll $c_{2}$-monogalactosyldiacylglyceride ester $\mathbf{3}$, which was extracted from Emiliania huxleyi and identified using mass spectrometry ${ }^{[72]}$. Similar compounds have also been identified in Chrysochroulina polyepis ${ }^{[73,74]}$. The best known example is perhaps tolyporphin A $\mathbf{4}$, one of a series of bacteriochlorin pigments ${ }^{[75,76]}$. It is the first glycoporphyrin isolated in nature from blue-green algae and was the target of a total synthesis (vide supra) ${ }^{[77]}$. Another such example includes the fluorescent glycosylated chlorophyll linear tetrapyrrole catabolite $\mathbf{5}$ which accumulates in senescent, yellow banana leaves. The structure was elucidated using 


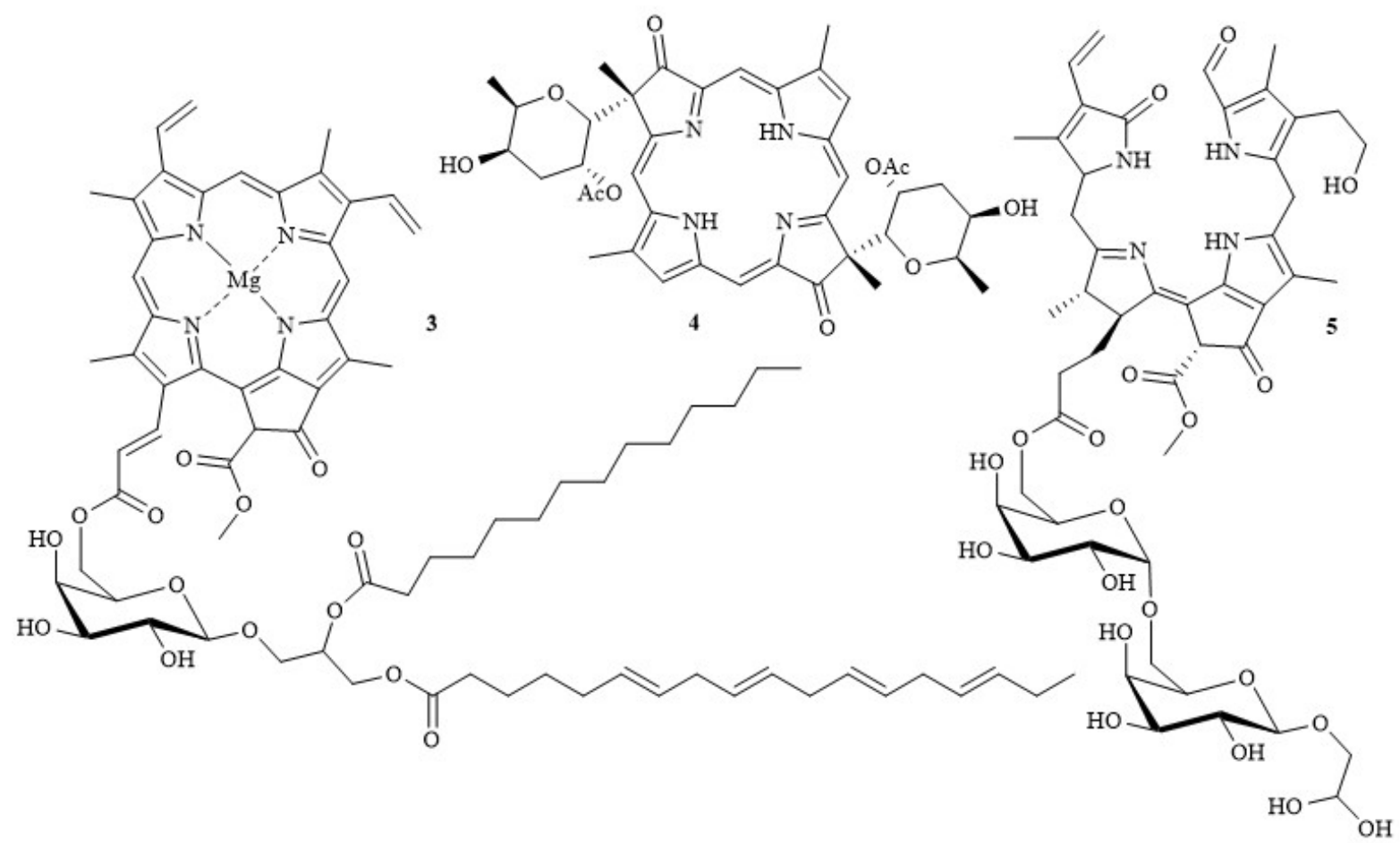

Formula 1.

NMR and mass spectrometry and proved to comprise a $6-\alpha-$ galactopyranosyl-( $1 \rightarrow 6)-\beta$-galactopyranosyl-( $1 \rightarrow 1)$-glyceryl unit ${ }^{[78]}$. The physiological function of these fluorescent chlorophyll catabolites is unknown, however, they are a source of blue luminescence in senescent banana leaves.

Porphyrin cofactors exhibit a structural partnership with proteins which provide vital roles in biochemistry. Note, that porphyrins are bound and transported in the serum by carbohydrate proteins and hemopoxin ${ }^{[79]}$. This is important for the tissue distribution and uptake of potential PS and has implications for the anti-angiogenic properties of PSs ${ }^{[80]}$. Porphyrins such as the heme cofactor of the glycoprotein hemoglobin are essential for gaseous transport ${ }^{[81]}$, while the glycoprotein transcobalamin provides facile transport of cofactor vitamin $\mathrm{B}_{12}$ (cobalamin or hydroxocobalamin) into the body ${ }^{[82]}$. The detailed interactions of glycoproteins and porphyrin PSs are beyond the scope of this review, however, several documents focusing on this area have been published ${ }^{[83-85]}$. One such example are the direct inhibition of ATPase activity of P-glycoprotein by porphyrin PSs ${ }^{[83]}$ and the interaction of sulfonated porphyrins with HIV glycoproteins gp120 causing photodamage and inhibition of antibody binding ${ }^{\left[{ }^{84]}\right.}$. One highlight of this research has focused recently on the fructation of hemoglobin (i.e. protein glycation with the monosaccharide fructose) which promotes the formation of endogenous reactive oxygen species resulting in the degradation of the heme macrocycle. Such research can provide insight into the effect of the accumulation of heme degradation products for hyperglycemia in diabetics ${ }^{[86]}$.

Similar to interactions with glycoproteins, porphyrins have been shown to exhibit other physiological interactions with, for example, lectins, i.e., specific carbohydrate recognition proteins. Several examples exist of metalloporphyrins binding to plant lectins where the association constant $\left(\mathrm{K}_{\mathrm{a}}\right)$ and stoichiometry of binding have been determined ${ }^{[87-89]}$. In most cases the addition of the binding sugar appears to have no effect on the binding of the porphyrin, i.e., their binding sites are different.

The studies into their non-covalent interactions lead to the investigation of porphyrin-lectin conjugates as drug delivery systems. Lectins are not just found on the surface of cells but are easily taken up by cells as well. While tumors overexpress lectins, solid tumors have also shown an abundance of $O$-glycosyl moieties such as T or Tn antigens ${ }^{[90,91]}$. Very few examples of lectins covalently bound to porphyrins exist. One such compound is 5-(4-(5-carboxy-1-butoxy)phenyl)-10,15,20-tris(4- $N$-methyl)-pyridiniumyl)-porphyrin covalently coupled with the plant lectin Morniga G, a galactose specific binding lectin which exhibited an increased photocytotoxicity (at $10 \mathrm{nM}$ concentration) compared to the free porphyrin in vitro through an $O$-glycan dependent process using Tn-positive Jurkat leukemia cells as well as targeting glycan alterations on tumor cells ${ }^{[92,93]}$. 


\subsection{Porphyrin Carbohydrate Interactions}

Although the focus of the present work is on covalently linked porphyrin-sugar systems, the possibility for noncovalently bound systems exists ${ }^{[94,95]}$. For example, boronic acid porphyrins have been used for sugar sensing [96-105], discrimination of sugars [106,107], and have been used to control sugar-DNA interactions ${ }^{[108]}$. Boronic acid porphyrins have also been employed in glycosylated steroid sensing ${ }^{[109]}$ and the detection of carbohydrates on the surface of cells using a photochemical approach ${ }^{[110]}$. Other studies included selective saccharide recognition using naphthyl substituted porphyrins [111,112], quinoline substituted porphyrins [113], steroid derivatives ${ }^{[114-117]}$, selective maltotetraose binding by meso-meso linked bisporphyrins [118,119], and a Ce(IV) double-decker system for saccharide binding ${ }^{[119,120]}$. Dimeric porphyrins have been exploited for their hydrogen bonding interactions with oligosaccharides for sensory applications ${ }^{[121]}$. Non-covalent host-guest interactions were also observed between cyclic porphyrin-cryptand conjugates sandwiched together and saccharides, i.e., the guest molecule which occupied the cavity between the assemblies in water ${ }^{[122]}$. Studies have also been carried out using aspartate ureaporphyrins ${ }^{[123]}$ and urea functionalized porphyrins with amino acid residues ${ }^{[124]}$ as artificial carbohydrate receptors. Urea appended porphyrins incorporated into molecular imprinted polymers displayed differentiation properties between closely related carbohydrates ${ }^{[125]}$.

TPP substituted with boronic acid groups, $\mathrm{NH}_{3}{ }^{+}$and $\mathrm{SO}_{3}{ }^{-}$ groups in solution and immobilized on cellulose fibers were used to identify and quantify rhamnose and other sugars which were known to be contained in the exosporium of bacterial endospores of some Clostridium and Bacillus species ${ }^{[126]}$. Picket fence type $\alpha \alpha \alpha \alpha$-TPP's containing urea, carbamate, or amide groups provided carbohydrate recognition through hydrogen-bonding and $\mathrm{CH}-\pi$ interactions ${ }^{[127,128]}$. Several studies have been carried out using bile acid conjugate porphyrins for carbohydrate recognition [129-131], one particular example exhibits the potential of such derivatives for direct targeting of bile acid porphyrins to sugar moieties which are expressed on malignant tumors. Both in vivo and in vitro studies displayed significant potential for PDT ${ }^{[129]}$. Likewise, positively charged amphiphilic cyclodextrins were investigated as supramolecular aggregates for the delivery of PSs to bacterial cells for antimicrobial PDT. Here the focus was on targeting methicillin-resistant Staphylococcus aureus (MRSA) and Escherichia coli Gram-positive and Gramnegative bacteria ${ }^{[132]}$. Supramolecular control of $\pi$-stacked aqueous phthalocyanines via host guest interactions with per- $O$-methylated $\alpha$-cyclodextrin were also achieved and observed by enhanced fluorescence ${ }^{[133]}$. Another example of non-covalently bound systems includes the use of chitosanbased nanoparticles as PS delivery vehicles which proved successful in sustained release of the PS in vitro and in vivo [134].

\section{SYNTHESIS OF GLYCOPORPHYRINS}

\subsection{Porphyrin Chemistry}

Porphyrins are a large group of organic compounds containing four pyrrole rings connected to form a highly conjugated, aromatic ring system. The $4 \mathrm{~N}$-core is able to complex various metal ions with cobalt, nickel, magnesium, iron and zinc complexes found in nature. They have absorption maxima in the UV-visible spectrum at $\sim 400 \mathrm{~nm}$ (Soret band) and several smaller absorption peaks from 500$800 \mathrm{~nm}$ (Q bands). The optical, chemical and photophysical properties can be varied significantly through substitution and/or macrocycle manipulation ${ }^{[135]}$.

Since the landmark synthesis of protoporphyrin IX by Fischer significant advances have been made in their synthesis. For aldehyde-pyrrole condensation reactions, this ranges from the early Rothemund condensation ${ }^{[136]}$ to the now standard Lindsey condensation ${ }^{[137]}$. Partial and total syntheses were facilitated first by the MacDonald condensation, a [2+2] condensation utilizing dipyrromethenes, dipyrroyl ketones and dipyrromethanes ${ }^{[138]}$. Condensation methods are continuously improving and almost any desired meso or $\beta$-substituted porphyrin can be prepared today ${ }^{[139-142]}$. In parallel the functionalization of porphyrins through functional group interconversions and direct substitution reactions has progressed dramatically in the past decades ${ }^{[140,143]}$. This is mainly a result of the use of organolithium ${ }^{[144,145]}$ and transition metal catalyzed reactions [143,146] which now allows the preparation of unsymmetrical ABCD-type meso substituted porphyrins and arrays $[139,147,148]$

Reports on the synthesis of glycoporphyrins mimic this progress. Thus, initial studies on rather simple glycoporphyrins have now progressed to more complex systems. Still, advances in porphyrin chemistry must be accompanied by progress in carbohydrate chemistry and the necessary linker chemistry.

Strategically glycoporphyrins may be synthesized via the following general methods:

- Condensation reactions using pyrrole and a protected carbohydrate aldehyde.

- Total synthesis with a protected carbohydrate unit linked to a $\beta$-pyrrole position.

- Partial synthesis using [2+2] or [3+1] condensation reactions and protected sugar moieties.

- Attachment of carbohydrate units to porphyrin linker groups.

With regard to the porphyrin macrocycle the linker unit can be attached to either the meso or $\beta$ positions or, in principle, through the metal center via axial ligation. Additionally, the number and regiochemical arrangement of the sugar units gives scope in fine tuning the hydrophobicity and hydrophilicity of these molecules ${ }^{[149,150]}$. 


\subsection{Total Synthesis of Tolyporphyin}

Tolyporphin A (4) is a bacteriochlorin, which is not involved in photosynthesis. It was isolated from the bluegreen microalga Tolypothrix nodosa by Moore et al. and was the first natural glycoporphyrin to be identified and fully characterized by ${ }^{1} \mathrm{H}$ NMR, ${ }^{13} \mathrm{C}$ NMR and extensively studied by $2 \mathrm{D} \mathrm{NMR}{ }^{[75,77]}$. In addition, the isolation and structural identification of ten other tolyporphins $\mathrm{B}-\mathrm{K}$ has been reported ${ }^{[75,76]}$. Compound $\mathbf{4}$ was found to be a multidrug resistant (MDR) reversing agent. For example, it increased the cytotoxicity of adriamycin or vinblastine, an intercalating chemotherapy drug, in vinblastine-resistant human ovarian adenocarcinoma and breast cell lines with doses as low as 1 $\mu \mathrm{g} . \mathrm{mL}^{-1}[77,151]$.

Next to heme and chlorophyll a, tolyporphin A presents one of the few natural porphyrins whose constitution was confirmed by total synthesis. Tolyporphins are comprised of an unsymmetrical hydroporphyrin macrocycle (dioxobacteriochlorin), $\beta$-linked to $C$-glycosides at C-2 and $\mathrm{C}-12$, which have hydroxyl or acetyl groups. A retrosynthetic analysis of the macrocyclic system $\mathbf{6}$ established the construction of tolyporphin via four monocyclic precursors (Scheme 2) where the 8 and 9 precursors were identical. The feasibility of this approach was demonstrated by Kishi's group through a total synthesis [152,153]. Compound 21 was synthesized via octahydroporphyrin $\mathbf{1 9}$, conversion to the bacteriochlorin $\mathbf{2 0}$<smiles>CC(C)O[C@@H]1C[C@H](O)[C@@H](C)O[C@@H]1[C@@]1(C)CC(=S)NC1(C)C</smiles><smiles>[Y4]C1(OCCCCC)CC(=C)NC1=O</smiles><smiles></smiles>

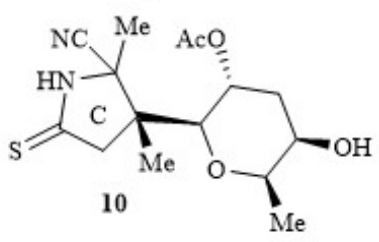<smiles>C=CC=C</smiles>

Scheme 2. Retrosynthesis of tolyporphin.<smiles>CC1(C)CC(=S)NC1(C)C#N</smiles>

11<smiles></smiles>

14<smiles>[X]C1([X])CC(=O)NC1=C</smiles>

12<smiles>CC1(C)CC(=S)NC1(C)C</smiles>

15<smiles>[Y]C1(C)CC(=O)N=C1/C=C1/CC(C)(C)C(=C)N1</smiles>

13<smiles>CCCCCCCC</smiles><smiles>[X]C1(C)CC(=S)N=C1/C=C1/CC(C)(C)C(C)(C)N1</smiles>

16<smiles>[X]C1(C)CC(C=C2CC([X])(C)C(C)(C)N2)=NC1=CC1=NC(=CC2=NC(=O)CC2(C)C)C(C)(C)C1</smiles>

viii), ix)

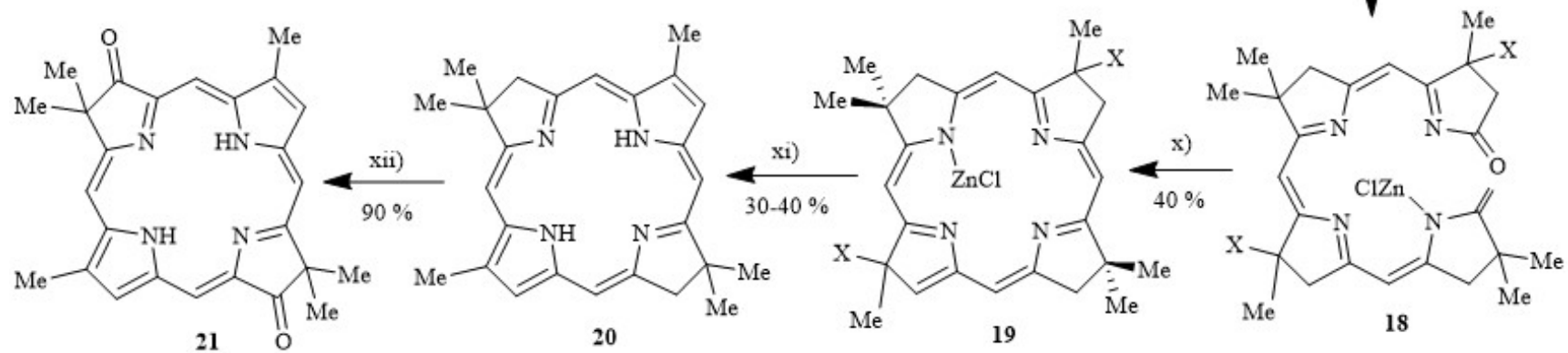

Scheme 3. Synthesis of the tolyporphin chromophore I. Conditions: i) NIS (1 equiv.), DBU (2 equiv.), $\mathrm{CH}_{3} \mathrm{CN}, 3$ h, r.t.; ii) (EtO) ${ }_{3} \mathrm{P}, \mathrm{xylenes}$ (1:4), 23 h, 125 ${ }^{\circ} \mathrm{C}$; iii) $t-\mathrm{BuOK} / t-\mathrm{BuOH}, 3 \mathrm{~h}, 85^{\circ} \mathrm{C}$; iv) Lawesson's reagent, toluene, $3 \mathrm{~h}, 80{ }^{\circ} \mathrm{C}$; v) NIS (1 equiv.), DBU ( 4 equiv.), $\mathrm{CH}_{3} \mathrm{CN}, 3$ h, r.t.; vi) $\mathrm{CDCl}_{3}, \mathrm{NaHCO}_{3}$, $\mathrm{PPh}_{3}, \mathrm{CH}_{3} \mathrm{CN}, 4$ h, r.t.; vii) $\mathrm{KCN}, \mathrm{MeOH}, 10$ min, r.t.; (viii/h) $t$ - $\mathrm{BuOK} / t$ - $\mathrm{BuOH}, 15$ min., $80{ }^{\circ} \mathrm{C}$; (ix/i) $\mathrm{Zn}\left(\mathrm{CIO}_{4}\right)_{2}, \mathrm{MeOH}$, r.t.; $(\mathrm{x} / \mathrm{j}) \mathrm{Et}_{3} \mathrm{OBF}_{4}, \mathrm{CH}_{2} \mathrm{Cl}_{2}, i-\mathrm{Pr}_{2} \mathrm{NEt}$, 20 h, r.t.; (xi/k) a: TFA, anisole, 1 h, r.t.; b: $\mathrm{MeOH}, 10 \mathrm{~min}$, c: $t$ - $\mathrm{BuOK} / t-\mathrm{BuOH}, 15 \mathrm{~min}$., d: $20 \% \mathrm{HCl}, 1$ min., r.t.; $x$ ii) $\mathrm{CrO}_{3} \cdot$ dimethyl pyrazole, $\mathrm{CH}_{2} \mathrm{Cl}_{2}$, r.t. 
by a double retroaldol/autoxidation reaction followed by site-specific oxidation ${ }^{[153]}$. Thus, with several modifications, the Eschenmoser sulfide contraction/iminoester cyclization method ${ }^{[154]}$ was incorporated in the assembly of the four monocyclic pyrrole precursors $(\mathbf{1 1}, \mathbf{1 2}, \mathbf{1 4}, \mathbf{1 5})$ to yield 19. Establishing protecting groups $\mathrm{X}$ at $\mathrm{C}-7$ and $\mathrm{C}-13$ allowed for double elimination yielding the tetrahydroporphyrin and upon oxidation gave the chromophore 21 (Scheme 3) ${ }^{[153]}$.

Two years later Kishi et al. synthesized (+)-tolyporphin$O, O$-diacetate derivatives using a similar methodology as reported above, however, replacing precursors 11, 15 with 7, $9{ }^{[155]}$. However, the structure of the final product did not match the comparative NMR of the natural $(+)$-tolyporphin A ultimately leading to a revised structure of the original natural product. Using extensive NOE experiments the true structure of the synthetic tolyporphin $O, O$-diacetate derivative was confirmed as $\mathbf{6}$ which is different from the natural tolyporphin derivative. Re-examination of the NMR data obtained for the natural (+)-tolyporphin A $O, O$-diacetate showed the NOE experiments to be identical. The only difference between the two derivatives was at the quaternary centers $\mathrm{C}-2$ and $\mathrm{C}-12$ resulting in four possible structures, depending on the orientation of the methyl and glycosyl units. Based on spectroscopic data and analysis of the various early syntheses $\mathbf{4}$ was proposed as the structure of natural (+)-tolyporphin $\mathrm{A}$ and this was finally confirmed by total synthesis ${ }^{[156,157]}$. These landmark syntheses are now classic examples of porphyrin total synthesis. However, in practical terms such approaches are not suitable for synthetic applications. In recent years, the total synthesis of structural models via $[3+1]$ condensation reactions was attempted with only a tricyclic fragment as a precursor for tolyporphin ${ }^{[158]}$.

\subsection{Synthesis via Condensation Reactions}

The synthesis of porphyrins via pyrrole condensation reactions is the most widely used method reported in the literature. Condensation reactions, notably using Lindsey conditions ${ }^{[137]}$ offer a simple entry into porphyrin systems and have been widely used, especially for meso substituted systems. This requires a combination of pyrrole (delivering the $\beta$-pyrrole units) and aldehyde (delivering the meso carbon unit) under acid-catalyzed conditions and obviously is a convenient method for the preparation of $\mathrm{A}_{4}$-type porphyrins with the same residue in all meso positions. For glycoporphyrins the utility of this method is limited to acidstable derivatives.

One of the oldest examples of glycoporphyrins synthesis involved the preparation of a $\beta$-glycoporphyrin. Based on his interest in etio-type porphyrin systems, Ono et al. prepared a Knorr-type pyrrole using a Barton-Zard reaction $(\mathbf{2 2} \rightarrow \mathbf{2 3})$ to yield the protected 3-glycopyrrole $\mathbf{2 3}$ and related systems in $50-70 \%$ yield (Scheme 4) ${ }^{[159]}$. Reduction to the pyrrole carbinol and followed by acid-catalyzed tetramerization then gave the etioporphyrin I 24 in $18 \%$ yield. Due to the steric hindrance imparted on the system through the sugar units no scrambling was observed in this case. Porphyrin $\mathbf{2 4}$ could easily be deprotected to the respective water-soluble derivative. Using a singlet oxygen trapping reaction the photosensitizing activity of the deprotected system was shown to be similar to Rose Bengal and haematoporphyrin, indicating its potential as a PS.

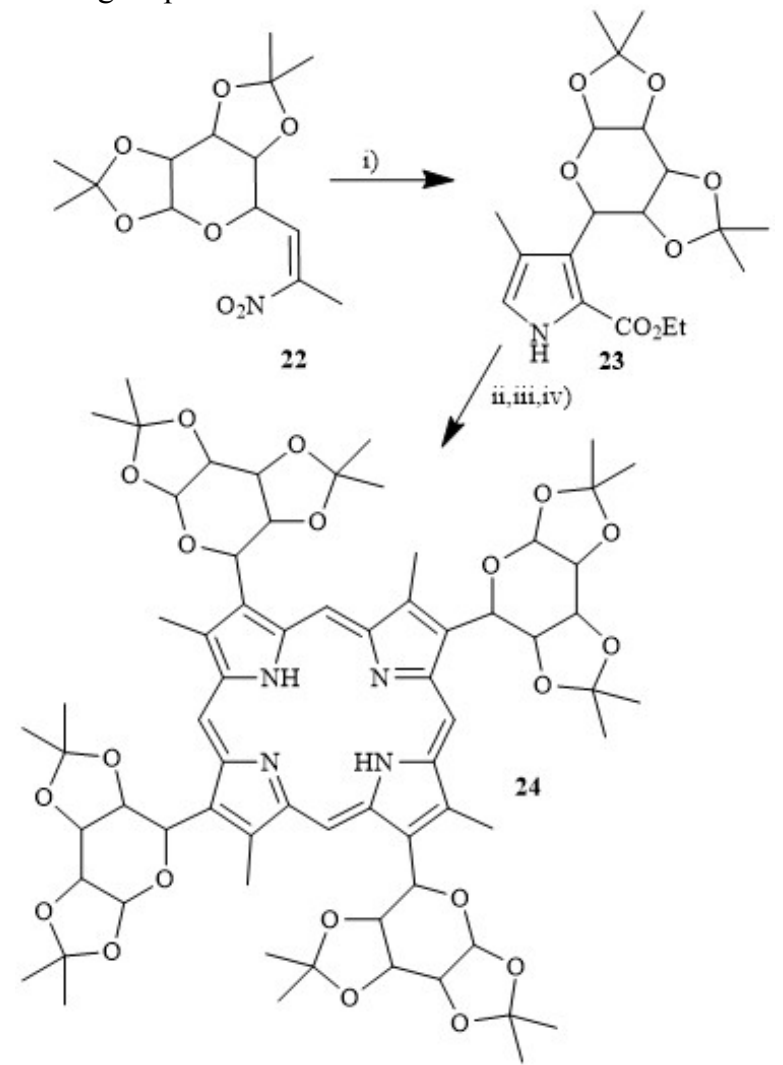

Scheme 4. Synthesis of $\beta$-substituted systems. Conditions: i) $\mathrm{CNCH}_{2} \mathrm{CO}_{2} \mathrm{Et}$, $\mathrm{DBU}$, THF, r.t., $8 \mathrm{~h}$; ii) $\mathrm{LiAlH}_{4}, 0{ }^{\circ} \mathrm{C} \rightarrow$ r.t., 2 h; iii) $p \mathrm{TsOH}$, r.t., $10 \mathrm{~h}$; iv) $p$ chloranil, r.t., $3 \mathrm{~h}$

\subsubsection{A A $_{4}$-type Porphyrins}

Due to the ease of synthesis most of the early reports focused on the synthesis of meso-aryl substituted glycoporphyrins. We have attempted to compile most of these syntheses into Table 1 (see Figure 2 for carbohydrate abbreviations used). For example, in one of the earlier studies condensation of tetraacetyl- $\beta$ - $D$-glucose-2benzaldehyde with pyrrole gave tetrasubstituted glycoporphyrin 25 in $42 \%$ yield. The compound was obtained as three atropisomers $(\alpha \beta \alpha \beta, \alpha \alpha \beta \beta$ and $\alpha \alpha \alpha \beta)$ in a ratio of 1:2:4 all of which were converted to the water soluble glycoporphyrins $\mathbf{2 6}$. The substitution of the glycosyl group on the ortho position of the meso phenyl was 
investigated for its steric hindrance, solubility in water, and atropisomerism. Later studies on the use of these compounds for the asymmetric oxidation of alkenes showed significant potential for the $\alpha \alpha \alpha \alpha$ atropisomer ${ }^{[160]}$ which could be prepared via atropisomerization ${ }^{[161]}$. The same methods are suitable for preparation of both glucosyl (26) and maltosyl (28) substituted TPPs. The substitution of the glycosyl group on the ortho position of the meso phenyl caused steric hindrance to metallation by zinc ${ }^{[160]}$

In extension of this work Maillard et al. synthesized a range of tetraglycosylated TPP's containing glucopyranosyl, galactopyranosyl, maltosyl, and glucosaminosyl residues $O$ linked at the para position under Lindsey condensation conditions (29-36) ${ }^{[162]}$. Similar compounds were prepared to determine the effect the glycosyl unit had on the singlet oxygen production for possible application in asymmetric photooxygenation. Compounds $\mathbf{3 0}$ and $\mathbf{3 8}$ were synthesized via trifluoride etherate catalyzed condensation of acetylated carbohydrate-substituted benzaldehydes and pyrrole with subsequent deprotection of the acetyl groups ${ }^{[163]}$. Their efficiency of singlet oxygen production was measured monitoring the oxidation of TEMP (2,2,6,6-tetramethyl-4piperidone) to TEMPO using ESR spectroscopy and compared to the non-glycosylated derivative 37. Higher reaction rates were observed for the sugar derivatives in the order $\mathbf{3 7}<\mathbf{3 8}<\mathbf{3 0}$. Both the glucosyl and cellobiosyl porphyrins exhibited longer triplet lifetimes compared to $\mathbf{3 7}$. Preliminary photooxygenation experiments with the ene reaction of ethyl trans-2-methyl-2-butenoate with $\mathbf{3 8}$ embedded in polystyrene matrices were promising, but only low ee values were observed.

Related compounds ${ }^{[164]}$ prepared via condensation methods include a series of 5,10,15,20-tetrakis[3- or 4-( $\beta$ - $D$ glycopyranosyloxy)phenyl]porphyrins $(\mathbf{3 0}, 32,39-44)$ and 45-52 ${ }^{[165]}$. The latter were prepared by condensation of pyrrole with $p$ - or $m$-peracetyl-glycosyl benzaldehydes via modification of Lindsey's method where $\mathrm{Zn}\left(\mathrm{CH}_{3} \mathrm{CO}_{2}\right)_{2} \cdot 2 \mathrm{H}_{2} \mathrm{O}$ was used as a templating agent affording the acetylated derivatives in 53-93\% yield, followed by demetallation to the free bases in 36-86\% yield. Both the metallated and demetallated derivatives were deprotected. The hydrophobicity parameters $(\log \mathrm{P})$ of the 16 acetylated derivatives of these systems were determined via reverse phase HPLC and ranged from 4.8 to $7.8^{[166]}$.

Simple variation of the aldehyde in porphyrin condensation reactions allows the preparation of porphyrins with more than four carbohydrate moieties. For example, Mikata et al. used a 3,5-di-O-glycopyranosylbenzaldehyde to generate the porphyrin $\mathbf{5 3}$ with eight sugar units after deprotection ${ }^{[167]}$.

The first example of directly meso linked glycosyl porphyrins were synthesized by Momonteau et al. via Lindsey condensation methods in $1992^{[168]}$. The four protected sugar substituents were directly linked to the meso positions via condensation with pyrrole and their pre-formed aldehyde analogues. Porphyrins (54-56) bearing xylofuranose, glucofuranose and galactopyranose were isolated in $4 \%$ yield compared to the porphyrins $(\mathbf{5 7}, \mathbf{5 8})$ bearing $O$-linked glucopyranose and galactopyranose, which were not stable.

Subsequently, these studies were extended by Cornia et al. to the synthesis of meso-tetraglycosylated porphyrins with varying degree of lipophilicity and hydrophilicity. Using a slightly modified Lindsey procedure, the acid catalyzed condensation of pyrrole with sugar aldehydes was achieved at high dilution using $\mathrm{BF}_{3} \cdot \mathrm{OEt}_{2}$, affording an easy route to $C$-meso-glycosylated porphyrin atropisomers. Pyrrole condensed with enantiopure 2,3- $O$-isopropylidene- $D$ glyceraldehyde yielded two major atropisomers, $\alpha \beta \alpha \beta \mathbf{5 9}$ and $\alpha \alpha \alpha \beta 60$ in $3 \%$ and $4 \%$ yield, respectively. This reaction was applied to different carbohydrates, e.g., 1,2-Oisopropylidene-3- $O$-methyl- $\alpha-D$-xylo-pentodialdofuranose yielding 61 as a single $\alpha, \beta, \alpha, \beta$-isomer in $6 \%$ and both enantiomers of 2,3:4,5-di- $O$-isopropylidenearabinose produced three major porphyrin compounds as the same $\alpha, \beta, \alpha, \beta$-isomer. These were the expected fully protected porphyrin 62 in $2 \%$ yield, accompanied by monodeprotected porphyrin in $1 \%$ and the bisdeprotected porphyrin in $4 \%$ yield. The other isomers and derivatives (63-65) were synthesized in similar yields. All derivatives were fully deprotected yielding water soluble compounds for possible use in chiral recognition and asymmetric catalysis in aqueous medium ${ }^{[169]}$.

Several other examples were reported. These include the benzyl protected derivatives 66 and $67^{[170]}$ and condensation products of commercially available di-aldose and pyrrole [171]. Metallation and deprotection of the glycosyl units provided compounds 68 and $\mathbf{6 9}$ in yields ranging from 63-90 $\%$. The latter compounds were used for DNA photocleavage studies with the $\mathrm{Pd}(\mathrm{II})$ complex 69 exhibiting the best activity ${ }^{[171]}$. More details on other examples of $\mathrm{A}_{4}$-type glycoporphyrins (70-108) will be given below and are listed in Table $1^{\text {[172-183] }}$

Pyrrole condensation reaction may also be employed for the preparation of dodecasubstituted porphyrins. Note, these highly substituted porphyrins are nonplanar and thus have bathochromically shifted absorption bands and altered excited state properties [135]. For example, the tetrabenzoporphyrin 113 bearing glucosyl moieties on the meso positions was synthesized from tetrahydroisoindole and glucosylaldehyde in $36 \%$ yield ${ }^{[184]}$. Followed by zinc metal complexation (62\%) and oxidative aromatization with DDQ to afford 112, this could be demetallated and deacetylated to 113 (Scheme 5). In addition, 5,10,15,20tetraphenylcarboxytetrabenzoporphyrin 109b was glycosylated via addition of 2,20-(ethylenedioxy)-bisethylamine as a linker which was substituted with triacetyl lactone and subsequently deprotected affording 111. All compounds display a high degree of photostability at light of fluence rate $2.5 \mathrm{~mW} / \mathrm{cm}^{2}$ and absence of photobleaching after $1 \mathrm{~h}$. The glycobenzoporphyrins 111 and 113 were found to be as efficient as $\mathrm{HP}$ in producing ${ }^{1} \mathrm{O}_{2}{ }^{[184]}$. 


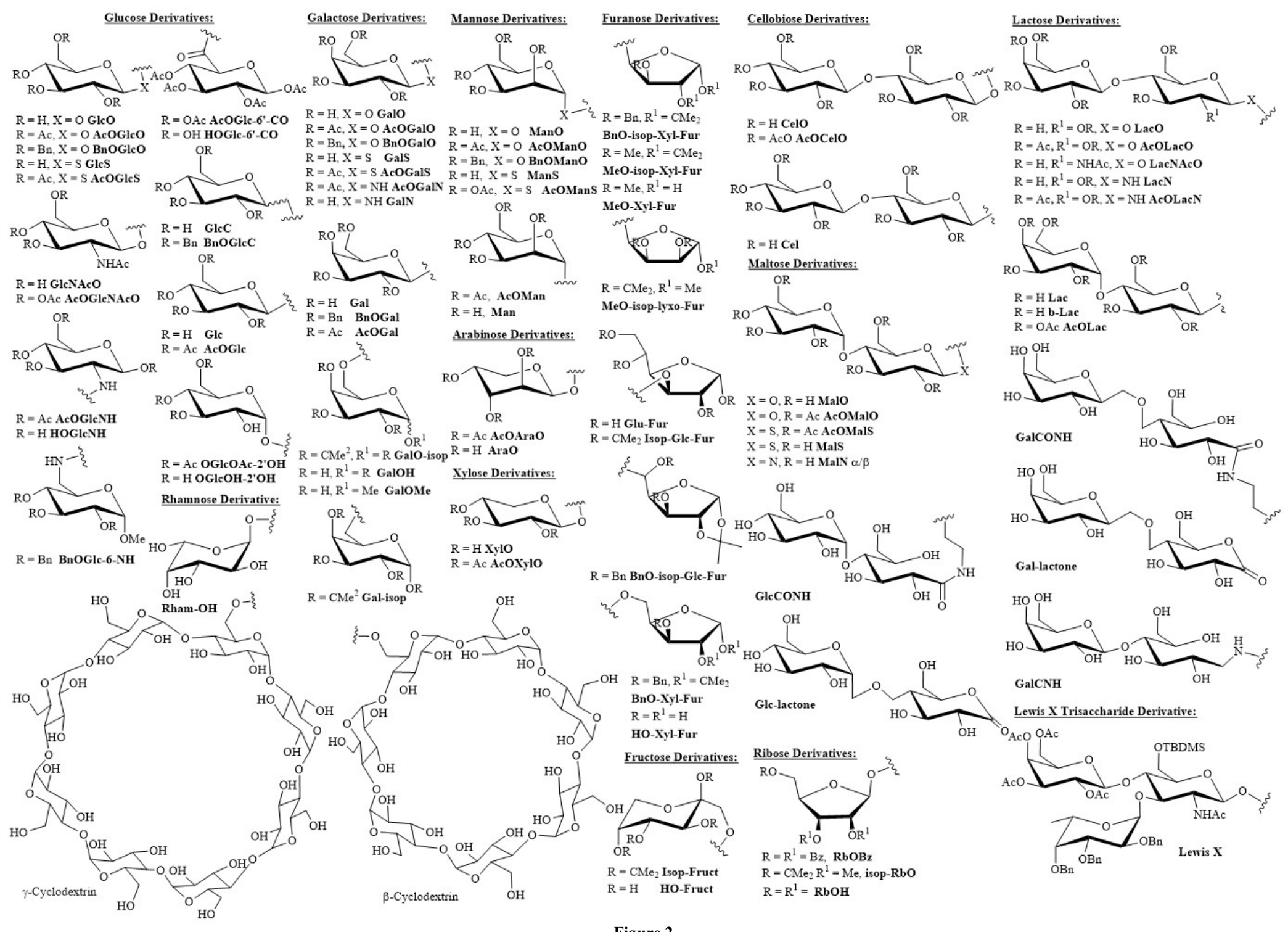


Table 1. Symmetric $\mathrm{A}_{4}$-type (5,10,15,20-tetrasubstituted) glycoporphyrins and reference compounds.

\begin{tabular}{|c|c|c|c|c|c|}
\hline Entry & \# & A substituent & Metal & Comments & Ref. \\
\hline 1 & 25 & 2-(AcOGlcO $)-\mathrm{C}_{6} \mathrm{H}_{4^{-}}$ & $2 \mathrm{H}$ & $\begin{array}{l}\text { Condensation of pyrrole and 2-(AcOGlcO)- } \mathrm{C}_{6} \mathrm{H}_{4}-\mathrm{CHO}, \mathrm{CH}_{2} \mathrm{Cl}_{2}, \mathrm{BF}_{3} \cdot \mathrm{OEt}_{2} \text {, then } \\
\text { of DDQ. Obtained as } \alpha \beta \alpha \beta, \alpha \alpha \beta \beta, \alpha \alpha \alpha \beta \text { atropisomers in } 2,7 \text {, and } 17 \% \text {, } \\
\text { respectively. }\end{array}$ & {$[160,255]$} \\
\hline 2 & 26 & $2-(\mathrm{GlcO})-\mathrm{C}_{6} \mathrm{H}_{4-}$ & $2 \mathrm{H}$ & $\begin{array}{l}\text { From } 25 \mathrm{NaOMe} / \mathrm{MeOH} \text {, Obtained as } \alpha \beta \alpha \beta, \alpha \alpha \beta \beta \text {, } \alpha \alpha \alpha \beta \text { atropisomers in } 90,80 \\
\text { and } 100 \% \text {, respectively. }\end{array}$ & {$[160]$} \\
\hline 3 & 27 & 2-(AcOMalO) $-\mathrm{C}_{6} \mathrm{H}_{4}-$ & $2 \mathrm{H}$ & $\begin{array}{l}\text { Condensation of pyrrole and 2-(AcOMalO)- } \mathrm{C}_{6} \mathrm{H}_{4}-\mathrm{CHO}, \mathrm{CH}_{2} \mathrm{Cl}_{2}, \mathrm{BF}_{3} \cdot \mathrm{OEt}_{2} \text {, then } \\
\text { of } p \text {-chloranill; } 10 \% \text {. }\end{array}$ & {$[160]$} \\
\hline 4 & 28 & 2-(MalO)- $\mathrm{C}_{6} \mathrm{H}_{4-}^{-}$ & $2 \mathrm{H}$ & From 27: $\mathrm{NaOMe} / \mathrm{MeOH} ; 90 \%$ & {$[160]$} \\
\hline 5 & 29 & $4-(\mathrm{AcOGlcO})-\mathrm{C}_{6} \mathrm{H}_{4}-$ & $2 \mathrm{H}$ & $\begin{array}{l}\text { Condensation of pyrrole and } 4 \text { - }(\mathrm{AcOGlcO})-\mathrm{C}_{6} \mathrm{H}_{4}-\mathrm{CHO}, \mathrm{CH}_{2} \mathrm{Cl}_{2}, \mathrm{BF}_{3} \cdot \mathrm{OEt}_{2} \text {, then } \\
\text { p-chloranil; best: } 28 \% \text {. }\end{array}$ & $\begin{array}{l}{[162,167,225,} \\
207]\end{array}$ \\
\hline 6 & 30 & $4-(\mathrm{GlcO})-\mathrm{C}_{6} \mathrm{H}_{4-}$ & $2 \mathrm{H}$ & From 29: $\mathrm{NaOMe} / \mathrm{MeOH}$ or condensation and in situ cleavage; $64-88 \%$ & $\begin{array}{l}{[162,160,} \\
163,165,167 \\
342]\end{array}$ \\
\hline 7 & 31 & $4-(\mathrm{AcOGalO})-\mathrm{C}_{6} \mathrm{H}_{4}-$ & $2 \mathrm{H}$ & $\begin{array}{l}\text { Condensation of pyrrole and } 4 \text { - }(\mathrm{AcOGalO})-\mathrm{C}_{6} \mathrm{H}_{4}-\mathrm{CHO}, \mathrm{CH}_{2} \mathrm{Cl}_{2}, \mathrm{BF}_{3} \cdot \mathrm{OEt}_{2} \text {, then } \\
\text { p-chloranil; } 20 \% \text {. }\end{array}$ & {$[162,167]$} \\
\hline 8 & 32 & 4-(GalO) $-\mathrm{C}_{6} \mathrm{H}_{4-}-$ & $2 \mathrm{H}$ & From 31: $\mathrm{NaOMe} / \mathrm{MeOH} ; 88 \%$ & {$[162,165,167]$} \\
\hline 9 & 33 & 4-( $\mathrm{AcOMalO})-\mathrm{C}_{6} \mathrm{H}_{4}-$ & $2 \mathrm{H}$ & $\begin{array}{l}\text { Condensation of pyrrole and } 4 \text {-(AcOMalO)- } \mathrm{C}_{6} \mathrm{H}_{4}-\mathrm{CHO}, \mathrm{CH}_{2} \mathrm{Cl}_{2}, \mathrm{BF}_{3} \cdot \mathrm{OEt}_{2} \text {, then } \\
\text { addition of } p \text {-chloranil; } 10 \% \text {. }\end{array}$ & [162] \\
\hline 10 & 34 & 4-(MalO)- $\mathrm{C}_{6} \mathrm{H}_{4-}^{-}$ & $2 \mathrm{H}$ & From 33: $\mathrm{NaOMe} / \mathrm{MeOH} ; 96 \%$ & {$[162]$} \\
\hline 11 & 35 & 4-(AcOGlcNAcO) $-\mathrm{C}_{6} \mathrm{H}_{4}-$ & $2 \mathrm{H}$ & $\begin{array}{l}\text { Condensation of pyrrole and } 4 \text { - }(\mathrm{AcOGlcNAcO})-\mathrm{C}_{6} \mathrm{H}_{4}-\mathrm{CHO}, \mathrm{CH}_{2} \mathrm{Cl}_{2}, \mathrm{BF}_{3} \cdot \mathrm{OEt}_{2} \text {, } \\
\text { then } p \text {-chloranil; } 15 \% \text {. }\end{array}$ & {$[162,167]$} \\
\hline 12 & 36 & 4-(GlcNAcO) $-\mathrm{C}_{6} \mathrm{H}_{4}-$ & $2 \mathrm{H}$ & From 35: $\mathrm{NaOMe} / \mathrm{MeOH} ; 97 \%$ & {$[162,167]$} \\
\hline 13 & 37 & $4-\mathrm{HO}-\mathrm{C}_{6} \mathrm{H}_{4-}^{-}$ & $2 \mathrm{H}$ & & \\
\hline 14 & 38 & $4-(\mathrm{CelO})-\mathrm{C}_{6} \mathrm{H}_{4}-$ & $2 \mathrm{H}$ & $\begin{array}{l}\text { Condensation of pyrrole and } 4-(\mathrm{AcOCelO})-\mathrm{C}_{6} \mathrm{H}_{4}-\mathrm{CHO}, \mathrm{CH}_{2} \mathrm{Cl}_{2}, \mathrm{BF}_{3} \cdot \mathrm{OEt}_{2} \text {, then } \\
\text {-chloranil, then } \mathrm{NaOMe} / \mathrm{MeOH} \text {. }\end{array}$ & {$[163]$} \\
\hline 15 & 39 & $3-(\mathrm{GlcO})-\mathrm{C}_{6} \mathrm{H}_{4-}^{-}$ & $2 \mathrm{H}$ & $\begin{array}{l}\text { Condensation of pyrrole and } 3-(\mathrm{AcOGlcO})-\mathrm{C}_{6} \mathrm{H}_{4}-\mathrm{CHO}, \mathrm{CHCl}_{3}, \mathrm{BF}_{3} \cdot \mathrm{OEt}_{2} \\
\mathrm{Zn}(\mathrm{OAc})_{2} \text {, then } p \text {-chloranil and } 4 \mathrm{M} \mathrm{HCl} ; 68 \% \text {. } \mathrm{NaOMe} / \mathrm{MeOH} \rightarrow \mathbf{3 9} ; 96 \% \text {. }\end{array}$ & {$[165,342]$} \\
\hline 16 & 40 & $3-(\mathrm{GalO})-\mathrm{C}_{6} \mathrm{H}_{4-}$ & $2 \mathrm{H}$ & Like entry 15 with $3-(\mathrm{AcOGalO})-\mathrm{C}_{6} \mathrm{H}_{4}-\mathrm{CHO} ; 57 \%$. NaOMe$/ \mathrm{MeOH} \rightarrow \mathbf{4 0} ; 76 \%$. & {$[165]$} \\
\hline 17 & 41 & $3-(\mathrm{XylO})-\mathrm{C}_{6} \mathrm{H}_{4}^{-}$ & $2 \mathrm{H}$ & Like entry 15 with $3-(\mathrm{AcOX} y \mathrm{lO})-\mathrm{C}_{6} \mathrm{H}_{4}-\mathrm{CHO} ; 86 \%$. NaOMe$/ \mathrm{MeOH} \rightarrow \mathbf{4 1} ; 91 \%$. & {$[165]$} \\
\hline 18 & 42 & $4-(\mathrm{XylO})-\mathrm{C}_{6} \mathrm{H}_{4}^{-}$ & $2 \mathrm{H}$ & Like entry 15 with $4-(\mathrm{AcOXylO})-\mathrm{C}_{6} \mathrm{H}_{4}-\mathrm{CHO} ; 64 \%$. NaOMe/MeOH $\rightarrow$ 42; $94 \%$. & {$[165,342,160]$} \\
\hline 19 & 43 & $3-(\mathrm{AraO})-\mathrm{C}_{6} \mathrm{H}_{4}^{-}$ & $2 \mathrm{H}$ & Like entry 15 with $3-(\mathrm{AcOAraO})-\mathrm{C}_{6} \mathrm{H}_{4}-\mathrm{CHO} ; 37 \%$. NaOMe$/ \mathrm{MeOH} \rightarrow \mathbf{4 3} ; 98 \%$. & {$[165]$} \\
\hline 20 & 44 & 4-(AraO)- $\mathrm{C}_{6} \mathrm{H}_{4^{-}}$ & $2 \mathrm{H}$ & Like entry 15 with $4-(\mathrm{AcOAraO})-\mathrm{C}_{6} \mathrm{H}_{4}-\mathrm{CHO} ; 36 \%$. NaOMe$/ \mathrm{MeOH} \rightarrow \mathbf{4 4} ; 96 \%$. & {$[165]$} \\
\hline 21 & 45 & 4-(GlcO) $-\mathrm{C}_{6} \mathrm{H}_{4-}^{-}$ & $\mathrm{Zn}(\mathrm{II})$ & $\begin{array}{l}\text { Condensation of pyrrole and } 4-(\mathrm{AcOGlcO})-\mathrm{C}_{6} \mathrm{H}_{4}-\mathrm{CHO}, \mathrm{CHCl}_{3}, \mathrm{BF}_{3} \cdot \mathrm{OEt}_{2} \\
\mathrm{Zn}(\mathrm{OAc})_{2} \text {, then } p \text {-chloranil; } 94 \% \text {. NaOMe} / \mathrm{MeOH} \rightarrow \mathbf{4 5} ; 93 \% \text {. }\end{array}$ & {$[165]$} \\
\hline 22 & 46 & $3-(\mathrm{GlcO})-\mathrm{C}_{6} \mathrm{H}_{4}-$ & $\mathrm{Zn}(\mathrm{II})$ & Like entry 21 with 3 -(AcOGlcO)- $\mathrm{C}_{6} \mathrm{H}_{4}-\mathrm{CHO} ; 93 \%$. NaOMe/MeOH $\rightarrow \mathbf{4 6} ; 89 \%$. & {$[165]$} \\
\hline 23 & 47 & $4-(\mathrm{GalO})-\mathrm{C}_{6} \mathrm{H}_{4}^{-}$ & $\mathrm{Zn}(\mathrm{II})$ & Like entry 21 with $4-\left(\right.$ AcOGalO) $-\mathrm{C}_{6} \mathrm{H}_{4}-\mathrm{CHO} ; 53 \%$. NaOMe$/ \mathrm{MeOH} \rightarrow 47 ; 90 \%$. & {$[165]$} \\
\hline 24 & 48 & $3-(\mathrm{GalO})-\mathrm{C}_{6} \mathrm{H}_{4}-$ & $\mathrm{Zn}(\mathrm{II})$ & Like entry 21 with $3-(\mathrm{AcOGalO})-\mathrm{C}_{6} \mathrm{H}_{4}-\mathrm{CHO} ; 72 \%$. NaOMe$/ \mathrm{MeOH} \rightarrow \mathbf{4 8} ; 90 \%$. & {$[165]$} \\
\hline 25 & 49 & $4-(\mathrm{XylO})-\mathrm{C}_{6} \mathrm{H}_{4}^{-}$ & $\mathrm{Zn}(\mathrm{II})$ & Like entry 21 with $4-(\mathrm{AcOX} y \mathrm{lO})-\mathrm{C}_{6} \mathrm{H}_{4}-\mathrm{CHO} ; 84 \%$. NaOMe$/ \mathrm{MeOH} \rightarrow \mathbf{4 9} ; 98 \%$. & {$[165]$} \\
\hline 26 & 50 & $3-(\mathrm{XylO})-\mathrm{C}_{6} \mathrm{H}_{4}-$ & $\mathrm{Zn}(\mathrm{II})$ & Like entry 21 with $3-(\mathrm{AcOXylO})-\mathrm{C}_{6} \mathrm{H}_{4}-\mathrm{CHO} ; 84 \%$. NaOMe/MeOH $\rightarrow \mathbf{5 0} ; 92 \%$. & {$[165]$} \\
\hline 27 & 51 & $4-(\mathrm{AraO})-\mathrm{C}_{6} \mathrm{H}_{4}^{-}$ & $\mathrm{Zn}(\mathrm{II})$ & Like entry 21 with $4-(\mathrm{AcOAraO})-\mathrm{C}_{6} \mathrm{H}_{4}-\mathrm{CHO} ; 63 \%$. NaOMe$/ \mathrm{MeOH} \rightarrow \mathbf{5 1} ; 98 \%$. & {$[165]$} \\
\hline 28 & 52 & $3-(\mathrm{AraO})-\mathrm{C}_{6} \mathrm{H}_{4-}^{-}$ & $\mathrm{Zn}(\mathrm{II})$ & Like entry 21 with $3-(\mathrm{AcOAraO})-\mathrm{C}_{6} \mathrm{H}_{4}-\mathrm{CHO} ; 60 \%$. NaOMe$/ \mathrm{MeOH} \rightarrow \mathbf{5 2} ; 96 \%$. & {$[165]$} \\
\hline 29 & 53 & $3,5-\mathrm{di}(\mathrm{GlcO})-\mathrm{C}_{6} \mathrm{H}_{3}-$ & $2 \mathrm{H}$ & $\begin{array}{l}\text { Modified Lindsey condensation with 3,5-(di-AcOGlcO)- } \mathrm{C}_{6} \mathrm{H}_{3}-\mathrm{CHO} \text { in } 32 \% \text {; } \\
\text { deprotection with } \mathrm{NaOMe} / \mathrm{MeOH} \rightarrow \mathbf{5 3} ; 100 \% \text {. }\end{array}$ & {$[167]$} \\
\hline 30 & 54 & (Bn-isop-Xyl-Fur)- & $2 \mathrm{H}$ & $\begin{array}{l}\text { Condensation of pyrrole and (Bn-isop-Xyl-Fur)-CHO, } \mathrm{CH}_{2} \mathrm{Cl}_{2}, \mathrm{BF}_{3} \cdot \mathrm{OEt}_{2} \text {, then } p \text { - } \\
\text { chloranil, } 4 \% \text { yield. }\end{array}$ & [168] \\
\hline 31 & 55 & (Bn-isop-Glc-Fur)- & $2 \mathrm{H}$ & Like entry 30 with (Bn-isop-Glc-Fur)-CHO; $4 \%$ & {$[168]$} \\
\hline 32 & 56 & (Gal-isop)- & $2 \mathrm{H}$ & Like entry 30 with (GalO-isop)-( $\left(\mathrm{CH}_{2}\right)-\mathrm{CHO} ; 4 \%$. & [168] \\
\hline 33 & 57 & $(\alpha-\mathrm{AcOGalO})-\left(\mathrm{CH}_{2}\right)-$ & $2 \mathrm{H}$ & Unstable, not characterized. & [168] \\
\hline 34 & 58 & (GalO-isop)- $\left(\mathrm{CH}_{2}\right)-$ & $2 \mathrm{H}$ & Unstable, not characterized. & [168] \\
\hline 35 & 59 & $\begin{array}{l}(1 S)-1,2-O-i s o p r o p y l-1,2- \\
\text { dihydroxyethyl }\end{array}$ & $2 \mathrm{H}$ & $\begin{array}{l}\text { Pyrrole, 2,3-O-isopropylidene- } D \text {-glyceraldehyde, } \mathrm{BF}_{3} \cdot \mathrm{OEt}_{2}, \mathrm{CH}_{2} \mathrm{Cl}_{2} \text {, then DDQ; } 3 \\
\% \alpha \beta \alpha \beta \text {. }\end{array}$ & [169] \\
\hline 36 & 60 & $\begin{array}{l}(1 S)-1,2-O-i s o p r o p y l-1,2- \\
\text { dihydroxyethyl }\end{array}$ & $2 \mathrm{H}$ & Like entry $35 ; 4 \%, \alpha \alpha \alpha \beta$ & [169] \\
\hline 37 & 61 & (MeO-isop-Xyl-Fur)- & $2 \mathrm{H}$ & Like entry 35 with (MeO-isop-Xyl-Fur)-CHO; $6 \%, \alpha \beta \alpha \beta$ & [169] \\
\hline 38 & 62 & $\begin{array}{l}2,3,4,5 \text {-Di- } O \text {-isopropylidene- } \\
D \text {-arabinose-tetritol-1-yl }\end{array}$ & $2 \mathrm{H}$ & Like entry 35 with $2,3,4,5$-di- $O$-isopropylidene- $D$-arabinose; $2 \%, \alpha \beta \alpha \beta$. & [169] \\
\hline 39 & 63 & $\mathrm{OH} \quad \mathrm{OH}$ & $2 \mathrm{H}$ & From 62: $50 \%$ aq. TFA, $\mathrm{CH}_{2} \mathrm{Cl}_{2}$, r.t., sonication, $2 \mathrm{~h} ; 90 \%, \alpha \beta \alpha \beta$. & {$[169]$} \\
\hline 40 & 64 & $\begin{array}{l}2,3,4,5 \text {-Di- } O \text {-isopropylidene- } \\
L \text {-arabinose-tetritol-1-yl }\end{array}$ & $2 \mathrm{H}$ & $\begin{array}{l}\alpha \beta \alpha \beta \text {; Pyrrole, } 2,3,4,5 \text {-di- } O \text {-isopropylidene- } L \text {-arabinose, } \mathrm{BF}_{3} \cdot \mathrm{OEt}_{2}, \mathrm{CH}_{2} \mathrm{Cl}_{2} \text {, then } \\
\text { DDQ; } 2 \% \text {. }\end{array}$ & {$[169]$} \\
\hline
\end{tabular}


<smiles>CC[C@H](O)[C@@H](C)[C@H](O)CO</smiles>

$$
\overline{\mathrm{O}} \mathrm{H}
$$

2-(AcOMalO) $-\mathrm{C}_{6} \mathrm{H}_{4}$ -

1043 -(AcOGlcO) $-\mathrm{C}_{6} \mathrm{H}_{4}$ -

\section{4-Pyridyl}

$3,5-(\mathrm{HO})-\mathrm{C}_{6} \mathrm{H}_{3}-$

$4-\mathrm{H}_{2} \mathrm{~N}-\mathrm{C}_{6} \mathrm{H}_{4}-$

$\mathrm{C}_{6} \mathrm{~F}_{5}-$

$\mathrm{C}_{6} \mathrm{~F}_{5}$ -

$\mathrm{C}_{6} \mathrm{~F}_{5^{-}}$

$\mathrm{C}_{6} \mathrm{~F}_{5}$ -

4-(AcOGlcS) $-\mathrm{C}_{6} \mathrm{~F}_{4}$ -

4-(AcOGlcS) $-\mathrm{C}_{6} \mathrm{~F}_{4}$ -

4-(AcOGlcS)- $\mathrm{C}_{6} \mathrm{~F}_{4-}$

4-(GlcS)- $\mathrm{C}_{6} \mathrm{~F}_{4}-$

4-(GlcS)- $\mathrm{C}_{6} \mathrm{~F}_{4-}$

4-(GlcS)- $\mathrm{C}_{6} \mathrm{~F}_{4-}$

4-(GlcS)- $\mathrm{C}_{6} \mathrm{~F}_{4-}$

3-(AcOMalO) $-\mathrm{C}_{6} \mathrm{H}_{4}$ -

$1063-(\mathrm{AcOLacO})-\mathrm{C}_{6} \mathrm{H}_{4-}$
$3,5-\left(\mathrm{NH}_{2} \mathrm{CH}_{2} \mathrm{CH}_{2}\right)-\mathrm{O}-\mathrm{C}_{6} \mathrm{H}_{3}-$

$3,5-\left(\mathrm{CH}_{2} \mathrm{CN}\right)-\mathrm{O}-\mathrm{C}_{6} \mathrm{H}_{3}-$

3,5-(di-GalCONH)-O- $\mathrm{C}_{6} \mathrm{H}_{3}-$

$3,5-\left(\right.$ di-GlcCONH)-O- $\mathrm{C}_{6} \mathrm{H}_{3}-$

$N$ - $\left(\alpha / \beta-M a l N-\mathrm{C}_{2} \mathrm{H}_{4}\right)-4$-pyridyl

$N$-( GalCNH- $\left.{ }_{3} \mathrm{H}_{6}\right)-4$-pyridyl

4-(AcOGlcS) $-\mathrm{C}_{6} \mathrm{~F}_{4}$ -

4-(GalS) $-\mathrm{C}_{6} \mathrm{~F}_{4}$ -

\begin{tabular}{|c|c|}
\hline 96 & 4-(AcOGalS)- $\mathrm{C}_{6} \mathrm{~F}_{4}-$ \\
\hline 97 & $4-\left[\mathrm{CH}_{3} \mathrm{SO}\right]-\mathrm{C}_{6} \mathrm{H}_{4-}^{-}$ \\
\hline 98 & $4-[\beta$-cyclodextrin $]-\mathrm{S}-\mathrm{C}_{6} \mathrm{H}_{4}-$ \\
\hline 99 & $4-[\beta$-cyclodextrin $]-\mathrm{S}-\mathrm{C}_{6} \mathrm{H}_{4}-$ \\
\hline 100 & $4-[\beta$-cyclodextrin $]-S-C_{6} F_{4}-$ \\
\hline 101 & 4- $(\alpha-\mathrm{GlcO})-\mathrm{CH}_{2}-\mathrm{C}_{6} \mathrm{H}_{4}-$ \\
\hline 102 & $2-(\mathrm{AcOGlcO})-\mathrm{C}_{6} \mathrm{H}_{4-}$ \\
\hline 103 & $2-(\mathrm{AcOMalO})-\mathrm{C}_{6} \mathrm{H}_{4}-$ \\
\hline 104 & 3-(AcOGlcO $)-\mathrm{C}_{6} \mathrm{H}_{4}-$ \\
\hline
\end{tabular}

$2 \mathrm{H}$

107 4-(GlcO- $\left.\left(\mathrm{CH}_{2}\right)_{3}-\mathrm{O}\right)-\mathrm{C}_{6} \mathrm{H}_{4}-$
$2 \mathrm{H}$

$\mathrm{Mn}(\mathrm{Cl})$

$\mathrm{Mn}(\mathrm{Cl})$

$2 \mathrm{H}$

$\mathrm{Mn}(\mathrm{Cl})$

$\mathrm{Mn}(\mathrm{Cl})$

$\mathrm{Mn}(\mathrm{Cl})$

Pyrrole, (BnOGal)- $\mathrm{CH}_{2}-\mathrm{CHO}, \mathrm{BF}_{3} \mathrm{OEt}_{2}, \mathrm{CH}_{2} \mathrm{Cl}_{2}$, then DDQ, $18 \%$.

Like entry 42 with (BnOGlcC)-CHO; $36 \%$.

From 61: $75 \%$ aq. TFA, $\mathrm{CH}_{2} \mathrm{Cl}_{2}$, sonication, $3 \mathrm{~h} ; 90 \%$.

From 61: $\mathrm{M}(\mathrm{OAc})_{2}, \mathrm{CH}_{3} \mathrm{OH} / \mathrm{CHCl}_{3}(1: 1, \mathrm{v} / \mathrm{v})$, sonication, then $75 \%$ aq. TFA,

$\mathrm{CH}_{2} \mathrm{Cl}_{2}$, r.t., 3 h; 63-90\%; $\mathrm{M}=\mathrm{Pd}(\mathrm{II}), \mathrm{Cu}(\mathrm{II}), \mathrm{Ni}(\mathrm{II}), \mathrm{Zn}(\mathrm{II})$.

Pyrrole and 4-(BnOGlcC)- $\mathrm{C}_{6} \mathrm{H}_{4}-\mathrm{CHO}, \mathrm{BF}_{3} \cdot \mathrm{OEt}_{2}, \mathrm{NaCl}$, r.t., 5 h, then DDQ, r.t., 30 min.; $53 \%$.

$2 \mathrm{H} \quad$ From 70: $\mathrm{H}_{2}$, Pd $10 \%$ on C, EtOAc-MeOH, r.t., 16 h; $98 \%$.

a) 3,5-Dihydroxybenzaldehyde, propargyl bromide, $\mathrm{K}_{2} \mathrm{CO}_{3}$, DMF, r.t.; b) 3,5dipropargyloxybenzaldehyde, pyrrole, TFA, $\mathrm{CH}_{2} \mathrm{Cl}_{2}$, r.t., then $p$-chloranil, r.t.; c) 5,10,15,20-tetra-3',5'-dipropargyloxyphenyl-porphyrin, $\beta$-(Lac)- $\mathrm{N}_{3}, \mathrm{CuBr}_{2}$, ascorbic acid, propylamine, r.t.

From 73: MeI, DMF, $5 \mathrm{~h}, 40^{\circ} \mathrm{C}$

From 76: $\mathrm{LiAlH}_{4}, \mathrm{AlCl}_{3}$, THF, 1 h, r.t.; $60 \%$.

From 77: $\mathrm{BrCH}_{2} \mathrm{CN}, \mathrm{K}_{2} \mathrm{CO}_{3}$, DMF, $40{ }^{\circ} \mathrm{C}, 2 \mathrm{~h} ; 98 \%$.

From 75: Gal-lactone, MeOH, reflux, 12 h; $63 \%$.

From 75: Glc-lactone, $\mathrm{MeOH}$, reflux, $12 \mathrm{~h} ; 92 \%$.

$[170]$

$[170]$

[171]

[175]

[175]

[175]

[175]

$[176]$

[176]

Pyrrole, pentafluorobenzaldehyde, $\mathrm{CH}_{2} \mathrm{Cl}_{2}, \mathrm{BF}_{3} \cdot \mathrm{OEt}_{2}, 4 \mathrm{~h}$ then $p$-chloranil, reflux, 12 h. $\mathrm{Zn}(\mathrm{OAc})_{2}, \mathrm{MeOH}, \mathrm{CHCl}_{3}$, reflux, $10 \mathrm{~h} ; 7 \%$.

From 83: 2,3,4,6-Tetra- $O$-acetyl- $1 S$-acetyl-1-thio- $\beta$ - $D$-glucopyranose,

$[178,177,200$

diethylamine, DMF, r.t., 24 h; $74 \%$.

206,296]

[177]

$[177]$

From 85: Like entry $63 ; 83 \%$

From 86: Like entry $63 ; 85 \%$.

From 87: $\mathrm{NaOMe}, \mathrm{MeOH}, \mathrm{CH}_{2} \mathrm{Cl}_{2}, 45-50{ }^{\circ} \mathrm{C}, 1 \mathrm{~h} ; 69 \%$.

[177]

$[178,177,206$,

$296,337]$

From 88: $\mathrm{NaOMe}, \mathrm{MeOH}, \mathrm{CH}_{2} \mathrm{Cl}_{2}, 45-50{ }^{\circ} \mathrm{C}, 1 \mathrm{~h} ; 81 \%$.

[177]

[177]

From 89: Like entry $69 ; 68 \%$

[177]

$[178,247]$ Or:

From 83: GalSNa, DMF, r.t., overnight; $92 \%$.

From 83: acetyl 2,3,4,6-tetra- $O$-acetyl-1-thio- $\beta$ - $D$-galactopyranoside, diethylamine, DMF, r.t., 24 h; $86 \%$

Pyrrole, $p$-(methylthio)benzaldehyde, EtCOOH, reflux; $15 \%$.

From 97, trifluoroacetic anhydride, $\mathrm{CH}_{2} \mathrm{Cl}_{2}, 40{ }^{\circ} \mathrm{C}, 30$ min., then $\beta$-cyclodextrin iodide, DMF, $\mathrm{MeOH}, \mathrm{Et}_{3} \mathrm{~N}, 60^{\circ} \mathrm{C}, 24 \mathrm{~h} ; 25 \%$.

From 98: $\mathrm{MnCl}_{2}, 2,6$-lutidine, DMF, $\Delta, 27 \mathrm{~h} ; 98 \%$.

From 83: 6-deoxy-6-mercapto- $\beta$-cyclodextrin, $\mathrm{K}_{2} \mathrm{CO}_{3}$, DMF, r.t.; $95 \%$; then $\mathrm{MnCl}_{2}$, 2,6-lutidine, DMF, $\Delta ; 93 \%$.

5,10,15,20-Tetrakis(4-bromomethylphenyl)porphyrin, 2,3,4,6-tetra- $O$-acetyl- $\alpha-D$ glucopyranotrichloroacetimidate, $\mathrm{CH}_{2} \mathrm{Cl}_{2}$, molecular sieves $4 \AA, \mathrm{ZnCl}_{2}$.

From 25: $\mathrm{MnCl}_{2}, 2$,6-lutidine,4-nitrophenol, DMF, $12 \mathrm{~h}, 40{ }^{\circ} \mathrm{C}$; obtained as $\alpha \beta \alpha \beta$, $\alpha \alpha \beta \beta, \alpha \alpha \alpha \beta$ atropisomers in 15,27 and $26 \%$, respectively.

From 27: $\mathrm{MnCl}_{2}$, 2,6-lutidine ,4-nitrophenol, DMF, $12 \mathrm{~h}, 40{ }^{\circ} \mathrm{C}$; obtained as mixture of $\alpha \beta \alpha \beta, \alpha \alpha \beta \beta, \alpha \alpha \alpha \beta$ atropisomers in $100 \%$.

- Condensation of pyrrole and 3-(AcOGlcO)- $\mathrm{C}_{6} \mathrm{H}_{4}-\mathrm{CHO}, \mathrm{CH}_{2} \mathrm{Cl}_{2}, \mathrm{BF}_{3} \cdot \mathrm{OEt}_{2}$, then ${ }^{\circ} \mathrm{C}, 64 \%$.

$\mathrm{Mn}(\mathrm{Cl}) \quad$ Like entry 81 with 3-(AcOMalO)- $\mathrm{C}_{6} \mathrm{H}_{4}-\mathrm{CHO} ; 2 \%$.Then $\mathrm{MnCl}_{2} ; 70 \%$ [182]

$\mathrm{Mn}(\mathrm{Cl}) \quad$ Like entry 81 with $3-(\mathrm{AcOLacO})-\mathrm{C}_{6} \mathrm{H}_{4}-\mathrm{CHO} ; 4 \%$. Then $\mathrm{MnCl}_{2} ; 73 \%$. [182]

$2 \mathrm{H} \quad$ Pyrrole and (AcOGlcO) $-\mathrm{C}_{6} \mathrm{H}_{4}-\mathrm{CHO}(1: 4), \mathrm{EtCOOH} / \mathrm{Ac}_{2} \mathrm{O} ; 36 \%$ yield; then $\mathrm{NaOMe}, \mathrm{MeOH}, \mathrm{CH}_{2} \mathrm{Cl}_{2}, 1 \mathrm{~h} ; 80 \%$.

$2 \mathrm{H} \quad$ Like entry 84 with (AcOMalO) $-\mathrm{C}_{6} \mathrm{H}_{4}-\mathrm{CHO} ; 16 \%$. Then $\mathrm{NaOMe}, \mathrm{MeOH} ; 80 \%$. 


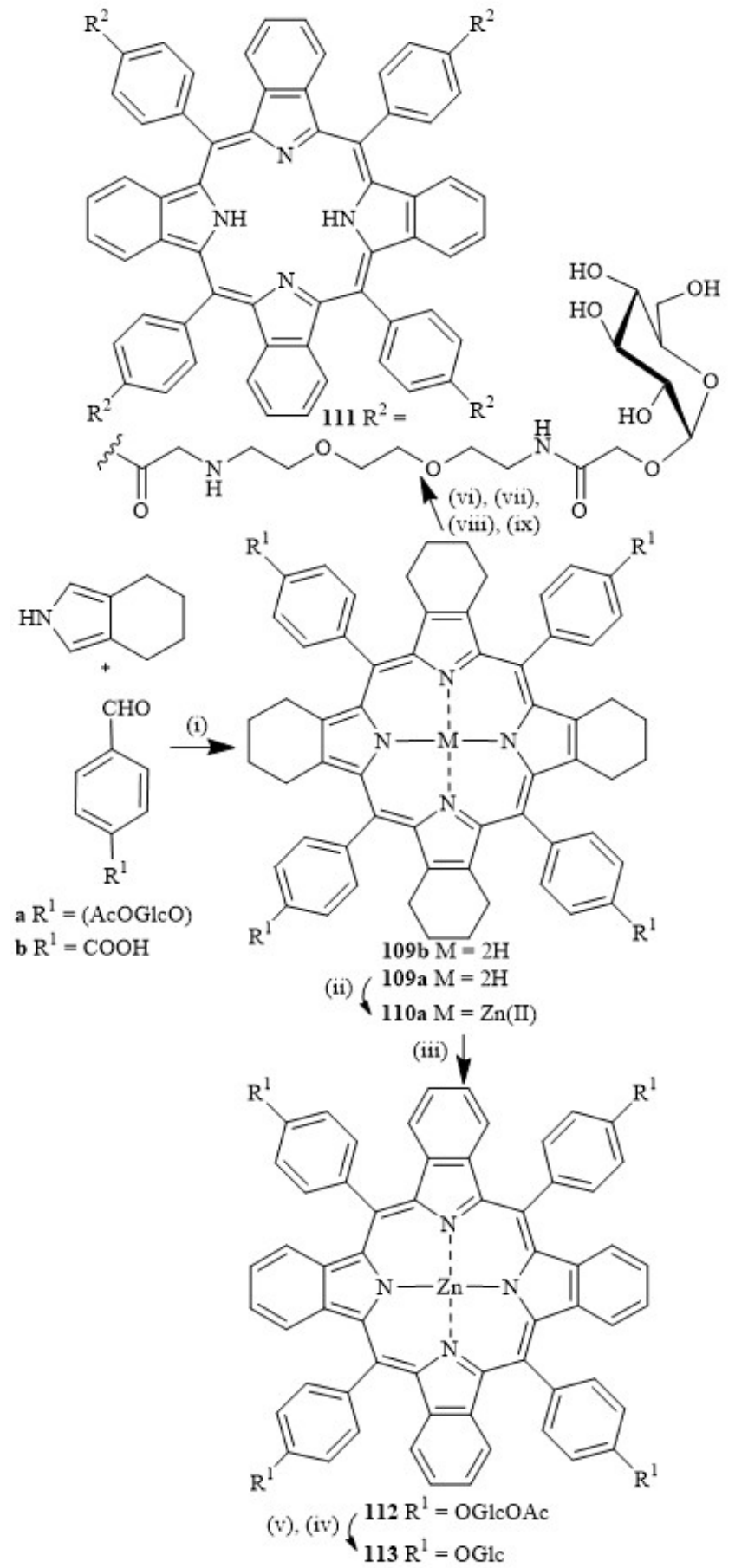

Scheme 5. Synthesis of glycoconjugated tetrabenzoporphyrins. i) $\mathrm{BF}_{3} \cdot \mathrm{OEt}_{2}$, $\mathrm{CH}_{2} \mathrm{Cl}_{2}$, r.t., $2 \mathrm{~h}$, then DDQ, $1 \mathrm{~h}$; ii) $\mathrm{Zn}(\mathrm{OAc})_{2}, \mathrm{CHCl}_{3} / \mathrm{MeOH}(9: 1), 1 \mathrm{~h}$; iii) DDQ (16 equiv.),THF, reflux, $1 \mathrm{~h}$; iv) $\mathrm{HCl}(10 \mathrm{M}), \mathrm{CH}_{2} \mathrm{Cl}_{2}, 5 \mathrm{~min}$; v) $\mathrm{MeONa}\left(0.5 \mathrm{M}, 3\right.$ equiv. per acetate group), $\mathrm{CH}_{2} \mathrm{Cl}_{2} / \mathrm{MeOH}(8: 2), 1 \mathrm{~h}$; vi) $\mathrm{H}_{2} \mathrm{~N}-\mathrm{CH}_{2}-\left(\mathrm{CH}_{2}-\mathrm{O}-\mathrm{CH}_{2}\right)_{2}-\mathrm{CH}_{2}-\mathrm{NHBoc}$, DCC (4.4 equiv.), HOBt (4.4 equiv.), DMF, r.t., $85 \%$; vii) $\mathrm{CF}_{3} \mathrm{CO}_{2} \mathrm{H} / \mathrm{CH}_{2} \mathrm{Cl}_{2}(1: 1)$, r.t., $2 \mathrm{~h}$, quantitative; viii) DMAP (16 equiv.), $\mathrm{CHCl}_{3}$, r.t., 2 weeks, $28 \%$; ix) $\mathrm{MeONa}(0.5 \mathrm{M}, 3$ equiv. per acetate group), $\mathrm{CH}_{2} \mathrm{Cl}_{2} / \mathrm{MeOH}(8: 2), 30 \mathrm{~min}$., $82 \%$.

\subsubsection{Unsymmetrically Substituted Glycoporphyrins}

Mixed condensations have been widely used for the construction of unsymmetrical glycoporphyrins of the $\mathrm{A}_{3} \mathrm{~B}$-, $\mathrm{A}_{2} \mathrm{~B}_{2^{-}}, \mathrm{A}_{2} \mathrm{BC}$ and ABCD-type (Figure 3) ${ }^{[160,185]}$. Most of the relevant compounds are listed in Table 2. Condensations with two or more different aldehydes with pyrrole can yield a variety of porphyrins including all the $\mathrm{A}_{3} \mathrm{~B}-, \mathrm{A}_{2} \mathrm{~B}_{2-}, \mathrm{A}_{2} \mathrm{BC}$ and $\mathrm{ABCD}$-types. Selectivity for a particular array can be achieved using specific equivalents of aldehyde versus pyrrole. Reactions using Lindsey's [2+2] and [3+1] methods can afford large scale syntheses of all types of substituted porphyrins ${ }^{[137,139,186]}$.

A classic study in this context is Maillard's work from $1995^{[162]}$. After the synthesis of the symmetric compounds 29-36 the importance of the amphiphilicity of such structures was becoming apparent and their synthetic studies then focused on mixed meso (glycosylated aryl) aryl porphyrins and mixed meso (glycosylated aryl) alkyl porphyrins bearing the same glycosyl moieties as the tetrasubstituted TPP's studied earlier (114-119). Optimized condensation conditions provided primarily the di- and triglycosylated derivatives and minimized the tetrasubstituted compounds.

The behavior of such tetra-, tri- and diglycosylated mesosubstituted porphyrins 114-119 and 30 were examined in aqueous media by absorbance and fluorescence spectroscopy $[162,187]$. All dimerized in aqueous solutions with the dimers stability dependent upon the substitution pattern. The diglycosylated derivative $\mathbf{1 1 5}$ was the most stable dimer with an offset stacked geometry similar to that reported for the dimer of an aminoglucosamide protoporphyrin in solution [31,187]. The fluorescence of all dimers was quenched, except for the 5,10-diglycosylated derivative 115. A linear dependence was determined between the number of the aryl groups of the glucosylated porphyrins and the singlet oxygen formation, with yields of singlet oxygen in the range 0.54 0.81 .

A similar study by Gaud et al. ${ }^{[183]}$ provided 13 meso glycosylporphyrins using mixed pyrrole condensation reactions and/or direct glycosylations of $o$ - and $p$ hydroxyalkoxyarylporphyrins. The series included $\mathrm{AB}_{3}$ (120-127), $\mathrm{A}_{3} \mathrm{~B}-(\mathbf{1 2 8}), 5,10-\mathrm{A}_{2} \mathrm{~B}_{2^{-}}$(129), 5,15- $\mathrm{A}_{2} \mathrm{~B}_{2^{-}}$(130), and $\mathrm{A}_{4}$-type $(\mathbf{1 0 7 , 1 0 8})$ porphyrins with $\mathrm{A}$ being either glucose, ribose, maltose or lactose residues. In all cases a $\beta$ configuration was found after basic deprotection. Due to the use of mixed condensation reactions for the underlying porphyrin framework yields were limited. Nevertheless, this presented a significant body of work and gave the first possibility for QSAR studies investigating either the influence of different sugar units and the regiochemistry and number thereof. Glycoporphyrins derived from simple mixed condensation can also serve as precursors for more complex 
1. Mixed condensation<smiles>[R]C(=O)c1ccc[nH]1</smiles>

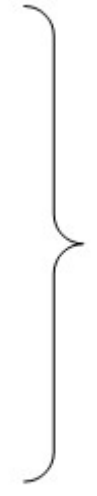<smiles>[B]CC(CC)CC(C)C</smiles><smiles>[Y]CC(CC)CBr</smiles>

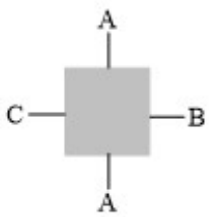<smiles>[Y]CC(CC)CBr</smiles><smiles>[2H]CC(CC)CCC</smiles>

2. $2+2$ condensation<smiles>[2H]C(O)c1ccc(C(C)c2ccc(C(O)Br)[nH]2)[nH]1</smiles><smiles>ClC(c1ccc[nH]1)c1ccc[nH]1</smiles>

Or<smiles>CC(=O)c1ccc[nH]1</smiles>

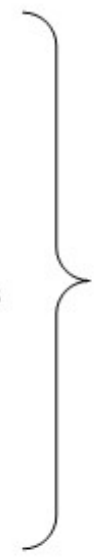

3. $3+1$ condensation<smiles>[Y]C(c1ccc(C([2H])O)[nH]1)c1ccc(C(Br)c2ccc(C(C)O)[nH]2)[nH]1</smiles><smiles>[Y]C(c1ccc(CO)[nH]1)c1ccc(C([Y])c2ccc(C([B])=O)[nH]2)[nH]1</smiles><smiles>[B]CC1CCCC1[B]C</smiles><smiles>CCCCCCC</smiles><smiles>[2H]CC(CC)CC</smiles>

Or<smiles>CCC(C)CBr</smiles>

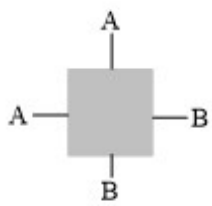

Figure 3. Synthesis of unsymmetric $\mathrm{ABCD}$-type glycoporphyrins via mixed condensation reactions.

target systems. For example, following on from their work on glycosylated haematoporphyrins ${ }^{[188]}$, [see below], Krausz et al. synthesized a range of amphiphilic cationic glycoporphyrins of the $\mathrm{A}_{3} \mathrm{~B}$-type ${ }^{[189]}$. Here, the amphiphilicity was introduced via varying lipophilic $N$ substituted entities including methyl, isopropyl, $n$-octyl groups. First, 5-(2-tetraacetyl- $\beta$ - $D$-glucopyranosylphenyl)10,15,20-tris(4-pyridyl)porphyrin 131 was synthesized via condensation of glycosyl benzaldehyde), 4- pyridinecarboxaldehyde and pyrrole in $7 \%$ yield. Next, compound 131 was alkylated at the pyridyl- $N$ using large excesses of methyl, isopropyl and $n$-octyl iodides affording 132-135 as the triiodides in 85, 90 and $75 \%$ yield, respectively. Finally, all derivatives were deprotected providing 135-138.

This work was later extended to the synthesis of a whole range of unsymmetrical free base cationic $O$-linked glycoporphyrins based on 5,10,15-tris\{4-(alkyl 
Table 2. Unsymmetric ABCD-type (5-A,10-B,15-C,20-D-substituted) glycoporphyrins and reference compounds.

\begin{tabular}{|c|c|c|c|c|c|c|c|c|}
\hline Entry & \# & A substituent & B substituent & C substituent & D substituent & Metal & Comments & Ref. \\
\hline 1 & 114 & $4-(\mathrm{GlcO})-\mathrm{C}_{6} \mathrm{H}_{4^{-}}$ & $4-(\mathrm{GlcO})-\mathrm{C}_{6} \mathrm{H}_{4^{-}}$ & 4-(GlcO)- $\mathrm{C}_{6} \mathrm{H}_{4^{-}}$ & Phenyl & $2 \mathrm{H}$ & $\begin{array}{l}\text { a) Pyrrole, 4-( } \mathrm{AcOGlcO})-\mathrm{C}_{6} \mathrm{H}_{4}-\mathrm{CHO} \text {, benzaldehyde, } \mathrm{CHCl}_{3}, \mathrm{BF}_{3} \cdot \mathrm{OEt}_{2} \text {, } \\
\text { r.t., } 24 \mathrm{~h} \text {, then } p \text {-chloranil, } 1 \mathrm{~h} \text {, reflux; } 10 \% \text {; b) } \mathrm{NaOMe}, \mathrm{MeOH}, 1 \mathrm{~h} \text {, } \\
\text { r.t.; } 83 \% \text {. }\end{array}$ & $\begin{array}{l}{[162,160} \\
210,342]\end{array}$ \\
\hline 2 & 115 & $4-(\mathrm{GlcO})-\mathrm{C}_{6} \mathrm{H}_{4}-$ & $4-(\mathrm{GlcO})-\mathrm{C}_{6} \mathrm{H}_{4-}$ & $n$-Butyl & $n$-Butyl & $2 \mathrm{H}$ & Like entry 1 with $n$-pentanal. a) $3 \%$; b) $82 \%$. & [162] \\
\hline 3 & 116 & $4-(\mathrm{GlcO})-\mathrm{C}_{6} \mathrm{H}_{4}-$ & 4-(GlcO) $-\mathrm{C}_{6} \mathrm{H}_{4}-$ & 4-(GlcO) $-\mathrm{C}_{6} \mathrm{H}_{4}-$ & $\mathrm{C}_{6} \mathrm{~F}_{5^{-}}$ & $2 \mathrm{H}$ & Like entry 1 with $\mathrm{C}_{6} \mathrm{~F}_{5}-\mathrm{CHO}$. a) $3 \%$; b) $87 \%$. & {$[162,342]$} \\
\hline 4 & 117 & $4-(\mathrm{GlcO})-\mathrm{C}_{6} \mathrm{H}_{4}^{-}$ & $4-(\mathrm{GlcO})-\mathrm{C}_{6} \mathrm{H}_{4}-$ & $4-(\mathrm{GlcO})-\mathrm{C}_{6} \mathrm{H}_{4}^{-}$ & $n$-Butyl & $2 \mathrm{H}$ & Like entry 1 with $n$-pentanal. a) $1 \%$; b) $78 \%$. & {$[162]$} \\
\hline 5 & 118 & 4-(GlcO) $-\mathrm{C}_{6} \mathrm{H}_{4}-$ & $n$-Butyl & 4-(GlcO) $-\mathrm{C}_{6} \mathrm{H}_{4}-$ & $n$-Butyl & $2 \mathrm{H}$ & Like entry 1 with $n$-pentanal. a) $2 \%$. & [162] \\
\hline 6 & 119 & 4-(GlcO) $-\mathrm{C}_{6} \mathrm{H}_{4-}^{-}$ & $4-(\mathrm{GlcO})-\mathrm{C}_{6} \mathrm{H}_{4}-$ & Undecyl & Undecyl & $2 \mathrm{H}$ & Like entry 1 with $n$-dodecanal. a) $2 \%$; b) $70 \%$. & [162] \\
\hline 7 & 120 & $2-\left[\mathrm{GlcO}-\left(\mathrm{CH}_{2}\right)_{3}-\mathrm{O}\right]-\mathrm{C}_{6} \mathrm{H}_{4^{-}}$ & $4-\mathrm{Me}^{-} \mathrm{C}_{6} \mathrm{H}_{4-}^{-}$ & $4-\mathrm{Me}-\mathrm{C}_{6} \mathrm{H}_{4-}$ & $4-\mathrm{Me}-\mathrm{C}_{6} \mathrm{H}_{4}^{-}$ & $2 \mathrm{H}$ & $\begin{array}{l}\text { a) 5-[2-(3-Hydroxylpropyloxyphenyl]-10,15,20-tritolylporphyrin, } \\
1,2,3,4,6 \text {-penta-O-acetyl- } \beta \text {-D-glucopyranose, } \mathrm{SnCl}_{4}, \mathrm{CH}_{2} \mathrm{Cl}_{2}, 18 \mathrm{~h} ; 35 \\
\% \text {. b) } \mathrm{NaOMe}, \mathrm{MeOH}, \mathrm{CH}_{2} \mathrm{Cl}_{2}, 1 \mathrm{~h} ; 80 \% \text {. }\end{array}$ & [183] \\
\hline 8 & 121 & 4-[GlcO- $\left.\left(\mathrm{CH}_{2}\right)_{3}-\mathrm{O}\right]-\mathrm{C}_{6} \mathrm{H}_{4^{-}}$ & $4-\mathrm{Me}-\mathrm{C}_{6} \mathrm{H}_{4}-$ & $4-\mathrm{Me}-\mathrm{C}_{6} \mathrm{H}_{4^{-}}$ & $4-\mathrm{Me}-\mathrm{C}_{6} \mathrm{H}_{4}^{-}$ & $2 \mathrm{H}$ & $\begin{array}{l}\text { Like entry } 7 \text { with } 5 \text {-[4-(3-hydroxylpropyloxyphenyl]-10,15,20- } \\
\text { tritolylporphyrin. a) } 2 \text { h; } 60 \% \text {. b) } 80 \% \text {. }\end{array}$ & {$[183,365]$} \\
\hline 9 & 122 & $2-\left[\mathrm{RbOH}-\left(\mathrm{CH}_{2}\right)_{3}-\mathrm{O}\right]-\mathrm{C}_{6} \mathrm{H}_{4}-$ & 4-Me- $\mathrm{C}_{6} \mathrm{H}_{4^{-}}$ & 4-Me- $\mathrm{C}_{6} \mathrm{H}_{4}^{-}$ & $4-\mathrm{Me}-\mathrm{C}_{6} \mathrm{H}_{4}^{-}$ & $2 \mathrm{H}$ & $\begin{array}{l}\text { Like entry } 7 \text { with } O \text {-acetyl- } 2,3,5 \text {-tribenzoate- } \beta \text { - } D \text {-ribofuranose. a) } 16 \mathrm{~h} \text {; } \\
60 \% \text {. b) } 85 \% \text {. }\end{array}$ & [183] \\
\hline 10 & 123 & $4-\left[\mathrm{RbOH}-\left(\mathrm{CH}_{2}\right)_{3}-\mathrm{O}\right]-\mathrm{C}_{6} \mathrm{H}_{4}-$ & 4-Me- $\mathrm{C}_{6} \mathrm{H}_{4^{-}}$ & $4-\mathrm{Me}-\mathrm{C}_{6} \mathrm{H}_{4^{-}}$ & 4-Me- $\mathrm{C}_{6} \mathrm{H}_{4}^{-}$ & $2 \mathrm{H}$ & $\begin{array}{l}\text { Like entry } 9 \text { with 5-[4-(3-hydroxylpropyloxyphenyl]-10,15,20- } \\
\text { tritolylporphyrin. a) } 2 \text { h; } 75 \% \text {. b) } 85 \% \text {. }\end{array}$ & [183] \\
\hline 11 & 124 & $2-\left[\mathrm{MalO}-\left(\mathrm{CH}_{2}\right)_{3}-\mathrm{O}\right]-\mathrm{C}_{6} \mathrm{H}_{4^{-}}$ & 4-Me- $\mathrm{C}_{6} \mathrm{H}_{4}-$ & $4-\mathrm{Me}-\mathrm{C}_{6} \mathrm{H}_{4-}$ & $4-\mathrm{Me}-\mathrm{C}_{6} \mathrm{H}_{4}-$ & $2 \mathrm{H}$ & $\begin{array}{l}\text { Like entry } 7 \text { with } 2,3,6,2,3,4,5,6 \text {-hepta- } O \text {-acetyl- } \beta \text { - } D \text {-maltopyranose. a) } \\
24 \text { h; } 12 \% \text {. b) } 85 \% \text {. }\end{array}$ & [183] \\
\hline 12 & 125 & 4-[MalO- $\left.\left(\mathrm{CH}_{2}\right)_{3}-\mathrm{O}\right]-\mathrm{C}_{6} \mathrm{H}_{4-}-$ & $4-\mathrm{Me}-\mathrm{C}_{6} \mathrm{H}_{4}-$ & $4-\mathrm{Me}-\mathrm{C}_{6} \mathrm{H}_{4^{-}}$ & $4-\mathrm{Me}-\mathrm{C}_{6} \mathrm{H}_{4^{-}}$ & $2 \mathrm{H}$ & $\begin{array}{l}\text { Like entry } 8 \text { with } 2,3,6,2,3,4,5,6 \text {-hepta- } O \text {-acetyl- } \beta \text { - } D \text {-maltopyranose. a) } \\
6 \text { h; } 38 \% \text {. b) } 90 \% \text {. }\end{array}$ & [183] \\
\hline 13 & 126 & $2-\left[\mathrm{LacO}-\left(\mathrm{CH}_{2}\right)_{3}-\mathrm{O}\right]-\mathrm{C}_{6} \mathrm{H}_{4-}-$ & $4-\mathrm{Me}-\mathrm{C}_{6} \mathrm{H}_{4-}$ & $4-\mathrm{Me}-\mathrm{C}_{6} \mathrm{H}_{4}-$ & $4-\mathrm{Me}-\mathrm{C}_{6} \mathrm{H}_{4^{-}}$ & $2 \mathrm{H}$ & $\begin{array}{l}\text { Like entry } 7 \text { with } 2,3,6,2,3,4,5,6 \text {-hepta- } O \text {-acetyl- } \beta \text { - } D \text {-lactopyranose. a) } \\
24 \text { h; } 21 \% \text {. b) } 85 \% \text {. }\end{array}$ & [183] \\
\hline 14 & 127 & $4-\left[\mathrm{LacO}-\left(\mathrm{CH}_{2}\right)_{3}-\mathrm{O}\right]-\mathrm{C}_{6} \mathrm{H}_{4^{-}}$ & $4-\mathrm{Me}-\mathrm{C}_{6} \mathrm{H}_{4}-$ & 4-Me- $\mathrm{C}_{6} \mathrm{H}_{4}-$ & 4-Me- $\mathrm{C}_{6} \mathrm{H}_{4}^{-}$ & $2 \mathrm{H}$ & $\begin{array}{l}\text { Like entry } 8 \text { with } 2,3,6,2,3,4,5,6 \text {-hepta- } O \text {-acetyl- } \beta \text { - } D \text {-lactopyranose. a) } \\
6 \text { h; } 42 \% \text {. b) } 85 \% \text {. }\end{array}$ & [183] \\
\hline 15 & 128 & $4-\left[\mathrm{GlcO}-\left(\mathrm{CH}_{2}\right)_{3}-\mathrm{O}\right]-\mathrm{C}_{6} \mathrm{H}_{4^{-}}$ & $\begin{array}{l}4-\left[\mathrm{GlcO}-\left(\mathrm{CH}_{2}\right)_{3}-\mathrm{O}\right]- \\
\mathrm{C}_{6} \mathrm{H}_{4^{-}}\end{array}$ & $\begin{array}{l}\left.\text { 4-[GlcO- }\left(\mathrm{CH}_{2}\right)_{3}-\mathrm{O}\right]- \\
\mathrm{C}_{6} \mathrm{H}_{4^{-}}\end{array}$ & 4-Me- $\mathrm{C}_{6} \mathrm{H}_{4}^{-}$ & $2 \mathrm{H}$ & $\begin{array}{l}\text { a) } 2 \times \text { Pyrrole, } 3 \times 4 \text {-(AcOGlcO)- } \mathrm{C}_{6} \mathrm{H}_{4}-\mathrm{CHO}, 1 \times 4-\mathrm{Me}_{-} \mathrm{C}_{6} \mathrm{H}_{4}-\mathrm{CHO} \text {, } \\
\text { EtCOOH} / \mathrm{Ac}_{2} \mathrm{O} ; 3 \% \text {. b) NaOMe, } \mathrm{MeOH}, \mathrm{CH}_{2} \mathrm{Cl}_{2}, 1 \mathrm{~h} ; 80 \% \text {. }\end{array}$ & [183] \\
\hline 16 & 129 & 4-[GlcO- $\left.\left(\mathrm{CH}_{2}\right)_{3}-\mathrm{O}\right]-\mathrm{C}_{6} \mathrm{H}_{4^{-}}$ & $\begin{array}{l}4-\left[\mathrm{GlcO}-\left(\mathrm{CH}_{2}\right)_{3}-\mathrm{O}\right]- \\
\mathrm{C}_{6} \mathrm{H}_{4^{-}}\end{array}$ & 4-Me- $\mathrm{C}_{6} \mathrm{H}_{4^{-}}$ & 4-Me- $\mathrm{C}_{6} \mathrm{H}_{4}-$ & $2 \mathrm{H}$ & $\begin{array}{l}\text { a) } 4 \times \text { Pyrrole, } 3 \times 4 \text {-(AcOGlcO)- } \mathrm{C}_{6} \mathrm{H}_{4}-\mathrm{CHO}, 1 \times 4-\mathrm{Me}-\mathrm{C}_{6} \mathrm{H}_{4}-\mathrm{CHO} \text {, } \\
\text { EtCOOH} / \mathrm{Ac}_{2} \mathrm{O} ; 2 \% \text { mixture of } 5,10 \text { and } 5,15 \text { regioisomers. b) } \\
\mathrm{NaOMe}, \mathrm{MeOH}, \mathrm{CH}_{2} \mathrm{Cl}_{2}, 1 \mathrm{~h} ; 75 \% \text {. }\end{array}$ & [183] \\
\hline 17 & 130 & $4-\left[\mathrm{GlcO}-\left(\mathrm{CH}_{2}\right)_{3}-\mathrm{O}\right]-\mathrm{C}_{6} \mathrm{H}_{4^{-}}$ & 4-Me- $\mathrm{C}_{6} \mathrm{H}_{4^{-}}$ & $\begin{array}{l}\left.4-[\mathrm{GlcO})-\left(\mathrm{CH}_{2}\right)_{3}-\mathrm{O}\right]- \\
\mathrm{C}_{6} \mathrm{H}_{4^{-}}\end{array}$ & 4-Me- $\mathrm{C}_{6} \mathrm{H}_{4}^{-}$ & $2 \mathrm{H}$ & $\begin{array}{l}\text { a) Like entry } 16 ; 2 \% \text { mixture of } 5,10 \text { and } 5,15 \text { regioisomers b) } \\
\mathrm{NaOMe}, \mathrm{MeOH}, \mathrm{CH}_{2} \mathrm{Cl}_{2}, 1 \mathrm{~h} ; 75 \%\end{array}$ & [183] \\
\hline 18 & 131 & 2-(AcOGlcO)- $\mathrm{C}_{6} \mathrm{H}_{4^{-}}$ & 4-Pyridyl & 4-Pyridyl & 4-Pyridyl & $2 \mathrm{H}$ & $\begin{array}{l}\text { Pyrrole, 4-pyridinecarboxaldehyde, 2-(AcOGlcO) }-\mathrm{C}_{6} \mathrm{H}_{4}-\mathrm{CHO} \text {, } \\
\text { EtCOOH} / \mathrm{Ac}_{2} \mathrm{O} \text {, reflux; } 7 \% \text {. }\end{array}$ & {$[189,190]$} \\
\hline 19 & 132 & $2-(\mathrm{AcOGlcO})-\mathrm{C}_{6} \mathrm{H}_{4^{-}}$ & $N$-Me-4-pyridyl & $N$-Me-4-pyridyl & $N$-Me-4-pyridyl & $2 \mathrm{H}$ & From 131: MeI, DMF, $\Delta ; 85 \%$ & {$[189,190]$} \\
\hline 20 & 133 & 2-( $\mathrm{AcOGlcO})-\mathrm{C}_{6} \mathrm{H}_{4}-$ & $N-i$ Pr-4-pyridyl & $N-i$ Pr-4-pyridyl & $N-i$ Pr-4-pyridyl & $2 \mathrm{H}$ & From 131: isopropyl iodide, DMF, $\Delta ; 90 \%$. & [189] \\
\hline 21 & 134 & 2-( $\mathrm{AcOGlcO})-\mathrm{C}_{6} \mathrm{H}_{4^{-}}$ & $N$-n-Octyl-4-pyridyl & $N-n$-Octyl-4-pyridyl & $N$-n-Octyl-4-pyridyl & $2 \mathrm{H}$ & From 131: $n$-octyl iodide, DMF, $\Delta ; 75 \%$ & {$[189,190]$} \\
\hline 22 & 135 & $2-(\mathrm{GlcO})-\mathrm{C}_{6} \mathrm{H}_{4-}^{-}$ & $N$-Me-4-pyridyl & $N$-Me-4-pyridyl & $N$-Me-4-pyridyl & $2 \mathrm{H}$ & From 132: $\mathrm{Et}_{3} \mathrm{~N}-\mathrm{MeOH}-\mathrm{H}_{2} \mathrm{O}(10 / 10 / 1, \mathrm{v} / \mathrm{v} / \mathrm{v}), 0{ }^{\circ} \mathrm{C}$. & {$[189,190]$} \\
\hline 23 & 136 & 2-(GlcO) $-\mathrm{C}_{6} \mathrm{H}_{4}-$ & $N-i$ Pr-4-pyridyl & $N-i$ Pr-4-pyridyl & $N-i$ Pr-4-pyridyl & $2 \mathrm{H}$ & From 133: Like entry 22. & {$[189,190]$} \\
\hline 24 & 137 & $2-(\mathrm{GlcO})-\mathrm{C}_{6} \mathrm{H}_{4}-$ & $N-n$-Octyl-4-pyridyl & $N-n$-Octyl-4-pyridyl & $N-n$-Octyl-4-pyridyl & $2 \mathrm{H}$ & From 134: Like entry 22. & {$[189,190]$} \\
\hline 25 & 138 & 2-( $\mathrm{GlcO})-\mathrm{C}_{6} \mathrm{H}_{4^{-}}$ & 4-Pyridyl & 4-Pyridyl & 4-Pyridyl & $2 \mathrm{H}$ & From 131: Like entry 22 & {$[189,190]$} \\
\hline 26 & 139 & 4-( AcOGlcO)- $\mathrm{C}_{6} \mathrm{H}_{4^{-}}$ & 4-Pyridyl & 4-Pyridyl & 4-Pyridyl & $2 \mathrm{H}$ & $\begin{array}{l}\text { Pyrrole, 4-pyridinecarboxaldehyde, 4-( } \mathrm{AcOGlcO})-\mathrm{C}_{6} \mathrm{H}_{4}-\mathrm{CHO} \text {, } \\
\text { EtCOOH} / \mathrm{Ac}_{2} \mathrm{O} \text {, reflux, } 1.5 \mathrm{~h} ; 7 \% \text {. }\end{array}$ & {$[189,190]$} \\
\hline 27 & 140 & 4-(GlcO)- $\mathrm{C}_{6} \mathrm{H}_{4^{-}}$ & 4-Pyridyl & 4-Pyridyl & 4-Pyridyl & $2 \mathrm{H}$ & From 139: $\mathrm{Et}_{3} \mathrm{~N}-\mathrm{MeOH}-\mathrm{H}_{2} \mathrm{O}(10 / 10 / 1, \mathrm{v} / \mathrm{v} / \mathrm{v}), 0{ }^{\circ} \mathrm{C}, 24 \mathrm{~h} ; 96 \%$. & [190] \\
\hline 28 & 141 & 4-( AcOGlcO) $-\mathrm{C}_{6} \mathrm{H}_{4^{-}}$ & $N$-Me-4-pyridyl & $N$-Me-4-pyridyl & $N$-Me-4-pyridyl & $2 \mathrm{H}$ & From 139: Methyl iodide, DMF, $40^{\circ} \mathrm{C}, 3 \mathrm{~h} ; 94 \%$ & {$[189,190]$} \\
\hline 29 & 142 & 4-( $\mathrm{AcOGlcO})-\mathrm{C}_{6} \mathrm{H}_{4}^{-}$ & $N-i \operatorname{Pr}-4$-pyridyl & $N-i \operatorname{Pr}-4$-pyridyl & $N-i \mathrm{Pr}-4$-pyridyl & $2 \mathrm{H}$ & From 139: Isopropyl iodide, DMF, $40^{\circ} \mathrm{C}, 3 \mathrm{~h} ; 93 \%$. & {$[189,190]$} \\
\hline 30 & 143 & 4-( $\mathrm{AcOGlcO})-\mathrm{C}_{6} \mathrm{H}_{4}^{-}$ & $N-n$-Octyl-4-pyridyl & $N-n$-Octyl-4-pyridyl & $N-n$-Octyl-4-pyridyl & $2 \mathrm{H}$ & From 139: $n$-octyl iodide, DMF, $40^{\circ} \mathrm{C}, 3 \mathrm{~h} ; 93 \%$. & [189] \\
\hline 31 & 144 & $4-(\mathrm{GlcO})-\mathrm{C}_{6} \mathrm{H}_{4^{-}}$ & $N$-Me-4-pyridyl & $N$-Me-4-pyridyl & $N$-Me-4-pyridyl & $2 \mathrm{H}$ & From 141: $\mathrm{Et}_{3} \mathrm{~N}-\mathrm{MeOH}-\mathrm{H}_{2} \mathrm{O}(10 / 10 / 1, \mathrm{v} / \mathrm{v} / \mathrm{v}), 0{ }^{\circ} \mathrm{C}, 24 \mathrm{~h} ; 91 \%$. & {$[189,190]$} \\
\hline
\end{tabular}




\begin{tabular}{|c|c|c|}
\hline 32 & 145 & 4-(GlcO)- $-\mathrm{C}_{6} \mathrm{H}_{4^{-}}$ \\
\hline 33 & 146 & 4-(GlcO)- $-\mathrm{C}_{6} \mathrm{H}_{4^{-}}$ \\
\hline 34 & 147 & 2-(AcOMalO) $-\mathrm{C}_{6} \mathrm{H}_{4}-$ \\
\hline 35 & 148 & 4-(AcOMalO) $-\mathrm{C}_{6} \mathrm{H}_{4}-$ \\
\hline 36 & 149 & 2-(MalO)- $\mathrm{C}_{6} \mathrm{H}_{4^{-}}^{-}$ \\
\hline 37 & 150 & 4-(MalO)- $-\mathrm{C}_{6} \mathrm{H}_{4^{-}}$ \\
\hline 38 & 151 & 2-( $(\mathrm{AcOLacO})-\mathrm{C}_{6} \mathrm{H}_{4-}$ \\
\hline 39 & 152 & 4-( $\mathrm{AcOLacO})-\mathrm{C}_{6} \mathrm{H}_{4-}$ \\
\hline 40 & 153 & 2-( $(\mathrm{LacO})-\mathrm{C}_{6} \mathrm{H}_{4^{-}}$ \\
\hline 41 & 154 & 4-( $(\mathrm{LacO})-\mathrm{C}_{6} \mathrm{H}_{4^{-}}$ \\
\hline 42 & 155 & 2-( $(\mathrm{AcOMalO})-\mathrm{C}_{6} \mathrm{H}_{4}-$ \\
\hline 43 & 156 & 4-( AcOMalO) $-\mathrm{C}_{6} \mathrm{H}_{4}-$ \\
\hline 44 & 157 & 2-(MalO)- $\mathrm{C}_{6} \mathrm{H}_{4^{-}}$ \\
\hline 45 & 158 & 4-( $\mathrm{MalO})-\mathrm{C}_{6} \mathrm{H}_{4^{-}}$ \\
\hline 46 & 159 & 2-( $(\mathrm{AcOLacO})-\mathrm{C}_{6} \mathrm{H}_{4^{-}}$ \\
\hline 47 & 160 & 4-( $(\mathrm{AcOLacO})-\mathrm{C}_{6} \mathrm{H}_{4-}$ \\
\hline 48 & 161 & 2-( $(\mathrm{LacO})-\mathrm{C}_{6} \mathrm{H}_{4^{-}}$ \\
\hline 49 & 162 & 4-( $(\mathrm{LacO})-\mathrm{C}_{6} \mathrm{H}_{4^{-}}-$ \\
\hline 50 & 163 & $4-(\mathrm{AcOGlcO})-\mathrm{C}_{6} \mathrm{H}_{4^{-}}$ \\
\hline 51 & 164 & 4-( $\mathrm{AcOGlcO})-\mathrm{C}_{6} \mathrm{H}_{4^{-}}$ \\
\hline 52 & 165 & 4-(AcOGlcO) $-\mathrm{C}_{6} \mathrm{H}_{4^{-}}$ \\
\hline 53 & 166 & 4-(AcOGlcO) $-\mathrm{C}_{6} \mathrm{H}_{4^{-}}$ \\
\hline 54 & 167 & 4-( $\mathrm{AcOGlcO})-\mathrm{C}_{6} \mathrm{H}_{4}-$ \\
\hline
\end{tabular}

\begin{tabular}{|c|c|}
\hline $\begin{array}{l}N-i \text { Pr-4-pyridyl } \\
N \text {-n-Octyl-4-pyridyl } \\
\text { 4-Pyridyl }\end{array}$ & $\begin{array}{l}N-i \text { Pr-4-pyridyl } \\
N-n \text {-Octyl-4-pyridyl } \\
\text { 4-Pyridyl }\end{array}$ \\
\hline 4-Pyridyl & 4-Pyridyl \\
\hline 4-Pyridyl & 4-Pyridyl \\
\hline 4-Pyridyl & 4-Pyridyl \\
\hline 4-Pyridyl & 4-Pyridyl \\
\hline 4-Pyridyl & 4-Pyridyl \\
\hline 4-Pyridyl & 4-Pyridyl \\
\hline 4-Pyridyl & 4-Pyridyl \\
\hline$N$-Me-4-pyridyl & $N$-Me-4-pyridyl \\
\hline$N$-Me-4-pyridyl & $N$-Me-4-pyridyl \\
\hline$N$-Me-4-pyridyl & $N$-Me-4-pyridyl \\
\hline$N$-Me-4-pyridyl & $N$-Me-4-pyridyl \\
\hline$N$-Me-4-pyridyl & $N$-Me-4-pyridyl \\
\hline$N$-Me-4-pyridyl & $N$-Me-4-pyridyl \\
\hline$N$-Me-4-pyridyl & $N$-Me-4-pyridyl \\
\hline$N$-Me-4-pyridyl & $N$-Me-4-pyridyl \\
\hline 4-( $\mathrm{AcOGlcO})-\mathrm{C}_{6} \mathrm{H}_{4-}$ & 4-( $\mathrm{AcOGlcO})-\mathrm{C}_{6} \mathrm{H}_{4}-$ \\
\hline 4-(AcOGlcO) $-\mathrm{C}_{6} \mathrm{H}_{4-}^{-}$ & 4-( $\mathrm{AcOGlcO})-\mathrm{C}_{6} \mathrm{H}_{4}-$ \\
\hline 4-(AcOGlcO) $-\mathrm{C}_{6} \mathrm{H}_{4}-$ & 4-( $\mathrm{AcOGlcO})-\mathrm{C}_{6} \mathrm{H}_{4-}$ \\
\hline 4-(AcOGlcO) $-\mathrm{C}_{6} \mathrm{H}_{4-}$ & 4-( $\mathrm{AcOGlcO})-\mathrm{C}_{6} \mathrm{H}_{4-}$ \\
\hline 4-( $(\mathrm{AcOGlcO})-\mathrm{C}_{6} \mathrm{H}_{4-}$ & 4-( $\mathrm{AcOGlcO})-\mathrm{C}_{6} \mathrm{H}_{4}-$ \\
\hline 4-(GlcO)- $-\mathrm{C}_{6} \mathrm{H}_{4^{-}}$ & 4-(GlcO) $-\mathrm{C}_{6} \mathrm{H}_{4^{-}}$ \\
\hline
\end{tabular}
-n-Octyl-4-pyridyl
$N-i \operatorname{Pr}-4$-pyridyl 4-Pyridyl

4-Pyridyl 4-Pyridyl 4-Pyridyl 4-Pyridyl 4-Pyridyl 4-Pyridyl 4-Pyridyl $N$-Me-4-pyridyl $\mathrm{N}$-Me-4-pyridyl $N$-Me-4-pyridyl $N$-Me-4-pyridyl $N$-Me-4-pyridyl $N$-Me-4-pyridyl $N$-Me-4-pyridyl $\mathrm{N}$-Me-4-pyridyl 2-HO- $\mathrm{C}_{6} \mathrm{H}_{4}-$

4-HO- $\mathrm{C}_{6} \mathrm{H}_{4-}$

2- $\left(\mathrm{I}^{(} \mathrm{CH}_{2}\right)_{3} \mathrm{O}-\mathrm{C}_{6} \mathrm{H}_{4}-$ 4- $\left(\mathrm{I}^{(} \mathrm{CH}_{2}\right)_{3} \mathrm{O}-\mathrm{C}_{6} \mathrm{H}_{4-}$ 4-Pyridyl

$o / p-\mathrm{C}_{6} \mathrm{H}_{4}$-Arg-GlyAsp

\begin{tabular}{|c|c|}
\hline 4-( $\mathrm{AcOLacO})-\mathrm{C}_{6} \mathrm{H}_{4^{-}}$ & $\mathrm{H}$ \\
\hline 4-MeO- $\mathrm{C}_{6} \mathrm{H}_{4}-$ & $\mathrm{H}$ \\
\hline $\begin{array}{l}\text { 4-(AcOLacO)- } \mathrm{C}_{6} \mathrm{H}_{4^{-}} \\
\text {4-MeO- } \mathrm{C}_{6} \mathrm{H}_{4^{-}} \\
\text {4-( } \mathrm{LacO})-\mathrm{C}_{6} \mathrm{H}_{4^{-}} \\
1,2,4 \text {-tri-O-blenyl- } D- \\
\text { arabino-tetritol-1-yl }\end{array}$ & $\begin{array}{l}\mathrm{H} \\
\mathrm{H} \\
\mathrm{H} \\
4-\mathrm{F}-\mathrm{C}_{6} \mathrm{H}_{4-}\end{array}$ \\
\hline
\end{tabular}

( $\alpha$-BnOGlcC)-

$(\alpha-\mathrm{Gal})-\mathrm{CH}_{2}-$

$2 \mathrm{H}$
From 142 Like entry $31 ; 91 \%$

From 143 Like enty $31 ; 90 \%$ EtCOOH $/ \mathrm{Ac}_{2} \mathrm{O}$, reflux, $1.5 \mathrm{~h} ; 6 \%$

ike entry 34 with 4-(AcOMalO)- $\mathrm{C}_{6} \mathrm{H}_{4}-\mathrm{CHO} ; 7 \%$

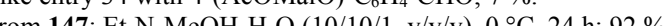
From 148: Like entry $36 ; 92 \%$

ike entry 34 with 2-(AcOLacO)- $\mathrm{C}_{6} \mathrm{H}_{4}-\mathrm{CHO} ; 6 \%$

Like entry 34 with 4-(AcOLacO)- $\mathrm{C}_{6} \mathrm{H}_{4}-\mathrm{CHO} ; 7 \%$

From 151: $\mathrm{Et}_{3} \mathrm{~N}-\mathrm{MeOH}-\mathrm{H}_{2} \mathrm{O}(10 / 10 / 1, \mathrm{v} / \mathrm{v} / \mathrm{v}), 0{ }^{\circ} \mathrm{C}, 24 \mathrm{~h} ; 92 \%$

From 152: Like entry 40; $92 \%$.

rom 147: Methyl iodide, DMF, $40^{\circ} \mathrm{C}, 3 \mathrm{~h} ; 90 \%$.

148: Like entry $42 ; 93 \%$.

rom 149: Like entry $42 ; 86 \%$.

From 150: Like entry $42 ; 98 \%$.

From 151: Like entry $42 ; 90 \%$

152: Like entry $42 ; 90 \%$.

rom 153: Like entry $42 ; 86 \%$

rom 154: Like entry $42 ; 98 \%$.

Pyrrole, 4-(AcOGlcO)- $\mathrm{C}_{6} \mathrm{H}_{4}$-CHO, 2-hydroxybenzaldehyde, $\mathrm{CH}_{2} \mathrm{Cl}_{2}$,

$\mathrm{BF}_{3} \cdot \mathrm{OEt}_{2}$, r.t. $24 \mathrm{~h}$, then $p$-chloranil, $1 \mathrm{~h}$, reflux; $5-13 \%$

ike entry 51 with 4-hydroxybenzaldehyde; $5-13 \%$.

From 163: $\mathrm{I}\left(\mathrm{CH}_{2}\right)_{3} \mathrm{I}, \mathrm{K}_{2} \mathrm{CO}_{3}, \mathrm{DMF}, 6 \mathrm{~h} ; 80-85 \%$

From 164: As entry $52 ; 80-85 \%$.

Pyrrole, 4-pyridinecarboxaldehyde, 4-(AcOGlcO)- $\mathrm{C}_{6} \mathrm{H}_{4}-\mathrm{CHO}$,

EtCOOH $/ \mathrm{Ac}_{2} \mathrm{O}$, reflux, $1 \mathrm{~h} ; 7 \%$.

a) $4 \times$ Pyrrole, $3 \times 4$-(AcOGlcO)- $\mathrm{C}_{6} \mathrm{H}_{4}-\mathrm{CHO}, 2$ or 4-(hydroxy)- $\mathrm{C}_{6} \mathrm{H}_{4}$ $\mathrm{CHO} \mathrm{CH}_{2} \mathrm{Cl}_{2}, \mathrm{BF}_{3} \cdot \mathrm{OEt}_{2}$, r.t., $24 \mathrm{~h}$, then $p$-chloranil, $1 \mathrm{~h}$, reflux or: EtCOOH $/ \mathrm{Ac}_{2} \mathrm{O}$, reflux, $1 \mathrm{~h} ; 8-12 \%$. b) Ethyl 4-bromobutyrate, $\mathrm{K}_{2} \mathrm{CO}_{3}$, DMF, r.t., 18 h; $82-85 \%$. c) TFA, $\mathrm{CH}_{2} \mathrm{Cl}_{2}$, r.t., 4 h; $95 \%$. d) Wang resin-COCH

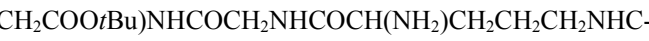

(NH)NH-(2,2,4,6,7-pentamethyldihydrobenzofuran-5-sulfonyl), $N, N^{\prime}$ diisopropylcarbodiimide, $\mathrm{HOBt}$, DMF, r.t., $24 \mathrm{~h} ; \mathrm{MeONa} / \mathrm{MeOH} /$

$\mathrm{CH}_{2} \mathrm{Cl}_{2}$, r.t., 2 h; $53-56 \%$.

$2 \mathrm{H}$ Dipyrromethane, 4-(AcOLacO)- $\mathrm{C}_{6} \mathrm{H}_{4}-\mathrm{CHO}, \mathrm{CH}_{2} \mathrm{Cl}_{2}, \mathrm{TFA}, 15 \mathrm{~h}$, then $p$-chloranil; $19 \%$.

$2 \mathrm{H} \quad$ Dipyrromethane, 4-anis aldehyde, $\mathrm{CH}_{2} \mathrm{Cl}_{2}, \mathrm{TFA}, 15 \mathrm{~h}$, then $p$-chloranil, $1 \mathrm{~h} ; 23 \%$

Fe(II) From 169: $\mathrm{FeCl}_{2}$, DMF, $60{ }^{\circ} \mathrm{C}, 6 \mathrm{~h} ; 78 \%$

$\mathrm{Fe}(\mathrm{II}) \quad$ From 170: Similar to entry $58 ; 69 \%$.

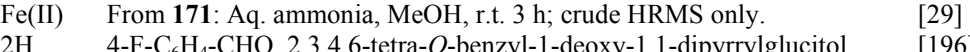
$\mathrm{CF}_{3} \mathrm{COOH}, \mathrm{CH}_{2} \mathrm{Cl}$, r.t., $3 \mathrm{~h}$, then DDQ; $20 \%$.

$2 \mathrm{H}$ 2,6-Anhydro-1,3,4,5-tetra- $O$-benzyl-7,8-dideoxy-8,8-di-1H-pyrrol-2yl-D-glycerol- $L$-galacto-octitol, $\mathrm{C}_{6} \mathrm{~F}_{5}$-CHO, $\mathrm{CH}_{2} \mathrm{Cl}_{2}$, TFA, r.t., $20 \mathrm{~h}$ then DDQ; $22 \%$. Or: Pentafluorophenyldipyrrylmethane, $(\alpha-\mathrm{BnOGal})-$ $\mathrm{CH}_{2}$-CHO, TFA, $\mathrm{CH}_{2} \mathrm{Cl}_{2}$, r.t., 20 h, then DDQ, $3 \mathrm{~h} ; 27 \%$.

$2 \mathrm{H}$ 2,6-Anhydro-1,3,4,5-tetra-O-benzyl-7,8-dideoxy-8,8-di-1 $\mathrm{H}$-pyrrol-2yl-D-glycero- $L$-gulo-octitol, $\mathrm{C}_{6} \mathrm{~F}_{5}$ - $\mathrm{CHO}, \mathrm{CH}_{2} \mathrm{Cl}_{2}$, TFA, r.t., $20 \mathrm{~h}$, then yl- $D$-glycero- $L$-gulo-octitol, $\mathrm{C}_{6} \mathrm{~F}_{2}-\mathrm{C}$
$\mathrm{DDQ}$, crude, MS and UV only.

2H From 175: $\mathrm{CH}_{2} \mathrm{Cl}_{2}, \mathrm{MeOH}, 10 \% \mathrm{Pd}-\mathrm{C}, \mathrm{H}_{2}$, r.t., 24 h; $63 \%$. 


\begin{tabular}{|c|c|c|c|c|c|}
\hline 65 & 178 & 4-( $\beta-\mathrm{BnOGlcC})-\mathrm{C}_{6} \mathrm{H}_{4^{-}}$ & Phenyl & Phenyl & Phenyl \\
\hline 66 & 179 & $4-(\beta-\mathrm{GlcC})-\mathrm{C}_{6} \mathrm{H}_{4^{-}}$ & Phenyl & Phenyl & Phenyl \\
\hline 67 & 180 & 4-(COOMe)- $\mathrm{C}_{6} \mathrm{H}_{4^{-}}^{-}$ & $4-\mathrm{Me}^{-} \mathrm{C}_{6} \mathrm{H}_{4}^{-}$ & 4-Me- $\mathrm{C}_{6} \mathrm{H}_{4^{-}}$ & $4-\mathrm{Me}-\mathrm{C}_{6} \mathrm{H}_{4^{-}}$ \\
\hline 68 & 181 & 4-(COOMe) $-\mathrm{C}_{6} \mathrm{H}_{4^{-}}$ & 4-Me- $\mathrm{C}_{6} \mathrm{H}_{4^{-}}$ & 4-(COOMe)- $\mathrm{C}_{6} \mathrm{H}_{4^{-}}$ & $4-\mathrm{Me}-\mathrm{C}_{6} \mathrm{H}_{4^{-}}$ \\
\hline 69 & 182 & 4-(COOMe)- $\mathrm{C}_{6} \mathrm{H}_{4^{-}}$ & 4-(COOMe)- $\mathrm{C}_{6} \mathrm{H}_{4^{-}}$ & 4-Me- $\mathrm{C}_{6} \mathrm{H}_{4^{-}}$ & $4-\mathrm{Me}-\mathrm{C}_{6} \mathrm{H}_{4-}$ \\
\hline 70 & 183 & 4-(COOMe) $-\mathrm{C}_{6} \mathrm{H}_{4^{-}}$ & 4-(COOMe) $-\mathrm{C}_{6} \mathrm{H}_{4^{-}}$ & 4-(COOMe)- $\mathrm{C}_{6} \mathrm{H}_{4^{-}}$ & $4-\mathrm{Me}-\mathrm{C}_{6} \mathrm{H}_{4^{-}}$ \\
\hline 71 & 184 & 4-(COOMe)- $\mathrm{C}_{6} \mathrm{H}_{4^{-}}$ & 4-(COOMe) $-\mathrm{C}_{6} \mathrm{H}_{4^{-}}$ & 4-(COOMe)- $\mathrm{C}_{6} \mathrm{H}_{4^{-}}$ & 4-(COOMe) $-\mathrm{C}_{6} \mathrm{H}_{4}$ \\
\hline 72 & 185 & 4-(GalO-isop-CO)- $\mathrm{C}_{6} \mathrm{H}_{4}-$ & 4-Me- $\mathrm{C}_{6} \mathrm{H}_{4^{-}}$ & 4-Me- $\mathrm{C}_{6} \mathrm{H}_{4^{-}}$ & $4-\mathrm{Me}-\mathrm{C}_{6} \mathrm{H}_{4}^{-}$ \\
\hline 73 & 186 & 4-(GalO-isop-CO)- $\mathrm{C}_{6} \mathrm{H}_{4}-$ & 4-Me- $\mathrm{C}_{6} \mathrm{H}_{4}-$ & $\begin{array}{l}\text { 4-(GalO-isop-CO)- } \\
\mathrm{C}_{6} \mathrm{H}_{4^{-}}\end{array}$ & $4-\mathrm{Me}-\mathrm{C}_{6} \mathrm{H}_{4}-$ \\
\hline 74 & 187 & 4-(GalO-isop-CO)- $\mathrm{C}_{6} \mathrm{H}_{4}-$ & $\begin{array}{l}\text { 4-(GalO-isop-CO)- } \\
\mathrm{C}_{6} \mathrm{H}_{4^{-}}\end{array}$ & 4-Me- $\mathrm{C}_{6} \mathrm{H}_{4^{-}}$ & $4-\mathrm{Me}-\mathrm{C}_{6} \mathrm{H}_{4}-$ \\
\hline 75 & 188 & 4-(GalO-isop-CO)- $\mathrm{C}_{6} \mathrm{H}_{4}-$ & $\begin{array}{l}\text { 4-(GalO-isop-CO)- } \\
\mathrm{C}_{6} \mathrm{H}_{4^{-}}\end{array}$ & $\begin{array}{l}\text { 4-(GalO-isop-CO)- } \\
\mathrm{C}_{6} \mathrm{H}_{4^{-}}\end{array}$ & $4-\mathrm{Me}-\mathrm{C}_{6} \mathrm{H}_{4}-$ \\
\hline 76 & 189 & 4-(GalO-isop-CO)- $\mathrm{C}_{6} \mathrm{H}_{4^{-}}$ & $\begin{array}{l}\text { 4-(GalO-isop-CO)- } \\
\mathrm{C}_{6} \mathrm{H}_{4^{-}}\end{array}$ & $\begin{array}{l}\text { 4-(GalO-isop-CO)- } \\
\mathrm{C}_{6} \mathrm{H}_{4^{-}}\end{array}$ & $\begin{array}{l}\text { 4-(GalO-isop-CO)- } \\
\mathrm{C}_{6} \mathrm{H}_{4^{-}}\end{array}$ \\
\hline 77 & 190 & 4-(HO)- $\mathrm{C}_{6} \mathrm{H}_{4^{-}}$ & Phenyl & Phenyl & Phenyl \\
\hline 78 & 191 & 4-( $\mathrm{AcOGlcO})-\mathrm{C}_{6} \mathrm{H}_{4^{-}}$ & Phenyl & Phenyl & Phenyl \\
\hline 79 & 192 & 4-(GlcO)- $\mathrm{C}_{6} \mathrm{H}_{4^{-}}$ & Phenyl & Phenyl & Phenyl \\
\hline 80 & 193 & 4-( GalO-isop-CO)- $\mathrm{C}_{6} \mathrm{H}_{4^{-}}$ & Phenyl & Phenyl & Phenyl \\
\hline 81 & 194 & 4- $(\alpha / \beta-\mathrm{GalOH}-\mathrm{CO})-\mathrm{C}_{6} \mathrm{H}_{4^{-}}$ & Phenyl & Phenyl & Phenyl \\
\hline 82 & 195 & 4-( $(\alpha-\mathrm{GalOMe}-\mathrm{CO})-\mathrm{C}_{6} \mathrm{H}_{4--}$ & Phenyl & Phenyl & Phenyl \\
\hline 83 & 196 & 4-( $\beta-\mathrm{GalOMe}-\mathrm{CO})-\mathrm{C}_{6} \mathrm{H}_{4^{-}}$ & Phenyl & Phenyl & Phenyl \\
\hline 84 & 197 & 4-( $(\mathrm{COOH})-\mathrm{C}_{6} \mathrm{H}_{4^{-}}^{-}$ & Phenyl & Phenyl & Phenyl \\
\hline 85 & 198 & $4-\mathrm{C}_{6} \mathrm{~F}_{5}-\mathrm{O}-\mathrm{C}_{6} \mathrm{H}_{4^{-}}$ & Phenyl & Phenyl & Phenyl \\
\hline 86 & 199 & 4-(4-GalO-isop- $\left.\mathrm{C}_{6} \mathrm{~F}_{4}-\mathrm{O}\right)-\mathrm{C}_{6} \mathrm{H}_{4^{-}}$ & Phenyl & Phenyl & Phenyl \\
\hline 87 & 200 & $4-\left(4-\alpha / \beta-\mathrm{GalOH}-\mathrm{C}_{6} \mathrm{~F}_{4}-\mathrm{O}\right)-\mathrm{C}_{6} \mathrm{H}_{4^{-}}$ & Phenyl & Phenyl & Phenyl \\
\hline 88 & 201 & 4-(GalO-isop)- $\mathrm{C}_{6} \mathrm{~F}_{4}$ & $\mathrm{C}_{6} \mathrm{~F}_{5^{-}}$ & $\mathrm{C}_{6} \mathrm{~F}_{5^{-}}$ & $\mathrm{C}_{6} \mathrm{~F}_{5^{-}}$ \\
\hline 89 & 202 & $4-(\alpha / \beta-\mathrm{GalOH})-\mathrm{C}_{6} \mathrm{~F}_{4^{-}}$ & $\mathrm{C}_{6} \mathrm{~F}_{5^{-}}$ & $\mathrm{C}_{6} \mathrm{~F}_{5^{-}}$ & $\mathrm{C}_{6} \mathrm{~F}_{5^{-}}$ \\
\hline 90 & 203 & 4-HO- $\left(\mathrm{C}_{2} \mathrm{H}_{4}-\mathrm{O}\right)_{2}-\mathrm{C}_{6} \mathrm{H}_{4}-$ & $\begin{array}{l}\text { 4-( } \mathrm{HO}-\left(\mathrm{C}_{2} \mathrm{H}_{4}-\mathrm{O}\right)_{2^{-}} \\
\mathrm{C}_{6} \mathrm{H}_{4^{-}}\end{array}$ & $\begin{array}{l}\text { 4-( } \mathrm{HO}-\left(\mathrm{C}_{2} \mathrm{H}_{4}-\mathrm{O}\right)_{2^{-}} \\
\mathrm{C}_{6} \mathrm{H}_{4^{-}}\end{array}$ & Phenyl \\
\hline 91 & 204 & 4-[GalO- $\left.\left(\mathrm{C}_{2} \mathrm{H}_{4}-\mathrm{O}\right)_{2}\right]-\mathrm{C}_{6} \mathrm{H}_{4^{-}}$ & $\begin{array}{l}\left.\text { 4-[GalO- }\left(\mathrm{C}_{2} \mathrm{H}_{4}-\mathrm{O}\right)_{2}\right]- \\
\mathrm{C}_{6} \mathrm{H}_{4^{-}}\end{array}$ & $\begin{array}{l}\left.\text { 4-[GalO- }\left(\mathrm{C}_{2} \mathrm{H}_{4}-\mathrm{O}\right)_{2}\right]- \\
\mathrm{C}_{6} \mathrm{H}_{4^{-}}\end{array}$ & Phenyl \\
\hline 92 & 205 & $4-\left[\alpha-\mathrm{GalO}-\left(\mathrm{C}_{2} \mathrm{H}_{4}-\mathrm{O}\right)_{2}\right]-\mathrm{C}_{6} \mathrm{H}_{4}-$ & $\begin{array}{l}\text { 4-[ } \alpha-\mathrm{GalO}-\left(\mathrm{C}_{2} \mathrm{H}_{4^{-}}\right. \\
\left.\mathrm{O})_{2}\right]-\mathrm{C}_{6} \mathrm{H}_{4^{-}}\end{array}$ & $\begin{array}{l}\text { 4-[ } \alpha-\mathrm{GalO}-\left(\mathrm{C}_{2} \mathrm{H}_{4^{-}}\right. \\
\left.\mathrm{O})_{2}\right]-\mathrm{C}_{6} \mathrm{H}_{4^{-}}\end{array}$ & Phenyl \\
\hline 93 & 206 & 4-[ManO- $\left.\left(\mathrm{C}_{2} \mathrm{H}_{4}-\mathrm{O}\right)_{2}\right]-\mathrm{C}_{6} \mathrm{H}_{4^{-}}$ & $\begin{array}{l}\left.\text { 4-[ManO- }\left(\mathrm{C}_{2} \mathrm{H}_{4}-\mathrm{O}\right)_{2}\right]- \\
\mathrm{C}_{6} \mathrm{H}_{4^{-}}\end{array}$ & $\begin{array}{l}\left.\text { 4-[ManO- }\left(\mathrm{C}_{2} \mathrm{H}_{4}-\mathrm{O}\right)_{2}\right]- \\
\mathrm{C}_{6} \mathrm{H}_{4^{-}}\end{array}$ & Phenyl \\
\hline 94 & 207 & 4-[GalS- $\left.\left(\mathrm{C}_{2} \mathrm{H}_{4}-\mathrm{O}\right)_{2}\right]-\mathrm{C}_{6} \mathrm{H}_{4}{ }^{-}$ & $\begin{array}{l}4-\left[\text { GalS- }\left(\mathrm{C}_{2} \mathrm{H}_{4}-\mathrm{O}\right)_{2}\right]- \\
\mathrm{C}_{6} \mathrm{H}_{4^{-}}\end{array}$ & $\begin{array}{l}4-\left[\mathrm{GalS}-\left(\mathrm{C}_{2} \mathrm{H}_{4}-\mathrm{O}\right)_{2}\right]- \\
\mathrm{C}_{6} \mathrm{H}_{4-}\end{array}$ & Phenyl \\
\hline 95 & 208 & 4-[ManS- $\left.\left(\mathrm{C}_{2} \mathrm{H}_{4}-\mathrm{O}\right)_{2}\right]-\mathrm{C}_{6} \mathrm{H}_{4^{-}}$ & $\begin{array}{l}4-\left[\mathrm{ManS}-\left(\mathrm{C}_{2} \mathrm{H}_{4}-\mathrm{O}\right)_{2}\right]- \\
\mathrm{C}_{6} \mathrm{H}_{4^{-}}\end{array}$ & $\begin{array}{l}\left.\text { 4-[ManS- }\left(\mathrm{C}_{2} \mathrm{H}_{4}-\mathrm{O}\right)_{2}\right]- \\
\mathrm{C}_{6} \mathrm{H}_{4^{-}}\end{array}$ & Phenyl \\
\hline 96 & 209 & $N$-(GalO-isop)-4-pyridyl & 4-pyridyl & 4-pyridyl & 4-pyridyl \\
\hline 97 & 210 & $N$-( $\alpha / \beta$-GalOH)-4-pyridyl & 4-pyridyl & 4-pyridyl & 4-pyridyl \\
\hline 98 & 211 & $N$-(GalOH)-4-pyridyl & $N$-Me-4-pyridyl & $\mathrm{N}$-Me-4-pyridyl & $\mathrm{N}$-Me-4-pyridyl \\
\hline 99 & 212 & $N$ - $(\alpha / \beta-\mathrm{GalOH})-4$-pyridyl & $N$-Me-4-pyridyl & $N$-Me-4-pyridyl & $N$-Me-4-pyridyl \\
\hline 100 & 213 & $2-\mathrm{HO}-\mathrm{C}_{6} \mathrm{H}_{4^{-}}$ & 4-Me- $\mathrm{C}_{6} \mathrm{H}_{4^{-}}$ & 4-Me- $\mathrm{C}_{6} \mathrm{H}_{4^{-}}$ & $4-\mathrm{Me}-\mathrm{C}_{6} \mathrm{H}_{4^{-}}$ \\
\hline
\end{tabular}
$2 \mathrm{H} 4$ Pyrrole +3 benzaldehyde $+14-\left(\beta-\mathrm{BnOGlcC}^{2}-\mathrm{C}_{6} \mathrm{H}_{4}-\mathrm{CHO}, \mathrm{BF}_{3} \cdot \mathrm{OEt}_{2}\right.$,

From 178: $\mathrm{H}_{2}$, Pd $10 \%$ on carbon, EtOAc-MeOH, r.t., 16 h; $98 \%$.

From 180: MeLi, Gal-isop-H, THF, $8 \mathrm{~h}, 80-90{ }^{\circ} \mathrm{C} ; 38 \%$.

From 181: MeLi, Gal-isop-H, THF, $2.5 \mathrm{~h}, 80-90^{\circ} \mathrm{C} ; 50 \%$.

From 182: Like entry $73 ; 28 \%$

From 183: Like entry 73, 5 h; $7 \%$.

From 184: Like entry $73,4.5 \mathrm{~h}, 80-90{ }^{\circ} \mathrm{C} ; 5 \%$.

$4 \times$ Pyrrole $+1.2 \times 4$-hydroxybenzaldehyde, $3 \times$ benzaldehyde,

EtCOOH, nitrobenzene, reflux, $1 \mathrm{~h} ; 15 \%$.

From 190: (AcOGlc-Br), $\mathrm{CH}_{2} \mathrm{Cl}_{2}, \mathrm{Na}_{2} \mathrm{SO}_{4}, \mathrm{Ag}_{2} \mathrm{CO}_{3}, 24$ h, r.t., TFA, 10 $\min$, r.t.; $66 \%$

From 191: $\mathrm{NaOMe}, \mathrm{MeOH}, \mathrm{CH}_{2} \mathrm{Cl}_{2}$, r.t. 6 h; $80 \%$

From 197: I-Gal-isop, DMF, $\mathrm{K}_{2} \mathrm{CO}_{3}, 30 \mathrm{~h}, 100{ }^{\circ} \mathrm{C} ; 50 \%$

From 193: TFA- $\mathrm{H}_{2} \mathrm{O}(9: 1, \mathrm{v} / \mathrm{v}), 30 \mathrm{~min}$.; $94 \%$.

From 194: $\mathrm{MeOH}, \mathrm{HCl}, \mathrm{CHCl}_{3}, 4$ h, r.t..; $45 \%$

From 194: $\mathrm{MeOH}, \mathrm{HCl}, \mathrm{CHCl}_{3}, 4$ h, r.t.; $40 \%$ nitrobenzene, reflux, $1 \mathrm{~h} ; 13 \%$

From 190: NaH, DMF, hexafluorobenzene, $70{ }^{\circ} \mathrm{C}, 4 \mathrm{~h} ; 85 \%$

From 198: $\mathrm{H}$-GalO-isop, DMF, NaH, $70{ }^{\circ} \mathrm{C}, 1.5 \mathrm{~h} ; 70 \%$.

From 199: TFA: $\mathrm{H}_{2} \mathrm{O}(9: 1, \mathrm{v} / \mathrm{v})$, r.t., 30 min.; $93 \%$.

$\mathbf{8 3}+$ H-GalO-isop, toluene, NaH, reflux, $24 \mathrm{~h} ; 40 \%$

From 201: TFA: $\mathrm{H}_{2} \mathrm{O}(9: 1, \mathrm{v} / \mathrm{v})$, r.t., 30 min.; $90 \%$.

5,10,15-Tris( $p$-hydroxyphenyl)-20-phenyl porphyrin, 2-(2-chloro-

ethoxy)-ethanal, $\mathrm{KI}, \mathrm{K}_{2} \mathrm{CO}_{3}$, DMF, $100{ }^{\circ} \mathrm{C}, 4 \mathrm{~h} ; 90 \%$

Like entry 88 with (AcOGal)- $\left(\mathrm{C}_{2} \mathrm{H}_{4}-\mathrm{O}\right)_{2}-\mathrm{Br}, \mathrm{K}_{2} \mathrm{CO}_{3}$, DMF, $60^{\circ} \mathrm{C}, 24 \mathrm{~h}$;

$38 \%$. Then: $\mathrm{NaOMe}, \mathrm{MeOH}, \mathrm{CH}_{2} \mathrm{Cl}_{2}$, r.t., $1.5 \mathrm{~h} ; 98 \%$.

Like entry 88 with ( $\alpha$-AcOGal)- $\left(\mathrm{C}_{2} \mathrm{H}_{4}-\mathrm{O}\right)_{2}-\mathrm{Br}$; $51 \%$. Then: $98 \%$;

contains $\sim 10 \%$ of the $\beta$-configuration.

$2 \mathrm{H} \quad$ Like entry 88 with (Man)- $\left(\mathrm{C}_{2} \mathrm{H}_{4}-\mathrm{O}\right)_{2}-\mathrm{Br} ; 23 \%$. Then: $\mathrm{NaOMe}, \mathrm{MeOH}$, $\mathrm{CH}_{2} \mathrm{Cl}_{2}$, r.t., $1.5 \mathrm{~h} ; 100 \%$

$2 \mathrm{H} \quad 5,10,15$-Tris $\{p$ - $O$-[2-(2-bromo-ethoxy)-ethoxy]phenyl $\}$-20-phenyl porphyrin, GalS-H, $\mathrm{K}_{2} \mathrm{CO}_{3}$, acetone, r.t., $24 \mathrm{~h} ; 73 \%$. Then: NaOMe, $\mathrm{MeOH}, \mathrm{CH}_{2} \mathrm{Cl}_{2}$, r.t., $1.5 \mathrm{~h} ; 98 \%$

$2 \mathrm{H} \quad$ Like entry 92 with 1-thio-2,3,4,6-tetraacetyl- $\alpha$-mannose; $86 \%$. Then: $\mathrm{NaOMe}, \mathrm{MeOH}, \mathrm{CH}_{2} \mathrm{Cl}_{2}, \mathrm{r}, \mathrm{t}, 1.5 \mathrm{~h} ; 100 \%$

I-Gal-isop (1 equiv.), 5,10,15,20-tetrakis(4-pyridyl)porphyrin (1

equiv.), 1,2,4-trichlorobenzene, $2 \mathrm{~h}$, reflux; $50 \%$.

From 209: TFA: $\mathrm{H}_{2} \mathrm{O}(9: 1, \mathrm{v} / \mathrm{v}), 1$ h, r.t.; $90 \%$.

From 209. Iodomethane, DMF, $40{ }^{\circ} \mathrm{C} ; 90 \%$

From 210. Iodomethane, DMF $40{ }^{\circ} \mathrm{C} \cdot 90 \%$

$4 \times$ pyrrole, 2 -hydroxybenzaldehyde, $3 \times 4$-tolualdehyde,
[172] 


\begin{tabular}{|c|c|c|c|c|c|}
\hline 101 & 214 & $4-\mathrm{HO}-\mathrm{C}_{6} \mathrm{H}_{4}-$ & 4-Me- $\mathrm{C}_{6} \mathrm{H}_{4}^{-}$ & $4-\mathrm{Me}-\mathrm{C}_{6} \mathrm{H}_{4^{-}}$ & 4-Me- $\mathrm{C}_{6} \mathrm{H}_{4}-$ \\
\hline 102 & 215 & $2-\left(\mathrm{OC}_{3} \mathrm{H}_{6}-\mathrm{NH}-\mathrm{Boc}\right)-\mathrm{C}_{6} \mathrm{H}_{4^{-}}$ & 4-Me- $\mathrm{C}_{6} \mathrm{H}_{4}^{-}$ & $4-\mathrm{Me}-\mathrm{C}_{6} \mathrm{H}_{4^{-}}$ & 4-Me- $\mathrm{C}_{6} \mathrm{H}_{4}-$ \\
\hline 103 & 216 & 4-( $\left(\mathrm{OC}_{3} \mathrm{H}_{6}-\mathrm{NH}-\mathrm{Boc}\right)-\mathrm{C}_{6} \mathrm{H}_{4}-$ & $4-\mathrm{Me}-\mathrm{C}_{6} \mathrm{H}_{4}^{-}$ & $4-\mathrm{Me}-\mathrm{C}_{6} \mathrm{H}_{4}^{-}$ & $4-\mathrm{Me}-\mathrm{C}_{6} \mathrm{H}_{4}-$ \\
\hline 104 & 217 & 2- $\left(\mathrm{OC}_{3} \mathrm{H}_{6}-\mathrm{NH}_{2}\right)-\mathrm{C}_{6} \mathrm{H}_{4^{-}}$ & $4-\mathrm{Me}-\mathrm{C}_{6} \mathrm{H}_{4^{-}}$ & $4-\mathrm{Me}-\mathrm{C}_{6} \mathrm{H}_{4^{-}}$ & 4-Me- $\mathrm{C}_{6} \mathrm{H}_{4^{-}}$ \\
\hline 105 & 218 & 4- $\left(\mathrm{OC}_{3} \mathrm{H}_{6}-\mathrm{NH}_{2}\right)-\mathrm{C}_{6} \mathrm{H}_{4^{-}}$ & 4-Me- $\mathrm{C}_{6} \mathrm{H}_{4^{-}}$ & $4-\mathrm{Me}-\mathrm{C}_{6} \mathrm{H}_{4^{-}}$ & 4-Me- $\mathrm{C}_{6} \mathrm{H}_{4-}^{-}$ \\
\hline 106 & 219 & 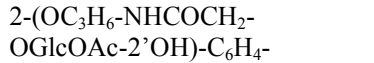 & $4-\mathrm{Me}-\mathrm{C}_{6} \mathrm{H}_{4}-$ & 4-Me- $\mathrm{C}_{6} \mathrm{H}_{4}-$ & $4-\mathrm{Me}-\mathrm{C}_{6} \mathrm{H}_{4-}$ \\
\hline 107 & 220 & $\begin{array}{l}\text { 4-( }\left(\mathrm{OC}_{3} \mathrm{H}_{6}-\mathrm{NHCOCH}_{2-}\right. \\
\text { OGlcOAc-2'OH)-- } \mathrm{C}_{6} \mathrm{H}_{4^{-}}\end{array}$ & $4-\mathrm{Me}-\mathrm{C}_{6} \mathrm{H}_{4}-$ & 4-Me- $\mathrm{C}_{6} \mathrm{H}_{4}-$ & $4-\mathrm{Me}-\mathrm{C}_{6} \mathrm{H}_{4-}$ \\
\hline 108 & 221 & $\begin{array}{l}2-\left(\mathrm{OC}_{3} \mathrm{H}_{6}-\mathrm{NHCOCH}_{2}-\mathrm{OGlcOH}-\right. \\
\left.2^{\prime} \mathrm{OH}\right)-\mathrm{C}_{6} \mathrm{H}_{4^{-}}\end{array}$ & $4-\mathrm{Me}-\mathrm{C}_{6} \mathrm{H}_{4}-$ & $4-\mathrm{Me}-\mathrm{C}_{6} \mathrm{H}_{4}-$ & $4-\mathrm{Me}-\mathrm{C}_{6} \mathrm{H}_{4-}$ \\
\hline 109 & 222 & $\begin{array}{l}4-\left(\mathrm{OC}_{3} \mathrm{H}_{6}-\mathrm{NHCOCH}_{2}-\mathrm{OGlcOH}-\right. \\
\left.2^{\prime} \mathrm{OH}\right)-\mathrm{C}_{6} \mathrm{H}_{4^{-}}\end{array}$ & $4-\mathrm{Me}-\mathrm{C}_{6} \mathrm{H}_{4-}$ & 4-Me- $\mathrm{C}_{6} \mathrm{H}_{4}-$ & 4-Me- $\mathrm{C}_{6} \mathrm{H}_{4}-$ \\
\hline 110 & 223 & $2-\mathrm{Br}\left(\mathrm{CH}_{2}\right)_{3}-\mathrm{O}-\mathrm{C}_{6} \mathrm{H}_{4^{-}}$ & $4-\mathrm{Me}-\mathrm{C}_{6} \mathrm{H}_{4}-$ & $4-\mathrm{Me}-\mathrm{C}_{6} \mathrm{H}_{4}-$ & $4-\mathrm{Me}-\mathrm{C}_{6} \mathrm{H}_{4^{-}}$ \\
\hline 111 & 224 & $4-\mathrm{Br}\left(\mathrm{CH}_{2}\right)_{3}-\mathrm{O}-\mathrm{C}_{6} \mathrm{H}_{4^{-}}$ & 4-Me- $\mathrm{C}_{6} \mathrm{H}_{4}-$ & $4-\mathrm{Me}-\mathrm{C}_{6} \mathrm{H}_{4}-$ & $4-\mathrm{Me}-\mathrm{C}_{6} \mathrm{H}_{4}-$ \\
\hline 112 & 225 & 2-[AcOGalS- $\left.\left(\mathrm{CH}_{2}\right)_{3}-\mathrm{O}\right]-\mathrm{C}_{6} \mathrm{H}_{4^{-}}$ & 4-Me- $\mathrm{C}_{6} \mathrm{H}_{4}^{-}$ & 4-Me- $\mathrm{C}_{6} \mathrm{H}_{4}^{-}$ & 4-Me- $\mathrm{C}_{6} \mathrm{H}_{4}-$ \\
\hline 113 & 226 & 4-[AcOGalS- $\left.\left(\mathrm{CH}_{2}\right)_{3}-\mathrm{O}\right]-\mathrm{C}_{6} \mathrm{H}_{4^{-}}$ & $4-\mathrm{Me}-\mathrm{C}_{6} \mathrm{H}_{4}-$ & $4-\mathrm{Me}-\mathrm{C}_{6} \mathrm{H}_{4}-$ & $4-\mathrm{Me}-\mathrm{C}_{6} \mathrm{H}_{4^{-}}$ \\
\hline 114 & 227 & 2-[AcOGlcS- $\left.\left(\mathrm{CH}_{2}\right)_{3}-\mathrm{O}\right]-\mathrm{C}_{6} \mathrm{H}_{4}-$ & 4-Me- $\mathrm{C}_{6} \mathrm{H}_{4-}^{-}$ & $4-\mathrm{Me}-\mathrm{C}_{6} \mathrm{H}_{4^{-}}$ & 4-Me- $\mathrm{C}_{6} \mathrm{H}_{4}-$ \\
\hline 115 & 228 & 4-[AcOGlcS- $\left.\left(\mathrm{CH}_{2}\right)_{3}-\mathrm{O}\right]-\mathrm{C}_{6} \mathrm{H}_{4-}$ & 4-Me- $\mathrm{C}_{6} \mathrm{H}_{4-}^{-}$ & 4-Me- $\mathrm{C}_{6} \mathrm{H}_{4-}$ & $4-\mathrm{Me}-\mathrm{C}_{6} \mathrm{H}_{4}-$ \\
\hline 116 & 229 & 2-[AcOManS- $\left.\left(\mathrm{CH}_{2}\right)_{3}{ }^{-} \mathrm{O}\right]-\mathrm{C}_{6} \mathrm{H}_{4^{-}}$ & $4-\mathrm{Me}-\mathrm{C}_{6} \mathrm{H}_{4}-$ & $4-\mathrm{Me}-\mathrm{C}_{6} \mathrm{H}_{4}-$ & 4-Me- $\mathrm{C}_{6} \mathrm{H}_{4-}^{-}$ \\
\hline 117 & 230 & 4-[AcOManS- $\left.\left.\left(\mathrm{CH}_{2}\right)_{3}\right)^{-} \mathrm{O}\right]-\mathrm{C}_{6} \mathrm{H}_{4}-$ & 4-Me- $\mathrm{C}_{6} \mathrm{H}_{4-}^{-}$ & 4-Me- $\mathrm{C}_{6} \mathrm{H}_{4-}^{-}$ & 4-Me- $\mathrm{C}_{6} \mathrm{H}_{4}-$ \\
\hline 118 & 231 & $2-\left[\mathrm{GalS}-\left(\mathrm{CH}_{2}\right)_{3}-\mathrm{O}\right]-\mathrm{C}_{6} \mathrm{H}_{4}{ }^{-}$ & $4-\mathrm{Me}-\mathrm{C}_{6} \mathrm{H}_{4}^{-}$ & $4-\mathrm{Me}-\mathrm{C}_{6} \mathrm{H}_{4}-$ & $4-\mathrm{Me}-\mathrm{C}_{6} \mathrm{H}_{4}^{-}$ \\
\hline 119 & 232 & 4-[GalS- $\left.\left(\mathrm{CH}_{2}\right)_{3}-\mathrm{O}\right]-\mathrm{C}_{6} \mathrm{H}_{4}-$ & $4-\mathrm{Me}-\mathrm{C}_{6} \mathrm{H}_{4-}$ & $4-\mathrm{Me}-\mathrm{C}_{6} \mathrm{H}_{4^{-}}$ & $4-\mathrm{Me}-\mathrm{C}_{6} \mathrm{H}_{4^{-}}$ \\
\hline 120 & 233 & $2-\left[\mathrm{GlcS}-\left(\mathrm{CH}_{2}\right)_{3}-\mathrm{O}\right]-\mathrm{C}_{6} \mathrm{H}_{4}-$ & $4-\mathrm{Me}-\mathrm{C}_{6} \mathrm{H}_{4-}$ & $4-\mathrm{Me}-\mathrm{C}_{6} \mathrm{H}_{4^{-}}$ & $4-\mathrm{Me}-\mathrm{C}_{6} \mathrm{H}_{4^{-}}$ \\
\hline 121 & 234 & 4-[GlcS- $\left.\left(\mathrm{CH}_{2}\right)_{3}-\mathrm{O}\right]-\mathrm{C}_{6} \mathrm{H}_{4^{-}}$ & $4-\mathrm{Me}-\mathrm{C}_{6} \mathrm{H}_{4^{-}}$ & $4-\mathrm{Me}-\mathrm{C}_{6} \mathrm{H}_{4^{-}}$ & 4-Me- $\mathrm{C}_{6} \mathrm{H}_{4^{-}}$ \\
\hline 122 & 235 & 2-[ManS- $\left.\left(\mathrm{CH}_{2}\right)_{3}-\mathrm{O}\right]-\mathrm{C}_{6} \mathrm{H}_{4}-$ & $4-\mathrm{Me}-\mathrm{C}_{6} \mathrm{H}_{4}-$ & $4-\mathrm{Me}-\mathrm{C}_{6} \mathrm{H}_{4}-$ & $4-\mathrm{Me}-\mathrm{C}_{6} \mathrm{H}_{4^{-}}$ \\
\hline 123 & 236 & 4-[ManS- $\left.\left(\mathrm{CH}_{2}\right)_{3}-\mathrm{O}\right]-\mathrm{C}_{6} \mathrm{H}_{4^{-}}$ & $4-\mathrm{Me}^{-} \mathrm{C}_{6} \mathrm{H}_{4^{-}}$ & $4-\mathrm{Me}-\mathrm{C}_{6} \mathrm{H}_{4^{-}}$ & 4-Me- $\mathrm{C}_{6} \mathrm{H}_{4^{-}}$ \\
\hline 124 & 237 & 4-(AcOGlcS)- $\mathrm{C}_{6} \mathrm{~F}_{4^{-}}$ & $\mathrm{C}_{6} \mathrm{~F}_{5^{-}}$ & $\mathrm{C}_{6} \mathrm{~F}_{5^{-}}$ & $\mathrm{C}_{6} \mathrm{~F}_{5^{-}}$ \\
\hline 125 & 238 & $4-(\mathrm{GlcS})-\mathrm{C}_{6} \mathrm{~F}_{4^{-}}$ & $\mathrm{C}_{6} \mathrm{~F}_{5^{-}}$ & $\mathrm{C}_{6} \mathrm{~F}_{5^{-}}$ & $\mathrm{C}_{6} \mathrm{~F}_{5^{-}}$ \\
\hline 126 & 239 & 4-(AcOGlcS) $-\mathrm{C}_{6} \mathrm{~F}_{4}{ }^{-}$ & 4-(AcOGlcS)- $\mathrm{C}_{6} \mathrm{~F}_{4}-$ & $\mathrm{C}_{6} \mathrm{~F}_{5^{-}}$ & $\mathrm{C}_{6} \mathrm{~F}_{5^{-}}$ \\
\hline 127 & 240 & $4-(\mathrm{GlcS})-\mathrm{C}_{6} \mathrm{~F}_{4^{-}}$ & 4-(GlcS)- $\mathrm{C}_{6} \mathrm{~F}_{4}-$ & $\mathrm{C}_{6} \mathrm{~F}_{5^{-}}$ & $\mathrm{C}_{6} \mathrm{~F}_{5^{-}}$ \\
\hline 128 & 241 & 4-(AcOGlcS) $-\mathrm{C}_{6} \mathrm{~F}_{4^{-}}$ & $\mathrm{C}_{6} \mathrm{~F}_{5^{-}}$ & 4-(AcOGlcS)- $\mathrm{C}_{6} \mathrm{~F}_{4^{-}}$ & $\mathrm{C}_{6} \mathrm{~F}_{5^{-}}$ \\
\hline 129 & 242 & $4-(\mathrm{GlcS})-\mathrm{C}_{6} \mathrm{~F}_{4^{-}}$ & $\mathrm{C}_{6} \mathrm{~F}_{5^{-}}$ & $4-(\mathrm{GlcS})-\mathrm{C}_{6} \mathrm{~F}_{4^{-}}$ & $\mathrm{C}_{6} \mathrm{~F}_{5^{-}}$ \\
\hline 130 & 243 & 4-(AcOGlcS)- $\mathrm{C}_{6} \mathrm{~F}_{4^{-}}$ & 4-(AcOGlcS) $-\mathrm{C}_{6} \mathrm{~F}_{4^{-}}$ & 4-(AcOGlcS)- $\mathrm{C}_{6} \mathrm{~F}_{4^{-}}$ & $\mathrm{C}_{6} \mathrm{~F}_{5^{-}}$ \\
\hline 131 & 244 & $4-(\mathrm{GlcS})-\mathrm{C}_{6} \mathrm{~F}_{4-}$ & 4-(GlcS)- $\mathrm{C}_{6} \mathrm{~F}_{4^{-}}$ & 4-(GlcS)- $\mathrm{C}_{6} \mathrm{~F}_{4^{-}}$ & $\mathrm{C}_{6} \mathrm{~F}_{5^{-}}$ \\
\hline 132 & 245 & 3,5-(di-GalCONH-O)- $\mathrm{C}_{6} \mathrm{H}_{3}-$ & Phenyl & Phenyl & Phenyl \\
\hline 133 & 246 & 3,5-(di-GlcCONH-O)- $\mathrm{C}_{6} \mathrm{H}_{3}-$ & Phenyl & Phenyl & Phenyl \\
\hline 134 & 247 & 4-(AcOGlcNH-CO)- $\mathrm{C}_{6} \mathrm{H}_{4^{-}}$ & Phenyl & Phenyl & Phenyl \\
\hline 135 & 248 & 4-(AcOGlcNH-CO)- $\mathrm{C}_{6} \mathrm{H}_{4^{-}}$ & Phenyl & $\begin{array}{l}\text { 4-(AcOGlcNH)-CO- } \\
\mathrm{C}_{6} \mathrm{H}_{4^{-}}\end{array}$ & Phenyl \\
\hline 136 & 249 & 4-(BnOGlc-6-NH)- $\mathrm{C}_{6} \mathrm{~F}_{4}-$ & $\mathrm{C}_{6} \mathrm{~F}_{5^{-}}$ & $\mathrm{C}_{6} \mathrm{~F}_{5^{-}}$ & $\mathrm{C}_{6} \mathrm{~F}_{5^{-}}$ \\
\hline 137 & 250 & 4-(BnOGlc-6-NH)- $\mathrm{C}_{6} \mathrm{~F}_{4}^{-}$ & 4-(BnOGlc-6-NH)- & $\mathrm{C}_{6} \mathrm{~F}_{5^{-}}$ & $\mathrm{C}_{6} \mathrm{~F}_{5^{-}}$ \\
\hline
\end{tabular}

EtCOOH $/ \mathrm{Ac}_{2} \mathrm{O}$, reflux, $1 \mathrm{~h} ; 3 \%$

$4 \times$ pyrrole, 4-hydroxybenzaldehyde, $3 \times 4$-tolualdehyde,

$2 \mathrm{H} \quad 4 \times$ pyrrole, 4-hydroxybenzaldehy

$2 \mathrm{H} \quad$ From 213: $\mathrm{Br}\left(\mathrm{CH}_{2}\right)_{3} \mathrm{NH}-\mathrm{Boc}\left(5\right.$ equiv.), $\mathrm{K}_{2} \mathrm{CO}_{3}$ (20 equiv.), DMF, 18

h, r.t.

$2 \mathrm{H} \quad$ From 214: Similar to entry 102 .

From 215: $\mathrm{CH}_{2} \mathrm{Cl}_{2} / \mathrm{TFA}(80 / 20, \mathrm{v} / \mathrm{v}), 3$ h, r.t.

$2 \mathrm{H} \quad$ From 216: like entry 104.

$2 \mathrm{H} \quad$ From 217: 3,4,6-Tri- $O$-acetyl-carboxymethyl- $\alpha-D$-glucopyranoside-2- [237]

$O$-lactone, $\mathrm{CH}_{2} \mathrm{Cl}_{2}$, DMAP, r.t., 16 h.

From 218: Like entry 104.

From 219: $\mathrm{NaOMe}, \mathrm{MeOH}$.

From 220: $\mathrm{NaOMe}, \mathrm{MeOH}$.

From 221: $\left(\mathrm{CH}_{2}\right)_{3} \mathrm{Br}_{2}, \mathrm{~K}_{2} \mathrm{CO}_{3}$, DMF; $91 \%$

From 214: $\left(\mathrm{CH}_{2}\right)_{3} \mathrm{Br}_{2}, \mathrm{~K}_{2} \mathrm{CO}_{3}$, DMF; $84 \%$

From 223: 2,3,4,6-Tetra- $O$-acetyl-1S-acetyl-1-thio- $\beta$-D-

galactopyranose, $\left(\mathrm{C}_{2} \mathrm{H}_{5}\right) \mathrm{NH}$, DMF, DEA, r.t., $15 \mathrm{~h} ; 66 \%$.

$2 \mathrm{H} \quad$ From 224: Like entry $112,6 \mathrm{~h} ; 67 \%$.

From 223: 2, 3, 6, 6-Tetra- $O$-acetyl-1 1 -acetyl-1-thio- $\beta$ - $D$-glucopyranose,

$\left(\mathrm{C}_{2} \mathrm{H}_{5}\right) \mathrm{NH}$, DMF, DEA, r.t., $15 \mathrm{~h} ; 84 \%$

From 224: Like entry $114,6 \mathrm{~h} ; 79 \%$.

From 223: 2,3,4,6-Tetra- $O$-acetyl- $1 S$-acetyl-1-thio- $\alpha-D-$

mannope, $\left(\mathrm{C}_{2} \mathrm{H}_{5}\right) \mathrm{NH}$, DMF, DEA, r.t., $15 \mathrm{~h} ; 75 \%$.

From 224: Like entry $116,6 \mathrm{~h} ; 76 \%$

From 225. $\mathrm{NaOMe}, \mathrm{MeOH}, \mathrm{CH}_{2} \mathrm{Cl}_{2}$, r.t., 1 h; $96 \%$

From 226: Like entry $118 ; 96 \%$.

From 227. Like entry 118,96\%.

From 228: Like entry 118, $96 \%$.

From 229. Like entry $118,96 \%$.

From 230: Like entry $118,96 \%$.

${ }^{2} \mathrm{PPF}_{20}, 2,3,4,6$-tetra- $O$-acetyl-1 $S$-acetyl-1-thio- $\beta$ - $D$-glucopyranose,

diethylamine, DMF, r.t., $24 \mathrm{~h} ; 42 \%$.

From 237: $\mathrm{CH}_{2} \mathrm{Cl}_{2}, \mathrm{CH}_{3} \mathrm{OH}, \mathrm{NaOCH}_{3}, 45-50{ }^{\circ} \mathrm{C}, 7 \mathrm{~min}$.; $61 \%$.

Like entry $124 ; 19 \%$.

From 239: Like entry $125 ; 55 \%$.

Like entry $124 ; 14 \%$.

From 241: Like entry $125 ; 51 \%$.

Like entry $124 ; 32 \%$.

From 243: Like entry $125 ; 53 \%$.

(AcOGlcNH)-OH, 5-(4-carboxyphenyl)-10,15,20-triphenylporphyrin, EDCL, DMAP, $\mathrm{CH}_{2} \mathrm{Cl}_{2}, 0{ }^{\circ} \mathrm{C}, 3 \mathrm{~h}$ then r.t., $24 \mathrm{~h} ; 65 \%$.

meso-[4-(1,3,4,6-Tetra- $O$-acetyl-2-amido-2-deoxy- $\beta=D-$

glucopyranose)]dipyrromethane, benzaldehyde, $\mathrm{CH}_{2} \mathrm{Cl}_{2}$, TFA, r.t., $2 \mathrm{~h}$, then 1 DDQ 1 ; $6 \%$

$2 \mathrm{H} \quad \mathrm{TPPF}_{20}$ methyl 6-amino-2,3,4-tri- $O$-benzyl-6-deoxy- $\alpha-D-$ glucopyranoside, 1,2,4-trichlorobenzene, reflux, $5 \mathrm{~h}$.; $40 \%$

Like entry $136 ; 14 \%$.

\section{[199]}

[199]

$[199$

199

$[199$

$[199]$

$[199$

[199]

[199]

[200]

$200]$

200

[200]

[200]

[200]

[200]

[175]

[201] 


\begin{tabular}{|c|c|c|c|c|c|c|c|c|}
\hline 138 & 251 & 4-(BnOGlc-6-NH)- $\mathrm{C}_{6} \mathrm{~F}_{4}-$ & $\begin{array}{l}\mathrm{C}_{6} \mathrm{~F}_{4^{-}} \\
\mathrm{C}_{6} \mathrm{~F}_{5^{-}}\end{array}$ & $\begin{array}{l}4-(\mathrm{BnOGlc}-6-\mathrm{NH})- \\
\mathrm{C}_{6} \mathrm{~F}_{4-}^{-}\end{array}$ & $\mathrm{C}_{6} \mathrm{~F}_{5^{-}}$ & $2 \mathrm{H}$ & Like entry $136 ; 7 \%$. & [202] \\
\hline 139 & 252 & 4-(BnOGlc-6-NH)- $\mathrm{C}_{6} \mathrm{~F}_{4^{-}}$ & $\begin{array}{l}4-(\mathrm{BnOGlc}-6-\mathrm{NH})- \\
\mathrm{C}_{6} \mathrm{~F}_{4^{-}}\end{array}$ & $\begin{array}{l}4-(\mathrm{BnOGlc}-6-\mathrm{NH})- \\
\mathrm{C}_{6} \mathrm{~F}_{4^{-}}\end{array}$ & $\mathrm{C}_{6} \mathrm{~F}_{5^{-}}$ & $2 \mathrm{H}$ & Like entry $136 ; 8 \%$. & [202] \\
\hline 140 & 253 & 4-(BnOGlc-6-NH)- $\mathrm{C}_{6} \mathrm{~F}_{4}-$ & $\mathrm{C}_{6} \mathrm{~F}_{5^{-}}$ & $\mathrm{C}_{6} \mathrm{~F}_{5^{-}}$ & $\mathrm{C}_{6} \mathrm{~F}_{5}-$ & Zn(II) & $\begin{array}{l}\mathrm{Zn}(\mathrm{II}) \mathrm{TPPF}_{20} \text {, methyl 6-amino-2,3,4-tri- } O \text {-benzyl-6-deoxy- } \alpha-D- \\
\text { glucopyranoside, 1,2,4-trichlorobezene, reflux, } 5 \mathrm{~h} ; 43 \% \text {. }\end{array}$ & [202] \\
\hline 141 & 254 & 4-(BnOGlc-6-NH)- $\mathrm{C}_{6} \mathrm{~F}_{4}-$ & $\begin{array}{l}4-(\mathrm{BnOGlc}-6-\mathrm{NH})- \\
\mathrm{C}_{6} \mathrm{~F}_{4^{-}}\end{array}$ & $\mathrm{C}_{6} \mathrm{~F}_{5}^{-}$ & $\mathrm{C}_{6} \mathrm{~F}_{5^{-}}$ & Zn(II) & Like entry $140 ; 8 \%$ & [202] \\
\hline 142 & 255 & 4-(BnOGlc-6-NH)- $\mathrm{C}_{6} \mathrm{~F}_{4-}^{-}$ & $\mathrm{C}_{6} \mathrm{~F}_{5^{-}}$ & $\begin{array}{l}4-(\mathrm{BnOGlc}-6-\mathrm{NH})- \\
\mathrm{C}_{6} \mathrm{~F}_{4^{-}}\end{array}$ & $\mathrm{C}_{6} \mathrm{~F}_{5}-$ & Zn(II) & Like entry $140 ; 5 \%$. & [202] \\
\hline 143 & 256 & 4-(BnOGlc-6-NH)- $\mathrm{C}_{6} \mathrm{~F}_{4}-$ & $\begin{array}{l}4-(\mathrm{BnOGlc}-6-\mathrm{NH})- \\
\mathrm{C}_{6} \mathrm{~F}_{4^{-}}\end{array}$ & $\begin{array}{l}4-(\mathrm{BnOGlc}-6-\mathrm{NH})- \\
\mathrm{C}_{6} \mathrm{~F}_{4^{-}}\end{array}$ & $\mathrm{C}_{6} \mathrm{~F}_{5-}^{-}$ & Zn(II) & Like entry $140 ; 1 \%$ & [202] \\
\hline 144 & 257 & $\begin{array}{l}4-\left[N-(\mathrm{GalO})-4^{\prime}-\left(\mathrm{OCH}_{2}\right)-1^{\prime}, 2^{\prime}, 3^{\prime}-\right. \\
\text { triazol-1'-yl]- } \mathrm{C}_{6} \mathrm{H}_{4^{-}}\end{array}$ & 4- $\mathrm{HO}-\mathrm{C}_{6} \mathrm{H}_{4^{-}}$ & $4-\mathrm{HO}-\mathrm{C}_{6} \mathrm{H}_{4}$ & $4-\mathrm{HO}-\mathrm{C}_{6} \mathrm{H}_{4}$ & Zn(II) & $\begin{array}{l}\text { i) Propargyl bromide, } \mathrm{K}_{2} \mathrm{CO}_{3}, 60^{\circ} \mathrm{C}, 24 \mathrm{~h} \text {; ii) } \mathrm{Zn}(\mathrm{OAc})_{2} \text {, } \\
\mathrm{CH}_{3} \mathrm{OH}, \mathrm{CHCl}_{3} \text {, reflux } 1 \mathrm{~h} ; 28 \% \text {; iii) } \mathrm{Gal}-\mathrm{N}_{3}, \mathrm{CuSO}_{4} \cdot 5 \mathrm{H}_{2} \mathrm{O} \text {, sodium } \\
\text { ascorbate, } t \mathrm{BuOH} / \mathrm{H}_{2} \mathrm{O}(1: 1, \mathrm{v} / \mathrm{v}), 70^{\circ} \mathrm{C}, 24 \mathrm{~h} ; 92 \% \text {. }\end{array}$ & [203] \\
\hline 145 & 258 & $\begin{array}{l}4-\left[N-(\mathrm{LaCO})-4^{\prime}-\left(\mathrm{OCH}_{2}\right)-\right. \\
1^{\prime}, 2^{\prime}, 3^{\prime} \text {-triazol-1'-yl]-- }{ }_{6} \mathrm{H}_{4^{-}}\end{array}$ & $4-\mathrm{HO}-\mathrm{C}_{6} \mathrm{H}_{4^{-}}$ & $4-\mathrm{HO}-\mathrm{C}_{6} \mathrm{H}_{4}$ & $4-\mathrm{HO}-\mathrm{C}_{6} \mathrm{H}_{4}$ & Zn(II) & Like entry $144 ; 88 \%$ & [203] \\
\hline 146 & 259 & $\begin{array}{l}4-\left[4^{\prime}-\left(\text { AcOGlcO-CH }{ }_{2}\right)-1^{\prime} N-\right. \\
\left.1^{\prime}, 2^{\prime}, 3^{\prime}-\text { triazol-1'- }-\mathrm{yl}\right]-\mathrm{C}_{6} \mathrm{H}_{4^{-}}\end{array}$ & $\mathrm{C}_{6} \mathrm{H}_{5^{-}}$ & $\mathrm{C}_{6} \mathrm{H}_{5^{-}}$ & $\mathrm{C}_{6} \mathrm{H}_{5^{-}}$ & Zn(II) & $\begin{array}{l}\text { 5-(4-Azido-phenyl), } 10,15,20 \text {-triphenylporphyrin, } \mathrm{CuCl} \text {, (AcOGlcO)- } \\
\mathrm{CH}_{2} \mathrm{CCH} \text {, toluene } / \mathrm{H}_{2} \mathrm{O}(1: 1, \mathrm{v} / \mathrm{v}), 140{ }^{\circ} \mathrm{C}, 20 \text { min., microwave; } 93 \% \text {. }\end{array}$ & [32] \\
\hline 147 & 260 & $\begin{array}{l}\left.\text { 4-[4'-(AcOManO-CH }{ }_{2}\right)-1 \text { ' } N- \\
1^{\prime}, 2^{\prime}, 3^{\prime} \text { 'triazol-1'-yl]- } \mathrm{C}_{6} \mathrm{H}_{4}\end{array}$ & $\mathrm{C}_{6} \mathrm{H}_{5^{-}}$ & $\mathrm{C}_{6} \mathrm{H}_{5^{-}}$ & $\mathrm{C}_{6} \mathrm{H}_{5^{-}}$ & Zn(II) & $\begin{array}{l}\text { Similar to entry } 146,\left(\text { AcOManO) }-\mathrm{CH}_{2} \mathrm{CCH} \text {, toluene } / \mathrm{H}_{2} \mathrm{O}(4: 1, \mathrm{v} / \mathrm{v}) \text {, }\right. \\
140^{\circ} \mathrm{C}, 20 \text { min., microwave; } 86 \% \text {. }\end{array}$ & [32] \\
\hline 148 & 261 & $\begin{array}{l}\left.\text { 4-[4'-(ManO- } \mathrm{CH}_{2}\right)-1^{\prime}{ }^{\prime} N-1^{\prime}, 2^{\prime}, 3^{\prime}- \\
\text { triazol-1'-yl]- } \mathrm{C}_{6} \mathrm{H}_{4}\end{array}$ & $\mathrm{C}_{6} \mathrm{H}_{5^{-}}$ & $\mathrm{C}_{6} \mathrm{H}_{5^{-}}$ & $\mathrm{C}_{6} \mathrm{H}_{5^{-}}$ & Zn(II) & $\begin{array}{l}\text { Similar to entry } 146 \text { with }(\mathrm{ManO})-\mathrm{CH}_{2} \mathrm{CCH} ; 61 \% \text {. Alternatively, } \\
\mathrm{Cu}(\mathrm{MeCN})_{4} \mathrm{PF}_{6} \text {, (ManO)-CH } \mathrm{CH}_{2} \mathrm{CCH} \text {, toluene/MeOH }(0.6: 0.2, \mathrm{v} / \mathrm{v}), 110 \\
{ }^{\circ} \mathrm{C}, 20 \text { min., microwave; } 92 \% \text {. }\end{array}$ & {$[32,33]$} \\
\hline 149 & 262 & $\begin{array}{l}4-\left[4^{\prime}-\left(\mathrm{AcOGlcO}-\mathrm{CH}_{2}\right)-1^{\prime} N-\right. \\
1^{\prime}, 2^{\prime}, 3^{\prime} \text {-triazol-1'-yl] }-\mathrm{C}_{6} \mathrm{H}_{4^{-}}\end{array}$ & $4-\left(\mathrm{N}_{3}\right)-\mathrm{C}_{6} \mathrm{H}_{4^{-}}$ & $\mathrm{C}_{6} \mathrm{H}_{5^{-}}$ & $\mathrm{C}_{6} \mathrm{H}_{5^{-}}$ & Zn(II) & $\begin{array}{l}\text { 5,10-Di(4-azido-phenyl),15,20-diphenylporphyrin, } \mathrm{CuCl} \text {, (AcOGlcO)- } \\
\mathrm{CH}_{2} \mathrm{CCH} \text {, toluene } / \mathrm{H}_{2} \mathrm{O}(4: 1, \mathrm{v} / \mathrm{v}), 140^{\circ} \mathrm{C}, 20 \mathrm{~min} \text {., microwave; } 87 \% \text {. }\end{array}$ & [32] \\
\hline 150 & 263 & $\begin{array}{l}4-\left[4^{\prime}-\left(\text { AcOGlcO- } \mathrm{CH}_{2}\right)-1^{\prime} N-\right. \\
1^{\prime}, 2^{\prime}, 3^{\prime}-\text { triazol-1'--yl]- } \mathrm{C}_{6} \mathrm{H}_{4}-\end{array}$ & $\begin{array}{l}4-\left[4^{\prime}-(\mathrm{AcOManO}-\right. \\
\left.\mathrm{CH}_{2}\right)-1^{\prime} N-1^{\prime}, 2^{\prime}, 3^{\prime}- \\
\text { triazol-1'-yl]- } \mathrm{C}_{6} \mathrm{H}_{4}\end{array}$ & $\mathrm{C}_{6} \mathrm{H}_{5^{-}}$ & $\mathrm{C}_{6} \mathrm{H}_{5^{-}}$ & Zn(II) & $\begin{array}{l}\text { From 262: } \mathrm{CuCl},(\mathrm{AcOManO})-\mathrm{CH}_{2} \mathrm{CCH} \text {, toluene } / \mathrm{H}_{2} \mathrm{O}(4: 1, \mathrm{v} / \mathrm{v}), 140 \\
{ }^{\circ} \mathrm{C}, 20 \text { min., microwave; } 53 \% \text {. }\end{array}$ & [32] \\
\hline 151 & 264 & $\begin{array}{l}4-\left[4^{\prime}-\left(\mathrm{AcOGlcO}-\mathrm{CH}_{2}\right)-1^{\prime} N-\right. \\
1^{\prime}, 2^{\prime}, 3^{\prime} \text {-'triazol-1'-yl] }-\mathrm{C}_{6} \mathrm{H}_{4^{-}}\end{array}$ & $\begin{array}{l}4-\left[4^{\prime}-\left(\mathrm{AcOG}{ }^{\prime} \mathrm{C}^{\prime} \mathrm{O}-\right.\right. \\
\left.\mathrm{CH}_{2}\right)-1^{\prime} N-1^{\prime}, 2^{\prime}, 3^{\prime}- \\
\text { triazol-1'- }\end{array}$ & $\begin{array}{l}4-\left[4^{\prime}-(\mathrm{AcOGlcO}-\right. \\
\left.\mathrm{CH}_{2}\right)-1^{\prime} N-1^{\prime}, 2^{\prime}, 3^{\prime}- \\
\text { triazol-1'- }\end{array}$ & $\mathrm{C}_{6} \mathrm{H}_{5^{-}}$ & Zn(II) & $\begin{array}{l}\text { 5,10,15-Tris(4-azidophenyl)-20-phenylporphyrin, } \mathrm{CuCl} \text {, (AcOGlcO)- } \\
\mathrm{CH}_{2} \mathrm{CCH} \text {, toluene } / \mathrm{H}_{2} \mathrm{O}(1: 1, \mathrm{v} / \mathrm{v}), 140^{\circ} \mathrm{C}, 20 \text { min., microwave; } 91 \% \text {. }\end{array}$ & [32] \\
\hline 152 & 265 & $\begin{array}{l}4-\left[4^{\prime}-(\text { AcOGlcO-CH }\right. \\
2)-1^{\prime} N- \\
1^{\prime}, 2^{\prime}, 3^{\prime}-\text { triazol-1'-yl]- } \mathrm{C}_{6} \mathrm{H}_{4^{-}}\end{array}$ & $\begin{array}{l}4-\left[4^{\prime}-(\mathrm{AcOGlcO}-\right. \\
\left.\mathrm{CH}_{2}\right)-1^{\prime} N-1^{\prime}, 2^{\prime}, 3^{\prime}- \\
\text { triazol-1'- }\end{array}$ & $\begin{array}{l}4-\left[4^{\prime}-(\mathrm{AcOG}) \mathrm{co}-\right. \\
\left.\mathrm{CH}_{2}\right)-1^{\prime} N-1^{\prime}, 2^{\prime}, 3^{\prime}- \\
\text { triazol-1'- }\end{array}$ & $\begin{array}{l}4-\left[4^{\prime}-(\mathrm{AcOG}) \mathrm{AcO}-\right. \\
\left.\mathrm{CH}_{2}\right)-1^{\prime} N-1^{\prime}, 2^{\prime}, 3^{\prime}- \\
\text { triazol-1'- }\end{array}$ & Zn(II) & $\begin{array}{l}\text { 5,10,15,20-Tetrakis(4-azidophenyl)porphyrin, } \mathrm{CuCl} \text {, (AcOGlcO)- } \\
\mathrm{CH}_{2} \mathrm{CCH} \text {, toluene } / \mathrm{H}_{2} \mathrm{O}(1: 1, \mathrm{v} / \mathrm{v}), 140^{\circ} \mathrm{C}, 20 \text { min., microwave; } 50 \% \text {. }\end{array}$ & [32] \\
\hline 153 & 266 & $\begin{array}{l}\left.\text { 4-\{[AcOManO- }\left(\mathrm{OCC}_{2} \mathrm{H}_{4}\right)\right]_{3-}^{-} \\
\left.\left.\mathrm{CNHCOCH}_{2} \mathrm{NHCO}\right\}\right\}-\mathrm{C}_{6} \mathrm{H}_{4-}^{-}\end{array}$ & Phenyl & Phenyl & Phenyl & $2 \mathrm{H}$ & $\begin{array}{l}\text { 5-(4-Carboxyphenyl)-10,15,20-triphenylporphyrin, [AcOManO- } \\
\left.\left(\mathrm{OC}_{2} \mathrm{H}_{4}\right)\right]_{3}-\mathrm{CNHCOCH}_{2} \mathrm{NH}_{2}, 1 \text {-ethyl-3-(3- } \\
\text { dimethylaminopropyl)carboiimide (EDC)/Hydroxybenzotriazole } \\
\text { ( } \mathrm{HOBt} \text { ), } \mathrm{Et}_{3} \mathrm{~N}, \mathrm{CH}_{2} \mathrm{Cl}_{2} ; 86 \% \text {. }\end{array}$ & [204] \\
\hline 154 & 267 & $\begin{array}{l}\left.\text { 4-\{[AcOManO- }\left(\mathrm{OC}_{2} \mathrm{H}_{4}\right)_{2}\right]_{3-} \\
\mathrm{CNHCOCH} \mathrm{NHCO}_{3}-\mathrm{C}_{6} \mathrm{H}_{4-}\end{array}$ & Phenyl & Phenyl & Phenyl & $2 \mathrm{H}$ & Like entry 153 with $\left[\mathrm{AcOManO}-\left(\mathrm{OC}_{2} \mathrm{H}_{4}\right)_{2}\right]_{3}-\mathrm{CNHCOCH}_{2} \mathrm{NH}_{2} ; 57 \%$ & [204] \\
\hline 155 & 268 & $\begin{array}{l}4-\left\{\left[\mathrm{ManO}-\left(\mathrm{OC}_{2} \mathrm{H}_{4}\right)\right]_{3-}^{-}\right. \\
\mathrm{CNHCOCH}\}_{2} \mathrm{NHCO}_{-}-\mathrm{C}_{6} \mathrm{H}_{4-}\end{array}$ & Phenyl & Phenyl & Phenyl & $2 \mathrm{H}$ & From 266: $\mathrm{NaOMe}, \mathrm{MeOH}, \mathrm{THF}$; quantitative. & [204] \\
\hline 156 & 269 & $\begin{array}{l}4-\left\{\left[\mathrm{ManO}-\left(\mathrm{OC}_{2} \mathrm{H}_{4}\right)_{2}\right]_{3-}^{-}\right. \\
\mathrm{CNHCOCH}{ }_{2} \mathrm{NHCO}_{-}-\mathrm{C}_{6} \mathrm{H}_{4-}\end{array}$ & Phenyl & Phenyl & Phenyl & $2 \mathrm{H}$ & From 267 like entry 155. & [204] \\
\hline 157 & 270 & $\begin{array}{l}4-\left\{\left[\mathrm{AcOGlcO}-\left(\mathrm{CH}_{2}\right)\right]_{3-}^{-}\right. \\
\left.\mathrm{CNHCOCH}_{2} \mathrm{NHCO}\right\}-\mathrm{C}_{6} \mathrm{H}_{4^{-}}\end{array}$ & Phenyl & Phenyl & Phenyl & $2 \mathrm{H}$ & Like entry 153 with $\left[\mathrm{AcOGlcO}-\left(\mathrm{CH}_{2}\right)\right]_{3}-\mathrm{CNHCOCH}_{2} \mathrm{NH}_{2} ; 33 \%$. & [205] \\
\hline 158 & 271 & $4-\left\{\left[\mathrm{GlcO}-\left(\mathrm{CH}_{2}\right)\right]_{3}-\right.$ & Phenyl & Phenyl & Phenyl & $2 \mathrm{H}$ & From 270: $\mathrm{NaOMe}, \mathrm{MeOH}, \mathrm{CH}_{2} \mathrm{Cl}_{2} ; 89 \%$. & [205] \\
\hline 159 & 272 & $\begin{array}{l}4-\left[\mathrm{CH}_{3} \mathrm{OOC}\left(\mathrm{CH}_{2}\right)_{3} \mathrm{CONH}\right]- \\
\mathrm{C}_{6} \mathrm{H}_{4^{-}}\end{array}$ & 4- $\mathrm{NH}_{2}-\mathrm{C}_{6} \mathrm{H}_{4^{-}}$ & $4-\mathrm{NH}_{2}-\mathrm{C}_{6} \mathrm{H}_{4^{-}}$ & $4-\mathrm{NH}_{2}-\mathrm{C}_{6} \mathrm{H}_{4}-$ & $2 \mathrm{H}$ & From 82: Methylglutaryl chloride, triethylamine, THF, $0{ }^{\circ} \mathrm{C}, 2 \mathrm{~h}$. & [206] \\
\hline
\end{tabular}




\begin{tabular}{|c|c|c|c|c|c|}
\hline 160 & 273 & $\begin{array}{l}4-\left[\mathrm{CH}_{3} \mathrm{OOC}\left(\mathrm{CH}_{2}\right)_{3} \mathrm{CONH}\right]- \\
\mathrm{C}_{6} \mathrm{H}_{4^{-}}\end{array}$ & $\begin{array}{l}\text { 4-[AcOGlc-6'-CO- } \\
\text { (NH)]-C } \mathrm{C}_{6} \mathrm{H}_{4}\end{array}$ & $\begin{array}{l}\text { 4-[AcOGlc-6'-CO- } \\
(\mathrm{NH})]-\mathrm{C}_{6} \mathrm{H}_{4}\end{array}$ & $\begin{array}{l}\text { 4-[AcOGlc-6'-CO- } \\
(\mathrm{NH})]-\mathrm{C}_{6} \mathrm{H}_{4}\end{array}$ \\
\hline 161 & 274 & $2-(\mathrm{AcOGlcO})-\mathrm{C}_{6} \mathrm{H}_{4^{-}}$ & $4-\mathrm{NO}_{2}-\mathrm{C}_{6} \mathrm{H}_{4}-$ & $4-\mathrm{NO}_{2}-\mathrm{C}_{6} \mathrm{H}_{4^{-}}$ & $4-\mathrm{NO}_{2}-\mathrm{C}_{6} \mathrm{H}_{4^{-}}$ \\
\hline 162 & 275 & 4-(AcOGlcO)- $\mathrm{C}_{6} \mathrm{H}_{4^{-}}$ & $4-\mathrm{NO}_{2}-\mathrm{C}_{6} \mathrm{H}_{4^{-}}$ & $4-\mathrm{NO}_{2}-\mathrm{C}_{6} \mathrm{H}_{4^{-}}$ & $4-\mathrm{NO}_{2}-\mathrm{C}_{6} \mathrm{H}_{4}^{-}$ \\
\hline 163 & 276 & 4-( $\mathrm{AcOGlcO})-\mathrm{C}_{6} \mathrm{H}_{4^{-}}$ & 4- $\mathrm{NO}_{2}-\mathrm{C}_{6} \mathrm{H}_{4-}$ & 4-( $\mathrm{AcOGlcO})-\mathrm{C}_{6} \mathrm{H}_{4^{-}}$ & $4-\mathrm{NO}_{2}-\mathrm{C}_{6} \mathrm{H}_{4}-$ \\
\hline 164 & 277 & 4-(AcOGlcO)- $-\mathrm{C}_{6} \mathrm{H}_{4}-$ & 4-(AcOGlcO)- $-\mathrm{C}_{6} \mathrm{H}_{4}-$ & $4-\mathrm{NO}_{2}-\mathrm{C}_{6} \mathrm{H}_{4^{-}}$ & $4-\mathrm{NO}_{2}-\mathrm{C}_{6} \mathrm{H}_{4}-$ \\
\hline 165 & 278 & 4-(AcOGlcO)- $\mathrm{C}_{6} \mathrm{H}_{4^{-}}$ & 4-(AcOGlcO)- $\mathrm{C}_{6} \mathrm{H}_{4^{-}}$ & 4-(AcOGlcO)- $\mathrm{C}_{6} \mathrm{H}_{4}-$ & $4-\mathrm{NO}_{2}-\mathrm{C}_{6} \mathrm{H}_{4}-$ \\
\hline 166 & 279 & 4-(AcOGlcO)- $-\mathrm{C}_{6} \mathrm{H}_{4}-$ & $4-(\mathrm{AcOGlcO})-\mathrm{C}_{6} \mathrm{H}_{4}-$ & 4-(AcOGlcO)- $-\mathrm{C}_{6} \mathrm{H}_{4}-$ & $2-\mathrm{NO}_{2}-\mathrm{C}_{6} \mathrm{H}_{4}-$ \\
\hline 167 & 280 & 2-(AcOGlcO) $-\mathrm{C}_{6} \mathrm{H}_{4}-$ & 4- $\mathrm{NH}_{2}-\mathrm{C}_{6} \mathrm{H}_{4^{-}}^{-}$ & 4- $\mathrm{NH}_{2}-\mathrm{C}_{6} \mathrm{H}_{4^{-}}^{-}$ & 4- $\mathrm{NH}_{2}-\mathrm{C}_{6} \mathrm{H}_{4^{-}}$ \\
\hline 168 & 281 & 4-(AcOGlcO)- $\mathrm{C}_{6} \mathrm{H}_{4^{-}}$ & 4- $\mathrm{NH}_{2}-\mathrm{C}_{6} \mathrm{H}_{4}^{-}$ & 4- $\mathrm{NH}_{2}-\mathrm{C}_{6} \mathrm{H}_{4^{-}}$ & 4- $\mathrm{NH}_{2}-\mathrm{C}_{6} \mathrm{H}_{4^{-}}$ \\
\hline 169 & 282 & 4-(AcOGlcO)- $\mathrm{C}_{6} \mathrm{H}_{4^{-}}$ & $4-\mathrm{NH}_{2}-\mathrm{C}_{6} \mathrm{H}_{4^{-}}$ & 4-(AcOGlcO)- $\mathrm{C}_{6} \mathrm{H}_{4^{-}}$ & 4- $\mathrm{NH}_{2}-\mathrm{C}_{6} \mathrm{H}_{4^{-}}$ \\
\hline 170 & 283 & 4-(AcOGlcO)- $\mathrm{C}_{6} \mathrm{H}_{4^{-}}$ & 4-(AcOGlcO)- $\mathrm{C}_{6} \mathrm{H}_{4^{-}}$ & $4-\mathrm{NH}_{2}-\mathrm{C}_{6} \mathrm{H}_{4^{-}}^{-}$ & 4- $\mathrm{NH}_{2}-\mathrm{C}_{6} \mathrm{H}_{4^{-}}$ \\
\hline 171 & 284 & 4-(AcOGlcO)- $\mathrm{C}_{6} \mathrm{H}_{4^{-}}$ & 4-(AcOGlcO)- $\mathrm{C}_{6} \mathrm{H}_{4^{-}}$ & 4-(AcOGlcO) $-\mathrm{C}_{6} \mathrm{H}_{4^{-}}$ & 4- $\mathrm{NH}_{2}-\mathrm{C}_{6} \mathrm{H}_{4^{-}}$ \\
\hline 172 & 285 & 4-(AcOGlcO)- $\mathrm{C}_{6} \mathrm{H}_{4^{-}}$ & 4-(AcOGlcO)- $\mathrm{C}_{6} \mathrm{H}_{4^{-}}$ & 4-(AcOGlcO) $-\mathrm{C}_{6} \mathrm{H}_{4^{-}}$ & $2-\mathrm{NH}_{2}-\mathrm{C}_{6} \mathrm{H}_{4^{-}}$ \\
\hline 173 & 286 & 2-( $\mathrm{AcOGlcO})-\mathrm{C}_{6} \mathrm{H}_{4^{-}}$ & $\begin{array}{l}\text { 4- } \mathrm{C}_{6} \mathrm{H}_{4}-\mathrm{NH}-\mathrm{L} \text {-Ala- } \\
\text { Fmoc }\end{array}$ & $\begin{array}{l}\text { 4- } \mathrm{C}_{6} \mathrm{H}_{4}-\mathrm{NH}-L-\mathrm{Ala}- \\
\text { Fmoc }\end{array}$ & $\begin{array}{l}4-\mathrm{C}_{6} \mathrm{H}_{4}-\mathrm{NH}-L-\mathrm{Ala}- \\
\text { Fmoc }\end{array}$ \\
\hline 174 & 287 & 4-(AcOGlcO)- $\mathrm{C}_{6} \mathrm{H}_{4^{-}}$ & $\begin{array}{l}\text { 4- } \mathrm{C}_{6} \mathrm{H}_{4}-\mathrm{NH}-\mathrm{L}-\mathrm{Ala}- \\
\text { Fmoc }\end{array}$ & $\begin{array}{l}\text { 4- } \mathrm{C}_{6} \mathrm{H}_{4}-\mathrm{NH}-\mathrm{L} \text {-Ala- } \\
\text { Fmoc }\end{array}$ & $\begin{array}{l}\text { 4- } \mathrm{C}_{6} \mathrm{H}_{4}-\mathrm{NH}-\mathrm{L}-\mathrm{Ala}- \\
\text { Fmoc }\end{array}$ \\
\hline 175 & 288 & 4-(AcOGlcO)- $-\mathrm{C}_{6} \mathrm{H}_{4}^{-}$ & $\begin{array}{l}\text { 4- } \mathrm{C}_{6} \mathrm{H}_{4}-\mathrm{NH}-L \text {-Ala- } \\
\text { Fmoc }\end{array}$ & 4-( $\mathrm{AcOGlcO})-\mathrm{C}_{6} \mathrm{H}_{4^{-}}$ & $\begin{array}{l}\text { 4- } \mathrm{C}_{6} \mathrm{H}_{4}-\mathrm{NH}-\mathrm{L}-\mathrm{Ala}- \\
\text { Fmoc }\end{array}$ \\
\hline 176 & 289 & 4-(AcOGlcO)- $-\mathrm{C}_{6} \mathrm{H}_{4}-$ & 4-( $\mathrm{AcOGlcO})-\mathrm{C}_{6} \mathrm{H}_{4^{-}}$ & $\begin{array}{l}\text { 4- } \mathrm{C}_{6} \mathrm{H}_{4}-\mathrm{NH}-L \text {-Ala- } \\
\text { Fmoc }\end{array}$ & $\begin{array}{l}\text { 4- } \mathrm{C}_{6} \mathrm{H}_{4}-\mathrm{NH}-\mathrm{L} \text {-Ala- } \\
\text { Fmoc }\end{array}$ \\
\hline 177 & 290 & 4-(AcOGlcO)- $-\mathrm{C}_{6} \mathrm{H}_{4}^{-}$ & 4-(AcOGlcO)- $\mathrm{C}_{6} \mathrm{H}_{4^{-}}$ & 4-( $\mathrm{AcOGlcO})-\mathrm{C}_{6} \mathrm{H}_{4^{-}}$ & $\begin{array}{l}\text { 4- } \mathrm{C}_{6} \mathrm{H}_{4}-\mathrm{NH}-\mathrm{L}-\mathrm{Ala}- \\
\text { Fmoc }\end{array}$ \\
\hline 178 & 291 & 4-(AcOGlcO)- $\mathrm{C}_{6} \mathrm{H}_{4}{ }^{-}$ & 4-(AcOGlcO)- $-\mathrm{C}_{6} \mathrm{H}_{4}-$ & 4-(AcOGlcO)- $\mathrm{C}_{6} \mathrm{H}_{4^{-}}$ & $\begin{array}{l}2-\mathrm{C}_{6} \mathrm{H}_{4}-\mathrm{NH}-L-\mathrm{Ala}- \\
\text { Fmoc }\end{array}$ \\
\hline 179 & 292 & 2-(GlcO)- $-\mathrm{C}_{6} \mathrm{H}_{4-}$ & $\begin{array}{l}\text { 4- } \\
{\left[\left(\mathrm{NH}_{2}\right)\left(\mathrm{CH}_{3}\right) \mathrm{CHCON}\right.} \\
\mathrm{H}]-\mathrm{C}_{6} \mathrm{H}_{4^{-}}\end{array}$ & $\begin{array}{l}\text { 4- } \\
{\left[\left(\mathrm{NH}_{2}\right)\left(\mathrm{CH}_{3}\right) \mathrm{CHCON}\right.} \\
\mathrm{H}]-\mathrm{C}_{6} \mathrm{H}_{4^{-}}\end{array}$ & $\begin{array}{l}\text { 4- } \\
{\left[\left(\mathrm{NH}_{2}\right)\left(\mathrm{CH}_{3}\right) \mathrm{CHCON}\right.} \\
\mathrm{H}]-\mathrm{C}_{6} \mathrm{H}_{4^{-}}\end{array}$ \\
\hline 180 & 293 & 4-(GlcO)- $\mathrm{C}_{6} \mathrm{H}_{4^{-}}$ & $\begin{array}{l}\text { 4- } \\
{\left[\left(\mathrm{NH}_{2}\right)\left(\mathrm{CH}_{3}\right) \mathrm{CHCON}\right.} \\
\mathrm{H}]-\mathrm{C}_{6} \mathrm{H}_{4^{-}}\end{array}$ & $\begin{array}{l}\text { 4- } \\
{\left[\left(\mathrm{NH}_{2}\right)\left(\mathrm{CH}_{3}\right) \mathrm{CHCON}\right.} \\
\mathrm{H}]-\mathrm{C}_{6} \mathrm{H}_{4^{-}}\end{array}$ & $\begin{array}{l}\text { 4- } \\
{\left[\left(\mathrm{NH}_{2}\right)\left(\mathrm{CH}_{3}\right) \mathrm{CHCON}\right.} \\
\mathrm{H}]-\mathrm{C}_{6} \mathrm{H}_{4^{-}}\end{array}$ \\
\hline 181 & 294 & 4-(GlcO) $-\mathrm{C}_{6} \mathrm{H}_{4^{-}}$ & $\begin{array}{l}\text { 4- } \\
{\left[\left(\mathrm{NH}_{2}\right)\left(\mathrm{CH}_{3}\right) \mathrm{CHCON}\right.} \\
\mathrm{H}]-\mathrm{C}_{6} \mathrm{H}_{4}-\end{array}$ & $4-(\mathrm{GlcO})-\mathrm{C}_{6} \mathrm{H}_{4^{-}}$ & $\begin{array}{l}\text { 4- } \\
{\left[\left(\mathrm{NH}_{2}\right)\left(\mathrm{CH}_{3}\right) \mathrm{CHCON}\right.} \\
\mathrm{H}]-\mathrm{C}_{6} \mathrm{H}_{4^{-}}\end{array}$ \\
\hline 182 & 295 & 4-(GlcO) $-\mathrm{C}_{6} \mathrm{H}_{4^{-}}$ & 4-(GlcO)- $\mathrm{C}_{6} \mathrm{H}_{4^{-}}$ & $\begin{array}{l}\text { 4- } \\
{\left[\left(\mathrm{NH}_{2}\right)\left(\mathrm{CH}_{3}\right) \mathrm{CHCON}\right.} \\
\mathrm{H}]-\mathrm{C}_{6} \mathrm{H}_{4^{-}}\end{array}$ & $\begin{array}{l}\text { 4- } \\
{\left[\left(\mathrm{NH}_{2}\right)\left(\mathrm{CH}_{3}\right) \mathrm{CHCON}\right.} \\
\mathrm{H}]-\mathrm{C}_{6} \mathrm{H}_{4^{-}}\end{array}$ \\
\hline 183 & 296 & 4-(GlcO) $-\mathrm{C}_{6} \mathrm{H}_{4-}$ & 4-(GlcO) $-\mathrm{C}_{6} \mathrm{H}_{4^{-}}$ & $4-(\mathrm{GlcO})-\mathrm{C}_{6} \mathrm{H}_{4-}$ & $\begin{array}{l}\text { 4- } \\
{\left[\left(\mathrm{NH}_{2}\right)\left(\mathrm{CH}_{3}\right) \mathrm{CHCON}\right.} \\
\mathrm{H}]-\mathrm{C}_{6} \mathrm{H}_{4^{-}}\end{array}$ \\
\hline 184 & 297 & 4-(GlcO)- $\mathrm{C}_{6} \mathrm{H}_{4^{-}}$ & 4-(GlcO)- $\mathrm{C}_{6} \mathrm{H}_{4}-$ & 4-(GlcO)- $\mathrm{C}_{6} \mathrm{H}_{4-}$ & $\begin{array}{l}2- \\
{\left[\left(\mathrm{NH}_{2}\right)\left(\mathrm{CH}_{3}\right) \mathrm{CHCON}\right.} \\
{\mathrm{H}]-\mathrm{C}_{6} \mathrm{H}_{4}}^{-}\end{array}$ \\
\hline 185 & 298 & $\begin{array}{l}\left.\text { 2-[GlcO- } \mathrm{CH}_{2} \mathrm{CH}\left(\mathrm{NH}_{2}\right) \mathrm{CONH}\right]- \\
\mathrm{C}_{6} \mathrm{H}_{4^{-}}\end{array}$ & $4-\mathrm{Me}-\mathrm{C}_{6} \mathrm{H}_{4}^{-}$ & $4-\mathrm{Me}-\mathrm{C}_{6} \mathrm{H}_{4-}^{-}$ & $4-\mathrm{Me}-\mathrm{C}_{6} \mathrm{H}_{4^{-}}$ \\
\hline 186 & 299 & $\begin{array}{l}\left.\text { 4-[GlcO- } \mathrm{CH}_{2} \mathrm{CH}\left(\mathrm{NH}_{2}\right) \mathrm{CONH}\right]- \\
\mathrm{C}_{6} \mathrm{H}_{4^{-}}\end{array}$ & 4-Me- $\mathrm{C}_{6} \mathrm{H}_{4^{-}}$ & 4-Me- $\mathrm{C}_{6} \mathrm{H}_{4^{-}}$ & 4-Me- $\mathrm{C}_{6} \mathrm{H}_{4}-$ \\
\hline 187 & 300 & $2-\left(\mathrm{NO}_{2}\right)-\mathrm{C}_{6} \mathrm{H}_{4^{-}}^{-}$ & $4-\mathrm{Me}-\mathrm{C}_{6} \mathrm{H}_{4}^{-}$ & $4-\mathrm{Me}-\mathrm{C}_{6} \mathrm{H}_{4}-$ & $4-\mathrm{Me}-\mathrm{C}_{6} \mathrm{H}_{4^{-}}$ \\
\hline
\end{tabular}

2H From 272: 1,2,3,4-Tetra- $O$-acetyl- $\beta$ - $D$-glucopyranuronoyl chloride, $4 \times$ Pyrrole, $1 \times 2-\left(\right.$ AcOGlcO) $-\mathrm{C}_{6} \mathrm{H}_{4}-\mathrm{CHO}, 3 \times 4-\mathrm{NO}_{2}-\mathrm{C}_{6} \mathrm{H}_{4}-\mathrm{CHO}$

$\mathrm{CH}_{2} \mathrm{Cl}_{2}, \mathrm{BF}_{3} \cdot \mathrm{OEt}_{2}, 18 \mathrm{~h}$, then $p$-chloranil; $14 \%$.

Like entry 161 with 4-(AcOGlc) $-\mathrm{C}_{6} \mathrm{H}_{4}-\mathrm{CHO}, 14 \%$. $4 \times$ Pyrrole, $2 \times 4-(\mathrm{AcOGlcO})-\mathrm{C}_{6} \mathrm{H}_{4}-\mathrm{CHO}, 2 \times 4-\mathrm{NO}_{2}-\mathrm{C}_{6} \mathrm{H}_{4}-\mathrm{CHO}$, $\mathrm{CH}_{2} \mathrm{Cl}_{2}, \mathrm{BF}_{3} \cdot \mathrm{OEt}_{2}, 18 \mathrm{~h}$, then $p$-chloranil; $16 \%$.

$4 \times$ Pyrrole, $3 \times 4$-(AcOGlcO)- $\mathrm{C}_{6} \mathrm{H}_{4}-\mathrm{CHO}, 1 \times 4-\mathrm{NO}_{2}-\mathrm{C}_{6} \mathrm{H}_{4}-\mathrm{CHO}$,

$\mathrm{CH}_{2} \mathrm{Cl}_{2}, \mathrm{BF}_{3} \cdot \mathrm{OEt}_{2}, 18 \mathrm{~h}$, then $p$-chloranil; $16 \%$

Like entry 165 with $2-\mathrm{NO}_{2}-\mathrm{C}_{6} \mathrm{H}_{4}-\mathrm{CHO} ; 14 \%$.

From 274: $\mathrm{H}_{2}, 10 \%$ Pd-C, THF, r.t., 5 h; $60 \%$

From 275: Like entry $167 ; 56 \%$.

From 276: Like entry $167 ; 62 \%$.

From 277: Like entry $167 ; 64 \%$.

From 278: Like entry $167 ; 76 \%$.

From 279: Like entry $167 ; 60 \%$.

From 280: Like entry 167; $54 \%$.

From 281: $\mathrm{CH}_{2} \mathrm{Cl}_{2}$, DCC, Fmoc-L-Ala, $15 \mathrm{~h} ; 89 \%$.

From 282: Like entry $174 ; 88 \%$.

From 283: Like entry $174 ; 85 \%$.

From 284: Like entry $174 ; 90 \%$.

From 285: Like entry $174 ; 85 \%$.

From 286: Morpholine, then $\mathrm{NaOMe} / \mathrm{MeOH} / \mathrm{CH}_{2} \mathrm{Cl}_{2} ; 71 \%$.

\section{[207]}

$[207$ 


\begin{tabular}{|c|c|c|c|c|c|}
\hline 188 & 301 & 4- $\left(\mathrm{NO}_{2}\right)-\mathrm{C}_{6} \mathrm{H}_{4^{-}}$ & $4-\mathrm{Me}-\mathrm{C}_{6} \mathrm{H}_{4^{-}}$ & $4-\mathrm{Me}-\mathrm{C}_{6} \mathrm{H}_{4}-$ & 4-Me- $\mathrm{C}_{6} \mathrm{H}_{4^{-}}$ \\
\hline 189 & 302 & 2-( $\left(\mathrm{NH}_{2}\right)-\mathrm{C}_{6} \mathrm{H}_{4^{-}}$ & 4-Me- $\mathrm{C}_{6} \mathrm{H}_{4^{-}}$ & 4-Me- $\mathrm{C}_{6} \mathrm{H}_{4^{-}}$ & 4-Me- $\mathrm{C}_{6} \mathrm{H}_{4^{-}}$ \\
\hline 190 & 303 & 4-( $\left(\mathrm{NH}_{2}\right)-\mathrm{C}_{6} \mathrm{H}_{4^{-}}$ & 4-Me- $\mathrm{C}_{6} \mathrm{H}_{4}^{-}$ & 4-Me- $\mathrm{C}_{6} \mathrm{H}_{4^{-}}$ & 4-Me- $\mathrm{C}_{6} \mathrm{H}_{4-}^{-}$ \\
\hline 191 & 304 & $\begin{array}{l}\text { 2-[NH-(AcOGlcO)Ser-Fmoc]- } \\
\mathrm{C}_{6} \mathrm{H}_{4}\end{array}$ & 4-Me- $\mathrm{C}_{6} \mathrm{H}_{4}^{-}$ & 4-Me- $\mathrm{C}_{6} \mathrm{H}_{4}^{-}$ & 4-Me- $\mathrm{C}_{6} \mathrm{H}_{4^{-}}$ \\
\hline 192 & 305 & $\begin{array}{l}\text { 4-[NH-(AcOGlcO)Ser-Fmoc]- } \\
\mathrm{C}_{6} \mathrm{H}_{4}\end{array}$ & $4-\mathrm{Me}^{-} \mathrm{C}_{6} \mathrm{H}_{4}-$ & 4-Me- $\mathrm{C}_{6} \mathrm{H}_{4}^{-}$ & $4-\mathrm{Me}-\mathrm{C}_{6} \mathrm{H}_{4^{-}}$ \\
\hline 193 & 306 & $3-(\mathrm{GlcO})-\mathrm{C}_{6} \mathrm{H}_{4}-$ & $3-(\mathrm{GlcO})-\mathrm{C}_{6} \mathrm{H}_{4}-$ & $3-(\mathrm{GlcO})-\mathrm{C}_{6} \mathrm{H}_{4-}$ & Phenyl \\
\hline 194 & 307 & $3-(\mathrm{HO})-\mathrm{C}_{6} \mathrm{H}_{4^{-}}$ & $3-(\mathrm{HO})-\mathrm{C}_{6} \mathrm{H}_{4-}$ & $3-(\mathrm{HO})-\mathrm{C}_{6} \mathrm{H}_{4-}$ & Phenyl \\
\hline 195 & 308 & $4-\left[\mathrm{GlcO}-\left(\mathrm{C}_{2} \mathrm{H}_{4}-\mathrm{O}\right)_{2}\right]-\mathrm{C}_{6} \mathrm{H}_{4}-$ & $\begin{array}{l}4-\left[\mathrm{GlcO}-\left(\mathrm{C}_{2} \mathrm{H}_{4}-\mathrm{O}\right)_{2}\right]- \\
\mathrm{C}_{6} \mathrm{H}_{4^{-}}\end{array}$ & $\begin{array}{l}4-\left[\mathrm{GlcO}-\left(\mathrm{C}_{2} \mathrm{H}_{4}-\mathrm{O}\right)_{2}\right]- \\
\mathrm{C}_{6} \mathrm{H}_{4^{-}}\end{array}$ & Phenyl \\
\hline 196 & 309 & 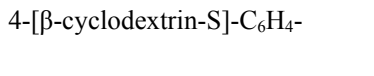 & Phenyl & $\begin{array}{l}4-[\beta \text {-cyclodextrin-S }]- \\
\mathrm{C}_{6} \mathrm{H}_{4^{-}}\end{array}$ & Phenyl \\
\hline 197 & 310 & 4-[ $\beta$-cyclodextrin- $\mathrm{S}]-\mathrm{C}_{6} \mathrm{H}_{4^{-}}$ & $\begin{array}{l}\text { 4-[ }[\beta \text {-cyclodextrin-S }]- \\
\mathrm{C}_{6} \mathrm{H}_{4^{-}}\end{array}$ & Phenyl - & Phenyl \\
\hline 198 & 311 & 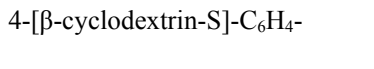 & Phenyl & 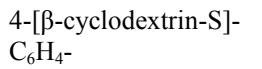 & Phenyl \\
\hline 199 & 312 & 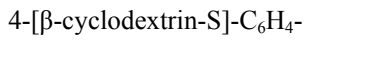 & $\begin{array}{l}\text { 4-[ } \beta \text {-cyclodextrin-S]- } \\
\mathrm{C}_{6} \mathrm{H}_{4^{-}}\end{array}$ & Phenyl - & Phenyl \\
\hline 200 & 313 & $4-[\beta$-cyclodextrin-O $]-\mathrm{C}_{6} \mathrm{H}_{4^{-}}$ & $4-\mathrm{Me}-\mathrm{C}_{6} \mathrm{H}_{4-}$ & $\begin{array}{l}\text { 4-[ }[\beta \text {-cyclodextrin- } O]- \\
\mathrm{C}_{6} \mathrm{H}_{4^{-}}\end{array}$ & 4-Me- $\mathrm{C}_{6} \mathrm{H}_{4-}$ \\
\hline 201 & 314 & $4-[\beta$-cyclodextrin- $O]-\mathrm{C}_{6} \mathrm{H}_{4^{-}}$ & $4-\mathrm{Me}-\mathrm{C}_{6} \mathrm{H}_{4}^{-}$ & $\begin{array}{l}\text { 4-[B-cyclodextrin- } O]- \\
\mathrm{C}_{6} \mathrm{H}_{4^{-}}\end{array}$ & 4-Me- $\mathrm{C}_{6} \mathrm{H}_{4-}$ \\
\hline 202 & 315 & 4-[ManO- $\left.\left(\mathrm{C}_{2} \mathrm{H}_{4}-\mathrm{O}\right)\right]-\mathrm{C}_{6} \mathrm{H}_{4^{-}}$ & $\begin{array}{l}\left.\text { 4-[ManO- }\left(\mathrm{C}_{2} \mathrm{H}_{4}-\mathrm{O}\right)\right]- \\
\mathrm{C}_{6} \mathrm{H}_{4^{-}}\end{array}$ & $\begin{array}{l}\left.\text { 4-[ManO- }\left(\mathrm{C}_{2} \mathrm{H}_{4}-\mathrm{O}\right)\right]- \\
\mathrm{C}_{6} \mathrm{H}_{4^{-}}\end{array}$ & Phenyl \\
\hline 203 & 316 & $\begin{array}{l}\left.\text { 4-[4'-(GlcO-CH } \mathrm{CH}_{2}\right)-1^{\prime}{ }^{\prime} N-1^{\prime}, 2^{\prime}, 3^{\prime}- \\
\text { triazol-1'-yl]- } \mathrm{C}_{6} \mathrm{H}_{4^{-}}\end{array}$ & $\mathrm{C}_{6} \mathrm{H}_{5^{-}}$ & $\mathrm{C}_{6} \mathrm{H}_{5^{-}}$ & $\mathrm{C}_{6} \mathrm{H}_{5^{-}}$ \\
\hline 204 & 317 & $\begin{array}{l}4-\left[4^{\prime}-\left(\mathrm{GlcNAcO}-\mathrm{CH}_{2}\right)-1^{\prime} N-\right. \\
1^{\prime}, 2^{\prime}, 3^{\prime}-\text { triazol-1'-vl]- } \mathrm{C}_{6} \mathrm{H}_{4-}\end{array}$ & $\mathrm{C}_{6} \mathrm{H}_{5^{-}}$ & $\mathrm{C}_{6} \mathrm{H}_{5^{-}}$ & $\mathrm{C}_{6} \mathrm{H}_{5^{-}}$ \\
\hline 205 & 318 & $\begin{array}{l}\left.\text { 4-[4'-(LacNAcO-CH }{ }_{2}\right)-1 \text { ' } N- \\
1,2,3^{\prime},- \text { triazol-1'-yl]- } \mathrm{C}_{6} \mathrm{H}_{4-}\end{array}$ & $\mathrm{C}_{6} \mathrm{H}_{5^{-}}$ & $\mathrm{C}_{6} \mathrm{H}_{5^{-}}$ & $\mathrm{C}_{6} \mathrm{H}_{5^{-}}$ \\
\hline 206 & 319 & $\begin{array}{l}4-\left[4^{\prime}-\left(\text { Lewis X-CH }{ }_{2}\right)-1^{\prime} N-\right. \\
1^{\prime}, 2^{\prime}, 3^{\prime} \text {-triazol-1'-yl] }-\mathrm{C}_{6} \mathrm{H}_{4}-\end{array}$ & $\mathrm{C}_{6} \mathrm{H}_{5^{-}}$ & $\mathrm{C}_{6} \mathrm{H}_{5^{-}}$ & $\mathrm{C}_{6} \mathrm{H}_{5^{-}}$ \\
\hline 207 & 320 & 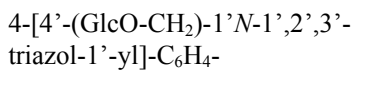 & 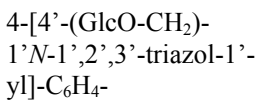 & $\mathrm{C}_{6} \mathrm{H}_{5^{-}}$ & $\mathrm{C}_{6} \mathrm{H}_{5^{-}}$ \\
\hline 208 & 321 & $\begin{array}{l}\left.\text { 4-[4'-(ManO- } \mathrm{CH}_{2}\right)-1^{\prime}{ }^{\prime} N-1^{\prime}, 2^{\prime}, 3^{\prime}- \\
\text { triazol-1'-yl]- } \mathrm{C}_{6} \mathrm{H}_{4^{-}}\end{array}$ & $\begin{array}{l}4-\left[4^{\prime}-\left(\mathrm{ManO}-\mathrm{CH}_{2}\right)-\right. \\
1^{\prime} N-1^{\prime}, 2^{\prime}, 3^{\prime} \text { 'triazol-1'- } \\
\text { yl]-C } \mathrm{C}_{6} \mathrm{H}_{4^{-}}\end{array}$ & $\mathrm{C}_{6} \mathrm{H}_{5^{-}}$ & $\mathrm{C}_{6} \mathrm{H}_{5^{-}}$ \\
\hline 209 & 322 & $\begin{array}{l}\left.\text { 4-[4'-(GlcNAcO-CH }{ }_{2}\right)-1^{\prime} N- \\
1^{\prime}, 2^{\prime}, 3^{\prime} \text {-triazol-1'-yl] } \mathrm{C}_{6} \mathrm{H}_{4^{-}}\end{array}$ & $\begin{array}{l}\text { 4-[4'-(GlcNAcO- } \\
\left.\mathrm{CH}_{2}\right)-1^{\prime} N-1^{\prime}, 2^{\prime}, 3^{\prime}- \\
\text { triazol-1'-yl]-- } \mathrm{C}_{6} \mathrm{H}_{4^{-}}\end{array}$ & $\mathrm{C}_{6} \mathrm{H}_{5^{-}}$ & $\mathrm{C}_{6} \mathrm{H}_{5^{-}}$ \\
\hline 210 & 323 & 4-[4'-( $\left.\mathrm{LacNAcO}-\mathrm{CH}_{2}\right)-1^{\prime} N-$ & 4-[4'-(LacNAcO- & $\mathrm{C}_{6} \mathrm{H}_{5^{-}}$ & $\mathrm{C}_{6} \mathrm{H}_{5^{-}}$ \\
\hline
\end{tabular}

$\mathrm{BF}_{3} \cdot \mathrm{OEt}_{2}, 18 \mathrm{~h}$, then $p$-chloranil, $1 \mathrm{~h} ; 15 \%$.

Like entry 187 ith $4-\left(\mathrm{NO}_{2}\right) \mathrm{C}_{1} \mathrm{H}_{4} \mathrm{CHO} ; 15 \%$

From 300: $\mathrm{SnCl}_{2} / \mathrm{HCl}$. reflux, $15 \mathrm{~h} ; 92 \%$.

From 301: Like entry $189 ; 90 \%$.

From 302: DCC, (AcOGlcO)Ser-Fmoc, 15 h, r.t.; $60 \%$. [207]

From 303: Like entry 191; $84 \%$.

Pyrrole, 3-(AcOGlcO)- $\mathrm{C}_{6} \mathrm{H}_{4}-\mathrm{CHO}$, benzaldehyde, $\mathrm{CHCl}_{3}, \mathrm{BF}_{3} \cdot \mathrm{OEt}_{2}$, r.t., 24 h, then $p$-chloranil, $1 \mathrm{~h}$, reflux; then $\mathrm{NaOMe}, \mathrm{MeOH}, 1 \mathrm{~h}$, r.t.

5,10,15-Tri( $p$-hydroxyphenyl)-20-phenyl porphyrin, 2[2-(2-bromoethoxy)-ethoxy]-O-2', 3', 4', 6'-tetraacetyl- $\beta$ - $D$-glucose, $\mathrm{K}_{2} \mathrm{CO}_{3}$, DMF, $60{ }^{\circ} \mathrm{C}, 24 \mathrm{~h}$; then $\mathrm{NaOMe}, \mathrm{MeOH}, \mathrm{CH}_{2} \mathrm{Cl}_{2}$, r.t., $1.5 \mathrm{~h}$.

$2 \mathrm{H}$ i) meso-Phenyldipyrromethane, $p$-(methylthio)benzaldehyde, $\mathrm{CHCl}_{3}$ $\mathrm{BF}_{3} \cdot \mathrm{OEt}_{2}$, r.t., $1 \mathrm{~h}$; then $p$-chloranil, $1 \mathrm{~h}$, reflux; $20 \%$, mixture of 5,15 and 5,10-derivatives. Then, $\mathrm{CH}_{2} \mathrm{Cl}_{2}, m$-chloroperoxybenzoic acid, $0^{\circ} \mathrm{C}$, $40 \mathrm{~min}$.; separation of isomers. ii) 5,15 isomer, trifluoroacetic anhydride, $\mathrm{CH}_{2} \mathrm{Cl}_{2}, 40{ }^{\circ} \mathrm{C}, 50 \mathrm{~min}$.; then $\beta$-cyclodextrin iodide, DMF, $\mathrm{MeOH}, \mathrm{Et}_{3} \mathrm{~N}, 70{ }^{\circ} \mathrm{C}, 24 \mathrm{~h} ; 30 \%$.

$2 \mathrm{H} \quad$ i) Like entry 196 ; ii) like entry 196 with 5,10 isomer; $45 \%$. [179]

$\mathrm{MnCl}$ From 309: $\mathrm{MnCl}_{2}$, 2,6-lutidine, DMF, $\Delta, 27 \mathrm{~h} . ; 80 \%$ [179]

$\mathrm{MnCl}$ From 310: Like entry $198 ; 40 \%$. [179]

$\mathrm{Zn}$ (II) $\quad\{5,15-(4-H y d r o x y p h e n y l)-10,20-d i(p$-tolyl)porphyrinato $\} z i n c(I I), 6-O-\quad[211]$

$\begin{array}{ll}\text { Ru(II) } & \text { Like entry } 200 \text { with }\{5,15-(4-\text {-hydroxyphenyl)-10,20-di }(p-\end{array}$ tolyl)porphyrinato $\}$ ruthenium(II)

$\mathrm{Zn}(\mathrm{II}) \quad$ 5-(4-Azidophenyl)-10,15,20-triphenylporphyrin, $\mathrm{Cu}(\mathrm{MeCN})_{4} \mathrm{PF}_{6}$, (GlcO)- $\mathrm{CH}_{2} \mathrm{CCH}$, toluene/ $\mathrm{MeOH}(0.6: 0.2, \mathrm{v} / \mathrm{v}), 110^{\circ} \mathrm{C}, 20 \mathrm{~min}$. microwave; $93 \%$.

$\mathrm{Zn}(\mathrm{II}) \quad$ Like entry 203 with (GlcNAcO)- $\mathrm{CH}_{2} \mathrm{CCH} ; 93 \%$.

$\mathrm{Zn}(\mathrm{II}) \quad$ Like entry 203 with (LacNAcO)-CH $2 \mathrm{CCH} ; 93 \%$.

$\mathrm{Zn}(\mathrm{II}) \quad$ Like entry 203 with (Lewis $\mathrm{X}$ )- $\mathrm{CH}_{2} \mathrm{CCH} ; 65 \%$.

$\mathrm{Zn}$ (II) 5,10-Bis(4-azidophenyl)-15,20-diphenylporphyrin, $\mathrm{Cu}(\mathrm{MeCN})_{4} \mathrm{PF}_{6}$, ( $\mathrm{GlcO})-\mathrm{CH}_{2} \mathrm{CCH}$, toluene/ $\mathrm{MeOH}(0.6: 1, \mathrm{v} / \mathrm{v}), 110^{\circ} \mathrm{C}, 20 \mathrm{~min}$., microwave; $91 \%$

$\mathrm{Zn}(\mathrm{II}) \quad$ Like entry 207 with (ManO)- $\mathrm{CH}_{2} \mathrm{CCH} ; 93 \%$.

$\mathrm{Zn}(\mathrm{II}) \quad$ Like entry 207 with (GlcNAcO)-CH $2 \mathrm{CCH} ; 82 \%$.

$\mathrm{Zn}(\mathrm{II}) \quad$ Like entry 207 with (LacNAcO)- $\mathrm{CH}_{2} \mathrm{CCH} ; 77 \%$. 
$\begin{array}{lll}1^{\prime}, 2^{\prime}, 3^{\prime}-\text { triazol-1'-yl]- } \mathrm{C}_{6} \mathrm{H}_{4^{-}} & \left.\mathrm{CH}_{2}\right)-1^{\prime} N-1^{\prime}, 2^{\prime}, 3^{\prime}- \\ \text { triazol-1'- }-\mathrm{yl}]-\mathrm{C}_{6} \mathrm{H}_{4}-\end{array}$ $N-1^{\prime}, 2^{\prime}, 3^{\prime}-\quad \mathrm{C}_{6} \mathrm{H}_{5}-$ triazol-1'-yl]- $\mathrm{C}_{6} \mathrm{H}_{4}-$

$\begin{array}{lll}212 & 325 & 4-\left[4^{\prime}-\left(\mathrm{LacNAcO}-\mathrm{CH}_{2}\right)-1^{\prime} N-\right. \\ & 1^{\prime}, 2^{\prime}, 3^{\prime} \text {-'triazol-1'-yl] }-\mathrm{C}_{6} \mathrm{H}_{4}\end{array}$

4-[4'-(GlcO-CH$\left.-\mathrm{CH}_{2}\right)-1^{\prime} N-1^{\prime}, 2^{\prime}, 3^{\prime}-$

$327 \quad$ triazol-1'-yl]- $\mathrm{C}_{6} \mathrm{H}_{4^{-}}-4^{\prime}-\left(\mathrm{ManO}-\mathrm{CH}_{2}\right)-1^{\prime} N-1^{\prime}, 2^{\prime}, 3^{\prime}-$

21 triazol-1'-yl]- $\mathrm{C}_{6} \mathrm{H}_{4}-$

215

328 4-[4'-(GlcNAcO-CH 2$)-1^{\prime} N-$

1',2',3'-triazol-1'-yl]- $\mathrm{C}_{6} \mathrm{H}_{4^{-}}$

216

1',2',3'-triazol-1'-yl]- $\mathrm{C}_{6} \mathrm{H}_{4}$

217

4-[4'-(ManO- $\left.\mathrm{CH}_{2}\right)-1^{\prime} N-1^{\prime}, 2^{\prime}, 3^{\prime}$ ' triazol-1'-yl]- $\mathrm{C}_{6} \mathrm{H}_{4}-$

218

4-[4'-(ManO-CH $\left.-\mathrm{CH}_{2}\right)-1^{\prime} N-1^{\prime}, 2^{\prime}, 3^{\prime}$ triazol-1'-yl]- $\mathrm{C}_{6} \mathrm{H}_{4}-$

219

220

221

222

223

224

225

226

227

340

4-( $\mathrm{AcOGlcO})-\mathrm{C}_{6} \mathrm{H}_{4-}^{-}$
$\mathrm{C}_{6} \mathrm{H}_{5-}^{-}$

4-[4'-( $\left.\mathrm{ManO}-\mathrm{CH}_{2}\right)-$

$1^{\prime} N-1$ ', 2',3'-triazol-1'.

yl]- $-\mathrm{C}_{6} \mathrm{H}_{4^{-}}$

4-[4'-(LacNAcO-

$\left.\mathrm{CH}_{2}\right)-1^{\prime} N-1^{\prime}, 2^{\prime}, 3^{\prime}$

triazol-1'-yl]- $\mathrm{C}_{6} \mathrm{H}_{4}$

4-[4'-(GlcO- $\left.\mathrm{CH}_{2}\right)$

$1^{\prime} N-1^{\prime}, 2^{\prime}, 3^{\prime}$-triazol-1'

yl] $-\mathrm{C}_{6} \mathrm{H}_{4-}$

$4-\left[4 '-\left(\mathrm{GlcO}-\mathrm{CH}_{2}\right)-\right.$

$1^{\prime} N-1$ ', 2', 3'-triazol-1'-

yl] $-\mathrm{C}_{6} \mathrm{H}_{4-}$

4-[4'-( $\left.\mathrm{ManO}-\mathrm{CH}_{2}\right)-$ 4-[4'-(ManO- $\left.\mathrm{CH}_{2}\right)-$

$1^{\prime} N-1^{\prime}, 2^{\prime}, 3^{\prime}$-triazol-1'- $\quad 1^{\prime} N-1^{\prime}, 2^{\prime}, 3^{\prime}$-triazol-1'yl] $-\mathrm{C}_{6} \mathrm{H}_{4}$

4-[4'-(GlcNAcO-

$\left.\mathrm{CH}_{2}\right)-1^{\prime} N-1^{\prime}, 2^{\prime}, 3^{\prime}-$

triazol-1'-yl]- $\mathrm{C}_{6} \mathrm{H}_{4-}$

4-[4'-(LacNAcO-

$\left.\mathrm{CH}_{2}\right)-1{ }^{\prime} N-1,22^{\prime}, 3^{\prime}$

triazol-1'-yl]- $\mathrm{C}_{6} \mathrm{H}_{4}$

yl]- $-\mathrm{C}_{6} \mathrm{H}_{4-}-$

44-[4'-(GlcNAcO-

$\left.\mathrm{CH}_{2}\right)-1^{\prime} N-1^{\prime}, 2^{\prime}, 3^{\prime}-$

triazol-1'-yl]- $\mathrm{C}_{6} \mathrm{H}_{4--}$

4-[4'-(LacNAcO-

$\left.\mathrm{CH}_{2}\right)-1^{\prime} N-1^{\prime}, 2^{\prime}, 3^{\prime}-$

triazol-1'-yl]- $\mathrm{C}_{6} \mathrm{H}_{4}$

$\mathrm{C}_{6} \mathrm{H}_{5-}$

4-[4'-(GlcNAcO-

$\left.\mathrm{CH}_{2}\right)-1^{\prime} N-1^{\prime}, 2^{\prime}, 3^{\prime}-$

triazol-1'-yll]- $\mathrm{C}_{6} \mathrm{H}_{4}-$

4-( $\mathrm{GlcO})-\mathrm{C}_{6} \mathrm{H}_{4}$

4-(GlcO)- $\mathrm{C}_{6} \mathrm{H}_{4}-$

$\mathrm{C}_{6} \mathrm{H}_{5-}$

$\mathrm{C}_{6} \mathrm{H}_{5^{-}}$

$\mathrm{C}_{6} \mathrm{H}_{5^{-}}$

$\mathrm{C}_{6} \mathrm{H}_{5^{-}}$

${ }^{\mathrm{C}_{6} \mathrm{H}_{5^{-}}}$

$\mathrm{C}_{6} \mathrm{H}_{13}-$

3,5- $\left(\mathrm{CF}_{3}\right)-\left(\mathrm{C}_{6} \mathrm{H}_{3}\right)-$

$\mathrm{C}_{6} \mathrm{H}_{5^{-}}$

$\mathrm{C}_{6} \mathrm{H}_{13^{-}}$

3,5- $\left(\mathrm{CF}_{3}\right)-\left(\mathrm{C}_{6} \mathrm{H}_{3}\right)-$

$\mathrm{C}_{6} \mathrm{H}_{5}$

4-( $\mathrm{AcOGlcO})-\mathrm{C}_{6} \mathrm{H}_{4^{-}} \quad 3,5-\left(\mathrm{CF}_{3}\right)-\left(\mathrm{C}_{6} \mathrm{H}_{3}\right)-$
$\mathrm{Zn}(\mathrm{II}) \quad$ 5,15-Bis(4-azidophenyl)-10,20-diphenylporphyrin, $\mathrm{Cu}(\mathrm{MeCN})_{4} \mathrm{PF}_{6}$, (ManO)- $\mathrm{CH}_{2} \mathrm{CCH}$, toluene/ $\mathrm{MeOH}(0.6: 1, \mathrm{v} / \mathrm{v}), 110^{\circ} \mathrm{C}, 20 \mathrm{~min}$. microwave; $85 \%$

$\mathrm{Zn}$ (II) Like entry 211 with ( $\mathrm{LacNAcO}$ )- $\mathrm{CH}_{2} \mathrm{CCH} ; 75 \%$.

$\mathrm{Zn}$ (II) Like entry 211 with (GlcO)- $\mathrm{CH}_{2} \mathrm{CCH} ; 70 \%$

$\mathrm{Zn}$ (II) 5,10,15-Tris(4-azidophenyl)-20-phenylporphyrin, $\mathrm{Cu}(\mathrm{MeCN})_{4} \mathrm{PF}_{6}$, (ManO) $-\mathrm{CH}_{2} \mathrm{CCH}$, toluene/ $\mathrm{MeOH}(0.6: 1, \mathrm{v} / \mathrm{v}), 110^{\circ} \mathrm{C}, 20 \mathrm{~min}$., microwave; $79 \%$.

$\mathrm{Zn}$ (II) Like entry 214 with (GlcNAcO)- $\mathrm{CH}_{2} \mathrm{CCH} ; 76 \%$.

$\mathrm{Zn}(\mathrm{II}) \quad$ Like entry 214 with ( $\mathrm{LacNAcO})-\mathrm{CH}_{2} \mathrm{CCH} ; 56 \%$.

$\mathrm{Zn}(\mathrm{II}) \quad$ 5,10-Bis(4-azidophenyl), 15,20-diphenylporphyrin, $\mathrm{Cu}(\mathrm{MeCN})_{4} \mathrm{PF}_{6}$ (ManO)- $\mathrm{CH}_{2} \mathrm{CCH}$, toluene/ $\mathrm{MeOH}(0.6: 1, \mathrm{v} / \mathrm{v}), 110^{\circ} \mathrm{C}, 20 \mathrm{~min}$. microwave; $67 \%$

$\mathrm{Zn}(\mathrm{II}) \quad$ From 330, $\mathrm{Cu}(\mathrm{MeCN})_{4} \mathrm{PF}_{6},(\mathrm{GlcNAcO})-\mathrm{CH}_{2} \mathrm{CCH}$, toluene $/ \mathrm{MeOH}$ $(0.6: 1, \mathrm{v} / \mathrm{v}), 110{ }^{\circ} \mathrm{C}, 20 \mathrm{~min}$, microwave; $85 \%$

$2 \mathrm{H}$ i) Pyrrole, $4-(\mathrm{AcOGlcO})-\mathrm{C}_{6} \mathrm{H}_{4}-\mathrm{CHO}$, benzaldehyde, $\mathrm{CHCl}_{3}, \mathrm{BF}_{3} \cdot \mathrm{OEt}_{2}$ r.t., $24 \mathrm{~h}$, then $p$-chloranil, $1 \mathrm{~h}$, reflux, $10 \%$. ii) $\mathrm{NaOMe}, \mathrm{MeOH}, 1 \mathrm{~h}$, r.t., $; 83 \%$.

$2 \mathrm{H} \quad 5$-(4-Aminophenyl)-10,15,20-triphenylporphyrin, per- $O$-acetyl- $\beta-D$ glucopyranosyl isothiocyanate, $\mathrm{CHCl}_{3}, 24 \mathrm{~h}$, reflux; $72 \%$. Or: $\mathrm{CHCl}_{3}$,

$2 \mathrm{H} \quad 5$-(4-Hydroxyphenyl)-10,15,20-triphenylporphyrin, per- $O$-acetyl- $\beta-D-$ glucopyranosyl isothiocyanate, TEA, $\mathrm{CHCl}_{3}, 48 \mathrm{~h}$, reflux; $23 \%$ ike entry 221,24 h, reflux; then $\mathrm{NaOH}, 24$ h, r.t.; $49 \%$. \{5-(3-Hydroxyphenyl)-10,15,20-triphenylporphyrinato $\}$ zinc(II), 2,3,4,6-tetra- $O$-acetyl- $\beta$ - $D$-glucopyranosyl trichloroacetimidate, $\mathrm{BF}_{3} \cdot \mathrm{OEt}_{2}, 15$ min. r.t.; then $\mathrm{THF}, \mathrm{HCl}, 10$ min., r.t.; $89 \%$. Then $\mathrm{THF} / \mathrm{MeOH} / \mathrm{NaOMe}, 2 \mathrm{~h} ; 97 \%$.

Like entry 223 with $\{5$-(3-hydroxyphenyl)-10,15,20trihexylporphyrinato zinc(II).

Like entry 223 with $\left\{5\right.$-(3-hydroxyphenyl)-10,15,20-tri [3,5-( $\left.\mathrm{CF}_{3}\right)$ $\left.\mathrm{C}_{6} \mathrm{H}_{3}\right)$ porphyrinato $\mathrm{zinc}$ (II)

\{5-(3-Hydroxyphenyl)-10,15,20-triphenylporphyrinato $\}$ zinc(II), 2,3,4,6-tetra- $O$-acetyl- $\beta$ - $D$-galactopyranosyl trichloroacetimidate, BF. $\mathrm{OEt}_{2}, 120 \mathrm{~min}, \mathrm{r} . \mathrm{t}$; then $\mathrm{THF}, \mathrm{HCl}, 10 \mathrm{~min}, \mathrm{r} . \mathrm{t} \cdot 84 \%$. Then $\mathrm{THF} / \mathrm{MeOH} / \mathrm{NaOMe}, 2 \mathrm{~h}$

5,10,15-(3-Hydroxyphenyl)-20-[3,5- $\left.\left(\mathrm{CF}_{3}\right)-\left(\mathrm{C}_{6} \mathrm{H}_{3}\right)\right]$ -

porphyrinato zinc(II), 2,3,4,6-tetra- $O$-acetyl- $\beta$ - $D$-galactopyranosyl porphride

4-(4-pyridyl-S)- $\mathrm{C}_{6} \mathrm{~F}_{4^{-}} \quad$ 4-(4-pyridyl-S)- $\mathrm{C}_{6} \mathrm{~F}_{4^{-}} \quad 2 \mathrm{H}$ 


\begin{tabular}{|c|c|c|c|c|c|c|c|c|}
\hline 229 & 342 & 4-(GlcS)- $\mathrm{C}_{6} \mathrm{~F}_{4^{-}}$ & 4-(4-pyridyl-S)- $\mathrm{C}_{6} \mathrm{~F}_{4^{-}}$ & 4-(GlcS)- $\mathrm{C}_{6} \mathrm{~F}_{4^{-}}$ & 4-(4-pyridyl-S)- $\mathrm{C}_{6} \mathrm{~F}_{4^{-}}$ & $2 \mathrm{H}$ & & [215] \\
\hline 230 & 343 & 4-(XylS)- $-\mathrm{C}_{6} \mathrm{~F}_{4^{-}}$ & $4-(\mathrm{GlcS})-\mathrm{C}_{6} \mathrm{~F}_{4^{-}}$ & 4-(4-pyridyl-S)- $-\mathrm{C}_{6} \mathrm{~F}_{4^{-}}$ & 4-(4-pyridyl-S)- $-\mathrm{C}_{6} \mathrm{~F}_{4}-$ & $2 \mathrm{H}$ & & [215] \\
\hline 231 & 344 & 4-(XylS)- $-\mathrm{C}_{6} \mathrm{~F}_{4^{-}}$ & 4-(4-pyridyl-S)- $\mathrm{C}_{6} \mathrm{~F}_{4}-$ & $4-(\mathrm{GlcS})-\mathrm{C}_{6} \mathrm{~F}_{4^{-}}$ & 4-(4-pyridyl-S)- $-\mathrm{C}_{6} \mathrm{~F}_{4}-$ & $2 \mathrm{H}$ & & [215] \\
\hline 231 & 345 & 4-(GlcS) $-\mathrm{C}_{6} \mathrm{~F}_{4^{-}}$ & $4-(\mathrm{GlcS})-\mathrm{C}_{6} \mathrm{~F}_{4^{-}}$ & 4-(XylS)- $\mathrm{C}_{6} \mathrm{~F}_{4^{-}}$ & 4-(4-pyridyl-S)- $-\mathrm{C}_{6} \mathrm{~F}_{4}-$ & $2 \mathrm{H}$ & & [215] \\
\hline 232 & 346 & 4-(GlcS) $-\mathrm{C}_{6} \mathrm{~F}_{4^{-}}$ & 4-(XylS)- $\mathrm{C}_{6} \mathrm{~F}_{4^{-}}$ & 4-(GlcS)- $\mathrm{C}_{6} \mathrm{~F}_{4^{-}}$ & 4-(4-pyridyl-S)- $\mathrm{C}_{6} \mathrm{~F}_{4}-$ & $2 \mathrm{H}$ & & [215] \\
\hline 233 & 347 & 4-(XylS)- $\mathrm{C}_{6} \mathrm{~F}_{4-}$ & $4-(\mathrm{XylS})-\mathrm{C}_{6} \mathrm{~F}_{4^{-}}$ & $4-(\mathrm{GlcS})-\mathrm{C}_{6} \mathrm{~F}_{4^{-}}$ & 4-(4-pyridyl-S)- $\mathrm{C}_{6} \mathrm{~F}_{4}-$ & $2 \mathrm{H}$ & & [215] \\
\hline 234 & 348 & 4-(XylS)- $-\mathrm{C}_{6} \mathrm{~F}_{4^{-}}$ & 4-(GlcS) $-\mathrm{C}_{6} \mathrm{~F}_{4^{-}}$ & 4-(XylS)- $\mathrm{C}_{6} \mathrm{~F}_{4^{-}}$ & 4-(4-pyridyl-S)- $\mathrm{C}_{6} \mathrm{~F}_{4}-$ & $2 \mathrm{H}$ & & [215] \\
\hline 235 & 349 & 4-(GlcS) $-\mathrm{C}_{6} \mathrm{~F}_{4^{-}}$ & 4-(GlcS) $-\mathrm{C}_{6} \mathrm{~F}_{4^{-}}$ & $4-(\mathrm{GlcS})-\mathrm{C}_{6} \mathrm{~F}_{4^{-}}$ & 4-(4-pyridyl-S)- $\mathrm{C}_{6} \mathrm{~F}_{4}-$ & $2 \mathrm{H}$ & & [215] \\
\hline 236 & 350 & 4-(GlcS)- $-\mathrm{C}_{6} \mathrm{~F}_{4^{-}}$ & 4-(GlcS)- $\mathrm{C}_{6} \mathrm{~F}_{4^{-}}$ & $\begin{array}{l}\text { 4-(N-Me-4-pyridyl-S })- \\
\mathrm{C}_{6} \mathrm{~F}_{4-}\end{array}$ & $\begin{array}{l}\text { 4-( } N \text {-Me-4-pyridyl- } \\
\text { S)- } \mathrm{C}_{6} \mathrm{~F}_{4^{-}}\end{array}$ & $2 \mathrm{H}$ & & [215] \\
\hline 237 & 351 & 4-(GlcS)- $\mathrm{C}_{6} \mathrm{~F}_{4^{-}}$ & $\begin{array}{l}\text { 4-(N-Me-4-pyridyl-S })- \\
\mathrm{C}_{6} \mathrm{~F}_{4^{-}}\end{array}$ & $4-(\mathrm{GlcS})-\mathrm{C}_{6} \mathrm{~F}_{4^{-}}$ & $\begin{array}{l}\text { 4-(N-Me-4-pyridyl- } \\
\mathrm{S})-\mathrm{C}_{6} \mathrm{~F}_{4}-\end{array}$ & $2 \mathrm{H}$ & & [215] \\
\hline 238 & 352 & $\mathrm{C}_{6} \mathrm{~F}_{5^{-}}$ & 4-pyridyl & 4-pyridyl & 4-pyridyl & $2 \mathrm{H}$ & $\begin{array}{l}\text { Pyrrole, pyridyl-4-carboxaldehyde, pentafluorobenzaldehyde, } \\
\text { propionic acid. }\end{array}$ & [215] \\
\hline 239 & 353 & 4-(GlcS)- $\mathrm{C}_{6} \mathrm{~F}_{4^{-}}$ & 4-pyridyl & 4-pyridyl & 4-pyridyl & $2 \mathrm{H}$ & $\begin{array}{l}\text { From 352: 2,3,4,6-Tetra- } O \text {-acetyl-glucosylthioacetate, diethylamine, } \\
\text { DMF, } \mathrm{CHCl}_{3} \text {, methanol, } 24 \mathrm{~h} \text {. }\end{array}$ & [215] \\
\hline 240 & 354 & $\mathrm{C}_{6} \mathrm{~F}_{5^{-}}$ & $\mathrm{C}_{6} \mathrm{~F}_{5^{-}}$ & 4-pyridyl & 4-pyridyl & $2 \mathrm{H}$ & Similar to entry 238. & [215] \\
\hline 241 & 355 & 4-(GlcS)- $\mathrm{C}_{6} \mathrm{~F}_{4^{-}}$ & $4-(\mathrm{GlcS})-\mathrm{C}_{6} \mathrm{~F}_{4^{-}}$ & 4-pyridyl & 4-pyridyl & $2 \mathrm{H}$ & $\begin{array}{l}\text { From 354: 2,3,4,6-Tetra- } O \text {-acetyl-glucosylthioacetate, diethylamine, } \\
\text { DMF, } \mathrm{CHCl}_{3}, \mathrm{MeOH}, 24 \mathrm{~h} ; 82 \% \text {. }\end{array}$ & [215] \\
\hline 242 & 356 & $\mathrm{C}_{6} \mathrm{~F}_{5^{-}}$ & 4-pyridyl & $\mathrm{C}_{6} \mathrm{~F}_{5^{-}}$ & 4-pyridyl & $2 \mathrm{H}$ & Similar to entry 238. & [215] \\
\hline 243 & 357 & 4-(GlcS)- $\mathrm{C}_{6} \mathrm{~F}_{4^{-}}$ & 4-pyridyl & $4-(\mathrm{GlcS})-\mathrm{C}_{6} \mathrm{~F}_{4^{-}}$ & 4-pyridyl & $2 \mathrm{H}$ & From 356: Like entry 241. & [215] \\
\hline 244 & 358 & $\mathrm{C}_{6} \mathrm{~F}_{5^{-}}$ & $\mathrm{C}_{6} \mathrm{~F}_{5^{-}}$ & $\mathrm{C}_{6} \mathrm{~F}_{5^{-}}$ & 4-pyridyl & $2 \mathrm{H}$ & Similar to entry 238. & [215] \\
\hline 245 & 359 & 4-(GlcS)- $\mathrm{C}_{6} \mathrm{~F}_{4^{-}}$ & 4-(GlcS)- $\mathrm{C}_{6} \mathrm{~F}_{4-}$ & $4-(\mathrm{GlcS})-\mathrm{C}_{6} \mathrm{~F}_{4-}$ & 4-pyridyl & $2 \mathrm{H}$ & From 358: Like entry $241 ; 90 \%$. & [215] \\
\hline 246 & 360 & 4-( $(\mathrm{GlcS})-\mathrm{C}_{6} \mathrm{~F}_{4^{-}}$ & $N$-Me-4-pyridyl & $N$-Me-4-pyridyl & $N$-Me-4-pyridyl & $2 \mathrm{H}$ & From 353: $\mathrm{CH}_{3} \mathrm{I}, \mathrm{CH}_{2} \mathrm{Cl}_{2}, 40^{\circ} \mathrm{C}, 17 \mathrm{~h}$. & [215] \\
\hline 247 & 361 & 4-(GlcS) $-\mathrm{C}_{6} \mathrm{~F}_{4^{-}}$ & 4-(GlcS)- $-\mathrm{C}_{6} \mathrm{~F}_{4^{-}}$ & $N$-Me-4-pyridyl & $N$-Me-4-pyridyl & $2 \mathrm{H}$ & From 355: Like entry 246. & [215] \\
\hline 248 & 362 & 4-(GlcS) $-\mathrm{C}_{6} \mathrm{~F}_{4^{-}}$ & $N$-Me-4-pyridyl & $4-(\mathrm{GlcS})-\mathrm{C}_{6} \mathrm{~F}_{4^{-}}$ & $N$-Me-4-pyridyl & $2 \mathrm{H}$ & From 357: Like entry 246. & [215] \\
\hline 249 & 363 & 4-(GlcS) $-\mathrm{C}_{6} \mathrm{~F}_{4^{-}}$ & 4-(GlcS)- $-\mathrm{C}_{6} \mathrm{~F}_{4^{-}}$ & 4-(GlcS) $-\mathrm{C}_{6} \mathrm{~F}_{4^{-}}$ & $N$-Me-4-pyridyl & $2 \mathrm{H}$ & From 359: Like entry 246. & [215] \\
\hline
\end{tabular}

Note: all are Beta isomers (apart from mannose which is alpha) unless stated as alpha (or beta for mannose) or a mixture of the two.

pyridiniumyl)\} porphyrin ${ }^{[190]}$. The respective $\mathrm{A}_{3} \mathrm{~B}$ neutral precursor porphyrins with one glycoside residue (glucoside, maltoside and lactoside) $(\mathbf{1 3 1}, \mathbf{1 3 9}, \mathbf{1 4 7}$, 148, 151, 152) were synthesized via the Little method ${ }^{[191]}$ by condensation of pyrrole, 4-pyridinecarboxaldehyde, and ortho- or para-peracetylated glycosyloxybenzaldehyde derivatives in 6-7 \% yield. Again, addition of methyl, isopropyl, $n$-octyl groups provided the cationic glycosylated $N$ alkylpyridinium porphyrins 132-134, 141-143, 155-162. Deacetylation then gave 135-138, 140, 149, 150, 153, 154 and 144-146, 155, 156 in quantitative yield.

Similar strategies were also employed to construct dimeric systems. Here, condensation reactions with a mixture of $4-\left(2^{\prime}, 3^{\prime}, 4^{\prime}, 6^{\prime}\right.$-tetra- $O$-acetyl- $\beta-D-$ glucopyranosyloxy)-benzaldehyde, ortho- or para-hydroxybenzaldehyde and pyrrole via Lindsey conditions, followed by oxidation with $p$-chloranil, afforded the neutral tri- $O$-glycosyl porphyrins 163 and 164 in $5-13 \%$ yield ${ }^{[192]}$. Alternatively, use of the Little method gave the same porphyrins in yields of 5$7 \%$. The neutral porphyrin dimers $\mathbf{3 6 4} o, p$ were prepared via two step ether linkage with good yields, followed by quantitative deprotection to provide $\mathbf{3 6 5}$ o, $p$. Related cationic dimers were prepared via Fleischer's method ${ }^{[193]}$ in

which the porphyrins were linked by a 1,3-dipyridiniumyl propane linkage. Here, the Little method was adapted to yield $\mathbf{1 6 7}$ in $7 \%$. The 1,3dipyridiniumylpropane-linked bisporphyrin $\mathbf{3 6 6}$ was synthesized in one step with 1,3-diiodopropane in $14 \%$ yield. Subsequently the sugar units were deprotected quantitatively yielding $\mathbf{3 6 7}$. Compounds $\mathbf{3 6 5} o, p$ and $\mathbf{3 6 7}$ exhibited significant fluorescence quenching in aqueous solutions due to aggregation ${ }^{[192]}$.

The same building blocks could also be used for the synthesis of mixed glycosylated neutral and cationic porphyrin dimers prepared with a flexible hydrocarbon chain linked at the meso position ${ }^{[194]}$. Within the series the two hydrophilic compounds $\mathbf{3 6 5} o, p$ differing by the ortho or para-substitution and an amphiphilic derivative $\mathbf{3 6 8}$ were synthesized. The second part of the series consisted of the synthesis of two $O$-glycosyl cationic dimers where the linkage at the pyridine nitrogen afforded its mono-cationic nature. Again, the monomer structures were synthesized by Lindsey's method via condensation of pyrrole, 4-pyridine carboxaldehyde and 4-(2, $3^{\prime}, 4^{\prime}, 6^{\prime}$-tetra- $O$-acetyl- $\beta$ - $D$ glucopyranosyloxy)benzaldehyde with propionic acid. The previously synthesized monomers were also incorporated into $\mathbf{3 6 9}$ and $\mathbf{3 7 0}$ which allowed for covalent attachment on one side via ether linkage and alkylation of the 


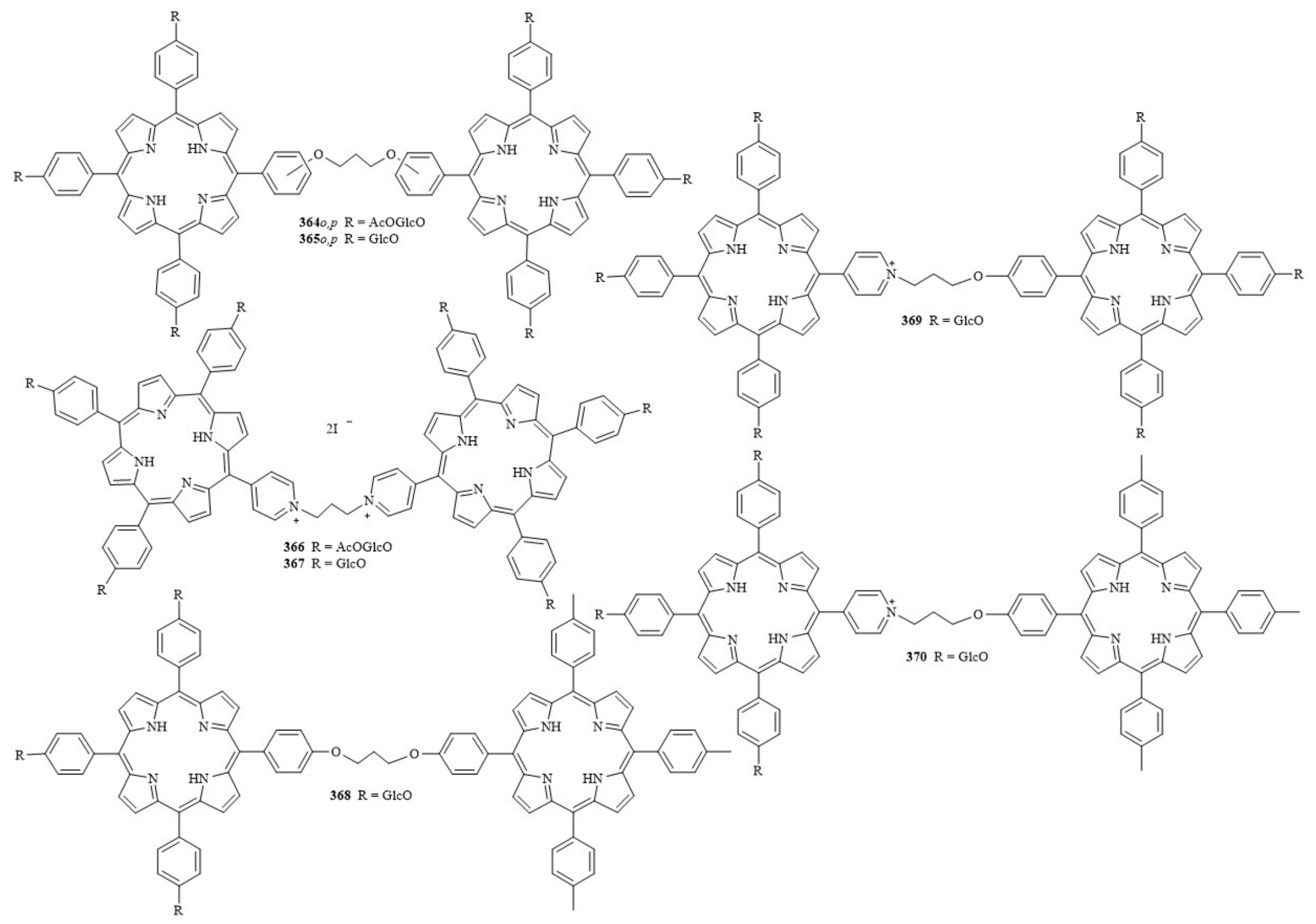

Formula 2. 
pyridine nitrogen on the other side. Deacetylation afforded the mono-cationic derivatives $\mathbf{3 6 9}$ and $\mathbf{3 7 0}$.

Mixed condensations may also be used as an entry for subsequent solid phase syntheses. For example, Krausz et al. carried out the solid phase synthesis of two mono-RGDtriglucosylporphyrins where RGD is a tripeptide sequence of arginine-glycine-aspartate ${ }^{[195]}$. Using Little's method the protected derivatives of glycoporphyrins 168 were synthesized through condensation of pyrrole, glucosylaldehyde and either para-hydroxybenzaldehyde or salicylaldehyde in propionic acid in 6 and $4 \%$, while the use of Lindsey's method doubled the yields. Conversion to the carboxylic acid provided the porphyrin scaffold for amide coupling to a solid phase Wang resin functionalized with a RGD residue. The porphyrin derivatives $168 o, p$ were obtained after detachment from the Wang resin and subsequent deprotection.

An example for the preparation of 5,15-disubstituted porphyrins involved the condensation under Lindsey conditions of tetraacetyl- $\beta-D$-lactosyl-benzaldehyde with dipyrromethane to afford 5,15-bis( $p$-per-acetyl- $\beta$ lactosylphenyl)porphyrin 169 after oxidation with $p$ chloranil ${ }^{[29]}$. The metallated porphyrin $\mathbf{1 7 1}$ was obtained after treatment with $\mathrm{FeCl}_{2}$ and followed by deacetylation yielded 173. The compound was tested in a range of solvents and was found to be mono-dispersed in aqueous DMF but formed aggregates in aqueous NMP ( $N$-methylpyrrolidone). It showed an unusual colorimetric response with the addition of calcium ions in tris- $\mathrm{HCl}$ buffer $(50 \mathrm{mM}, \mathrm{pH} 9.6,50 \mathrm{v} / \mathrm{v} \%$ NMP) changing the color from orange to pink over a $10 \mathrm{~h}$ equilibration period. The same response was not observed for any other ion including potassium, sodium or magnesium. The calcium ion response was dependent on the $\mathrm{pH}$ and the co-solvent used. A comparison with chloro(2,3,7,8,12,13,17,18-octaethylporphyrinato)iron(III) and the non-conjugated derivative 172, both of which displayed no response, confirmed the carbohydrate units being responsible for the response ${ }^{[29]}$.

Naturally, mixed condensations may also be employed for the synthesis of unsymmetrical $C$-glycosylated porphyrins. Often, this involves initial multi-step syntheses of appropriate dipyrromethanes. A typical example is the preparation of $\alpha, \alpha^{\prime}$-dipyrrylalditols (371) via condensation of pyrrole with glyceraldehyde, arabinofuranose or glucopyranose using a mixture of $\mathrm{EtMgBr}$ and $\mathrm{TiCl}_{4}$. This yields synthetically useful precursors for the synthesis of glycoporphyrins, incorporating carbon-linked sugar moieties (Scheme 6). Condensation of dipyrryl derivative 371b with $p$-fluorobenzaldehyde was achieved in $20 \%$ yield with a single isomer identified to yield the corresponding $\mathrm{A}_{2} \mathrm{~B}_{2}$ porphyrin $174^{[196]}$.
Other dipyrrylglycosides (e.g., 372 and $\mathbf{3 7 3}$ were easily prepared by reaction of pyrrole with aldoses in 30-40\% yield ${ }^{[216]}$. Standard [2+2] condensation with aryl aldehydes then yielded the respective meso-glycoconjugated porphyrins in $6-16 \%$ yield. Note, that the presence of branched meso<smiles></smiles>

371<smiles>[R][CH][C@H]1COC(C)(C)O1</smiles>

Scheme 6. Synthesis of $\alpha, \alpha^{\prime}$-dipyrrylalditol derivatives.

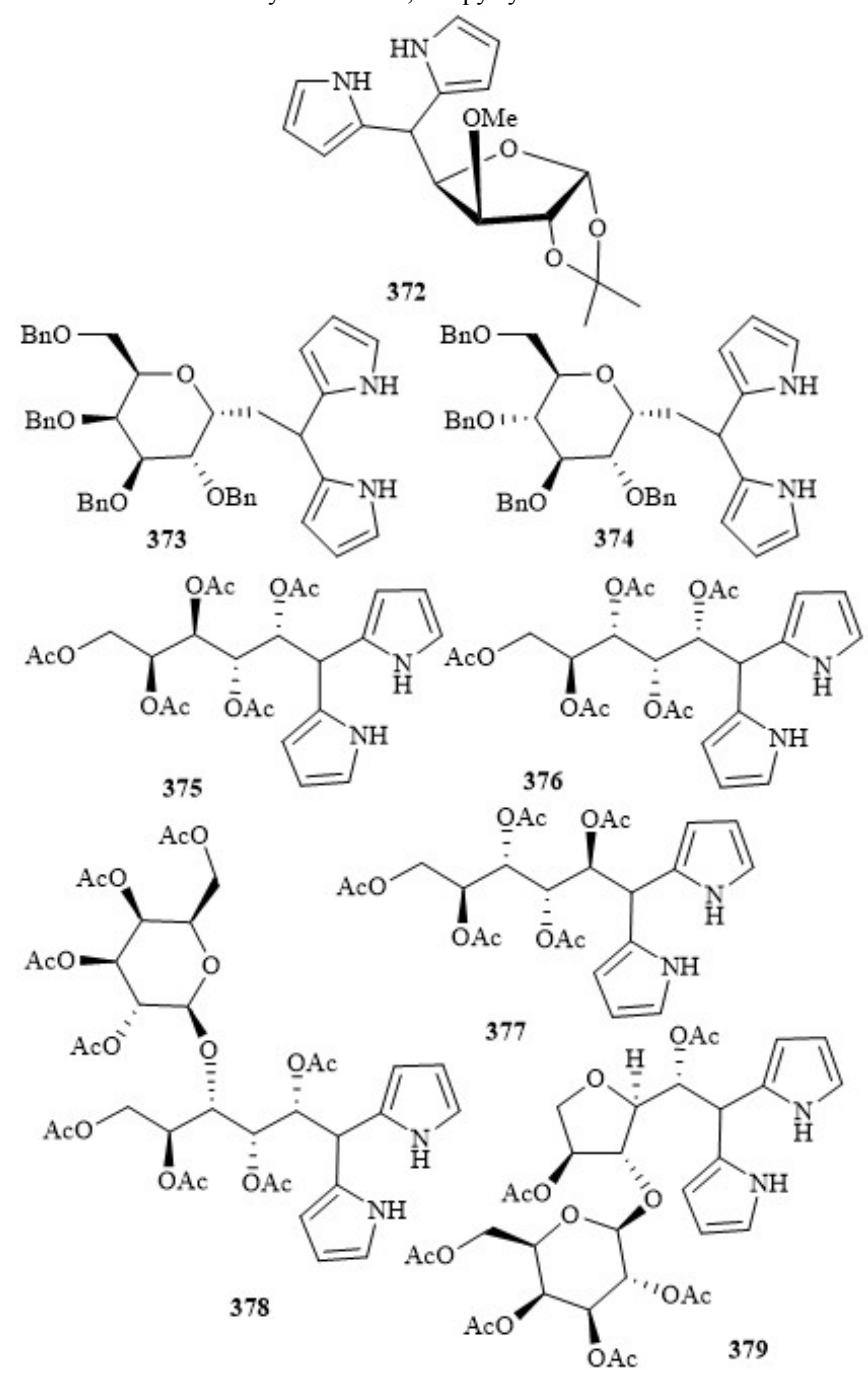

Formula 3. 
alkyl residues from the sugar units results in conformationally constrained systems ${ }^{[217]}$. Here, a dynamic saddle conformation was proposed to exist in solution ${ }^{[216]}$. These studies were later extended to the related $\mathrm{A}_{4}$-type systems (namely the four atropisomers of 5,10,15,20tetrakis-(1,2-O-isopropylidene-1,2-dihydroxyethyl)-

porphyrins) which were investigated in detail with regard to their atropisomerism ${ }^{[218]}$. Selective deprotection pathways were found for the acetonide precursor systems which allowed conversion of the lipophilic protected systems into amphiphilic, partially deprotected and water soluble, fully deprotected systems. A unique method for the synthesis of $C$-dipyrrylglycosides incorporates the use of dithioacetal glycosyl derivatives by reaction with pyrrole to afford monoand disaccharidic $C$-1-deoxyalditol moieties ${ }^{[219]}$. The glycosyl starting materials $D$-galactose, $D$-mannose, $D$ glucose, $D$-lactose and agarose underwent a mercaptolysisacetylation to form the peracetylated diethyl dithioacetals. The carbonyl of these residues were deprotected and subsequently reacted via pyrrole condensation to afford $\mathbf{3 7 5}$ 379 in an overall yield of $22-49 \%{ }^{[219]}$.

In extension of the $\mathrm{A}_{4} C$-glycosyl porphyrins 66 and 67 diglycosylated porphyrins were synthesized to investigate their ability to form chiral supramolecular structures in different aqueous-organic solvent mixtures ${ }^{[170]}$. The 5,15substitution pattern was achieved using the [2+2] MacDonald type procedure where the dipyrryl derivatives were prepared by condensing the respective aldehydes with pyrrole affording $\mathbf{3 7 3}$ and $\mathbf{3 7 4}$ in 49 and $84 \%$ yield, respectively. Condensation with pentafluorobenzaldehyde gave $\mathbf{1 7 5}$ in $22 \%$. The same reaction was carried out for the glucose derivative 176. An alternative method used a condensation of pentafluorophenyl-dipyrrylmethane with the sugar aldehydes which afforded $\mathbf{1 7 5}$ in a higher yield. Debenzylation was achieved by hydrogenation over $\mathrm{Pd} / \mathrm{C}$ giving the hydroxylated derivative $\mathbf{1 7 7}$ in $63 \%$ yield. Note, these structures form chiral super-structures due to selfaggregation in aqueous-organic solvent mixtures with the morphology of the aggregates depending on the porphyrin structure and the bulk conditions of aggregation ${ }^{[170]}$.

Another example of $C$-glycosylated tetraarylporphyrins was reported by Drain and coworkers ${ }^{[172]}$. Their synthesis of the glycosyl benzaldehyde required several steps to convert the initial $S$-glycosyl linkage to the more stable $C$-linkage. $\alpha, \alpha$-Dibromo- $p$-xylene was reacted with $2,3,4,6$-tetra- $O$ acetyl-1-thio- $\beta$ - $D$-glucopyranoside providing the thioglucoside $\mathbf{3 8 0}$ which was converted to the silyl derivative 381 and the protecting groups were changed from the acetyl to benzyl groups for subsequent steps. Next, the sulfone of compound $\mathbf{3 8 3}$ was synthesized and used in a RambergBäcklund synthesis of the exo-glucal 384 (two isomers with a $Z / E$ ratio of 8:2). Subsequent hydrogenation afforded the $\beta$ $C$-glycoside $\mathbf{3 8 5}$ followed by cleavage of the silyl protecting group and Swern oxidation to the required aldehyde (Scheme 7). The aldehyde $\mathbf{3 8 6}$ was then used to synthesize $\mathbf{7 0}$ and $\mathbf{1 7 8}$ in 53 and $15 \%$ yields, respectively, using Lindsey conditions. Hydrogenolysis of the benzyl protecting groups provided $\mathbf{7 1}$ and $\mathbf{1 7 9}$ in quantitative yields ${ }^{[172]}$.

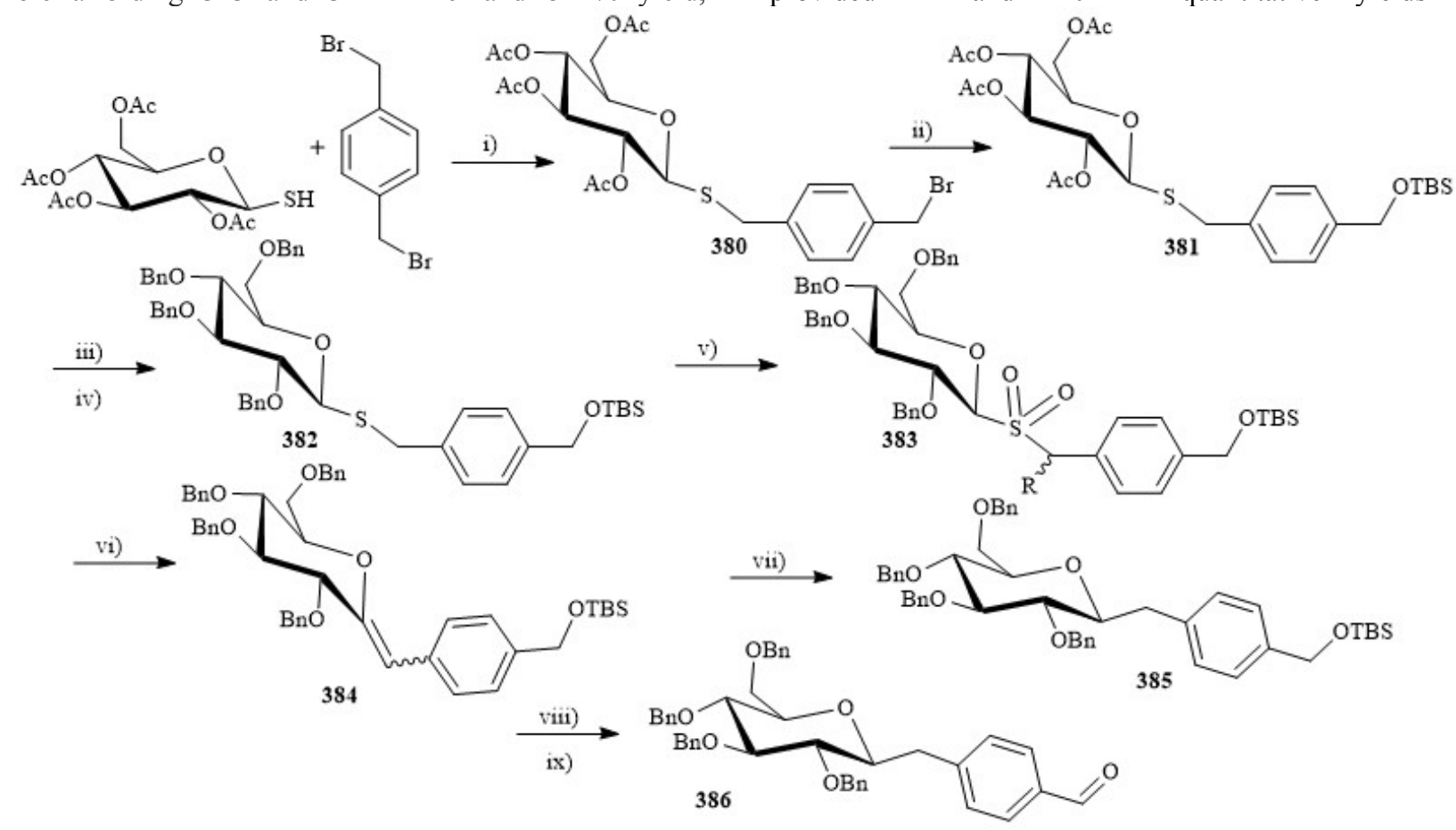

Scheme 7. Synthesis of mono- and tetra- $C$-glycosyl substituted TPP. i) NaH, THF, r.t., 2 h, 90 \%; ii) TBDMSOH, $\mathrm{Ag}(\mathrm{OTf}), 2,6$-di-tert-butylpyridine, $\mathrm{CH}_{2} \mathrm{Cl}_{2}$, r.t., 3 h, $60 \%$; iii) MeONa, MeOH, r.t., 3 h; iv) NaH, BnBr, Bu 4 NI, THF-DMF, r.t., 8 h, $80 \%$ for 2 steps; v) MMPP, THF-EtOH-H ${ }_{2} \mathrm{O}, 60{ }^{\circ} \mathrm{C}, 2$ h, $87 \%$; vi) $\mathrm{CBr}_{2} \mathrm{~F}_{2}, \mathrm{KOH} 25 \%$ on alumina, $\mathrm{CH}_{2} \mathrm{Cl}_{2}-t$ - $\mathrm{BuOH}, 0{ }^{\circ} \mathrm{C}$ to r.t., 3 h, $88 \%$; vii) $\mathrm{H}_{2}$, Pd $5 \%$ on alumina, EtOAc, r.t., 12 h, $95 \%$; viii) Bu 4 NF, THF, r.t., 2 h, $98 \%$; ix) oxalyl chloride, DMSO, $\mathrm{CH}_{2} \mathrm{Cl}_{2}, \quad$ r.t., then $\mathrm{Et}_{3} \mathrm{~N}, \quad-78 \quad{ }^{\circ} \mathrm{C} \quad$ to $\quad$ r.t., $1.5 \quad$ h, $85 \quad$. 
Condensation reactions can also be employed to construct geometrically more complex structures. For example Krausz and coworkers synthesized glycosyl strapped porphyrins to determine the effect of strapping on its efficacy as a PS (Scheme 8) ${ }^{[220]}$. The synthesis of the two glycosyl strapped porphyrin $389(5,15$, 'trans') and 391 (5,10, 'cis') used standard Lindsey conditions with meso- $(p$ tolyl)dipyrromethane (2 equiv.) (388) and glycosyl bisaldehyde $6,6^{\prime}$-di- $O$-(3-formyl)benzoyl,2,3,4,2',3',4'-hexa$O$-trimethylsilyl- $\alpha, \alpha$-trehalose ( 1 equiv.) (387) to yield $\mathbf{3 8 9}$ and 391 which were subsequently deprotected giving 390 and 392 in $8 \%$ and $5 \%$ yield, respectively.

\subsection{Synthesis via Functionalization Reactions}

Due to the low stability of carbohydrates under many of the conditions used for porphyrin synthesis the preparation of more complex systems is better achieved by first constructing the porphyrin macrocycle and then appending the desired sugar units. The most basic approach is the use of porphyrins with simple functional groups such as $-\mathrm{OH}$, $\mathrm{NH}_{2}$, - $\mathrm{COOH}$ and standard coupling methods [221]. Functionalization of porphyrins by functional group interconversion and direct substitution of the porphyrin scaffold have facilitated the assembly of several glycoporphyrins via different ligation strategies including, glycosylation, olefin metathesis, amide coupling, organometallic coupling and are summarized in Figure 4. All of which afford $O-, N-, S$ - $C$-linkage of the glycoconjugate for $\mathrm{A}_{3} \mathrm{~B}-, \quad \mathrm{A}_{2} \mathrm{~B}_{2^{-}}, \quad \mathrm{A}_{2} \mathrm{BC}$ and $\mathrm{ABCD}$-type porphyrins including $\beta$-substituted and dimeric porphyrins, too.

\subsection{1 $\beta$-Substituted Porphyrins}

\subsubsection{Glycosidation Reactions}

Diglucoside 396 and digalactoside 397 derivatives of isohematoporphyrin were the first water soluble glycoporphyrins to be synthesized as efficient PSs (Scheme 9) ${ }^{[222]}$. Both 394 and $\mathbf{3 9 5}$ were synthesized via diastereoselective $O$-glycosylations using a solid surface active silver catalyst and acetyl groups for neighboring group participation yielding the $\beta$-diglycosides in $51 \%$ and $85 \%$ yields, respectively. The glycosylations with $\alpha-D-$ acetobromoglucose or $\alpha$ - $D$-acetobromogalactose were carried out at room temperature over 1-7 days followed by deprotection of the acetyl groups yielding the stable $\operatorname{bis}(D-$ glucosyl)isohematoporphyrin 396 and the $\operatorname{bis}(D-$ galactosyl)isohematoporphyrin 397. A similar approach to amphiphilic porphyrins was taken with hematoporphyrin dimethyl ester. It underwent glycosylation with 2,3,4,6-tetra$O$-acetyl- $\alpha$ - $D$-glucopyranosyl bromide in the presence of mercuric salts $\left(\mathrm{HgBr}_{2}\right.$ and $\left.\mathrm{Hg}(\mathrm{CN})_{2}\right)$ for $6 \mathrm{~h}$ to yield the mono-glycosylated porphyrin in $30 \%$ when using two equivalents of the glucosyl residue while using 4 equivalents
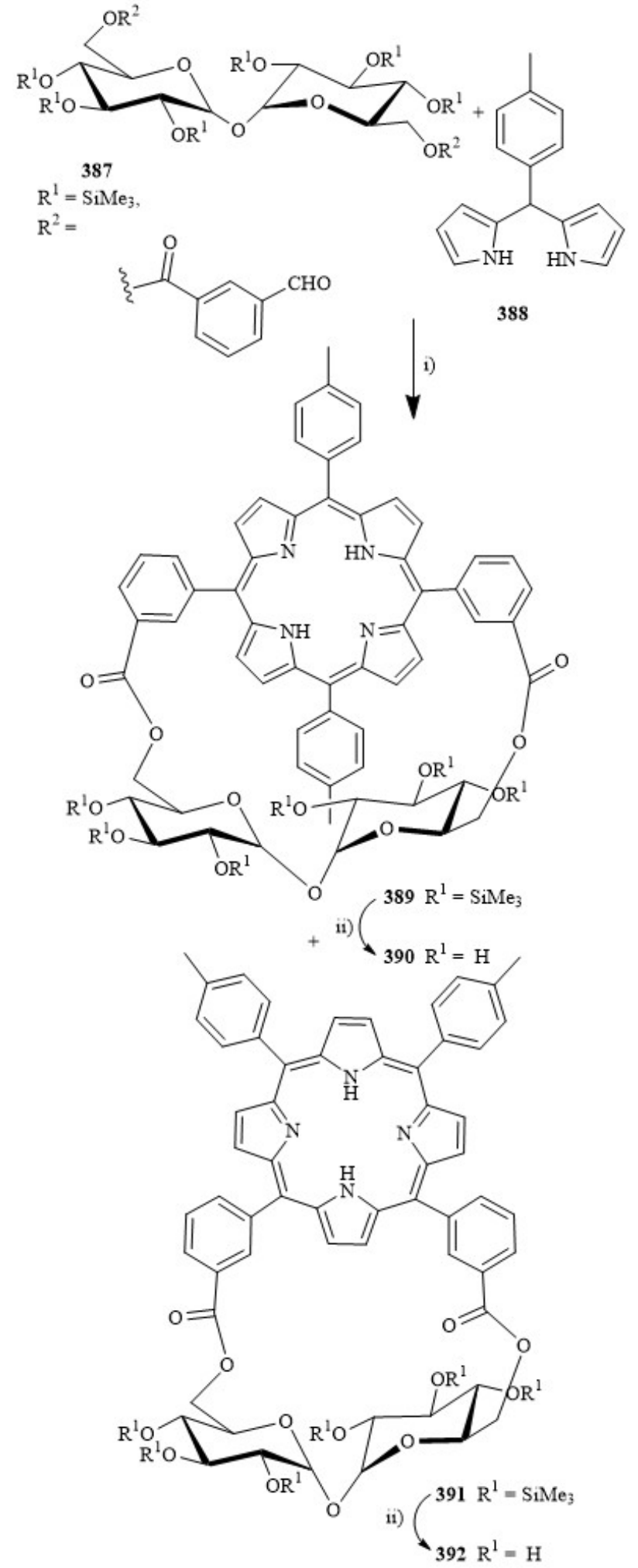

Scheme 8. Synthesis of glycosyl-strapped porphyrins. i) $\mathrm{BF}_{3} \cdot \mathrm{OEt}_{2}, p$ chloranil, dichloromethane; ii) TBAF. 


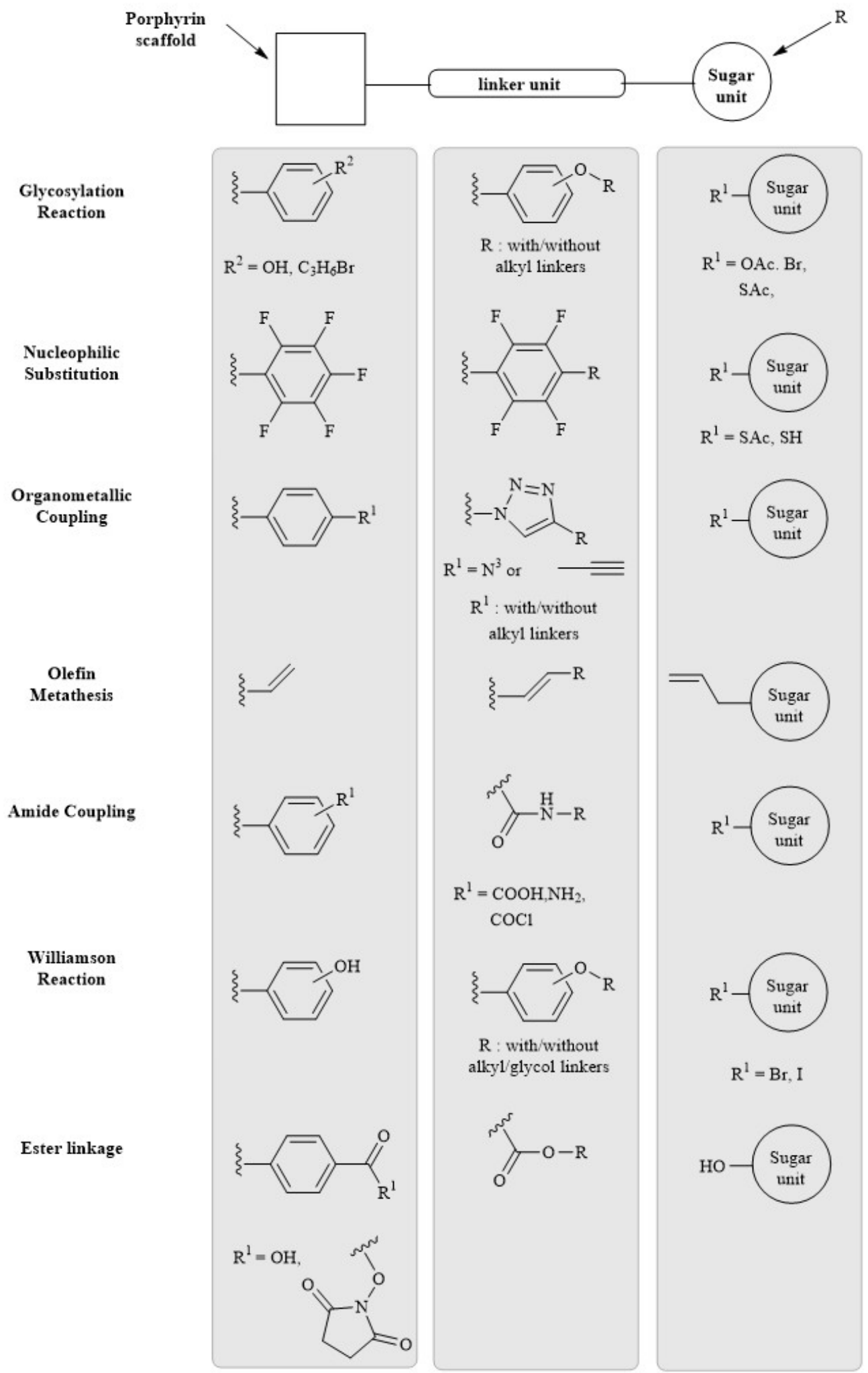

Figure 4. Synthesis of glycoporphyrins via porphyrin FGI. 
of the sugar unit afforded the diglycosylated derivative in 52 $\%$ yield. Deprotection of the acetyl groups using either KCN or $\mathrm{NaOMe}$ could be achieved in 70-80\% yields ${ }^{[188]}$.

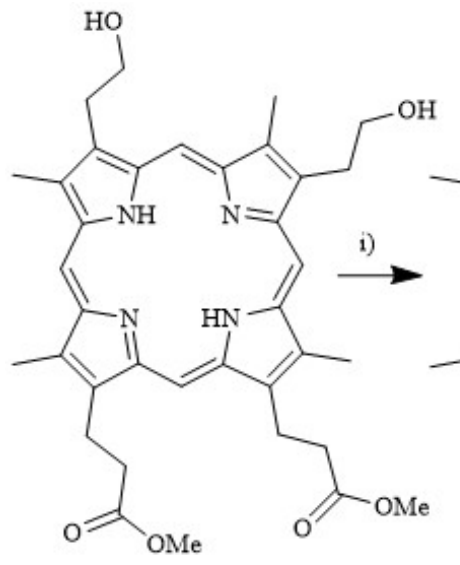

393

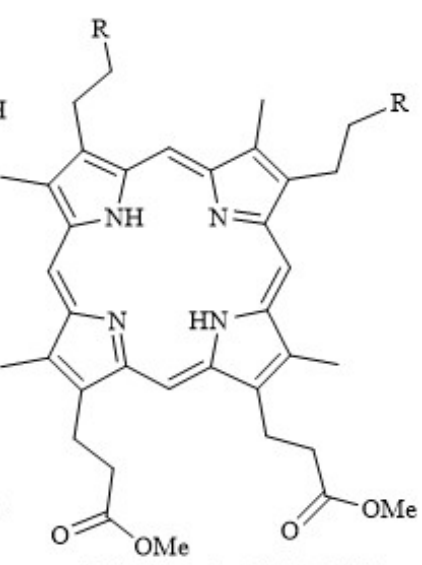

$394 \mathrm{R}=\beta$-AcOGlcO, $51 \%$

ii) $395 \mathrm{R}=\beta-\mathrm{AcOGalO}, 85 \%$
$396 \mathrm{R}=\beta-\mathrm{GlcO}, 87 \%$

$$
397 \mathrm{R}=\beta-\mathrm{GalO}, 77 \%
$$

Scheme 9. Synthesis of bis(D-glucosyl)isohematoporphyrin and $\operatorname{bis}(D-$ galactosyl)isohematoporphyrin. i) $\alpha$-AcOGlcOAc or $\alpha$-AcOGalOAc, $\mathrm{Ag}_{2} \mathrm{CO}_{3}, \mathrm{Na}_{2} \mathrm{SO}_{4}, \mathrm{CH}_{2} \mathrm{Cl}_{2}, 20^{\circ} \mathrm{C}, 1-7$ days; ii) $\mathrm{KOH}, \mathrm{MeOH}, 100{ }^{\circ} \mathrm{C}, 24 \mathrm{~h}$.

Naturally, the functional group used for coupling may also be positioned on a meso substituent. Hombrecher reported the synthesis of several galatopyranosyl substituted porphyrins using different procedures, the first of which was the treatment of porphyrinic carboxylic esters (180-184) with 1,2,3,4-di- $O$-isopropylidene- $\alpha$ - $D$-galactopyranose. The reaction proceeded in moderate yields of 28-50\% for porphyrins 185-187. Compounds 188 and 189, however, were produced in $7 \%$ yield due to increased formation of the carboxylic acid ${ }^{[197]}$. Alternative routes were explored using condensation reactions which produced compound 189 in a yield of $20 \%$ by using the 4-[di- $O$-isopropylidene- $\alpha-D$ galactopyranosyl]benzaldehyde. They also used Lindsey's procedure, treating the aldehyde in a mixed condensation with $p$-tolyl aldehyde and pyrrole which proved unsuccessful in increasing the yields ${ }^{[197]}$. Hombrecher later extended his studies with the porphyrin framework shown for 402. After preparation of the hydroxmethylphenylporphyrin 399 this was reacted with a variety of glycosyl imidates 398 to yield the nickel(II) porphyrins 400. These were then deprotected and demetallated to the free bases 401 (Scheme 10). Notably, this was one of the earlier papers that attached disaccharides to a porphyrin core ${ }^{[223]}$. In aqueous solution these systems formed very stable aggregates. However, the porphyrins could be incorporated easily as monomers into membrane model systems (liposomes).

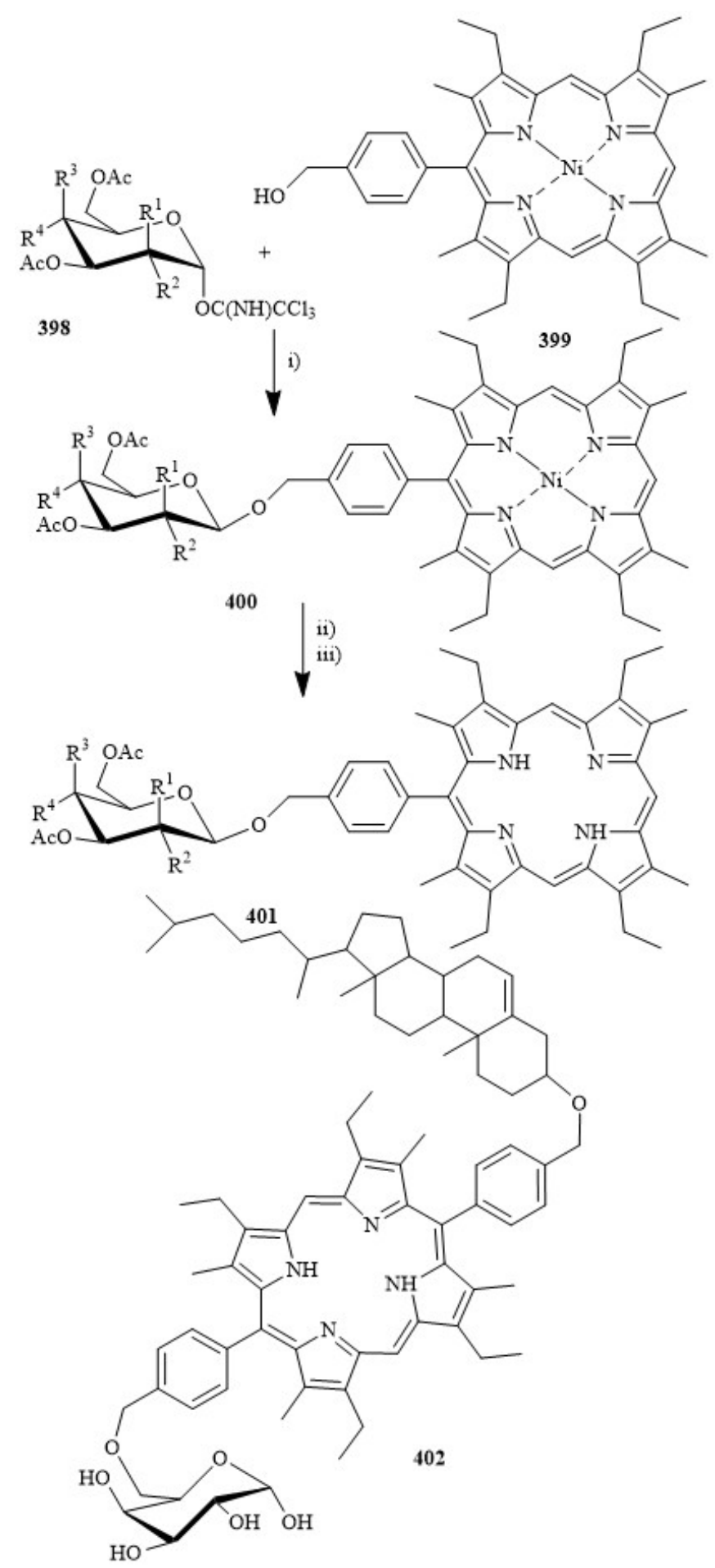

Scheme 10. Synthesis of porphyrins with a carbohydrate unit appended to the meso position. Conditions: i) $\mathrm{CH}_{2} \mathrm{Cl}_{2}, \mathrm{ZnCl}_{2}, 22-33 \%$; ii) $\mathrm{NaOMe}$, $\mathrm{MeOH}, 5$ min.; iii) TFA, 1,3-propanedithiol, 61-99\%. $\mathrm{R}^{1}=\mathrm{OH}, \mathrm{H}, \mathrm{OAc} ; \mathrm{R}^{2}$ $=\mathrm{H}, \mathrm{OH}, \mathrm{NHAc}, \mathrm{OAc} ; \mathrm{R}^{3}=\mathrm{H}, \mathrm{OH}, \mathrm{OAc} ; \mathrm{R}^{4}=\mathrm{H}, \mathrm{OAc}, \alpha(1,4) \mathrm{Glc}(\mathrm{OAc})_{4}$, $\beta(1,4) \mathrm{Gal}(\mathrm{OAc})_{4}, \beta(1,4) \mathrm{Glc}(\mathrm{OAc})_{4}, \alpha(1,4) \mathrm{Glc}, \beta(1,4) \mathrm{Gal}, \beta(1,4) \mathrm{Glc}$. 


\subsubsection{Organometallic Coupling Reactions}

Organometallic couplings can also be used to introduce bioconjugate groups in the $\beta$-position. One example comprises the synthesis of $\beta$-substituted glycoporphyrins using second generation Grubbs catalyst, vinylporphyrins, and allylic acetonide carbohydrate units ${ }^{[224]}$. The $\mathrm{Zn}(\mathrm{II})$ complexes of 2-vinyl-5,10,15,20-tetraphenylporphyrin 403a and protoporphyrin-IX dimethyl ester $\mathbf{( 4 0 3 b )}$ were reacted with allylic acetonides of the $D$-ribose $(\mathbf{4 0 4 c}), D$-galactose (404d), $D$-glucose (404e), and two isomeric derivatives of $D$ fructose (404f,g) using $25 \mathrm{~mol} \%$ of Grubbs catalyst. The yields for 405a-(c-g) were quantitative, however the yields for 405b-(c-g) varied from 74-93\% with no dimerization of either starting substrate observed. The reaction displayed a significantly high $E$-stereoselectivity due to steric hindrance provided by the large macrocycle (Scheme 11).
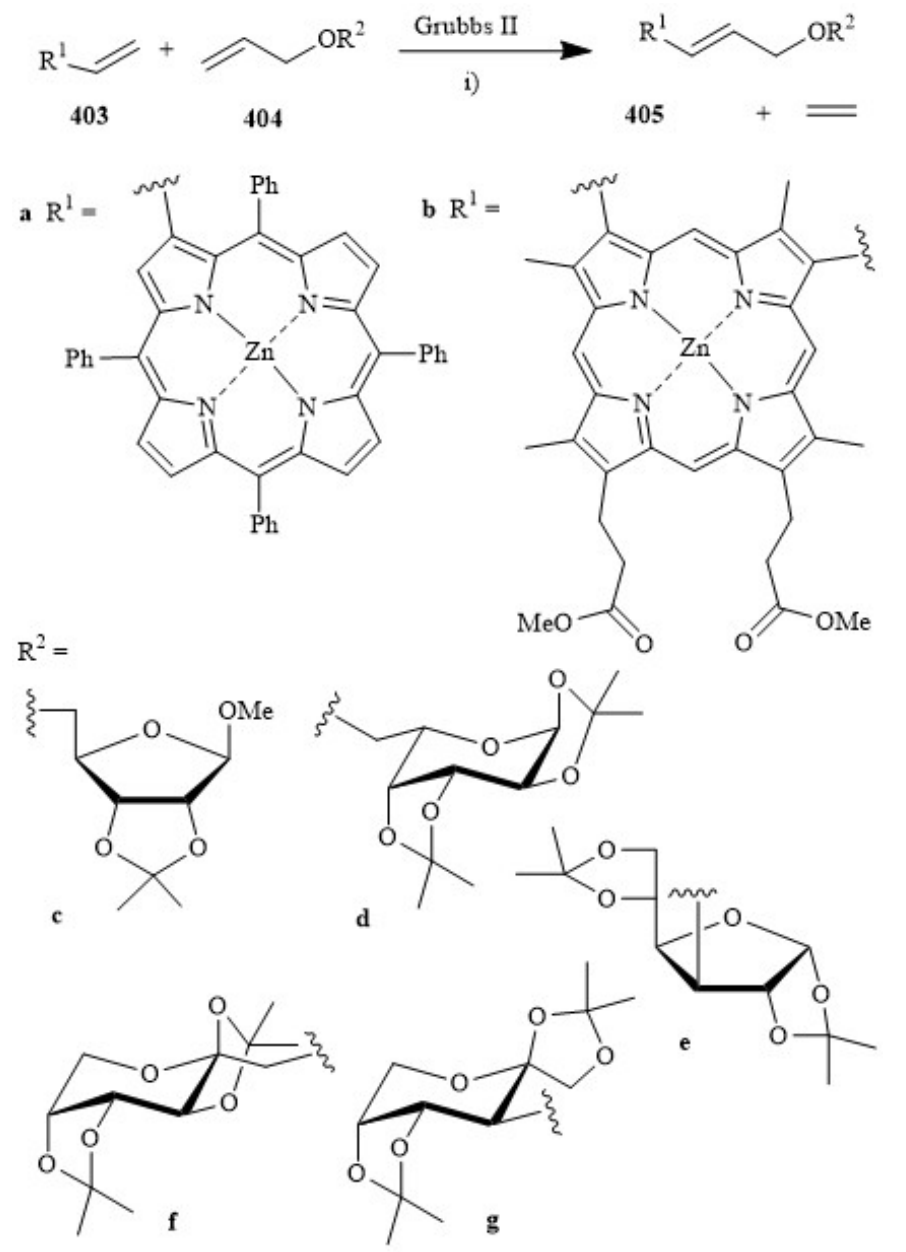

Scheme 11. Synthesis of glycoporphyrins via cross-metathesis between vinylporphyrins and allyl sugars. (i) $\mathrm{CH}_{2} \mathrm{Cl}_{2}$, reflux.

Another example is the one pot synthesis of a water soluble (octa- $\beta$-lactosylated porphyrinato)copper(II) 72 via copper-catalyzed Husigen click chemistry using an octaalkyne porphyrin and $\beta$-lactosyl azide ${ }^{[173]}$. Its spatial structure and lectin affinity were determined. It was observed that the eight $\beta$-lactoside-appendages formed a thick hydrophilic shell which shielded the hydrophobic surface of porphyrin-core forming the most stable spherical conformation. These (porphyrinato)copper(II) derivatives have poor singlet oxygen yields and are not suitable for PDT [173]

\subsection{2 meso-Substituted Porphyrins}

The most significant body of work is available for derivatives of $5,10,15,20$-tetrasubstituted porphyrins. The ease of their synthesis makes them suitable precursors for glycosidation and functionalization reactions.

\subsubsection{Glycosidation Reactions}

O-linked systems: A typical example is the analysis of two series of glycosylated porphyrins which were synthesized and studied using solid secondary ion mass spectroscopy (SIMS) [189,225,226]. The first series of monosubstituted tristolyl glycoporphyrins were prepared starting from the mono-hydroxyphenyl-tolyl porphyrins 406 $o, p$ obtained with propionic acid via the Little method followed by substitutions with 3-bromo-propan-1-ol and glycosylation with four protected mono- and disaccharides affording 408-411 in 20-70\% yield (Scheme 12) ${ }^{[226]}$. The second series, unsymmetrical pyridyl mono-glycosylated porphyrins $(131,139,147,148,151,152)$ substituted with protected mono- and disaccharides, were synthesized according to the Adler-Long method via glycosylated benzaldehydes in $\sim 6 \%$ yield ${ }^{[189,190]}$. For a comparative study the symmetric tetraglucosyl derivative 29 was also synthesized ${ }^{[225]}$. SIMS was evaluated as a good study for the rapid screening of compounds during synthesis. It avoids problems displayed by FABMS (fast atom bombardment mass spectroscopy) which can result in contamination of the ion source and chemical reactions occurring in a matrix. Positive and negative spectra were obtained of all glycoporphyrins. The simple spectra identified the molecular weight and showed the groups appended at the meso positions unlike DCI-CID (desorption chemical ioization collision-induced dissociation) which does not yield mass spectra of higher mass compounds ${ }^{[225]}$.

Similarly, four series of meso-tetraaryl-glycoporphyrins were synthesized with protected and de-protected hydroxyl groups to evaluate their photo-inactivation of HSV-1 and HSV-2 herpes simplex virus ${ }^{[30]}$. Three different classes of porphyrins $(83,190,197)$ were prepared by Rothemund or cross-Rothemund reactions and porphyrin 190 was reacted with hexafluorobenzene affording $\mathbf{1 9 8}$ in addition to the series. The first two series of glycoporphyrins were synthesized via the coupling of tetraphenylporphyrins with a hydroxyl group (190) or a carboxylic group (197) with 1bromo-2,3,4,6-tetra- $O$-acetyl- $\alpha$ - $D$-glucopyranose and 6-iodo1,2:3,4-di- $O$-isopropylidene- $\alpha$ - $D$-galactopyranose,

respectively affording 191 and 193 in $66 \%$ and $50 \%$ yields. The other two series were synthesized via substitution of one 


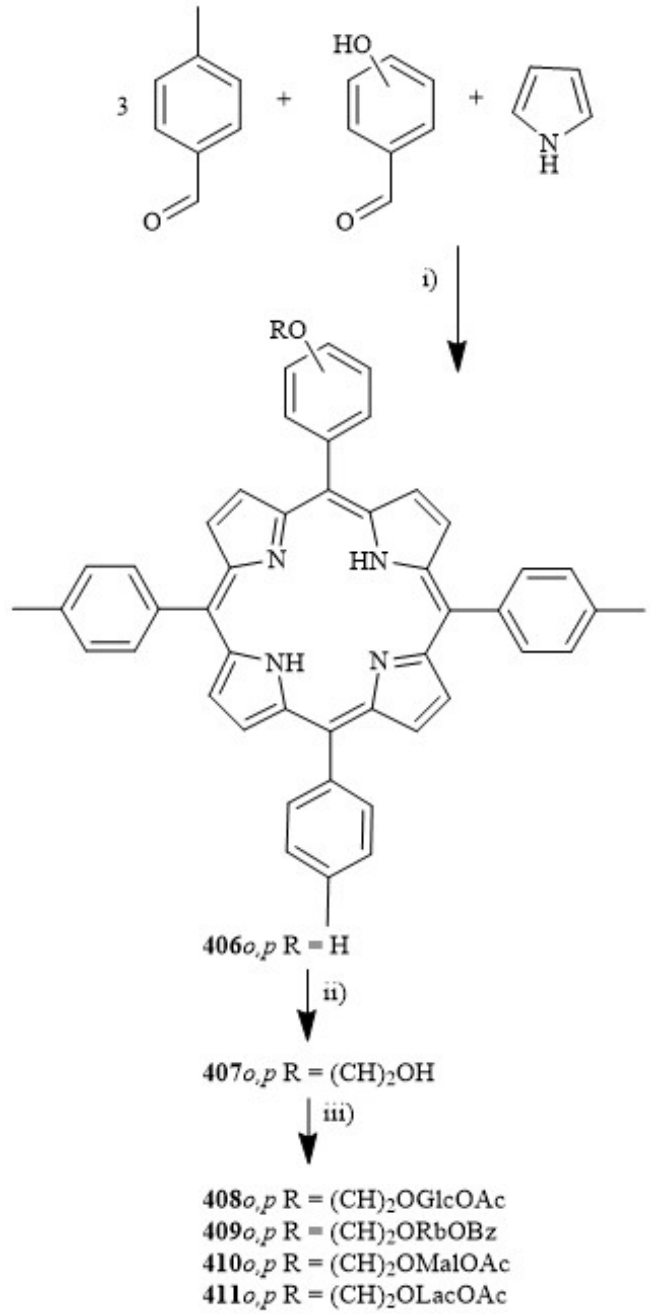

Scheme 12. Synthesis of mono-glycosylated tristolyl porphyrins. i) EtCOOH, reflux; ii) $\mathrm{Br}(\mathrm{CH})_{2} \mathrm{OH} / \mathrm{K}_{2} \mathrm{CO}_{3} / \mathrm{DMF}$; iii) 408: 2,3,4,6-tetra-Oacetyl- $\alpha$ - $D$-glucopyranosyl bromide, $\mathrm{Hg}(\mathrm{CN})_{2}, \mathrm{HgBr}_{2}$, toluene/nitromethane; 409: 1-O-acetyl-2,3,5-tribenzoate- $\beta-D$-ribofuranose, $\mathrm{SnCl}_{4}, \mathrm{CH}_{2} \mathrm{Cl}_{2} ; \mathbf{4 1 0}$ : peracetylated maltose, $\mathrm{SnCl}_{4}, \mathrm{CH}_{2} \mathrm{Cl}_{2} ; 411$ : peracetylated lactose, $\mathrm{SnCl}_{4}$, $\mathrm{CH}_{2} \mathrm{Cl}_{2}$.

para fluorine atom on $\mathbf{8 3}$ or on a meso-tetraphenylporphyrin containing a pentafluorobenzene spacer (198) with 1,2:3,4di- $O$-isopropylidene- $\alpha$ - $D$-galactopyranose providing 201 and 199. All series were deprotected yielding the amphiphilic derivatives 192, 194, 200 and 202. Compound 194 was converted to the methyl galactopyranosides 195 and 196 in $43 \%$ and $40 \%$ yields, respectively ${ }^{[30]}$. All porphyrin derivatives were tested against HSV-1 and HSV-2 in Vero cells. These compounds also proved to be suitable materials for detailed electrospray tandem mass spectrometry ${ }^{[227,228]}$.

Similar concepts can also be employed to prepare unsymmetrical cationic glycoporphyrins. In an extension of previous work on the synthesis of neutral glycoporphyrins which displayed good inactivation of herpes simplex virus (HSV-1 and HSV-2) ${ }^{[30]}$ Tomé et al. synthesized glycosylated neutral and cationic tripyridylporphyrins and evaluated their antiviral activity ${ }^{[229]}$. The porphyrin starting material 412 was synthesized via crossed Rothemund reaction which underwent a one-pot conversion to the activated ester 413 by reaction with thionyl chloride and addition of $N$-hydroxysuccinimide. Coupling of the activated ester afforded the glycoporphyrin $\mathbf{4 1 4}$ which was subsequently deprotected to give 415 . Treatment with methyl iodide afforded the tris $(N$-methylpyridinium)porphyrins 416a-c (Scheme 13). All compounds are good singlet oxygen generators, however, the cationic porphyrin derivatives are slightly better than the neutral ones.

Related studies targeted cationic galactosyl porphyrins and their inactivation potential of Micrococcus sp. [Gram $(+)$ ] and Pseudomonas sp. [Gram (-)] which were resistant to UV inactivation ${ }^{[230]}$. Glycoporphyrins $416 \mathbf{b}, \mathbf{c}^{[229]}$ and the non-glycoconjugate $\mathbf{7 3}^{[174]}$ served as controls. The latter compound was used as a precursor for the synthesis of the mono-charged derivatives 209 and 210 and tetra-charged porphyrin derivatives $(\mathbf{7 4}, \mathbf{2 1 1}, \mathbf{2 1 2})$. Compound 209 was synthesized via $\mathrm{N}$-alkylation of 73 with 6-iodo-1,2:3,4-di- $O$ isopropylidene- $\alpha$ - $D$-galactopyranose which was subsequently deprotected to afford the $\alpha / \beta$ mixture of galactoporphyrin 210 in $90 \%$ yield. To form the cationic derivatives 74, 211 and 212 methylation of the pyridyl groups of $\mathbf{7 3}$ and $\mathbf{2 0 9}$ and 210 was carried out [230]. All of the galactoporphyrin derivatives proved to be more efficient at singlet oxygen production compared to 73 . The tri-cationic derivatives 416b,c displayed higher production than the tetra-cationic ones. The unprotected PSs 416c and 212 have a higher efficiency to generate singlet oxygen compared to the protected derivatives $\mathbf{4 1 6 b}$ and $211^{[230]}$.

Other linker units may be employed as well. Thus, a library of diethylene glycol linked glycoporphyrins TPP (204-206) and their $S$-analogues $(\mathbf{2 0 7}, \mathbf{2 0 8})$ were synthesized and their PDT efficacy tested against retinoblastoma cells ${ }^{[198]}$. All $O$-glycosylated diethylene glycol porphyrins and the reference compound (203) were synthesized from 5,10,15tris( $p$-phenol)-20-phenylporphyrin and reacted with the bromo-diethylene glycol substituted glycosides followed by deacetylation or with 2-(2-chloroethoxy)ethanol for 203. The thiosugar derivatives $\mathbf{2 0 7}$ and 208 were obtained by reacting 5,10,15-tris( $p$-phenol)-20-phenylporphyrin with 1-bromo-2(2-bromo-ethoxy)-ethane which was subsequently condensed with 1-thio-tetraacetate- $\beta$ - $D$-galactose and 1-thiotetraacetate- $\alpha-D$-mannose, followed by deacetylation.

Diethylene glycol linkers also found use in Maillard's synthesis of a range of glycosylated zinc dimer/trimer derivatives (418-421) for application in 2-photon absorption PDT ${ }^{[231]}$. All derivatives were substituted with three $\alpha$ mannose units on each porphyrin in the hope of targeting tumor cells which contained over-expressed lectin-type membrane receptors. The four oligomers (418-421) were all synthesized from a single parent monomer $\mathbf{4 1 7}$ with varying $\pi$-conjugation linkers between the porphyrin moieties. The parent monomer 417 was pre-formed in five steps and the trimethylsilyl group was deprotected for oligomer synthesis. The butadiyne core dimer $\mathbf{4 1 8}$ was synthesized via Glaser- 
<smiles>O=C(O)c1ccc(C(c2ccncc2)=c2ccc(=C(c3ccncc3)c3ccc(C(=C4C=CC(C(=C5C=Cc6ccc5[nH]6)c5ccncc5)=N4)c4ccncc4)[nH]3)[nH]2)cc1</smiles>

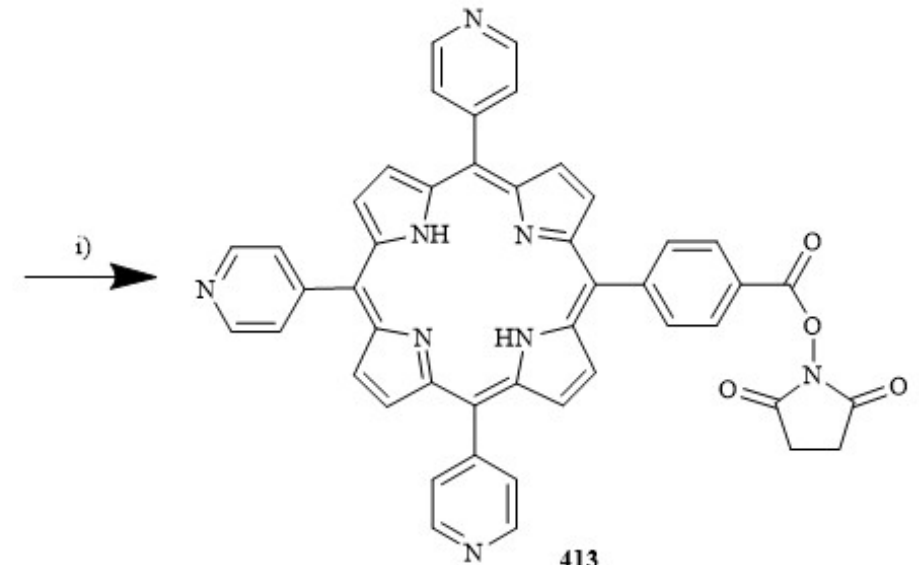

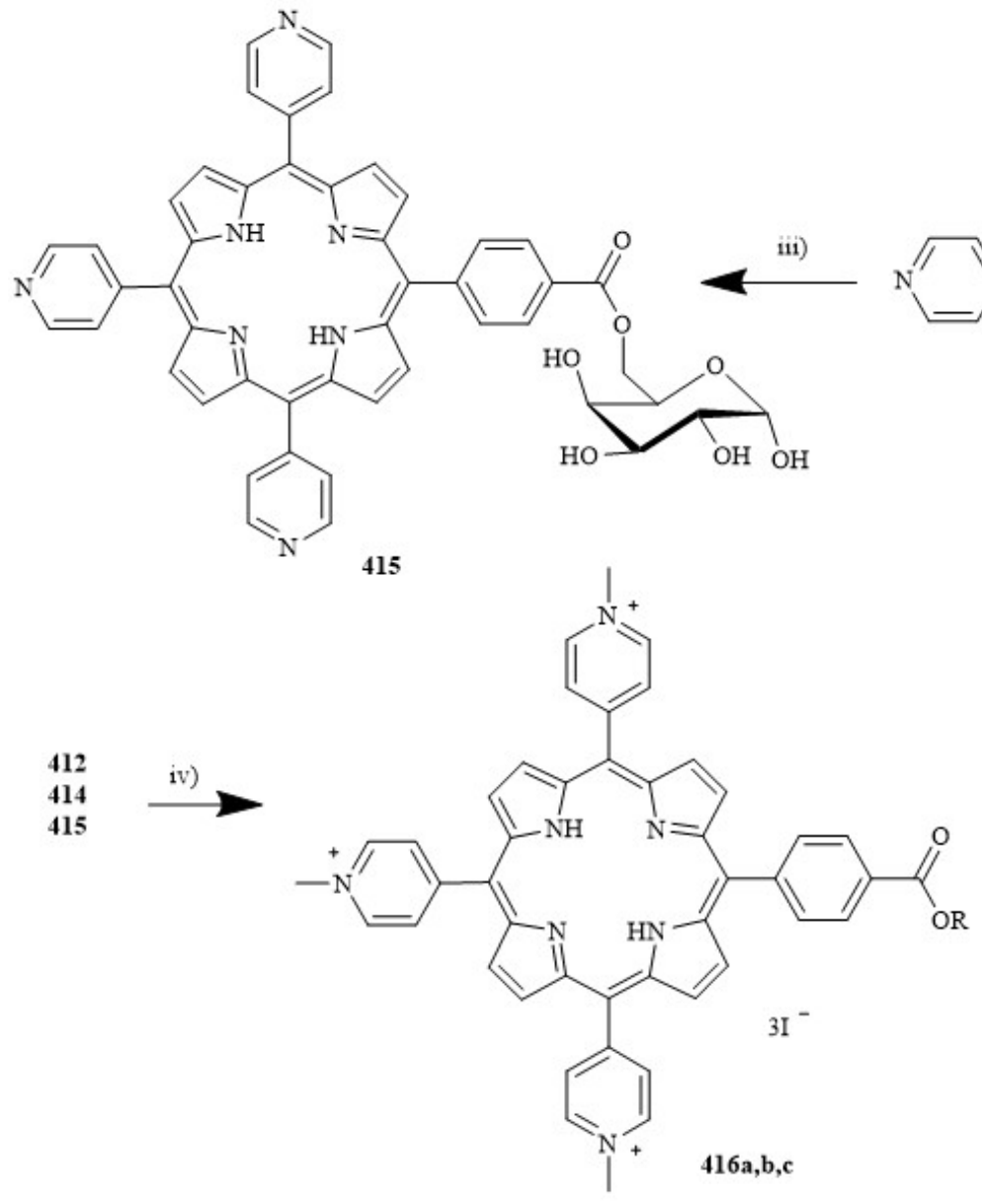

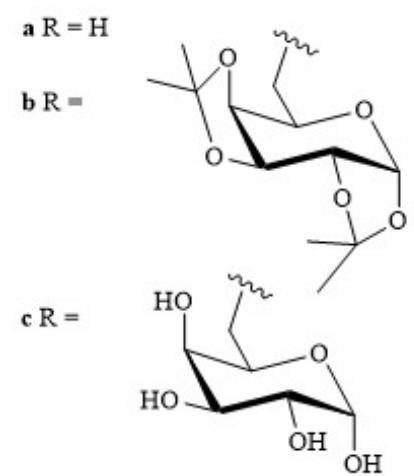

Scheme 13. Synthesis of glycosyl tris $\left(N\right.$-methylpyridinium)porphyrins. i) $\mathrm{SOCl}_{2}$, pyridine, $50{ }^{\circ} \mathrm{C}, 30 \mathrm{~min}$., then $N$-hydroxysuccinimide, $50{ }^{\circ} \mathrm{C}, 3 \mathrm{~h}$; ii) $1,2: 3,4-$ di- $O$-isopropylidene- $\alpha$ - $D$-galactopyranose, $\mathrm{NaH}$, toluene, r.t., 90 min.; iii) TFA, $\mathrm{H}_{2} \mathrm{O}$, r.t., 30 min.; iv) $\mathrm{CH}_{3} \mathrm{I}, \mathrm{DMF}, 40^{\circ} \mathrm{C}, 3 \mathrm{~h}$. 
Hay oxidative coupling in $72 \%$ yield. The dimers 419, 420 and trimer 421 were prepared via Heck cross-coupling reactions with the corresponding halogenated derivatives in $31 \%, 42 \%$ and $80 \%$ yields, respectively. Compounds 418 and $\mathbf{4 2 1}$ were deprotected using Zemplén conditions which proved impossible to characterize via NMR and photophysical data due to solubility problems.

Next, the photophysical properties of glycoporphyrins $(418,419,421)$ were compared to data of 1PA (one-photon absorption) and 2PA (two-photon absorption) nonglycosylated derivatives (422) ${ }^{[231-233]}$. The 1PA absorption studies of 418 and 419 are very similar to that of $\mathbf{4 2 2}$ and 423, however, all the transitions of the former were slightly<smiles>[R]C#Cc1ccc(N(c2ccc(C#C[R])cc2)c2ccc(C#C[R])cc2)cc1</smiles>
$\mathrm{R}^{1}=$

422

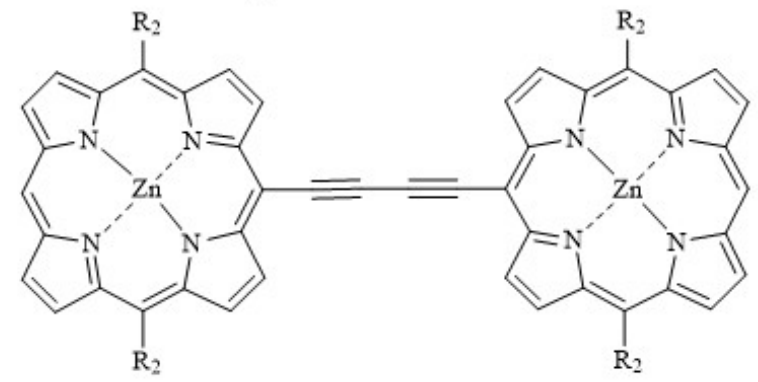

423<smiles>[R][CH]C(C)(C)c1cc(I)cc(C(C)(C)C)c1</smiles>
AcOManO- $\left(\mathrm{C}_{2} \mathrm{H}_{4}\right) \mathrm{O}$

blue-shifted compared to the latter pair. This is indicative of a weaker conjugation along the $\pi$-system of the glycosylated derivatives. Compound $\mathbf{4 3 1}$ also displayed similar results to dimer $419^{[232]}$. The carbohydrate moieties caused a twisted conformation of the dimers which subsequently decreased the conjugation and the 2PA cross-section, but this interpretation needs further proof. Looking at the $2 \mathrm{PA}$ properties of the trimer $\mathbf{4 2 1}$ it exhibits two fluorescence emission bands which show different behavior upon 2PA excitation, i.e., they have two different 2PA excitation pathways. The 2PA cross sections of 418 and 421 are above the average cross section of typical 2PA PSs ${ }^{[232]}$.

Subsequent extension of this work by Maillard focused on

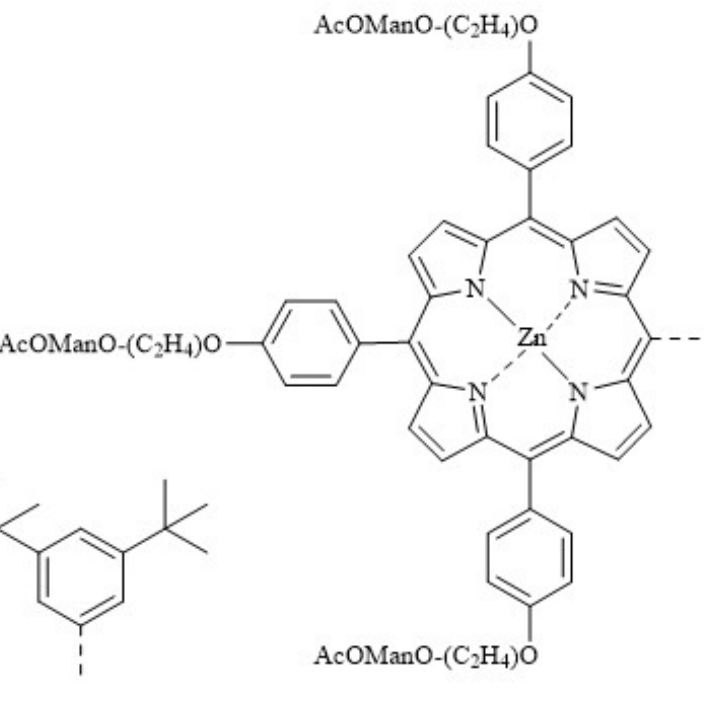

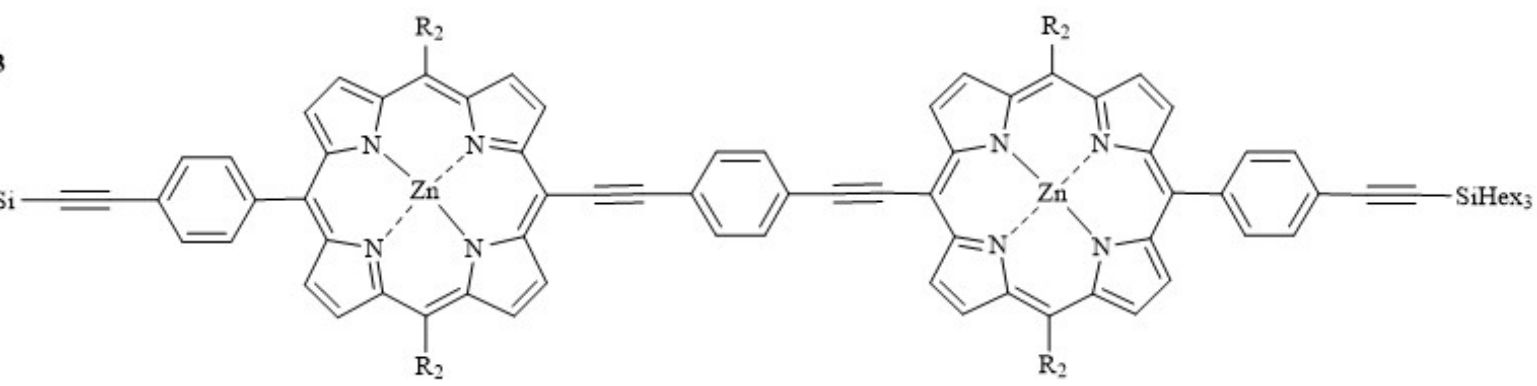

Formula 4. 


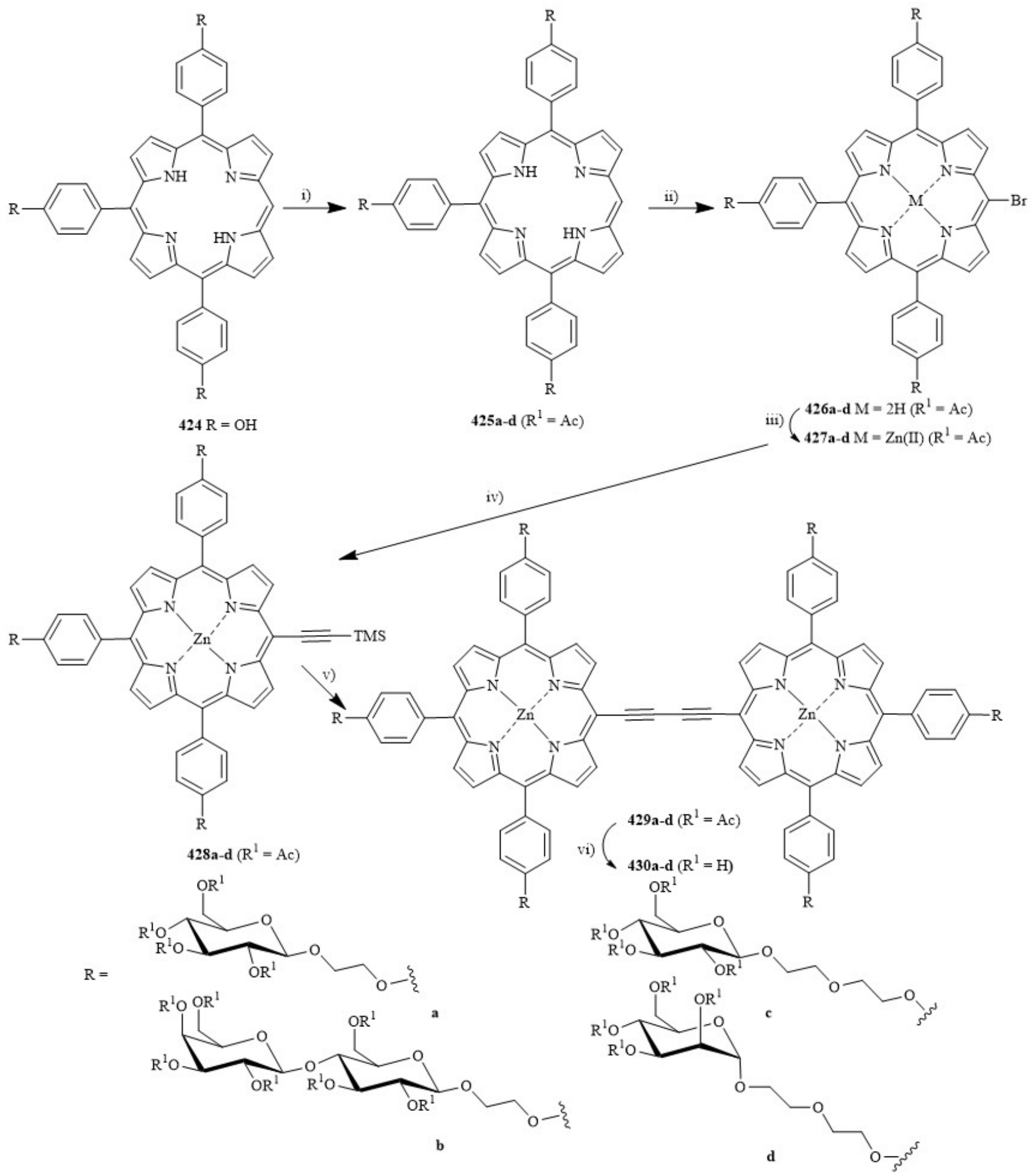

Scheme 14. Synthesis of glycoconjugated porphyrin dimers. i) bromo-carbohydrate derivatives, $\mathrm{CsCO}_{3}$, DMF, r.t., 24 h; ii) $\mathrm{NBS}, \mathrm{CHCl}_{3}$, pyridine, 15 min., 0 ${ }^{\circ} \mathrm{C}$; iii) $\mathrm{Zn}(\mathrm{OAc})_{2}, \mathrm{MeOH}, \mathrm{CHCl}_{3}$, reflux, 1 h; iv) TMSA, CuI, $\mathrm{Pd}\left(\mathrm{PPh}_{3}\right)_{2} \mathrm{Cl}_{2}, \mathrm{THF}, \mathrm{NEt}_{3},-180{ }^{\circ} \mathrm{C} \rightarrow$ r.t., 12 h; v) TBAF $1 \mathrm{M}, \mathrm{CuI}, \mathrm{Pd}\left(\mathrm{PPh}_{3}\right)_{2} \mathrm{Cl}_{2}, \mathrm{CH}_{2} \mathrm{Cl}_{2}, \mathrm{THF}$,

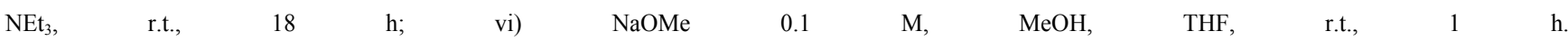


derivatives with carbohydrate variation and with different polyethylene glycol linkers, which is illustrated in Scheme $14^{[234]}$. The one-photon PDT efficiency was examined and compared to the free base monomer $\mathbf{2 0 6}$ which is used for in vivo PDT treatment [234-236]. All glycoporphyrins were synthesized from $\mathbf{4 2 4}$ which underwent a Williamson reaction with various bromo-polyethyleneglycol protected glycosyl units affording 425a-d in yields ranging from 54-78 $\%$. Mono-bromination was carried out on the trisubstituted macrocycles in 83-97 \% yields followed by metallation with zinc acetate in quantitative yield affording 427a-d. Sonogashira cross coupling reactions were carried out to provide 428a-d in 49-78\%. The trimethylsilyl group was subsequently removed and self-dimerization was carried out using a palladium catalyst yielding 429a-d in 38-69\%. Zémplen deprotection was performed to provide the final dimers 430a-d.

An example for the use of amide linker units was reported in 2006. Here two new mono-glucosyl tritolylporphyrins $\mathbf{( 2 2 1 , 2 2 2 )}$ were synthesized with the glycosyl moiety linked via a carboxymethyl glycosidic $\alpha$ - $D$-linkage. The monohydroxyphenyltritolylporphyrins 213/214 were prepared by Little's method ${ }^{[191]}$ through condensation of pyrrole with $p$-tolualdehyde and the ortho- or parahydroxybenzaldehyde in propionic acid. Incorporation of a terminal amine (217/218) allowed for coupling with 3,4,6tri- $O$-acetyl-carboxymethyl- $\alpha$ - $D$-glucopyranoside- $2-O$ -

lactone and transesterification afforded 221, $222^{[237]}$.

In addition to the increase in water solubility glycoporphyrins also offer an entry into amphiphilic systems. A typical example is a study on amphiphilic porphyrins with three maltohexaose units and a single alkyl chain (ethyl, $n$-butyl, $n$-hexyl, $n$-decyl and hexadecyl) ${ }^{\text {[231a] }}$. Here 3-iodopropyl nonadeca- $O$-acetyl- $\beta$ - $D$-maltohexaoside was reacted with 5,10,15,20-tetrakis(4hydroxyphenyl)porphyrin ( $p$ THPP) to yield trisugar substituted porphyrins followed by alkylation of the remaining free HO-group. Alternatively, monoalkylation of one $\mathrm{OH}$ group followed by introduction of three carbohydrates was possible as well. Deprotection then provided a library of amphiphilic porphyrins (431). The derivatives exhibited high water solubility, however, the Soret band broadening was dependent on the concentration in solution compared to solutions of DMSO which exhibited sharp Soret bands and inactive CD. The aggregation of the derivatives was examined by absorbance and circular dichroism (CD) spectra. Trismaltohexaosylated porphyrins with long alkyl chains formed stable aggregates in water and did not exhibit concentration dependency. In aqueous media, the intensity of the CD cotton effect signals decreased with increasing alkyl chain length. A chiral face to face aggregation was observed for short alkyl chain derivatives while an edge to edge aggregation was observed in the long alkyl chain derivatives. These observations show the potential to create photofunctional nanomaterials ${ }^{[231 \mathrm{a}]}$.

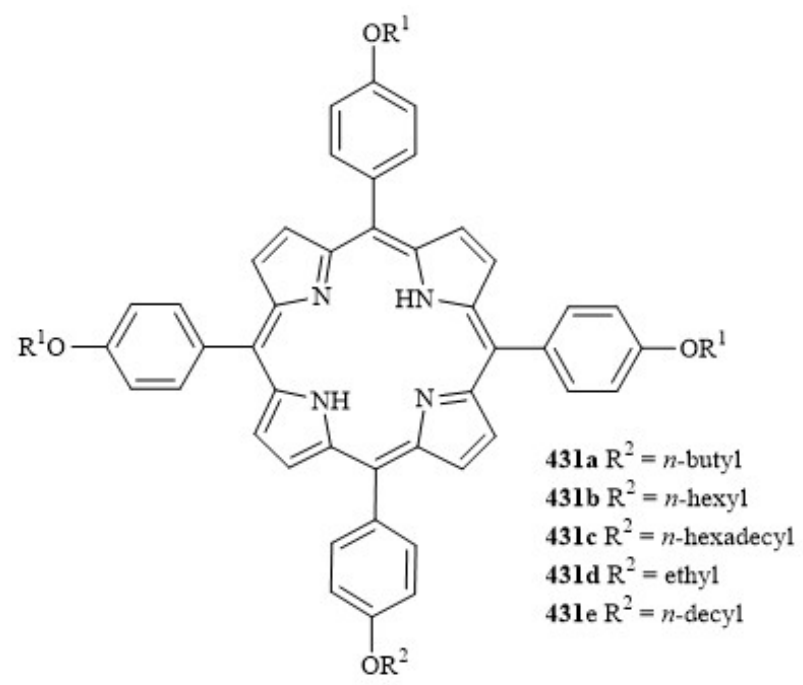

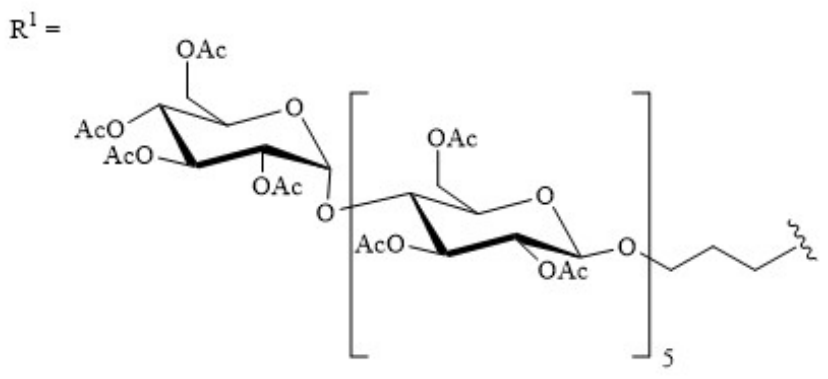

Formula 5.

Similar strategies were used for the derivatization of $m$ THPP. $m$ THPP itself was identified by Bonnet et al. to be a promising PS. A significant screen was carried out showing $m$ THPP to be 25-30 times better than HpD in tumor models as a PS ${ }^{[238,239]}$. Several in vitro and in vivo studies have been carried out displaying the advantages of $m$ THPP ${ }^{[239-242]}$. The derivatization of $m$ THPP with 3iodopropylperacetylmaltohexaoside yielded a series of mono-, di-, tri- and tetra-maltohexaose substituted $m$ THPP (432-436) as highly water soluble PSs for PDT applications [202a]. All derivatives were deprotected affording 437-441 either in form of the free base of the zinc(II) complexes (Scheme 15). The water solubility of which rose with the increasing number of sugar moieties while the zinc derivatives had higher water solubility compared to the corresponding free base derivatives.

A robust methodology for direct glycosylation of a range of different mono-hydroxyl porphyrins with trichloroacetimidate glycosyl donors afforded high yields and faster reaction times ${ }^{[214]}$. Synthesis of 336-352 was 


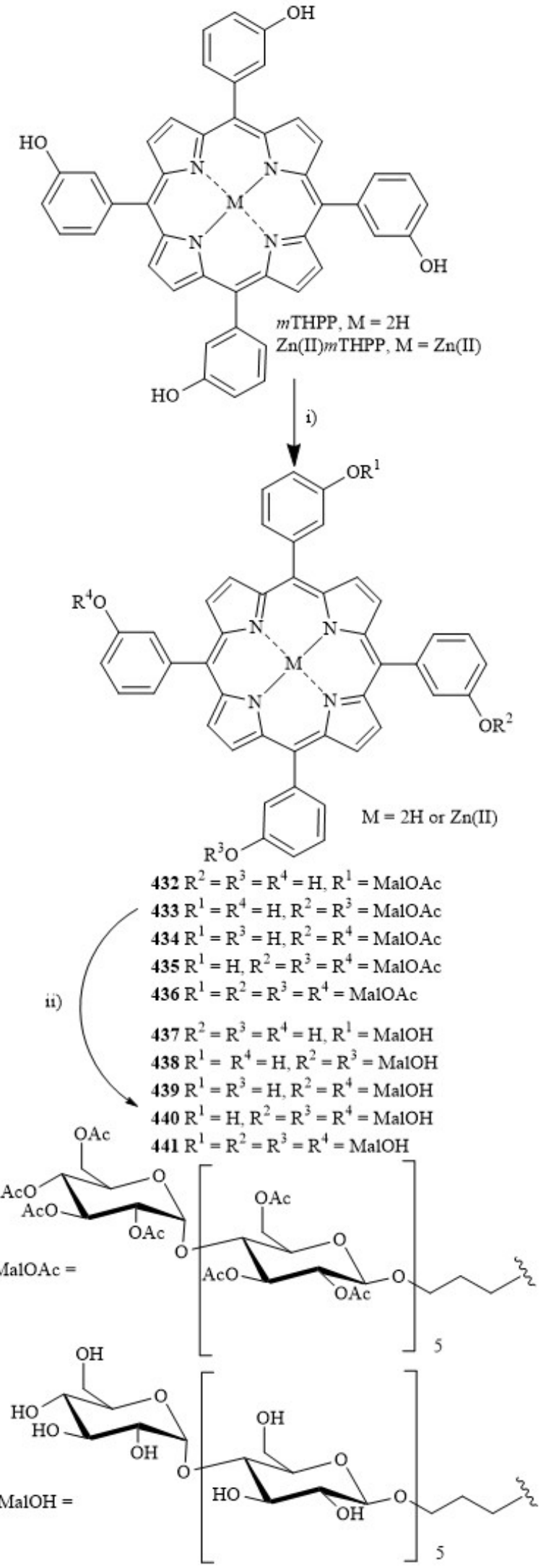

Scheme 15. Synthesis of maltohexose $m$ THPP. i) MalOAc-I, $\mathrm{K}_{2} \mathrm{CO}_{3}$, DMF, r.t. ii) $\mathrm{NaOMe}, \mathrm{MeOH}-\mathrm{CHCl}_{3}$, reflux. achieved via direct glycosylation in the presence of $\mathrm{BF}_{3} \bullet \mathrm{OEt}_{2}$ as the Lewis acid producing the alkyl and aryl derivatives in high yields $84-92 \%$ followed by subsequent deprotection in 94-97\% yield. This procedure was also extended to tri-hydroxyl porphyrins and gave the glycosyl derivative $\mathbf{3 4 0}$ in $90 \%$ yield $^{[214]}$.

S-linked systems: In comparison to the $O$-linked glycoporphyrins $S$-glycosyl bonds might be more resistant towards endogenous hydrolysis by glycosidases. A typical synthesis is outlined in Scheme 16. It involved the addition of a 1,3-dibromopropane linker unit to monohydroxytolylporphyrins (442) in $90 \%$ and $85 \%$ yields for $\mathbf{4 4 3}$, respectively ${ }^{[243]}$. Glycosylation using a method from Bennett et al. ${ }^{[244]}$ gave 444-446 in 60-80\% yields. Quantitative deacetylation gave 447-449. Similarly, a range of meso-aryl porphyrins and protoporphyrin IX derivatives appended with deprotected $S$-glucose, mannose and galactose residues were synthesized ${ }^{[199]}$. Series one was synthesized from preformed ortho- and para-hydroxylphenyl precursors $(213,214)$ using Little conditions, to yield 223 and 224 in $91 \%$ and $84 \%$ yields when reacted with 1,3,dibromopropane and subsequently underwent glycosylation with 2,3,4,6-tetra- $O$ acetyl- $1 S$-acetyl-1-thio- $\beta$ - $D$-galactopyranose, $\quad-\beta-D$ glucopyranose and $-\alpha$ - $D$-mannopyranose in $64-84 \%$, followed by deacetylation in quantitative yields producing 230-236. The second series of thio-glycosylated porphyrins was synthesized from protoporphyrin IX dimethylester $\mathbf{4 5 0}$ which was converted to the isohematoporphyrin $\mathbf{4 5 1}$ via modifications using methods from Smith's group [245,246] (Scheme 17). The latter was brominated to form $\mathbf{4 5 2}$ and was subsequently glycosylated yielding $\mathbf{4 5 3 - 4 5 5}$ in 64-93\%. This was followed by full deprotection (456a-458a) and partial deprotection $\mathbf{( 4 5 6 b - 4 5 8 b )}$ in quantitative yields. The relative stability of $\beta$-thioglucosides versus $\beta$-glucosides towards hydrolysis was tested by $\beta$-glucosidases finding $\beta$ thioglucoside to be a more stable residue ${ }^{[199]}$.

5,10,15,20-Tetra(pentafluorophenyl)porphyrin $\left(\mathrm{TPPF}_{20}\right)$ is a useful porphyrin precursor as the p-position of the pentafluorophenyl residue is susceptible to nucleophilic substitution. Thus, it can be used directly in glycosylation reactions without prior activation. For example, Drain et al. synthesized nonhydrolyzable tetrasubstituted glycoporphyrins (e.g., 87, 95) in high yields from $\mathrm{TPPF}_{20}$ in a nucleophilic substitution reaction with thio-glucose and thio-galactose derivatives to decrease the acid hydrolysis of the glycosyl bond using $C$ - or $S$-glycoside linkages to reduce drug dosage requirements ${ }^{[172,247]}$. $S$ - or $C$-glycoside type PSs were previously synthesized in relatively low yields $[171,199,218,243]$. However, significantly higher yields $>85 \%$ were observed with both the acetyl protected or the free hydroxyl sugar present with such an approach ${ }^{[172,247]}$.

These strategies can also be employed for cationic water soluble systems. Here, an example is Boyle et al. 's synthesis of a series of 5-( $N$-alkyl-4-pyridyl)- $-10,15,20$-tris(4thioglycosyl-2,3,5,6-tetrafluorophenyl)porphyrins (Scheme $18)^{[248]}$. The synthesis of the key intermediate, 5-(4-pyridyl)- 


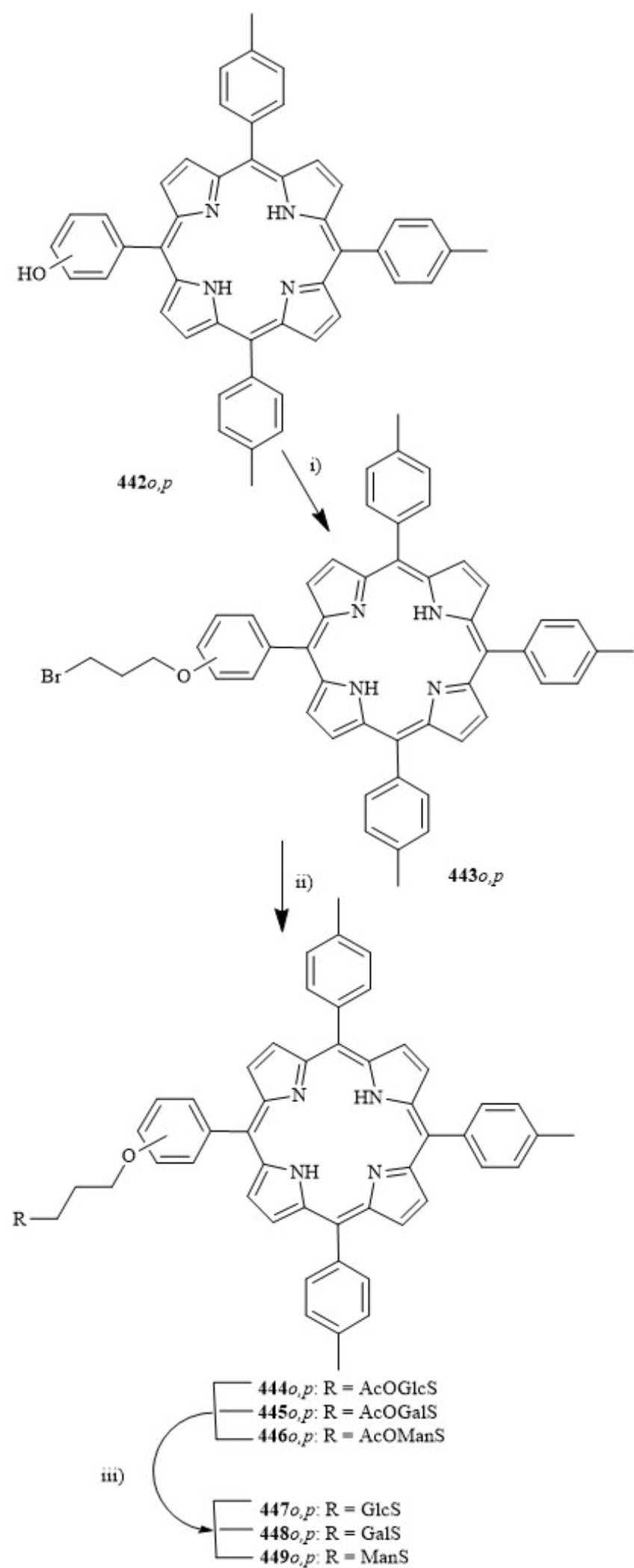

Scheme 16. Synthesis of thioglycosyl tolylporphyrins. (i) $\mathrm{Br}-(\mathrm{CH})_{3} \mathrm{Br}$, DMF, r.t., 20 h. ii) $2,3,4,6$ tetra- $O$-acetyl- $1 S$-acetyl-thio- $\beta-D$ galactopyranose, $\beta$ - $D$-glucopyranose, or $\alpha$-D-mannopyranose, $\left(\mathrm{C}_{2} \mathrm{H}_{5}\right)_{2} \mathrm{NH}$, DMF, r.t., 6 h, 60-80\%; iii) $\mathrm{MeONa} / \mathrm{MeOH}$, r.t., 1 h 10,15,20-tris(pentafluorophenyl)porphyrin $\mathbf{4 5 8}$ was carried out using the Alder-Longo method with 4pyridinecarboxaldehyde, pentafluoro-benzaldehyde and pyrrole. Replacing the fluorine of the para position with a thioglucosyl residue yielded 459 in $72 \%$. Quaternization of the pyridine nitrogen with methyl, $n$-hexyl, $n$-octadecyl and benzyl functionalities yielded 460a-d in 39-71\%. Deprotection of the sugar residue provided the water soluble glycosyl cationic porphyrins 461a-d. Cationic porphyrin monomers such as these have the potential to be characterized by electrospray ionization tandem mass spectrometry (ESI-MS/MS) ${ }^{[228,249]}$.

For satisfactory clinical use a PDT agent must generate sufficient ROS in addition to efficient cellular uptake. Several metalloporphyrin derivatives or analogues have been approved for PDT or are under clinical trial, for example $\mathrm{SnEt}_{2}$, TOOKAD ${ }^{\circledR}$ (Padeliporfin - Pd bacteriopheophorbide monolysotaurine) and Lutrin where the heavy atoms can increase the ${ }^{1} \mathrm{O}_{2}$ quantum yield. Severe conditions are usually required for complexation, especially for late transition metal complexes and these are incompatible with porphyrins having sugar moieties. Therefore complexation with the heavy metal ion should be done prior to introduction of sugar units. Thus, 5,10,15,20-tetrakis(4-(2,3,4,6-tetra- $O$ acetyl- $\beta$ - $D$-glucopyranosylthio)-2,3,5,6-

tetrafluorophenyl)porphyrin $\mathbf{8 7}$ was synthesized via Lindsey's condensation method of pyrrole and pentafluorobenzaldehyde and underwent nucleophilic substitution with 1,2,3,4,6-penta- $O$-acetyl-1-thio- $\beta$ - $D$ glucopyranose ${ }^{[177]}$. Its $\mathrm{Zn}(\mathrm{II}), \mathrm{Pd}(\mathrm{II})$, and $\mathrm{Pt}(\mathrm{II})$ complexes 88-90 were synthesized from $\mathrm{TPPF}_{20}$ via conventional complexation methods with $\mathrm{Zn}\left(\mathrm{CH}_{3} \mathrm{CO}_{2}\right)_{2}, \mathrm{PdCl}_{2}$, or $\mathrm{K}_{2}\left[\mathrm{PtCl}_{4}\right]$ in $64 \%, 70 \%$ and $76 \%$ yield, respectively, followed by nucleophilic substitution of the $S$-glucoside. All compounds were deprotected to give the $S$-glucosylated porphyrin 91 and its metal complexes 92-94. The singlet oxygen production increased in the order of free-base fluoroporphyrins $(\mathbf{8 7}, 0.08$ and 91, 0.06) $<\mathrm{Zn}$ (II) complexes $(\mathbf{8 8}, 0.055$ and $\mathbf{9 2}, 0.056)<\mathrm{Pd}(\mathrm{II})$ complexes $(\mathbf{8 9}, 0.003$ and 93, 0.002) due to the heavy-atom effect. However, the ${ }^{1} \mathrm{O}_{2}$ production of $\mathrm{Pt}(\mathrm{II})$ complexes (90, 0.001 and 94, 0.001) were very low ${ }^{[177]}$.

A 'complete set' (mono-, 5,10-bis-, 5,15-bis-, tris- and tetrakis) of tetrakis(perfluorophenyl)porphyrins substituted with $\beta$ - $D$-glucopyranosylthio groups on the phenyl ring was also synthesized. Nucleophilic substitution of $\mathrm{TPPF}_{20}$ with the $S$-glycoside affording all five derivatives 237, 239, 241, 243 and 87. The extent of $S$-glycosylation could be controlled by the equivalents of $\mathrm{TPPF}_{20}$ and $S$-glycoside used, optimum conditions were developed for each derivative and afforded maximum yields of $42 \%$ (237), 19 \% (239), $14 \%$ (241), $32 \%$ (243) and $83 \%$ (87). All of which were deprotected in quantitative yields ${ }^{[200]}$.

Several series of $\mathrm{TPPF}_{20}$ 's substituted with glycosyl and amphiphilic moieties totaling 742 in number were 

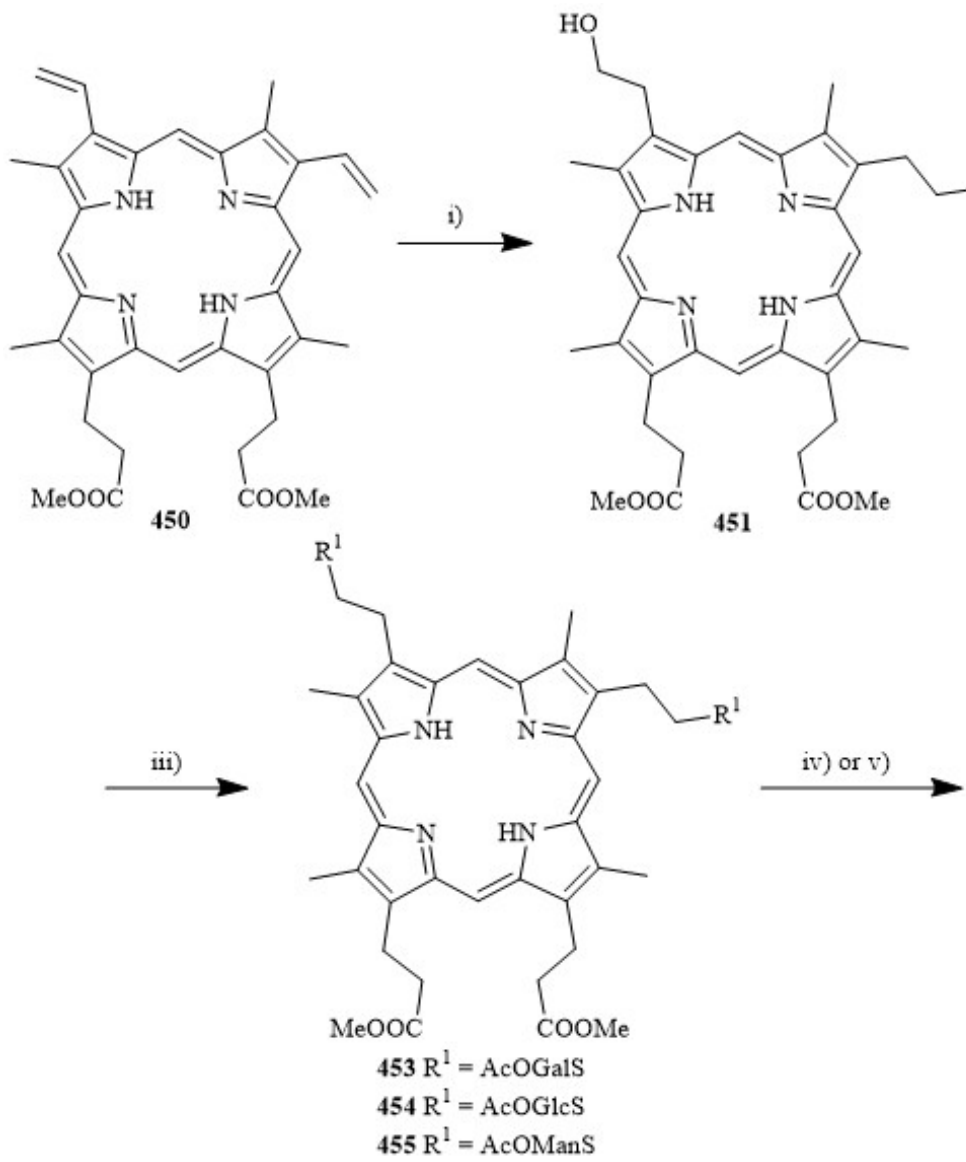
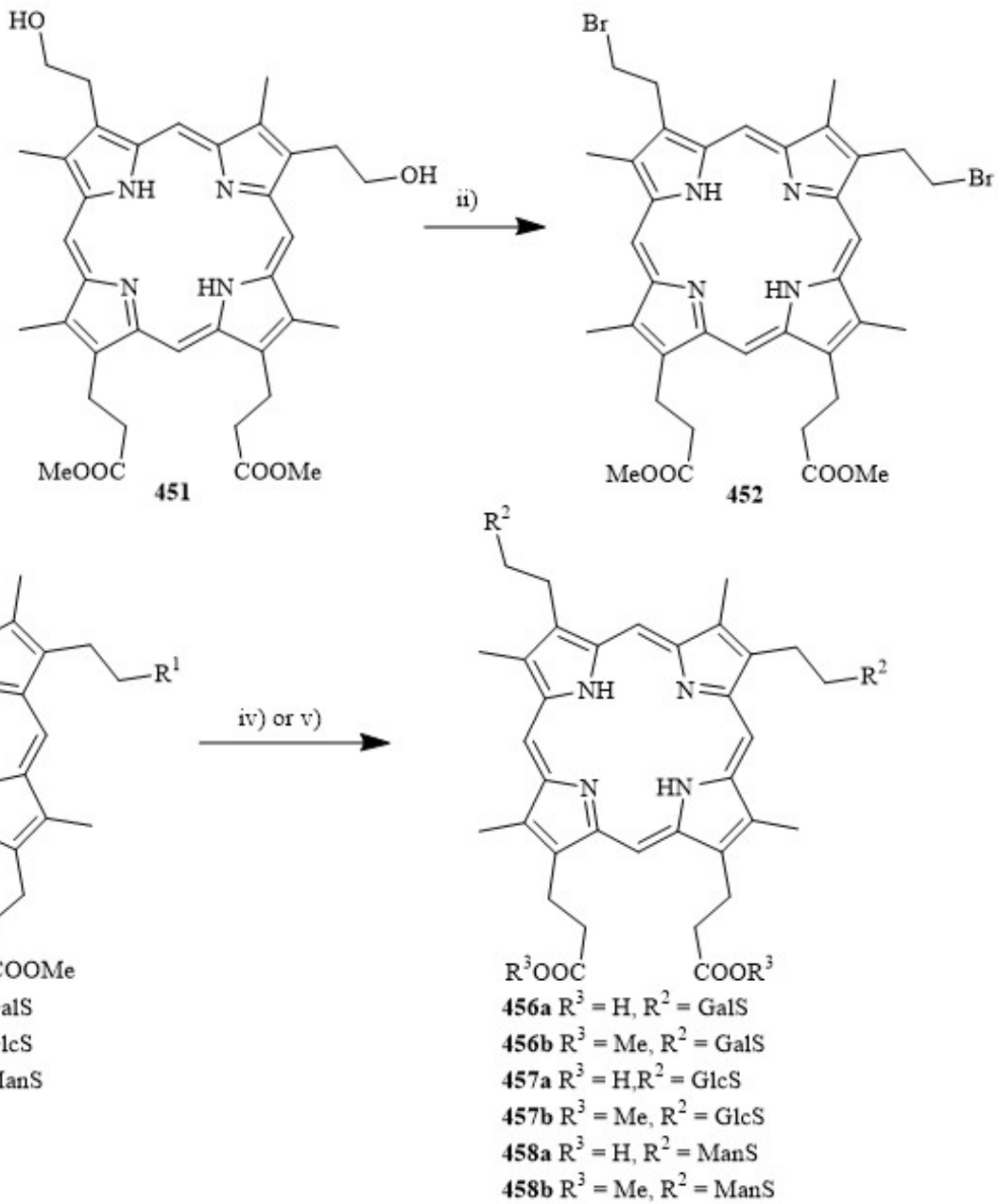

Scheme 17. Synthesis of thioglycosylated isohematoporphyrin derivatives. i) a) $\mathrm{Tl}\left(\mathrm{NO}_{3}\right)_{3}, 3 \mathrm{H}_{2} \mathrm{O}, \mathrm{MeOH}$, b) $\mathrm{HCOOH}$ c) $\mathrm{NaBH}_{4}$; ii) $\mathrm{SOBr}_{2} / \mathrm{K}_{2} \mathrm{CO}_{3}$; iii) $2,3,4,6-$ tetra- $O$-acetyl- $1 S$-acetyl-1-thio- $\beta$ - $D$-galactopyranose, $-\beta$ - $D$-glucopyranose and - $\alpha$-D-mannopyranose $\left(\mathrm{C}_{2} \mathrm{H}_{5}\right) \mathrm{NH}$, DMF; iv) $\mathrm{NaOMe}, \mathrm{MeOH}$ or v) $\mathrm{KOH}$.

synthesized via combinatorial synthesis and identified by HPLC and mass spectrometry. From the series conjugates with the most promising PDT efficacy were identified by in vitro analysis. The viable derivatives (341-363) were subject to direct synthesis and further testing ${ }^{[215]}$.

$N$-linked systems: A range of $N$-linked glycoporphyrins have been prepared as well. For example, amide-linked diand octagalactosyl and glucosyl tetraphenylporphyrins (78, 79, 245, 246) were synthesized for a comparative study of their uptake in hepatocytes which have receptors (asialoglycoproteins) for terminal galactose residues. The highly water-soluble amide-linked octagalactose and octaglucose derivatives of TPP were synthesized by reacting lactonolactone or maltonolactone with octaamine TPP $\mathbf{7 5}$, which was formed from the octa-ol TPP 77 via the octanitrile TPP $76^{[175]}$.
Aminoglycosamide TPP and chlorin were studied for the effect symmetric or asymmetric glycoconjugation had on the photophysical properties, photosensitivity and cellular internalization compared to the parent porphyrin TPP in HT29 human adenocarcinoma cells ${ }^{[201]}$. Synthesis of $\mathbf{2 4 7}$ was carried out by coupling $O$-acetylated glucosamine with 5-(4-carboxyphenyl)-10,15,20-triphenyl-porphyrin which was previously prepared via the Little condensation conditions in $7 \%$ yield ${ }^{[191]}$. The corresponding monocarboxyphenyl chlorin was prepared by the diimide reduction of 5-(4-carboxyphenyl)-10,15,20triphenylporphyrin in $70 \%$ yield which was subsequently coupled with $O$-acetylated glucosamine affording the inseparable isomeric 2,3- and 7,8- monoglucosylated chlorins. The trans-biglucosylated porphyrin $\mathbf{2 4 8}$ was synthesized from glucosylated dipyrromethane and benzaldehyde. The number of glycosylated groups altered 


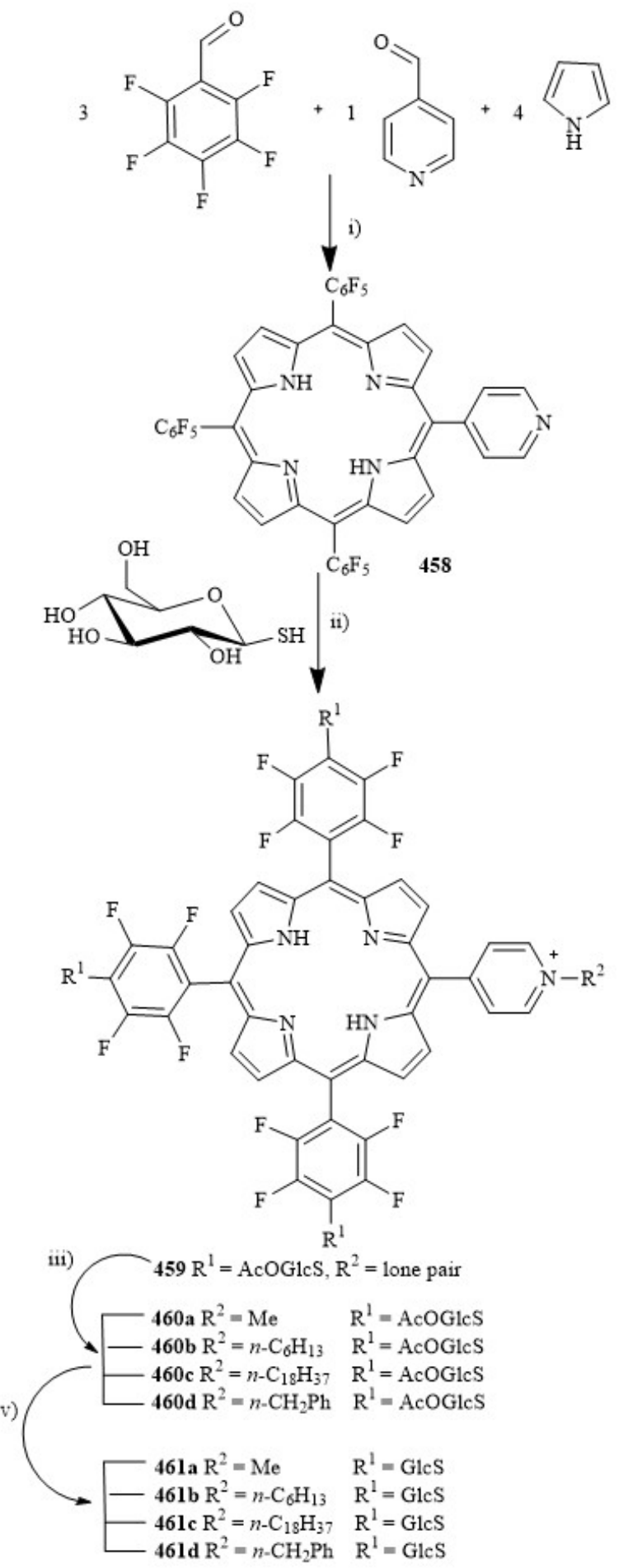

Scheme 18. Synthesis of a series of 5-( $N$-alkyl-4-pyridyl)-10,15,20-tris(4thioglycosyl 2,3,5,6-tetrafluorophenyl)porphyrins. i) DMF, r.t., $16 \mathrm{~h}$; ii) $\mathrm{R}^{1} \mathrm{I}$, DMF, r.t., 16 h; iii) $\mathrm{NaOMe}, \mathrm{MeOH}$, r.t., 1 h.

the singlet oxygen quantum yield showing the effect of structural changes on the triplet quantum yield ${ }^{[201]}$.

In a more exhaustive study a range of glucosylated mesotetraarylporphyrins were synthesized from mesoaminophenyl-substituted, $\beta$-amino-substituted and meso- pentafluorophenyl-substituted porphyrin derivatives [202]. Amide coupling of 5-(4-aminophenyl)-10,15,20triphenylporphyrin with glucuronic acid afforded the $\mathrm{N}$ linked glycoconjugate $\mathbf{4 6 2}$ after deprotection. Due to difficulty in hydrogenation of the benzyl protecting groups small amounts of monobenzylated derivatives were still present. A similar coupling was carried out via $\beta$-amino substituted porphyrins using nickel complexes which provided easier deprotection of the benzyl groups affording 463. Another approach for the coupling of glycosyl units was achieved via nucleophilic aromatic substituted with mesopentafluorophenyl-substituted porphyrin selectively at the para position affording a mixture of products $\mathbf{2 4 9 - 2 5 2}$ in 40 $\%, 7 \%, 14 \%$ and $8 \%$, respectively. In addition, zinc derivatives 253-259 provided similar results.

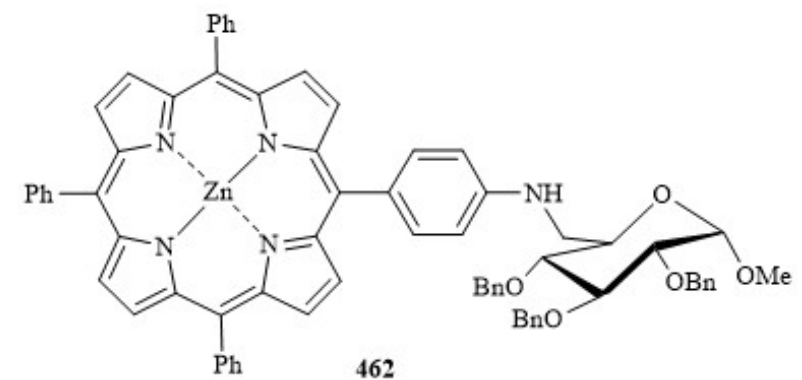

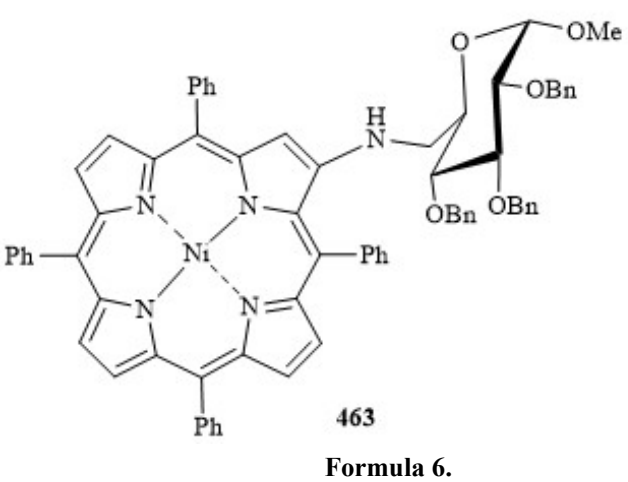

Thiourea, thiocarbamate and carbamate linkages have been investigated for their chemical stability and PDT efficiency in the form of glycoconjugates 333-335 ${ }^{\text {[213] }}$. They were prepared from the amino or hydroxyl substituted tetraaryl porphyrins and per- $O$-acetyl- $\beta$ - $D$-glucopyranosyl isothiocyanate under reflux or ultrasonication in good yields $72 \%, 23 \%$ and $49 \%$, respectively. Examining the photophysical properties 335 displayed the best singlet oxygen production in the range $335>$ Photofrin $^{\circledR}>333>$ 334 while the photostability increased in the same order. In conclusion, the carbamate derivative $\mathbf{3 3 5}$ has the most promising potential ${ }^{[213]}$.

\subsubsection{Organometallic Coupling Reactions}

The use of "click chemistry" as a mode of chemoselective linkage of azido appended polysaccharides and alkyne terminated porphyrins was successfully carried out and has now turned into a mainstay of carbohydrate porphyrin 
chemistry. The native linear $(1 \rightarrow 3)-\beta$ - $D$-glucan (curdlan, 464) was converted to the 6-bromo-6-deoxycurdlan 465 with subsequent azidation afforded 6-azido-6-deoxycurdlan $\mathbf{4 6 6}$. Coupling of 466 with the alkyne porphyrin was achieved using $\mathrm{CuBr}_{2}$ and ascorbic acid (Scheme 19) ${ }^{[250]}$. By now, this method has been extended to generate a wide variety of glycoporphyrins. These include systems with either one or four galactose or lactose units $(\mathbf{2 5 7}, \mathbf{2 5 8})$ from Vicente's group ${ }^{[203]}$. Note, the click reaction can also be used to prepare suitable aldehydes for condensation reactions as exemplified in the synthesis of $\mathbf{4 7 2}$ (Scheme 20) ${ }^{[203]}$.

A collaboration between our groups focused on the development of robust methodology for the synthesis of mono-, di-, tri- and tetra-modified glycoporphyrins (259268) ${ }^{[32]}$. TPP was functionalized with an azido moiety and subsequently reacted with commercially available $\beta$ propargyl glucose and $\alpha$-propargyl mannose either with acetylated or free hydroxyl groups under conventional and microwave-mediated heating conditions. The microwave conditions reduced reaction times from 3 days to $20 \mathrm{~min}$. and was optimized to allow functionalization with both protected and deprotected carbohydrates in high yields. A sequential "double-click" process was used to obtain a new class of bismodified 5,10-diglycoporphyrins (263) with heterogeneous carbohydrates. Reacting a propargyl glucoside with three equivalents of 5,10-di(4-azido-phenyl)-15,20diphenylporphyrin provided $\mathbf{2 6 2}$ as the only product in $87 \%$ yield and was subsequently reacted with propargyl mannoside via a second 'click' reaction affording 263 in 53 $\%$ yield.

In continuation of this initial work, a significantly large library of mono-, di- and trideprotected glycoporphyrins (316-331,261) were synthesized with monosaccharides and for the first time, synthetic disaccharides and trisaccharide ${ }^{[33]}$. These studies also provide the first examples of bismodified glycosylated porphyrins and a significant highlight was the ligation of the synthetic trisaccharide Lewis ${ }^{\mathrm{X}}$, a histo-blood group antigen. Optimization studies were conducted to achieve a high yield ligation with the deprotected saccharide units all synthesized with a propargyl unit at the anomeric position. Again TPP was functionalized with an azido moiety and subsequently reacted with synthetic mono-, di- and tri- saccharide via optimized Huisgen 'click' cycloaddition reaction in very high yields. With a sequential "double-click" process used again to obtain bis-modified 5,10-diglycoporphyrins (331). This study was the first example of synthetic carbohydrates including $N$-Ac-lactosamine and Lewis ${ }^{\mathrm{X}}$ conjugated to the porphyrin scaffold ${ }^{[33]}$.

Similar work by Maillard prepared a series of porphyrins with three glycosyl units linked via triazole groups using microwave heating conditions ${ }^{[251]}$. Several parameters were examined including the sugar type, the length of the spacer, and the position of the triazole ring for changes in photobiological properties. All derivatives were synthesized from the precursor $\mathbf{4 7 3}$ which was synthesized previously by

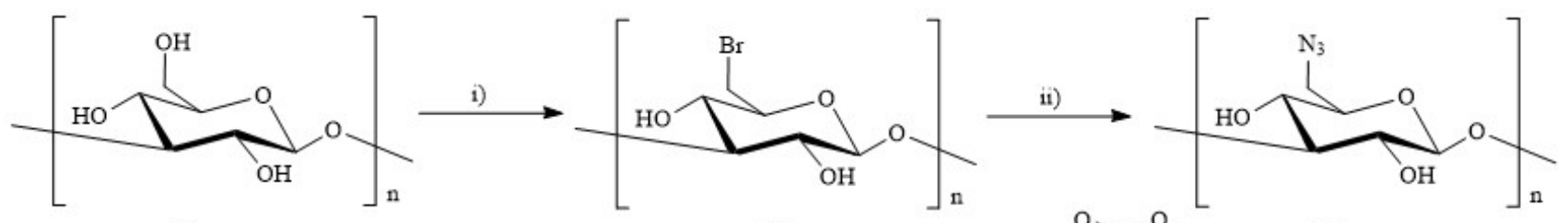

464

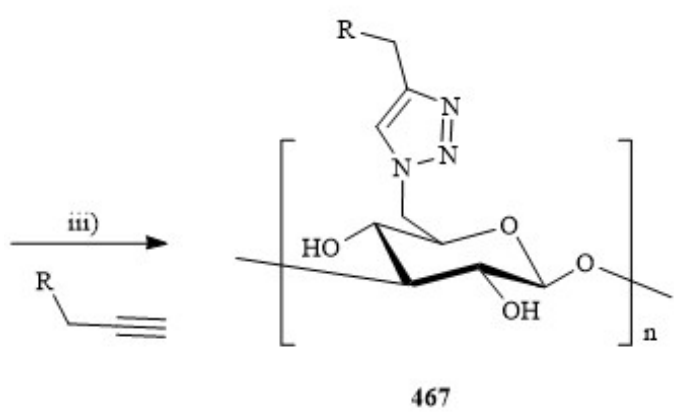

467
465

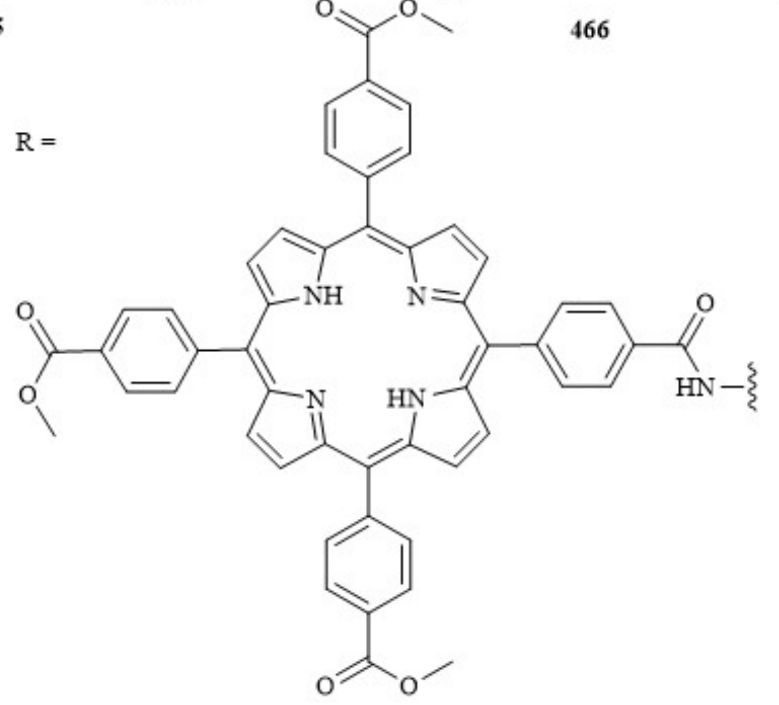

Scheme 19. Synthesis of $(1 \rightarrow 3)-\beta$ - $D$-glucan conjugated methoxycarbonylphenyl-porphyrin. i) Triphenylphosphine, DMF, LiCl, r.t., 3 h, and then, carbon tetrabromide, $60{ }^{\circ} \mathrm{C}, 24 \mathrm{~h}$; ii) sodium azide, $\mathrm{Me}_{2} \mathrm{SO}, 80^{\circ} \mathrm{C}, 36 \mathrm{~h}$; iii) alkyne-terminated functional modules, CuBr 2 , ascorbic acid, propylamine, r.t., $12 \mathrm{~h}$, $\mathrm{Me}_{2} \mathrm{SO}$ or NMP. 


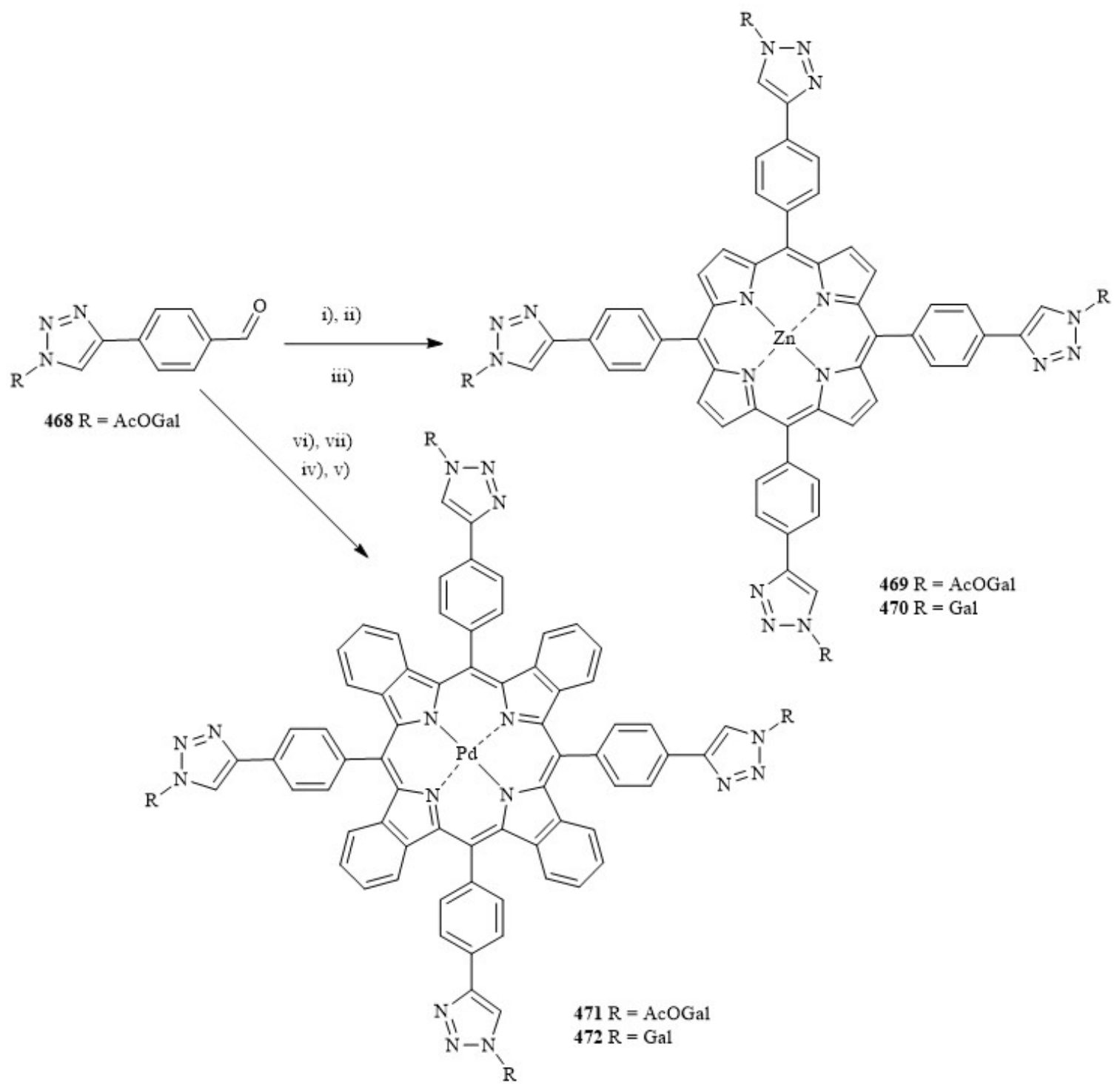

Scheme 20. Synthesis of tetrasubstituted glycoconjugated porphyrins. i) Pyrrole, $\mathrm{BF}_{3} \cdot \mathrm{OEt}_{2}, \mathrm{DDQ}$; ii) $0.5 \mathrm{M} \mathrm{CH}_{3} \mathrm{ONa} / \mathrm{MeOH}$; iii) $\mathrm{Zn}(\mathrm{OAc})_{2}$; iv) tetrahydroisoindole, $\mathrm{BF}_{3} \cdot \mathrm{OEt}_{2}$; v) DDQ; vi) $\mathrm{Pd}(\mathrm{OAc})_{2}$; $\mathrm{DDQ}$; vii) $0.5 \mathrm{M} \mathrm{CH}_{3} \mathrm{ONa} / \mathrm{MeOH}$.

a modified Senge procedure ${ }^{[144,252]}$. It was substituted with 1,3-dibromo propane which was converted to the azido groups or propargyl bromide to provide two different scaffolds 474, 475 for glycosyl conjugations. The compounds were protected by zinc(II) complexation before carrying out the Cu-catalyzed Huisgen 1,3-dipolar cycloaddition with copper salts affording 476, 477 in quantitative yields. Likewise, 478, 479, 482-484 were obtained via copper-catalyzed azide-alkyne cycloadditions with optimized conditions for the different substituents on the porphyrins 476,477 . Coupling of $\mathbf{4 7 6}$ with propargyl glycosides was carried out using $\mathrm{CuCl}$ affording 478, 479 in $65 \%$ and $68 \%$ yield while coupling of 477 with azido glycosides required a $\mathrm{CuSO}_{4} /$ sodium $L$-ascorbate catalyst system producing 482-485 in 40-80\%. Zinc was removed from all glycoporphyrins quantitatively and followed by deacetylation to afford 480, 481, 486-489 (Scheme 21).

A series of dendritic glycoporphyrins (491a-494a) and 491b-494b) were obtained from a multi-step procedure via coupling of the glycodendritic precursors and the alkynefunctionalized porphyrin core by a convergent methodology using click chemistry (Scheme 22). The porphyrin core was obtained via Lindsey-type condensation and subsequent functionalization with propargyl group and metallation with zinc. This porphyrin core was coupled through $\mathrm{Cu}(\mathrm{I})$ catalyzed click reactions with the azidic glycodendritic 


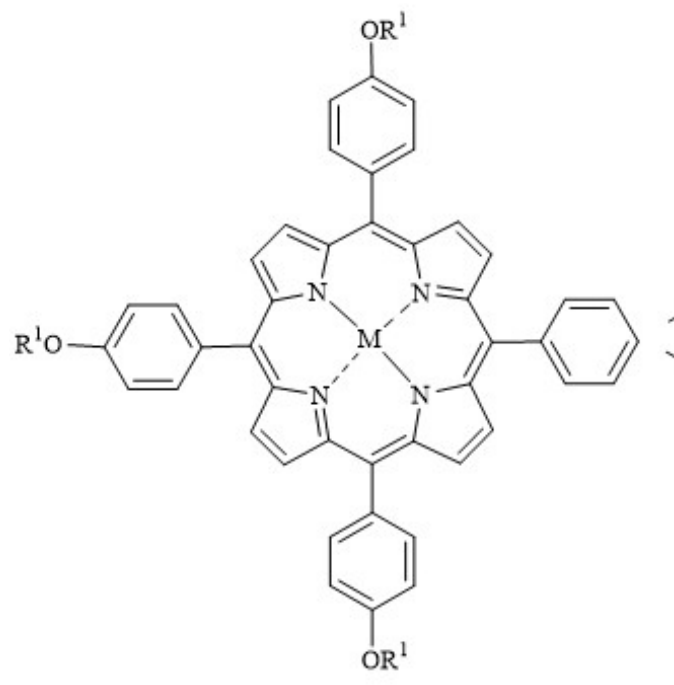
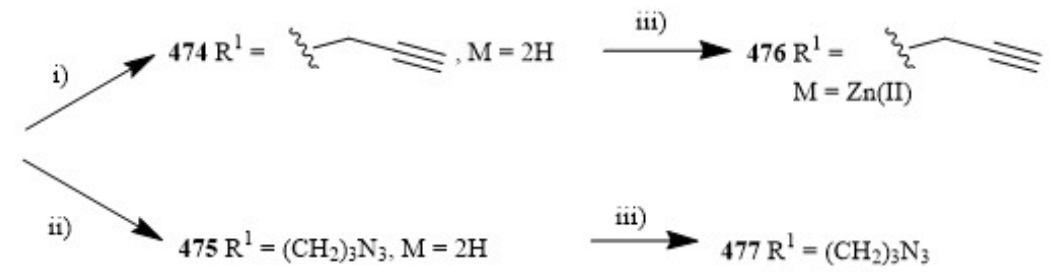

$473 \mathrm{R}^{1}=\mathrm{H}, \mathrm{M}=2 \mathrm{H}$

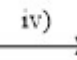

$\mathrm{R}^{2}$<smiles>[R]Cc1cn(CCCOC)nn1</smiles>

$479 \mathrm{R}^{2}=\mathrm{AcOManO}, \mathrm{M}=\mathrm{Zn}(\mathrm{II})$

ii) $475 \mathrm{R}^{1}=\left(\mathrm{CH}_{2}\right)_{3} \mathrm{~N}_{3}, \mathrm{M}=2 \mathrm{H}$
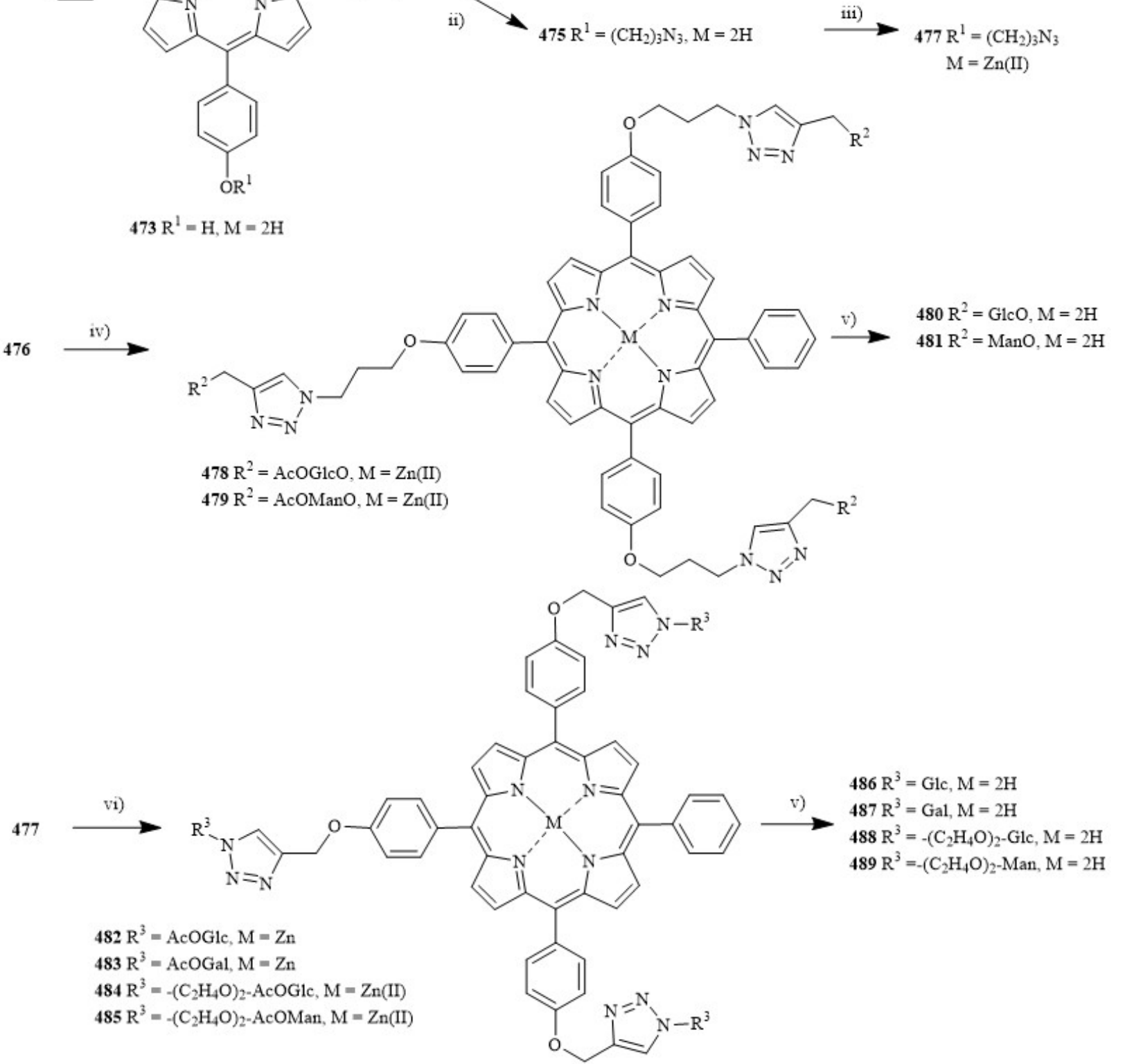

Scheme 21. Synthesis of tri-subsubstituted glycoporphyrins via Huisgen 'click' chemistry. i) 1,3-Dibromopropane, $\mathrm{K}_{2} \mathrm{CO}_{3}$, dry $\mathrm{DMF}$, r.t., $60 \mathrm{~h}, 77 \%$, then $\mathrm{NaN}_{3}$, dry DMF, r.t., 16 h, 59 \%; ii) propargyl bromide, $\mathrm{K}_{2} \mathrm{CO}_{3}$, dry DMF, r.t., 18 h, 98 \%; iii) ZnOAc, $\mathrm{MeOH}$, reflux, 1 h; iv) CuCl, propargyl glycosyl derivatives, toluene, microwave irradiation $\left(100 \mathrm{~W}, 140{ }^{\circ} \mathrm{C}, 20 \mathrm{~min}\right.$.); v) TFA (demetalation) and then $\mathrm{MeONa} / \mathrm{MeOH}, \mathrm{CH}_{2} \mathrm{Cl}_{2}, 1 \mathrm{~h}$; (vi) $\mathrm{CuSO}_{4}$, sodium $L$ ascorbate, glycosyl derivatives, $\mathrm{THF} / \mathrm{t}-\mathrm{BuOH} / \mathrm{H}_{2} \mathrm{O}$, microwave irradiation $\left(80 \mathrm{~W}, 85^{\circ} \mathrm{C}, 3 \mathrm{~min}\right.$.). 


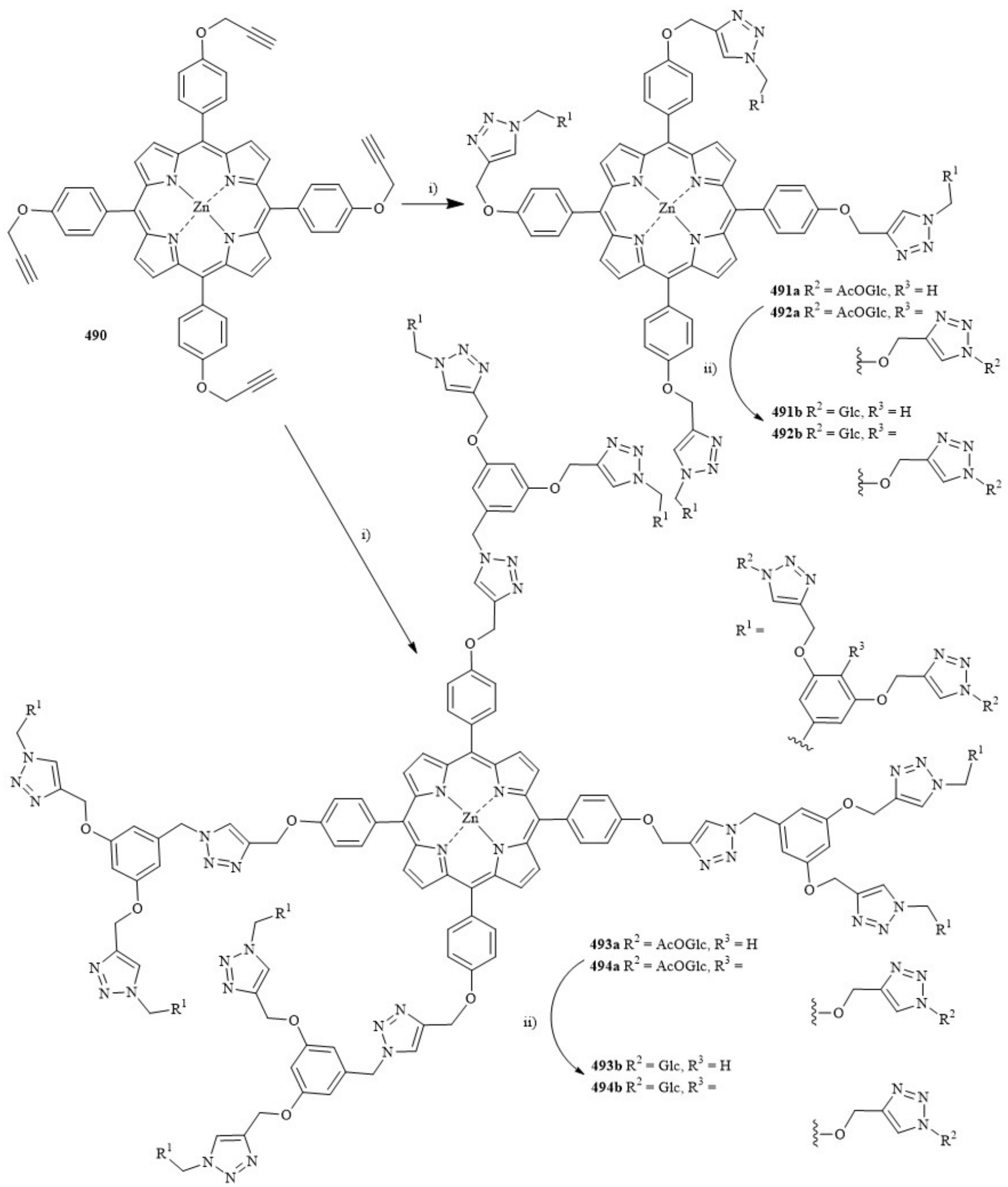

Scheme 22. Synthesis of tetra- and octa-subsubstituted glycoporphyrins via Huisgen 'click' chemistry. i) $\mathrm{CuSO}_{4}$, sodium $L$-ascorbate, glycosyl dendron, $\mathrm{THF} / \mathrm{H}_{2} \mathrm{O}, 18$ h r.t.; ii) $\mathrm{MeONa} / \mathrm{MeOH}, \mathrm{CH}_{2} \mathrm{Cl}_{2}$, overnight. 
precursors using $\mathrm{CuSO}_{4}$ and sodium $L$-ascorbate to form the dendritic glycoporphyrins (491a-494a) in 40-60\% yield followed by deprotection to afford the water soluble derivatives $491 b-494 b^{[253]}$.

\subsubsection{Dendritic Systems}

The first glycoporphyrin with branched carbohydrate units was reported by Stoddart and coworkers using amide bond forming reactions ${ }^{[254]}$. Highly water soluble, tetrasubstituted porphyrins with peracetylated and perbenzylated $\beta$-D-glucopyranosyl modalities were synthesized in $16 \%$ and $39 \%$ yield and then deprotected under Zemplén conditions to 495 and $\mathbf{4 9 6}$.

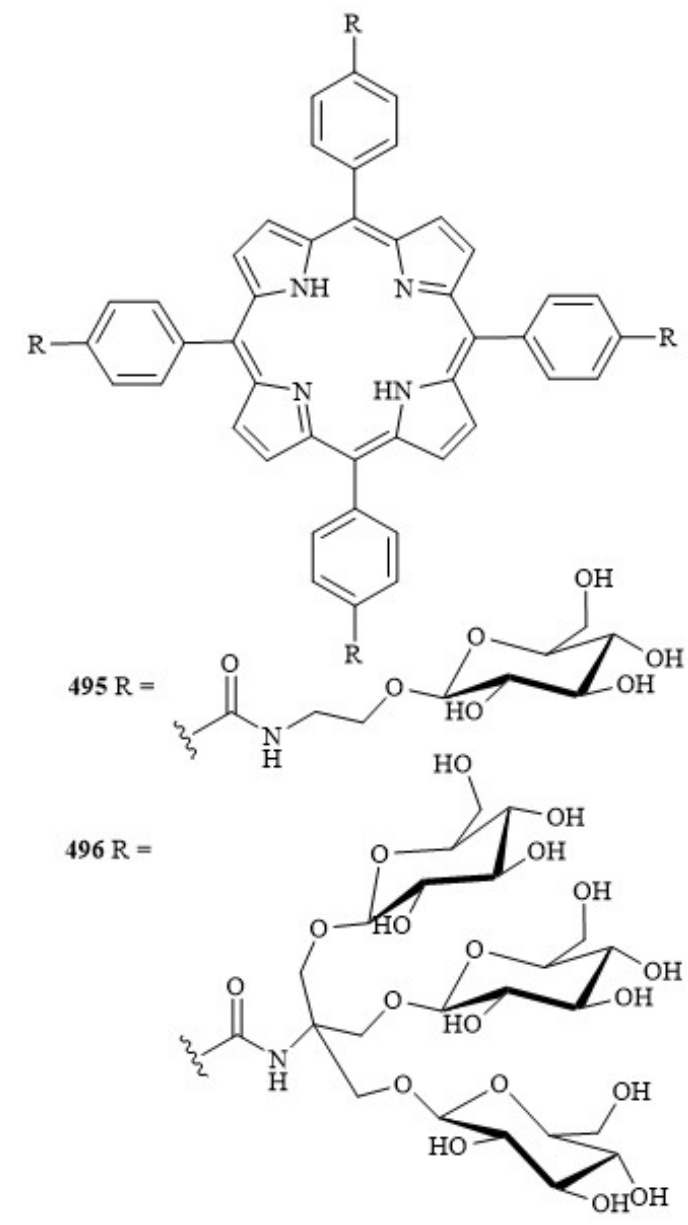

Formula 7.

In order to gain an entry into amphiphilic dendritic porphyrins Maillard prepared two amphiphilic glycodendrimeric TPPs bearing only one glycodendrimer moiety with variable linkers ${ }^{[204]}$. The glycodendrimeric derivatives 266 and $\mathbf{2 6 7}$ were synthesized via amide coupling of preformed glycodendrimers with 5-( $p$-benzoic acid)-10,15,20-triphenylporphyrin in good yields of $86 \%$ and $57 \%$, respectively. De-acetylation afforded amphiphilic glycodendrimeric porphyrins 268 and $269^{[204]}$.

Maillard's group also synthesized two families of glycoporphyrins, the first with sterically hindered sugar moieties with reduced mobility (271) and the second (499b511b) with flexible linkers reducing constraints observed in $271{ }^{[205]}$. Compound 271 with reduced mobility was synthesized by coupling 5-(4-benzoic acid)-10,15,20triphenylporphyrin with the aminoglucodendrimer using EDC-HOBt in $49 \%$ yield. Compound 270 subsequently underwent deacetylation in quantitative yield. The second family (499b-511b, Scheme 23) were prepared by condensing porphyrin $\mathbf{4 9 7}$ with several aminoglycodendrimers using EDC-HOBt as a coupling agent in $\sim 40 \%$ yield followed by quantitative deacetylation. The non-glycosylated dendron was also coupled with 497 affording 512a,b for comparative biological studies. Taking into account their previous work on glycoporphyrins $[160,162,198,204,205,255]$ they concluded that the best compounds appeared to have three $\alpha$-glycosyl groups on the paraposition of the meso-phenyl ring via a flexible linker. However, the presence of three sugars via a glycodendrimer decreased the phototoxicity ${ }^{[256]}$.

\subsubsection{Chlorins}

Chlorins and bacteriochlorins having strong absorption in the red region of the visible spectrum are ideal candidates for deep tissue penetration with light (Figure 5) ${ }^{[257]}$.
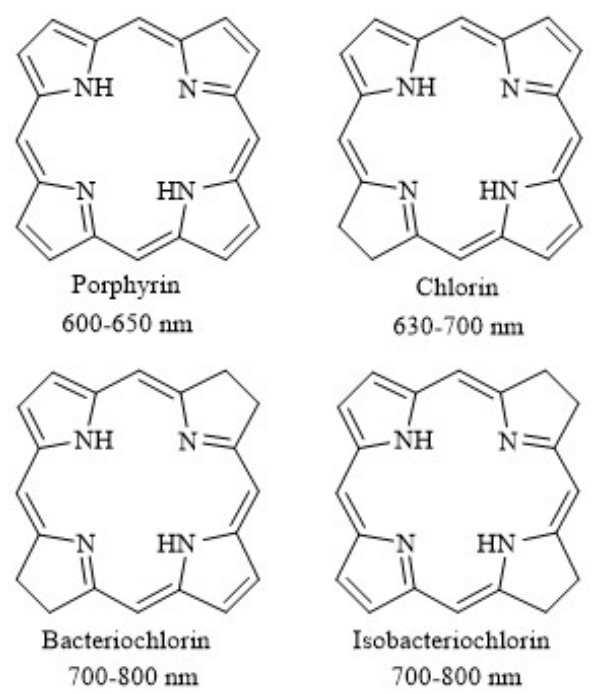

Figure 5.

\subsubsection{Reduction}

The simplest method for the preparation of chlorins is reduction of a parent porphyrin. However, yields can be low 


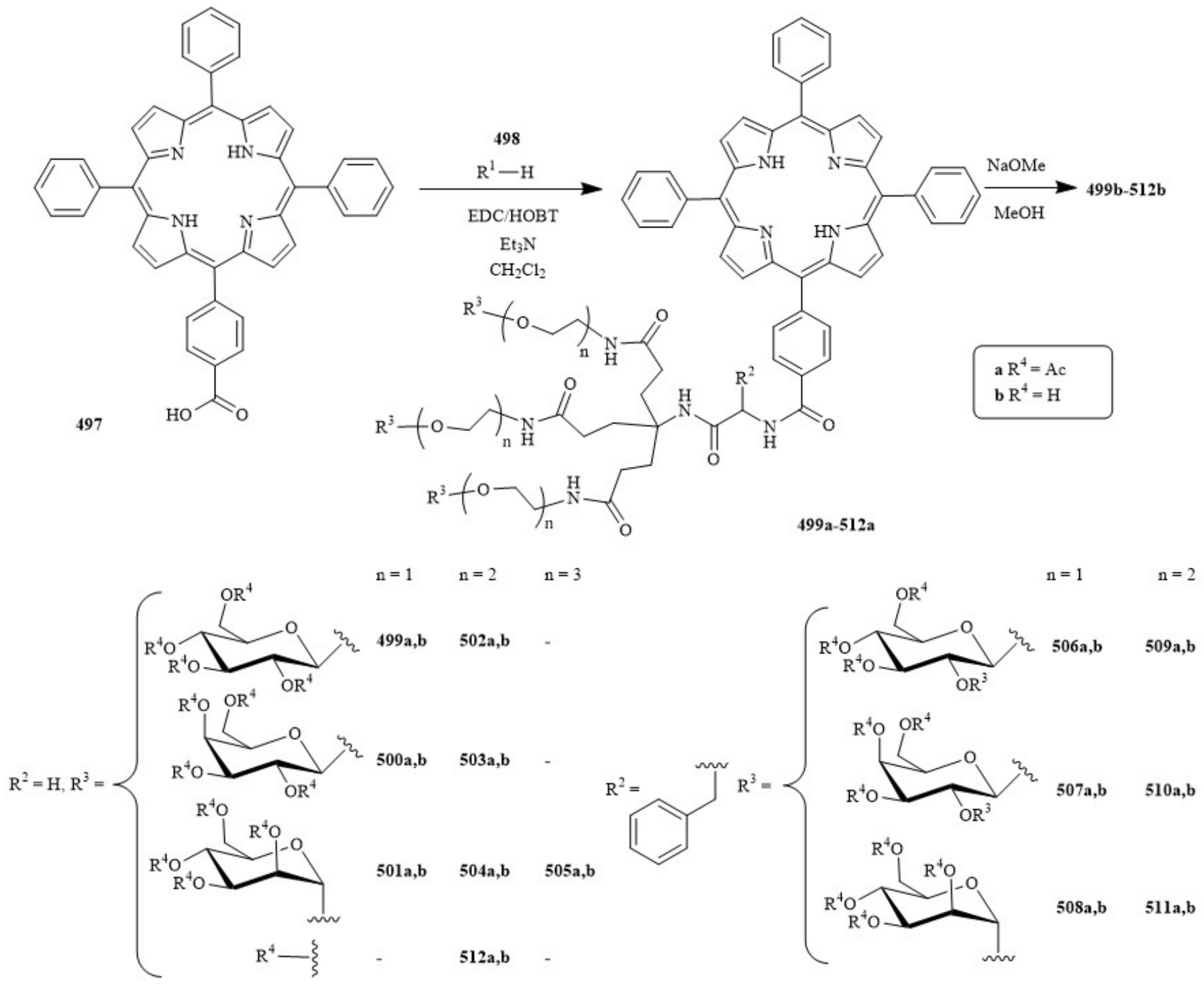

Scheme 23. Synthesis of glycodendrimeric porphyrins. $\mathrm{R}^{1}=$ the corresponding aminoglycodendron.

and for unsymmetrical porphyrins different regioisomers will be formed. For example, Mikata et al. synthesized chlorin derivatives via diimide reduction from their previously synthesized acetylated-glycosyl porphyrins 29-32 and 53 $[167,258]$. The tetra- and octaglycosyl-tetraphenylchlorins 513a-c were obtained in 57-73\% yield and deprotected to the water soluble derivatives 514a-c. A similar synthesis of the $N$-acetylglucosamine derivative ${ }^{[258,259]}$ could not be isolated due to solubility problems with the product. Similar $m$ THPC glycoconjugates were prepared as illustrated in Scheme 24. However, reduction of the trisubstituted porphyrin yielded two inseparable isomers 2,3- and 7,8meta-triglucosylated chlorins $(\mathbf{5 1 6}, \mathbf{5 1 7})$ while the metasubstituted tetraglucosylated chlorin $\mathbf{5 1 4 f}$ was formed as a mixture of four atropisomers at r.t. ${ }^{[260]}$. By now, many other glycochlorins have been prepared using the Whitlock method, including a series of $5,10,15,20$-tetrakis[3- or 4-( $\beta$ $D$-glycopyranosyloxy)phenyl]chlorins with glucopyranosyl, galactopyranosyl, xylopyranosyl and arabinopyranosyl groups $(\mathbf{5 1 4 a}, \mathbf{b}, \mathbf{d}-\mathbf{i}){ }^{[261,262]}$. Note, that reduction can afford a mixture of chlorins and bacteriochlorins which was the case in this study. Purification of the glycochlorins required reverse phase-TLC to give the glycochlorin derivatives in $14-49 \%$ yield. UV-vis spectroscopy of the crude mixture showed for the meta-substituted glycoporphyrins the yield of the bacteriochlorins to be considerably higher than the chlorins unlike the para-substituted derivatives ${ }^{[261]}$. 


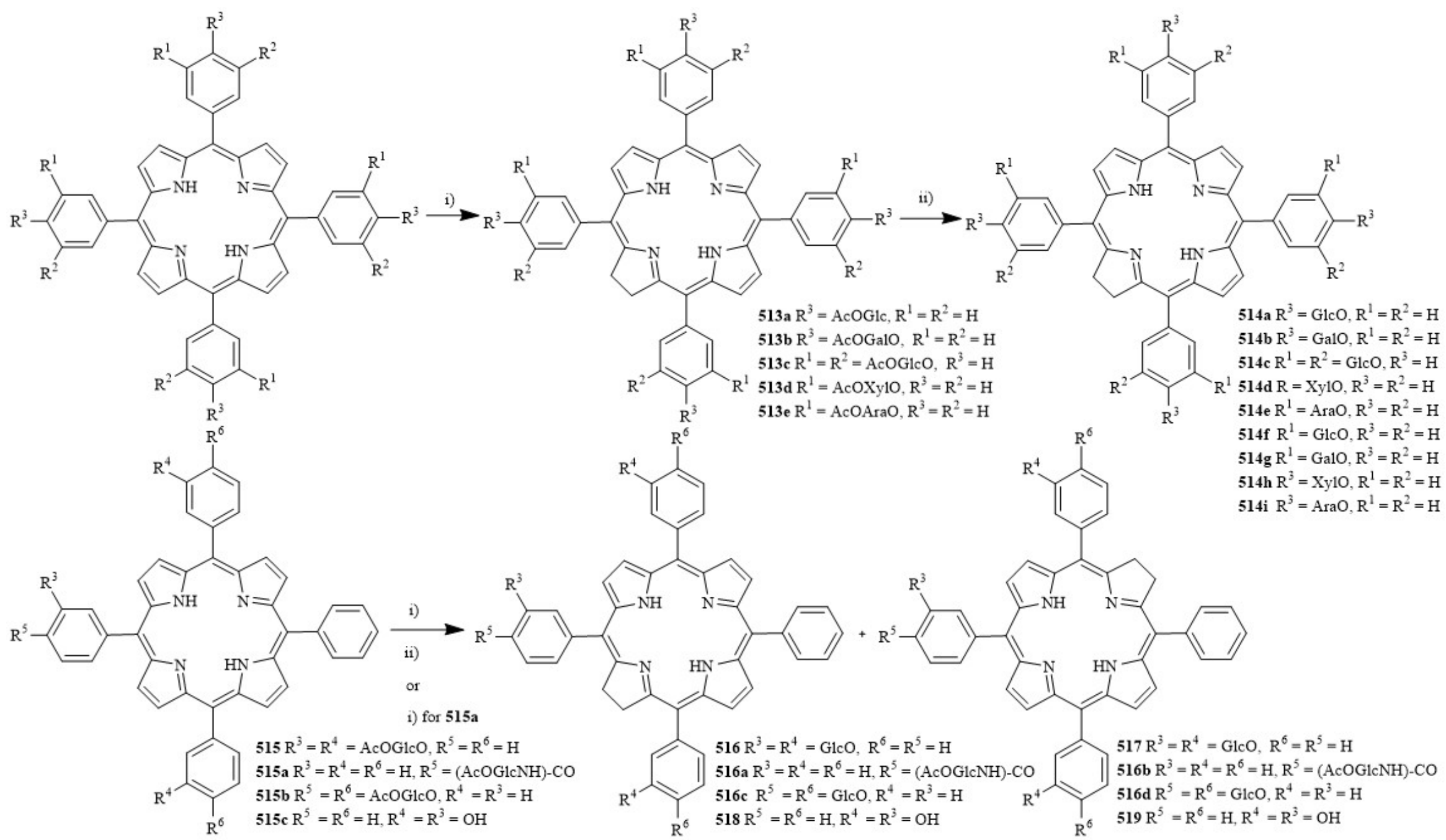

Scheme 24. Synthesis of carbohydrate derivatives of $m$ THPC. i) (a) toluene- 4 sulfonohydrazide, anhydrous $\mathrm{K}_{2} \mathrm{CO}_{3}$, dry pyridine, $100{ }^{\circ} \mathrm{C}-110{ }^{\circ} \mathrm{C}$, (b) $o$-chloranil, ethyl acetate or benzene, $20{ }^{\circ} \mathrm{C}$; ii) $\mathrm{CHCl}_{3} / \mathrm{MeONa} / \mathrm{MeOH}, 20^{\circ} \mathrm{C}$.

\subsubsection{Dihydroxylation}

More synthetically useful is the 2,3-dihydroxylation of porphyrins and follow-up chemistry [263-265]. Some of the earlier examples of hydroxychlorins derived from octaethylporphyrin were substituted with glycerol, $D$-glucose and $D$-mannitol via ether linkages as outlined in Scheme $25^{[39,266]}$. The 2,3-dihydroxychlorin $\mathbf{5 2 0}$ undergoes a pinacol 
rearrangement yielding the oxochlorin $\mathbf{5 2 1}$ in $75 \%$ yield. This is an advantageous reaction as the geminal dimethyl unit stabilizes the system and prevents reoxidation to the porphyrin. Reduction in an excess of sodium borohydride in ethanol yielded the hydroxychlorin $\mathbf{5 2 2}$ in $87 \%$ yield with conversion to the bromide derivative which was reacted immediately with the desired alcohol. Glycerol 524 and $D$ mannitol 526 derivatives yielded in both cases two diastereoisomers while the $D$-glucose derivative $\mathbf{5 2 5}$ yielded four diastereoisomers due to the $\mathrm{C}-2$ on the chlorin and the anomeric carbon of the sugar ${ }^{[39]}$.

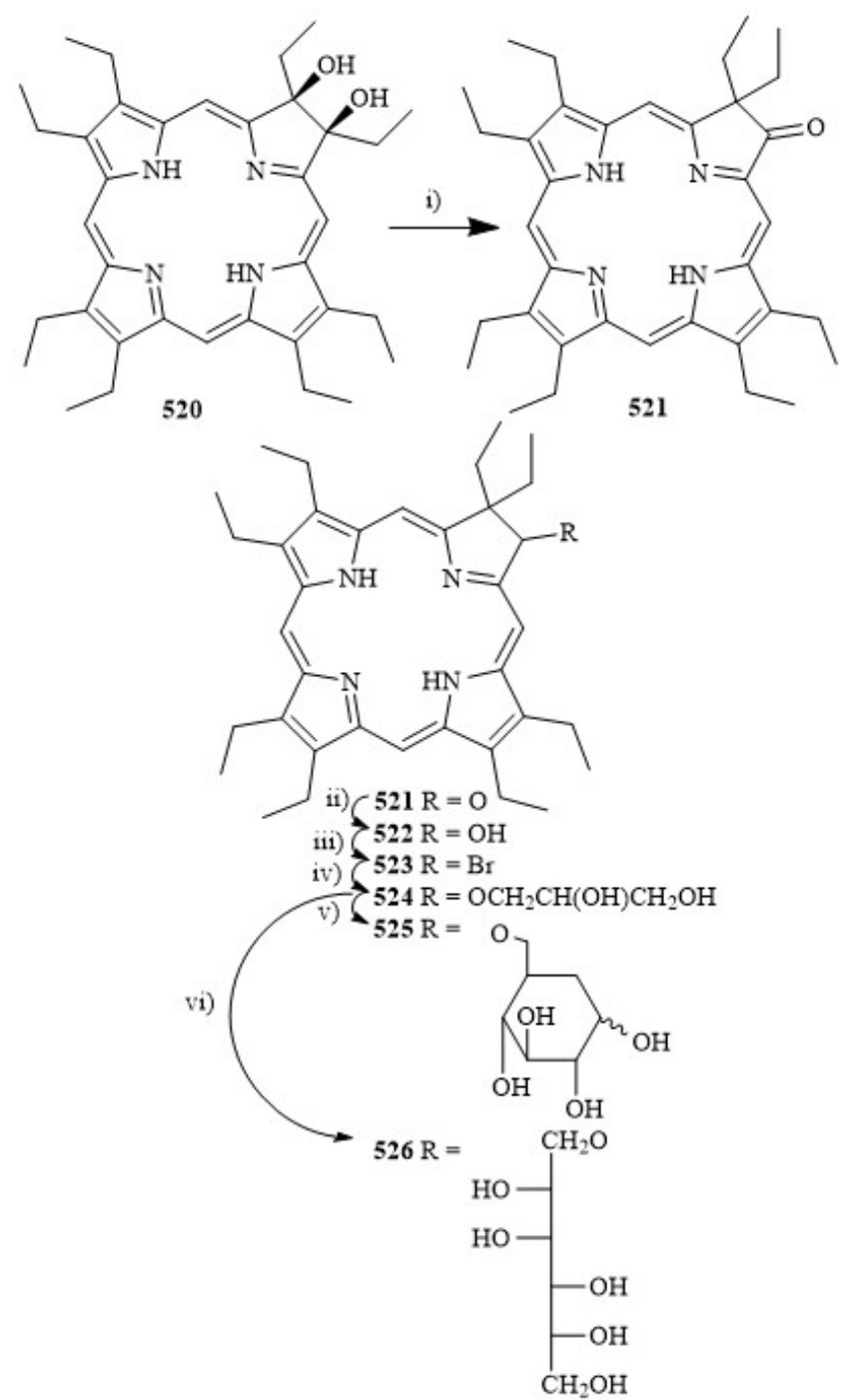

Scheme 25. Synthesis of hydroxychlorins derived from $2,3,7,8,12,13,17,18$ octaethylporphyrin substituted with glycerol, $D$-glucose and $D$-mannitol via ether linkages. i) Fuming $\mathrm{H}_{2} \mathrm{SO}_{4}$; ii) sodium borohydride, $1 \mathrm{~h}, \mathrm{EtOH} / \mathrm{H}_{2} \mathrm{O}$; iii) $50 \% \mathrm{HBr}-\mathrm{HOAc}$ for $1 \mathrm{~h}$ at $20{ }^{\circ} \mathrm{C}$; iv) glycerol; v) $D$-glucose; vi) $D$ mannitol.
The method can also be applied to meso-substituted porphyrins as was exemplified by functionalization of 5,10,15,20-tetra( $p$-aminophenyl)porphyrin $\quad 82^{[206]}$. The glucosyl TPP derivative $\mathbf{2 7 3}$ was synthesized from the monosubstituted protected carboxylic acid functionality (272) and subsequent addition of the $\beta$ - $D$-glucopyranuronoyl chloride and additional base afforded the desired compound in $33 \%$ yield. Conversion to the corresponding chlorins and bacteriochlorins was determined by the equivalents of osmium tetroxide-mediated dihydroxylation. Reacting porphyrin 273 with two equivalents of $\mathrm{OsO}_{4}$ afforded four osmate ester isomers, two sets of regioisomers each of which consisted of two stereoisomers. The regioisomers were separated and subsequently treated with hydrogen sulfide affording vic-dihydroxychlorins $\mathbf{5 2 7 a / a}$ and $\mathbf{5 2 7 b} / \mathbf{b}$ ' (Scheme 26). Following with hydrolysis of the sugar acetyl groups and glutaryl methyl ester of $\mathbf{5 2 7} \mathbf{a} / \mathbf{a}^{\prime}$ and $\mathbf{5 2 7 b} / \mathbf{b}$ ' afforded the hydrophilic chlorins $\mathbf{5 2 8 a} / \mathbf{a}$ ' and $\mathbf{5 2 8 b} / \mathbf{b}$ '. The related 2,3,12,13-(vic-dihydroxy)bacteriochlorins were synthesized with a large excess of $\mathrm{OsO}_{4}$ from porphyrin 273 affording four isomers which were separated into two components. The two fractions consisted of two isomers which were treated with hydrogen sulphide providing bis(vic-dihydroxy)bacteriochlorins $\mathbf{5 2 9}$ which were subsequently hydrolyzed to the deprotected bacteriochlorins 530. The carboxylic acid functionality allows for conjugation to biomolecules and nanoscaffolds which was displayed by the conjugation of the chlorins to a dextran-coated nanoscaffold. It displayed good suspension stability and did not show differences in excited state quenching at any loading compared to chlorin $\mathrm{e}_{6}{ }^{[206]}$.

\subsubsection{1,3-Dipolar Cycloadditions}

More significant structural variations to the porphyrin core can be introduced with 1,3-dipolar cycloaddition reactions at the $\beta$-pyrrole positions ${ }^{[267,268]}$.

In this context a typical example are pyrrolidinoporphyrin glycoconjugates with $\mathrm{N}$ - or $\mathrm{C}$-linked galactose derivatives prepared using the 1,3-dipolar cycloaddition strategy with sugar azomethine ylides by Cavaleiro and coworkers ${ }^{[269]}$. A typical example is the synthesis of glycosylated isoxazolidine-fused chlorins and bacteriochlorins via 1,3dipolar cycloaddition reactions of $\mathrm{TPPF}_{20}$ and glycosyl nitrones ${ }^{[270]}$. A mixture of porphyrin $\mathrm{TPPF}_{20}$ and an excess of the galactosyl nitrone were reacted at $60{ }^{\circ} \mathrm{C}$ in a concentrated solution in toluene for 5 days and afforded a mixture of three compounds. The major product, chlorin 531a in $66 \%$, displayed endo addition. Two other products were the result of bis addition to the porphyrin affording the bacteriochlorins $\mathbf{5 3 2 a} / \mathbf{b}$ or $\mathbf{5 3 3 a} / \mathbf{b}$ in $21 \%$ and $9 \%$ yields, respectively. The relative yield was dependent on the the reaction time ${ }^{[270]}$. The cycloaddition of other sugar 


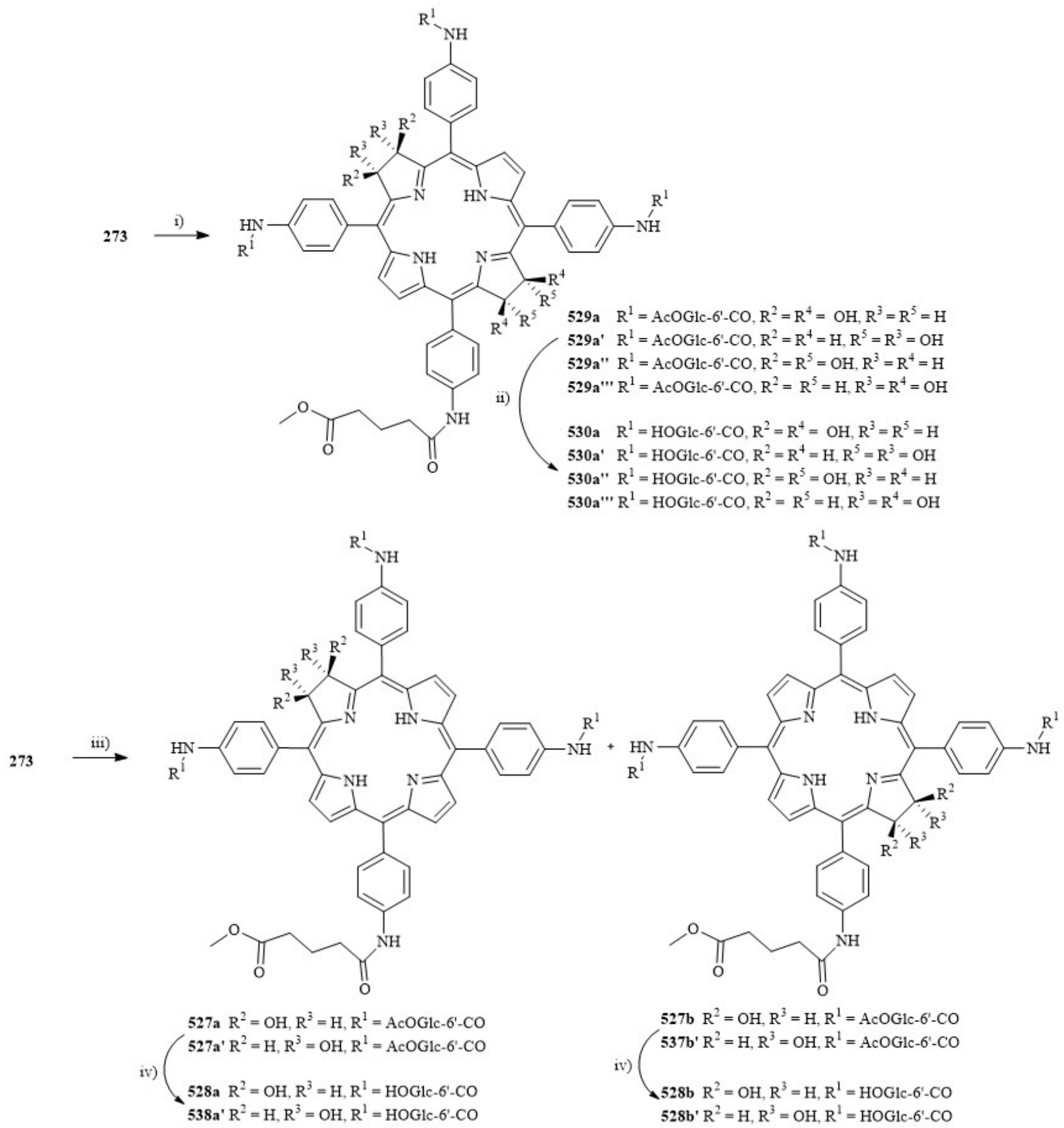

Scheme 26. Synthesis of vic-dihydroxychlorins and -bacteriochlorins. i) excess $\mathrm{OsO}_{4}$; ii) $0.3 \mathrm{M} \mathrm{LiOH}, 2$ h; iii) (a) $\mathrm{OsO}_{4}$, pyridine; b) $\mathrm{H}_{2} \mathrm{~S}$; iv) $0.3 \mathrm{M} \mathrm{LiOH}, 2$ h.

residues yielded the chlorin $\mathbf{5 3 1}$ and in some cases the bacteriochlorins. Reacting ribosyl nitrone at $60{ }^{\circ} \mathrm{C}$ for 5 days provided only the chlorin derivative $\mathbf{5 3 1 b}$ in $30 \%$ yield. The xylose derivative reacting at $100{ }^{\circ} \mathrm{C}$ for $10 \mathrm{~h}$ yielded the chlorin 531c in $37 \%$ and only traces of the bacteriochlorins.
The lyxosyl nitrone gave a single bacteriochlorin (532) in 61 $\%$ yield and the chlorin 531d in $29 \%$. All glycosylated chlorins and bacteriochlorins isopropylidene acetals could be deprotected in quantitative yields. 


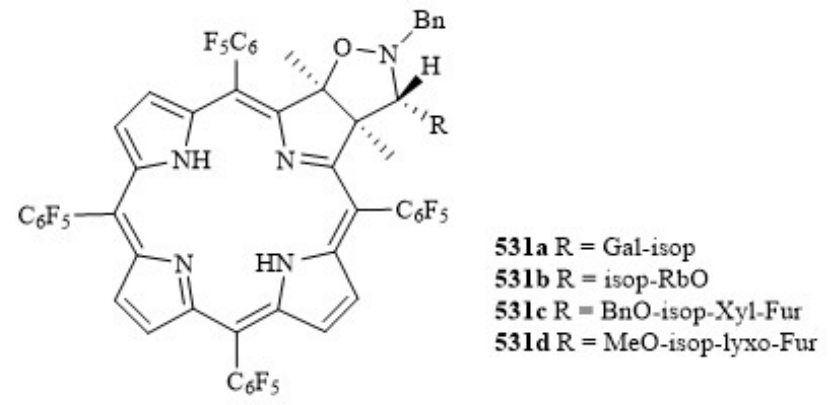

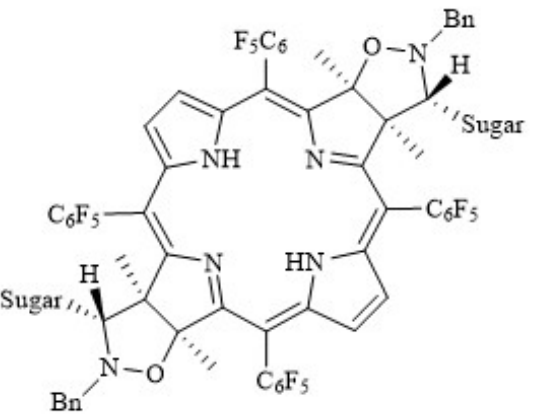

$532 a$

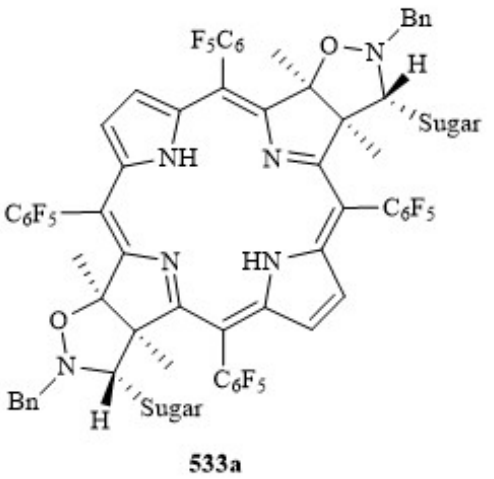

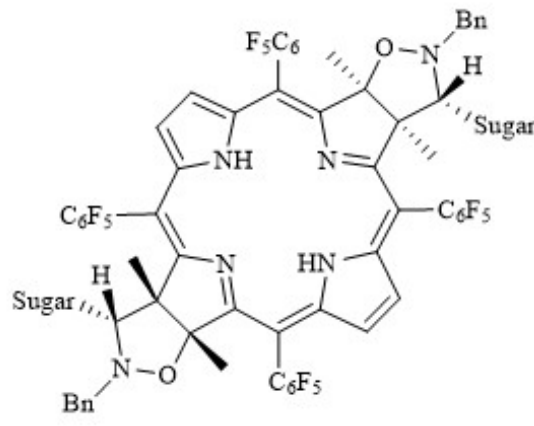

$532 \mathrm{~b}$

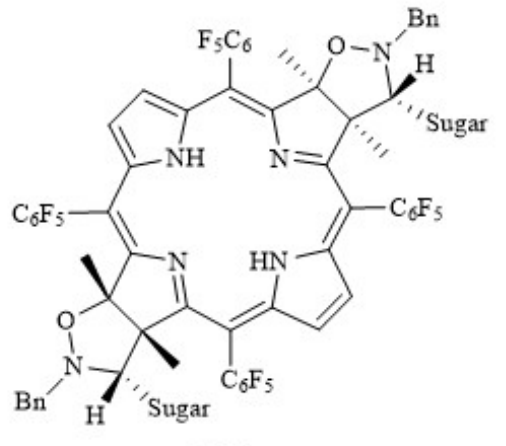

533b

Formula 8 .

Subsequently, this work was extended to azomethine ylides and diazoacetates ${ }^{[271]}$. A new method for the synthesis of the glyco-conjugates (536-539) was developed using $\mathrm{CuCl}$ to catalyze the cyclopropanation reactions of $\{5,10,15,20$-tetra(pentafluorophenyl)porphyrinato $\}$ zinc(II)

(534) with $\alpha$-diazoacetates (535a-d) of glucofuranose, fructopyranose, galactopyranose and xylofuranose (Scheme 27). It was found that the distribution of products (536-539) was dependent upon the carbohydrate moiety with the transchlorin 536 being the major product in all cases. The highest selectivity was obtained with 535a. The new chlorins 536a-d and the isobacteriochlorin $\mathbf{5 3 8 b}$ were characterized while the other structures present in trace amounts were only identified by UV-vis spectra. Removal of the protecting groups and metal complexes of 536a-d was carried out in aq. TFA affording 540a-d. Singlet oxygen production studies showed the chlorins 540a-d to be better than the well-known singlet oxygen producer methylene blue with compound 540b providing the best production. No significant difference in Log P was observed, but chlorins 540a and 540c were only slightly more hydrophilic than $\mathbf{5 4 0 b}, \mathbf{d}^{[271]}$.

Similar methods were used by Hirohara et al. to study a range of $O$-glycosylated tetraarylporphyrins and -chlorins, which showed several advantages including reduced dark cytotoxicity, improved water-solubility, better cellular uptake, and sugar-dependent photocytotoxicity $[165,261,262,272,273]$. The $O$-glycosylated chlorins had promising photocytotoxicity in HeLa cells but were difficult to purify. In expansion of these results, research focused on the photochemical evaluation of $S$-glycosylated chlorins. Eight 


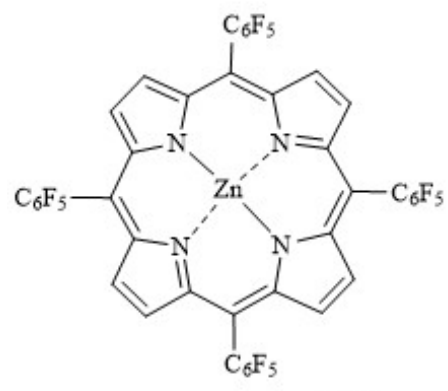

534<smiles>[R]C(=O)C=N</smiles>

537

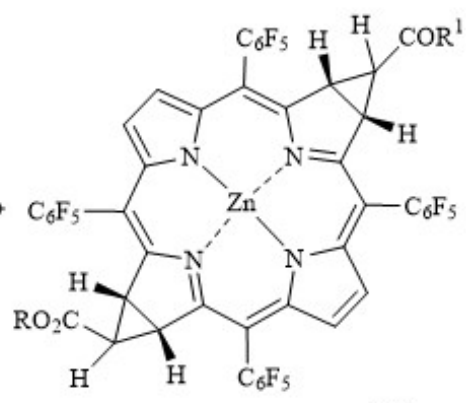

539

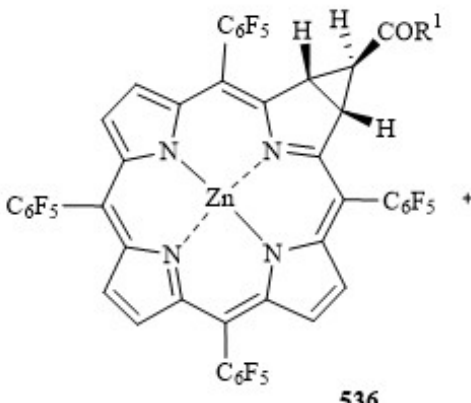

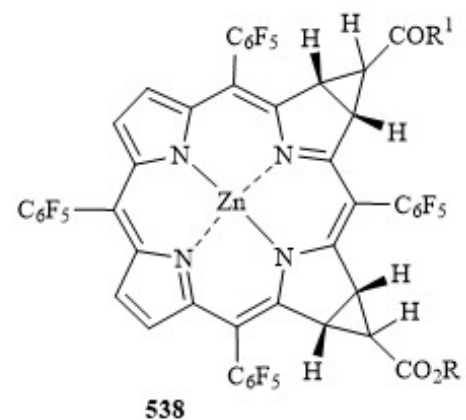

ii)

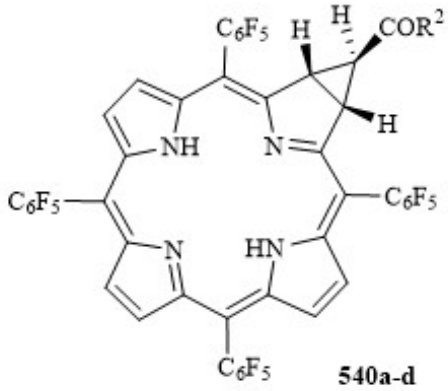

a $\mathrm{R}^{2}=\alpha / \beta$-Glc-Fur

b $\mathbf{R}^{2}=\alpha / \beta$-HO-Xyl-Fur

$c \mathrm{R}^{2}=$ HO-Fruct

$\mathbf{d R}^{2}=\alpha / \beta-\mathrm{Gal}-\mathrm{OH}$

Scheme 27. Synthesis of deprotected glyco-chlorins and bacteriochlorins. i) $\mathrm{CuCl}(2 \mathrm{~mol} \%), \mathrm{CH}_{2} \mathrm{Cl}_{2}, 40{ }^{\circ} \mathrm{C}$; ii) $\mathrm{TFA} / \mathrm{H}_{2} \mathrm{O}(9: 1)$, r.t., $30 \mathrm{~min}$.

$S$-glycosylated $\mathrm{TPPF}_{20}$ 's $(\mathbf{8 7}, \mathbf{9 1}, \mathbf{9 5}, \mathbf{9 6})$ and their 1,3-dipolar cycloadducts, i.e. chlorins 542a', 542b', 542a and 542b were synthesized by nucleophilic substitution of the pentafluorophenyl groups $\mathbf{( 8 3 )}$ with $S$-glycosides. $S$ glycosylated porphyrins 87 and 96 and chlorins 542a' and $\mathbf{5 4 2 b}$ ' were isolated in excellent yields of $74 \%, 86 \%, 94 \%$ and $82 \%$, respectively. Subsequent removal of the acetyl groups via alkaline hydrolysis afforded the free-hydroxyl derivatives $(\mathbf{9 1}, \mathbf{9 5}, \mathbf{5 4 2 a}$ and $\mathbf{5 4 2 b})$. The fused chlorins were found to be significantly more stable than those prepared via diimide reduction. The photophysical properties showed the $S$-glycosylated chlorins to have molar extinction coefficients of the Q band twice that of the corresponding unconjugated derivative. These compounds were also investigated for their in vitro photocytotoxicity in HeLa cells ${ }^{[178]}$.

In a similar way, Drain and coworkers extended from previous work on the synthesis of thioglycosylated porphyrins (95 and 91) ${ }^{[247]}$ to form their 1,3-dipolar cycloadducts, i.e. chlorins (542a, a'), isobacteriochlorins (545a,a') and bacteriochlorins (546a,a') via the same procedure ${ }^{[274]}$. The photophysical properties showed that the lowest energy Q band of 542a is 25 times greater than that of the corresponding porphyrin 91 while that of the isobacteriochlorin 545a is only 5 times greater than 91. All these compounds were evaluated against MDA-MB-231 and K:Molv NIB 3 T3 mouse fibroblast cells ${ }^{[274]}$. 


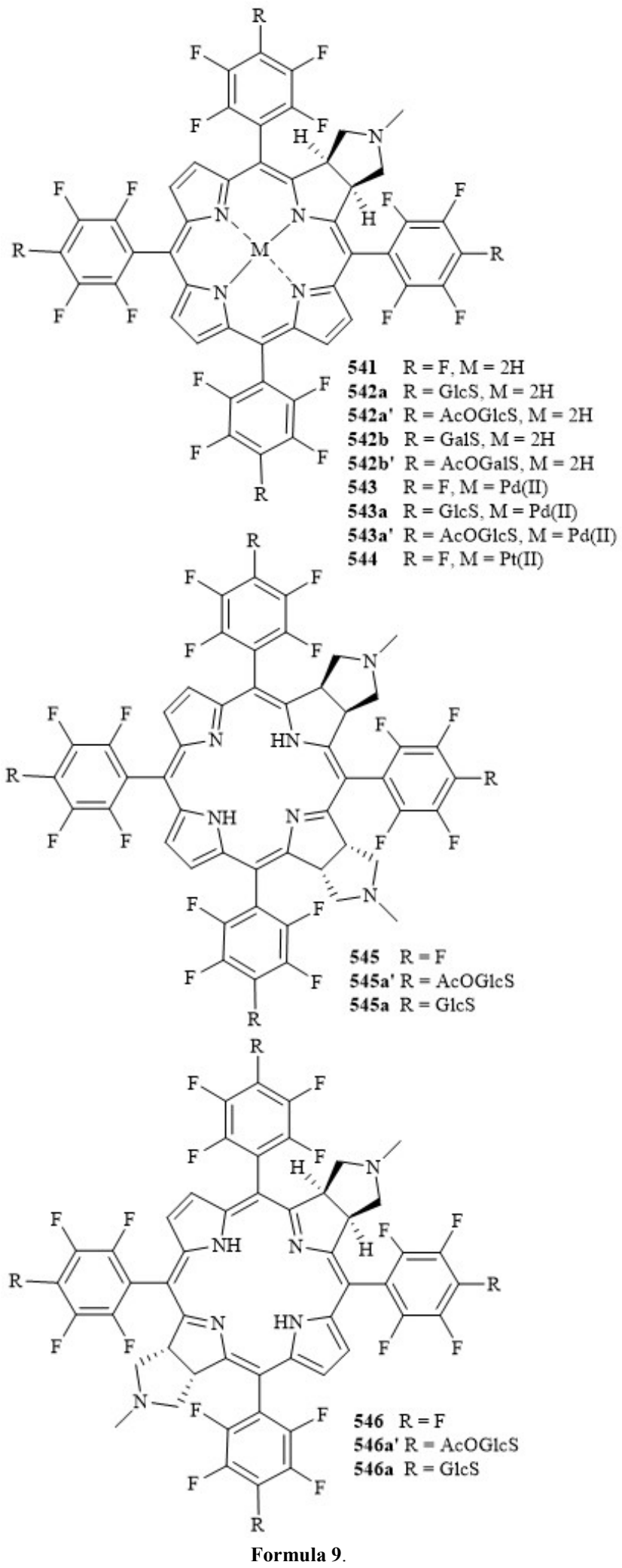

\subsubsection{Benzochlorins}

A more 'classic' approach is the preparation of benzochlorins ${ }^{[275,276]}$. Maillard et al. regioselectively synthesized an amphiphilic glycoconjugated meso-monoarylbenzochlorin from the meso-monoaryl- nickel(II) porphyrin [277]. This involved a somewhat regiospecific Vilsmeier reaction of $\mathbf{5 4 7}$ to provide two isomeric nickel(II) porphyrins 548 and 549 (85:14) in $85 \%$ yield. The former was subjected to inert acid conditions and underwent cyclization yielding the nickel(II) benzochlorin $\mathbf{5 5 0}$ in $\mathbf{5 8 \%}$. Followed by dealkylation of the methoxy group, demetallation and subsequent glycosylation of the free hydroxyl gave the glycosylated benzochlorin 552a. The amphiphilic benzochlorin 552b was obtained after quantitative deacetylation under Zemplén conditions (Scheme 28).

Pandey et al. expanding on their previous work ${ }^{[278]}$ synthesized an extended library of 569a,b and $\mathbf{5 7 0}$ incorporating compounds which are known for their photoactivity. A benzochlorin derivative of octaethylporphyrin (OEP) condensed with a glucosamine, galactosamine, and lactosamine were investigated for their galectin-binding ability ${ }^{[279]}$. The carboxylic acid functionality on the fused exocyclic benzene ring of the benzochlorin 553a was substituted with acetylated galactosamine, glucosamine, and lactosamine affording the glycoconjugate derivatives 554a, 555a and 556a in 40 \%, 97 $\%$ and $43 \%$ yields, respectively. All of which were deprotected in quantitative yields and the free base derivatives were converted into the $\mathrm{Zn}$ (II) analogues $\mathbf{( 5 5 4 b}$, $\mathbf{5 5 5 b}, \mathbf{5 5 6 b}$ ) as they had been previously shown to have better PDT efficacy ${ }^{[280]}$. Studies have shown that the $C$ glycosyl derivatives are more stable than the $\mathrm{O}$ - or $\mathrm{N}$ glycosyl compounds ${ }^{[281]}$. To determine the difference in PDT efficacy a benzochlorin- $C$-galactose conjugate was prepared as well (557) ${ }^{[279]}$.

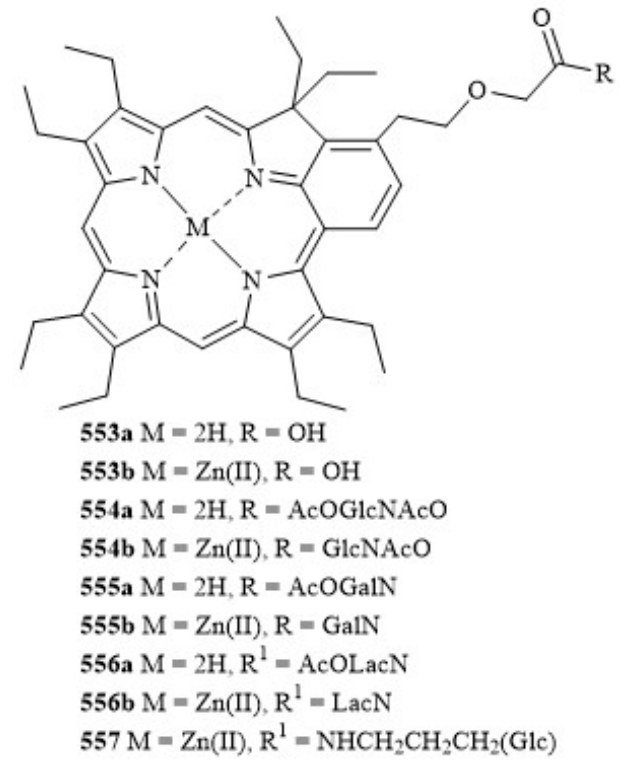

Formula 10. 


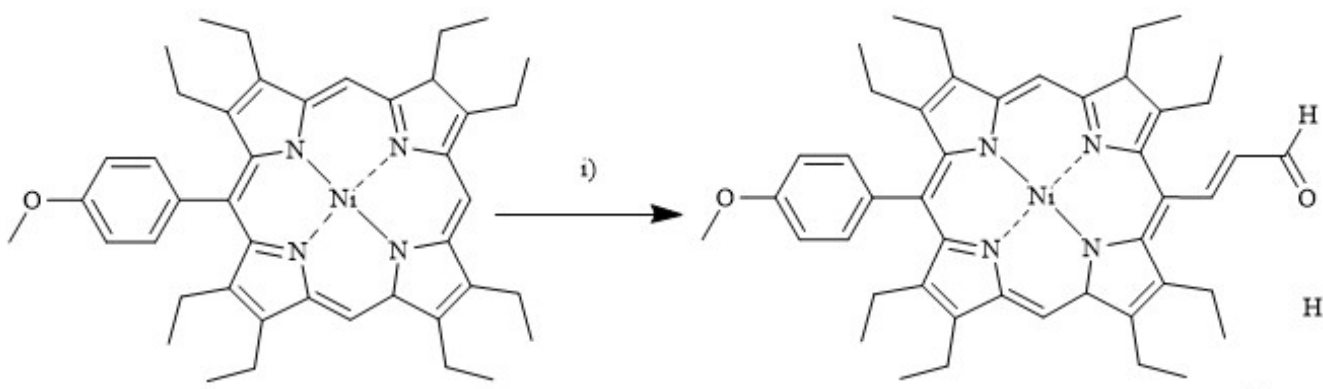

547

548

ii)
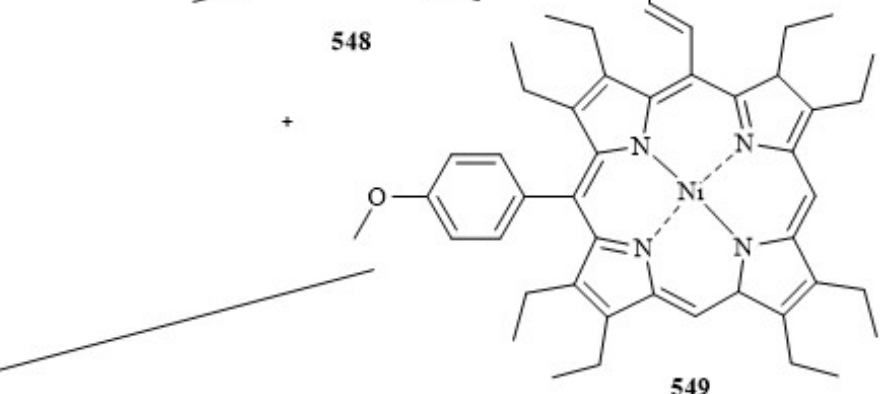

549

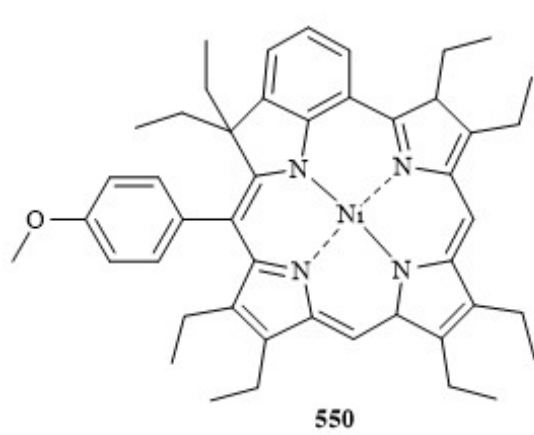

550

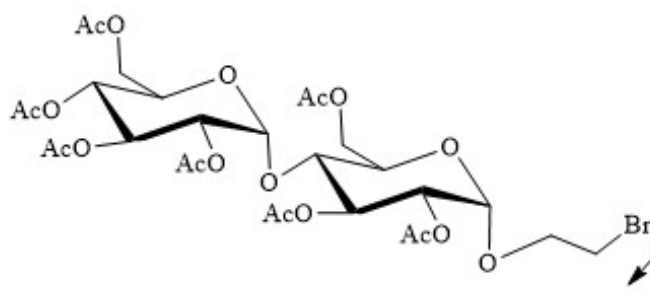

iii)

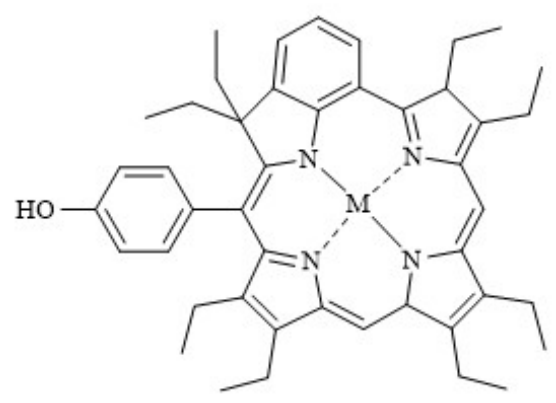

iv)

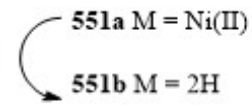

\section{v)}

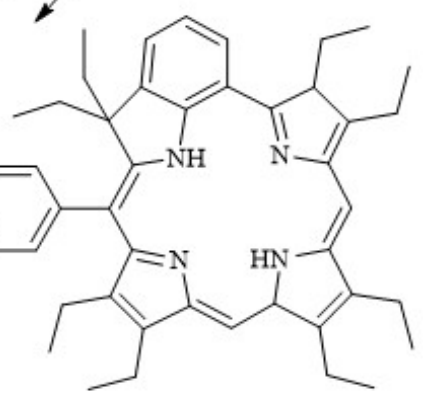

vi) $\left\{\begin{array}{l}552 a \mathrm{R}=\mathrm{Ac} \\ 552 \mathrm{~b} \mathrm{R}=\mathrm{H}\end{array}\right.$

Scheme 28. Synthesis of an amphiphilic glycosylated benzochlorin. i) 3-(Dimethylamino)acrolein/ $\mathrm{POCI}_{3} ;$ ii) $\mathrm{CF}_{3} \mathrm{CO}_{2} \mathrm{H} / \mathrm{Ar}$;ii) $\mathrm{BBr}_{3} / \mathrm{dry} \mathrm{CH}_{2} \mathrm{Cl}_{2}$; iv) $\mathrm{H}_{2} \mathrm{SO}_{4}$; v) $\mathrm{K}_{2} \mathrm{CO}_{3}$ in $\mathrm{DMF} / 60{ }^{\circ} \mathrm{C}$; vi) $\mathrm{MeONa} / \mathrm{MeOH}$. 


\subsubsection{Chlorophyll, Phytochlorin, and Rhodochlorin} Derivatives

Chlorophyll derivatives offer another intriguing avenue for new PS ${ }^{[150,282,283]}$. Their reduced character makes them promising candidates for PDT, although this is somewhat counteracted by their low stability. Mironov used his expertise in chlorophyll chemistry to prepare pheophorbide a 559 derivatives, where the sugar moiety was linked via the 3 -vinyl group ${ }^{[284]}$. Synthetically this required transformation of the vinyl group in $\mathbf{5 5 9}$ into the 2-bromoethyl derivatives with $\mathrm{HBr}$, which were reacted in situ with 1,2;3,4-di-Oisopropylidenegalactose to yield, for example, the glycolpheophorbide a 560. These compounds were insoluble in nonpolar solvents, displayed good solubility in alcohol and limited solubility in water ${ }^{[284]}$.

Naturally, the $\mathrm{C} 17$ side chain can be used for conjugation as well. Pyropheophorbide 2-deoxyglucosamide 563 was synthesized for applications in fluorescence imaging and for PDT. It was obtained through pyropheophorbide succinimidyl ester $\mathbf{5 6 2}$ by reacting the parent pyropheophorbide 561 with $N$-hydroxysuccinimide which subsequently underwent glycosylation with $D$-glucosamine affording the bioconjugate in an overall yield of $50 \%$ (Scheme 29). Synthesized as a glucose transporter targeting PS it can take advantage of the intracellular trapping mechanism displayed by some tumor cells for 2deoxyglucosamide ${ }^{[285]}$.

The carboxylic acid function also offers a convenient handle for the improvement of "established" PSs. In the phytochlorin series one such compound is HPPH (Photochlor) which displays strong absorbtions at $408 \mathrm{~nm}$ and $665 \mathrm{~nm}$, the latter of which is important for its PDT efficacy ${ }^{[286]}$. HPPH is under investigation in several clinical trials for esophageal cancer, non-small cell lung cancer, basal cell skin cancer, head and neck cancer. HPPH, like Photofrin $^{\circledR}$, is lipophilic with slow clearance from the body [286]. 3-Devinyl-3-(1'-hexyloxyethyl) pyropheophorbide-a (HPPH) was conjugated with mono-, di-, or tetra-saccharides to determine the effect the carbohydrate unit had on the PDT efficacy of the basic macrocycle ${ }^{[287]}$. To determine the effect $\beta$-galactose carbohydrates have on tumor-selectivity and its dependence on galectin 3 binding both galactose conjugates (galactose and lactose) and non-galactose conjugates (glucose and cellobiose) of HPPH were synthesized. The first series of HPPH derivatives were synthesized by coupling HPPH with 1-aminotetra-acetogalactose, 1aminotetra-acetoglucose, 1-aminohepta-acetolactose and 1aminoheptaacetocellobiose and deacetylated affording 558ad. The second series synthesized incorporated mono- and multivalent rigidified lactose units which were coupled to HPPH with an extended amine linker $(\mathbf{5 5 8 e})$ followed by

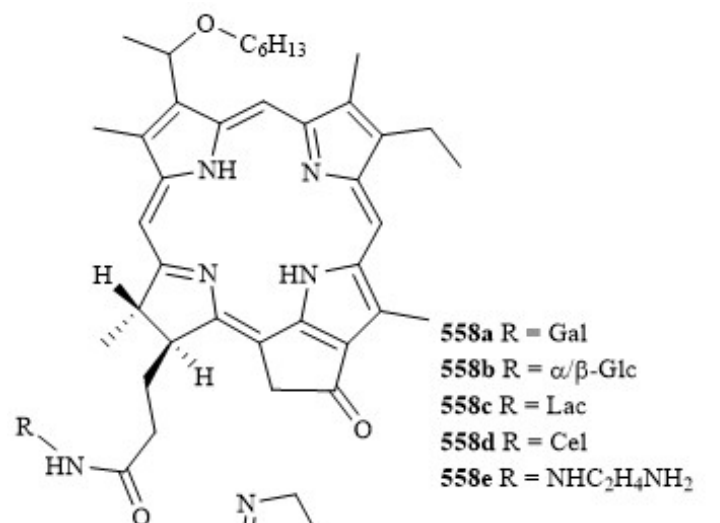

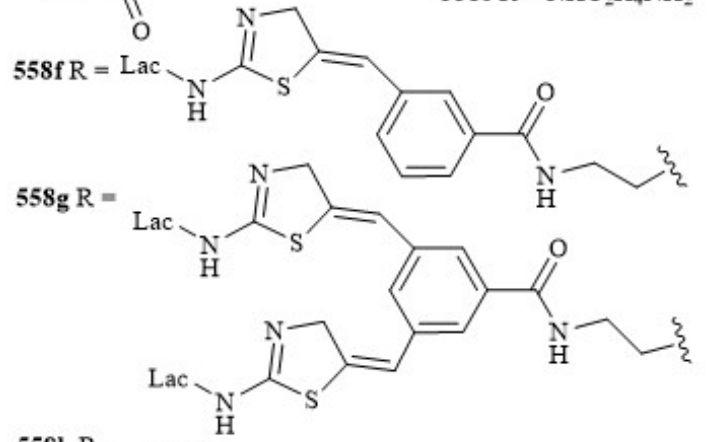

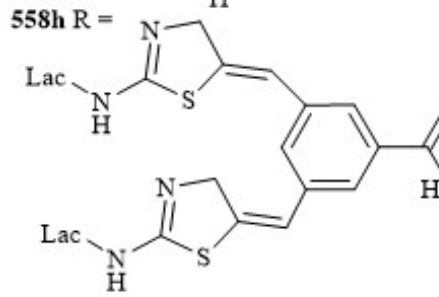

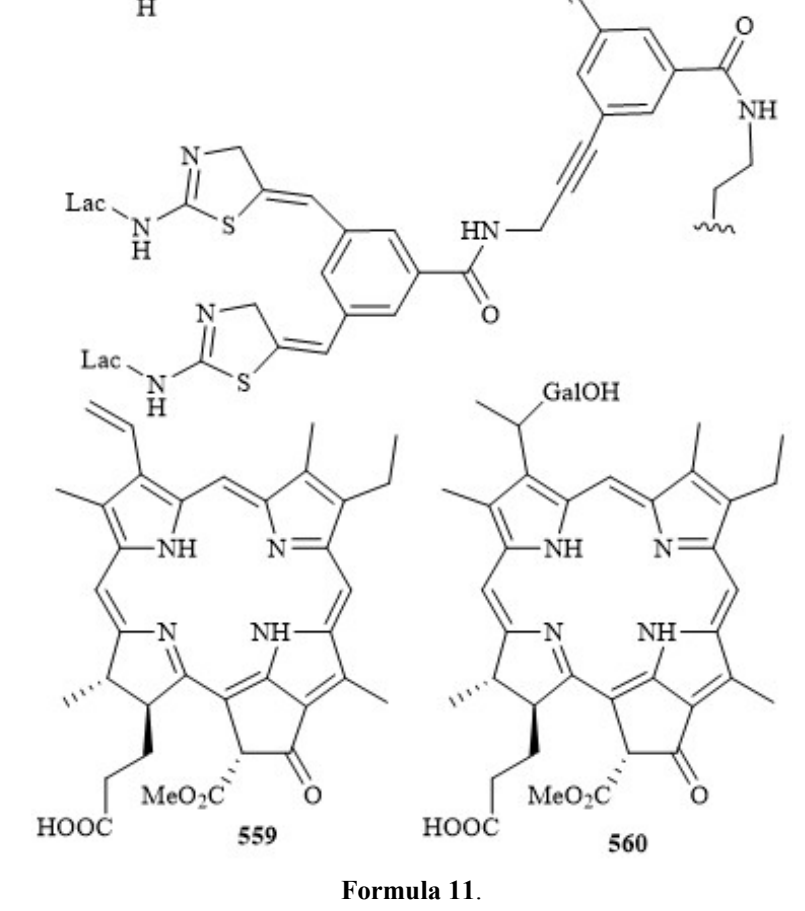

(C) 2014 Bentham Science Publishers 


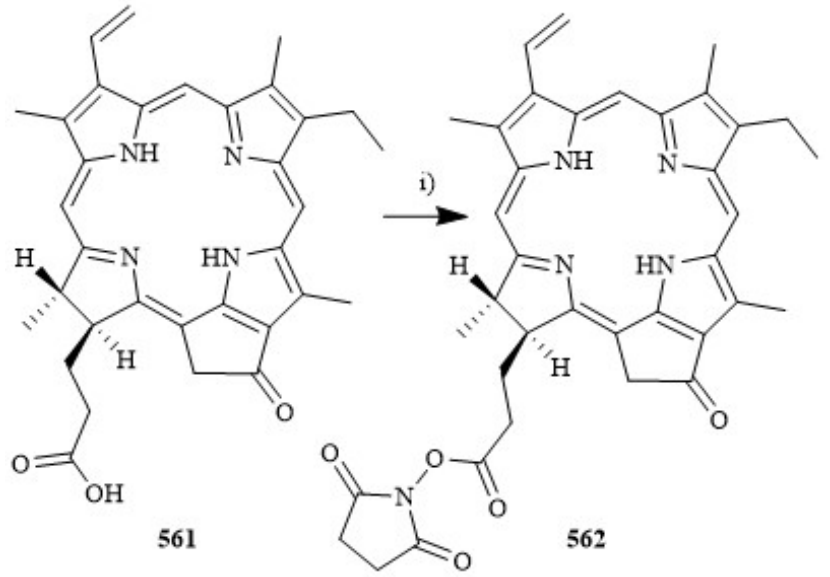

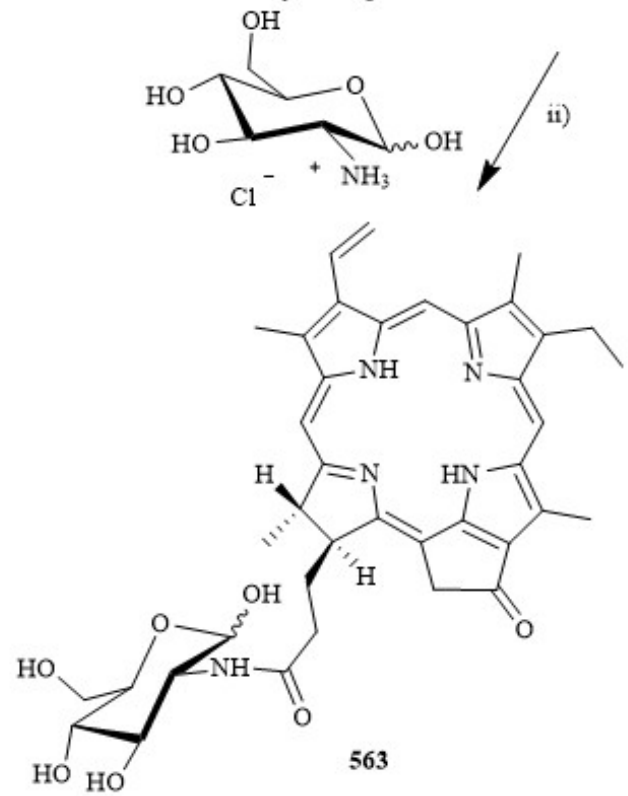

Scheme 29. Synthesis of pyropheophorbide 2-deoxyglucosamide. i) DCC; ii) $\mathrm{NaOMe}$, DMSO

deacetylation afforded 558f-h. All were tested for their affinity for galectins overexpressed on cancer cells ${ }^{[287]}$.

Similar systems were also employed in a study on the effect of the carbohydrate unit had on PET imaging and PDT efficacy a range of glycosylated phytochlorin derivatives were synthesized. Galactose conjugated HPPH had previously shown higher photosensitizing efficacy than HPPH in mice bearing RIF tumors ${ }^{[285,287]}$. Following this 3(1'-m-iodobenzyloxyethyl)pyropheophorbide-a methyl ester (564), $3-\left(1^{\prime}-m\right.$-iodobenzyloxyethyl $)-17^{2}-\{(2-$ deoxy)glucose $\}$ pyropheophorbide-a (565), and 3-(1'-miodobenzyloxyethyl)-172-\{(1-deoxy)-galactose $\}$

pyropheophorbide-a (566) were synthesized and converted into the corresponding ${ }^{124} \mathrm{I}$-labeled analogues via reacting trimethyltin analogues with $\mathrm{Na}^{124} \mathrm{I}$. All pyropheophorbide-a analogues 564-566 were examined in vivo for PET imaging, biodistribution and therapeutic potential in a comparative study in BALB/c mice bearing Colon-26 tumors ${ }^{[288]}$.
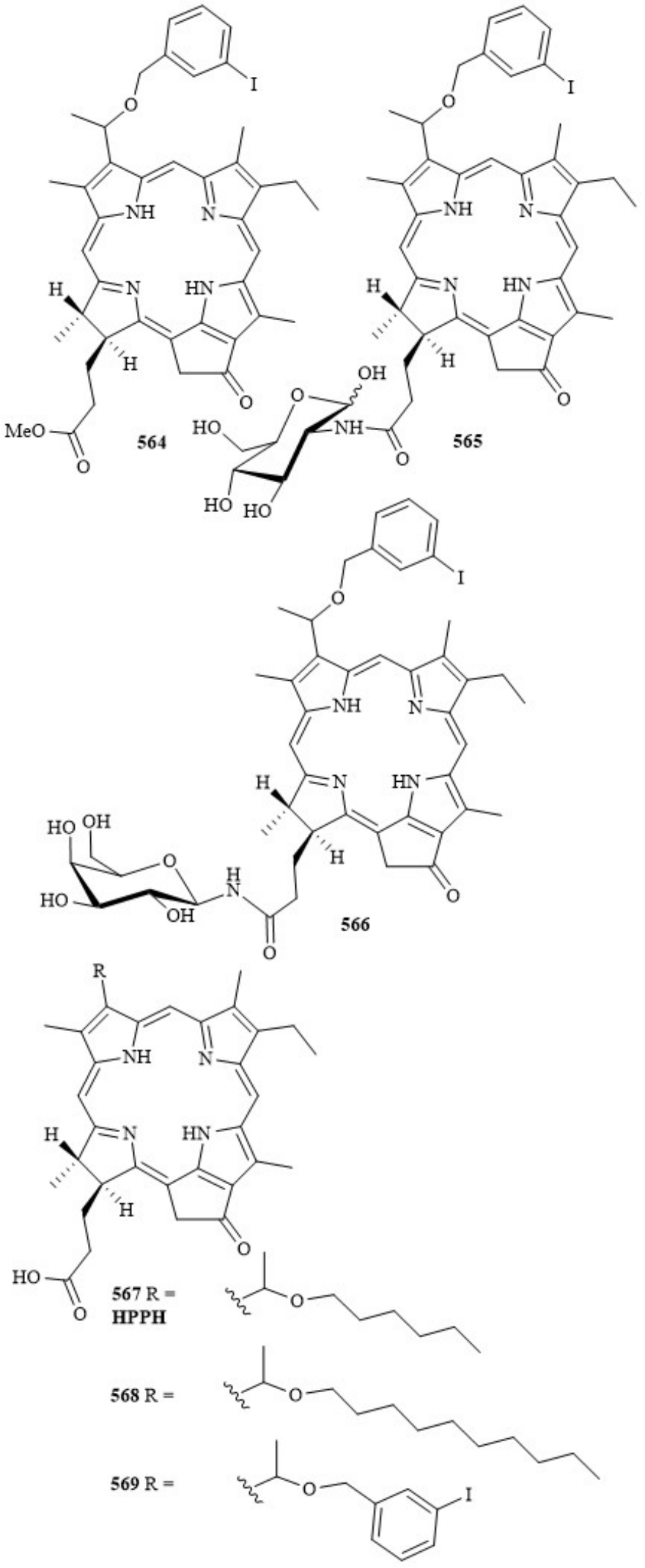

Formula 12 
Pandey's group also spent considerable efforts on the preparation of purpurin derivatives. Purpurin-18 is a derivative of chlorophyll which can be readily isolated from spinach ${ }^{[289]}$. It has three distinct features: a vinyl group, a fused anhydride ring and a propanoic acid side chain. Thus, upon modification it can provide a series of PSs including purpurinimides [35]. Pandey's group first synthesized $\beta$ galactose conjugated purpurinimides $(\mathbf{5 6 9} \mathbf{a}, \mathbf{b}, \mathbf{5 7 0})$ via enyne metathesis for more specific targeting to the Gal-1 (galectin1) receptor. Molecular modeling studies indicated that once the PS was connected via the anomeric position it had no effect on the galectin-carbohydrate recognition. The quantitative structure-activity relationship evaluation of the galactosylated derivatives identified that the presence and positioning of the sugar moiety was important for photobiological activity ${ }^{[278]}$. Subsequently, purpurinimides were substituted at varying positions with different linkers and glycosyl units to determine their effect on the PDT

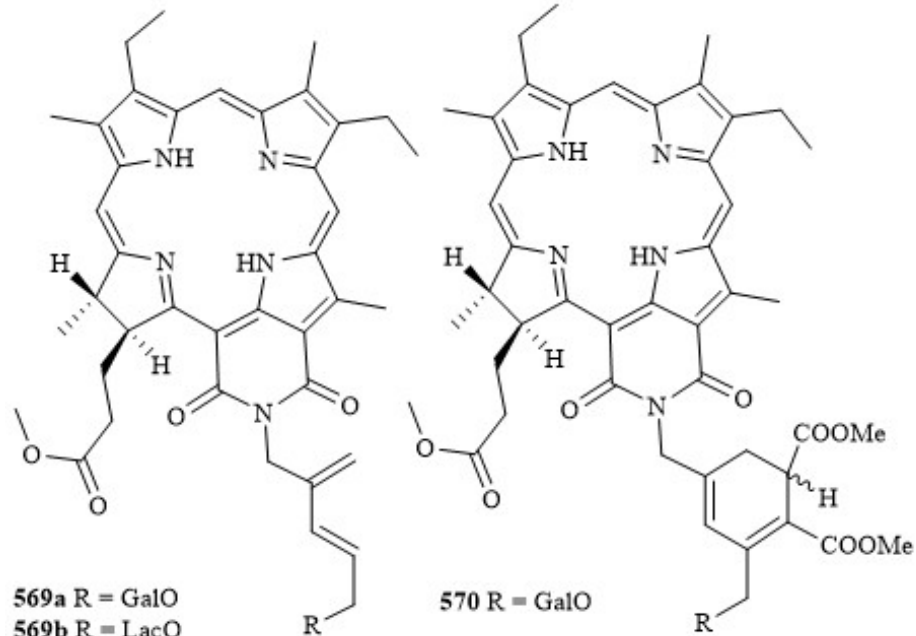

efficacy in vitro and in vivo ${ }^{[290]}$. The lactose unit was linked with a diene $(\mathbf{5 6 9 b})$, an amide (574), an ethylene (575) or was regioselectively introduced at positions 3,8 and 12 forming the positional isomers $(\mathbf{5 7 1 b}, \mathbf{5 7 2}, \mathbf{5 7 3})$. Compounds 571c and 571d were prepared for comparison. A facile methodology allowed for the synthesis of both $N$-alkyl/aryl analogues of monocarbohydrate-purpurinimides with varying lipophilicity.

Other chlorophyll derivatives potentially suitable for PDT are the rhodochlorins, notably chlorin $\mathrm{e}_{6}$. For example, Mironov and coworkers used 1,3-dipolar cycloaddition Huisgen click chemistry for the synthesis of a glycoconjugate of chlorin $\mathrm{e}_{6}$ providing an amphiphilic PS for application in PDT. The synthesis of 577a was achieved by conversion of methyl pheophorbide $a$ (576) to the chlorin $\mathrm{e}_{6}$ derivative via ring-opening of the pentanone exocycle using propargylamine forming the terminal triple bond (Scheme 30) ${ }^{[291]}$. The ethynyl derivative 577a underwent a

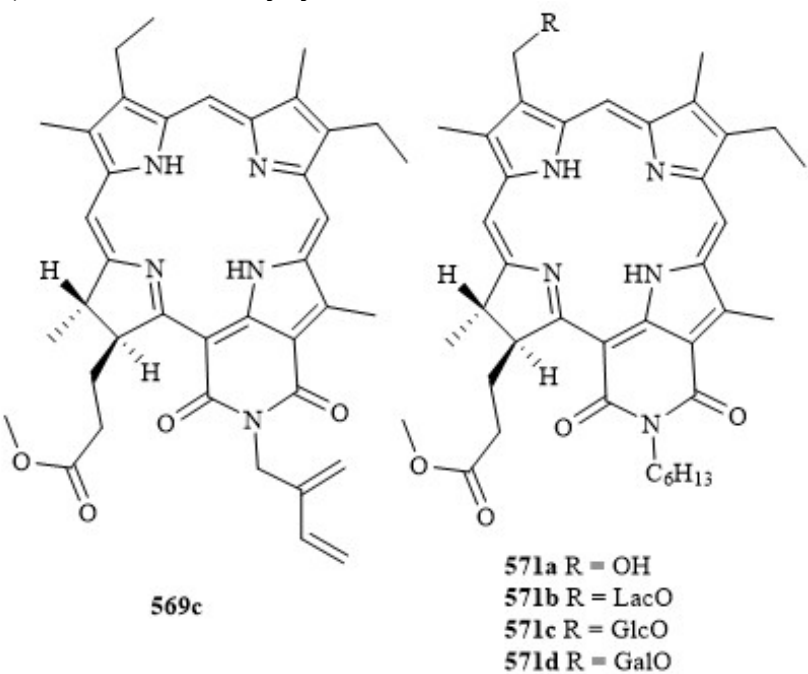

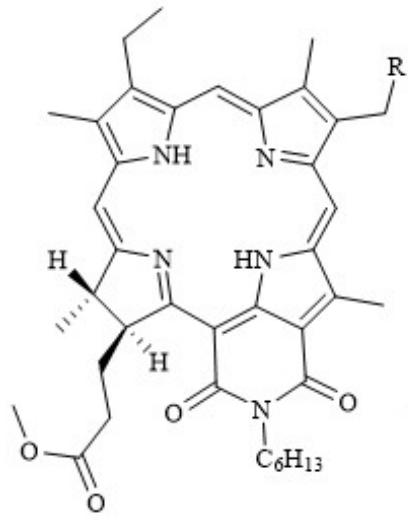

$572 \mathrm{R}=\mathrm{LacO}$

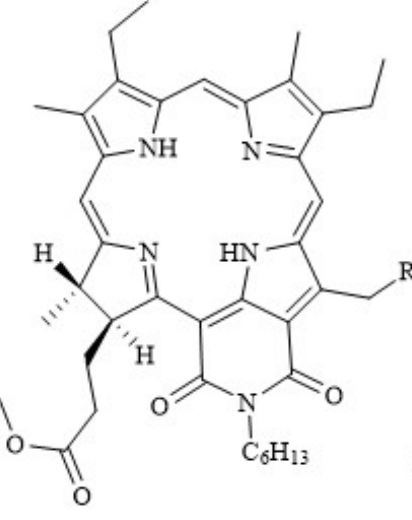

$573 \mathrm{R}=\mathrm{LacO}$

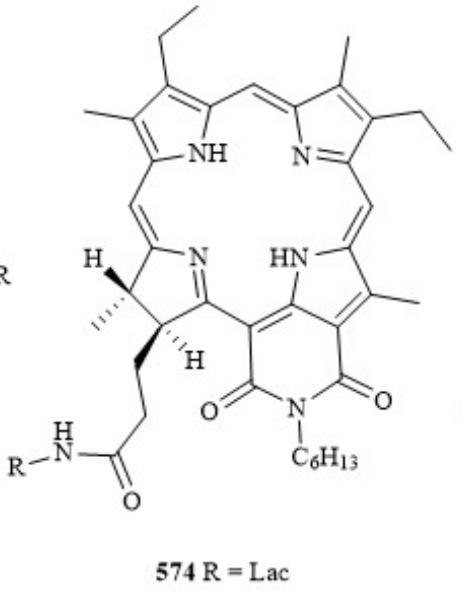

$574 \mathrm{R}=\mathrm{Lac}$

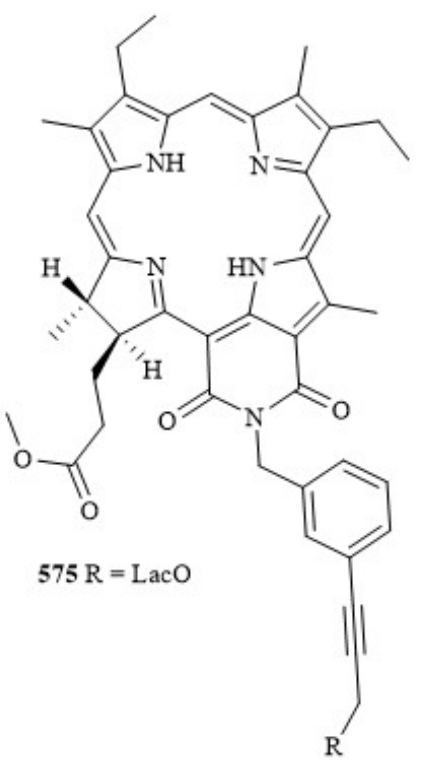


cycloaddition reaction with $1-O$-(2-azidoethyl)- $\beta$ - $D$-lactose peracetate using copper(I) iodide as a catalyst affording the glycochlorin 578a in $82 \%$ yield. The removal of the copper from the macrocycle caused destruction of the glycochlorin. Therefore, the $\mathrm{Zn}$ (II) complex $\mathbf{5 7 7 b}$ was prepared to protect the macrocycle and was easily removed following the cycloaddition reaction. Compound 578c was subsequently deacetylated to give the water soluble glycochlorin 578d. The photophysical properties of $\mathbf{5 7 8 c}$ were examined and the quantum yields of fluorescence $(\Phi \sim 0.25)$ and singlet oxygen $\left(\Phi^{1} \mathrm{O}_{2} \sim 0.75\right)$ were not changed by addition of the sugar residue compared to 577a. This work was later extended to prepare a series of derivatives with glucose, galactose and lactose residues $(\mathbf{5 7 9}, \mathbf{5 7 8 b}, \mathbf{d}){ }^{[292,293]}$ as well as the chlorine $\mathrm{p}_{6}$ glycoconjugate $\mathbf{5 8 1}{ }^{[293]}$. In addition glycoconjugates of a bacteriochlorin cyclic imide were prepared from bacteriopurpurin yielding the glycosylated bacteriochlorophyll a (580a, 582) derivatives. Quantum yields of fluorescence and singlet oxygen of all three 579a,c and 578b derivatives were $\Phi \sim 0.25$ and $\Phi^{1} \mathrm{O}_{2} \sim 0.75$

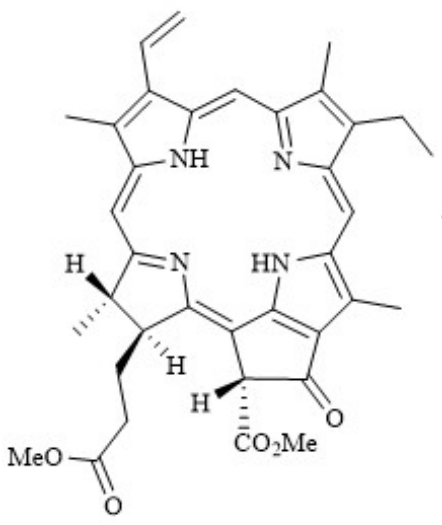

576

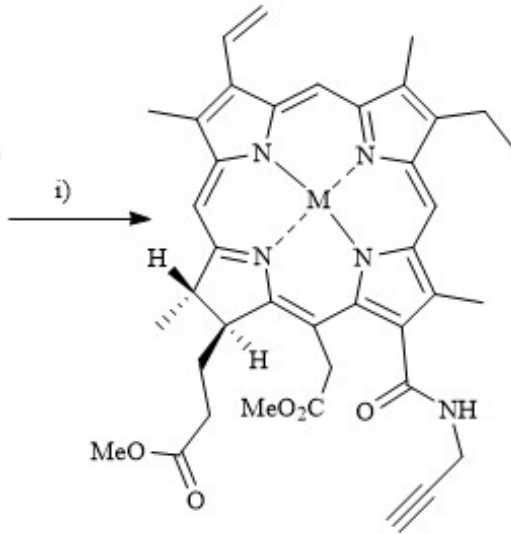

ii)

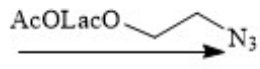

iii)

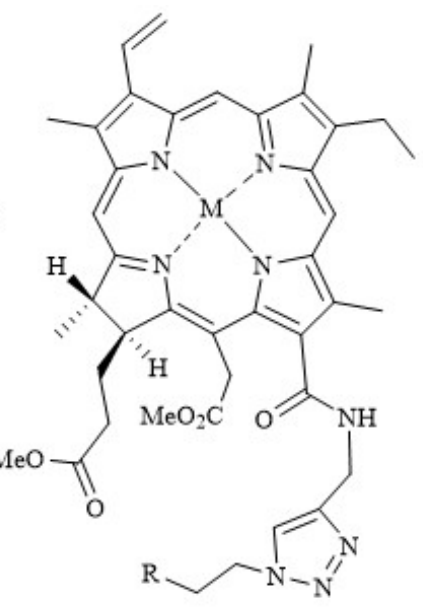

578a $\mathrm{M}=\mathrm{Cu}(\mathrm{II}), \mathrm{R}=\mathrm{AcOL}$ acO

iv)

v)<smiles></smiles>

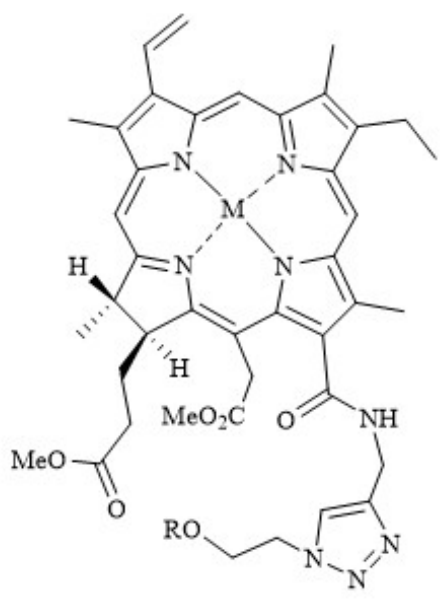

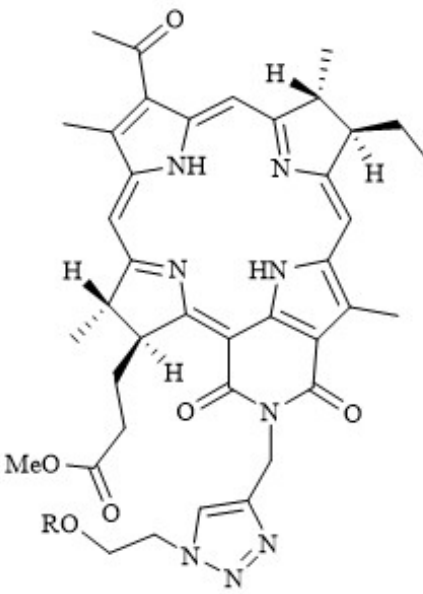

580a R = AcOLac

$580 \mathrm{~b} \mathrm{R}=\mathrm{Lac}$

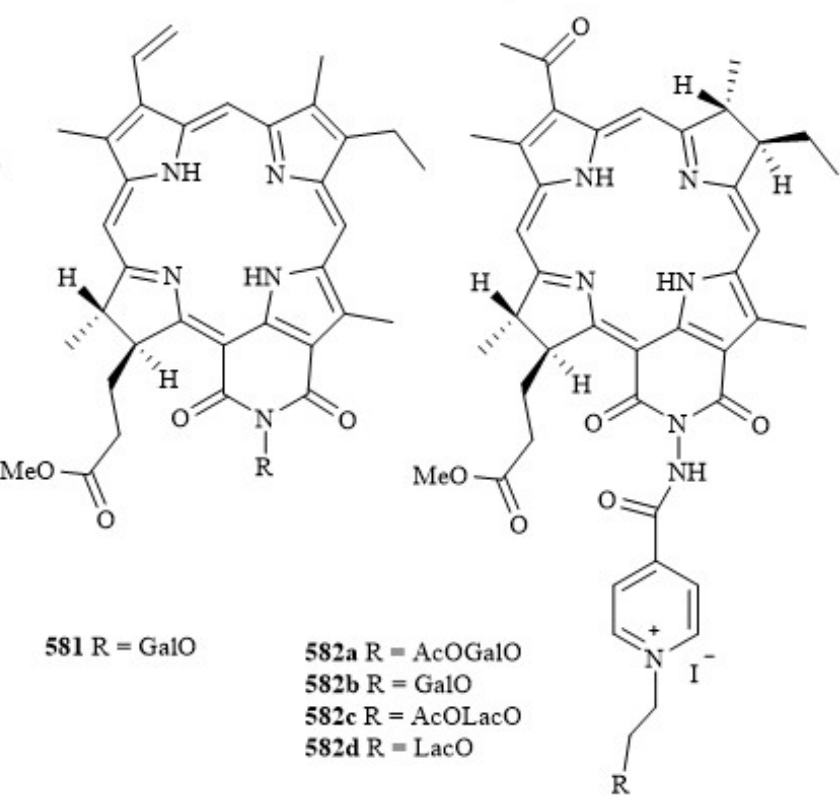

stic $\mathrm{M}=\mathrm{Zn}(\mathrm{II}), \mathrm{R}=\mathrm{AcOGlc}$

579b $\mathrm{M}=2 \mathrm{H}, \mathrm{R}=\mathrm{Glc}$

579c $\mathrm{M}=\mathrm{Zn}$ (II), $\mathrm{R}=\mathrm{AcOG}$ al

$582 \mathrm{~b}$ R $=\mathrm{Ga} 1 \mathrm{O}$

$582 \mathrm{~d} R=\mathrm{LacO}$

Scheme 30. Synthesis of chlorin e6 glycoconjugates and metal complexes. i) Propargylamine, $\mathrm{CH}_{2} \mathrm{Cl}_{2} ;$ ii) $\mathrm{Zn}(\mathrm{OAc})_{2}, \mathrm{CHCl}_{3}-\mathrm{MeOH}$; iii) $\mathrm{CuI}, \mathrm{MeCN}$, $\mathrm{Et} 3 \mathrm{~N}$; iv) $\mathrm{HCl}$; v) $\mathrm{MeONa}, \mathrm{MeOH}$. 
compared to meso-tetrakis(4-sulfophenyl)porphyrin ${ }^{[292,293]}$.

More complex carbohydrates have also been appended to chlorophyll derivatives. For example, a significantly advanced glycosyl unit in the form of hyaluronic acid, consisting of repeating disaccharide units of $N$-acetyl- $D$ glucosamine and $D$-glucuronic acid was adhered to several Pheophorbide a PSs. The synthesis involved acetylation of hyaluronic acid with acetic anhydride, followed by conjugation with pheophorbide a via carbodiimide coupling reaction to yield $\mathbf{5 8 3}{ }^{[294]}$. In addition a similar polysaccharide nanogel was synthesized containing pullulan/folate conjugated to Pheophorbide a PSs [295]. Pullulan is a maltotriose isolated from the fungus Aureobasidium pullulans and can be used as a drug carrier and, combined via DCC or DMAP-mediated ester formation with folate which can target overexpressed receptors on the surface of several human cancer cells, provides a selforganized nanogel. When coupled with pheophorbide a again with a carbodiimide reaction they afford nanostructures $\sim 170$ $\mathrm{nm}$ in size with three derivatives isolated with varying degrees of PS attached to the sugar chain ${ }^{[295]}$.

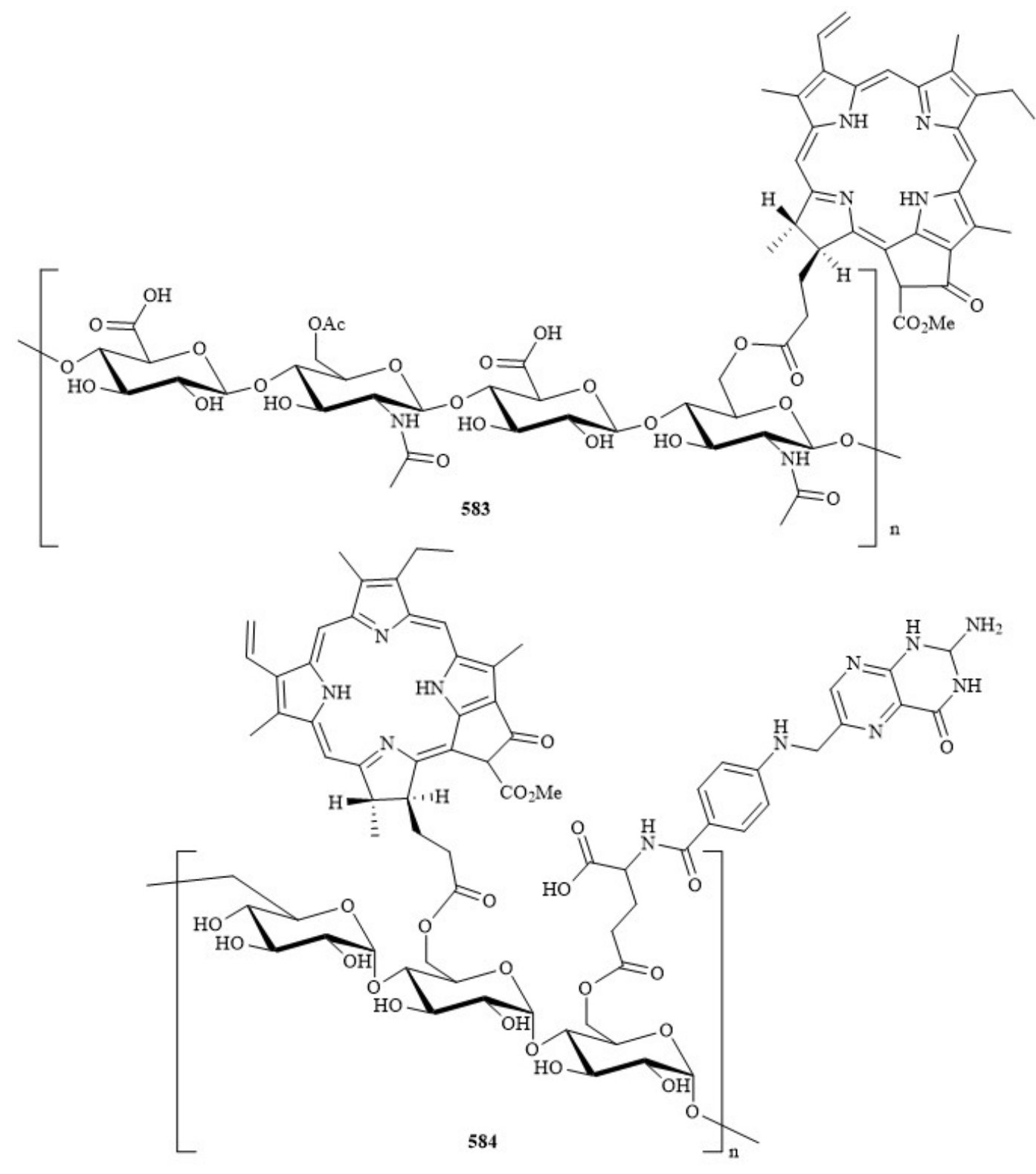

Formula 14. 


\subsection{Related Dyes}

\subsubsection{Pyrrole Derivatives}

Many other dye systems have been investigated for PDT. With the exception of systems such as hypericin, most of these are chemically related to porphyrins. Consequently some examples of expanded, contracted and isomeric glycoporphyrins have been investigated as PS. However, synthetic examples of carbohydrate conjugates for these systems are limited.

One such example is 585a, a sapphyrin derivative, which exhibited a singlet oxygen quantum yield of 0.18 and showed phototoxicity against Staphylococcus aureus similar to that of HpD. Although initially promising, interest in PDT use of sapphyrins has waned in recent years ${ }^{[296]}$. Král et al. also synthesized similar expanded dyes (587 and 586b) via preformed sapphyrins functionalized with glycosyl residues by amine coupling using DMAP as well as the disubstituted glyco-protoporphyrin $\mathbf{5 8 7}$ which were tested in vivo ${ }^{[297,298]}$.

Two water soluble glucosamine sapphyrin derivatives were synthesized via EDC-mediated coupling of sapphyrin diacid 586a and either $D$-glucosamine $(\mathbf{5 8 6 d}, 13 \%)$ or $N$ methyl- $D$-glucosamine $(\mathbf{5 8 6 c}, 10 \%)$ to investigate their anion binding properties ${ }^{[299]}$. The compounds formed $\mathrm{H}$ aggregates in neutral aqueous solutions and the extent of aggregation was anion dependent with the protonated sapphyrin acting as an anion binding agent. In particular, addition of phosphate anions at $\mathrm{pH} 7$ afforded considerable formation of monomeric species. The concentration dependent behavior of this effect indicates the possible use of glycosylated sapphyrins as fluorescent phosphate anion sensors. Several examples of mono-, di- and triglycosylated fluorophenyl corroles were synthesized via combinatorial approaches with a total of 24 products ${ }^{[215]}$.

An example of a deprotected mono-glycosylated calix[4]pyrrole derivative was synthesized by Drašer et al. via $C$-glycosyl linkage to the meso-positions of oligopyrrole. The synthesis of the protected mono- and diglycosylated calix[4]pyrrole derivatives was achieved in a two-step procedure (Scheme 31). First, acid catalyzed condensation of pyrrole and the carbohydrate ketone derivative 588a gave the dipyrryl derivative 589a and secondly, acid-catalyzed MacDonald reaction with acetone provided a mixture of diastereoisomers of 5,15-syn and 5,15-anti calix[4]pyrrol10,20-diyl-bis-D-glycero- $D$-gulo-heptitols $\quad \mathbf{( 5 9 1 )}$ and monosubstituted calix[4]pyrrole (590a) in $16 \%$ yield. In a similar procedure, the deprotected carbohydrate ketone $\mathbf{5 8 8 b}$ was used with methane sulfonic acid as a catalyst affording the mono-substituted 590b in $11 \%$ with no disubstituted product found after purification. Ketone $\mathbf{5 8 8 b}$ and 5,5dimethyldipyrromethane gave 590b in $10 \%$ yield. Usually dipyrrylmethane derivatives provide the symmetric

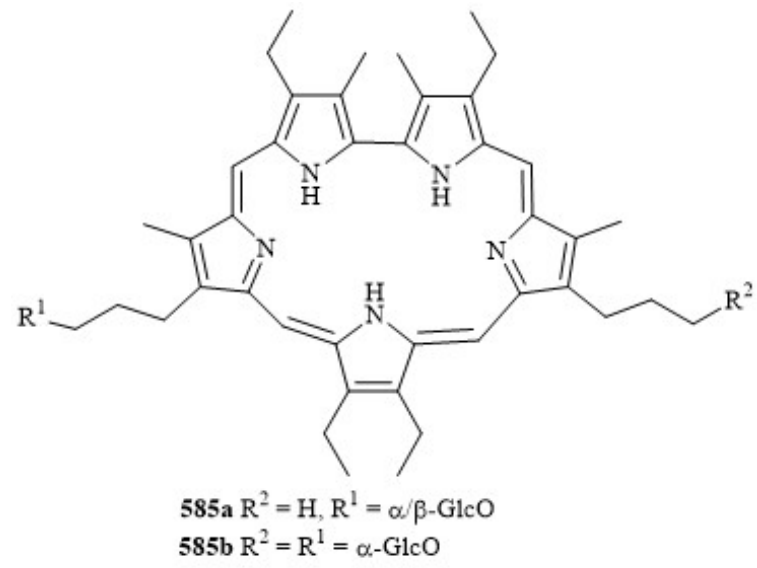

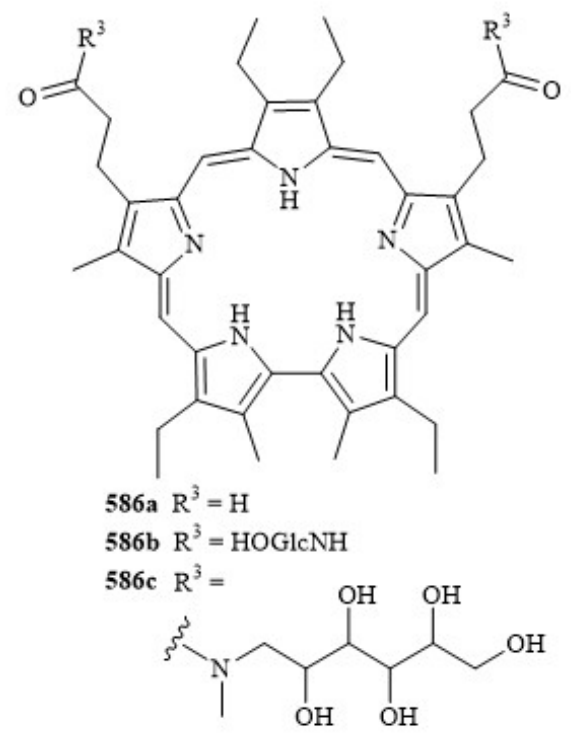

$586 \mathrm{~d} \mathrm{R}=$<smiles>C#CCNCC(O)C(O)C(O)C(O)CO</smiles>

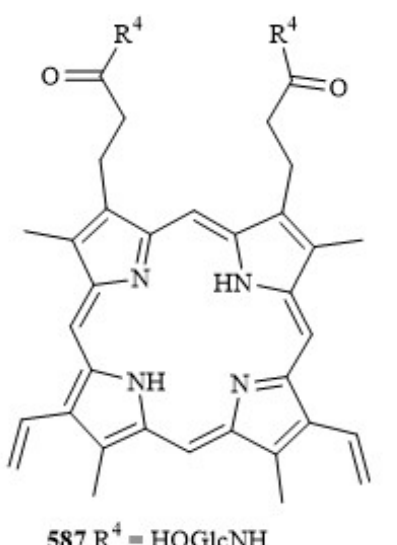

Formula 15. 
<smiles>[R]C[C@H]1O[C@H](CC(C)=O)[C@@H]([R])[C@H]([R])[C@@H]1[R]</smiles><smiles>CCCCCCC</smiles><smiles>[R]C[C@H]1O[C@H](CC(C)(c2ccc[nH]2)c2ccc[nH]2)[C@H]([R])[C@H]([R])[C@@H]1[R]</smiles>
589a $\mathrm{R}=\mathrm{OAC}$ $589 \mathrm{~b} \mathrm{R}=\mathrm{OH}$

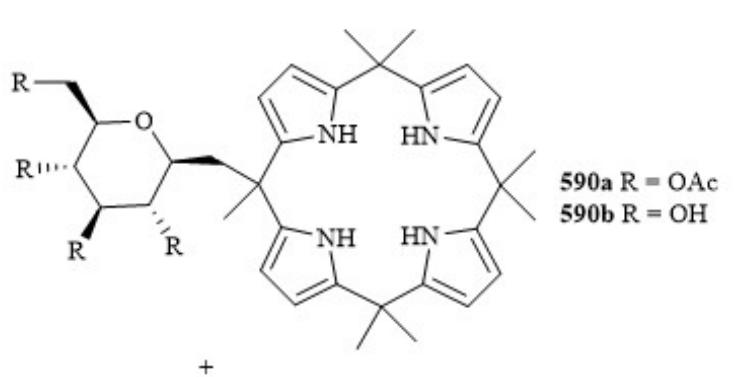

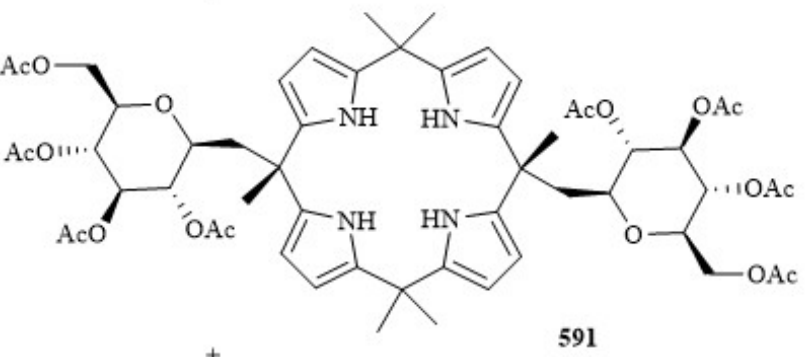

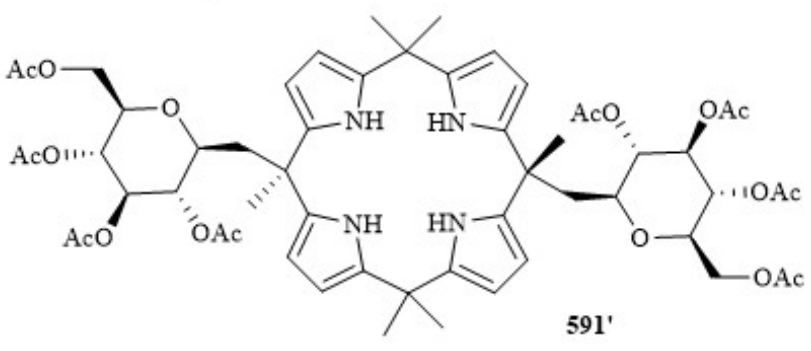

Scheme 31. Asymmetrical/symmetrical glycosyl substituted calix[4]pyrrole. i) TFA, $\mathrm{CH}_{2} \mathrm{Cl}_{2}$ for $\mathbf{5 9 1}$ or ii) $\mathrm{MeHSO}_{3}, \mathrm{MeOH}_{\text {for }} \mathbf{5 8 8 b}-\mathbf{5 9 0 b}$.

disubstituted oligopyrroles in $2+2$ additions; however, a $3+1$ addition forming the mono-substituted calix[4]pyrrole is usually only achieved by mixed condensations ${ }^{[300]}$.

\subsubsection{Phthalocyanines}

Phthalocyanines $\quad(5,10,15,20$-tetraazabenzoporphyrins $)$ have also found wide interest as potential PSs. They are normally more photostable than porphyrins but the synthesis of unsymmetrically substituted phthalocyanines lags behind that of the porphyrins. The synthesis of their glycoconjugates follows similar strategies to those employed for porphyrins ${ }^{[301]}$. However, as the meso position is now a nitrogen atom the carbohydrate unit is solely introduced at the $\beta$-pyrrole positions and often there is ambiguity as to the exact regiochemistry. For condensation reactions this requires the use of dedicated precursors. For example, the phthalocyanine derivative 592 was synthesized in $61 \%$ yield from 592a and zinc acetate and was subsequently deprotected to afford $\mathbf{5 9 3}$ [255]. A good example of the synthesis of glycophthalocyanines is illustrated in Scheme 32. Here, 3,4dicyanophenyl $O$ - and $S$ - glucosides, galactosides, lactosides, and cellobiosides (594a-g) could be prepared quantitatively via NAS of 4- nitrophthalonitrile with acetylated glycosides and 1-thio-glycosides. While the glycosyl oxide gave mainly the $\alpha$-glycoside, the glycosyl thiolates gave only the $\beta$ - thioglycosides. Also prepared were 2-(3,4dicyanophenoxy)ethyl 2,3,4,6-tetra- $O$-acetyl- $\beta$ - $D$-gluco- and galacto-pyranosides via nucleophilic substitution of 2(tosyloxy)ethyl 2,3,4,6-tetra- $O$-acetyl- $\beta$ - $D$-gluco- and galacto-pyranoside with 3,4-dicyanophenol. All compounds were subjected to Zemplén deacetylation and reacted under classical phthalocyanine template condensation chemistry to form the glycosylated zinc(II) phthalocyanines 595a-g in 42$54 \%$ yields (Scheme 32) ${ }^{[302]}$. In extension of such work similar derivatives were synthesized for naphthalocyanines [303]

With the choice of the right precursors unsymmetrically glycosylated phthalocyanines are accessible through condensation reactions as well. Such an amphiphilic glycophthalocyanine was synthesized via the crosscondensation of tetrakis(1,2:3,4-di- $O$-isopropylidene- $\alpha-D$ galactopyranos-6-yl)phthalonitrile with phthalonitrile ${ }^{[304]}$. All carbohydrate units linked to the same isoindolyl group at C- 6 of the galactose residue afforded a single phthalocyanine isomer (Scheme 33). The glycophthalonitrile was prepared by nucleophilic substitution of tetrafluorophthalonitrile with $1,2: 3,4$-di- $O$-isopropylidene- $\alpha$ - $D$-galactopyranose which was used to form phthalocyanine $\mathbf{5 9 7}$ in $30 \%$ though crosscondensation with zinc chloride. Deprotection yielded the amphiphilic phthalocyanine 598. Amphiphilic derivatives 


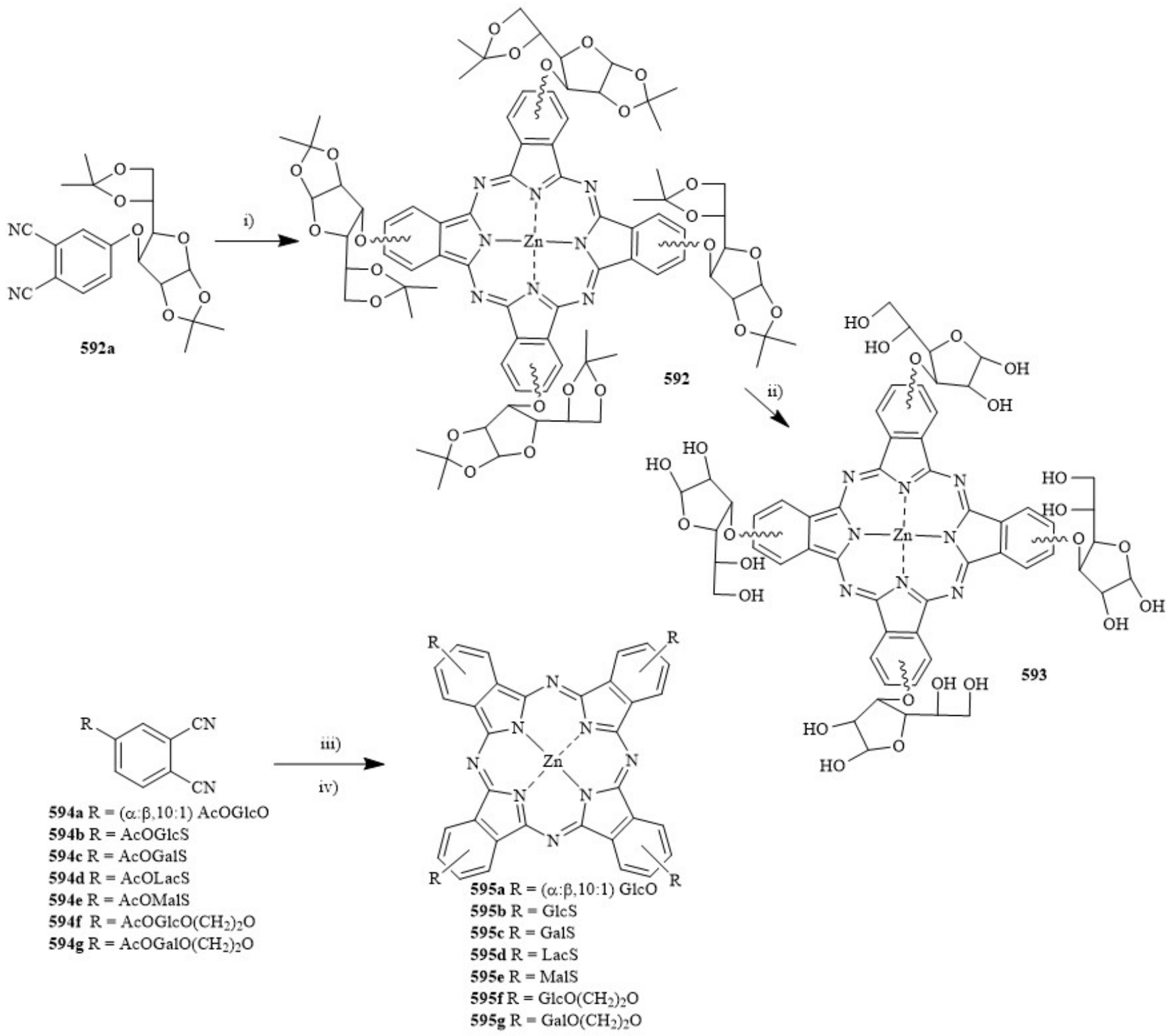

Scheme 32. Synthesis of glycosylated phthalocyanines. i) $\mathrm{Zn}(\mathrm{OAc})_{2}$, dimethylaminoethanol $(\mathrm{DMAE}), \mathrm{reflux}, 6 \mathrm{~h}$; ii) $\mathrm{CF}_{3} \mathrm{COOH}_{\mathrm{H}} \mathrm{O}(9: 1)$, $30 \mathrm{~min}$.; iii) $\mathrm{MeOH} / \mathrm{NaOMe}$ r.t., $1 \mathrm{~h}$; iv) $\mathrm{Zn}(\mathrm{OAc})_{2}, \mathrm{Me}_{2} \mathrm{NCH}_{2} \mathrm{CH}_{2} \mathrm{OH}, n$ - $\mathrm{BuOH}, 100{ }^{\circ} \mathrm{C}, 24$ h.

can be prepared as well, for example by cross-condensation of $\quad 1,2: 5,6$-di- $O$-isopropylidene- $\alpha$ - $D$-glucofuranosyl phthalonitrile and octadecyloxyl phthalonitrile in the presence of zinc chloride with subsequent deprotection in 25 $\%$ yield to afford an amphiphilic derivative with three sugar units and a $\mathrm{C} 18$ side chain ${ }^{[305]}$.

Unprotected phthalonitriles can be employed in such condensation reactions as well. In a novel glycosylation reaction nucleophilic aromatic substitution was used to synthesize the first glycophthalonitrile linked via the anomeric position of the sugar unit in high yields ${ }^{[306]}$.
Starting from 4-nitrophthalonitrile, which underwent glycosylation with 2,3,4,6-tetra- $O$-benzyl-, benzoyl- and acetyl-protected glucopyranoses using $\mathrm{NaH}$ for 599a and $\mathrm{K}_{2} \mathrm{CO}_{3}$ for 599b,c, afforded preferentially the $\alpha$ anomers in 600a $(\alpha / \beta=9 / 1), 600 b(\alpha / \beta=10 / 1)$ and 600c $(\alpha / \beta=10 / 1)$ (Scheme 34). Use of classical template synthesis with these protected glycophthalonitriles (600a-c), followed by deprotection, gave poor conversion. However, preliminary deprotection of the tetraacetylated derivative $\mathbf{6 0 0 c}$ under Zemplén conditions to $\mathbf{6 0 1 a}$ and template synthesis with zinc acetate gave 602a in $51 \%{ }^{[307]}$. A similar method of direct 
anomeric linkage was applied by Boyle et al. to synthesize a tetragalactose-substituted $\mathrm{Zn}$ (II) phthalocyanine (602b) ${ }^{[308]}$. Similarly, water soluble glycophthalocyanines with four or eight unprotected $D$-galactose moieties were synthesized for application in PDT ${ }^{[309]}$. The glycophthalonitrile $\mathbf{6 0 3}$ was tetramerized in the presence of $\mathrm{ZnCl}_{2}$, affording phthalocyanine 604 in $43 \%$ yield. Compound 604 had been previously synthesized via DBU-promoted self-cyclisation in the presence of zinc acetate ${ }^{[310]}$. The glycophthalocyanine with eight $D$-galactose units $\mathbf{6 0 8}$ was synthesized using a similar route with glycophthalonitrile 606, whereby phthalocyanine $\mathbf{6 0 7}$ was obtained in $62 \%$ yield. Treatment of compound $\mathbf{6 0 4}$ and 607 with aqueous TFA gave the unprotected compounds $\mathbf{6 0 5}$ and $\mathbf{6 0 8}$ (Scheme 35). Phthalocyanine $\mathbf{6 0 7}$ was later also synthesized through cyclotetramerization of the isoindoline derivative of 4,5bis(1,2:3,4-di- $O$-isopropylidene- $\alpha$ - $D$-galactopyranos-6-

yl)phthalonitrile in presence of zinc acetate and DMAE in 29 $\%$ yield and subsequent deprotection ${ }^{[311]}$.

In addition to the octa substituted glycophthalocyanine 600c, a highly glycosylated dendritic glycophthalocyanine (611a) containing 16 glycosyl units was synthesized by diglycosylation of 2,4,6-trichloro-1,3,5-triazine followed by substitution with 1,3-dimercaptopropane and subsequent nucleophilic substitution with (hexadecafluorophthalocyaninato)zinc(II) in $81 \%$ yield. The porphyrin dervatives (610a) was synthesized in a similar way using $\mathrm{TPPF}_{20}$. Hydrolysis of both produced an $\alpha / \beta$ mixture of the unprotected glycodendrimer conjugates $\mathbf{6 1 0 b}$ or $611 b$ (Scheme 36$)^{[312]}$.<smiles>[R]c1c([R])c(C#N)c([R])c(C#N)c1[R]</smiles>

$596 \mathrm{R}=$ GalO-isop

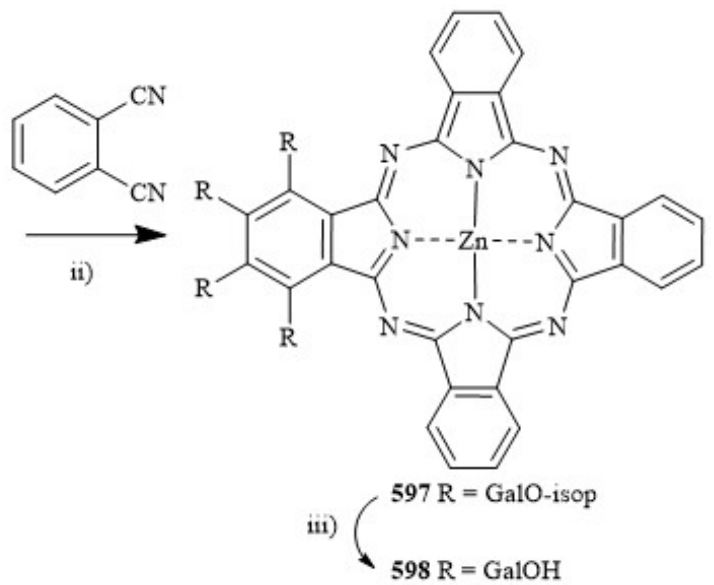

Scheme 33. Synthesis of tetrasubstituted galactosyl phthalocyanine. i) (Gal-isop)-OH, $\mathrm{NaH}$, toluene, $\mathrm{N}_{2}, 70{ }^{\circ} \mathrm{C}$; ii) $\mathrm{ZnCl}_{2}, \mathrm{DMAE}, \mathrm{N}_{2}, \mathrm{reflux}$; iii) $\mathrm{TFA} / \mathrm{H}_{2} \mathrm{O}$ $(9: 1)$, r.t.

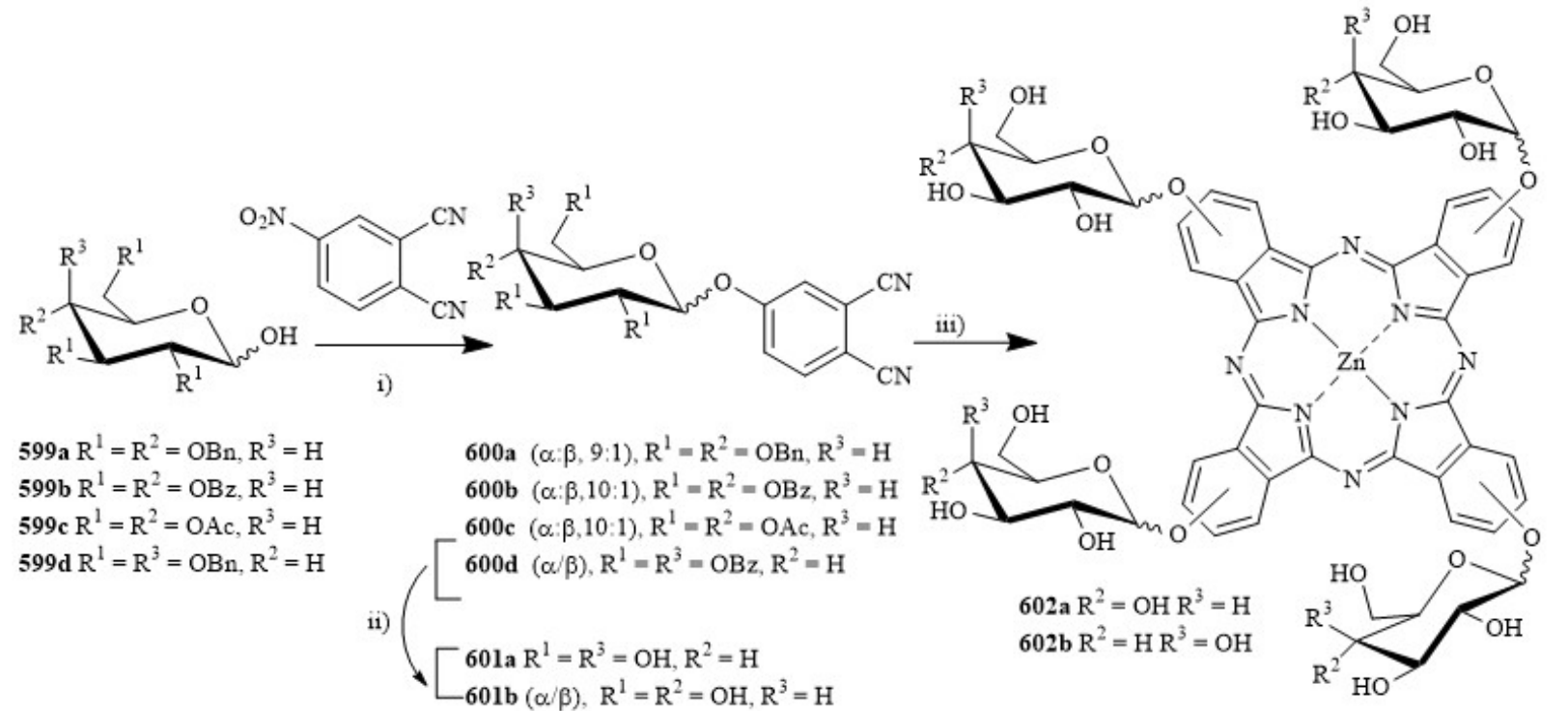

Scheme 34. Synthesis of tetra-substituted glucosylated phthalocyanine. i) For 599a: $\mathrm{NaH}$ (1 equiv.), dry DMF, r.t., 1 h; for 599 b,c: $\mathrm{K}_{2} \mathrm{CO}_{3}(6$ equiv.), DMF, r.t., $12 \mathrm{~h}$; for 599d: $\mathrm{K}_{2} \mathrm{CO}_{3}$, DMF, r.t.; ii) for $\mathbf{6 0 0 c}$ and $\mathbf{6 0 0 d}$ : cat. $\mathrm{NaOMe}$, dry $\mathrm{MeOH}$, r.t., 1 h; iii) DMAE $/ n$-butanol $(2: 1)$, dry zinc acetate $\left(\mathrm{Zn}(\mathrm{OAc})_{2}, 100{ }^{\circ} \mathrm{C}, 24\right.$ 


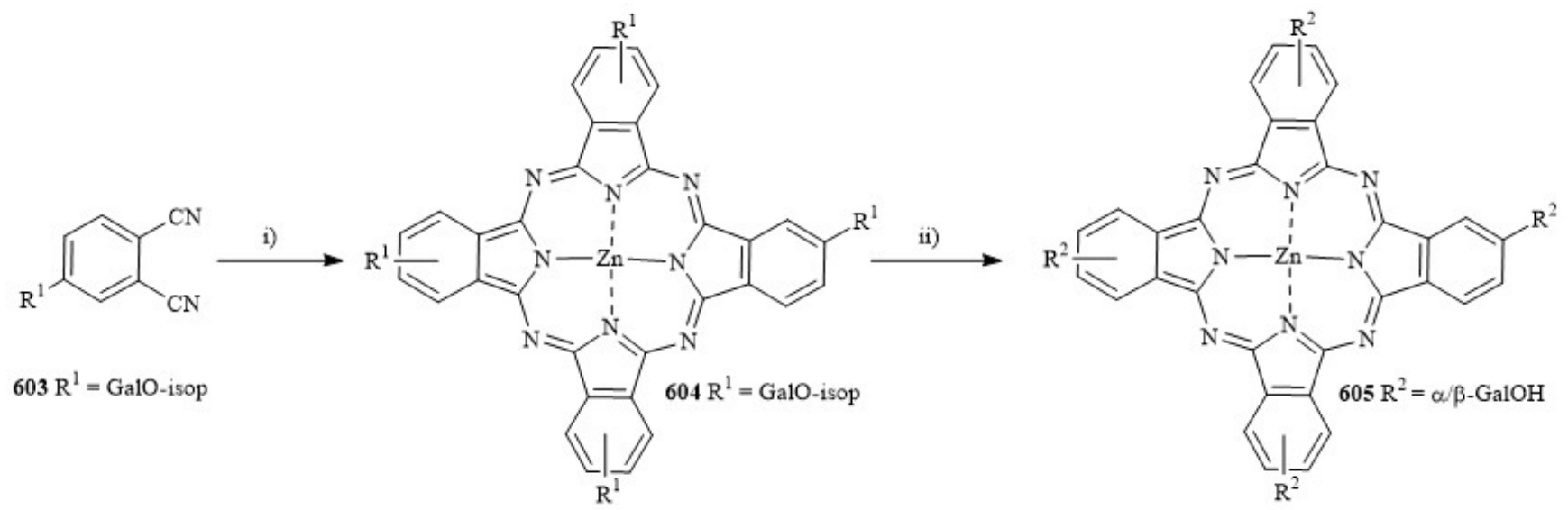

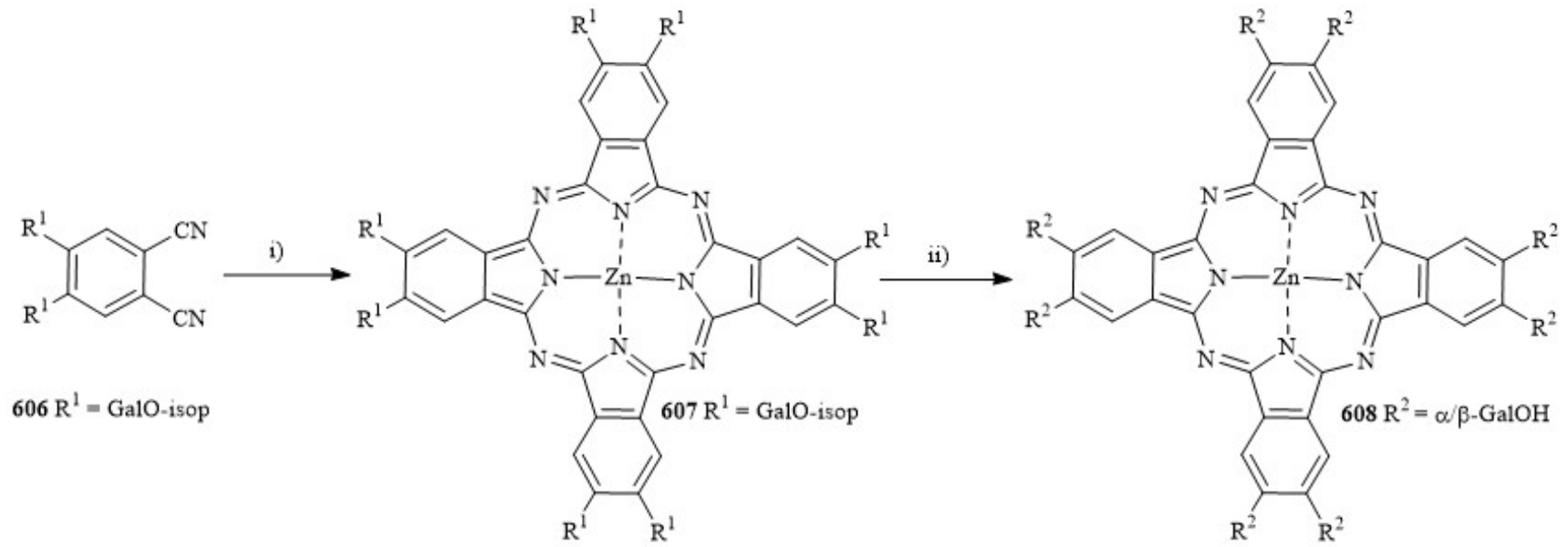

Scheme 35. Synthesis of glycoconjugated phthalocyanines. $\quad$ i) $\quad \mathrm{ZnCl}_{2}, \quad \mathrm{DMAE}, \quad \mathrm{N}_{2}, \quad 140 \quad{ }^{\circ} \mathrm{C} ; \quad$ ii) $\quad \mathrm{TFA} / \mathrm{H}_{2} \mathrm{O} \quad$ (9:1), $\quad$ r.t.

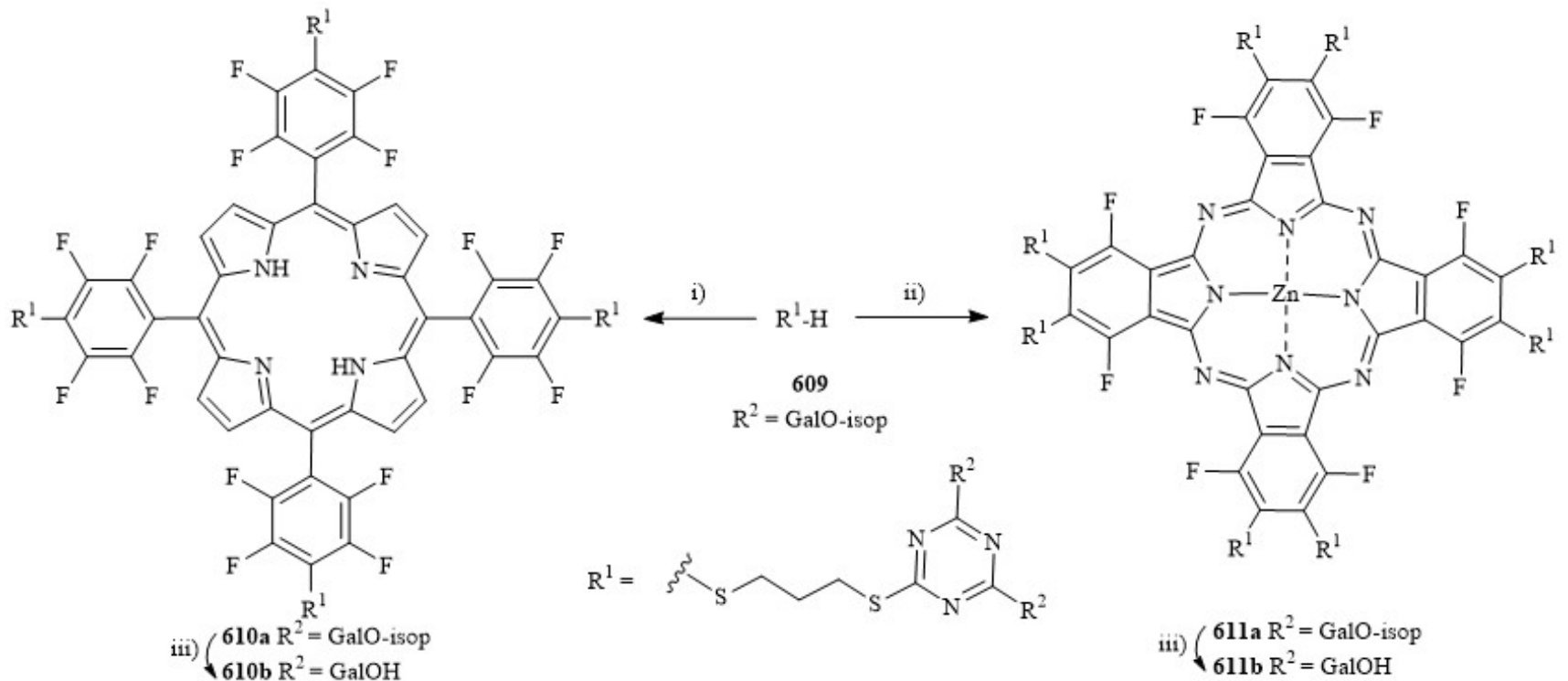

Scheme 36. Synthesis of glycoconjugated phthalocyanines. i) $\mathrm{TPPF}_{20}, \quad N, N^{\prime}$-diisopropylethylamine, $\mathrm{DMF}, \quad 50 \quad{ }^{\circ} \mathrm{C}, \quad \mathrm{N}_{2}, \quad 24 \quad \mathrm{~h}, \quad 78 \quad \%$; $\quad$ ii) (hexadecafluorophthalocyaninato)zinc(II), $N, N$ '-diisopropylethylaimne, DMF, $50{ }^{\circ} \mathrm{C}, \mathrm{N}_{2}, 24 \mathrm{~h}, 81 \%$; iii) $\mathrm{TFA} / \mathrm{H}_{2} \mathrm{O}(9: 1), 78-80 \%$. 
Organometallic couplings can also be employed for these tetrapyrroles. For example, the synthesis of the deoxyribosephthalocyanine conjugate 612, where the sugar moiety is connected via a C-C bond to the phthalocyanine, was achieved via Sonogashira coupling of (tetraiodophthalocyaninato)zinc(II) with $\{2$-deoxy-3,5-bis $[O-(p-$ toluoyl)]- $\alpha, \beta$ - $D$-ribofuranosyl $\}$ ethyne which was subsequently $p$-toluoyl deprotected affording the free hydroxyl derivative. The sugar unit improved water solubility without decreasing the photophysical properties [313]. Other organometallic couplings were used in the synthesis of tetraglucosyl zinc phthalocyanine (613) via click chemistry ${ }^{[314]}$. Likewise, axial coordination can be used for binding of the carbohydrate units. This is illustrated in a series of galactose conjugated silicon(IV) phthalocyanines which were synthesized via substitution of dichloro(phthalocyaninato)silicon(IV) 616 with 1,2:3,4-di$O$-isopropylidene- $\alpha$ - $D$-galactopyranose using $\mathrm{NaH}$ in toluene for the disubstitution product $(\mathbf{6 1 7}, 38 \%)$ or a mixed substitution of $1,2: 3,4$-di- $O$-isopropylidene- $\alpha-D$ galactopyranose and several alcohols for the unsymmetric derivatives (618, 11-18 \%, Scheme 37).

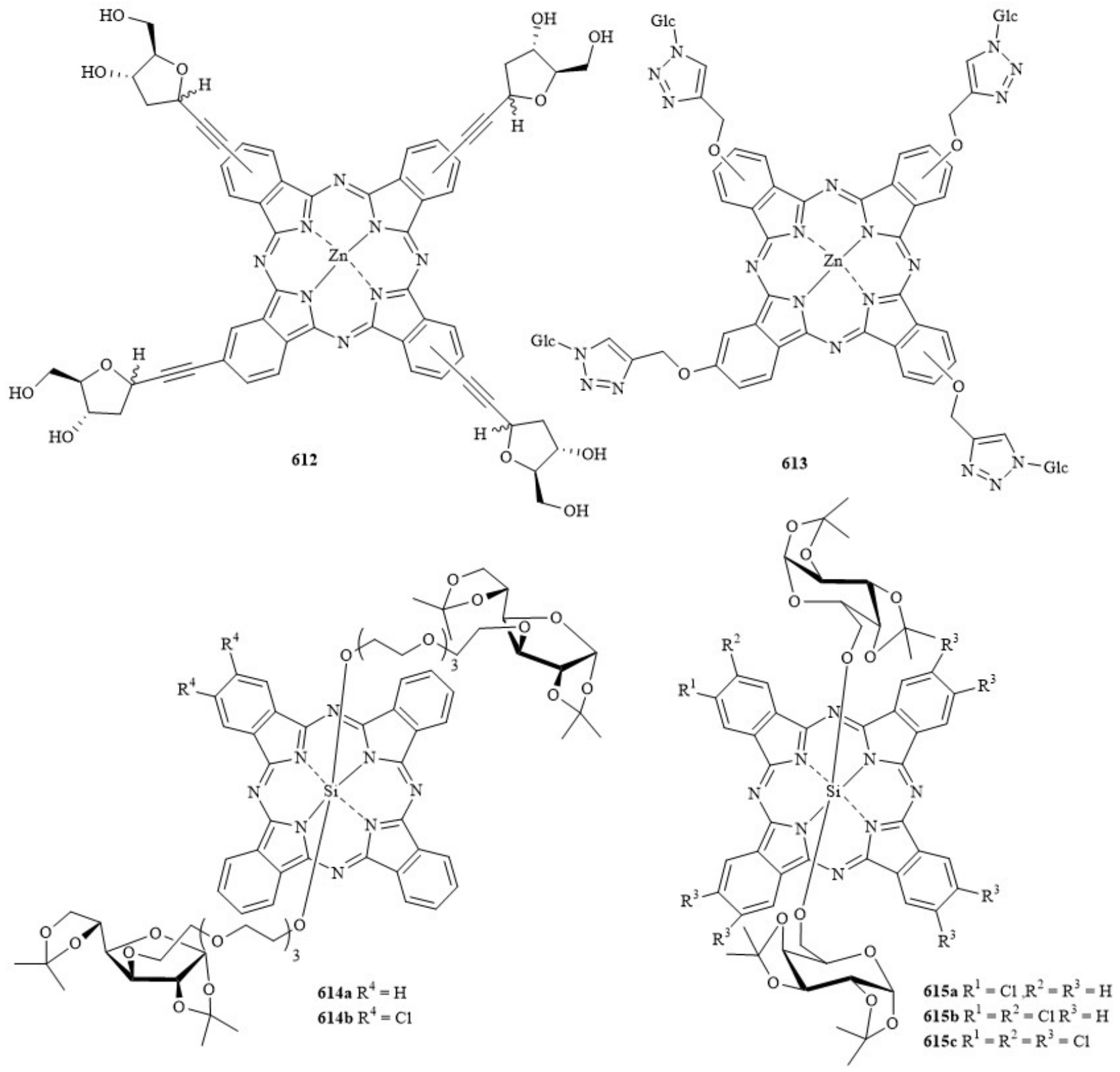


In a similar manner, the glucosylated silicon(IV) phthalocyanines derivatives $614 \mathbf{a}, \mathbf{b}$ were synthesized via two axial 1,2:5,6-di- $O$-isopropylidene- $\alpha$ - $D$-glucofuranose linked by a tetraethylene glycol chain to increase water solubility. The dichloro derivative $\mathbf{6 1 4 b}$ was synthesized by a $3+1$ mixed cyclization of 1,3-diiminoisoindoline and dichloro-1,3-diiminoisoindoline in the presence of $\mathrm{SiCl}_{4}$, subsequently was treated directly with 1,2:5,6-di-Oisopropylidene- $\alpha$ - $D$-glucofuranose with a tetraethylene glycol chain ${ }^{[315]}$. In addition, the galactose derivatives $\mathbf{6 1 5 b}, \mathbf{c}$ were prepared with di- and octachloro substitution via mixed- and self-cyclization with dichloro 1,3diiminoisoindoline and 1,3-diiminoisoindoline in the presence of $\mathrm{SiCl}_{4}$ and subsequent additions of the protected galactose with $\mathrm{NaH}$ in toluene at $120^{\circ} \mathrm{C}$ for two days. Increasing the temperature to $170^{\circ} \mathrm{C}$ for four days the monosubstituted derivative 615a could be formed in $4 \%$ yield [316]. All derivatives displayed significant PDT activity against HepG2 human hepatocarcinoma ${ }^{[315,317,318]}$. Larger constructs are also accessible, for example in the form of cyclodextrin-phthalocyanine dyads. This involved the synthesis of 4-( $\beta$-cyclodextrin) phthalonitrile by coupling $\beta$ cyclodextrin with 4-nitrophthalonitrile, followed by crosscondensation of 619 with excess of phthalonitrile or 4,5dibutoxyphthalonitrile affording 620a and 620b (Scheme 38) [319].

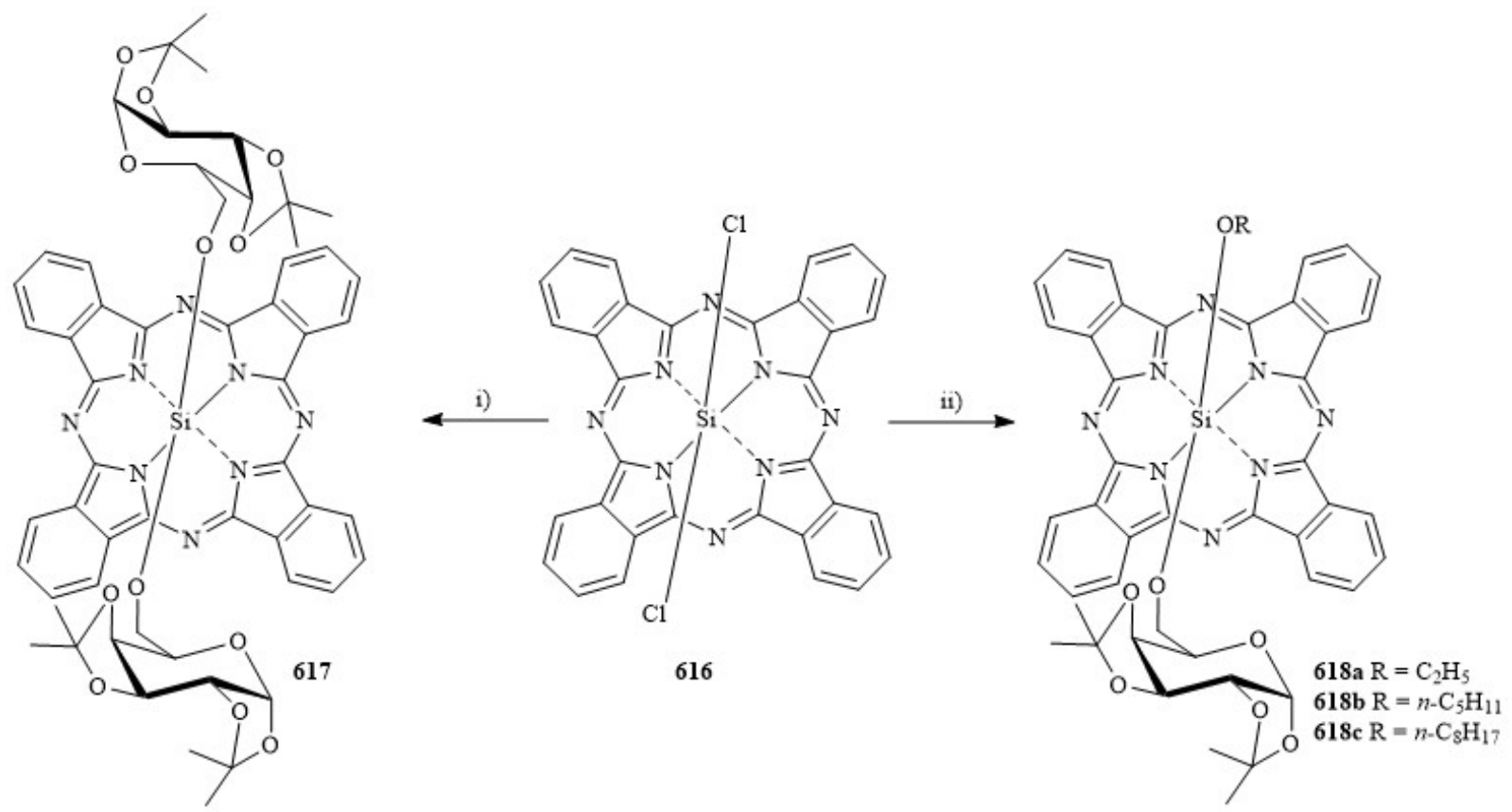

Scheme 37. Synthesis of amphiphilic silicon(IV) glycosylated phthalocyanines. i) (Gal-isop)-OH, NaH, toluene, reflux, 24 h; ii) (a) (Gal-isop)-OH, NaH, toluene, reflux, $24 \mathrm{~h}$ (b) $\mathrm{NaOC}_{2} \mathrm{H}_{5}$ or $\mathrm{NaOC}_{5} \mathrm{H}_{11}$ or $\mathrm{NaOC}_{8} \mathrm{H}_{17}$, toluene.

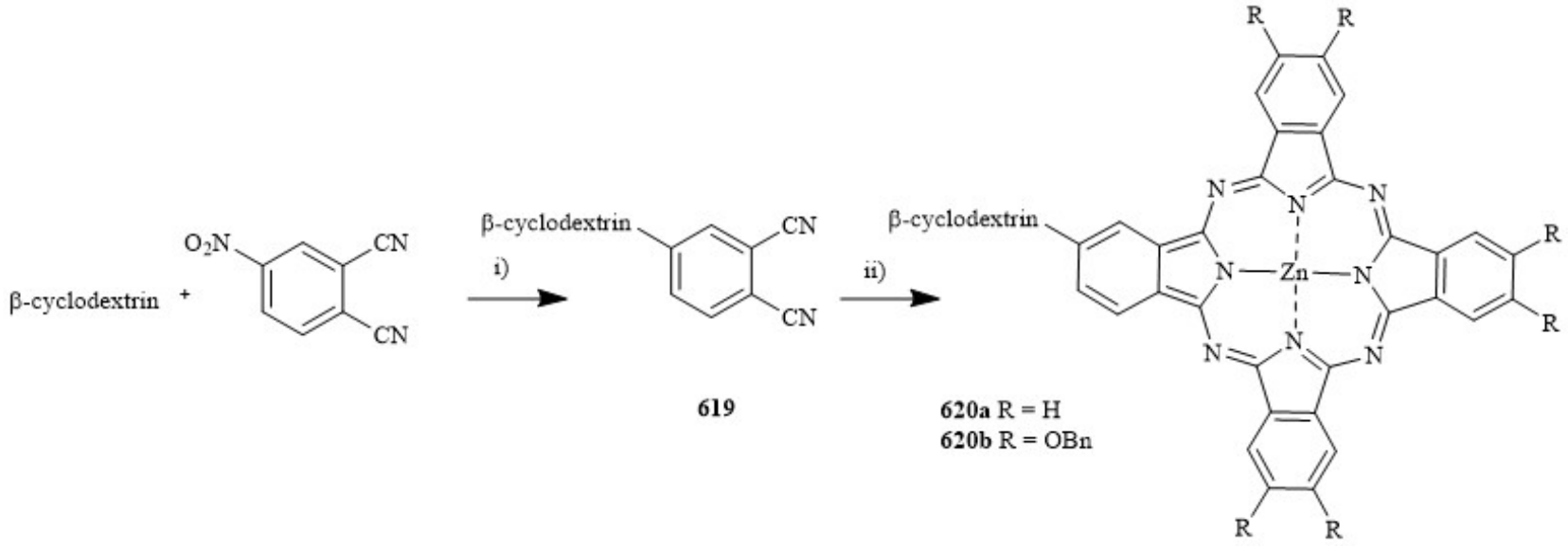

Scheme 38. Synthesis of the first cyclodextrin phthalocyanine dyad. i) $\mathrm{DMF}, \mathrm{K}_{2} \mathrm{CO}_{3}$, r.t.; ii) $\mathrm{DMAE}, \mathrm{ZnCl}_{2}$, reflux. 
With the success of the Si-phthalocyanine derivatives focus turned to the synthesis of the more stable tetra- and monoglycosylated zinc(II) phthalocyanines (Scheme 39). Both galactosyl (also reported by Soares et al. $\left.{ }^{[309]}, 604\right)$ and glucosyl (622) tetra-substituted derivatives were synthesized via DBU-promoted self-cyclisation in the presence of zinc acetate. In addition selectivity for the 1,8,15,22 isomer was observed when bulky groups were placed in the $\alpha$ position affording 625a and 625b from cerium promoted cyclisation reactions followed by metallation by zinc. Mixed condensations with the glycosubstituted phthalonitriles and phthalonitrile afforded $\mathrm{AB}_{3}$ type substituted derivatives 627a-d deprotection of the isopropylidene protecting groups proved problematic and was not carried out ${ }^{[310]}$.

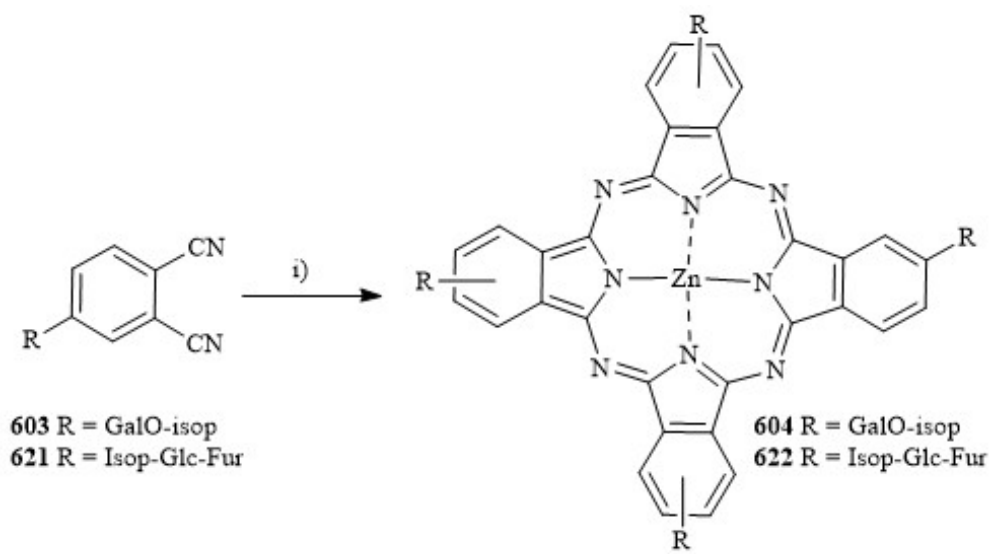<smiles>[R]c1cccc(C#N)c1C#N</smiles><smiles>[R]c1cccc(C#N)c1C#N</smiles>

Scheme 39. Synthesis of glycoconjugated phthalocyanines. i) $\mathrm{Zn}(\mathrm{OAc})_{2}$, DBU, $n-\mathrm{C}_{5} \mathrm{H}_{11} \mathrm{OH}$; ii) $\mathrm{CeCl}_{3}$, DBU, $n$ - $\mathrm{C}_{5} \mathrm{H}_{11} \mathrm{OH}$; iii) $\mathrm{Zn}(\mathrm{OAc})_{2}$, DMF; iv) Li, $n$ $\left.\mathrm{C}_{5} \mathrm{H}_{11} \mathrm{OH} ; \mathrm{v}\right) \mathrm{Zn}(\mathrm{OAc})_{2}$. 
In addition, mono- $\beta$, di- $\alpha-$, di- $\beta$ - and tetra-substituted glycosylated zinc(II) phthalonitriles (627-630) were synthesized with tetraethylene-glycol-linked saccharide units via self- and mixed cyclisation of glycosyl phthalonitrile and phthalonitrile in the presence of zinc acetate and DBU. Both the di- and tetra- derivatives were converted to the watersoluble conjugates $628 \mathrm{~b}-630 \mathrm{~b}$ and investigated to determine the effect of the number and position of glycosyl units on PDT activity ${ }^{[320]}$.
Organometallic couplings and glycosylations were also used for the synthesis of two series of amphiphilic nickel(II) phthalocyanines $(\mathbf{6 3 1}, 632)$ displaying an $\mathrm{AB}_{3}$ substitution pattern. A common hydroxylated phthalocyanine-nickel(II) was substituted with an ethyleneglycol spacer (633), a tetraethyleneglycol spacer (631) or an azidotetraethyleneglycol spacer (632). The both former underwent direct glycosylation in 70-90\% yield to afford glucose, galactose, mannose and lactose glycoconjugates

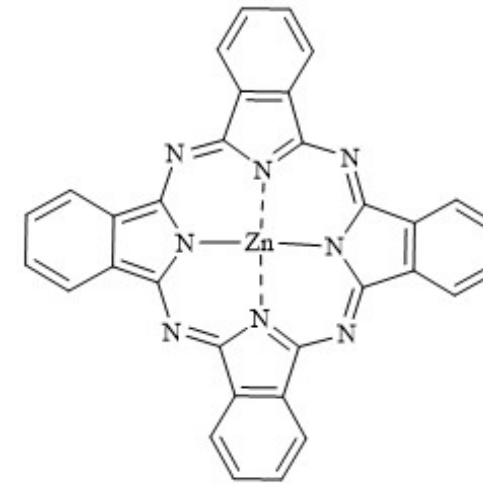

$627 \mathrm{R}=\left[\left(\mathrm{OC}_{2} \mathrm{H}_{4}\right)_{4}-\right.$ Isop-Glc-Fur $]$

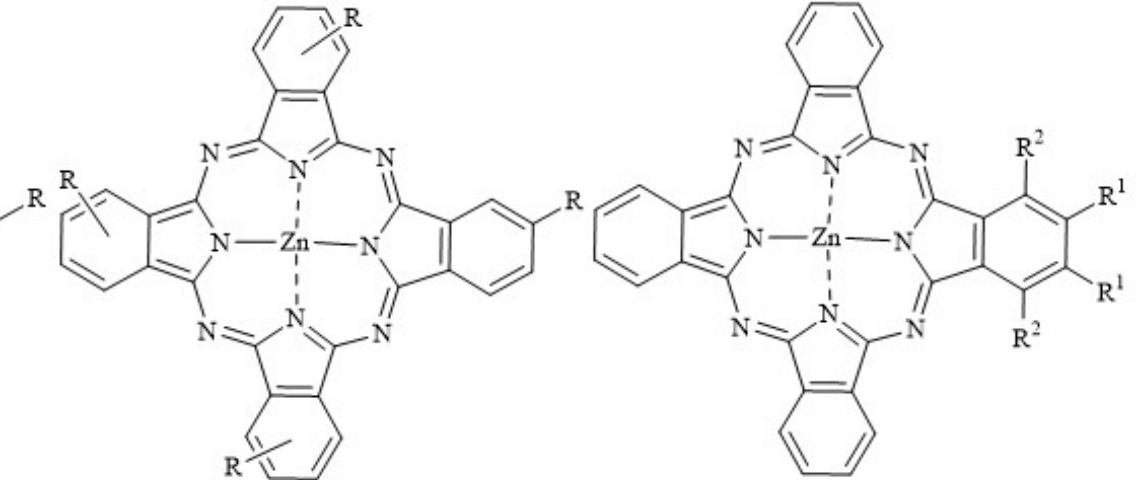

628a R $=\left[\left(\mathrm{OC}_{2} \mathrm{H}_{4}\right)_{4}-\right.$ Isop-Glc-Fur $]$ $628 \mathrm{~b} \mathrm{R}=\left[\left(\mathrm{OC}_{2} \mathrm{H}_{4}\right)_{4}-\mathrm{Glc}-\mathrm{Fur}\right]$

629a $\mathrm{R}^{1}=\left[\left(\mathrm{OC}_{2} \mathrm{H}_{4}\right)_{4}-\right.$ Isop-Glc-Fur $], \mathrm{R}^{2}=\mathrm{H}$ $629 b \mathrm{~b}^{1}=\left[\left(\mathrm{OC}_{2} \mathrm{H}_{4}\right)_{4}-\mathrm{Glc}-\mathrm{Fur}\right], \mathrm{R}^{2}=\mathrm{H}$ $630 \mathbf{a ~ R}^{2}=\left[\left(\mathrm{OC}_{2} \mathrm{H}_{4}\right)_{4}-\right.$ Isop-Glc-Fur $], \mathrm{R}^{1}=\mathrm{H}$ $630 \mathrm{bR}^{2}=\left[\left(\mathrm{OC}_{2} \mathrm{H}_{4}\right)_{4}-\mathrm{Glc}-\mathrm{Fur}\right], \mathrm{R}^{1}=\mathrm{H}$

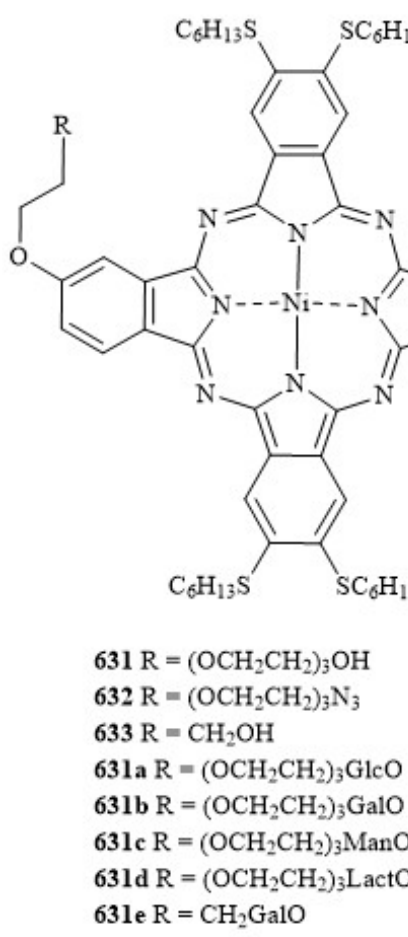

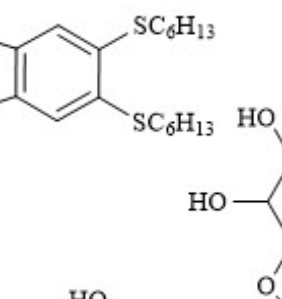<smiles>[R]C1CCCC1</smiles><smiles>CCc1cn(CCCOc2ccccc2)nn1</smiles>

$\mathrm{C}_{6} \mathrm{H}_{13} \mathrm{~S}$<smiles>[AsH2]c1ccccc1</smiles>

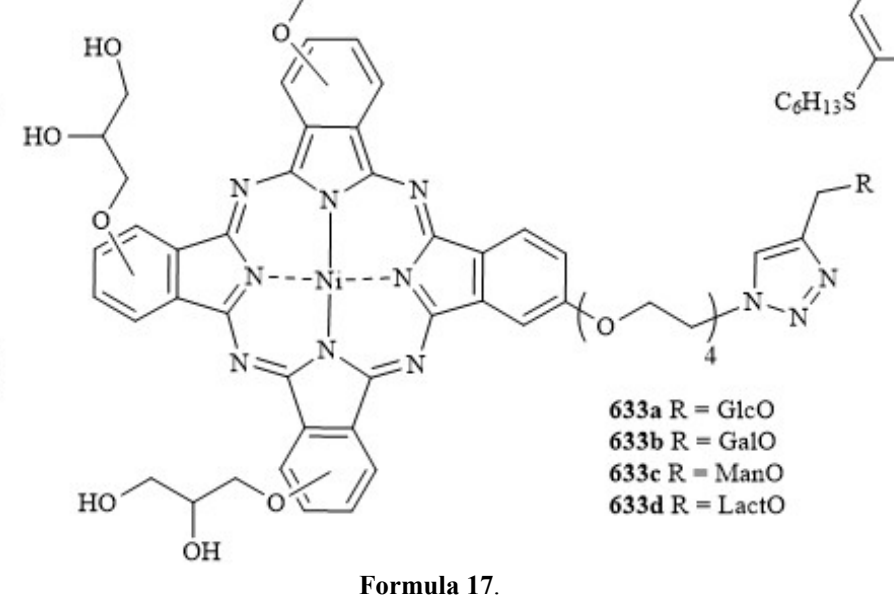


which were subsequently deprotected. With the benzoylated imidates providing better yields then the corresponding acetylated derivatives. The latter azido functionality underwent a click cycloadditon reaction with propargyl sugar units with subsequent deprotection providing 632a-d ${ }^{[321,322]}$. In expansion of this work, a series of water soluble asymmetric $\mathrm{AB}_{3}$ type glycophthalocyanine were synthesized with a single glycosyl unit and three glycerol moieties using the same click cycloadditon reaction. Glycoconjugates 633a-d were prepared in $\sim 95 \%$ yield and subsequently deprotected $^{[323]}$.

In extension of such work, Dumoulin et al. also synthesized two sets of zinc metallated glycosylated phthalocyanines to determine the difference in PDT efficacy of the glycosyl linked sugar unit versus the triazole bridge (Scheme 40 and 41) ${ }^{[324]}$. The compounds were synthesized

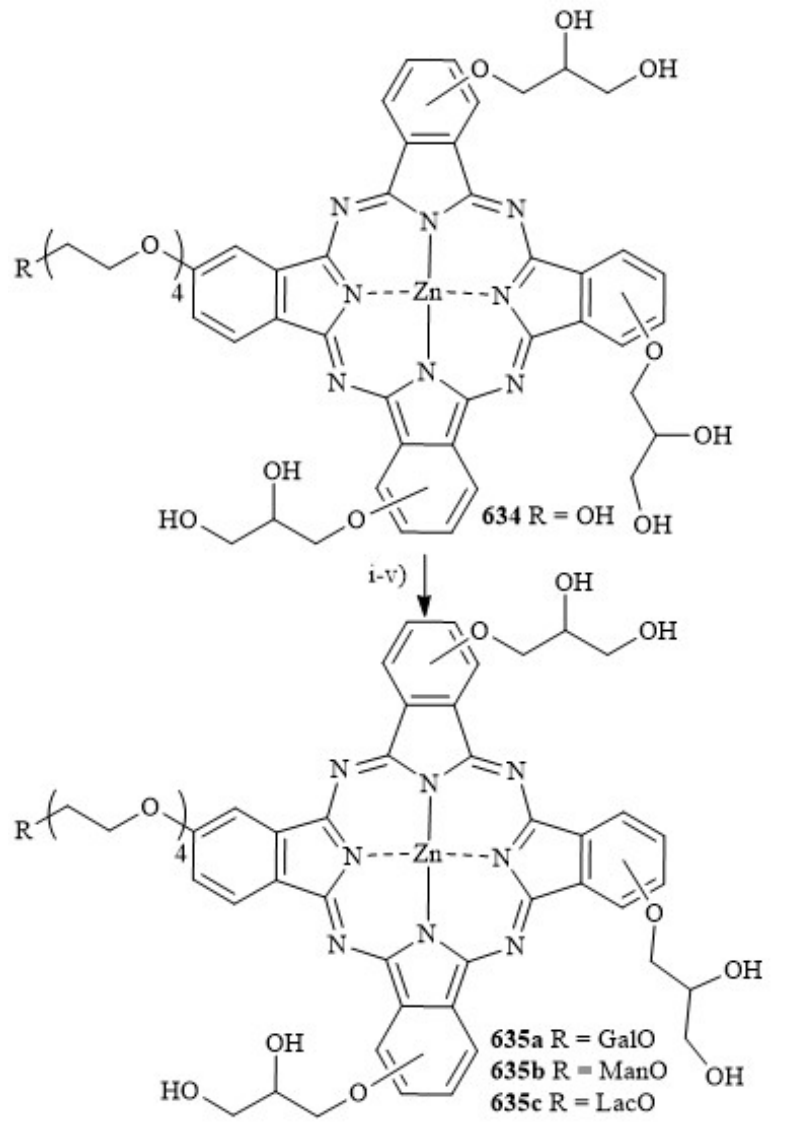

Scheme 40. Synthesis of glycoconjugated phthalocyanines. i) For 635a: 2,3,4,6-tetra- $O$-benzoyl- $\alpha$ - $D$-galactopyranosyl trichloroacetimidate, for 635b: 2,3,4,6-tetra- $O$-benzoyl- $\alpha-D$-mannopyranosyl trichloroacetimidate and for 635 : $\quad 4-O-(2,3,4,6$-tetra- $O$-benzoyl- $\beta$ - $D$-galactopyranosyl)-2,3,6-tri- $O$ benzoyl- $\alpha$ - $D$-glucopyranosyl trichloroacetimidate, TMSOTf ( 2 equiv.), -10 ${ }^{\circ} \mathrm{C}, \mathrm{CH}_{2} \mathrm{Cl}_{2}, 16 \mathrm{~h}$; ii) $80 \% \mathrm{AcOH}, 70{ }^{\circ} \mathrm{C}, 6 \mathrm{~h}$; iii) $\mathrm{MeONa}, \mathrm{MeOH}$; iv) $\mathrm{Ac}_{2} \mathrm{O}, \mathrm{C}_{5} \mathrm{H}_{5} \mathrm{~N}$; v) 2:1:1 MeOH-H $\mathrm{H}_{2} \mathrm{O}-\mathrm{Et}_{3} \mathrm{~N}, 2$ d, r.t. from mixed condensation of solketal and tetraethyleneglycol substituted phthalonitriles in the presence of $\mathrm{Zn}(\mathrm{OAc})_{2}$ affording $\mathbf{6 3 4}$ which could be converted to the alkyne derivative $\mathbf{6 3 6}$ for the triazole linkage. Glycosylation of $\mathbf{6 3 4}$ with 2,3,4,6-tetra- $O$-benzoyl- $\alpha$ - $D$-galactopyranosyl trichloroacetimidate, 2,3,4,6-tetra- $O$-benzoyl- $\alpha$-Dmannopyranosyl trichloroacetimidate and 4-O-(2,3,4,6-tetra$O$-benzoyl- $\beta$ - $D$-galactopyranosyl)-2,3,6-tri- $O$-benzoyl- $\alpha-D$ glucopyranosyl trichloroacetimidate was carried out in the presence of trimethylsilyl trifluoromethanesulfonate and subsequent deprotection affording 635a-c. Compounds 637a-c were synthesized via similar procedure by Lafont $e t$ al. ${ }^{[321,324]}$. Another example of post-cyclization functionalization includes the synthesis of octasubstituted carbamoyl glucosyl phthalocyanines and 1,4-substituted triazole glucosyl and galactosyl phthalocyanines all of which retained their $\beta$-configuration at the anomeric centre of the sugar units ${ }^{[325]}$.

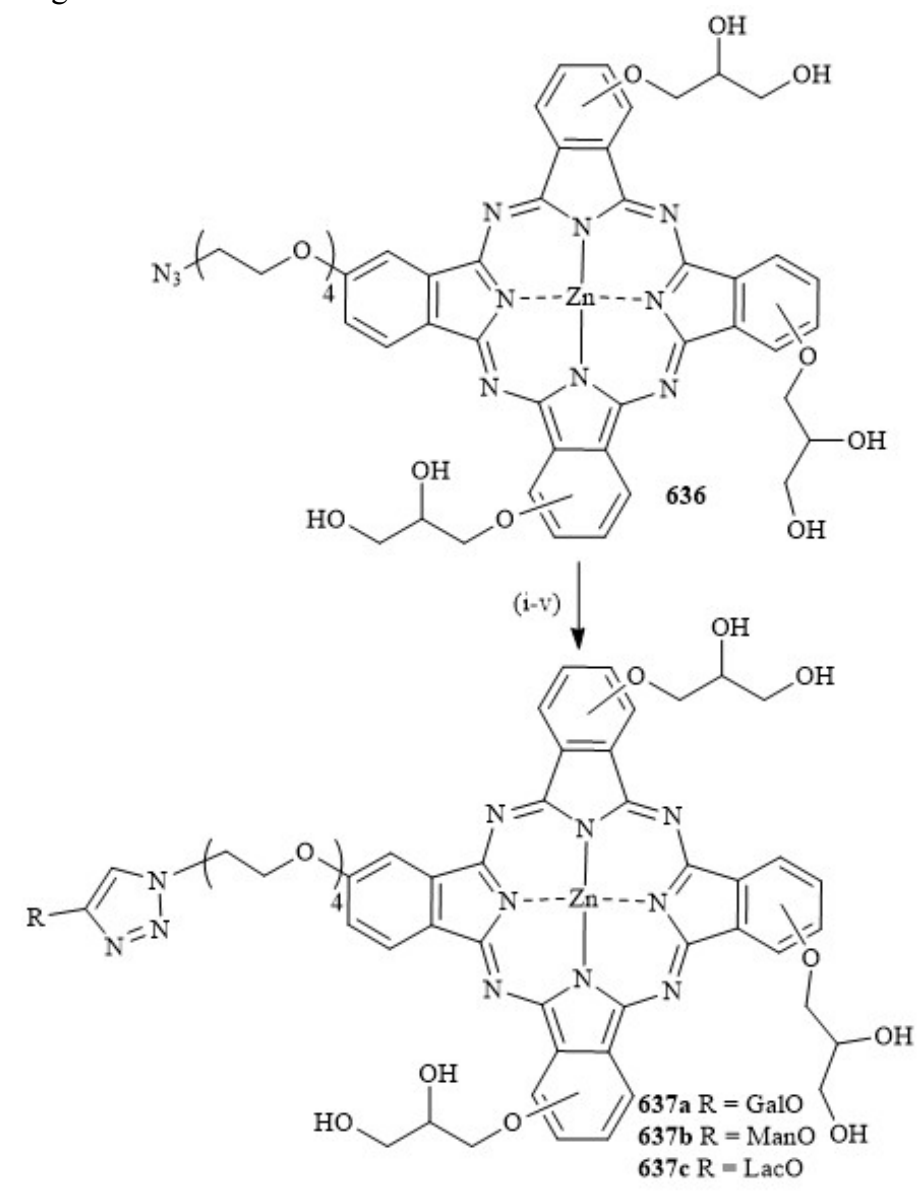

Scheme 41. Synthesis of glycosylated phthalocyanines via triazole linkage. i) $\mathrm{CuSO}_{4}$, sodium ascorbate, $\mathrm{CH}_{2} \mathrm{Cl}_{2}, \mathrm{H}_{2} \mathrm{O}$; ii) $80 \% \mathrm{AcOH}, 70{ }^{\circ} \mathrm{C}, 6 \mathrm{~h}$; iii) $\mathrm{MeONa}, \mathrm{MeOH}$; iv) $\mathrm{Ac}_{2} \mathrm{O}, \mathrm{C}_{5} \mathrm{H}_{5} \mathrm{~N}$; v) 2:1:1 $\mathrm{MeOH}-\mathrm{H}_{2} \mathrm{O}-\mathrm{Et}_{3} \mathrm{~N}, 2$ d, r.t. 


\subsection{Dual Modality Systems}

Increasingly attention is focused on dual modality systems where two or more different bioconjugate groups of different properties are present which have intrigued a number of researchers, including ourselves (Figure 6) ${ }^{[326]}$. As mentioned, some of the earlier studies on bioconjugates aimed to target porphyrins to steroid receptors. A classic example is Hombrecher's work to increase the degree of amphiphilicity in the target systems through preparation of a porphyrin with both a galactopyranosyl and a cholesterin unit. The porphyrin $\mathbf{4 0 2}$ was prepared in $5 \%$ yield using a mixed $2+2$ condensation of a sugar- and steroid dipyrromethane ${ }^{[68]}$. The porphyrin was easily incorporated into phosphatidyl ethanolamine vesicles and formed vesicles or aggregates in water ${ }^{[69]}$.

The first ever porphyrin-carbohydrate bioconjugate synthesized for a specific medicinal application remains to date one of the most complex and elaborate systems. Rakestraw et al. covalently linked a $\mathrm{Sn}(\mathrm{IV})$ chlorin $\mathrm{e}_{6}$ derivative to monoclonal antibody using a dextran linker group ${ }^{[327]}$. They first prepared a dextran carrier system through synthesis of a dextran carbazate with a terminal hydrazide which was functionalized by reaction with a hydroxybenzotriazole active ester of (chlorinato $\mathrm{e}_{6}$ )tin(IV). The tetrapyrrole unit was trace-labelled with ${ }^{113} \mathrm{Sn}$ to facilitate quantitation and the chlorin-dextran carrier system with a terminal hydrazide group was then linked to antimelanoma and anti-lymphoma monoclonal antibodies. The system showed excellent dose-dependent phototoxicity against SKMEL-2 human melanoma cells an effect only observed upon antibody binding to the cells. Note, this immunoconjugate system is also an efficient PS delivery system as about 10-15 tetrapyrrole units were bound per conjugate.
Clearly the use of targeting peptides in conjunction with carbohydrate units presents an intriguing possibility for the construction of new drugs. The first comprehensive study of this approach was provided by Sol et al. ${ }^{[207]}$. They prepared a series of porphyrins with meso glycosyl-aryl and alanylphenyl residues (292-297) and a number of compounds where the sugar unit was separated from the aryl residue by a serine unit $(\mathbf{2 9 8}, \mathbf{2 9 9})$. In the first approach, the nitrophenylporphyrin derivatives (274-279) were synthesized via Lindsey condensation, followed by oxidation with $p$ chloranil. Use of $p$-nitrobenzaldehyde and 2- or 4(2',3',4',6'-tetra- $O$-acetyl- $\beta$ - $D$ -

glucopyranosyloxy)benzaldehyde gave 274 and 275 in $14 \%$ and $16 \%$ yield, respectively. Different ratios of reagents, pyrrole, nitrobenzaldehyde and 4-(2',3',4',6'-tetra- $O$-acetyl$\beta$ - $D$-glucopyranosyloxy)benzaldehyde afforded maximum yields of compounds 275 (16\%), 276 (14\%), 277 (10\%) and $278(16 \%)$. Compound 29 was isolated as a side product in a yield of $11 \%$. The nitro functionalities were reduced using $\mathrm{H}_{2} / \mathrm{Pd}-\mathrm{C}$ yielding glycosylated aminoporphyrins $\mathbf{2 8 0}$ 285 in 56-76 \% yields followed by coupling with $N$-Fmoc- $L$ alanine provided 286-291. Deprotection of Fmoc and acetate groups yielded the respective mono-, di- and triamino acid glycoporphyrins 292-297 in 75-64\% yield. A second series of glycosylated amino acid porphyrins was synthesized where two tristolylporphyrins were substituted with glycosylated serine [259]. The synthesis of the mononitrophenyltristolylporphyrins $(\mathbf{3 0 0}, \mathbf{3 0 1})$ was carried out via condensation of pyrrole and $p$-tolylaldehyde with 2- or 4nitrobenzaldehyde in a ratio of $4 / 3 / 1$ to yield the mononitro derivatives in 14 and $15 \%$ yield. The nitro moieties were reduced $(302,303)$ and coupled with an $O$-glycosylated SerFmoc derivative, followed by deprotection of Fmoc and acetate groups providing monoglycosylamino acid porphyrins $(\mathbf{2 9 8}, \mathbf{2 9 9})^{[207,259]}$.
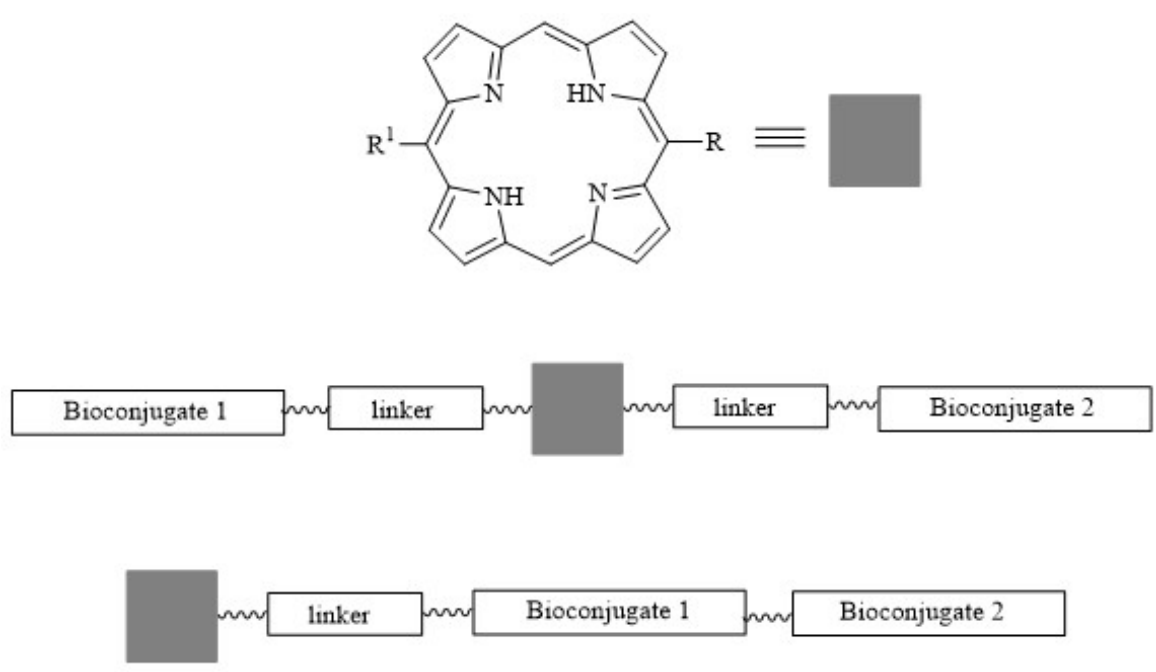

Figure 6. Dual modality systems. 


\section{MEDICINAL APPLICATIONS}

\subsection{Photodynamic Cancer Therapy (PDT)}

\subsubsection{Basic Principle of PDT}

Photodynamic therapy is used for the selective treatment of tumor cells. Intravenous or topological administration of a tumor specific PS drug, e.g., porphyrin derivatives, causes moderate retention of the drug in tumor tissue. The uptake of such PSs into neoplastic tissue is often greater than that of non-neoplastic tissue due to the EPR effect, previously discussed. Intracellular localization of PSs can be determined by fluorescence microscopy and some have been identified to localize in the mitochondria, endoplasmic reticulum, lysosomes, nuclei, plasma membranes and tumor vasculature ${ }^{[328]}$. Several documented cases suggest destruction of tumor vascular systems is of vital importance to the efficacy of PDT ${ }^{[12]}$. Most PDT PSs tend not to localize in the nuclei, reducing the risk of DNA damage and future carcinomas [329]. The localization of each individual PS is different. $m$ THPC has been identified to localize in the nuclear envelope ${ }^{[330]}$ while $\delta$-aminolevulinic acid (ALA) localizes in the mitochondria and methylene blue in lysosomes ${ }^{[331]}$.

The mechanism of uptake of PSs has not been clarified. It has been reported that the excess of low density lipoproteins (LDL) receptors on cancer cells, suggest LDL easily enter tumor cells. Due to the amphiphilic character of porphyrins it is known that they are carried by plasma lipoproteins ${ }^{[332]}$.

After localization the PS can be activated with visible or near IR light. De-excitation can then occur either via relaxation to the ground state (fluorescence, which may be used for diagnostic purposes) or via a photosensitization reaction with oxygen. The latter yields singlet oxygen (and other reactive oxygen species) which can damage cells within about $0.03 \mathrm{~nm}$ radius ${ }^{[7]}$ and has a lifetime of $\sim 3 \mu \mathrm{s}$ $[329,333]$. Singlet oxygen is a highly reactive species which causes irreversible photodamage to proteins, lipids, and nucleic acids in the affected cells, and plays a major role in vascular break down and immune response. Porphyrins derivatives are ideal PSs as they can produce singlet oxygen quite efficiently and absorb in the red region of the electromagnetic spectrum.

The method is non-invasive, mostly non-scarring, can be applied in an outpatient setting, and may be used in conjunction with other treatments such as surgery, radio- or chemotherapy. Side effects are often pain and prolonged photosensitivity. Its historical development is well documented ${ }^{[6,12]}$ and the potential of this approach is excellent.

At present PDT is an approved modality for the treatment of non small cell lung, cervical, bladder, head and neck, gastric and esophageal cancers ${ }^{[13,15]}$. It also has applications in the treatment of acne, skin cancer, cosmetic skin improvements, wrinkles and psoriasis [15,334,335]. Some examples of approved drugs for PDT include intravenous drugs Photofrin ${ }^{\circledR}$ (porfirmer sodium), Foscan ${ }^{\circledR}(m$ THPC) and topically drugs Levulan (ALA), Metvix (methyl aminolevulinate). In addition, PDT applications are currently under investigation in clinical trials for cancers of the prostate gland, cervix, head and neck, bladder, larynx, abdominal cavity, basal cell carcinoma, and brain with a range of second generation PSs.

Porphyrin based PSs traditionally dominated the field of PDT. Yet, the first generation PSs have many disadvantages including poor light absorption, long retention times, low tumor-tissue ratios, low water solubility and cause prolonged photosensitization of patients ${ }^{[150]}$. As a result an expansion of the field to second and third generation PSs has ensued to tackle the efficacy of PDT.

With regards to the development of new PSs specific characteristics apply to an 'ideal' PS which must be considered. The chemical purity and stability of a PS must be determined, i.e., the exact composition of the substance must be known, unlike first generation Photofrin ${ }^{\circledR}$ which consists of mixtures of monomers, dimers and oligomers, where only some of the components are biologically effective [7]. Knowledge of the optimum absorption, distribution, metabolism and excretion (ADME) properties are desirable. Minimal dark toxicity should be observed and the PS should only be toxic when activated by light of specific wavelengths. The PS should not produce toxic metabolites in vivo which itself could cause mutagenicity or carcinomas ${ }^{[15]}$. Fast clearance from cells and the body is optimum to reduce severe photosensitization side effects that can be quite debilitating to patients.

The diffusion of singlet oxygen is predicted as $2-4 \times 10^{-6}$ $\mathrm{cm}^{2} \mathrm{~s}^{-1}[336]$, therefore, tissue penetration of the light is extremely important for effective PDT treatment. As the absorption of light by tissue increases with wavelength decrease, longer excitation wavelengths are essential ${ }^{[150]}$. However, wavelengths beyond $800 \mathrm{~nm}$ are not desirable as the formation of singlet oxygen is difficult. $700-800 \mathrm{~nm}$ is the optimum range of wavelengths to increase light penetration, reduce light scattering and provide sufficient energy for singlet oxygen formation ${ }^{[150,337]}$. Porphyrins have $22 \pi$-electrons in an aromatic macrocycle which exhibit $\pi-\pi^{*}$ transition around $400 \mathrm{~nm}$ (Soret bands) and Q bands in the visible region. Two of the double bonds are not involved in the aromatic system and can therefore be reduced providing chlorins and bacteriochlorins ${ }^{[338]}$. This change in symmetry causes a bathochromic shift of the Q bands which exhibit higher extinction coefficients. Such shifts can be seen in the tolyporphin spectrum explained earlier. Bacteriochlorins such as tolyporphyrin have long wavelengths of 700-800 $\mathrm{nm}$ but they have the disadvantage 
that they are quite susceptible to oxidation making them the least stable derivative. In vivo, exposure to light can cause oxidation of these compounds making the chlorin derivative and changing the absorption of the chromophore leading to reduced efficiency of PDT.

Also, the PS must produce high singlet oxygen quantum yields for production of singlet oxygen and other ROS but undergo minimal photobleaching. Changes of the absorption of the molecules can be easily modified, however, the bioavailability and pharmacokinetics of such molecules is much harder to control ${ }^{[15]}$. For the development of a drug it is necessary to identify a target which will improve the therapeutic action of that drug. New PSs are required which exhibit significant selectivity for neoplastic tissue in high concentration leading to the development of third generation PSs i.e. target specific PSs. In the synthesis of such derivatives care must be taken not to lose the pharmacological properties of both moieties.

The solubility of PSs in aqueous media is essential for administration, however, the importance of amphiphilicity of such derivatives is related to their photodynamic activity. It has been proven by several groups that amphiphilic molecules versus hydrophilic and hydrophobic molecules show a greater degree of activity and can localize at the interface of hydrophobic-hydrophilic membranes ${ }^{[339,340]}$. Amphiphilicity can also effect the aggregation of PSs and in turn affect the photophysical properties of a PS ${ }^{[12]}$. Subcellular localization within organelles like the mitochondria is beneficial to obtain apoptotic instead of necrotic cell death which is favored.

The search for a novel PS that meets all the above chemical, physical and biological criteria is still ongoing. Also keeping in mind its production should be inexpensive and starting materials commercially available so as to sustain widespread production.

\subsubsection{Glycoporphyrins in PDT}

At a first glance one might assume that peripheral binding of sugar units to a porphyrin should not drastically alter its photophysical properties while gaining the benefit of increase water solubility and/or amphiphilicity. As always the reality is more complex. For example one of the earlier studies with a series of glucose or galactose (2- or 4-O) appended porphyrins of the $\mathrm{A}_{3} \mathrm{~B}$ - or $\mathrm{A}_{4}$-type showed that the singlet-state properties are affected by the configuration of the molecules. Likewise aggregation properties and hydrophobicity are influenced significantly. Even more importantly for drug delivery with liposomes an amphiphilic character was found to be beneficial. Thus, the $A_{3} B$ systems are clearly superior for incorporation into liposomes ${ }^{[341]}$. Clearly, both photophysics, uptake and photobiological studies must go hand in hand.

Some insight into the PDT utility of new compounds can also be gained from membrane incorporation experiments. Thus, during the initial phase of investigations on glycoporphyrins several studies investigated their incorporation into model membranes, notably into liposomes. For example, a comparative study by Voszka et al. utilized a series of glyco-tetraphenylporphyrin derivatives with glycosyl $\left(\mathbf{1 1 4}, \mathbf{1 1 6}\right.$ and 30) or xylosyl (42) residues ${ }^{[342]}$. The compounds differed in their degree of symmetry and hydrophobic character. Utilizing either neutral or negatively charged liposomes they found a strong effect of the liposomal surface charge and the negative surface charges of DMPC/DMPG liposomes helped with the association of the symmetrically substituted hydrophilic porphyrins to the liposomes. As expected, the association constants of the various compounds with liposomes followed their lipophilicity.

Likewise, tetra- and tri-meta-substituted TPP (39, 306) were investigated for their interaction with cell membranes compared to the non-glycoconjugate parent derivative $\mathbf{3 7}$ ${ }^{[208]}$. They were tested with a range of phospholipids spread at the air-water interface or grafted onto an RP-LC column. In the absence of phospholipids all porphyrin derivatives were able to form monolayers at the air-water surface interface. Although 37 and $\mathbf{3 0 6}$ have completely different chemical structures both lowered the surface tension to the same extent. However, 306 displayed reduced stability over time with an increase in surface tension observed after $10 \mathrm{~h}$ as a result of rearrangement of its asymmetric structure. For 39 the decrease in surface tension was very low compared to the other derivatives but appeared more stable over time compared to 306. The interfacial behavior of the three porphyrin derivatives were very different in their interactions in mixtures with distearoylphosphatidylcholine (DSPC) and diarachidoylphosphatidylcholine (DAPC) phospholipids and the phospholipid monolayer model is a good initial method to determine cellular uptake of such glycoporphyrins ${ }^{[208]}$.

Many of the more basic PDT-related studies have been described above. However, as a natural compound tolyporphin A deserves a special mention in investigations of glycoporphyrins as PSs. It was identified to be potent against EMT-6 tumor cell lines, where it localizes in the endoplasmic reticulum (ER), and also in vivo in tumors implanted on the backs of immunodeficient mice ${ }^{[343]}$. Its long wavelength absorption at $675 \mathrm{~nm}$ and its relatively good water solubility made it an excellent candidate for PDT. It showed exceptional activity and produced more effective photosensitization compared to Photofrin ${ }^{\circledR}$ both in vitro and in vivo. The higher photocytotoxicity was not attributed to a higher quantum yield of singlet oxygen or absorbance, as they are similar, if not the same as Photofrin ${ }^{\circledR}$, therefore, other factors played a role i.e. the localization of the compound within the cell ${ }^{[343]}$. Irradiation for $1 \mathrm{~h}$ at $0.1-0.5$ $\mathrm{J} / \mathrm{cm}^{-2}$ at drug dose concentrations of $0.02-0.1 \mu \mathrm{M}$ showed Tolyporphin to be $\sim 5000$ times more effective in PDT then Photofrin ${ }^{\circledR}$. Photofrin ${ }^{\circledR}$ localizes in the plasma membrane, lysosomes and mitochondria compared to tolyporphin which is exclusively in the ER and nuclear membrane ${ }^{[344]}$. As well as its exceptional activity in vivo with regard to PDT, also highlighted was the unusual biodistribution of tolyporphin 
compared to Photofrin ${ }^{\circledR}$ or other second generation PS. It displayed higher delivery to tumor tissue compared to significantly less extraction by the liver where the opposite applies to Photofrin ${ }^{\circledR}$. Its amphiphilicity is also essential for its pharmacokinetic behavior and its biodistribution. Further investigations into the use of tolyporphin derivatives for PDT are limited due to the tedious preparation via total synthesis ${ }^{[343]}$.

\subsubsection{In Vitro Studies}

A significant number of in vitro studies have been reported for glycoporphyrins and related bioconjugates. The main results of the individual studies are compiled in Table 3 and, for ease of access, have been sorted by cell line. For the subsequent discussion an ordering by basic porphyrin type has been choosen.

Table 3. In vitro PDT studies with glycoporphyrin conjugates.

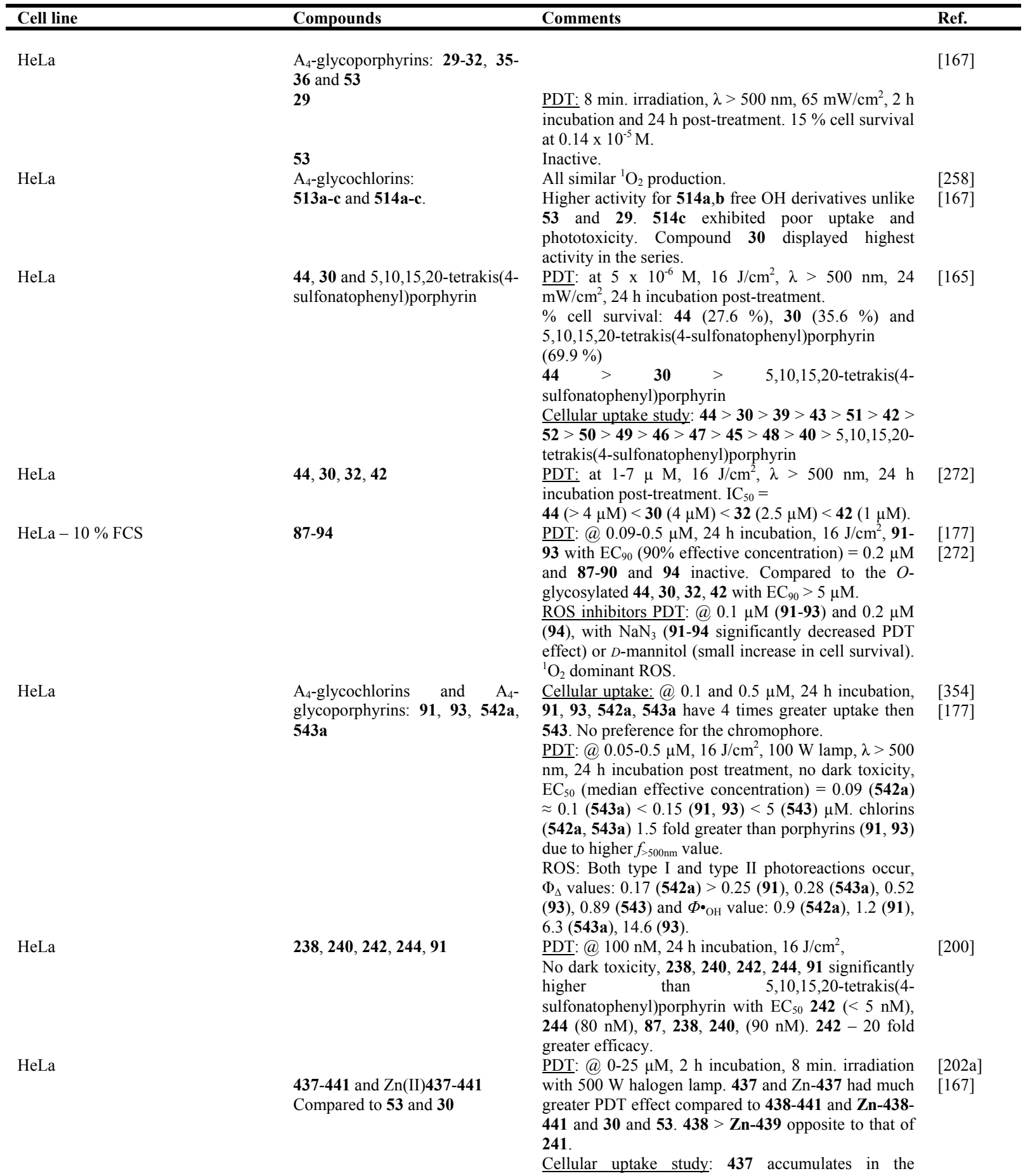


HeLa

HeLa

HeLa

HeLa

HeLa

HeLa
Cyclodextrinporphyrins:

638d and 638a

A 4 -glycochlorins:

514d-g

$\mathrm{A}_{4}$-glycochlorins: 514a,b, 514d-

i

PDT: 514d,f $A_{4}$-glycoporphyrin 30

PDT: 30, 32, 39-44, (514a,b, 514d-i), 45-52 and 5,10,15,20tetrakis(4-

sulfonatophenyl)porphyrin

$\mathrm{A}_{4}$-glycochlorins glycoporphyrins: 542a, 542b, 542a', 542b' and 87, 91, 95, 96

Glycophthalocyanines: 605, 608 and 598

583 - 3 derivatives of varying content of Pheophorbide a cytoplasm. 441 displayed no uptake. Compounds 438 showed considerable uptake.

PDT: @ 5 and $10 \mu \mathrm{M}, 0-4.2 \mathrm{~J} / \mathrm{cm}^{2}, 24$ incubation, no dark toxicity 638d $>$ 638a. Via both apoptosis and necrosis.

${ }^{1} \mathrm{O}_{2} \Phi_{\Delta}: 1.3$ to 0.6 .

PDT: @ $0.2 \mu \mathrm{M}, 16 \mathrm{~J} / \mathrm{cm}^{2}, 100 \mathrm{~W}$ lamp, $\lambda>500 \mathrm{~nm}$, $24 \mathrm{~h}$ incubation, no dark toxicity, 514f $>$ 514d $>$ 514e $>\quad \mathbf{5 1 4 g}, \quad 5,10,15,20$-tetrakis(4sulfonatophenyl)porphyrin

Cellular uptake studies: @ $5 \mu \mathrm{M}, 24$ h incubation, $514 d>514 i>514 \mathrm{e}>514 \mathrm{a}>514 \mathrm{~b}>514 \mathrm{~h}>514 \mathrm{f}>$

$\mathbf{5 1 4 g}>\quad 5,10,15,20$-tetrakis(4sulfonatophenyl)porphyrin. 514d 50 times greater than 5,10,15,20-tetrakis(4-sulfonatophenyl)porphyrin. $30 \approx 514 f$

PDT: @ $0.2 \mu \mathrm{M}, 25 \mathrm{~mW} / \mathrm{cm}^{2}, 100$ Watt lamp, $\lambda$ > $500 \mathrm{~nm}, 48 \mathrm{~h}$ incubation, $\mathrm{LD}_{50}=0.2(\mathbf{5 1 4 f}), 5(\mathbf{3 0})$ $\mu$ M. 514f 25 times greater than 30 .

PDT: @ $5 \mu \mathrm{M}, 16 \mathrm{~J} / \mathrm{cm}^{2}, 24 \mathrm{~h}$ incubation, no dark toxicity, $\mathrm{EC}_{50}<5 \mu \mathrm{M}(\mathbf{4 9}, \mathbf{4 6}, \mathbf{5 0}, \mathbf{5 2}, \mathbf{4 2}, \mathbf{3 9}, \mathbf{4 0}, \mathbf{4 1}$, 43, 514a, 514b, 514f, 514g, 514d, 514e), $\mathrm{EC}_{90}=0.2$ $(\mathbf{5 1 4 f}), 0.5(\mathbf{3 0}, \mathbf{5 1}) \mu \mathrm{M}$. 514d-g proved potent @ 5 $\mu \mathrm{M}$.

$\mathrm{EC}_{50}=$ Zinc porphyrin $(5 \mu \mathrm{M}, 45)<$ porphyrin $(4 \mu \mathrm{M}$, 30) $<$ chlorin $(0.7 \mu \mathrm{M}$, 514a $)$ i.e. chlorin $6 \times$ more potent. $\mathrm{EC}_{50}=$ meta-position $(39 @ 0.5 \mu \mathrm{M})<$ paraposition (30@4 $\mu \mathrm{M})$.

514a molar extinction co-efficient 3.7 fold greater than 30.

${ }^{1} \mathrm{O}_{2}$ dominant ROS

PDT: @ $0.5 \mu \mathrm{M}, 1-24 \mathrm{~h}$ incubation, 0 or $16 \mathrm{~J} / \mathrm{cm}^{2}, 30$ min., 100 Watt lamp, $\lambda>500 \mathrm{~nm}, 24 \mathrm{~h}$ incubation post treatment, no dark toxicity,

Glycoconjugates $>\mathbf{5 4 1}>\mathbf{8 4}$

87,96 and 542a' 542b' - inactive

91 and 542a 542b - potent

$542 a>542 b, 541>542 a$ '

$542 \mathrm{a}$ - apoptotic cell death

${ }^{1} \mathrm{O}_{2}$ dominant $\mathrm{ROS}$

PDT: @ $0.05-3 \mu \mathrm{M}, 0$ or $16 \mathrm{~J} / \mathrm{cm}^{2}, 30 \mathrm{~min} ., 100 \mathrm{~W}$ lamp, $\lambda>500 \mathrm{~nm}, 24 \mathrm{~h}$ incubation post treatment, $\mathrm{EC}_{50}=0.07$ (542a), 0.15 (91), 0.3 (542b), 1.5 (95) $\mu \mathrm{M}$. Glucosyl (542a) > galactosyl (542b) and chlorin (542a) > porphyrin $(91)$

Cellular uptake: @ $0.2 \mu \mathrm{M}, 1-24$ h incubation, 542b, 95 saturated at $12 \mathrm{~h}, \mathbf{5 4 2 a}, 91$ increase uptake up to 24 h. Glucosyl (542a) > galactosyl (542b)

Cellular uptake: @ 4 and 18 h incubation,

Low level-605, 608@4 h 598

High level@18h 598

PDT: $5 \mu \mathrm{M}$ and $10 \mu \mathrm{M}(605,608), 0.5$ and $1 \mu \mathrm{M}$ (598), 5-30 min., $\lambda=636 \mathrm{~nm}, 1.86-11.16 \mathrm{~J} / \mathrm{cm}^{2}$, no dark toxicity, Low activity for $\mathbf{6 0 5}$, extensive toxicity with 598, $\Phi_{\Delta}=0.69$ (605), $0.76(\mathbf{6 0 8}), 0.82$ (598). Localization: Golgi apparatus and lysosomes $\mathbf{- 5 9 8 .}$

Cellular morphology 598: $0.5 \mu \mathrm{M}, 4 \mathrm{~h}$ incubation, 15 min.,

(a) $3 \mathrm{~h}$ mitotic arrest - gradual cell retraction

(a) 24/48 h-apoptotic cells.

$1 \mu \mathrm{M}, 4 \mathrm{~h}$ incubation, $30 \mathrm{~min}$. - necrotic cells death.

Autoquenching in aqueous medium: - decrease with increased pheophorbide a content.

$\Phi \Delta: \sim 0.5$ similar for all in DMF, 0 in aqueous medium.

Cellular uptake: $6 \mathrm{~h}$ incubation: very rapid uptake, forming perinuclear spots in cytoplasm; possible internalization via endocytosis.

Addition of a hyaluronic acid polymer reduced $\mathbf{5 8 3}$ uptake; hyaluronic acid receptor mediated uptake. Fluorescence increases over time; 583 disintegrated by enzymes and release of Pheophorbide a.

PDT: @ 0.0025- $2.5 \mu \mathrm{g} / \mathrm{mL}, 1 \mathrm{~mW} / \mathrm{cm}^{2}, 10 \mathrm{~min}$. 
HeLa

HeLa Cells - STxB receptor (Gb3) cell line
584 - 3 derivatives of varying content of Pheophorbide a

114, 332-STxB and STxB-Cys irradiation, slight dark toxicity, $\mathbf{5 8 3} \approx$ Pheophorbide a.

Autoquenching in aqueous medium:

In organic solvent: - fluorescence similar to

Pheophorbide a

Aqueous medium: Autoquenching

Addition of esterase to aqueous medium: $8.5 \mathrm{U} / \mathrm{mL}$, surfactant $\left(0.1 \%\right.$ Tween $\left.80^{\circledR}\right)$; increase fluorescence.

Cellular uptake: $30 \mathrm{~min}$ - $24 \mathrm{~h}$ : increase fluorescence over time; enzymatic cleavage.

Localization: @ $1.5 \mu \mathrm{g} / \mathrm{mL}$ of Pheophorbide a content, 1, 6, $12 \mathrm{~h}, 37^{\circ} \mathrm{C} ; \mathbf{5 8 4}$ - endosomes to lysosomes

PDT: @ 0.125 - $1.25 \mu \mathrm{g} / \mathrm{mL}$ of Pheophorbide a content, $12 \mathrm{~h}$ incubation, $1.2 \mathrm{~J} / \mathrm{cm}^{2}, 670 \mathrm{~nm}$;

$\mathbf{5 8 4} \approx$ Pheophorbide a, $\mathrm{IC}_{50}=\sim 0.2 \mu \mathrm{g} / \mathrm{mL}$. Apoptotic cell death.

Immunofluorescence analysis: @ $1 \mu \mathrm{M}$ 332-STxB or STxB-Cys, 30 min. incubation @ $4{ }^{\circ} \mathrm{C}, 45 \mathrm{~min}$. incubation @37 ${ }^{\circ} \mathrm{C} ; 332-\mathrm{STxB} \approx \mathrm{STxB}-\mathrm{Cys}$

Receptor binding: $20 \mathrm{nM}\left[{ }^{125} \mathrm{I}\right] \mathrm{STxB}-\mathrm{Glyc}-\mathrm{KDEL}, 30$ min. incubation, 0 or $4 \mathrm{nM}-2.5 \mu \mathrm{M} 332-\mathrm{STxB}$ or STxB-Cys; 332-STxB $>$ STxB-Cys.

$\Phi_{\Delta}=0.13$ (332-STxB), 0.4 (114); 332-STxB 4 times lower.

PDT: @ 1,6 $\mu \mathrm{M}, \lambda>540 \mathrm{~nm}, 15 \mathrm{~min}$. irradiation, 1.8 $\overline{\mathrm{J} / \mathrm{cm}^{2}}, 2 \mathrm{~mW} / \mathrm{cm}^{2}, 24 \mathrm{~h}$ incubation, no dark toxicity; $\mathrm{LD}_{50}=0.6$ (332-STxB), 3 (114) $\mu \mathrm{M}$.

332-STxB > 114: Golgi apparatus - higher PDT.

Presence of Gb3 inhibitor (1-phenyl-2-hexadecanoylamino-3-morphorpholin-1-propanol

(PPMP)): 114 - no change

332-STXB - inactive.

Presence of brefeldin A (induces the accumulation of STxB in early endosomes): reduced PDT of $\mathbf{3 3 2}$ STxB - early endosomes

Human breast adenocarcinoma Tetrabenzoporphyrins: 111 and PDT: $9 \times 10^{-6}-10^{-2} \mathrm{mg} / \mathrm{mL}, 2 \mathrm{~h}$ irradiation, $5.5 \times 10^{-2}$ MCF-7 113

Human breast adenocarcinoma 4 MCF-7 - drug sensitive

Human breast adenocarcinoma 4 $\mathrm{MCF}-7 / \mathrm{ADR}$ - drug resistant

Human breast cancer cell lines 91 and $\mathbf{9 5}$ MDA-MB-231

Cellular uptake study: @ $10 \mu \mathrm{M}, 24$ h incubation, 91
$\mathrm{mW} / \mathrm{cm}^{2}, \quad \mathrm{IC}_{50}=78.7$ (Photofrin $\AA$ ), 971.5 (111) $\mathrm{ng} / \mathrm{mL}$.

$111<$ Photofrin; 113 ineffective.

Chemosensitization by $\mathbf{4}$ and verapamil:

Absence of anticancer drugs: $\mathrm{IC}_{50}=\sim 5.4 \pm 1.1 \mu \mathrm{M}$.

Presence of anticancer drugs (daunomycin, taxol, actinomycin $\mathrm{D}$, colchicine, cisplatin, melphalan): 0,1 , $2 \mu \mathrm{M}$ of 4 compared to $10 \mu \mathrm{M}$ of verapamil. - no significant effects on the $\mathrm{IC}_{50}$ values.

$\left[{ }^{3} \mathrm{H}\right]$-vinblastine transport:

$60 \mathrm{~min}$. incubation - control

$60 \mathrm{~min}$. incubation, 2 or $5 \mu \mathrm{M}$ of 4 or $10 \mu \mathrm{M}$ of verapamil. - no increase.

Chemosensitization by 4 and verapamil:

Absence of anticancer drugs: $\mathrm{IC}_{50}=6.8 \pm 0.2 \mu \mathrm{M}$

Presence of anticancer drugs (daunomycin, taxol, actinomycin $\mathrm{D}$, colchicine, cisplatin, melphalan): 0,1 , $2 \mu \mathrm{M}$ of 4 compared to $10 \mu \mathrm{M}$ of verapamil significantly reduced the resistance for daunomycin, taxol, actinomycin D and colchicine. No increased toxicity for cisplatin and melphalan.

$\left.{ }^{3} \mathrm{H}\right]$-vinblastine transport:

60 min. incubation - significantly lower concentration of $\left[{ }^{3} \mathrm{H}\right]$-vinblastine.

$60 \mathrm{~min}$. incubation, 2 or $5 \mu \mathrm{M}$ of 4 or $10 \mu \mathrm{M}$ of verapamil. $4>>$ verapamil; $>\mathbf{9 5}, 2.3$ fold greater

91 PDT: @ 10 or $20 \mu \mathrm{M}, \sim 11.3 \mathrm{~kJ} / \mathrm{m}^{2}$ (20 min. at $0.94 \mathrm{~mW} / \mathrm{cm}^{2}$ ): $20 \%$ and $>60 \%$ of the cells, respectively, were necrotic immediately and $100 \%$ at $24 \mathrm{~h}$ via apoptosis.

95 PDT: @ $20 \mu \mathrm{M}, \sim 1.6 \mathrm{~kJ} / \mathrm{m}^{2}$ (10 min. at 0.27 $\left.\mathrm{mW} / \mathrm{cm}^{2}\right)$ cell death in part by apoptosis.

Cell migration study: @ $10 \mu \mathrm{M}, 0.75 \mathrm{~kJ} / \mathrm{m}^{2}$ (5 min. at $\left.0.25 \mathrm{~mW} / \mathrm{cm}^{2}\right)-$ migration was reduced. @ $1 \mu \mathrm{M}$ - 
Human breast cancer cell lines MDA-MB-231

Human breast cancer cell lines MDA-MB-231
$\mathrm{A}_{4}$-glycochlorins: 542a,a $\mathrm{A}_{4}$-glycoisobacteriochlorins: 545a,a'

$\mathrm{A}_{4}$-glycobacteriochlorins:

546a,a'

Combinatorial library

341-363, 91

91 and 95

partially transformed $3 \mathrm{Y} 1$ overexpressing c-Src $\left(3 \mathrm{Y}^{\mathrm{c}-\mathrm{Src}}\right)$ and fully transformed $3 \mathrm{Y} 1$ cells (3Y $1^{\mathrm{v}}$ Src)

Human chronic leukemia K562

Human chronic leukemia K562

Human chronic leukemia K562

221,222

Human chronic leukemia K562

Human chronic leukemia K562

Human chronic leukemia K562

Human chronic leukemia K562

meso-Strapped glycoporphyrins: 390 and 392

231-236 and 456b-458b

Human keratinocyte cell line $\mathrm{HaCaT}$

Tetrabenzoporphyrins: 111 and 113

Human keratinocyte cell line $\mathrm{HaCaT}$ - non tumorigenic

Human leukemia T-cells Molt4
Glycophthalocyanines: $\mathbf{5 9 8}$

Purpurin imides:569a/b, 570 no necrosis or apoptosis however, significantly less aggressive in cell migration.

Fluorescence imaging: @ 25 nM of 546a; high [274]

fluorescence quantum yield

$\Phi_{\Delta}=0.28(\mathbf{5 4 2} \mathbf{a}$, DMSO) $<0.32(\mathbf{5 4 2 a}$, ethanol $)<$ 0.59 (545a) $<0.85(\mathbf{9 1})$.

Cellular uptake: @ 1-5 $\mu \mathrm{M}$ each porphyrin, $100 \mu \mathrm{M}$ total porphyrin content, $24 \mathrm{~h}$ incubation, up-taken porphyrins were isolated.

Fluorescence imaging of 91, 341, 342, 350, 351: @

2.5 or $10 \mu \mathrm{M}, 24 \mathrm{~h}$ incubation, @ $24 \mathrm{~h}$ and 1 week imaging@10 $10 \mathrm{M} 1$ day and 1 week $91 \approx 350>341$. Poor uptake of 342, 351. @ $2.5 \mu \mathrm{M}, 24 \mathrm{~h}$ imaging: all weak fluorescence $@ 2.5 \mu \mathrm{M}, 1$ week imaging: 91 > $350>$ 341. Conjugates display initial uptake by aggregation.

PDT of 91, 350, 341: @ 2.5 or $10 \mu \mathrm{M}, 20 \mathrm{~h}$ incubation, $20 \mathrm{~min}$. irradiation, $13 \mathrm{~W}$ fluorescent bulb, $0.9 \mathrm{~mW} / \mathrm{cm}^{2}, 10.8 \mathrm{~kJ} / \mathrm{m}^{2}$,

@ $10 \mu \mathrm{M}:$ 91, 350, $34125 \%$ immediate necrotic cell death, after 24 h 91, $350>80 \%$ apoptotic cell death and $341<60 \%$

(a) $2.5 \mu \mathrm{M}, 91,35040 \%$ necrotic, $>60 \%$ apoptotic. Cellular uptake of 352-363,91: @ $5 \mu \mathrm{M}, 20 \mathrm{~h}$ incubation, 361, 91 - best uptake: - cationic species better binders.

Cellular uptake study 91: no uptake in 3Y1,

Increase in the order $3 \mathrm{Y} 1<3 \mathrm{Y} 1^{\mathrm{c}-\mathrm{Src}}<3 \mathrm{Y} 1^{\mathrm{v}-\mathrm{Src}}$ with affinity $1: 2.3: 3.2$

91 PDT: $7 \mathrm{~min} ., \lambda>500 \mathrm{~nm}, 0.84 \mathrm{~mW} / \mathrm{cm}^{2}(3.5$

$\mathrm{kJ} / \mathrm{m}^{2}$ ), $24 \mathrm{~h}$ incubation with 91 , both apoptosis and necrosis in the order

$3 \mathrm{Y}^{\mathrm{v}-\mathrm{Src}}>3 \mathrm{Y}^{\mathrm{c}-\mathrm{Src}}>3 \mathrm{Y} 1$

PDT: $2 \mu \mathrm{M}, 50 \mathrm{~W} / \mathrm{m}^{2}, 24 \mathrm{~h}$ incubation -

No effect due to poor cell permeability.

PDT: $2 \mu \mathrm{M}, 30,60,90$ and $120 \mathrm{~min} ., 10 \mathrm{~mW} / \mathrm{cm}^{2}, 24$

h incubation, Photofrin ${ }^{\circledR} \approx \mathbf{3 6 8}>\mathbf{3 7 0}$.

$\mathbf{3 6 5} o, p$ and $\mathbf{3 6 9}$ - low activity.

Similar ${ }^{1} \mathrm{O}_{2}$ generation as HP.

PDT activity: @ $2 \mu \mathrm{M}(\mathbf{2 2 1}, \mathbf{2 2 2})$ or $1.25 \mu \mathrm{g} \cdot \mathrm{mL}^{-1}$ $\left(\right.$ Photofrin $\mathrm{II}^{\text {R }}$ ), $18 \mathrm{~h}$ incubation, white light, 0-120 minutes. Photofrin ${ }^{\mathbb{R}}>\mathbf{2 2 1}>\mathbf{2 2 2}$. No dark toxicity and early necrosis observed but absence of late necrosis/apoptosis

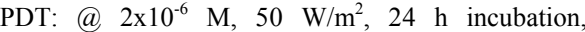
irradiation time $0-100 \mathrm{~min}$., less effective than Photofrin. ${ }^{1} \mathrm{O}_{2}$ production similar to HP. Primarily, apoptotic cell death.

PDT: @ $2 \mu \mathrm{M}, 10 \mathrm{~mW} / \mathrm{cm}^{2}, 30,60,90,120 \mathrm{~min} ., 0 \mathrm{~h}$ and $24 \mathrm{~h}$ incubation, at $120 \mathrm{~min}$. proved less effective than Photofrin; induced apoptosis.

${ }^{1} \mathrm{O}_{2}$ production similar to HP.

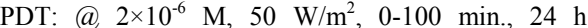
incubation, $\mathrm{HP}>\mathbf{3 9 0}>\mathbf{3 9 2}$

PDT: @ $2 \times 10^{-6} \mathrm{M}(\mathbf{2 3 1 - 2 3 6}$ and 456b-458b) and 1.2 $\mu \mathrm{g} / \mathrm{mL}$ (Photofrin ${ }^{\circledR}$ ), white bulb, $60 \mathrm{~W} / \mathrm{m}^{2}, 0-120$ min., $24 \mathrm{~h}$ incubation, 231, 233-235 all comparable to Photofrin $^{(B)}$ when irradiation time $>60$ min. 456b458b $>$ Photofrin ${ }^{\circledR}$.

Early necrosis and induced apoptosis.

PDT: $9 \times 10^{-6}-10^{-2} \mathrm{mg} / \mathrm{mL}, 2 \mathrm{~h}$ irradiation, $5.5 \times 10^{-2}$ $\mathrm{mW} / \mathrm{cm}^{2}, \mathrm{IC}_{50}=1224$ (Photofrin $\left.{ }^{\circledR}\right), 8414.6$ (111) $\mathrm{ng} / \mathrm{mL}$

$111<$ Photofrin $^{\circledR} ; 113$ ineffective

PDT: 0.5 (598), 5-30 $\min ., \lambda=636 \mathrm{~nm}, 5.58 \mathrm{~J} / \mathrm{cm}^{2}$, no dark toxicity,

Low activity with $\mathbf{5 9 8}$

PDT: @ $3.25 \mu \mathrm{M}, 16 \mathrm{~mW} / \mathrm{cm}^{2}, \lambda=695 \mathrm{~nm}, \mathbf{5 6 9 b}>$

569a, $570>$ non-glycoconjuate parent compound.

Activity of $569 \mathrm{a} / \mathbf{b}, 570$ could be suppressed by preincubation with lactose. 
Human hepatocarcinoma HepG2

Human esophageal squamous-cell carcinoma cell line OE21

Human ovarian carcinoma SKOV3 - drug sensitive

Human ovarian carcinoma SKVLB1 - drug resistan

Human retinoblastoma cells Y79

Human retinoblastoma cells Y79

Human retinoblastoma cells Y79

Human retinoblastoma cells Y79

Human retinoblastoma cells Y79

Human colorectal adenocarcinoma cells HT 29
Glycophthalocyanines 617 and 618a-c

Glycoporphyrins: 320-331, 261
203-208, 315

4

4

499b-511b, 271

$480,481,486-489$

$\mathrm{A}_{4} \quad \mathrm{AB}_{3}$-glycoporphyrins - $\mathbf{3 0}$, 39, 35, 114, 203-206, 306-308

$\mathrm{A}_{4} \quad \mathrm{AB}_{3}$-glycochlorins - $\mathbf{5 1 6}$ 517,516c/d, 514f, 518/519

A $_{6}$-glycoporphyrins dimers 430a-d deacetylated version of 418

A -glycoporphyrins dimers 430a-d deacetylated version of 418
PDT: $\lambda>610 \mathrm{~nm}, 40 \mathrm{~mW} / \mathrm{cm}^{2}, 48 \mathrm{~J} / \mathrm{cm}^{2} ; \mathrm{IC}_{50}=0.1$ (617, 618a), $0.28(618 b), 0.79(618 \mathrm{c}) \mu \mathrm{M}$ $\Phi^{1} \mathrm{O}_{2}=0.79-0.94$

PDT: $0.5-3 \mu \mathrm{M}, 2 \mathrm{~min}$., white light, $1.7 \mathrm{~mW} / \mathrm{cm}^{2}, 4$ $\mathrm{h}$ incubation post treatment.

320-331, 261- inactive

Cellular uptake: $5 \mu \mathrm{M}, 0-24 \mathrm{~h}$ incubation, 320-324 and $331<$ Foscan $^{\circledR}$. 5,10 derivatives showed greater uptake.

${ }^{1} \mathrm{O}_{2}$ production of $\mathbf{3 2 3}<$ Foscan $^{\circledR}$

Localization: similar to pattern of Golgi apparatus.

Chemosensitization by $\mathbf{4}$

Absence of anticancer drugs: $\mathrm{IC}_{50}=0.8 \mu \mathrm{M}$

Presence of anticancer drugs (daunomycin, taxol, cisplatin): $0,0.1,0.25,0.5 \mu \mathrm{M}$ of 4 - only slight effects on the $\mathrm{IC}_{50}$ values.

$\left[{ }^{3} \mathrm{H}\right]$-vinblastine transport:

60 min. incubation - contro

$60 \mathrm{~min}$. incubation, 2 or $5 \mu \mathrm{M}$ of 4 or $10 \mu \mathrm{M}$ of verapamil. - no increase.

Chemosensitization by 4

Absence of anticancer drugs: $\mathrm{IC}_{50}=4 \mu \mathrm{M}$; only $\sim 5$ fold less sensitive to resistance cell lines.

Presence of anticancer drugs (daunomycin, taxol, cisplatin): $0,0.1,0.25,0.5 \mu \mathrm{M}$ of 4 - significantly reduced the resistance for daunomycin and taxol. No increase sensitivity was observed for Cisplatin, i.e. non P-glycoprotein transport drug.

$\left.{ }^{3} \mathrm{H}\right]$-vinblastine transport:

60 min. incubation - significantly lower concentration of $\left[{ }^{3} \mathrm{H}\right]$-vinblastine.

$60 \mathrm{~min}$. incubation, 2 or $5 \mu \mathrm{M}$ of 4 or $10 \mu \mathrm{M}$ of verapamil. $4>$ verapamil (10 fold)@2 $2 \mu \mathrm{M}$ (4) and $10 \mu \mathrm{M}$ (verapamil) - total inhibition of efflux.

Cellular uptake study: @ $4 \mu \mathrm{M}, 4 \mathrm{~h}$ incubation. Intracellular concentration ranging from $0.78 \pm 0.05 \times$ $10^{-6}$ to $0.26 \pm 0.03 \times 10^{-6} \mathrm{nmol} /$ cell for 206 (highest) to $\mathbf{2 0 3}$ (lowest) and $\mathbf{3 1 5}$ poor internalization. Absence of glucose inhibited uptake by $40-45 \%$ for 204 and 206 - uptake via active mechanism

PDT: @ $2 \mu \mathrm{M}, \lambda=514 \mathrm{~nm}, 1 \mathrm{~J} / \mathrm{cm}^{2}, 4 \mathrm{~h}$ incubation, no dark toxicity, $\mathrm{LD}_{50}=0.05$ (205), 0.35 (206), 0.6 (315, 204, 207), 0.65 (208), > 2 (203) $\mu \mathrm{M}$. Localization in ER and mitochondria for 204. 203 distributed through-out cytoplasm. All similar ${ }^{1} \mathrm{O}_{2}$ quantum yields.

PDT: @ $\lambda>540 \mathrm{~nm}, 2 \mathrm{~J} / \mathrm{cm}^{2}, 24 \mathrm{~h}$ incubation, no [205] dark toxicity, $\mathrm{LD}_{50}=0.43(\mathbf{2 0 6}), 2.5-2.7(\mathbf{4 9 9 b}, \mathbf{5 0 1 b}, \quad[198]$ 503b), 3-4.5 (500b, 506b-508b, 511b), 4.9-6 (502b, 504b, 505b, 509b, 512b), > $10(271) \mu \mathrm{M}$.

$-0.1>\log \mathrm{P}>0.4$

PDT: @ $\lambda \geq 590 \mathrm{~nm}, 2 \mathrm{~J} / \mathrm{cm}^{2}, 24 \mathrm{~h}$ incubation, no dark toxicity, $\mathrm{IC}_{50}=0.35$ (206), 0.4 (489), 0.5 (486), $1.2-1.5$ (481, 487), 2 (488), 15 (480) $\mu \mathrm{M}$.

@ $0.1-15 \mu \mathrm{M}, 24 \mathrm{~h}$ incubation, $15 \mathrm{~min}$. @ $1.8 \mathrm{~J} / \mathrm{cm}^{2}$, $\lambda>540,3$ day incubation post treatment, , 517, 306, 30, 39, 35, $\quad[198]$ 203, 514f, $m$ THPC), > 10 (204-206, 308), > 15 ( $m$ THPP, 307, 114, 518/519) $\mu \mathrm{M}$.

Phototoxicity: $\mathrm{IC}_{50}=0.05$ (205), 0.35 (206), 0.5-0.7 ( $m$ THPP, 308, 204, $m$ THPC, 518/519), 0.8-1.9 (203, $114,307,39,306), 2-3.8(35,516,517,30,514 f$ 516c/d) $\mu \mathrm{M}$

PDT: $0.15-7.5 \mu \mathrm{M}, \lambda>540 \mathrm{~nm}, 11 \mathrm{~min} ., 2 \mathrm{~J} / \mathrm{cm}^{2}, 3$ $\mathrm{mW} / \mathrm{cm}^{2}, 3$ day incubation, all displayed dark toxicity $\mathrm{IC}_{50}=1$ (430d), 4 (430c), > $7.5 \quad$ (430a-b, deacetylated-418) - all inactive PDT.

Cellular uptake of 430d: $4 \mu \mathrm{M}, 24 \mathrm{~h}$ incubation, poor uptake of 430d due to solubility.

PDT: $0.15-7.5 \mu \mathrm{M}, \lambda>540 \mathrm{~nm}, 11 \mathrm{~min} ., 2 \mathrm{~J} / \mathrm{cm}^{2}, 3$

$\mathrm{mW} / \mathrm{cm}^{2}, 3$ day incubation, all displaced dark toxicity $\mathrm{IC}_{50}=0.5$ (430a), 4 (430d), 5.3 (430b), $6.3(\mathbf{4 3 0 c}),>$ 7.5 (deacetylated-418) 
Human colorectal adenocarcinoma cells HT 29

Human colorectal adenocarcinoma cells HT-29
Benzochlorins: 552b

203-208, 315

271 and $499 b-512 b$

cells HT-29

Human colorectal adenocarcinoma cells HT-29

Human colorectal adenocarcinoma cells HT-29

Human colorectal adenocarcinoma cells HT-29

\section{1a-d}

480, 481, 486-489

$\mathrm{A}_{4^{-}, \mathrm{A}} \mathrm{B}$-glycochlorins: 516, 517 and $\mathbf{5 1 4 f}$ Metabolite: $\mathbf{3 0 7}$
Human colorectal adenocarcinoma cells HT-29

Human colorectal adenocarcinoma cells HT-29
$\mathrm{A}_{4} \quad \mathrm{AB}_{3}$-glycoporphyrins - 30, 39, 35, 114, 203-206, 306-308 $\mathrm{A}_{4} \quad \mathrm{AB}_{3}$-glycochlorins - 516, $517,516 \mathrm{c} / \mathrm{d}, 514 \mathrm{f}, 518 / 519$

$\mathrm{AB}_{3}, \mathrm{~A}_{2} \mathrm{~B}_{2}$-glycochlorins 247, 248 and 514a,b
Human colorectal adenocarcinoma cells HT-29 deacetylated-418 and $\mathbf{4 3 0 b}$ - slightly PDT active but dark toxicity - unviable.

PDT: $\mathrm{IC}_{50}=8 \mu \mathrm{M}$ (white light); $\mathrm{IC}_{50}=5.7 \mu \mathrm{M}(\lambda>$ $590 \mathrm{~nm}) . \mathbf{5 5 2}$ b - active

Cellular uptake study: @ $4 \mu \mathrm{M}, 4 \mathrm{~h}$ incubation. Highest uptake by 206 compared to 208 and 203. The low cellular uptake of $\mathbf{2 0 5}(\log \mathrm{P}=0.5)$ and $208(\log$ $\mathrm{P}=0.46)$ is clearly related to their higher lipophilicity compared to those with high amphiphilicity, e.g., 206 $(\log \mathrm{P}=0.02)$

PDT: @ $2 \mu \mathrm{M}, \lambda=514 \mathrm{~nm}, 5 \mathrm{~J} / \mathrm{cm}^{2}, 4 \mathrm{~h}$ incubation, no dark toxicity, $\mathrm{LD}_{50}=0.1$ (205), 0.43 (206), 0.68 (204), 0.85 (315), 2 (207, 208) $\mu$ M. All similar ${ }^{1} \mathrm{O}_{2}$ quantum yields.

PDT: $@ \lambda>540 \mathrm{~nm}, 2 \mathrm{~J} / \mathrm{cm}^{-2}, 24 \mathrm{~h}$ incubation, no dark toxicity, $\mathrm{LD}_{50}=0.35(\mathbf{2 0 6}), 3(\mathbf{5 0 3 b}), 3.7(\mathbf{4 9 9 b}$, 501b), 5-6 (500b, 504b-508b, 511b, 512b) >10 (502b, 509b, 510b, 271) $\mu \mathrm{M}$

PDT: @ $2 \mu \mathrm{M}, \lambda=630 \mathrm{~nm}, 3.6 \mathrm{~J} / \mathrm{cm}^{2}, 24 \mathrm{~h}$ incubation, no dark toxicity, $\mathrm{LD}_{90}=25(461 \mathrm{c}), 35$ (461a), 45 (461b), 50 (461d) $\mu \mathrm{M}$

$-0.1>\log \mathrm{P}>0.4$

PDT: $@ \lambda \geq 590 \mathrm{~nm}, 2 \mathrm{~J} / \mathrm{cm}^{2}, 24 \mathrm{~h}$ incubation, no dark toxicity, $\mathrm{IC}_{50}=0.43$ (206), 11 (489), > 15 (480, 481, 486, 487) $\mu \mathrm{M}$.

Cellular uptake: $2 \mathrm{~h}$ incubation saturation after. @ 6 $\mu \mathrm{M}, 3 \mathrm{~h}$ incubation, drug concentrations $3.57 \times 10^{-6}$ $(m$ THPC $), 2.4 \times 10^{-6}(\mathbf{5 1 6}, \mathbf{5 1 7})$ and $1.03 \times 10^{-6}(\mathbf{5 1 4 f})$ $\mu \mathrm{mol} / \mu \mathrm{g} . \quad m \mathrm{THPC}>\mathbf{5 1 6}, \mathbf{5 1 7}(30 \%$ less $)>\mathbf{5 1 4 f}(80$ $\%$ less). 516, 517 temperature depend, active process. 516, 517 uptake more energy consuming then $m$ THPC tested via inhibition of mitochondrial respiratory chain via $\mathrm{NaN}_{3}$

516, 517 and $514 f$ localization in mitochondria.

PDT :@ $514 \mathrm{~nm}, 5,10,25 \mathrm{~J} / \mathrm{cm}^{2}$, no dark toxicity for 516, 517 however, 514f, exhibited dark toxicity dependent on FCS concentration (@ $4 \mu \mathrm{M}, 3 \mathrm{~h}$ incubation, $2 \% \mathrm{FCS}$ in the dark $-51 \%$ cell survival, compared to $100 \% @ 10 \%$ FCS).

(a) $2 \mu \mathrm{M}, 5 \mathrm{~J} / \mathrm{cm}^{2}$, cell survival fraction for $\mathbf{5 1 6}, \mathbf{5 1 7}$

$(2 \pm 0.6 \%), m$ THPC $(16 \pm 2 \%), \mathbf{5 1 4 f}(22 \pm 4 \%)$, photoactivity of $\mathbf{5 1 4 f}$ is poor.

PDT of metabolic 307: $0.5-2 \mathrm{mM}, 3 \mathrm{~h}$ incubation, $\lambda=$ $514 \mathrm{~nm}, 5 \mathrm{~J} / \mathrm{cm}^{2}, \mathrm{LD}_{50}=0.7 \mu \mathrm{M}$ of both $\mathbf{3 0 7}$ and $\mathbf{5 1 6}$, 517 but cellular drug conc. $307\left(5.9( \pm 0.8) \times 10^{-6}\right.$ $\mu \mathrm{mol} / \mu \mathrm{g})>\mathbf{5 1 6}, \mathbf{5 1 7}\left(2.5( \pm 0.8) \times 10^{-6} \mu \mathrm{mol} / \mu \mathrm{g}\right)$.

(a) $0.1-15 \mu \mathrm{M}, 24 \mathrm{~h}$ incubation, $15 \mathrm{~min}$. @ $1.8 \mathrm{~J} / \mathrm{cm}^{2}$, [210] $\lambda>540 \mathrm{~nm}, 24 \mathrm{~h}$ incubation post treatment, [71] cytotoxcity: $\mathrm{IC}_{50}=6-8$ (non-glycoconjugates: 35, [198] $m$ THPC, 307, 203, 518/519), > $10(\mathbf{3 0 8}, \mathbf{2 0 3 - 2 0 6}), \quad[260]$ $>15$ ( $m$ THPP, 30, 39, 514f, 306, 114, 516, 517, 516c/d) $\mu \mathrm{M}$

Phototoxicity: $\mathrm{IC}_{50}=0.1$ (205), 0.4 (206), 0.5 (203) 0.7 (204), 0.8 ( $m$ THPC), 1-2.2 ( $m$ THPP, 308, 114, 518/519, 35), 3.5-4.8 (514f, 516, 517, 306-307), 7.8 $(\mathbf{5 1 6 c} / \mathbf{d}),>10(\mathbf{3 0}, 39) \mu \mathrm{M}$

Cellular uptake study: @ $10 \mu \mathrm{M}, 24$ h incubation. $\mathbf{2 4 7}>\mathbf{5 1 4 a}, b>\mathbf{2 4 8}>$ TPP. 24711.5 fold greater than TPP.

$\Phi^{1} \mathrm{O}_{2}=0.73$ (514a,b), 0.55 (247, TPP), 0.33 (248)

Cytotoxicity: @ 0.5 to $30 \mu \mathrm{M}, 24$ h incubation, 247 and 514a,b no dark toxicity $\leq 10,248$ slight cytotoxicity.

Phototoxicity: @ $1 \mu \mathrm{M}, 24 \mathrm{~h}$ incubation, $\lambda=650 \mathrm{~nm}$, $4.54 \mathrm{~mW} / \mathrm{cm}^{2}, \mathrm{LD}_{50}=4.4(\mathbf{2 4 7}), 5.2(\mathbf{5 1 4 a}, \mathbf{b}), 11.1$ (248), 30.2 (TPP) $\mathrm{J} / \mathrm{cm}^{2}$.

247: Localization in the ER

PDT: $\lambda>610 \mathrm{~nm}, 40 \mathrm{nM} / \mathrm{cm}^{2}, 20 \mathrm{~min} ., 48 \mathrm{~J} / \mathrm{cm}^{2}$, no dark toxicity, $\mathrm{IC}_{50}=28 \mathrm{nM}(\mathbf{6 1 7})>7.5 \mu \mathrm{g} / \mathrm{mL}$ (Photofrin $^{(\mathbb{R}}$ )

Localization: granular spots through-out the cytoplasm.

PDT: $\lambda>610 \mathrm{~nm}, 40 \mathrm{nM} / \mathrm{cm}^{2}, 48 \mathrm{~J} / \mathrm{cm}^{2}$, no dark 
cells HT-29

$614 a, b$

Human colorectal adenocarcinoma cells HT-29

Human colorectal adenocarcinoma cells HT-29

Human colorectal adenocarcinoma cells HT-29

Human colorectal adenocarcinoma cells HT-29

Human colorectal adenocarcinoma cells HT-29

Human colon adenocarcinoma cells T84

Human carcinoma HEp2

$\begin{array}{lll}\text { Human } & \text { hepatocarcinoma cells } \\ \text { HepG2 } & & \\ \text { Human } & \\ \text { HepG2 } & & \end{array}$

Human hepatocarcinoma cells

HepG2

Human hepatocarcinoma cells Glycophthalocyanines:

HepG2

Human chronic myelogenous leukaemia K562 627-630, 628-630b

Cyclodextrinporphyrins:

638d and 638a

Glyco-Si- phthalocyanines: 615a-c, 617

Glycophthalocyanines: 602b

Glycophthalocyanines: 627-630, 628-630b

Glycophthalocyanines: 635a-c and 637a-c

Glycophthalocyanines 617

$\mathrm{AB}_{3}$-type triazole-linked glycoporphyrins: $\mathbf{2 5 7 , 2 5 8}$

$A_{4}$-type triazole-linked glycoporphyrin: $\mathbf{4 7 0}$

Tetrabenzoporphyrin: $\mathbf{4 7 2}$

257, 258, 470 and 472

$614 a, b$

Glyco-Si- phthalocyanines:

615a-c, 617

Glycophthalocyanines: $\mathbf{6 2 4 a} \mathbf{a} / \mathbf{b}$, $625 a / b, 604,622$ and $627 a-d$

203-208, 315 toxicity, $\mathrm{IC}_{50}=6 \mathrm{nM}(\mathbf{6 1 4 a}), 17 \mathrm{nM}(\mathbf{6 1 4 b}), 7.5$ $\mu \mathrm{g} / \mathrm{mL}$ (Photofrin ${ }^{\circledR}$ )

614a $>614 b>$ Photofrin

(a) $8 \mathrm{~J} / \mathrm{cm}^{2}: \mathrm{IC}_{50}=7 \mathrm{nM}(614 a)$

(a) $2 \mathrm{~J} / \mathrm{cm}^{2}: \mathrm{IC}_{50}=46 \mathrm{nM}(\mathbf{6 1 4 a})$.

$\Phi_{\Delta}=0.32$ (614a) - 0.41 (614b)

Presence of $D$-glucose did not impede uptake - not transported via glucose uptake.

Localization : lysosomes (614a)

PDT: $\lambda>610 \mathrm{~nm}, 40 \mathrm{nM} / \mathrm{cm}^{2}, 48 \mathrm{~J} / \mathrm{cm}^{2}$, no dark

toxicity, $\mathrm{IC}_{50}=0.03(\mathbf{6 1 7}), 0.09(\mathbf{6 1 5 a}), 0.87(\mathbf{6 1 5 b})$

$\mu \mathrm{M}$.

$615 \mathrm{c}$ - inactive

$\Phi_{\Delta}=0.08$ (615c), 0.35 (617), 0.37 (615a), 0.41

(615b).

Localization: 617 in lysosomes.

615a-c - no localization in lysosomes.

PDT: $0-8 \mu \mathrm{M}, \lambda>610 \mathrm{~nm}, 40 \mathrm{~mW} / \mathrm{cm}^{2}, 48 \mathrm{~J} / \mathrm{cm}^{2}$, no dark toxicity, $\mathrm{IC}_{50}=0.9(627 \mathbf{c}), 1(627 \mathbf{b}), 1.7(627 \mathbf{a})$, 2 (627d), 3.5 (625a), 4 (625b).

604, 622 - inactive.

PDT efficacy $>$ Photosense ${ }^{\circledR}$

Cellular uptake: 604, 622 - no uptake.

$627 \mathrm{a} \approx 627 \mathrm{c}>625 \mathrm{a}$.

ROS generation: $627 \mathrm{c}>627 \mathrm{a}>\mathbf{6 2 5 a}>\mathbf{6 0 4}$

PDT: $\leq 100 \mu \mathrm{M}, 3.6 \mathrm{~J} / \mathrm{cm}^{2}, 24 \mathrm{~h}$ incubation,

$\overline{\mathbf{6 0 2 b}}$ - inactive and no cellular uptake.

Cellular uptake: $8 \mu \mathrm{M}, 2$ h, 629a,b -no uptake,

$630 a>629 a>627>630 b$.

PDT: $0-8 \mu \mathrm{M}, \lambda>610 \mathrm{~nm}, 40 \mathrm{~mW} / \mathrm{cm}^{2}, 48 \mathrm{~J} / \mathrm{cm}^{2}$, no dark toxicity, $\mathrm{IC}_{50}=0.03(\mathbf{6 3 0 a}), 0.26(\mathbf{6 2 9 a}), 1.38$ (627), 2.97 (630b).

629ab - inactive

$\Phi_{\Delta}=0.43(627)-0.86(630 a)$

Cellular uptake: $635 \mathrm{~b}<635 \mathrm{a} \approx 637 \mathrm{a}<637 \mathrm{~b}<635 \mathrm{a}-$ c $635 \mathrm{c}<637 \mathrm{c}$.

PDT: $1-500 \mu \mathrm{M}, \mu \mathrm{M}, \lambda>600 \mathrm{~nm}, 3.6 \mathrm{~J} / \mathrm{cm}^{2}, 4.6$ $\mathrm{mW} / \mathrm{cm}^{2}, 24 \mathrm{~h}$ incubation post treatment, $\mathrm{LD}_{50}=110$ (635b), 219 (635a), 300 (637a), 386 (637b), 428 $(637 \mathrm{c})>500(635 \mathrm{c})$

635c-inactive@500 $\mu \mathrm{M}$

PDT: $\lambda>610 \mathrm{~nm}, 40 \mathrm{nM} / \mathrm{cm}^{2}, 20 \mathrm{~min} ., 48 \mathrm{~J} / \mathrm{cm}^{2}, \mathrm{IC}_{50}$ $=68 \mathrm{nM}(617)>7.5 \mu \mathrm{g} / \mathrm{mL}\left(\right.$ Photofrin $\left.^{\circledR}\right)$.

Uptake study: $10 \mu \mathrm{M}, 0,1,2,4,8,24 \mathrm{~h}$ incubation,

4725 -fold higher accumulation compared to

$257 \approx 258>470$.

PDT: 472, 257, 258, 470 - all inactive @ $100 \mu \mathrm{M}$.

257, 258 - localization in ER and endosomes.

470/472 - localization in lysosomes

PDT: $\lambda>610 \mathrm{~nm}, 40 \mathrm{nM} / \mathrm{cm}^{2}, 48 \mathrm{~J} / \mathrm{cm}^{2}$, no dark toxicity, $\mathrm{IC}_{50}=6 \mathrm{nM}(614 \mathrm{a}), 21 \mathrm{nM}(\mathbf{6 1 4 b}), 7.5$ $\mu \mathrm{g} / \mathrm{mL}\left(\right.$ Photofrin $\left.^{\circledR}\right)$.

PDT: $\lambda>610 \mathrm{~nm}, 40 \mathrm{nM} / \mathrm{cm}^{2}, 48 \mathrm{~J} / \mathrm{cm}^{2}$, no dark toxicity, $\mathrm{IC}_{50}=0.1(617), 0.4(615 a), 1.0(615 b) \mu \mathrm{M}$. $615 c$ - inactive

$\Phi_{\Delta}=0.08$ (615c), 0.35 (617), 0.37 (615a), 0.41 (615b).

PDT: $0-8 \mu \mathrm{M}, \lambda>610 \mathrm{~nm}, 40 \mathrm{~mW} / \mathrm{cm}^{2}, 48 \mathrm{~J} / \mathrm{cm}^{2}$, no dark toxicity, $\mathrm{IC}_{50}=1.1(\mathbf{6 2 7 b}), 1.5-1.8(\mathbf{6 2 7 a}, \mathbf{c}, \mathbf{d})$, 4.7 (625b), 5.1 (625a)

604, 622 - inactive.

PDT efficacy $>$ Photosense $^{\circledR}$

PDT: $0-8 \mu \mathrm{M}, \lambda>610 \mathrm{~nm}, 40 \mathrm{~mW} / \mathrm{cm}^{2}, 48 \mathrm{~J} / \mathrm{cm}^{2}$, no dark toxicity, $\mathrm{IC}_{50}=0.04$ (630a), $0.28(\mathbf{6 2 9 a}), 1.52$ (627), 2.48 (630b). 628a,b - inactive. PDT efficacy: 630a $(50 \mathrm{ng} / \mathrm{mL})>$ Photofrin ${ }^{\circledR}(4.5 \mu \mathrm{g} / \mathrm{mL})$.

PDT: @ 5 and $10 \mu \mathrm{M}, 0-4.2 \mathrm{~J} / \mathrm{cm}^{2}, 24 \mathrm{~h}$ incubation, no dark toxicity. $\mathrm{EC}_{50}=5 \mu \mathrm{M} @ 2.5 \mathrm{~J} / \mathrm{cm}^{2}(638 d), 10$ $\mu \mathrm{M} @ 3.4 \mathrm{~J} / \mathrm{cm}^{2}$ (638a) and $5 \mu \mathrm{g} / \mathrm{mL} @ 3.4 \mathrm{~J} / \mathrm{cm}^{2}$ $\left(\right.$ Photofrin $^{\circledR}$ ). Via apoptotic cell death.

Cellular uptake study: @ $4 \mu \mathrm{M}, 4 \mathrm{~h}$ incubation. Highest uptake by 206 compared to 208 and 203. The low cellular uptake of $205(\log \mathrm{P}=0.5)$ and $208(\log$ 
Melanoma cells B16-

Highly metastatic - B16-BL6

Weakly metastatic - B16-F1

Radiation-induced fibrosarcoma

(RIF) tumor cells

Radiation-induced

(RIF) tumor cells

fibrosarcoma

Murine

radiation-induced

fibrosarcoma (RIF) tumor cells

Mouse mammary carcinoma 4T1

mammary carcinoma 4T1

Mouse colon carcinoma CT26.CL25
Purpurin imides: 570,

569c,571a-d, 572-575

Cyclodextrinporphyrins:

638d and 638a

A -glycochlorins 542a, 543a

OEP-benzochlorins:

553-556b, 557

HPPH-glyco-conjugates: 558a-h

$\mathrm{A}_{4}$-glycochlorins 542a, 543a

Cyclodextrinporphyrins:

638d and 638a

Cyclodextrinporphyrins: 638a-d
$\mathrm{P}=0.46)$ is clearly related to their higher lipophilicity compared to those with high amphiphilicity, e.g., 206 $(\log \mathrm{P}=0.02)$.

PDT: @ $2 \mu \mathrm{M}, \lambda=514 \mathrm{~nm}, 5 \mathrm{~J} / \mathrm{cm}^{2}, 4 \mathrm{~h}$ incubation, no dark toxicity, $\mathrm{LD}_{50}=0.25$ (204), 0.35 (206), 0.44 (315), $0.82(\mathbf{2 0 5}, \mathbf{2 0 7}),>2$ (208) $\mu \mathrm{M}$. All similar ${ }^{1} \mathrm{O}_{2}$ quantum yields.

PDT: @ $0.05-0.1 \mu \mathrm{M}$

$\mathrm{EC}_{50}=0.5 \mu \mathrm{M}$ in B16-BL6 542a $>$ 543a

Galectin binding studies: ELISA showed 553-556b, 557 bind 141-387 times greater than lactose $\left(\mathrm{IC}_{50}=\right.$ $1200 \mu \mathrm{M}) . \mathrm{IC}_{50}=3.1$ (557), 4.5 (556b), 5.1 (555b), 6.2 (553b), 8.5 (554b), 1200 (lactose) $\mu \mathrm{M}$.

PDT: Good PDT effect: $1.0 \mu \mathrm{M}$ PS, $4 \mathrm{~h}$ incubation, $1-$ $\overline{5 \mathrm{~J} / \mathrm{cm}^{2}}$. Active: $\mathbf{5 5 3 - 5 5 6 b}, \mathbf{5 5 7}$

Activity of 555b, 556b, 557 could be suppressed by pre-incubation with lactose while $\mathbf{5 5 3 b}$ was unaffected.

PDT: @1 J/ $\mathrm{cm}^{2}$

572 - dark toxicity $>0.5 \mu \mathrm{M}$

Positional isomers 571b, 572, 573@ 0-1 $\mu \mathrm{M}, 1$ $\mathrm{J} / \mathrm{cm}^{2}, \mathbf{5 7 1 b}(0.21 \mu \mathrm{M}) 4-5$ times greater than $\mathbf{5 7 2 - 5 7 3}$ $(0.91 \mu \mathrm{M}, 0.84 \mu \mathrm{M})$

Linkers 569c, 571b, 574, 575: @ 0-1 $\mu \mathrm{M}, 1 \mathrm{~J} / \mathrm{cm}^{2}$,

571b - highest active

Sugar unit 571b, 571c, 571d, 571a: @ 0-1 $\mu \mathrm{M}, 1$ $\mathrm{J} / \mathrm{cm}^{2}, \mathrm{LD}_{50}=0.21($ 571b, 571c, 571d), 1 (571a) $\mu \mathrm{M}$.

5 times more effective.

Sugar unit 571b, 571c, 571d, 571a: @ 0-1 $\mu \mathrm{M}, 0.5$ $\mathrm{J} / \mathrm{cm}^{2}, \mathrm{LD}_{50}=0.42$ (571c), 0.47 (571d), 0.59 (571b), $>1$ (571a) $\mu \mathrm{M}$.

Cellular uptake 570,569c,571-575: @ 3 h and 24 h

(a) 3 h: 571b, 571c, 571d, 571a - high uptake

@ 24 h: 571c, 571d $>$ 571b

Overall 571c $>$ 571d $>$ 571b $>$ 571a $>$ 575 $>570>$

$\mathbf{5 7 4}>\mathbf{5 7 2}>\mathbf{5 7 3}$.

571b localized in lysosomes and the Golgi apparatus while 571a localized in only in the Golgi apparatus.

Cellular uptake: @ 3 h or 24 h 558a-d > HPPH $\approx$ 558f $>$ 558g,h

558a $>$ HPPH - due to HPPH efflux ABCG2 pump.

PDT: @ 0-1 $\mu \mathrm{M}, 24$ incubation $\lambda=665 \mathrm{~nm}, 48 \mathrm{~h}$ incubation post treatment, no dark toxicity, 558a,b $>$ $\mathbf{5 5 8 c}, \mathbf{d}>\mathrm{HPPH}$

$558 \mathrm{c}>\mathbf{5 5 8 f} \approx \mathrm{HPPH}$

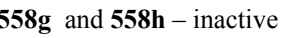

No improvement of efficacy due to galectin binding.

Localization: HPPH - mitochondria, 558a,c,d,f and-

lysosomes, 558c - Golgi apparatus and lysosomes 558g,h - mitochondria, Golgi apparatus and lysosomes

PDT:@ 5 and $10 \mu \mathrm{M}, 0-4.2 \mathrm{~J} / \mathrm{cm}^{2}, 24 \mathrm{~h}$ incubation, no dark toxicity. $\mathrm{EC}_{50}=5 \mu \mathrm{M} @ 2.5 \mathrm{~J} / \mathrm{cm}^{2}(638 d), 10$ $\mu \mathrm{M} @ 3.4 \mathrm{~J} / \mathrm{cm}^{2}(638 a)$ and $5 \mu \mathrm{g} / \mathrm{mL} @ 3.4 \mathrm{~J} / \mathrm{cm}^{2}$ $\left(\right.$ Photofrin $^{\circledR}$ ). Via both apoptosis and necrosis.

Uptake study: @ $10 \mu \mathrm{M}, 16 \mathrm{~h}$ incubation, determined via $\left(\mathrm{C}_{\mathrm{o}}-\mathrm{C}_{\mathrm{c}} / \mathrm{C}_{\mathrm{o}}\right), 45 \%$ (638d) and $14 \%$ (638a); localization in lysosomes.

PDT: @ 0.05 - $0.1 \mu \mathrm{M}$, 542a > 543a

PDT:@ 5 and $10 \mu \mathrm{M}, 0-4.2 \mathrm{~J} / \mathrm{cm}^{2}, 24 \mathrm{~h}$ incubation, no dark toxicity. 638d $>638 \mathrm{a}$.

PDT: 638a-c complexation with particular drugs, $1 \mathrm{~h}$, r.t., $4 \mathrm{~J} / \mathrm{cm}^{2}, 24 \mathrm{~h}$ incubation, dark toxicity due to conjugated drug. 638a,b conjugates with taxanes gave increase cell mortality 5-8 \% compared to $\beta$ cyclodextrin/drug. Combined therapy observed in most except kinase activation inhibitors (imatinib, sunitinib) and approved advantageous.

small molecule delivery: @ pH 7 - strong binding of the drug complex versus $\mathrm{pH} 5$ - significantly diminished binding.

$638 \mathrm{a}$ and $638 \mathrm{c}>>638 \mathrm{~b}>638 \mathrm{~d}$. 
Mouse colon adenocarcinoma Colon26

Mouse fibroblasts K:Molv NIH 3T3

$\mathrm{A}_{4}$-glycochlorins: 542a, 542a' $\mathrm{A}_{4}$-glycoisobacteriochlorins: 545a, a'

$\mathrm{A}_{4}$-glycobacteriochlorins: 546a, a'

Mouse mammary carcinoma 4T1

Murine mammary adenocarcinoma 4T1 containing a subset of ABCG2 expressing side population (SP) of cells versus no expression.
Rat hepatoma liver cells RLC-16 Transformed human embryonic kidney cells HEK-293 482R ABCG2 expressing
Cyclodextrinporphyrins: 638a-d

$\mathrm{HPPH}$ and 558a

245, 246

Pyropheophorbides, HPPH and purpurinimides: 558a-d, 558f-h, 559, 570, 569c, 571a-d, 572-575 , 565-569,
PDT: @ 0-1 $\mu \mathrm{M}, 24 \mathrm{~h}$ incubation, $\lambda=665 \mathrm{~nm}, 48 \mathrm{~h}$

Transformed human embryonic
kidney cells HEK-293 PcDNA ABCG2 non-expressing.

Saccharomyces cerevisiae, strain FL100

Jurkat T leukemia Tn-positive cells

Pyropheophorbides, HPPH and purpurinimides: 558a-d, 558f-h, 559, 570, 569c, 571a-d, 572575, 565-566

Thio-glycosylated

tolylporphyrins 247-249

TrMPyP-MorG

TrMPyP

MorG
Cellular uptake: $@ 3$ h or 24 h 558a $\approx \mathrm{HPPH}$ incubation post treatment, no dark toxicity,

(a) 24 h incubation: 558a $\approx \mathbf{5 5 8 b} \approx \mathrm{HPPH}$

(a) 4 h incubation: 558a $\approx \mathbf{5 5 8 b}>$ HPPH.

Uptake and confocal microscopy: @ $2.5 \mu \mathrm{M}, 20 \mathrm{~h}$ incubation, 1:2:7 91: 542a: 545a

(a) $10 \mu \mathrm{M}, 24 \mathrm{~h}$ incubation, for $\mathbf{5 4 6 a}$

PDT: @ $10 \mu \mathrm{M}, 10 \mathrm{~min}$. irradiation, $13 \mathrm{~W}$ fluorescent bulb; 542a and 545a - active at twice the light power. $545 a$ - significantly high fluorescence quantum yield therefore - optimum for tagging and sensor application.

Red absorption for PDT: 546a $>$ 542a $>$ 545a and 91

No photobleaching.

PDT: 638a-d complexation with particular drugs, $1 \mathrm{~h}$, r.t., $4 \mathrm{~J} / \mathrm{cm}^{2}, 24 \mathrm{~h}$ incubation, dark toxicity due to conjugated drug. 638a,b conjugates with taxanes gave increase cell mortality 5-8\% compared to $\beta$ cyclodextrin/drug. Combined therapy observed in most except kinase activation inhibitors (imatinib, sunitinib) and proved advantageous .

Small molecule delivery: @ pH 7 - strong binding of the drug complex versus pH 5 - significantly diminished binding.

$638 \mathrm{a}$ and $638 \mathrm{c}>>638 \mathrm{~b}>638 \mathrm{~d}$.

SP analysis: cells treated with imatinib mesylate and fumitremorgin found $4-15 \%$ of side population present.

PDT for 4T1: @ $800 \mathrm{nM}, 1 \mathrm{~J} / \mathrm{cm}^{2}, 3.2 \mathrm{nW} / \mathrm{cm}^{2}$, presence or absence of $10 \mu \mathrm{M}$ imatinib mesylate (ABCG2 inhibitor), $48 \mathrm{~h}$ incubation, for HPPH and 558a.

Absence of inhibitor: HPPH displayed an increase of SP in survival cells and 558a showed a decrease. Presence of inhibitor: decrease in SP for $\mathbf{5 5 8 a}$.

PDT for SP and non-SP 4T1: @ 200, 400 and 800 $\mathrm{nM}, 1 \mathrm{~J} / \mathrm{cm}^{2}, 3.2 \mathrm{nW} / \mathrm{cm}^{2}, 48 \mathrm{~h}$ incubation Confirm resistance of HPPH-PDT for SP cells Uptake: $245>246$ - receptors for galactose residues Affinity: with $10 \mu \mathrm{M}$ imatinib mesylate, pyropheophorbides: $559 \approx 567$ (position-3) $>\mathbf{5 6 9}$ $($ position-3) $>\mathbf{5 6 8}($ position-3) $>>\mathbf{5 6 6}($ position- 17$)>$ 565 (position-17).

566 and 565 - non-substrates

HPPH - substrate, 558a-d, 558f-h, 559

(position-17) - non substrate.

Purpurinimides: 571c, 571a - substrate (lesser affinity compared to $\mathbf{5 5 9}$ ),

Lactose at position-17 (574), 12 (573), $13_{2}$ (575) non substrate.

Glucose and galactose at position 3 (571c, 571d) substrate.

Linker change: $\mathbf{5 7 0}, \mathbf{5 7 1 b}, \mathbf{5 7 2}, \mathbf{5 7 3}, \mathbf{5 7 4}, \mathbf{5 7 5}, \mathbf{5 5 8 c}$,

$\mathbf{5 5 8 f}$ - non-substrate.

Lactose at position-3 (571b) - non substrate.

Affinity: with $10 \mu \mathrm{M}$ imatinib mesylate,

PDT: $1 \times 10^{-5} \mathrm{M}, 150 \mathrm{~W} / \mathrm{cm}^{2}:$ delayed effect compared to Photofrin. $o$-derivatives more effective than $p$.

Cellular uptake: $30 \mathrm{~min} ., 4{ }^{\circ} \mathrm{C}$, very high uptake.

Addition of $D$-glucose and $D$-mannose - no inhibitory effect, Addition of $D$-galactose or $N$-acetyl- $\alpha-D-$ galactosamine) - caused inhibition of uptake.

$1 \mu \mathrm{M}$ Bovine submaxillary mucin (glycoprotein containing high-density $O$-linked cryptic polyvalent T Tn glycotopes) - complete inhibition.

PDT: $10-10^{5} \mathrm{nM}, 7.5 \mathrm{~min} ., 16 \mathrm{~h}$ incubation,

No dark toxicity $\leq 10^{2} \mathrm{nM}$,

TrMPyP@10 nM - active 
Jurkat T leukemia Tn-positive cells size.

Normal T lymphocytes - CD1dnegative cells with low size. $5,10,15,20$-tetrakis $(4$ sulfonatophenyl)porphyrin MorG, TrMPyP -MorG, $\mathrm{AlPcS}_{4^{-}}$MorG and chlorin $\mathrm{e}_{6^{-}}$MorG, TrMPyP, tetrakis(4sulfonatophenyl)porphyrin derivative $\mathrm{AlPcS}_{4}$ derivative and chlorin $\mathrm{e}_{6}$

5,10,15,20-tetrakis(4sulfonatophenyl)porphyrinMorG, and $\mathrm{AlPcS}_{4}-$ MorG
TrMPyP -MorG@10 nM - active.

PDT: white light, $7.5 \mathrm{~min} ., 1.7 \mathrm{~J} / \mathrm{cm}^{2}$, or $\lambda=660 \mathrm{~nm}$,

$2 \mathrm{~min} ., 140 \mathrm{~W} / \mathrm{m}^{2}, 1.7 \mathrm{~J} / \mathrm{cm}^{2}, 24 \mathrm{~h}$ incubation, no dark toxicity,

Ratio of PS/Morg

white light: $\mathrm{LD}_{50}=2 \quad(5,10,15,20$-tetrakis $(4$ sulfonatophenyl)porphyrin -MorG, 3/1), 4 (tetrakis(4sulfonatophenyl)porphyrin -MorG, 1/1), 7 (chlorin $\mathrm{e}_{6}$ MorG, 9/1) < 10 (TrMPyP-MorG, 1/1) 16 (chlorin $\mathrm{e}_{6}-$ MorG, 1/1), 35 (AlPcS $_{4}-$ MorG, 10/1) $\geq 50$ $\left(\mathrm{AlPcS}_{4}-\mathrm{MorG}, 1 / 1\right)$

red light: $\mathrm{LD}_{50}=2$ (chlorin $\left.\mathrm{e}_{6}-\mathrm{MorG}, 9 / 1\right), 4\left(\mathrm{AlPcS}_{4}-\right.$ MorG, 1/1), 5 (AlPcS $_{4^{-}}$MorG, 10/1), 7 (chlorin $\mathrm{e}_{6}-$ MorG, $1 / 1), \quad 12 \quad$ (tetrakis(4sulfonatophenyl)porphyrin-MorG, 3/1), $\quad 25$ (tetrakis(4-sulfonatophenyl)porphyrin-MorG, 1/1) PS alone: 5,10,15,20-tetrakis(4sulfonatophenyl)porphyrin derivative, $\mathrm{AlPcS}_{4}$ derivative and chlorin $\mathrm{e}_{6}$ - poor activity

PDT: white light, $7.5 \mathrm{~min}$., $1.7 \mathrm{~J} / \mathrm{cm}^{2}$, or $\lambda=660 \mathrm{~nm}$,

$2 \mathrm{~min} ., 140 \mathrm{~W} / \mathrm{m}^{2}, 1.7 \mathrm{~J} / \mathrm{cm}^{2}, 24 \mathrm{~h}$ incubation,

White light for 5,10,15,20-tetrakis(4-

sulfonatophenyl)porphyrin-MorG, 1/1

Red light for $\mathrm{AlPcS}_{4}-\mathrm{MorG}, 1 / 1$

Very resistant to PS

\subsubsection{1 meso-Substituted Porphyrins}

Due to their easier synthesis the first PDT studies on glycoporphyrins used symmetrically meso substituted systems, e.g., $\mathrm{A}_{4}$-type porphyrins. However, it became apparent quickly that the nature, number and linkage position of the glycosyl unit, i.e., their amphiphilic character is of critical importance for their PDT efficacy ${ }^{[345]}$. In preliminary investigation of their PDT efficacy, the amphiphilic molecules 114-116 were found to be better than $\mathrm{HpD}$, i.e. those with strong amphiphilic character ${ }^{[162,345]}$. Still, symmetric systems should not be discounted nor should the protected "precursor" compounds. The latter became clear in an early study from 1998 where the meso substituted porphyrins 29-32, 35-36 and 53 were studied in HeLa cells $[162,167,345]$. Interestingly, in all cases the acetylated, i.e. protected derivatives, showed significantly higher phototoxicity than the deprotected species. The octaglycosylated derivative $\mathbf{5 3}$ gave no improvement; rather its effect was weaker. In general more globular water soluble systems appear to be disfavored for uptake by the cells. "Flatter", more hydrophobic derivatives such as $\mathbf{2 9}$ appear to be superior. The singlet oxygen quantum yield was comparable in all compounds and thus, the PDT effect is mainly the result of different uptake ${ }^{[167]}$

The first comprehensive study of 30, 32, 39-52 in HeLa cells showed that all 16 glycoporphyrins displayed better cellular uptake compared to 5,10,15,20-tetrakis(4sulfonatophenyl)porphyrin except $\mathbf{4 1}$ and $\mathbf{3 2}$ which exhibited precipitation. The para-substituted porphyrins showed much higher uptake compared to the meta-substituted derivatives. Improved uptake was shown for the $\beta$ - $D$-xylopyranosyl and $\beta$ - $D$-arabinopyranosyl groups compared to $\beta$ - $D$ glucopyranosyl and $\beta$ - $D$-galactopyranosy groups ${ }^{[165]}$. The order of photocytotoxicity was $44>\mathbf{3 0}>5,10,15,20$ - tetrakis(4-sulfonatophenyl)porphyrin in agreement with the order of cellular uptake.

Subsequently, these derivatives were further investigated to determine the role of the sugar moiety and their photocytotoxicity was found to follow the order $\mathbf{4 4}<\mathbf{3 0}<\mathbf{3 2}$ $<\mathbf{4 2}$ where the $\mathrm{IC}_{50}$ of $\mathbf{4 4}$ was 5 times that of $\mathbf{4 2}{ }^{[272]}$. The photophysical properties of the glycoconjugated porphyrins are also dependent on the sugar moieties in physiological media such as phosphate buffered saline (PBS) containing 10 wt.\% bovine serum albumin (BSA). The oscillator strength in the order of $\mathbf{4 4}=\mathbf{3 0}<\mathbf{3 2}<\mathbf{4 2}$ is in a similar order to the photocytotoxicity in HeLa cells. This suggests the sugar dependence of PDT activity could be due to interactions between glycoporphyrins and endogenous biomolecules such as albumin. Glycochlorin interactions with serum albumin had previously been shown to affect cellular uptake in HT29 cells ${ }^{[260]}$. The interaction between the glycoporphyrins and BSA was evaluated by electronic absorption, fluorometric and circular dichroic titrations ${ }^{[272]}$. No differences in the binding constants were observed for the glycoporphyrins. However, the number of binding sites depended on the sugar unit and increased in the order $32<30<44<42$. The number of binding sites is poorly related to the photophysical properties and phototoxicity but clearly the photophysical properties of the glycoporphyrins are strongly determined by the physiological media resulting in the sugardependent photocytotoxicity ${ }^{[272]}$

It is worth noting however, carbohydrate units do not necessarily always improve on PDT activity. For example, a study of thio-glycosylated tolylporphyrins (247-249) against yeast Saccharomyces cerevisiae, showed that the photocytotoxicity was un-related to the specific glycosyl unit [243]

Rather detailed investigations focused on the $S$ glycosylated $\mathrm{A}_{4}$-type porphyrins 85 and $\mathbf{8 7}$. In comparison to 
5,10,15,20-tetrakis(4-methoxyphenyl)porphyrin they exhibited enhanced cellular binding in human breast cancer cell lines (MDA-MB-231). The glucose derivative showed higher uptake and enhanced PDT activity and induced both apoptosis and necrosis ${ }^{[247]}$. The PDT activity was dependent upon the drug dosage, length of irradiation and the degree of uptake with the glucose derivative $\mathbf{8 5}$ displaying the best activity. Characteristically malignant cells have increased uptake of glucose for metabolic activity and human breast cancer cells have a higher expression of glucose transporters then normal cells which could be attributed to the higher uptake of $\mathbf{8 5}$. The migration of cancerous cells was significantly reduced and thus reducing the aggressiveness of the cancer when exposed to low levels of PDT. It was also determined that a normal rat fibroblast cell line (3Y1) exhibited less uptake of the conjugates than those overexpressing tyrosine kinase transformed version of the cell line. Showing a $>3$-fold affinity for cancer cells over non-cancerous ones reinforcing the results obtained in the MDA MB-231 cell line ${ }^{[247,346]}$. Subsequent localization studies showed that $\sim 90 \%$ of the PS accumulated in the ER and caused ER-stress inducing apoptosis upon irradiation. This caused the release of free calcium, followed by release of cytochrome $\mathrm{c}$ from the mitochondria, caspase 3 activation, PARP cleavage and chromation condensation; all of which are indicative of the apoptosis cascade process ${ }^{[346]}$. A similar range of thio-glycosylated derivatives (341-363) synthesized by combinatorial synthesis was evaluated against human breast cancer cells MDA-MB-231 and those prospective derivatives analyzed for PDT ${ }^{[215]}$.

Metalloporphyrins are used less frequently in PDT, the high fluorescence of the free bases often provides a good, additional analytical tool. One study utilizing metallo derivatives looked at the in vitro photocytotoxicity of $\mathbf{8 7 - 9 4}$ in HeLa cells ${ }^{[177]}$. However, all protected derivatives were inactive, while 91-94 exhibited potent photocytotoxicity with an $\mathrm{EC}_{90}$ of $\sim 0.2 \mu \mathrm{M}$ in each case. This $\mathrm{EC}_{90}$ value is much lower than that of $O$-glucosylated tetraphenylporphyrin derivatives $\left(\mathrm{EC}_{90}>5 \mu \mathrm{M}\right)^{[272]}$. Thus, $\mathrm{Zn}(\mathrm{II})$ and $\mathrm{Pd}(\mathrm{II})$ complexes show potential as PSs with enhanced cellular uptake due to their glycosylation and high ${ }^{1} \mathrm{O}_{2}$-production due to the heavy-atom effect ${ }^{[177]}$.

Most of the more recent studies employ either series of porphyrins with varying number and position of carbohydrate units $\left(\mathrm{A}_{\mathrm{x}} \mathrm{B}_{\mathrm{y}}\right.$-type $)$ or, from the beginning, focus on unsymmetric derivatives, e.g. $\mathrm{A}_{3} \mathrm{~B}, \mathrm{AB}_{3}$, etc.

For example, excellent results were also obtained with the $S$-glycosylated porphyrin 242 within the series 238, 240, 242, 244, 91. It showed excellent photocytotoxicity $\left(\mathrm{EC}_{50}<\right.$ $5 \mathrm{nM}$ ) against HeLa cells with 20 times higher efficacy and an uptake threefold greater than other $S$-glucosylated porphyrins. The photocytotoxicity of all compounds were examined in drug dose concentrations of $100 \mathrm{nM}$ in HeLa cells with photoirradiation at $16 \mathrm{~J} / \mathrm{cm}^{2}$ with $24 \mathrm{~h}$ incubation post treatment with no dark toxicity observed. All $S$ glucosylated porphyrins displayed much higher PDT effect then compared to 5,10,15,20-tetrakis(4sulfonatophenyl)porphyrin ${ }^{[200]}$. The 5,15-disubstituted porphyrin 242 displayed higher phototoxicity with larger $\mathrm{f}_{>500}$ and $\Phi_{\mathrm{DCFDA}} / \mathrm{f}_{>500}$ values compared to the other $S$ glycosylated porphyrin in the series. Additionally, the mode of cell death and morphological change induced by photoirradiation with $\mathbf{2 4 2}$ plays a key role. This compound exhibited enhanced cell membrane penetration and upon irradiation HeLa cells appeared shrunken with several apoptotic cells observed within $3 \mathrm{~h}$ and ultimately all of which were destroyed. Glycoconjugate PSs characteristically undergo apoptotic cell death as was reiterated here ${ }^{[200]}$.

A series maltohexaose substituted $m$ THPP derivatives (437-441 and $\mathrm{Zn}(\mathrm{II})$ 437-441) proved interesting with regard to the number of sugar units required for PDT effect. Investigations, again in HeLa cells, revealed that porphyrins containing a single maltohexaose (free base and $\mathrm{Zn}(\mathrm{II}) \mathbf{4 3 7}$ ) had significantly higher phototoxicity compared to all other maltohexaose derivatives and $\mathbf{3 0}$ and $\mathbf{5 3}{ }^{[167,202 a]}$. The 5,10disubstituted free base derivative $\mathbf{4 3 8}$ was more active than the 5,15-derivative 439. On the other hand, the singlet oxygen production of the free bases and zinc(II) complexes were comparable. Thus, the better PDT efficiency of $\mathbf{4 3 7}$ is the result of higher cellular uptake and accumulation in the cytoplasm.

For amide linked $\mathrm{AB}_{3}$-type systems $(\mathbf{2 2 1}, \mathbf{2 2 2})$ the orthosubstituted derivative $\mathbf{2 2 1}$ displayed much higher activity compared to the para derivative $\mathbf{2 2 2}$ but was still less active then Photofrin ${ }^{\circledR}$ against K562 human chronic myelogenous leukemia cells. Here, the glucosyl unit was essential for the PDT efficacy as the non-glycosyl derivative $\mathbf{2 1 5}$ displayed significantly less activity ${ }^{[237]}$. Also, compounds 221 and 222 showed similar singlet oxygen production to HP.

The in vitro behavior of $N$-linked glycoporphyrins $\mathbf{7 8 , 7 9}$ in rat hepatoma (liver cancer) cells (RLC-16) was determined in comparison to non-glycosyl water soluble porphyrin meso-tetrakis(4-sulfophenyl)porphyrin and $\mathbf{7 4}$ and the slightly water soluble digalactose and diglucose derivatives $\mathbf{2 4 5}$ and 246. Octaglucosyl TPP lacked cellular affinity while the galactose derivative displayed significant affinity which was related to saccharide specificity for hepatocytes of the galactose residue ${ }^{[175]}$.

A number of $\mathrm{A}_{3} \mathrm{~B}$-type systems with diethyleneglycol $\mathbf{( 2 0 3 , 2 0 4 - 2 0 8 )}$ and monoethyleneglycol (315) linkages were investigated for their cellular uptake, localization and photoactivity in Y79, HT-29 and B16 cell lines. The extent of cellular uptake was dependent on the sugar residue (206 $>$ 204, 205, 207), its anomeric configuration $(204 / 207>205)$ and the linker $(206>315)$ with the unconjugated derivative 203 exhibiting poor internalization in comparison ${ }^{[198]}$. These cell lines over-express lectins for specific glycosides but the uptake of the glycoporphyrins was independent of such receptors. Initial work carried out on the pre-incubation of the cells in glycosylated albumin showed a 40-45\% inhibition of uptake for $\mathbf{2 0 4}$ and 206 indicating a cell-sugarreceptor saturation. With regard to phototoxicity of the PSs 
(203-208, 315), the most significant result was the high uptake of 206 and its good photoactivity $\left(\mathrm{LD}_{50}=0.35 \mu \mathrm{M}\right)$ compared to the parent compound 203. Interestingly, 205 which only exhibited twice the internalization as $\mathbf{2 0 3}$ showed a significantly high phototoxicity with $\mathrm{LD}_{50}=0.05 \mu \mathrm{M}$ in the Y79 cell line ${ }^{[198]}$ due to the variation in localization within a cellular structure playing a major role in photodynamic efficiency. Cellular metabolic degradation of all compounds was minimal however, possible in vivo degradation can not be excluded.

It might appear logical to extent the carbohydrate units for improved biological effects. Here, a study of glycodendrimeric $A_{3} B$ porphyrins (499-512b and 271) used HT 29 and Y79 cell lines with $>540 \mathrm{~nm}$ light with a fluence of $2 \mathrm{~J} / \mathrm{cm}^{2}$ and $24 \mathrm{~h}$ incubation of the PS ${ }^{[205]}$. The glycodendritic porphyrins 499b, 501b and 502b had the highest $\mathrm{LD}_{50}(2.5$ to $2.7 \mu \mathrm{M})$ in HT29 cells and 503b had an $\mathrm{LD}_{50}=3 \mu \mathrm{M}$, in Y79 cells. However, in comparison to the previously synthesized glycoporphyrin 206 the compounds were six times less phototoxic in HT29 cells and 10 times less phototoxic in Y79 cell lines; clearly more is not always better ${ }^{[205]}$.

Cationic porphyrins of the $\mathrm{A}_{3} \mathrm{~B}$-type (461a-d) showed good PDT activity against HT-29 cells with $\mathrm{LD}_{90}$ ranging from $25(\mathbf{4 6 1 c})$ to $50 \mu \mathrm{M}(\mathbf{4 6 1 d})$. Here the carbohydrate units proved to be important for lowering the dark toxicity, as comparable systems without the sugar unit had much higher $\mathrm{LD}_{90}(5 \mu \mathrm{M})$ indicated the importance of the sugar unit in reducing dark toxicity ${ }^{[248]}$

Another series of $\mathrm{A}_{3} \mathrm{~B}$ porphyrins (480, 481, 486-489) substituted with three glycosyl units was based on 'clickchemistry' and was used to investigate the effect of the sugar, the length of the spacer arm and the position and orientation of the triazole core on phototoxicity [251]. Cytotoxicity and phototoxicity were examined in HT29 and Y79 cells. All compounds exhibited better photocytotoxicity in Y79 cells than HT29 and SAR were difficult to establish. The nature of the glycosyl units appeared to have no effect on the phototoxicity. Porphyrin $489\left(\mathrm{IC}_{50}=11\right.$ and $0.4 \mu \mathrm{M}$ on HT 29 and Y79 cell lines) is an analogy of a compound previously synthesized 206 which showed good PDT efficacy in vitro $\left(\mathrm{IC}_{50}=0.43\right.$ and $0.35 \mu \mathrm{M}$ on HT29 and Y79 cell lines, respectively) ${ }^{[198]}$. Although 489 exhibited good photodynamic activity it was still lower than 206 in HT29 cell lines. The other glycoporphyrins exhibited little or no activity compared to the reference compounds. In summary, the triazole core decreased in vitro phototoxicity independently of the spacer or its orientation (1',4'-triazole versus 4',1'-triazole).

Attempts to utilize both carbohydrate and amino acid residues in bioconjugates failed. For example, studies on porphyrins (292-299) bearing both carbohydrate and amino acid residues in K562 leukemia cells gave discouraging results. All compounds showed lower phototoxicity than hematoporphyrin. Only at very long illumination times and with a significant delay ( $24 \mathrm{~h})$ did the cell killing count reach that of $\mathrm{Hp}{ }^{[207,259]}$. A similar study employed $\mathrm{A}_{3} \mathrm{~B}$-type glycoporphyrins carrying the RGD tripeptide as unit $b$ $(\mathbf{1 6 8} o, p)$. The compounds exhibited a similar singlet oxygen production to that of hematoporphyin. However, their photocytotoxic activity reached that of Photofrin ${ }^{\circledR}$ only at very long irradiation times of $2 \mathrm{~h}$. Unlike Photofrin ${ }^{\circledR}$, they did not induce immediate cell death but delayed apoptotic cell death ${ }^{[195]}$.

A large library of $\mathrm{A}_{3} \mathrm{~B}, \mathrm{~A}_{2} \mathrm{~B}_{2}$ and $\mathrm{AB}_{3}$ deprotected glycoporphyrins (316-331, 261) was synthesized to investigate the nature and distribution of carbohydrates on the periphery of the porphyrin and how this affected their biological properties on esophageal squamous-cell carcinoma cell line OE21 ${ }^{[33]}$. The different carbohydrate units controlled the biological behavior. All glycoconjugates were tested for their PDT efficacy except for the monosubstituted derivatives (316-319, 261) which proved too insoluble for the study. None of the glycoporphyrins showed PDT efficacy under these experimental conditions. Similar results for triazole linked glycoporphyrins have been reported ${ }^{[251,324]}$, while no biological data are available for related cationic bioconjugates ${ }^{[347]}$. However, the $D_{2}$ symmetric $\quad 5 \alpha, 10 \beta, 15 \alpha, 20 \beta$-tetrakis $(1,2: 3,4$-di- $O$ isopropylidene- $\alpha$ - $D$-galactopyranose-6yl)porphyrin localized in lysosomes and caused cell death by apoptosis via caspasedependent apoptotic pathways ${ }^{[348]}$.

Cyclodextrin-porphyrin systems have elicited significant interest as drug carriers and for binding studies. For example, $\beta$-cyclodextrin sandwiched porphyrins were synthesized to mimic holohemoproteins ${ }^{[349]}$. This involved the synthesis of atropisomers of 5,10,15,20-tetrakis( $o$ thioacetoxyphenyl)porphyrins and linkage with A,D-diiodo$\beta$-cyclodextrin $\left(10-15 \%\right.$ yield ${ }^{[349,350]}$. A first detailed PDT study involved mono- (638d) and bis-cyclodextrin-appended (638a) tetrakis(pentafluorophenyl)porphyrins derivatives, which were tested on several tumor cell lines. Both PSs localized in lysosomes and caused delayed cell death by apoptosis in HL-60 cells compared to 4T1 cell lines which were subject to both apoptotic and necrotic cell death. The mono-cyclodextrin porphyrin derivative 638d showed higher in vitro phototoxicity than the bis-cyclodextrin derivative 638a ${ }^{[351]}$.

Further studies using the $\beta$-cyclodextrin conjugates 638a,b,d focused on paclitaxel delivery while the $\gamma$ cyclodextrin conjugate 638c was used for doxorubicin transport. Thus, these systems were designed to act as dualmodality systems with the cyclodextrin functioning as a drug delivery unit while the porphyrin fragment acted as a PDT agent. It was anticipated that the $\mathrm{pH}$-dependent cyclodextrin binding could be used to release the small molecule drugs in the slightly acidic cancer cells. The monosubstituted derivative 638d exhibited low binding affinity and was not studied further. The tetrasubstituted compound $\mathbf{6 3 8 b}$ was found to be more effective in vitro when tested against 4T1 and K562 cells compared to the same animal model as used 


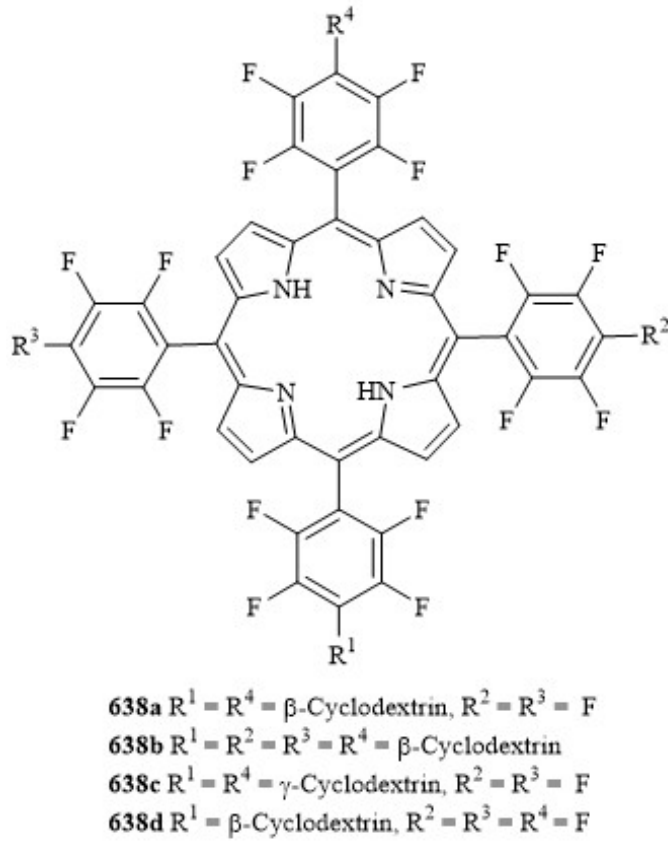

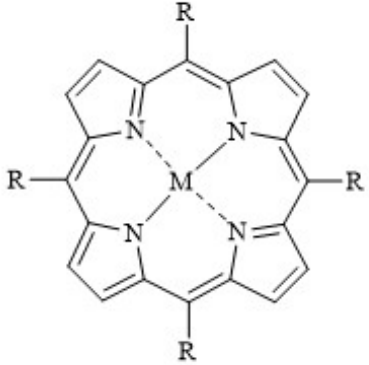

$639 \mathrm{R}$ $\mathrm{M}=2 \mathrm{H}(\mathbf{a}), \mathrm{Zn}(\mathrm{II})(\mathbf{b})$

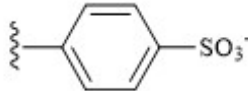

$640 \mathrm{R}=$ $\mathrm{M}=2 \mathrm{H}(\mathbf{a}), \mathrm{Cu}(\mathrm{II})(\mathbf{b})$

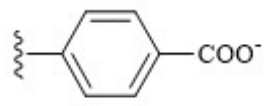

$641 \mathrm{R}=$

$\mathrm{M}=\mathrm{Cu}(\mathrm{II})(\mathbf{a}), \mathrm{Mn}(\mathrm{III})(\mathbf{b})$ $\mathrm{Au}(\mathrm{III})(\mathrm{c})$

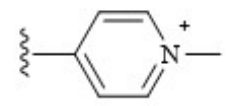

Formula 18

before. Best results were obtained with the disubstituted derivatives carrying two $\beta$ - (638a) or $\gamma$-cyclodextrins (638c) which gave very good in vitro and in vivo PDT activity combined with drug complexation at physiological $\mathrm{pH}$ conditions and release in cancer cells ${ }^{[351,352]}$.

An alternative method to present carbohydrate units is through strapping of two meso residues. A comparison of 5,10- and 5,15-strapped compounds (390 and 392) showed that 390 is significantly more active than $\mathbf{3 9 2}$ when tested on K562 human chronic myelogenous leukemia cells. The 5,15strapped porphyrin 390 showed significant delayed activity compared to $\mathrm{Hp}^{[220]}$.

\subsubsection{Hydroporphyrins}

Many studies focusing on chlorins utilized the basic framework of $m$ THPC, i.e, a $m$-tetra-" $O "$-phenylporphyrin core ${ }^{[353]}$. For example, the glycosylated tetraphenylchlorins 513a-c and 514a-c were tested for their PDT ability against HeLa cells ${ }^{[258]}$. They were more effective in generating singlet oxygen compared to 639a while within the series no marked difference in singlet oxygen production was observed. However, clear differences in cellular uptake were found. The highest activity was observed for the deprotected chlorins 514a,b in marked contrast to the respective parent porphyrin derivatives $[167,258]$. Interestingly, the octaglycosylated derivative 514c exhibited poor uptake and minimal PDT activity due to its high hydrophilicity.
Both tri- and tetra- glucosyl $m$ THPC conjugates (514f, 516, 517) were investigated for their photoactivity and internalization compared to $m$ THPC in HT29 human colorectal adenocarcinoma cells. The glycosyl substitution modified the photoactivity of $m$ THPC. Typically the symmetrical tetra-glucosylated chlorin $\mathbf{5 1 4 f}$ displayed poor internalization and photoactivity compared to the asymmetric, more amphiphilic derivatives $(\mathbf{5 1 6}, \mathbf{5 1 7})$ which showed better photoactivity compared to $m$ THPC with light

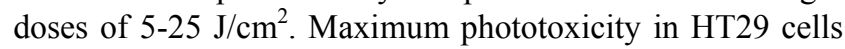
was observed with concentrations as low as $2 \mu \mathrm{M}$ of $\mathbf{5 1 6}$, 517 at light doses of $5-25 \mathrm{~J} / \mathrm{cm}^{2}$ compared to $m$-THPC required four times the concentration to achieve the same toxicity. Surprisingly, the cellular uptake of 516, $\mathbf{5 1 7}$ was 30 $\%$ lower than $m$ THPC and exhibited saturation. This increase in toxicity was due to the higher accumulation in mitochrondria, approximately a 2-fold increase in mitochondrial affinity for the glycoconjugated derivative $\mathbf{5 1 6}, 517^{[260]}$.

Next to the basic PDT activity the metabolism of the PS must be considered as well. Depending on the glycosyl unit these may be cleaved in vivo resulting in changes in the biodistribution, amphiphilicity and drug-cell interaction and possibly their PDT activity. Thus, the possible enzymatic metabolism of TPC $(m-O-G l c)_{3}(\mathbf{5 1 6}, \mathbf{5 1 7})$ was investigated in vitro in HT29 cells using MALDI-TOF mass spectrometry and HPLC ${ }^{[209]}$. This chlorin is four times more photoactive 
in vitro than Foscan ${ }^{\circledR}$ itself and thus of potential interest in the PDT treatment of tumors ${ }^{[260]}$. The three sugar units of 516, 517 were shown to undergo sequential hydrolysis with subsequent oxidation of the chlorin to the corresponding porphyrins. A cellular extract treated with $\mathbf{5 1 6}$ and $\mathbf{5 1 7}$ showed deglycosylation indicating that the cellular metabolism was independent of the substitution pattern. The cellular metabolism clearly effects the PDT activity of $\mathbf{5 1 6}$, 517 with the final metabolite $\mathbf{3 0 7}$ requiring 2-3 times the concentration to provide the same PDT effect ${ }^{[209]}$.

A comprehensive study expanding on work on 516, 517 [260] and $206{ }^{[198]}$ studied a range of related hydroxyphenylporphyrins and -chlorins and their glycoconjugated counter parts in HT29 and human retinoblastoma Y79 cell lines. The glycoconjugated derivatives had lower PDT activity compared to $m$ THPC as well as their respective parent compounds, displaying $\mathrm{IC}_{50}=$ 2.4-0.05 $\mu \mathrm{M}$ against Y79 cells. Among all the derivatives studied the tris- $p$ - $O$ - $\beta$-glucose porphyrin derivative 114 was found to have the best phototoxicity versus cytotoxicity activity compared to the parent porphyrin system with an $\mathrm{IC}_{50}=0.9 \mu \mathrm{M}^{[210]}$. However, this compound is less PDT active compared to the diethyleneglycol porphyrin conjugates described before (204-206) [198] including the glucose derivative $\mathbf{3 0 8}$. The extension of the linker between the glycoconjugate and the chromophore causes a significant increase in the PDT activity. Also highlighted was the difference in the anomeric configuration the $p$ - $O$-Deg- $O-\alpha-$ Gal compound $\left(\mathbf{2 0 5}, \mathrm{IC}_{50}=0.05 \mu \mathrm{M}\right){ }^{[198]}$ which shows a 10 fold greater phototoxicity than the $\beta$-glucose conjugate (308, $\left.\mathrm{IC}_{50}=0.6 \mu \mathrm{M}\right)$ in Y79 cells as well as $m$ THPC $\mathrm{IC}_{50}=0.6$ $\mu \mathrm{M}$. Here, porphyrins $\mathbf{2 0 5}$ and $\mathbf{1 1 4}$ were identified as good PDT PS ${ }^{[210]}$.

Similarly, the photochemical properties and the in vitro photocytotoxicity in HeLa cells of the four chlorin derivatives 514d-g were investigated ${ }^{[262]}$. Fluorescence intensities in phosphate-buffered saline of $\mathbf{5 1 4 f}$ and $\mathbf{5 1 4 g}$ (hexose groups) were $\sim 2$-times greater than 514d and 514e (pentose groups) proving a sugar-dependent difference of aggregation behavior. All the glycochlorins except 514g were spread throughout the cytoplasm. Compound $\mathbf{5 1 4 f}$ displayed the highest photocytotoxicity in HeLa cells while no phototoxicity was observed for $\mathbf{5 1 4 g}$.

Uptake studies in HeLa cells had shown that the chlorins 514a,b,d-i exhibited higher uptake then 639a and either equal or higher uptake then the corresponding porphyrin derivatives [261]. E.g., the 5,10,15,20-tetrakis[3-( $\beta$ - $D$ xylopyranosyloxy)phenyl]chlorin 514d showed 50 times higher uptake than 639a and the overall order of uptake was $514 d>514 i>514 e>514 a>514 b>514 h>514 f>514 g>$ 639a. The photocytotoxicity was unrelated to the cellular uptake as evidenced by the fact that the photocytotoxicity of the glycoconjugated chlorin $\mathbf{5 1 4 f}\left(\mathrm{LD}_{50} \sim 2 \times 10^{-7} \mathrm{M}\right)$ is almost 25 times that of the porphyrin $30\left(\mathrm{LD}_{50}=5 \times 10^{-6} \mathrm{M}^{\prime}\right.$ ${ }^{[165]}$ even though the cellular uptake of the two compounds are the same and there is no difference in their $\Phi^{1} \mathrm{O}_{2}$.
Other studies looked at the impact of physiological properties (i.e. cellular uptake and ROS) and structural diversity of the sugar unit ${ }^{[273]}$. The photocytotoxicity profiles poorly related to the amount of cellular uptake of the PSs. The cytotoxicity of 24 glycoconjugated PSs (30, 32, 3952, 524a,b,d-i and 639a) was evaluated in HeLa cells at a

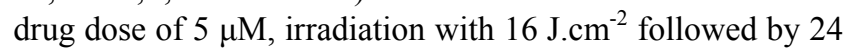
$\mathrm{h}$ incubation post treatment. All derivatives displayed no dark cytotoxicity and better photocytotoxicity than 639a. For 15 of the PSs (39-43, 46, 49, 50, 52, 514a,b, 514d-g) the $\mathrm{EC}_{50}$ was less than $5 \mu \mathrm{M}$. At $0.5 \mu \mathrm{M}$, the meta-substituted chlorins 514d-g still showed potent photocytotoxicity. $\mathrm{EC}_{90}$ values varied from about $0.5 \mu \mathrm{M}$ for $\mathbf{5 1}$ and $\mathbf{3 0}$ to less than $0.2 \mu \mathrm{M}$ for $\mathbf{5 1 4 f}$.

Examining the structural units the sugar moieties, the light-absorbing moiety and the substitution position of the sugar moiety in detail. The PSs bearing $D$-xylose residues usually showed higher photocytotoxicity than the other PSs, while those with $D$-arabinose units usually showed lower photocytotoxicity. Looking at the light-absorbing moiety the phototoxicity increased in the order of zinc porphyrin < porphyrin $<$ chlorin. For example, taking $\mathrm{EC}_{50}$ values 514a $(0.7 \mu \mathrm{M})<30(4.0 \mu \mathrm{M})<\mathbf{4 5}(5.0 \mu \mathrm{M})$ this showed the chlorin to be almost 6 times more phototoxic than the porphyrins ${ }^{[273]}$. On the other hand, no difference in the quantum yields of the porphyrin and chlorin derivatives were identified. However the molar extinction coefficient of 514a is $\varepsilon=2.4 \times 10^{-4} \mathrm{M}^{-1} . \mathrm{cm}^{-1}$ at $652 \mathrm{~nm}$ which is 3.7 times that of $30\left(\varepsilon=0.67 \times 10^{-4} \mathrm{M}^{-1} \cdot \mathrm{cm}^{-1}\right.$ at $\left.647 \mathrm{~nm}\right)$. The metasubstitution appears to increase photocytotoxicity for almost all PSs. Regarding the $\mathrm{EC}_{50}$ values for $\mathbf{3 0}$ at $4 \mu \mathrm{M}$ and $\mathbf{3 9}$ at $0.5 \mu \mathrm{M}$, switching the substitution position from para to meta changes the photocytotoxicity $\sim 8$-fold.

Significant results can also be obtained with one carbohydrate unit in chlorins. For example, a study with $\mathbf{2 4 7}$, 248 and 514a,b in HT29 cells clearly indicated that unsymmetric and less hydrophobic glucosylated PSs are superior to TPP ${ }^{[201]}$. The chlorin $\mathbf{5 1 4 a , b}$ gave the best PDT activity, probably due to its good singlet oxygen quantum yield $\left(\Phi^{1} \mathrm{O}_{2}=0.73\right.$ compared to 0.55 in $\mathbf{2 4 7}, 0.33$ in 248 and 0.55 for TPP). However, porphyrin 247 displayed 11.5 fold higher cellular uptake compared to TPP while the uptake of the chlorin was only five times better. Compound $\mathbf{2 4 7}$ localized in the ER. In general, the compounds with asymmetric substitution of saccharide units are more phototoxic than symmetrical compounds ${ }^{[201]}$.

An example of a different class of meso substituted chlorins are compounds 541 a', 542b', 542a and 542b which were tested for their photocytotoxicity in HeLa cells ${ }^{[178]}$. No dark toxicity was found at $0.5 \mu \mathrm{M}$. Only the deprotected PSs 91, 95, 542a and 542b were photocytotoxic. The $S$ glucosylated PSs 91 and 542a showed higher photoactivity than the $S$-galactosylated compounds 95 and 542b. The cellular uptake of the former increased up to $24 \mathrm{~h}$, while that of 95 and 542b was saturated by $12 \mathrm{~h}$. The photocytotoxicity of 542a was higher than that of $\mathbf{5 4 2 b}$ even at the same level 
of cellular uptake; clearly the glucose unit plays an important role in PDT effect.

Wavelengths $>500 \mathrm{~nm}$ are usually employed for PDT treatment, therefore the $S$-glycosylated chlorins have better photophysical properties for PDT compared to the $O$ glycosylated TPCs ${ }^{[178]}$. The difference in photoactivity is not attributed to the photophysics as all the compounds have similar properties therefore is due to biological effect.

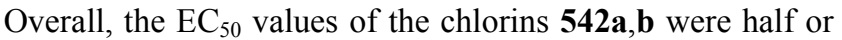
one-fifth of the porphyrins 91 and 95. Also, the $\beta-D-$ glucosyl-thio derivatives (91) showed a better photodynamic effect compared to $\beta$ - $D$-galactosyl-thio systems in MDAMB-231 human breast cancer and 3Y1 rat fibroblasts observed by Drain et al. ${ }^{[247]}$.

In addition meso substituted chlorins (542a,a'), isobacteriochlorins (545a,a') and bacteriochlorins (546a, a') were evaluated against human breast cancer cells MDA-MB231 and K:Molv NIB 3T3 mouse fibroblast cell lines ${ }^{[274]}$. Compound 542a showed a significant red shift compared to 91 however, the bacteriochlorins 546a displayed the greatest red shift near $730 \mathrm{~nm}$ and 50 times greater absorbance optimum for PDT.

Following from this, a comprehensive cellular uptake study of compounds 543, 543a,a', 542a, 93 and 91 was carried out showing that the glyco-conjugates had four times the uptake of $\mathbf{5 4 3}$ and with no difference in cellular uptake between the porphyrin and the chlorin macrocycles in HeLa cells ${ }^{[177,354]}$. The photocytotoxicity of the glycoconjugated PSs were 40 times greater than $\mathbf{5 4 3}$ where the $\mathrm{EC}_{50}$ values decreased in the order of $\mathbf{5 4 3}(5.0 \mu \mathrm{M})>\mathbf{9 3}$ and $91(0.15$ $\mu \mathrm{M})>$ 543a $(0.1 \mu \mathrm{M}) \approx 5$ 542a $(0.09 \mu \mathrm{M})$. The photocytotoxicity of the chlorin derivatives 543 and 542a was $\sim 1.5$ times greater than the corresponding porphyrin derivatives 93 and $91{ }^{[354]}$. Tests of 542a and 543a were also performed with B16-BL6 melanoma cells, weakly metastatic $\mathrm{B} 16 \mathrm{~F} 1$ cells and metastatic $4 \mathrm{~T} 1$ breast cancer cells. The free base chlorins gave the best results in all cell lines. Thus, any heavy atom effect on the photocytotoxicity was negligible. While often heavy atom effects are noted in solution of in vitro studies, there is no guarantee that it is maintained in a multicellular environment ${ }^{[307]}$. It is more efficient to attempt to improve the PS efficacy through the sugar unit ${ }^{[177,178,354]}$.

Benzochlorins have been of interest in PDT for a significant time ${ }^{[355]}$. The amphiphilic benzochlorin 552b displayed good photocytotoxicity in vitro against HT29 tumor cells when irradiated either with white light and a better one with red light irradiation to be more efficient. In comparison the parent, unconjugated derivative 551b exhibited no phototoxicity ${ }^{[277]}$. HT29 is also susceptible to a range of other amphiphilic porphyrins, benzochlorins and azaporphyrins; again glycosylated benzochlorin and azaporphyrins displayed the best photocytotoxcity when irradiated at $590 \mathrm{~nm}^{[356]}$.

In another study on OEP-type benzochlorins, all PSs $(\mathbf{5 5 3 - 5 5 6 b}, \mathbf{5 5 7})$ were found to be active in vitro on radiation-induced fibrosarcoma (RIF) tumor cells. These cells are known for their galectin-3 (Gal-3) expression. In control experiments the tumor cells were pre-incubated with lactose to block the $\beta$-galactoside binding sites before treatment with the PS. Both the galactose- and lactosebenzochlorin conjugates $(\mathbf{5 5 5 b}, \mathbf{5 5 6 b}, \mathbf{5 5 7})$ exhibited $100 \%$ loss of their PDT efficacy in the presence of free lactose, whereas 553b retained its activity. This indicates a possible $\beta$-galactoside-recognized protein specificity for $\mathbf{5 5 5 b}, \mathbf{5 5 6} \mathbf{b}$ and $\mathbf{5 5 7}^{\text {[279]. }}$

Due to their excellent uptake and photophysical properties phyto- and rhodochlorin derivatives have remained at the forefront of efforts to develop better PS ${ }^{[283]}$. Thus they present ideal candidates to investigate the influence of sugar units. For example, initial studies on purpurin imide conjugates compared the free purpurin imide to those conjugated with galactose $(\mathbf{5 6 9 a}, \mathbf{5 7 0})$ and lactose $(\mathrm{Gal}(\beta 1-$ 4)-Glc) (569b) which showed a significant increase in photosensitizing efficacy in vitro under similar light and drug doses in Molt-4 cells with the conjugate derivatives. The lactose derivate gave an increase in PDT efficacy compared to the galactose one ${ }^{[278]}$. Pre-incubation of the cell line with lactose showed a significant decrease in photoactivity, highlighting target-specificity in relation to the carbohydrate derivatives.

Expanding from these previous studies ${ }^{[278]}$, the effect of glycosyl regiochemistry, linker and glycosyl unit in $N$-hexyl mesopurpurimide glycoconjugates (569b,c, 571-575) was tested in radiation-induced fibrosarcoma (RIF) tumor cell lines ${ }^{[290]}$. A comparison of the three isomers $\mathbf{5 7 1 b}, \mathbf{5 7 2}, \mathbf{5 7 3}$ indicated that the 3-lactose purpurin-18- $N$-hexylimide (571b) provided the best efficacy; it was $\sim 4$ times more potent than $\mathbf{5 7 2}$ and $\mathbf{5 7 3}$. The effect of different linker units was examined with the lactose derivatives bound by an amide bond at $17^{2}(\mathbf{5 7 4})$, an $N$-benzyl group via an alkyne at $13^{2}(\mathbf{5 7 5}), \mathbf{5 6 9 b}$ and the non-conjugated purpurinimide $(569 c)$. These showed poor in vitro photoactivity compared to $\mathbf{5 7 1 b}$. Changing the sugar unit at position 3 showed the glucose conjugate 571c to be better than 571b and 571d. Overall the conjugate with lactose at position 3 (571b) proved the most effective ${ }^{[290]}$. Molecular modeling studies showed favorable binding affinity interactions of 3- and 12lactose-purpurinimide analogues with both galectin-1 and galectin-3. An ELISA study of the lactose conjugates compared to free lactose showed binding of all carbohydrate purpurinimides to both gal-1 and gal-3 galectins however there was a significant variation between the batches of galectins therefore requiring additional investigations.

One of the currently most promising candidates under trials is 2-(1'-hexyloxyethyl) pyropheophorbide a (HPPH), an effective PSs itself in phase I/II clinical trials ${ }^{[286]}$. Here, the dependence on galectins-3 binding was evaluated on both galactose conjugates $(\mathbf{5 5 8 a}, \mathbf{5 5 8 c})$ and nongalactose conjugates $(\mathbf{5 5 8 b}, \mathbf{5 5 8 d})$ of $\mathrm{HPPH}^{[287]}$. All in vitro tests were carried out in murine radiation-induced fibrosarcoma (RIF) and colon carcinoma (Colon26) cell lines; both express similar levels of the $\beta$-galactose-recognizing galectin- 1 and 
galectin-3. They differ, however, in the levels of the ATPdependent transporter ABCG2 which is responsible for the removal of HPPH in the cells. RIF cells express high levels of the protein, while Colon26 cells have no ABCG2 expression. All conjugates gave higher PDT activity than $\mathrm{HPPH}$ in RIF cells and 558a,b were twice as effective as $558 c, d$.

Notably, the introduction of a rigid linker in $\mathbf{5 5 8 f}$ proved to be several times less effective than amide bound lactose analogue 558c but similar to that of non glycosylated HPPH. Increasing the saccharide moiety $(\mathbf{5 5 8 g}, \mathbf{h})$ still proved ineffective. No difference was observed between the glucose (558a) and galactose (558b) derivatives therefore ruling out any contribution to uptake with the presence of galectins-3. Lower activity for HPPH was observed in RIF cells which was attributed to the action of the ABCG2 pump for which HPPH is a substrate. Both $\mathbf{5 5 8 c , d}$ and $\mathbf{5 5 8 f - h}$ all displayed lower activity. This was attributed to the larger carbohydrate units and the rigidity of the latter structures affecting the mode of cellular uptake and intracellular distribution. While $\mathbf{5 5 8 c}$ localized in the Golgi apparatus and lysosomes, 558g, $\mathbf{h}$ was also present in mitochondria as well. Several of the carbohydrate conjugates exhibited superior PDT activity which may be determined by subcellular localization and the signaling mechanisms initiated by PDT ${ }^{[287]}$.

The role of the ATP-dependent transporter ABCG2 was investigated in some detail. It is a multidrug resistant pump expressed at variable levels in cancerous cells and can cause efflux of a range of PS, thus lowering intracellular accumulation of the PS in the tumor tissue. Pandey's group investigated structural factors that affect the affinity of a PS for $\mathrm{ABCG} 2$ in an ABCG2-expressing cell line (HEK 293 482R) and a non-expressing cell line (HEK-293 PcDNA) with the goal of improving accumulation and photocytoxicity in ABCG2 expressing cells ${ }^{[357]}$. A series of conjugated PS with different groups attached at different positions of the macrocycle were examined, to test whether a change in affinity for the pump occurred. PSs without substitutions including pheophorbides (559) HPPH and purpurinimides (569c, 571a) were good substrates for ABCG2 efflux. However, carbohydrate groups conjugated at positions $8(\mathbf{5 7 2}), 12(\mathbf{5 7 3}), 13^{2}(\mathbf{5 6 9 b}, \mathbf{5 7 5})$, and $17(\mathbf{5 6 5}$, 566, 558a-d, 558f-h, 574) but not at position 3 repelled ABCG2 interactions independent of the structure/ type of the attached sugar unit ${ }^{[278,287,288,290,357]}$. At position 3, affinity for ABCG2 was maintained with the addition of iodobenzene (569), alkyl chains $(\mathbf{5 6 7}, \mathbf{5 6 8})$ and monosaccharides (571c,d), but not with disaccharides $(\mathbf{5 7 1 b})^{[278,290,357]}$. These results highlighted the possible importance of characteristics of position 3 influencing binding to ABCG2 ${ }^{[357]}$.

Self organized nanogels of $\mathbf{5 8 3} \sim 125 \mathrm{~nm}$ in size were evaluated for the auto-quenching properties and in vitro PDT efficacy against HeLa cells ${ }^{[294]}$. The auto-quenching of these materials decreased with increase Pheophorbide a content. Internalization of $\mathbf{5 8 3}$ was mediated via hyaluronic acid receptors with subsequent cleavage by intracellular enzymes releasing the free Pheophorbide a. Compound $\mathbf{5 8 3}$ exhibited similar PDT efficacy as the lone Pheophorbide a ${ }^{[294]}$. Similar results were obtained for compound 584, the fluorescence and singlet oxygen production were measured in DMF and aqueous medium where self-quenching was evident only in the latter. 584 was internalized via folate receptors and the PS cleaved by intracellular enzymes or extracellular matrix producing an active PS. Fluorescence was detected 30 minutes after injection and increased over $12 \mathrm{~h}$ period thus exhibiting similar phototoxic properties as the free pheophorbide $\mathrm{a}^{[295]}$.

\subsubsection{Related Systems}

Similar to that observed with the efflux of PSs by the ABCG2 expressing cell lines ${ }^{[357]}$, P-glycoproteins also act as energy dependent drug efflux pumps causing multi-drug resistance. $\beta$-glycosylated Tolyporphin (5) was investigated for its reversal of multidrug resistance of daunomycin, taxol and cisplatin in human ovarian (SKOV3 and SKVLB1) and breast cancer (MCF-7 and MCF-7/ADR) cell lines, the latter two of which over-express P-Glycoprotein ${ }^{[151]}$. Tolyporphin chemosensitizes SKVLB1 and MCF-7/ADR cells to drugs effluxed by P-glycoprotein but not those nontransported drugs. Similar to other reversing agents including verapamil, Tolyporphin acts as an antagonist binding to P-glycoprotein and inhibiting the removal of cytotoxic drugs. In comparison to verapamil for example Tolyporphin is significantly more potent at lower concentrations and the efficacy of both Tolyporphin and verapamil vary depending on the drug tested. Tolyporphin is particularly promising as a potential MDR agent ${ }^{[151]}$.

Although Hp and similar systems have often been used for comparison in the studies outlined here only one paper appears to have directly compared meso and $\beta$-substituted glycoporphyrins. Sylvain et al. determined the photocytotoxicities of two compound series (231-236 and 456b-458b) against K562 chronic leukaemia cells with Photofrin $^{\circledR}$ as a reference ${ }^{[199]}$. The compounds required $24 \mathrm{~h}$ post-treatment incubation in the dark at $37{ }^{\circ} \mathrm{C}$ and only with irradiation times of $>60$ minutes were they comparable to Photofrin ${ }^{\circledR}$. They initially displayed necrosis but time delayed apoptosis gave further cell death over the post treatment incubation period. All the thioglycosylated mesoaryl porphyrins ortho isomers $(\mathbf{2 3 1}, \mathbf{2 3 3}, \mathbf{2 3 5})$ and only the glucose para isomer 234 were found to be photocytotoxic. The partially deprotected isohematoporphyrin derivatives (456b-458b) were also found to be photocytotoxic and were more efficient then Photofrin ${ }^{\circledR}$ indicating that their PDT effect is dependent upon their amphiphilicity rather than the glycosyl moiety ${ }^{[199]}$.

One of the first few examples of sugar-containing phthalocyanines tested for their photoactivity were 617 and 618a-c which were studied on HepG2 human hepatocarcinoma cells ${ }^{[317]}$. They displayed very good singlet oxygen quantum yields ranging from 0.79 to 0.94 . The axially bisglycosylated compound $\mathbf{6 1 7}$ showed high 
photocytotoxicity providing $\mathrm{IC}_{50}$ value of $0.10 \mu \mathrm{M}$. The unsymmetric analogues (618) gave similar results and showed a slight decrease in PDT activity with the alkoxy chain length providing $\mathrm{IC}_{50}$ values ranging from 0.10-0.79 $\mu$ M. $\mathrm{Si}(\mathrm{IV}) \mathrm{Pc}\left(\mathrm{OC}_{6} \mathrm{H}_{13}\right)_{2}$ was tested for its PDT activity for comparison and showed no cytotoxicity. Thus, the PDT activity of 618 is primarily dependent upon the galactose residue and higher cellular uptake. Compound $\mathbf{6 1 7}$ was also examined in HT29 and T84 human colon adenocarcinoma cells with the $\mathrm{IC}_{50}$ showing it to more effective in the HT29 cells and significantly better than Photofrin ${ }^{\circledR}[318]$

Two glucosylated silicon(IV) phthalocyanines derivatives $(614 a, b)$ were examined against two different cell lines HT29 human colorectal carcinoma and HepG2 human hepatocarcinoma cells for their PDT efficacy ${ }^{[315]}$. Due to the heavy atom effect the dichloro glucosylated silicon(IV) phthalocyanine derivative (614b) displayed greater singlet oxygen generation but lower fluorescence emission compared to that of the non-chlorinated derivative (614a). Compound 614a showed higher photocytotoxicity with $\mathrm{IC}_{50}$ values of $6 \mathrm{nM}$ compared to $17-21 \mathrm{nM}$ for $\mathbf{6 1 4 b}$ with the effects greater in HT29 cells compared to HepG2 ${ }^{[315]}$. The photocytotoxicity of $\mathbf{6 1 4 a , b}$ were significantly higher compared to the PSs Photofrin ${ }^{\circledR}$ and pheophorbide $a^{[358]}$ with 614a highlighted as an excellent potential PS ${ }^{[315]}$. A comparative study of the galactose derivatives 617 and 615 against HT29 human colon adenocarcinoma and HepG2 human hepatocarcinoma cells was also carried out. It was observed that the octa-substituted derivative $\mathbf{6 1 5} \mathbf{c}$ displayed no photocytoxicity while the $\mathrm{IC}_{50}$ values ranged from 0.1 $1.05 \mu \mathrm{M}$ (HepG2) and 0.03-0.87 $\mu \mathrm{M}$ (HT29) for the other analogues in the series $617<615 \mathbf{a}<6 \mathbf{6 1 5 b}$. Concluding the chloro-derivatives are less potent than $617^{[316]}$.

Glycophthalocyanines $\mathbf{6 0 5}, \mathbf{6 0 8}$ and $5988^{[304,309]}$ were investigated for their PDT effect and influence on cell death of HeLa and HaCat cells ${ }^{[359]}$. The symmetric glycophthalocyanine $\mathbf{5 9 8}$ showed selective targeting with significant higher photocytotoxicity in the cancer cell line compared to non-malignant $\mathrm{HaCaT}$ cells. Compounds $\mathbf{6 0 5}$ and 608, even at higher concentrations, displayed very low PDT efficacy. The extent of cell damage was dependent upon both concentration and light dosage. The difference in PDT efficacy was presumed to be due to the difference in the behavior i.e., aggregation of the glycophthalocyanines $\mathbf{6 0 5}$ and 608 in solution ${ }^{[359]}$. The tetra galactose-substituted $\mathrm{Zn}$ (II) phthalocyanine 602b was also tested against HT-29 human adenocarcinoma cells but provided no $\mathrm{LD}_{90}$ values at concentrations as high as $100 \mu \mathrm{M}^{[308]}$.

The $\alpha$-substituted zinc(II) phthalocyanines $(\mathbf{6 2 5 a} / \mathbf{b})$ gave higher singlet oxygen quantum yields ranging from 0.40 0.66 compared to the metal free derivatives $\left(\mathbf{6 2 4 a} / \mathbf{b}, \Phi_{\Delta}=\right.$ 0.12-0.14) ${ }^{[310]}$. The glycoconjugated zinc(II) phthalocyanines 625a/b, 604, 622 and 627a-d were investigated for their PDT efficacy against HT29 human colon adenocarcinoma and HepG2 human hepatocarcinoma cells. The tetra- $\beta$-substituted glycosylated phthalocyanines
$(604,622)$ were not cytotoxic up to $8 \mu \mathrm{M}$ while $625 \mathrm{a} / \mathbf{b}$ and in particularly the mono-substituted analogues (627a-d) proved good PSs with $\mathrm{IC}_{50}$ values ranging from $2.0-0.9 \mu \mathrm{M}$ ${ }^{[310]}$. Unfortunately, 625a/b and $\mathbf{6 2 7 a - d}$ were less effective compared to the silicon(IV) analogues prepared previously $[315,317,318]$

Zinc (II) glucosyl phthalonitriles (627-630) were studied for their PDT activity against HT29 human colon adenocarcinoma and HepG2 human hepatocarcinoma cells ${ }^{[320]}$. The mono- and di-glycosyl derivatives $627,629 \mathbf{a} / \mathbf{b}$ and $\mathbf{6 3 0 a} / \mathbf{b}$ exhibited significant phototoxicity with the order of 630a $>629 a>627>630 b$ compared to Photofrin ${ }^{\circledR}$. The isopropyl derivative $\mathbf{6 3 0 a}$ proved significantly potent displaying $\mathrm{IC}_{50}$ values of 0.03 and 0.04 against HT29 human colon adenocarcinoma and HepG2 human hepatocarcinoma cells, respectively.

Zinc glycosylated phthalocyanines were investigated for their PDT efficacy against human colon adenocarcinoma cells (HT-29) ${ }^{[324]}$. The most potent derivative was the mannose conjugate $\mathbf{6 3 5} \mathbf{b}$ with an $\mathrm{LD}_{50}$ value of $110 \mu \mathrm{M}$ compared to $\mathbf{6 3 5} \mathbf{c}$ which failed to give an $\mathrm{LD}_{50}$ value even at concentrations as high as $500 \mu \mathrm{M}$. In comparing the linkage of glycosyl substitution versus triazole linkage, it was observed that a significant decrease in PDT activity was displayed by the $\mathbf{6 3 7}$ derivatives especially for the mannose conjugate $\mathbf{6 3 7 b}$ with a greater than three fold decrease in activity despite having similar cellular uptake ${ }^{[324]}$. This is in accordance with previous work carried out on triazole linkages for PDT by Maillard et al. ${ }^{[251]}$ and Daly et al. ${ }^{[33]}$

The highly glycosylated phthalocyanine $(\mathbf{6 1 1 b})$ and its porphyrin derivative $\mathbf{6 1 0 b}$ were evaluated for their photostability, singlet oxygen generation and their interaction with human serum albumin (HSA) ${ }^{[312]}$. Both displayed significant photostability over irradiation period of 30 minutes and singlet oxygen production similar to TPP. Fluorescence quenching of the intrinsic tryptophan fluorescence of HSA on addition of the two compounds was indicative of PS binding sites. In DMSO, 611b forms unusual needle like structures and under the electron microscopy, they self-assemble in irregular fashion forming sponge like material ${ }^{[312]}$.

Glucosyl-tetraaryltetrabenzoporphyrin conjugates (111 and 113) were investigated for their photocytotoxicity against the human cell lines $\mathrm{HaCaT}$ and MCF-7. Compound 113 was less active than Photofrin ${ }^{\circledR}$ and $\mathbf{1 1 1}$ proved to be an inefficient PS against both cell lines [184]. Tetrabenzoporphyrins also formed part of a study on triazole-linked glycoconjugates. The cellular uptake kinetics of 257, 258, $\mathbf{4 7 0}$ and $\mathbf{4 7 2}$ were evaluated in HEp2 cells. The tetrabenzoporphyrin conjugate 472 displayed a $\sim 5$-fold higher accumulation compared to the TPP conjugates at all time points. However, no conjugates exhibited toxicity up to $100 \mu \mathrm{M}$ concentrations ${ }^{[203]}$.

The $O$-glucosyl porphyrin dimers 365o,p and 367 were tested for their photocytotoxicity against the promyelocytary cell line K562 and compared to haematoporphyrin. 
However, no significant cell death was observed with all symmetrical glycosylated neutral and cationic porphyrin dimers possibly due to poor cellular permeability [192]. Subsequent studies on 3650, p, 368-370 confirmed this. This series of porphyrins showed the same efficiency for ${ }^{1} \mathrm{O}_{2}$ production as haematoporphyrin. The more hydrophilic dimers 3650,p, 369 exhibited very low photocytotoxic activity. The more amphiphilic compounds $\mathbf{3 6 8}$ and $\mathbf{3 7 0}$ were active against K562 cells. The former was the most active but still only equivalent to Photofrin ${ }^{\circledR}{ }^{[194]}$.

Another study found that all oligomeric derivatives (418421) displayed exceptionally high two-photon absorption at wavelengths suitable for biological tissues as well as significantly high singlet oxygen quantum yields $\left(\Phi_{\Delta}\right.$ from 0.43 up to 0.75 ) which make them promising PSs for twophoton excited PDT to treat tumor cells with overexpressing lectin-type receptors ${ }^{[231]}$. The rate of ${ }^{1} \mathrm{O}_{2}$ generation in a series of dimers (430a-d) was also examined by monitoring the quenching of DPBF in DMF. Apart from the deacetylated version of $\mathbf{4 1 8}$ all dimers produced ${ }^{1} \mathrm{O}_{2}$ more or less twice compared to $206^{[234]}$. Thus, conjugation had no effect on the ${ }^{1} \mathrm{O}_{2}$ generation as it was dependent on the number of macrocycles in the species. PDT studies with HT29 and Y79 cells revealed that the deacetylated version of 418 and 430b were only slightly phototoxic at high concentrations toward the Y79 cell line but also exhibited dark toxicity. The HT29 cell line overexpresses lectins for $\beta$ glucose residues and Y79 overexpresses lectins for $\alpha$ mannose residues. Here, no correlation was observed between the molecular structure and the lectins overexpressed. Additionally, these compounds have low solubility and are difficult to handle ${ }^{[234]}$.

The most complex carbohydrate unit being linked to a porphyrin involved the plant lectin Morniga G (MorG). This is a galactose specific binding lectin which was covalently bound to 5-(4-(5-carboxy-1-butoxy)-phenyl)-10,15,20-tris(4$N$-methyl)-pyridiniumyl)-porphyrin (TrMPyP) ${ }^{\left[{ }^{[2]}\right.}$. This system was used to determine its tumor-associated $\mathrm{T} / \mathrm{Tn}$ antigen specificity in Tn-positive (Jurkat lymphoid leukemia) cells and PDT efficacy. Several tumors show over-expression of $O$-glycans such as T (CD176) (Gal $\beta 1$ 3GalNAca1-O-Ser/Thr) or Tn (CD175) (GalNAca1-O-Ser/ Thr) antigens. A sugar dependent binding and uptake of fluorescein-labelled MorG was shown in Tn-positive cells as well as for the PS covalently linked MorG. The PS conjugate was bound and taken up very quickly. Within 5 minutes a dramatic increase in cytotoxicity at $10 \mathrm{nM}$ concentrations and with white light illumination was observed. The free PS was non-toxic in vitro. Normal lymphocytes were resistant to the conjugates; thus, these compounds are promising targeting agents for tumor cells ${ }^{[92]}$.

Subsequently, more therapeutically relevant PSs were synthesized with significant differences observed. These included conjugation to $\mathrm{Al}(\mathrm{III})$-phthalocyanin $\left(\mathrm{AlPcS}_{4}\right)$ and chlorin $\mathrm{e}_{6}$. Again, all conjugates displayed a similar increase in toxicity with $\mathrm{LD}_{50}$ in the nanomolar range compared to the

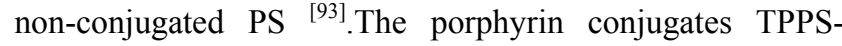
MorG and TrMPyP-MorG displayed similar phototoxicity with white light showing the porphyrin charge had no effect on the PDT efficacy. The AlPcS 4 -MorG conjugate showed weak toxicity on exposure to white light, however, displayed good activity with red light with $\mathrm{LD}_{50}=4 \mathrm{nM}$ a promising potential agent for PDT. $\mathrm{Ce}_{6}-\mathrm{MorG}$ was also found to be more potent under red light but not to the same extent. Conjugates of $\mathrm{AlPcS}_{4}$ and $\mathrm{Ce}_{6}$ have 9 and $10 \mathrm{PS}$ conjugated per protein, respectively, were tested for comparison with the $1 / 1$ ratio and only identified a slight increase in phototoxicity. In conclusion, the higher porphyrin conjugation the increase in ability of the conjugate to bind to specific clusters of antigens therefore no huge little increase in PDT efficacy was observed ${ }^{[93]}$.

The conjugate 332-STXB was evaluated for its receptor binding and PDT efficacy in STxB receptor cell line HeLa cells. It was found to have significantly higher PDT efficacy then $\mathbf{1 1 4}$ in such cell lines ${ }^{[212]}$. Concluding STxB conjugation offers an interesting tool for delivery of PSs.

\subsubsection{In Vivo Studies}

Similar to other porphyrin classes in vivo PDT results are more limited. Often different animal models and/or illumination protocols are used and thus a comprehensive QSAR analysis of in vivo data from different research groups is not possible.

For meso substituted glycoporphyrins initial in vivo studies on pharmacokinetics and biodistribution were performed in healthy rats with the triglucoconjugated porphyrin $306(0.25-1 \mathrm{mg} / \mathrm{kg})$. $m$ THPC $(0.3 \mathrm{mg} / \mathrm{kg})$ was used as a control ${ }^{[260,360]}$. The glycoporphyrin was cleared three times faster from the animals than $m$ THPC with mean residence times of 5 and $20 \mathrm{~h}$, respectively. In contrast to in vitro studies ${ }^{[209]}$ no deglycosylated or oxidized metabolites were found. In vivo, compound $\mathbf{3 0 6}$ was primarily concentrated in lung, liver and spleen, i.e. reticuloendothelial cells. The maximum PS concentration was observed at $14 \mathrm{~h}$ post-injection and elimination from the organs was complete within $48 \mathrm{~h}$. As glycosylation per se should not affect the photophysical properties this study underlines one of the more fundamental properties of glycoporphyrins in PDT, namely their rapid elimination. In a clinical setting this would shorten the post-treatment time of photosensitivity.

A similar study focused on the PS $\mathbf{2 0 6}$ which had shown affinity for human retinoblastoma cells and is specifically activated by green light at $514 \mathrm{~nm}{ }^{[198]}$. The flexible linkers increased the photodynamic activity in both HT29 and Y79 cell lines exhibiting a particularly high phototoxicity in human retinoblastoma cells Y79 and very low doses of the drug are required for a sufficient photobiological effect ${ }^{[198]}$. The compound was investigated in a mouse model of subcutaneously implanted retinoblastomas and ${ }^{1} \mathrm{H}$ and ${ }^{23} \mathrm{Na}$ magnetic resonance imaging (MRI) were used to monitor 
tumor tissue variations. ${ }^{23} \mathrm{Na}$ MRI is suitable for tracking the sodium concentrations through-out the PDT treatment ${ }^{[235]}$. It can be used to map extracellular compartments and characterize the degree of cell packing. PDT first caused a direct oxidative effect on the cells and this was then followed by a biological effect spreading cell damage to surrounding areas by signal transmission to neighboring cells, i.e. a bystander effect. A similar approach was taken to investigate the bystander effect in colorectal xenografted tumors. Here, the cellular tissue is less compact and the bystander effect was more difficult to induce. It could only be obtained using a double PDT treatment $4 \mathrm{~d}$ apart ${ }^{[236]}$.

One of the earliest studies in our context investigated the selective accumulation of the deoxyglucosamide pyropheophorbide 563 in a 9L glioma rat model ${ }^{[285]}$. The main purpose of the carbohydrate residue was to target the GLUT (glucose transporter) and hexokinase pathway which can be used to distinguish tumor tissue from normal tissue. Illumination of the tumor area in the animals occurred 30 minutes post-infection $(\lambda=670 \mathrm{~nm})$. Selective tumor accumulation could be shown via the PS fluorescence at 720 $\mathrm{nm}$ and illumination caused mitochondrial damage and alteration in the redox state in the irradiated region of the tumor.

In vivo studies on HPPH displayed slightly higher tumor uptake compared to 558a. It exhibited a several fold increase in liver retention due to faster clearance of the galactose derivative compared to HPPH. Likewise, it showed an elevated light dose-dependent activity compared to HPPH in both mice bearing RIF and Colon26 tumors. In comparison, the poor photocytotoxicity of $\mathbf{5 5 8 g}, \mathbf{h}$ could be attributed to their poor uptake, where 558f had similar uptake to HPPH [287]. Likewise, in vivo testing of a purpurinimide library (569b,c, 571-575) in $\mathrm{C} 3 \mathrm{H}$ mice bearing RIF tumors at (drug dosage of $5 \mu \mathrm{mol} / \mathrm{kg}^{1}, 700 \mathrm{~nm}, 137 \mathrm{~J} / \mathrm{cm}^{2}, 75 \mathrm{~mW} / \mathrm{cm}^{2}, 24 \mathrm{~h}$ post-injection) showed compound $\mathbf{5 7 1 b}$ to have the best long-term activity compared to the related glucose 571c and galactose 571d conjugates, which were ineffective ${ }^{[290]}$.

An important part of any in vivo studies is the effect of PS transporter systems. Above we described the relevance of ABCG2 activity, and it was noted that some cell lines had negligible ABCG2 activity in bulk. However, a small ABCG2-expressing side population appeared to be present that caused tumor regrowth. This side population was examined for its PDT resistance with ABCG2 substrates in vitro and in vivo in the murine mammary tumor $4 \mathrm{~T} 1$. The substrate PS HPPH was compared to the non-substrate PS HPPH-gal (558a) in vivo and showed selective preservation of the side population which was responsible for the regrowth in vitro. Therefore, PDT resistant side populations may be the cause of recurrences seen preclinically and clinically. To prevent $\mathrm{ABCG} 2$ resistance, using a nonsubstrate PS or administering an ABCG2 inhibitor with a substrate PS might be advantageous when treating ABCG2expressing tumors with PDT ${ }^{[357]}$.
Comprehensive in vivo studies were also reported for glycosylated pyropheophorbides $(\mathbf{5 6 4 - 5 6 6})^{[288]}$. Again, using $\mathrm{C} 3 \mathrm{H}$ mice bearing RIF tumors it was observed that in fact the non-carbohydrate analogue $\mathbf{6 5 4}$ was more effective in vivo, completely contrasting the in vitro findings which showed galactose and glucose derivatives to have enhanced cell killing compared to the parent compound. At the lowest drug dose $(0.50 \mu \mathrm{mol} / \mathrm{kg}), \mathbf{5 6 5}$ showed one out of five mice to be tumor-free compared to the non-conjugate 564 and galactose conjugate $\mathbf{5 6 6}$ which gave no tumor response. Increasing the dose to $1.5 \mu \mathrm{mol} / \mathrm{kg}, 564$ showed an increase in photosensitizing activity where four out of five mice were tumor-free at day 60 . At $1 \mu \mathrm{mol} / \mathrm{kg} 566$ was too toxic, killing the mice after $24 \mathrm{~h}$ post-treatment and at $1.5 \mu \mathrm{mol} / \mathrm{kg} \mathbf{5 6 5}$ showed some toxicity with two mice dying within $24 \mathrm{~h}$ after PDT and two out of the remaining three mice being tumor free by day 60 . Clearly, $\mathbf{5 6 4}$ has better PDT activity than the glycosylated derivatives ${ }^{[288]}$. The in vivo biodistribution of the three compounds were measured at $24,48,72,96 \mathrm{~h}$ postinjection. Compound $\mathbf{5 6 4}$ gave the best uptake into the tumor tissue at $24 \mathrm{~h}$ and $48 \mathrm{~h}$ with $4.1 \%$ IDg (percent injected dose per unit weight) at $24 \mathrm{~h}$ post-injection. However, 566 showed higher uptake at $72 \mathrm{~h}$ and $96 \mathrm{~h}$ but exhibited high liver and spleen uptake at these times, too. Lastly, $\mathbf{5 6 5}$ provided the worst uptake at all times. The poor accumulation of the glycosylated derivatives makes it unlikely that these PS will find application in PDT. Compound 564 was also tested in $\mathrm{BALB} / \mathrm{c}$ mice bearing Colon-26 tumors. It showed higher tumor accumulation in Colon-26 tumors than the RIF tumors at every time point. The clearance rate was higher in RIF tumors and the PS showed slightly higher blood uptake in $\mathrm{BALB} / \mathrm{c}$ than $\mathrm{C} 3 \mathrm{H}$ mice $^{[288]}$.

Pandey et al. synthesized purpurinimides substituted at varying positions with different linkers and glycosyl units to determine their effect on the PDT efficacy. In vivo testing in $\mathrm{C} 3 \mathrm{H}$ mice transplanted with RIF tumors showed the nonconjugated derivative to be ineffective at drug dosage of 5 $\mathrm{mm} . \mathrm{kg}^{-1}$ and light dosage of $130 \mathrm{~J} / \mathrm{cm}^{2}$ treated $24 \mathrm{~h}$ postinjection and under similar conditions after 30 days the carbohydrate derivatives $\mathbf{5 6 9 b}$ and 570 provided $50 \%$ of mice free of tumors ${ }^{[278]}$

The hydroxylated chlorin derivatives $\mathbf{5 2 4 - 5 2 6}$ were subject to an in vivo bioassay for tumors photonecrosis in PC6 myceloma. The most hydroxylated compounds, the tetrahydroxybacteriochlorin and the mannityl chlorin ether were the most effective in promoting tumors photonecrosis compared to Photofrin ${ }^{\circledR}$.The chlorin-glucose derivative is an effective PS but showed no selectivity for the neoplastic tissue in vivo ${ }^{[39]}$.

A detailed in vivo analysis of the mono-cyclodextrin porphyrin derivative 638d showed higher in vitro phototoxicity than the bis-cyclodextrin derivative 638a. The opposite result was obtained in vivo in $\mathrm{BALB} / \mathrm{c}$ mice bearing subcutaneously transplanted syngeneic mouse mammary carcinoma 4T1. Compound 638a exhibited faster and more 
selective tumor uptake and caused tumor eradication ${ }^{[351]}$. Clearly, even within a set study the in vivo activity of PS cannot simply be predicted from in vitro data. Another study of $\beta$ - and $\gamma$-cyclodextrins were investigated for their drug carrying ability with In vivo studies of $\mathbf{6 3 8}$ complexed with specific drugs carried out on $\mathrm{BALB} / \mathrm{c}$ mice bearing subcutaneously growing mammary carcinoma, $4 \mathrm{~T} 1$, and the nude mouse model with human amelanotic melanoma C32 at $100 \mathrm{~J} / \mathrm{cm}^{2}$ after $6 \mathrm{~h}$ incubation. The most efficient PDT combined therapy was observed in mice treated with 638apaclitaxel or 638c-doxorubicin however, tumor relapse occurred after 10 days. Repeating the therapy after 1 week had a higher therapeutic effect ${ }^{[352]}$.

In vivo study was carried out to determine if the ester cleavage of $\mathbf{5 8 3}$ to produce the free PS Pheophorbide a observed in vitro occurred in non-tumor-bearing Balb/Cnude mice ${ }^{[295]}$. It was demonstrated that the initial release of Pheophorbide a was slow but over time increased with the maximum achieved after $12 \mathrm{~h}$ incubation in agreement with the in vitro study. The retention of $\mathbf{5 8 3}$ was greater than for Pheophorbide a alone its presence was still observed 30 days after injection. The quantum yield of singlet oxygen was maintained at 0.43 for 30 days with irradiation at 1,7 and 30 days carried out ${ }^{[295]}$.

A glucosylated zinc phthalocyanine $\mathbf{6 1 3}$ was investigated for its potential as a near-infrared imaging agent with liver tumor-bearing athymic nude mice. The compound displayed a relatively high photostability. Significant luminescence was observed $12 \mathrm{~h}$ post injection with $2 \times 10^{-4} \mathrm{M}$ of 613 in the lung, kidney and liver i.e., the main metabolic organs. Tumor fluorescence was substantial and no toxicity was observed confirming the dye as a potential near infrared fluorescent probe ${ }^{[314]}$

Král et al. found that sapphyrins bearing neutral water solubilizing groups were the most selective for pancreatic carcinoma tissue in a xenographic murine model and showed the best tumor to liver ratio in vivo including the glycoconjugate 586b when compared against a disubstituted glyco-protoporphyrin $\mathbf{5 8 7}{ }^{[297]}$. The high tumor to liver ratio of 585a and 586 bould be due to the faster metabolism of the sugar unit causing faster clearance. In further studies, 585a was found to display the highest uptake in intact mitochondria in vitro compared to a series of six expanded porphyrins (metallotexaphyrins and free-base sapphyrins) and 5,10,15,20-tetrakis(4-sulfonatophenyl)porphyrin ${ }^{[298]}$.

The photochemical properties of $\mathbf{5 8 6}$ were investigated in aqueous media determining their self-aggregation and also interactions with target receptors. It displayed absorbance in the far visible region $(>600 \mathrm{~nm}$ ) which allowed for deeper tissue penetration in biological systems. The biolocalization of 586b had been described previously and showed good tumor localization but poor clearance ${ }^{[297,298]}$. However, it has a poor singlet oxygen generation with $\Phi_{\Delta}=0.04$, due possibly to self-aggregation. Studies on its self-aggregation properties in aqueous media at concentrations ranging from $0.15 \mathrm{nmol} / \mathrm{L}^{1}$ to $2.5 \mu \mathrm{mol} / \mathrm{L}^{1}$ showed a significant change in the Soret bands, indicative of dissociation from selfaggregates in dilute solutions ${ }^{[361]}$. Addition of a large excess of BSA caused monomerization of the aggregated species while at low concentrations highly aggregated dimeric and monomer BSA bound derivatives were evident. Its photochemical activity was low; on irradiation it was unable to damage BSA, poly(Trp) and GMP in aqueous media due to self-aggregation. Only in the presence of a large excess of BSA and calf thymus DNA did compound $\mathbf{5 8 6 b}$ result in effective photodamage.

The glycosapphyrin 586c ${ }^{[299]}$ was investigated for its localization in normal and tumor human pancreatic adenocarcinoma-bearing mice using resonance raman (RR) and UV-Visible spectroscopy ${ }^{[362]}$. These methods offer a means of identifying localization without tissue destruction and were used to identify the presence and to estimate the relative content of sapphyrin in various tissues at various times post-injection. A maximum preferential localization of sapphyrin in tumor tissue was observed at 6 days postinfection and sapphyrin metabolites were identified in the liver and kidneys.

\subsection{Other Photomedical Uses}

\subsubsection{Antiviral and Antifungal PDT}

Several of the compounds described so far have been tested for their antiviral or antifungal PDT activity ${ }^{[363,364]}$. These include sapphyrins ${ }^{[296]}$ and various porphyrin derivatives. An example for the latter was a study by Carré et $a l$. who investigated the antifungal properties of various meso aryl glycoporphyrins the synthesis of which was described previously ${ }^{[183,365]}$. Similar to the results found in PDT studies the amphiphilic derivatives $(\mathbf{1 2 9}, \mathbf{1 2 1})$ showed much better uptake by and phototoxicity for Saccharomyces cerevisia.

Another study investigated the antiviral activity of 191, 193, 194, 199, 200, 201, 202 and 195, 196 against herpes simplex virus type1 (HSV-1) and type 2 (HSV-2) in Vero cells at different stages of cell culture infection in comparison to acyclovir and foscarnet (antiviral controls) ${ }^{\text {[30] }}$. The antiviral activity of the porphyrin derivatives was determined by the virucidal effect inhibition and the decrease of the virus yield. The highest inhibitory effect on viral yield for both viruses was displayed by the deprotected porphyrin derivatives 192, 194, 200, 202 and the methyl galactopyranosides 195 and 196 which were comparable to those of the controls. The strong inhibition of viral yields was observed at concentrations lower than their maximum non-cytotoxic concentrations. Compounds 192 and 194 in particularly have very similar inhibitory effects as acyclovir or foscarnet. These compounds display significant inhibitory effects and offer potential for the treatment of herpes virus infections ${ }^{[30]}$.

Another broad study compared the antiviral effect of neutral porphyrins $(\mathbf{4 1 2}, \mathbf{4 1 4}, 415)$ and their cationic analogues (416a-c) against HSV-1 ${ }^{[229]}$. With the exception of $416 \mathrm{a}$ all compounds gave $\mathrm{IC}_{50}>0.5 \mu \mathrm{M}$ where the neutral 
porphyrin displayed higher toxicity with $\mathrm{IC}_{50}$ values just below $1.0 \mu \mathrm{M}$ possibly due to different interactions with the viral envelope. The neutral compounds $(\mathbf{4 1 2}, \mathbf{4 1 4}, \mathbf{4 1 5})$ were more effective than the cationic ones, with $90 \%$ viral inactivation at irradiation for 15 minutes and $50 \mathrm{~mW} . \mathrm{cm}^{-2}$. Earlier it had been shown that the fully deprotected derivative have the best activity ${ }^{[30]}$. Here, both $416 \mathrm{c}$ and $\mathbf{4 1 5}$ significantly reduced the HSV-1 yield in vero cells; additionally the cationic derivative exhibited dark toxicity at all times of the viral replication cycle ${ }^{[229]}$.

Another approach used supramolecular aggregates of positively charged amphiphilic cyclodextrins with 5-[4-(1dodecanoylpyridinium)]-10,15,20-triphenylporphyrin

(TDPyP). The cyclodextrin complexes displayed a very high singlet oxygen quantum yield $\left(\Phi_{\Delta}=0.90\right)$ and gave good inactivation of the Gram-positive methicillin-resistant bacterium Staphylococcus aureus and Gram-negative bacterial pathogens, such as Escherichia coli. The monomeric porphyrin-cyclodextrin complex showed excellent stability with no release of the porphyrin species from the host-guest system. The anti-bacterial PDT results indicate the utility of positively charged carrier systems for PSs, resulting in faster diffusion to intracellular compartments. Photokilling of antibiotic-resistant pathogens could be achieved at concentrations of $5 \mu \mathrm{M}$ upon irradiation compared to the uncomplexed PS which proved ineffective [132].

Cationic porphyrins 416b and 416c, 209-212, 74 were also examined for their potential photodynamic inactivation of Micrococcus $s p$. and all were found to cause inactivation at drug dosage of $0.2 \mu \mathrm{mol} / \mathrm{L}^{1}$ and inactivation values ranging between 4.0 and $8.0 \log$ after 15 minutes of irradiation. PSs $\mathbf{7 4}$ and 211 showed the lowest inactivation values compared to $212,416 \mathrm{~b}$ and $416 \mathrm{c}$ which were significantly more effective. Compound $\mathbf{4 1 6 b}$ gave the best results as it was able to inactivate Micrococcus $s p$. at 10 minutes. Inactivation of Pseudomonas $s p$. was also achieved by all the PSs however, the rate of inactivation was considerably lower after 15 minutes irradiation with the inactivation values ranging between 3.4-6.0 log. The porphyrins 212 and 416b were the best against Pseudomonas $s p$. causing similar inactivation after 15 minutes with inactivation values of 5.3 and $6.0 \log { }^{[230]}$. The best inactivation was displayed by $\mathbf{4 1 6 b}$ in both bacteria strains exhibiting the higher ${ }^{1} \mathrm{O}_{2}$ production and cellular binding. In conclusion the PSs can be used against multi-drug resistant and UV-B resistant bacteria.

\subsubsection{DNA Cleavage}

DNA cleavage studies have been reported with some compounds which were prepared to simultaneously behave as nucleic acid binders and destructive entities. The deprotected sugar units in close proximity to the porphyrin scaffold provides non covalent binding to the nucleic acids and the porphyrin scaffold upon irradiation causes selective double stranded DNA cleavage to form II nicked, circular
DNA ${ }^{[171]}$. Notably, the $\operatorname{Pd}(\mathrm{II})$ complex (69, M = Pd(II)) showed high activity in this regard.

DNA photosensitization by pyropheophorbide derivatives has not been extensively studied. One example, the watersoluble cationic pyropheophorbide 642, was investigated as a photonuclease on both single and double stranded oligodeoxynucleotides (ODN), it was confirmed that singlet oxygen caused damage to sites in the DNA mainly guanine residues. Also observed were the Frank scissions of the ODN from a radical process through hydrogen extraction and sugar oxidation initiated by the photodegradation of the pyropheophorbide derivative. The photosensitization of ODN causes two lesions, base oxidation and sugar oxidation [366].

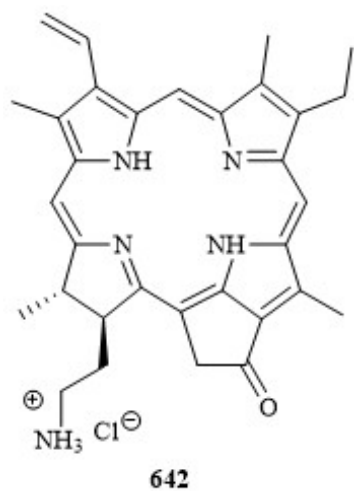

Formula 19.

The study of porphyrins DNA binding capacity is extensive [367-369], especially in the area of cationic porphyrins since the discovery of their ability to form several different complexes with DNA ${ }^{[370]}$. In recent years this research has expanded to incorporate bioactive functionalities for targeting. One such example was the introduction of amino acids/peptide conjugates to either increase cellular uptake of the porphyrin derivative or delivery of the peptidic units to nucleic acids ${ }^{[44,371-374]}$. One such recent example includes cationic porphyrins with Mn complexation which have been covalently linked with peptide cell signaling sequences. This conjugated derivative displaced significantly enhanced cellular uptake and DNA cleavage compared to the non-conjugated derivative ${ }^{[369]}$

Some studies have also reported on the synthesis of water soluble porphyrinyl nucleosides. For example, tri-, di- and mono- $p$-phenylene-5'-O-uridine substituted meso-(4-pyridyl) porphyrins were synthesized through conversion of the meso-(4-pyridyl $)_{4-\mathrm{n}}(p \text {-methoxyphenyl })_{\mathrm{n}}$-porphyrin to the $p$ hydroxyl derivatives. Next coupling with the uridine nucleoside gave the meso-(4-pyridyl) $)_{4-\mathrm{n}}\left(5^{\prime}-O-p\right.$-phenylene2'-3-O-isopropylidene-uridine $)_{\mathrm{n}}$-porphyrins which underwent $N$-methylation to afford the desired products 643$645^{[375]}$. The first examples of per(trifluoroethoxy) phthalocyanine-deoxyribonucleoside conjugates 646a, 647a were synthesized and evaluated as PDT agents. The twelve trifluoroethoxy substitutions prevent aggregation of the 
phthalocyanine and it was hoped that the nucleoside unit provides a key role for binding at the DNA recognition sites and induces radical selective cleavage of the DNA strand in the tumor cells under irradiation. The deoxyribonucleoside unit was connected to the phthalocyanine ring via an ethynyl linker as ethynyl-purine and pyrimidine nucleosides have significant potential due to their biological activities. The synthesis of 646 and 647 was achieved using Sonogashira cross-coupling of iodides and terminal alkynes in good yields. Additionally, the fluorinated conjugates can be advantageous in vivo studies using ${ }^{19} \mathrm{~F}$ NMR techniques ${ }^{[376]}$.

Pasetto et al. investigated a different approach to DNA photocleavage using $C$ - and $S$ - linked glycosylated tetraarylporphyrins. DNA photocleavage assays were used to evaluate the photoactivity of several porphyrins 71, 179, 91 and 95 all of which exhibited poor activity compared to 5,10-bis(4-methylpyridinium)-15-(4-methylphenyl)-20-(4hydroxyphenyl)porphyrin $\quad\left(\mathrm{DiMePy}^{+} \mathrm{MeOHP}\right)$

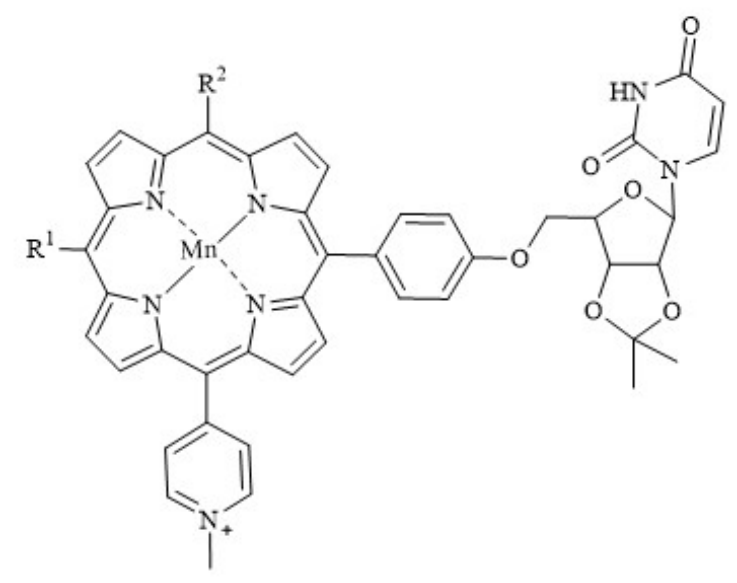

$643 \mathrm{R}^{1}=\mathrm{R}^{2}=$

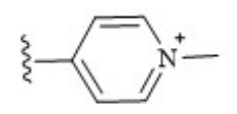<smiles></smiles><smiles>[Y]c1ccc(OC[C@H]2O[C@H]3OC(C)(C)O[C@H]32)cc1</smiles><smiles></smiles><smiles>[Y]c1cc[n+](CC)cc1</smiles>

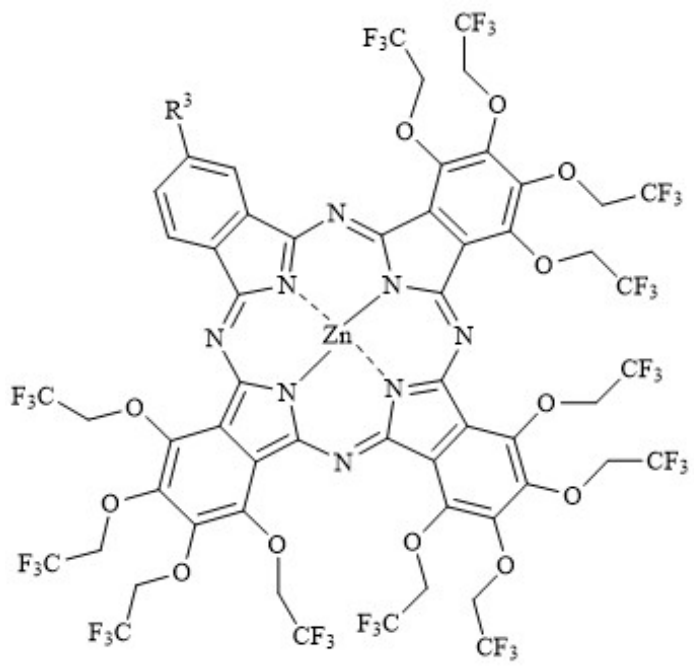

$646 R^{3}=$

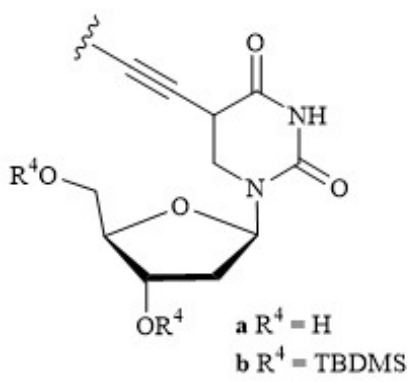

$647 \mathrm{R}^{3}=$

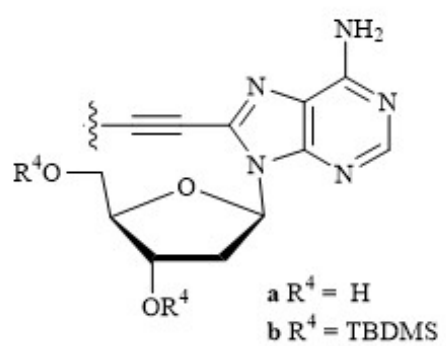

Formula 20. 


\section{Other Applications of Glycoporphyrins}

\subsection{Membrane and Protein Interactions}

PSs have been shown to bind to several plasma proteins depending on their amphiphilicity ${ }^{[377]}$. The more hydrophobic PSs bind to lipoproteins ${ }^{[378]}$ while hydrophilic PSs bind to albumin ${ }^{[379]}$. Albumin is the most important drug carrier protein. Preliminary observations from cellular uptake and phototoxicity tests indicate that 516, 517 showed more intermolecular association with albumin compared to $m$ THPC which is an important parameter to consider for in vivo testing ${ }^{[260]}$.

Membrane interaction studies are also important, as they can serve as models for more complex biological systems. For example, 269 and newly synthesized 649 prepared in the same manner were compared against the non-glycosylated dendrimeric porphyrin 648 for their interactions with biomimetic models of retina cancerous cell membranes which were absent of any lectin receptors on the surface and had increasing cholesterol content. It was observed that increasing the cholesterol content had no impact on the penetration of the PS into the lipid layers which was studied by surface pressure measurements and fluorescence spectroscopy ${ }^{[380]}$. The non-glycosylated derivative penetrated to a greater extent into the membranes at high lipid/porphyrin ratios only. The presence of the sugar moiety played an important role as the glycosylated derivatives could penetrate into the membranes even at low lipid/porphyrin ratios. Interactions of the sugar moiety with the polar phospholipid head groups limit their penetration depth. This work highlighted that glycoporphyrins can penetrate by passive diffusion independent of specific lectin interactions.

Another example is the use of glycosylated protoporphyrin IX derivatives as micellular fibers ${ }^{[31]}$. The amphiphilic glycosylated porphyrins $\mathbf{6 5 2 - 6 5 5}$ were synthesized from the porphyrin-formic acid mixed anhydride (651a) and the acetyl-2-deoxy-2-aminopyranosides which on subsequent deprotection afforded the desired amphiphilic compounds. All four compounds, as well as the racemate 653a:654a showed a broad and unsymmetric Soret band as a clear indication of some porphyrin aggregation, i.e. colloidal formation. These colloidal aqueous solutions showed significant stability over several months. Circular dichroism spectra revealed the more stable aggregate $\mathbf{6 5 2 a}$ to have greater rotational strength than the less stable aggregates of 653a and 654a thus providing strong evidence of only one dominant type of porphyrin aggregate. Electron micrography identified ribbons for 652a and 653a, 654a with approximate widths of $4 \mathrm{~nm}$ and lengths of $20 \mathrm{~nm}$ and $5 \mu \mathrm{m}$, respectively. On mixing 652b with photoactive dimeric porphyrins the aggregates do not quench their fluorescence which makes them candidates for electron acceptor or donor systems. The type and stability of molecular aggregates of glycoporphyrins strongly depends on the type and number of sugar units ${ }^{[381]}$.

Amphiphilic dendritic porphyrins 268 and 269 were tested for their interaction with phospholipids at the air-water interface and liposome bilayers were studied. Both compounds were incorporated in liposome membranes when

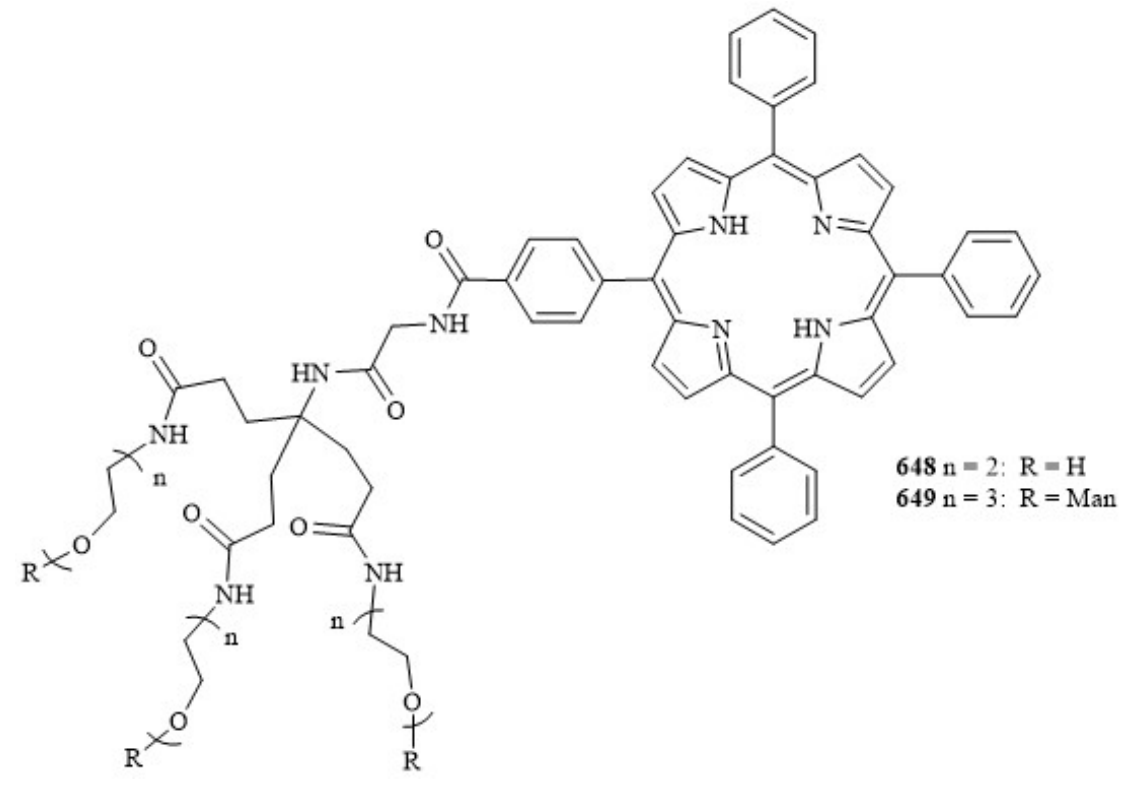

Formula 21. 

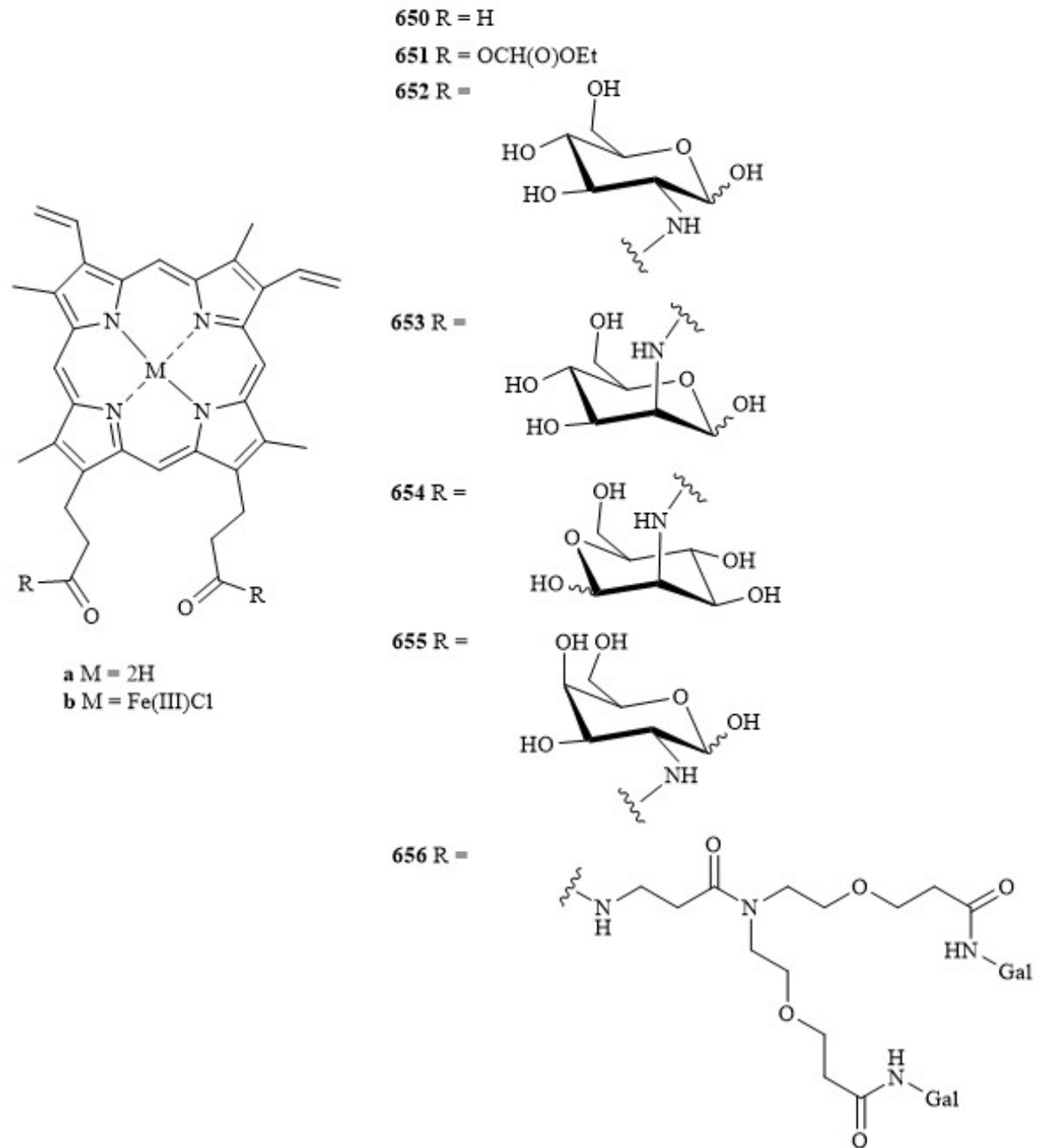

Formula 22

mixed with dimyristoylphosphatidylcholine (DMPC). Compound 269 mixed to a better extent than 268 and led to larger vesicles then DMPC alone with higher stability over time. Porphyrin 269 and DMPC mixed liposomes aggregated on addition of $\alpha$-mannose specific concanavalin $A$ suggesting that the macrocycle was embedded into the phospholipid bilayer while the sugar unit extended out into the aqueous phase ${ }^{[204]}$.

A range of hydroxylated ( $m$ THPP, $p$ THPP, 473, 512b) and glycosylated $(\mathbf{3 0}, \mathbf{3 9}, \mathbf{2 0 6}, \mathbf{5 0 4 b})$ derivatives of TPP were also examined for the effect their different structural characteristics had on their binding affinity to DMPC liposomes and HSA ${ }^{[382]}$. The glycosyl derivatives displayed greater solubility in aqueous medium but such conjugation decreased the affinity for the phospholipid bilayer of DMPC in all cases but increased binding to HSA with $\mathbf{3 9}$ displaying the best results. The hydroxylated derivatives proved to have the best affinity for DMPC liposomes with $m$ THPP and $p$ THPP affording the highest affinity. The dendrimeric structures 512b and 504b displayed significant selfaggregation and low affinity to both DMPC and HSA. Those conjugates with intermediate lipophilicity had greater affinity for DMPC and those of higher polarity displayed larger binding to HSA. The extent of penetration into the phospholipid bilayer of DMPC was dependent on the number of phenyl groups present and the distance of the glycosyl unit from the macrocyle. The dendrimeric structures showed the deepest penetration while the presence of diethylene glycol spacer increased the insertion of macrocycle compared to those without the linker ${ }^{[382]}$.

The incorporation of glycosyl units into a hemoprotein is quiet rare. One such example is the substitution of $\beta-N$ galactosyl dendritic units on the propionates of a ferric heme macrocycle $(\mathbf{6 5 2 b})$ with the ultimate goal of insertion into 
sperm whale apomyoglobin ${ }^{[383]}$. Porphyrin 652b was synthesized via an alaine type linker attached to the propionates of $\mathrm{Pp}$ IX which was coupled with $O$-acetylated 1 -amino- $D$-galactose by BOP in $42 \%$ yield. Subsequent insertion of iron and deprotection of the acetyl groups afforded the highly water soluble $\mathbf{6 5 2} \mathbf{b}$. The glycosylated ferric PpIX was inserted into sperm whale apomyoglobin with a yield of $40 \%$ and was purified by Sephadex G-25 gel filtration. The incorporation of $\mathbf{6 5 2 b}$ in the apomyoglobin was monitored by the UV-visible spectroscopy where the band shift at $396 \mathrm{~nm}$ to $408 \mathrm{~nm}$ is indicative of such events and ES-MS spectrum which identified 19,065 \pm 1 as the reconstituted myoglobin containing 652b [383]. The immunoprecipitation of $\mathbf{6 5 2} \mathbf{b}$ was tested in comparison to the wild-type myoglobin with biotin-labelled peanut lectin specific for $\beta$-galactose on streptavidin-modified sepharose. A decrease in heme content was observed for lectin combined with $\mathbf{6 5 2}$ b whereas not significant change was displayed for the wild-type myoglobin ${ }^{[383]}$.

One paper investigated the interaction of glycoporphyrins with toxins. Porphyrin 332 was covalently linked to the nontoxic homopentamer STxB subunit B of the bacterial Shiga toxins and verotoxins to take advantage of its retrograde delivery route. Shiga toxins and verotoxins comprise of an enzymatic component i.e. subunit A which inhibits protein biosynthesis and nontoxic homopentamer STxB i.e. Subunit B which promotes plasma membrane binding and internalization of subunit $\mathrm{A}^{[212]}$. STxB delivers holotoxin from the plasma membrane to the endoplasmic reticulum by endosomes and Golgi apparatus escaping degradation i.e. the retrograde route. Porphyrin 332 was synthesized via condensation of pyrrole with 4-O- $\left(2^{\prime}, 3^{\prime}, 4^{\prime}, 6^{\prime}-\right.$ tetraacetyl- $\beta$ - $D$-glucopyranosyloxy)benzaldehyde and 4bromomethylbenzaldehyde using $\mathrm{BF}_{3} \cdot \mathrm{OEt}_{2}$ in $31 \%$ yield and subsequent deprotection. It was then coupled with STxB-Cys via a cysteine residue ${ }^{[212]}$. The conjugate 332STxB was evaluated for its receptor binding and PDT efficacy in STxB receptor cell line HeLa cells ${ }^{[212]}$.

\subsection{Lectin Binding}

The interaction of porphyrin carbohydrate bioconjugates with lectins presents one of the more fundamental aspects of clinically relevant research in this area. Thus, many related studies have been reported. A classic topic is the use of glycosylated porphyrins $(\mathbf{5 8 7}, \mathbf{1 0 1})$ and sapphyrins $(\mathbf{5 8 5 b}$, 586b) for their non-covalent reversible binding to concanavalin A as a monitor of saccharide-lectin interactions. The addition of a natural substrate (e.g., Dmannose), which has a higher affinity for Con A compared to the non-covalent complex of lectin-glycomacrocycle causes the release of the weakly bound glycoporphyrin. This could be monitored by fluorescence spectroscopy due to quenching of fluorescence upon aggregation of the macrocycles. Here, compounds 586b, 587 and 101 showed good results for noncovalent fluorescent labeling of proteins ${ }^{[181]}$. In addition, the lectin-affinity of $\mathbf{7 3}$ was determined and exhibited strong specific binding with suppression of fluorescent intensity of fluorescein isothiocyanate (FITC)labeled lectins, FITC-RCA ${ }_{120}$ (Recinus comunus agglutinin, Lac-specific) while no change was observed with the $\alpha$ mannose and $\alpha$-glucose specific lectin FITC-ConA (Concanavalin A) ${ }^{[173]}$.

In a more recent study glycodendrimeric porphyrins (269, 649) ${ }^{[204,380]}$ were examined for their ability to be recognized by Con A. Their interactions were monitored by surface pressure measurements, fluorescence spectroscopy, dynamic light scattering, and QCM-D ${ }^{[384,385]}$. Binding capacity to Con A was dependent on the chemical structure with porphyrins 649 and 269 showing the highest binding constants. Immobilization of Con A as a monolayer covering a QCM-D gold sensor with addition of porphyrin-bearing liposomes determined the specific interaction via the sugar moiety [384,385].

Note, that previously glycodendrimeric porphyrins had given poor results in PDT as a result of their tendency to aggregate and to interact with blood proteins $[198,204,205,235,256,380,384]$. Such glycodendrimers have been also tested by dynamic light scattering and QCM-D for their affinity for a synthetic biomimietic lipid bilayer membrane of similar composition to retinoblastoma cell membranes containing Con A. The interactions proved promising affording specificity for mannosylated porphyrins with the spacer length between a porphyrin core and the mannose moiety playing an important role in their interaction ${ }^{[384,386]}$. Such models afforded better mimics of in vivo conditions and insight into the mechanisms of interaction between the glycoconjugates and retinoblastoma cells.

Expanding on the grafting of Con A bilayer systems ${ }^{[386]} \mathrm{a}$ detailed study was carried out to evaluate the interaction of porphyrins with classical Y79 cell lines and the adhesion of porphyrin-bearing liposomes to Y79 cells immobilized onto the sensor of a quartz crystal microbalance (QCM-D) in the absence of fetal calf serum ${ }^{[387]}$. QCM-D allowed for analysis of the mechanism of interaction of the glycodendrimeric porphyrins with the lectins which the classical cell cultures were not. The mannosylated porphyrins were able to interact specially with the mannose receptors similar to the results with Con A grafted onto artificial lipid bilayers ${ }^{[386,387]}$.

Noncovalent porphyrin protein systems have also been suggested as carrier vehicles. Thus, legume lectins, which show preferentially binding to tumor cells were found to bind simple porphyrins such as 5,10,15,20-tetrakis(4sulfonatophenyl)porphyrin with a binding constant of about 1.2-6.3 $\times 10^{4} \mathrm{M}^{-1}{ }^{[388]}$. Binding occurred in a binding site distant from the carbohydrate binding region. Studies with the galactose-specific lectin from snake gourd revealed two porphyrin binding sites with association constants of $1.7 \times 10^{4}$ - $6.2 \times 10^{5} \mathrm{M}^{-1}$ with higher affinities for metalloporphyrins compared to free base systems ${ }^{[389]}$. The binding appears to be predominantly through hydrophobic interactions as both cationic and anionic porphyrins were bound. Similar results were found for Jacalin, which recognizes the tumor 
associated T-antigenic structure Gal $\beta 1 \rightarrow 3$ GalNAc and could bind one porphyrin ${ }^{[89]}$.

Another example is the interaction of water soluble freebase and metalloporphyrins $(639 a / b, 640 a / b, 641 a$ and 74$)$ with Momordica charantia (bitter gourd) lectin (MCL), a tetrameric galactose-specific glycoprotein. Here, significant changes in the Soret band region of the electronic absorption spectra of the porphyrins were observed upon binding to MCL ${ }^{[390]}$. The association constants for the interaction of different porphyrins with $\mathrm{MCL}$ at $25{ }^{\circ} \mathrm{C}$ varied between $5 \times 10^{3} \mathrm{M}^{-1}$ and $10^{5} \mathrm{M}^{-1}$. Porphyrin binding affinity to $\mathrm{MCL}$ was not affected by addition of galactose, indicating that porphyrin and carbohydrate ligands bind at different sites. Likewise porphyrin binding did not significantly change the secondary or tertiary structures. 640b, 74 and 641a had significant affinity for MCL which identified MCL as a potential carrier for targeting these porphyrins to tumor tissues ${ }^{[390]}$.

The 639a-peanut lectin (PNA) interaction was analyzed crystallographically and provided considerable information on the porphyrin protein interaction ${ }^{[391]}$. The PNAporphyrin-lactose ternary complex and the PNA-porphyrin binary complex were determined at $3.10 \AA$ and $3.12 \AA$ resolutions, respectively. PNA is a tetrameric lectin, however, the four subunits are not related by 4-fold symmetry but do have similar tertiary structures and have equivalent carbohydrate binding sites which are specific for galactopyranose units at the monosaccharide level. The porphyrin binding partially overlaps with the carbohydrate binding site of two subunits while the two other subunits remain free for carbohydrate binding. Eleven molecules of 639a bind to the tetramer of PNA in five different stacking arrangements in both the PNA-639a binary complex and PNA-639a-lactose ternary complex. A comparison of the two structures revealed conformational changes in the presence and absence of lactose in the carbohydrate-binding site of PNA. Further details are given in a comprehensive review of carbohydrate-lectin non-covalent interactions (Figure 7) ${ }^{[392]}$.

a
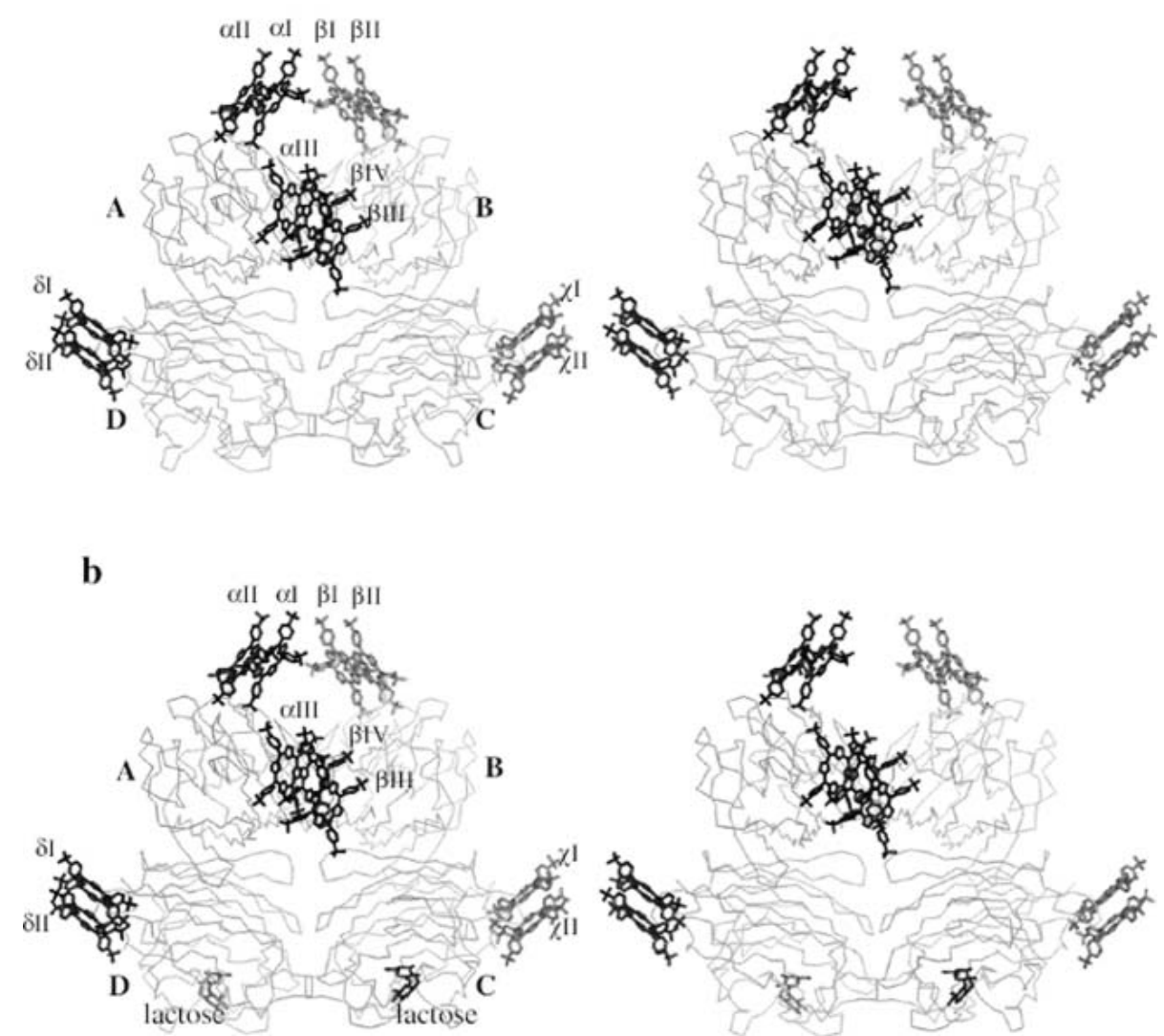

Figure 7. Crystal structures of (a) $11 \mathrm{H}_{2}$ TPPS (black) molecules interacting with tetramer PNA (in grey) and (b) the $\mathrm{H}_{2}$ TPPS-PNA-lactose ternary complex. Lactose (black) occupies the carbohydrate-binding site in subunits C and D of the PNA ternary complex but not in subunits A and B. Reproduced from ${ }^{\text {[397]. }}$ (Copyright, 2005, American Chemical Society).

Thermodynamic studies were carried out on the interaction of water soluble free base and metalloporphyrins with pea (Pisum sativum) lectin (PSL). Here, cationic and anionic porphyrins displayed similar affinity while 639b showed the strongest affinity for PSL compared to other porphyrin derivatives, possibly due to axial interactions of 
the $\mathrm{Zn}(\mathrm{II})$ metal ion with the protein. Introduction of the methyl- $\alpha$ - $D$-mannopyranoside, a specific substrate for PSL, showed no effect on the porphyrin binding determining the presence of different binding sites on the lectin ${ }^{[393]}$. The temperature dependence of the $K_{a}$ values were used to evaluate the thermodynamic forces that influence the interaction. It was concluded that the porphyrin binding to PSL was driven mainly by enthalpic forces with the entropic contribution being negative. Enthalpy-entropy compensation was observed in some interaction and highlighted the role of water structures in the overall binding process. Circular dichroism and differential scanning calorimetric studies showed porphyrin binding caused no change in the lectin structure or thermal stability however, carbohydrate binding has a stabilizing effect on the protein structure.

Several investigations addressed the interaction of glycoporphyrins with galectins. For example using the enzyme-linked immunosorbent assay it was shown that compounds 553-556b, 557 bind to Galectin with an enhanced affinity 141-387 times that of lactose $50 \%$ inhibitory concentration $\left(\mathrm{IC}_{50}\right)=1.2 \mathrm{mM}$ which is the standard $\beta$-galactoside used as a reference ${ }^{[279]}$. The benzochlorin galactose conjugates $\left(\mathbf{5 5 5 b}, \mathrm{IC}_{50}=5.1 \mu \mathrm{M}\right.$ and 557, $\left.\mathrm{IC}_{50}=3.1 \mu \mathrm{M}\right)$ and the benzochlorin lactose conjugate $\left(\mathbf{5 5 6 b}, \mathrm{IC}_{50}=4.3 \mu \mathrm{M}\right)$ had a two-fold advantage over the benzochlorin glucose conjugate $\mathbf{5 5 4 b}$ and the nonconjugated derivative 553b. This suggests a specificity of galactose and lactose conjugates for proteins that are known for their $\beta$-galactose recognition and overexpression on tumor cells.

In another study the binding activity of the tumor specific lectin-recombinant human galectin-1 (hGal-1) to three simple porphyrin compounds $(639 b, 641 b, c)$ was reported ${ }^{[394]}$. High binding affinities were observed with dissociation constants of $0.6-1.5 \mathrm{nM}$, similar to that for Con $\mathrm{A}$ and porphyrin binding. This reaffirms previous crystallographic studies which indicated that galectin and legume lectins such as Con A have similar topologies even though they have different sequences ${ }^{[357]}$. Experiments with human galectin-3 (hGal-3) gave similar results with $639 \mathrm{~b}(\mathrm{KD}=0.18-0.2 \mu \mathrm{M}$, both with and without lactose present). Thus, addition of lactose showed no inhibitory effect on binding showing the carbohydrate binding domain to be separate from noncarbohydrate binding sites. Circular dichroism spectra exhibited that binding of such hydrophobic compounds changed the hGal-3 secondary structure. As hGal-3 binds to important cancer carbohydrate antigens, it has the potential to operate as a target delivery molecule for anti-cancer therapy ${ }^{[395]}$.
Contemporary studies focus on related but more complex systems. For example, a series of tetravalent lactosylated glycoconjugates were synthesized via $\mathrm{Cu}(\mathrm{I})$-catalyzed azidealkyne cycloaddition in high yields. The glycoconjugates consist of a glycoporphyrin (657a) and several calix[4]arenes which were prepared from propargyl $m$ THPP and three topological isomers of tetra- $O$-propargylated calix[4]arenes, respectively via microwave mediated azide-alkyne cycloaddition with an acetylated azido-lactose derivative which was subsequently deprotected to afforded the free hydroxyl conjugates. They were evaluated for their binding to two lectins, ECA a lactose/fucosyllactose specific lectin from the legume plant Erythrina cristagalli and recombinant human galectin-1(gal-1). The inhibitory properties of these multivalent glycoconjugates were compared to the monovalent species (658) to determine selectivity of the glycoconjugates between the two lectins based on their topology. The glycoconjugates displayed high affinity for ECA and Galectin-1 with the porphyrin derivative 657a providing the best results for both in hemagglutination assays (HIA), only for ECA in enzyme-linked lectin assays experiments. A high selectivity was observed for the porphyrin derivative under HIA analysis but the same was not observed with surface plasmon resonance experiments. In conclusion this approach was not fully appropriate for multivalent ligands ${ }^{[396]}$.

An intriguing but complex approach promotes the use of phycocyanin in PDT. Phycocyanin (Pc) is a non-toxic, noncarcinogenic, water soluble phycobiliprotein present in the microalgae, Spirulina platensis. It is a pigment protein with a similar structure to bilirubin and is involved in light harvesting in photosynthesis of cyanobacteria, red alga and

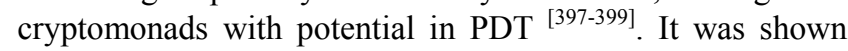
that the tumor-specific lectin jacalin, binds to Pc at a site independent of the carbohydrate active site and with affinities greater than that observed for porphyrins. Thus, the lectin has the potential to be a carrier for $\mathrm{Pc}$ for direct targeting of tumor tissue ${ }^{[397]}$.

Another approach focused on the use of carbon nanotubes (CNTs) for the electronic detection of carbohydrate-protein interactions. It is based on the effect that a single walled carbon nanotube field-effect transistor (NTFET) device can electronically transduce interactions between proteins and other biomolecules for example carbohydrates ${ }^{[400]}$. The NTFET were functionalized non-covalently with glycoporphyrins which were obtained using "click" azidealkyne chemistry. The specific binding of two bacterial lectins PA-IL, PA-IIL from Pseudomonas aeruginosa and a plant lectin Con A to three glycoporphyrins substituted with 


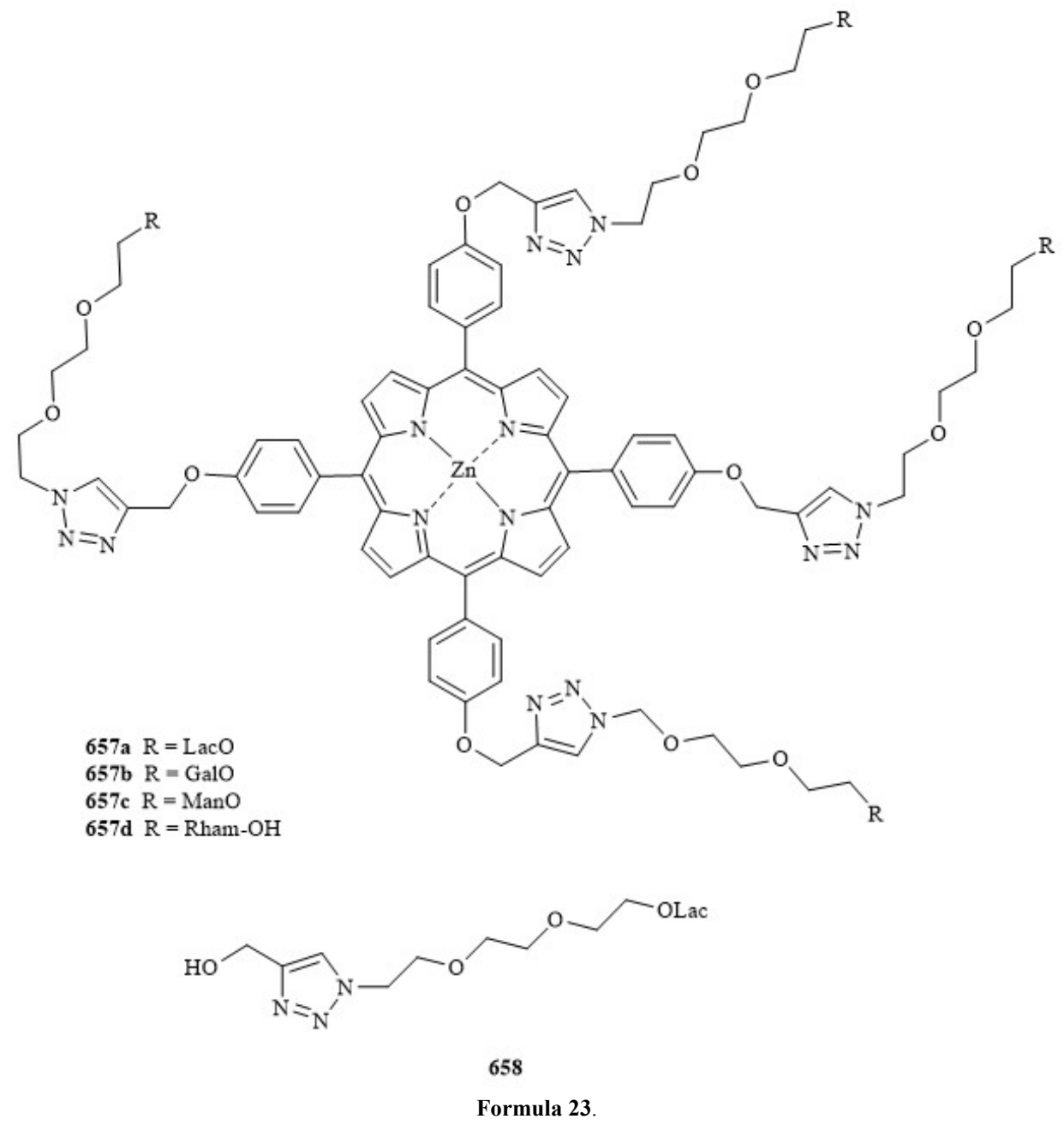

$\beta$ - $D$-galactose $(\mathbf{6 5 7 b}), \alpha$ - $L$-fucose $(657 \mathbf{d})$ and $\alpha$ - $D$-mannose $(\mathbf{6 5 7 b})$, respectively, were examined by changes in electrical conductance where the SWNT networks acted as conducting channels which transduced the binding between glycoporphyrins and lectins into electrical signals. Noncovalent functionalization of the devices with the glycoporphyrins prevented non-specific binding of the proteins with significant selective lectin carbohydrate binding. For example, the binding of PA-IL to galactosylated NTFETs was very sensitive $(2 \mathrm{nM})$ with a measured dissociation constant $\left(K_{\mathrm{d}}=6.8 \mu \mathrm{M}\right){ }^{[400]}$. Further investigations utilized both SWNTs and chemically converted graphene (CCG) as nano based biosensors ${ }^{[401]}$.

\subsection{Catalysis}

Metalloglycoporphyrins have been investigated in some detail as potential catalysts. While catalytic applications have no direct relevance for biomedical uses such studies do indicate the degree to which porphyrin-conjugates can be fine-tuned and modified for a specific purpose, such as substrate or receptor binding. The catalytic ability of metalloporphyrins is well known ${ }^{[402-404]}$ and use of glycoporphyrins might result in either water soluble catalysis and/or enhance catalyst selectivity. Such studies can also be of relevance for understanding natural porphyrin based oxidations.

A typical example is the use of chloro[tetra $(o-2,3,4,6-$ tetraacetyl- $\beta$ - $D$-glucopyranosyl-1- $O$ -

phenyl)porphyrinato]iron(III) ${ }^{[405]}$. The acetylated sugar derivatives exhibited improved turn-over-numbers and better oxidation stability in the cyclohexane oxidation with $\mathrm{PhIO}$ compared to the nonglycosylated ones. Likewise, related chloro-manganese(III) derivatives were investigated for the catalytic asymmetric epoxidation of $p$-chlorostyrene ${ }^{[406]}$. The steric hindrance induced by the protected sugar units prohibited a close contact between two porphyrins and allowed for stereoselective oxidation of the alkene. While all compounds induced asymmetric epoxidation it was lower 
than with reference compounds and the conversion to the epoxide decreased as the catalytic environment became asymmetric $^{[407]}$.

Detailed studies were performed with manganese and iron complexes of $O$-acetylated sugar TPP derivatives which provided stereoselective epoxidation of 4-chlorostyrene [160,406]. Initial catalysts were susceptible to $\mathrm{H}_{2} \mathrm{O}_{2}$ and thus (chloro)(5,10,15,20-tetrakis[2-chloro-6-(2,3,4,6-tetraacetyl$O$ - $\beta$-glucosyl)-phenyl]porphyrinato)manganese derivatives $(661 a-c)$ were synthesized (Scheme 42$)^{[408]}$. Use of Lindsey conditions gave 660a $(\alpha \beta \alpha \beta), 660 \mathrm{~b}(\alpha \alpha \beta \beta)$ and 660c $(\alpha \alpha \alpha \beta)$ in 1,3 and $5 \%$ yields, respectively. Attempts at thermal atropisomerisation to form $\alpha \alpha \alpha \beta$ atropisomer were unsuccessful in contrast to the previously synthesized ortho glycosylated compounds without the chloro group ${ }^{[161]}$. Conversion to the manganese complexes 661a-c was achieved via treatment with $\mathrm{MnCl}_{2}$ and 4-nitrophenol. The glycosyl group at the ortho position provides the chiral environment while the strong electron withdrawing groups provide stability to the catalyst. Using PhIO in the reactions provided 4-chlorostyrene epoxide in yields similar to the ones with nonchlorinated glycoporphyrins tested previously. Catalytic oxidation of 4-chlorostyrene was examined by two methods using either 4-tert-butylpyridine as axial ligand and benzoic acid as cocatalyst or using 2-methylimidazole as an axial ligand. Both required addition of a base to enhance the activity ${ }^{[408,409]}$. The epoxidation of 4-chlorostyrene by $\mathrm{H}_{2} \mathrm{O}_{2}$ with 4-tert-butylpyridine gave the epoxide in 25-30\% yield, similar to the unchlorinated glycoporphyrins ${ }^{[182]}$. In contrast, epoxidation with $\mathrm{H}_{2} \mathrm{O}_{2}$ with 2-methylimidazole afforded very low yields $4-5 \%$. The presence of the chloro groups did however, reduce the rapid autooxidation observed by the unchlorinated glycoporphyrins ${ }^{[160,408]}$. Generally speaking, the glycosyl groups of such derivatives provide a chiral environment and steric hindrance on one or both faces of the porphyrin which influenced the complexes catalytic properties. The enantioselectivity of the epoxidation was moderate and was dependent on the position of the chiral sugar unit. The $m$-glycosylated derivatives (104-106) are further from the porphyrin metal center and do not produce significant ee's. As the ortho substituted derivatives (102, 103) are closer to the active center of the porphyrin they afford higher enantioselectivity. The best result was obtained using $\mathrm{H}_{2} \mathrm{O}_{2}$ at low temperatures achieving enantiomeric excess of $20 \%{ }^{[182]}$.

A significant body of work on catalysis used cyclodextrin derivatives. One of the first examples of regioselective and stereoselective oxidation of linoleic acid via cyclodextrin sandwiched thioporphyrin mimicked lipoxygenase activity provided the stepping stone for the following work ${ }^{[410,411]}$. As well as initial work on selective electron transfer between cyclodextrin sandwiched thioporphyrin and quinone ${ }^{[412]}$.

Tetra- and dicyclodextrin substituted $\mathrm{Mn}(\mathrm{III})$ porphyrins were synthesized to investigate their ability to bind substrates for selective catalytic oxidation ${ }^{[179]}$. The starting
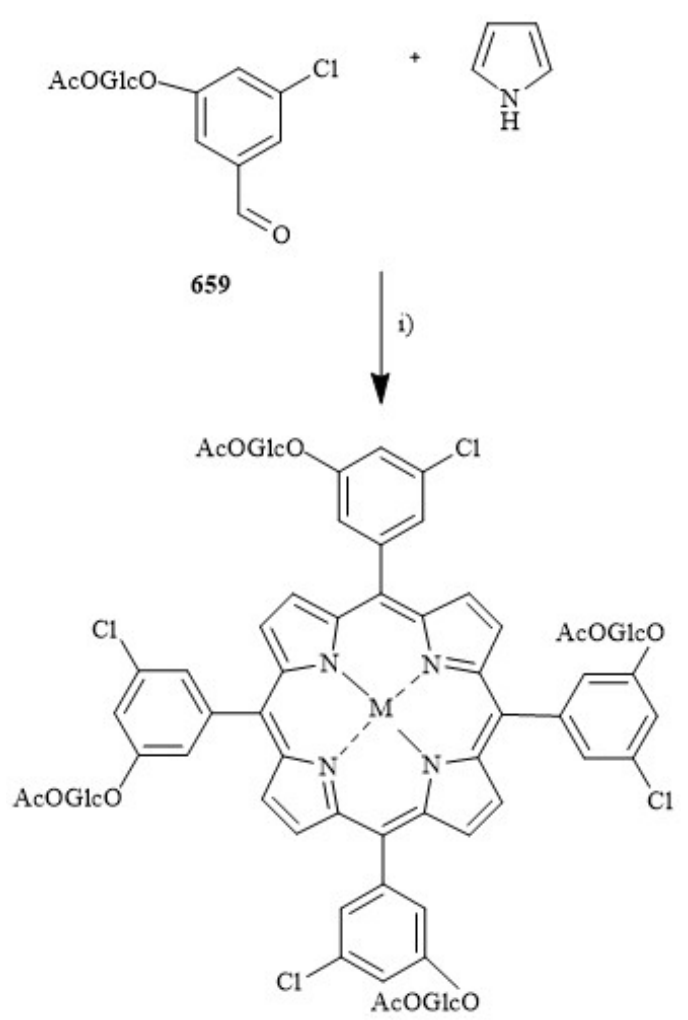

ii)

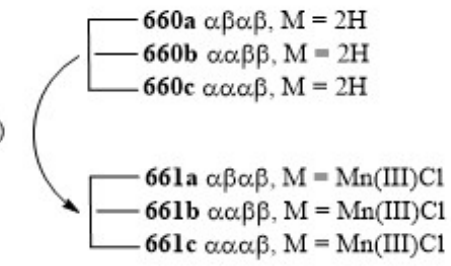

Scheme 42. Synthesis of tetrasubstituted Mn(III)glycoporphyrins. i) (a) $\mathrm{CH}_{2} \mathrm{Cl}_{2}, \mathrm{BF}_{3} \cdot \mathrm{OEt}_{2}$ b) $p$-chloranil, ii) $\mathrm{MnCl}_{2}$, 4-nitrophenol, DMF.

material 97 was synthesized from $p$ (methylthio)benzaldehyde by oxidation to the sulfoxide and followed by condensation with pyrrole. Compound 97 underwent Pummerer rearrangement and deacetylation to yield the tetrathiol which was coupled with 6-iodo-6deoxycycloheptamylose to afford the tetra-cyclodextrin derivative 98. Compound $\mathbf{3 0 9}$ was synthesized from dipyrrylmethane and condensed with $p$ (methylthio)benzaldehyde which was subjected to the same coupling conditions as 97. Scrambling of the reaction of $\mathbf{3 0 9}$ afforded some of the isomer 310. All of the free base porphyrins were subjected to binding studies with a variety of stilbene substrates and depending on the substrates a $2 / 1$ or $1 / 1$ ratio with 98 and a $1 / 1$ ratio with 309 was found. Conversion to their $\mathrm{Mn}$ (III) complexes allowed for selective oxidation of the substrates in good conversions, especially for 99 and $311^{[179]}$.

Studies with these and related catalysts (e.g., 662-665, 100) or $P 450$ mimics ${ }^{[413]}$ focused on several different substrates. For example, catalyst $\mathbf{6 6 2}$ was used for studies on steroid hydroxylation ${ }^{[414]}$ and comparative reactions with 
stilbenes ${ }^{[179,415]}$. Pioneering studies by Breslow indicated that catalyst $\mathbf{6 6 2}$ provided the best results for hydroxylation at C-9 of steroids with 90 turnovers ${ }^{[416]}$. Later on, an improved catalyst (663) was synthesized which incorporated the pyridine ring within its structure and was found to provide a turnover of 2000 . The precursor 664 performed the $6 \alpha$-hydroxylation of a steroid ligand with even better turnovers of $3000{ }^{[417]}$. In the natural P-450 enzymes the iron porphyrin is axially coordinated by a cysteine thiolate sulfur and the oxygen adds to the sixth coordination position. Initially, imidazole had been incorporated as the fifth ligand

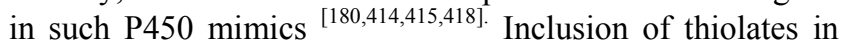
the P450 mimic either covalently attached (see 665) or via hydrogen bounding to the catalyst allowed the use of $\mathrm{H}_{2} \mathrm{O}_{2}$ as oxidant ${ }^{[419]}$. However, the turnovers were modest (15 with $\mathrm{NaOCl}, 10$ with hydrogen peroxide), which may be due to the incorporation of only two perfluorinated phenyl rings. Also the thiol group itself is an oxidizable point in the catalyst, too.

Intriguingly porphyrin cyclodextrin conjugates $(\mathbf{3 1 3}, \mathbf{3 1 4})$ were also used as synthetic receptors for $\beta, \beta$-carotene and for the regioselective catalytic cleavage of the $\mathrm{C} 15-\mathrm{C} 15$ ' bond to afford retinol (vitamin $\mathrm{A}$ ). The ruthenium derivative $\mathbf{3 1 4}$ was chosen to provide better cleavage capacity compared to zinc(II). The first step in the cleavage of the double bond is epoxide formation followed by tert-butyl hydroperoxide / ruthenium porphyrin mediated fragmentation. The ruthenium catalyst afforded cleavage at $\mathrm{C} 15-\mathrm{C} 15$ ', $\mathrm{C} 12$ '-C11', and at C10'-C9' in an overall yield of $30 \%$. However, the selective cleavage of $\mathrm{C} 15-\mathrm{C} 15$ ' was observed when one of the cyclohexene end groups of $\beta, \beta$-carotene was replaced by an ortho-dimethylphenyl group ${ }^{[211]}$.

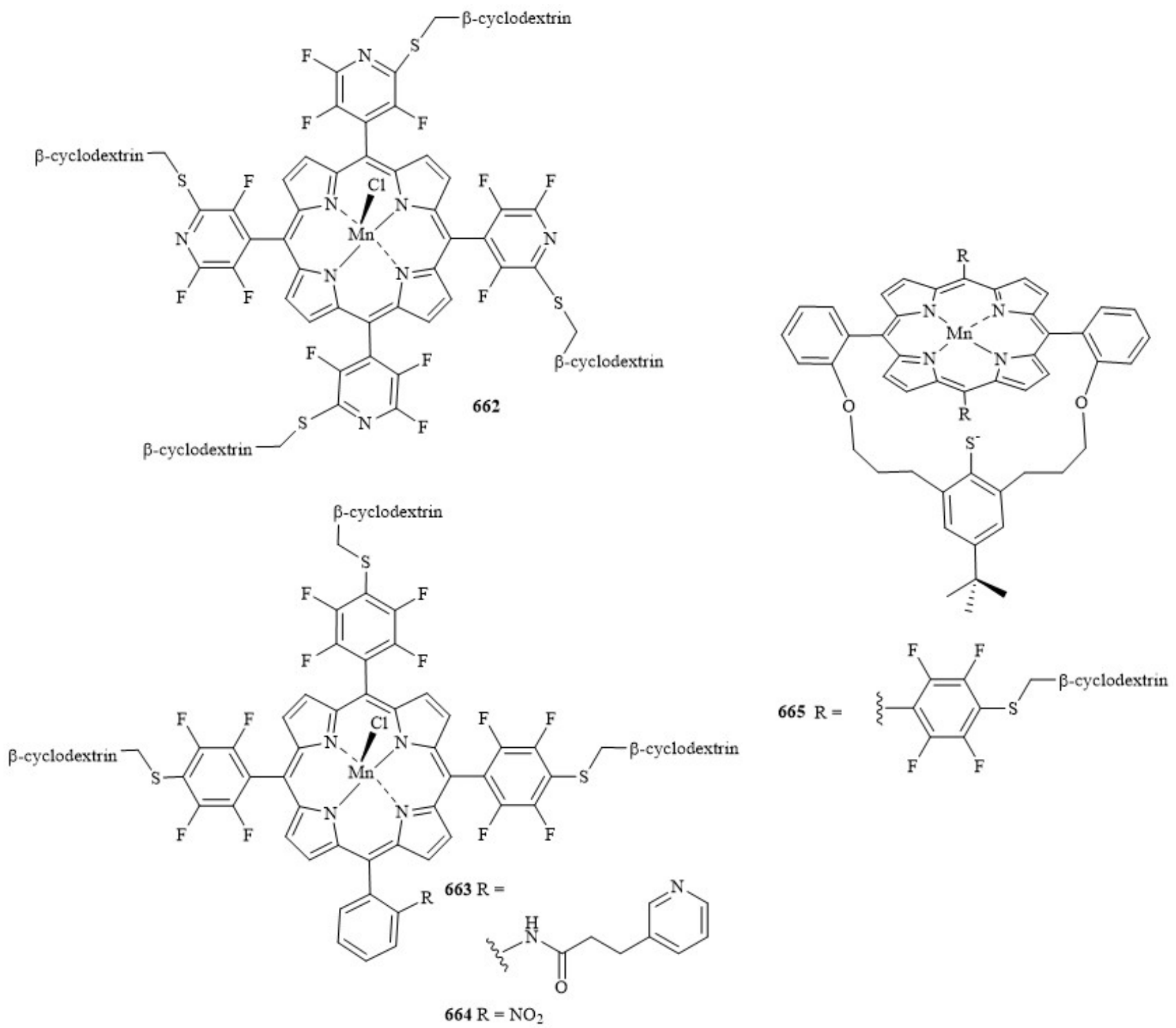

Formula 24 
Other catalytic investigations targeted the electrochemical oxidation of sugars for applications as fuel in fuel cells. This has now expanded into the use of $\mathrm{Rh}$ porphyrins on carbon black as a catalyst to promote such oxidation ${ }^{[420]}$. Certain types of $\mathrm{Rh}$ porphyrins were found to be good catalysts for oxidizing aldose in low-potential regions and exhibited higher activity for aldose than ketose derivatives. Also investigated for such application were cobalt phthalcyanines [420-424].

\subsection{Other uses of glycoporphyrins}

Carbohydrate appended porphyrins have also been used to construct biomimetic electron transfer (ET) systems. Examples are porphyrin-saccharide-anthraquinone systems, where a change in the sugar unit was found to affect the ET properties ${ }^{[425]}$. The porphyrin cyclodextrin conjugate $\mathbf{6 3 8 d}$ was used for photophysical and ET studies ${ }^{[426]}$. Resonance light scatter experiments in aqueous media showed that exciton coupling occurred between adjacent porphyrin units due to the formation of chiral assemblies. The cyclodextrins are of crucial importance for the overall structure and properties. For example, addition of cyclodextrin inclusion compounds (e.g., 1,4-benzoquinone) resulted in a quenching of fluorescence. This could be partially reversed through addition of 1-adamantanecarboxylic acid, which is known to have a high affinity for the cyclodextrin cavity but has no significant effect on the fluorescence. Fluorescence monitoring of the displacement of quenchers allowed a determination of the oxidative and reductive electron transfer potential of benzoquinone and others ${ }^{[426]}$.

Also reported was the immobilization of $\mathbf{6 3 8 d}$ on a solid surface of 3-aminopropylated silica particles and its interactions with several achiral aromatic compounds investigated. The influence of the achiral porphyrin spacer on the chiral recognition of binaphthyl derivatives by the $\beta$ cyclodextrin was investigated ${ }^{[427]}$. Other examples of applications include use as potential antioxidants [176], molecular receptors ${ }^{[428]}$ or as imaging ${ }^{[429]}$ and gelator materials ${ }^{[430,431]}$.

\section{Superoxide dismutase mimic}

Mn-porphyrin bioconjugates were synthesized as antioxidants for selective targeting of ROS overproducing sites. They had been previously identified to mimic superoxide dismutase (SOD) but they lack the selectivity to target cells that overproduce ROS ${ }^{[432]}$. The maltose derivative $\mathbf{8 0}$ was synthesized via connecting a maltose derivative to the Mnporphyrin via the Maillard reaction with little information on the selectivity at the anomeric position of maltose in the paper. Compound $\mathbf{8 0}$ showed effective SOD activity due to the cationic pyridyl group and carbohydrate recognition with Con A, a lectin specific for $\alpha$ - $D$-glucosyl or $\alpha$ - $D$-mannosyl residues ${ }^{[176]}$. Likewise, a $\mathrm{Mn}$ porphyrin lactose conjugate was synthesized which could also facilitated carbohydrate receptor binding. For example, a triantennary carbohydrate which has three terminal $\beta$-galactose residues showed significant affinity for a hepatic lectin asialoglycoprotein receptor (ASGP-R) ${ }^{[433]}$. Introducing several $\beta$-galactose residues around the periphery of the SOD mimic could allow for recognition of the ASGP-R expressed on hepatocytes. Hence the $\mathrm{Mn}$ porphyrin lactose conjugate $\mathbf{8 1}$ was synthesized via the initial introduction of cationic pyridyl groups connected to primary amino groups which subsequently underwent reductive amination between the amino group of the porphyrin scaffold and the reducing end of the lactose residue followed by $\mathrm{Mn}$ insertion [434] Compound $\mathbf{8 1}$ showed significant SOD activity despite the addition of the targeting ligands. It displayed no cytotoxicity at $100 \mu \mathrm{M}$ concentration and showed cell recognition on a human hepatoma cell line (Hep G2) which expresses ASGP$\mathrm{R}$ on the cell surface. In the presence of free lactose residues the recognition of the lactose Mn porphyrin derivative was decreased showing inhibition of the derivatives binding when in competition with lactose molecules ${ }^{[434]}$.

\section{Outlook}

Porphyrin bioconjugates specifically carbohydrate conjugates have remained at the forefront of third generation PS research. It has been established that porphyrins and carbohydrates play a key role in nature. Both of which can serve several medicinal applications. Carbohydrate biological research still lags behind that of porphyrins in understanding their complex biological function. Only with advances in the knowledge of both the chemical and biological properties of such building blocks, a possible key glycoporphyrin derivative will emerge.

This review offers an extremely detailed look at the synthesis as well as the biological evaluation of such derivatives. The range of synthetic conjugations of these molecules follows that of the evolution of porphyrin and carbohydrate chemistry concluding that the postfunctionalization of the carbohydrate unit on the porphyrin scaffold is the most advantageous method developed in recent times. Offering high yields with the stability of sensitive carbohydrate units remaining intact. The main synthetic focus has been that of mono- to tetrasubstitution at the meso positions with few examples of $\beta$-substitutions explored on the porphyrin periphery. One of the significant highlights of carbohydrate-lectin interaction is that of the cluster effect, while interesting comparisons of the effect the distance of meso di-substituted derivatives versus those glycodendrimer derivatives have on the binding potential to lectins is yet to be fully understood.

The emphasis of this review is that of PDT efficacy of glycoporphyrins, however for such conjugates to be efficient PDT agents their targeting ability and amphiphilic characteristics also needs to be examined in detail alongside any PDT applications. In summary, glycoporphyrins offer a range of tetrapyrrolic systems with quite varying degrees of 
aqueous solubility. In general, those derivatives with the greatest water solubility can afford poor PDT activity while those with amphiphilic character offer more scope as PDT agents. For systemic PDT a water soluble formulation is the prime desirable development as well as improved selectivity. Glycoporphyrins have the potential for such criteria. Not only has the carbohydrate residue the potential for improved amphiphilic character of the desired porphyrin scaffold, the unit itself can pose as a potential targeting agent. At a cellular level specific transporters and proteins can be targeted. Little understanding of lectin carbohydrate interactions exist and more precisely those interactions of glycosyl units with protein receptors over-expressed on malignant tissue. Specifically such third generation PSs have excellent potential in the PDT field however, only with significant advances in the understanding of the oncogenic transformations of surface lectins and glycobiology can such a field advance to surpass those of the current PDT standards while eradicating the problems observed in $1^{\text {st }}$ and $2^{\text {nd }}$ generations. With PSs displaying ideal photophysical characteristics including high singlet oxygen production, absorption in the near infra-red and requirements for a good delivery agent are all essential.

No reported glycoporphyrin has excelled nor shown true potential for medicinal trials or such advances, however, these derivatives have an excellent potential in the field with some fine-tuning of biological procedures. As seen from the analysis of the biological literature in Table 3 on in vitro testing, no standard protocols have been outlined. It is difficult to draw comparisons from one glycoporphyrin to another due to variance in light influence, irradiation time and incubation. Derivatives prove considerably difficult to evaluate as some contradictions apply to several of the same glycoporphyrin conjugates due to diverse variance in glycoporphyrin biological protocol. Most are reported in comparison to their non-conjugated parent analogues which has very little stance in PDT efficacy even if they display higher PDT activity. A detailed protocol is essential for such a field with direct comparison to approved PDT agents similar to procedures carried out in our group. Each PDT agent in clinical use has their own protocols to afford the best results for said agent. It is noteworthy that very few examples have been examined with endogenous biomolecules e.g., albumin. Such interactions have a clear impact on the photophysical properties in physiological media for such glycoporphyrins and approved PSs and are an essential investigation.

One of the main highlights includes tolyporphin which is the gold standard for this field. Localizing in the ER and nuclear membrane it affords excellent targeting and cell death 5000 times greater than Temoporfin through apoptotic cell death characteristic of most glycoporphyrins. Again this is an excellent example of the inconsistency of biological testing with no direct comparison to clinical conditions investigated as an excess of $1 \mathrm{~h}$ irradiation was required to achieve these results. A standard procedure similar to that outlined here for many chemical syntheses could afford a fitting protocol for such biological studies. There is significant scope in this area for research with the development of the correct protocols affording direct comparisons. Amphiphilic structures similar to that of tolyporphin could provide interesting results but with preparations less tedious as that of the total synthesis of such a complex molecule. It can be seen through the synthetic pathway that the nature, number and linkage positions of the glycosyl unit is extremely important affording amphiphilic character. Those issues highlighted if addressed by future research have the potential to afford an excellent third generation PS.

\section{LIST OF ABBREVIATIONS}

ALA - $\delta$-aminolevulinic acid; BOP - Benzotriazol-1yloxy)tris(dimethylamino)phosphoniumhexafluorophosphate; DMPC - dimyristoylphosphatidylcholine; DPBF - 1,3-diphenylisobenzofuran; DCC - N,N'dicyclohexylcarbodiimide; DCI-CID - desorption chemical ioization - collision-induced dissociation; DMAE dimethylaminoethanol; $\mathrm{EC}_{50} \quad-\quad$ median effective concentration; $\quad \mathrm{EC}_{90}-90 \%$ effective concentration; EDC/HOBt - 1-ethyl-3-(3dimethylaminopropyl)carboiimide/ Hydroxybenzotriazole; ELISA - enzyme linked immunosorbent assay; FCS - fetal calf serum; Fmoc - Fluorenylmethyloxycarbonyl; Gal galactosyl; Glc - glucosyl; Hp - haematoporphyrin; HpD, haematoporphyrin derivative; HPPH - 3-Devinyl-3-(1'hexyloxyethyl) pyropheophorbide-a; HSA- human serum albumin; HSV - herpes simplex virus; MDR - multidrug resistant; NAS- nucleophilic aromatic substitution; PA photon absorption; PC - Phycocyanin, PS - photosensitizer; PDT - Photodynamic therapy; PDD - Photodynamic detection; Pp IX - Protoporphyrin IX; $p$ THPP - 5,10,15,20tetrakis(4-hydroxyphenyl)porphyrin; EPR - enhanced permeability and retention; ROS - reactive oxygen species; ER - endoplasmic reticulum; TPP - 5,10,15,20tetraphenylporphyrin; $\quad \mathrm{TPPF}_{20} \quad-\quad 5,10,15,20$ tetra(pentafluorophenyl)porphyrin; DMF - N,Ndimethylformamide; NMP- $N$-methylpyroridone; QCM-D quartz crystal microbalance with dissipation; SAR structure-activity relationship.

\section{ACKNOWLEDGEMENTS}

Our work was supported by grants from Science Foundation Ireland (SFI P.I. 09/IN.1/B2650, 12/TIDA/B2381, and IvP 13/IA/1894) and the School of Chemistry (TCD).

\section{REFERENCES}

[1] Milgrom, L.R. The Colours of Life. Oxford University Press: Oxford, UK, 1997.

[2] Battersby, A.R. Tetrapyrroles: The pigments of life. Nat. Prod. Rep., 2000, 17, 507-526.

[3] Wasielewski M.R. Photoinduced electron-transfer in supramolecular systems for artificial photosynthesis. Chem. Rev., 1992, 92, 435-461. 
[4] Dolphin, D. 1993 Syntex award lecture - Photomedicine and photodynamic therapy. Can. J. Chem., 1994, 72, 1005-1013.

[5] James, M.F.M.; Hift, R.J. Porphyrias, Br. J. Anaesth., 2000, 85, 143153

[6] Ackroyd, R.; Kelly, C.; Brown, N.; Reed, M. The history of photodetection and photodynamic therapy. Photochem. Photobiol., 2001, 74, 656-669.

[7] Dougherty, T.J.; Gomer, C.J.; Henderson, B.W.; Jori, G.; Kessel, D.; Korbelik, M.; Moan, J.; Peng, Q. Photodynamic Therapy. J. Natl. Cancer Inst., 1998, 90, 889-905.

[8] Sternberg, E.T.; Dolphin, D.; Brückner, C. Porphyrin-based photosensitizers for use in photodynamic therapy. Tetrahedron, 1998, 54, 4151-4202.

[9] Matsumura, Y.; Maeda, H. A new concept for macromolecular therapeutics in cancer chemotherapy: Mechanism of tumoritropic accumulation of proteins and the antitumor agent smancs. Cancer Res., 1986, 46, 6387-6392.

[10] Khawar, I.A.; Kim, J.H.; Kuh, H.-J. Improving drug delivery to solid tumors: Priming the tumor microenvironment. J. Control. Release, 2015, 201, 78-89.

[11] Kobayashi, H.; Turkbey, B.; Watanabe, R.; Choyke, P.L. Cancer Drug Delivery: Considerations in the Rational Design of Nanosized Bioconjugates. Bioconj. Chem., 2014, 25, 2093-2100.

[12] MacDonald, I.J.; Dougherty, T.J. Basic principles of photodynamic therapy. J. Porphyrins Phthalocyanines, 2001, 5, 105-129.

[13] Dolmans, D.E.J.G.J.; Fukumura, D.; Jain, R.K. Photodynamic therapy for cancer, Nat. Rev. Chem., 2003, 3, 380-387.

[14] Senge, M.O.; Brandt, J.W. Temoporfin $\left(\right.$ Foscan $^{\circledR}, 5,10,15,20-\operatorname{Tetra}(m-$ hydroxyphenyl)chlorin)-A Second-generation Photosensitizer. Photochem. Photobiol., 2011, 87, 1240-1296.

[15] O'Connor, A.E.; Gallagher, W.M.; Byrne, A.T. Porphyrin and nonporphyrin photosensitizers in oncology: Preclinical and clinical advances in photodynamic therapy. Photochem. Photobiol., 2009, 85, 10531074 .

[16] Sear, P.; Wong, C.-H. Carbohydrate Mimetics: A New Strategy for Tackling the Problem of carbohydrate-mediated Bioloical Recognition. Angew. Chem. Int. Ed., 1999, 38, 2300-2324.

[17] Lee, Y.C.; Lee, R.T. Carbohydrate-Protein Interactions: Basis of Glycobiology. Acc. Chem. Res., 1995, 28, 321-327.

[18] Varki, A. Biological roles of oligosaccharides: all of the theories are correct. Glycobiology, 1993, 3, 97-130.

[19] Dwek, R.A. Glycobiology: Toward understanding the function of sugars. Chem. Rev., 1996, 96, 683-720.

[20] Sharon, N.; Lis, H. Lectins as Cell Recognition Molecules. Science, 1989, 246, 227-234

[21] Birnbaum, M.J.; Haspel, H.C.; Rosen, O.M. Transformation of rat fibroblasts by FSV rapidly increases glucose transporter gene transcription. Science, 1987, 235, 1495-1498.

[22] Zheng, X.; Pandey, R.K. Porphyrin-Carbohydrate Conjugates: Impact of Carbohydrate Moieties in Photodynamic Therapy (PDT). Anti-Cancer Agents Med. Chem., 2008, 8, 241-268.

[23] Medina, R.A.; Owen, G.I. Glucose Transporters: Expression, Regulation and Cancer. Biol. Res., 2002, 35, 9-26.

[24] Loris, R. Principles of structures of animal and plant lectins. Biochim. Biophys. Acta, 2002, 1572, 198-208.

[25] Lotan, R.; Ito, H.; Yasui, W.; Yokozaki, H.; Lotan, D.; Tahara, E. Expression of a 31-kDa Lactoside-Binding Lectin in Normal Human Gastric Mucosa and in Primary and Metastatic Gastric Carcinomas. Int. J. Cancer, 1994, 56, 474-480.

[26] Xu, X.C.; El-Naggar, A.K.; Lotan, R. Differential expression of galectin-1 and galectin-3 in thyroid tumors. Potential diagnostic implications. Am. J. Pathol., 1995, 147, 815-822.

[27] Gabius, H.J.; André, S.; Kaltner, H.; Siebert, H.C. The sugar code: Functional lectinomics. Biochim. Biophys. Acta, 2002, 1572, 165-177.

[28] Beat, E.; Magnani, J.L. From Carbohydrate Leads to Glycomimetic Drugs. Nature Rev. Drug Discov., 2009, 8, 661-677.

[29] Hasegawa, T.; Numata, M.; Asai, M.; Takeuchi, M.; Shinkai, S. Colometric calcium-response of [beta]-lactosylated [mu]-oxo-bis-[5,15meso-diphenyl porphyrinatoiron (III)]. Tetrahedron, 2005, 61, 7783-7788.

[30] Tomé, J.P.C.; Neves, M.G.P.M.S.; Tomé, A.C.; Cavaleiro, J.A.S.; Mendonça, A.F.; Pegado, I.N.; Duarte, R.; Valdeira, M. L. Synthesis of glycoporphyrin derivatives and their antiviral activity against herpes simplex virus types 1 and type 2. Bioorg. Med. Chem., 2005, 13, 3878-3888.
[31] Fuhrhop, J.-H.; Demoulin, C.; Boettcher, C.; Koening, J.; Siggel, U. Chiral micellar porphyrin fibers with 2-aminoglycosamide head groups. $J$. Am. Chem. Soc., 1992, 114, 4159-4165.

[32] Locos, O.B.; Heindl, C.C.; Corral, A.; Senge, M.O.; Scanlan, E.M. Efficient Synthesis of Glycoporphyrins by Microwave-Mediated "Click" Reactions. Eur. J. Org. Chem., 2010, 1026-1028.

[33] Daly, R.; Vaz, G.; Davies, A.M.; Senge, M.O.; Scanlan, E.M. Chem. Eur. J., 2012, 18, 14671-14679.

[34] Henderson, B.W.; Bellnier, D.A.; Greco, W.R.; Sharma, A.; Pandey, R.K.; Vaughan, L.A.; Weishaupt, K.R.; Dougherty T.J. An in Vivo Quantitative Structure-Activity Relationship for a Congeneric Series of Pyropheophorbide Derivatives as Photosensitizers for Photodynamic Therapy. Cancer Res., 1997, 57, 4000-4007.

[35] Zheng, G.; Potter, W.R.; Sumlin, A.; Dougherty, T.J.; Pandey, R.K. Photosensitizers related to purpurin-18- $\mathrm{N}$-alkylimides: a comparative in vivo tumoricidal ability of ester versus amide functionalities. Bioorg. Med. Chem. Lett., 2000, 10, 123-127.

[36] Rungta, A.; Zheng, G.; Missert, J.R.; Potter, W.R.; Dougherty, T.J.; Pandey, R.K. Purpurinimides as Photosensitizers: Effect of the Presence and Position of the Substituents in the in Vivo Photodynamic Efficacy. Bioorg. Med. Chem. Lett., 2000, 10, 1463-1466.

[37] Zheng, G.; Potter, W.R.; Camacho, S.H.; Missert, J.R.; Wang, G.; Bellnier, D.A.; Henderson, B.W.; Rodgers, M.A.J.; Dougherty, T.J.; Pandey, R.K. Synthesis, Photophysical Properties, Tumor Uptake, and Preliminary in Vivo Photosensitizing efficacy of a Homologous Series of 3(1'-Alkyloxy)ethyl-3-devinylpurpurin-18- $N$-alkylimides with Variable Lipophilicity. J. Med. Chem., 2001, 44, 1540-1559.

[38] Moan, J.; Peng, Q.; Evensen, J.F.; Berg, K.; Western, A.; Rimington, C. Photosensitizing Efficiencies, Tumor- and Cellular Uptake of Different Photosensitizing Drugs Relevant for Photodynamic Therapy of Cancer. Photochem. Photobiol., 1987, 46, 713-721.

[39] Adams, K.R.; Berenbaum, M.C.; Bonnett, R.; Nizhnik, A.N.; Salgado, A.; Vallés, M.A. Second generation tumour photosensitisers: the synthesis and biological activity of octaalkyl chlorins and bacteriochlorins with graded amphiphilic character, J. Chem. Soc., Perkin Trans. 1, 1992, 14651470

[40] Ben-Dror, S.; Bronshtein, I.; Wiehe, A.; Röder, B.; Senge, M. O.; Ehrenberg, B. On the correlation between hydrophobicity, liposome binding and cellular uptake of porphyrin sensitizers. Photochem. Photobiol., 2006, $82,695-701$.

[41] Wiehe, A.; Simonenko, E.J.; Senge, M.O.; Röder, B. Hydrophilicity vs hydrophobicity-varying the amphiphilic structure of porphyrins related to the photosensitizer $m$-THPC. J. Porphyrins Phthalocyanines, 2001, 5, 758761 .

[42] Rancan, F.; Wiehe, A.; Nöbel, M.; Senge, M.O.; Al Omari, S.; Böhm, F.; John, M.; Röder, B. Influence of substitutions on asymmetric dihydroxychlorins with regard to intracellular uptake, subcellular localization and photosensitization of Jurkat cells. J. Photochem. Photobiol. B: Biol., 2005, 78, 17-28.

[43] Sibani, S.A.; McCarron, P.A; Woolfson, A.D.; Donnelly, R.F. Photosensitiser delivery for photodynamic therapy. Part 2: systemic carrier platforms. Exp. Opin. Drug Deliv., 2008, 5, 1241-1254.

[44] Giuntini, F.; Alonso, C.M.A.; Boyle, R.W. Synthetic approaches for the conjugation of porphyrins related macrocycles to peptides and proteins Photochem. Photobiol. Sci., 2011, 10, 759-791.

[45] Sibrian-Vazquez, M.; Jensen, T.J.; Vincente, M.G.H. Synthesis, characterization and metabolic stability of porphyrin-peptide conjugates bearing bifunctional signaling sequences. J. Med. Chem., 2008, 51, 29152923.

[46] Sehgal, I.; Sibrian-Vazquez, M.; Vicente, M.G.H. Photoinduced cytotoxicity and biodistribution of prostate cancer cell-targeted porphyrins. J. Med. Chem., 2008, 51, 6014-6020.

[47] Sibrian-Vazquez, M.; Jensen, T.J.; Fronczek, F.R.; Hammer, R.P.; Vicente, M.G.H. Synthesis and Characterization of Positively Charged Porphyrin-Peptide Conjugates. Bioconj. Chem., 2005, 16, 852-863.

[48] Sutton, J.M.; Clarke, O.J.; Fernandez, N.; Boyle, R.W. Porphyrin, Chlorin, and Bacteriochlorin Isothiocyanates: Useful Reagents for the Synthesis of Photoactive Bioconjugates. Bioconj. Chem., 2002, 13, 249-263. [49] Perrée-Fauvet, M.; Verchére-Béaur, C.; Tarnuad, E.; AnneheimHerbelin, G.; Bône, N.; Gaudemer, A. New amino acid porphyrin derivatives. Part I: Synthesis. Tetrahedron, 1996, 52, 13569-13588. [50] Verchére-Béaur, C.; Perrée-Fauvet, M.; Tarnuad, E.; AnneheimHerbelin, G.; Bône, N.; Gaudemer, A. New amino acid porphyrin 
derivatives. Part II: DNA binging studies. Tetrahedron, 1996, 52, 1358913604.

[51] Geier III, G.R.; Sasaki, T. The design, synthesis and characterisation of a porphyrin-peptide conjugate. Tetrahedron Lett., 1997, 38, 3821-3824.

[52] Konovalova, N.V.; Karavaeva, N.A.; Gribkov, A.A.; Luzgina, V.N.; Evstigneeva, R.P. The synthesis of diporphyrin systems on the basis of tetraphenylporphyrin derivatives bridged with peptide spacers. Russ. J. Bioorg. Chem., 2000, 26, 98-103; Bioorg. Khim., 2000, 26, 112-117.

[53] Juillerat-Jeanneret, L.; Lohm, S.; Hamou, M.F.; Pinet, F. Regulation of aminopeptidase A in human brain tumor vasculature: evidence for a role of transforming growth factor- $\beta$. Lab. Invest., 2000, 80, 973-980.

[54] Berger, Y.; Greppi, A.; Siri, O.; Neier, R.; Juillerat-Jeanneret, L. Ethylene Glycol and Amino Acid Derivatives of 5-Aminolevulinic Acid as New Photosensitizing Precusors of Protoporphyrin IX in Cells. J. Med. Chem., 2000, 43, 4738-4746.

[55] Bullous A.J.; Alonso, C.M.; Boyle, R.W. Photosensitiser-antibody conjugates for photodynamic therapy. Photochem. Photobiol. Sci., 2011, 10, 721-750.

[56] Keinan, E.; Sinha, S.C.; Sinha-Bagchi, A.; Benory, E.; Ghozi, M.C.; Eshhar, Z.; Green, B.S. Towards antibody-mediated metallo-porphyrin chemistry. Pure Appl. Chem., 1990, 62, 2013-2019.

[57] Mew, D.; Lum, V.; Wat, C.-K.; Towers, G.H.N.; Sun, C.-H.C.; Walter, R.J.; Wright, W.; Berns, M.W.; Levy, J.G. Ability of specific monoclonal antibodies and conventional antisera conjugated to hematoporphyrin to label and kill selected cell lines subsequent to light activation. Cancer Res., 1985, 45, 4380-4386.

[58] Pikuz, S.S.; Sebyakin, Y.L. Model systems based on lipid-porphyrin assemblies and lipoporphyrins in biochemical studies (review). Russ. J. Bioorg. Chem., 1996, 22, 621-631; Bioorg. Khim., 1996, 22, 725-736.

[59] Song, X.-Z.; Jia, S.-L.; Miura, M.; Ma., J.-G.; Shelnutt, J.A. Electron transfer photosensitized by a tin lipoporphyrin in solution, micelles and at water-organic solvent interfaces, J. Photochem. Photobiol. A: Chem., 1998, $113,233-241$.

[60] Fedulova, I.N.; Bragina, N.A.; Novikov, N.V.; Ugol'nikova, O.A.; Mironov, A.F. Synthesis of lipophilic porphyrins to design lipid-porphyrin ensembles. Russ. J. Bioorg. Chem., 2007, 33, 589-593; Bioorg. Khim., 2007, $33,635-639$.

[61] Komatsu, T.; Moritake, M.; Nakagawa, A.; Tsuchida, E. SelfOrganized Lipid-Porphyrin Bilayers Membranes in Vesicular Form: Nanostructure, Photophysical Properties, and Dioxygen Coordination. Chem. Eur. J., 2002, 8, 5469-5480.

[62] Komatsu, T.; Moritake, M.; Tsuchida, E. Molecular Energy and Electron Transfer Assemblies Made of Self-Organized Lipid-Porphyrin Bilayer Vesicles. Chem. Eur. J., 2003, 9, 4626-4633.

[63] Milic, T.; Garno, J.C.; Batteas, J.D.; Smeureanu, G. Drain, C.M. SelfOrganization of Self-Assembled Tetrameric Porphyrin Arrays on Surfaces. Langmuir, 2004, 20, 3974-3983.

[64] Katsonic, N.; Vicario, J.; Kudernac, T. Self-Organized Monolayer of meso-Tetradodecylporphyrin Coordinated to $\mathrm{Au}(111), \mathrm{J}$. Am. Chem. Soc., 2006, 128, 15537-15541

[65] Paszko, E.; Ehrhardt, C.; Senge, M.O.; Kelleher, D.P.; Reynolds, J.V. Nanodrug applications in photodynamic therapy. Photodiagn. Photodyn. Ther., 2011, 8, 14-29.

[66] Tsuchida, E.; Nishide, H. Hemoglobin model- Articial oxygen carrier composed of porphinatoiron, Top. Curr. Chem., 1986, 132, 63-99.

[67] Dannhauser, T.J.; Nango, M.; Oku, N.; Anzai, K.; Loach, P.A. Transmembrane electron transfer as catalyzed by poly(ethylenimine)-linked manganese porphyrins, J. Am. Chem. Soc., 1986, 108, 5865-5871.

[68] Hombrecher, H. K.; Schell, C.; Thiem, J. Synthesis and investigation of galactosylpyranosyl-cholesteroyloxy substituted porphyrin. Bioorg. Med. Chem. Lett., 1996, 6, 1199-1202.

[69] Schell, C.; Hombrecher, H.K. Synthesis and Investigation of Glycosylated Mono- and Diarylporphyrins for Photodynamic Therapy. Bioorg. Med. Chem., 1999, 7, 1857-1865.

[70] Montforts, F.-P.; Meier, A.; Scheurich, G.; Haake, G.; Bats, J.W. Chlorins designed for photodynamic tumor therapy and as model systems for photosynthesis. Angew. Chem. Int. Ed. Engl., 1992, 31, 1592-1594.

[71] Boyle, R.W.; Dolphin, D.; Johnson C.K. Iodination and Heckalkynylation of 5,15-diphenylporphyrin - A convenient entry to asymmetrically meso substituted porphyrins. J. Chem. Soc., Chem. Commun., 1995, 527-528.

[72] Garrido, J.L.; Otero, J.; Maestro, M.A.; Zapata, M. The main nonpolar chlorophyll $c$ from Emiliania huxleyi (prymnesiophyceae) is a chlorophyll $c_{2}$-monoglactosyldiacylglyceride ester: A mass spectrometry study. $J$ Phycol., 2000, 36, 497-505.

[73] Zapata, M.; Garrido, J.L.; Jeffrey, S.W. In: Chlorophylls and bacteriochlorophylls: biochemistry, biophysics, functions and applications; Grimm, B.; Porra, R.J.; Rüdiger, W.; Scheer, H., Eds.; Springer: Dordrecht, 2006, Advances in photosynthesis and respiration, Vol. 25, pp. 39-53.

[74] Zapata, M.; Edvardsen, B.; Rodríguez, F.; Maestro, M.A.; Garridos, J.L. Chlorophyll $C_{22}$ monogalactosyldiacylglyceride ester (chl ${ }_{C_{2}}$-MGDG). A novel marker pigment for Chrysochromulina species (Haptophyta). Mar. Ecol. Prog. Ser., 2001, 219, 85-98.

[75] Prinsep, M.R.; Patterson, G.M.L.; Larsen, L.K.; Smith, C.D. Further tolyporphyrins from the blue-green alga tolypothrix nodosa. Tetrahedron, 1995, 51, 10523-10530

[76] Prinsep, M.R.; Patterson, G.M.L.; Larsen, L.K.; Smith, C.D. Tolyporphyrins $\mathrm{J}$ and $\mathrm{K}$, two further porphinoid metabolites from the cyanobacteria tolypothrix nodosa. J. Nat. Prod., 1998, 61, 1133-1136.

[77] Prinsep, M.R.; Caplan, F.R.; Moore, R.E.; Patterson, G.M.; Smith, C.D Tolyporphyrin, a novel multidrug resistance reversing agent from the bluegreen alga Tolypothrix nodosa. J. Am. Chem. Soc., 1992, 114, 385-387.

[78] Banala, S.; Moser, S.; Müller, T.; Kreutz, C.; Holzinger, A.; Lütz, C.; Kräutler, B. Hypermodified Fluorescent Chlorophyll Catabolites: Source of Blue Luminescence in Senescent Leaves. Angew. Chem. Int. Ed., 2010, 49, 5174-5177.

[79] Müller, E.U.; Morgan, W.T. Porphyrin binding proteins in serum. Ann. N.Y. Acad. Sci., 1975, 244, 624-650.

[80] Senge, M.O.; Radomski, M.W. Platelets, photosensitizers and PDT. Photodiagn. Photodyn. Ther., 2013, 10, 1-16.

[81] Corwin, A.H.; Erdman, J.G. Asynthetic ferroporphyrin complex that is passive to oxygen. J. Am. Chem. Soc., 1946, 68, 2473-2478

[82] Hall, C.A. Transcobalamins I and II as natural transport proteins of vitamin B12. J. Clin. Invest., 1975, 56, 1125-1131.

[83] Gibson, S.L.; Al-Shawi, M.K.; Senior, A.E.; Hilf, R. Inhibition of the ATPase activity of P-glycoprotein by porphyrin photosensitization of multidrug resistant cells in vitro. Photochem. Photobiol., 1995, 61, 390-396.

[84] Dairou, J.; Vever-Bizet, C.; Brault, D. Interaction of sulfonated anionic porphyrins with HIV glycoprotein gp 120: photodamages revealed by inhibition of antibody binding to V3 and C5 domains. Antiviral Res., 2004, 61,37-47.

[85] El-Far, M.A.; Pimstone, N.R. The interaction of tumour-localising porphyrins with collagen, Elastin, Gelatin, Fibrin and Fibrinogen. Cell Biochem. Funct., 1985, 3, 115-119.

[86] Goodarzu, M.; Moosavi-Movahedi, A.A.; Habibi-Rezaei, M.; Shourian, M.; Ghourchian, H.; Ahmad, F.; Farhadi, M.; Saboury, A.A.; Sheibani, N. Hemoglobin fructation promotes heme degradation through the generation of endogenous reactive oxygen species. Spectrochim. Acta A, 2014, 130, $561-567$.

[87] Kenoth, R.; Reddy, D.R.; Maiya, B.G.; Swamy, M.J. Thermodynamic and kinectic analysis of porphyrin binding to Trichosanthes cucumerina seed lectin. Eur. J. Biochem., 2001, 268, 5541-5549.

[88] Goel, M.; Anuradha,P.; Kaur, K.J.; Maiya, B.G.; Swamy, M.J.; Salunke, D.M. Porphyrin binding to jacalin is facilitated by the inherent plasticity of the carbohydrate-binding site: novel mode of lectin-ligand interaction. Acta Crystallogr., 2004, D60, 281-288.

[89] Goel, M.; Jain, D.; Kaur, K.J.; Kenoth, R.; Maiya, B.G.; Swamy, M.J.; Salunke, D.M. Functional Equalityin the Absence of Structural Similarity. $J$. Biol. Chem., 2001, 276, 39277-39281.

[90] Cao, Y.; Merling, A.; Karsten, U.; Goletz, S.; Punzel, M.; Kraft, R.; Butschak, G.; Schwartz-Albiez, R. Expression of CD175(Tn), CD175s (sialosyl-Tn) and CD176 (Thomsen-Friedenreich antigen) on malignant human hematopoietic cells. Int. J. Cancer, 2008, 123, 89-99.

[91] Komath, S.S.; Bhanu, K.; Maiya, B.G.; Swamy, M.J. Binding of porphyrins by the tumor-specific lectin, jacalin [jack fruit (Artocarpus integrifolia) agglutinin]. Biosci. Rep., 2000, 20, 265-276.

[92] Poiroux, G.; Pitié, M.; Culerrier, R.; Ségui, B.; Van Damme, E.J.M.; Peumans, W.J.; Bernadou, J.; Levade, T.; Rougé, P.; Barre, A.; Benoist, H.L. Morniga G: A Plant Lectin as an Endocytic Ligand for Photosensitizer Molecule Targeting Toward Tumor-Associated T/Tn Antigens. Photochem. Photobiol., 2011, 87, 370-377.

[93] Evangelio, E.; Poiroux, G.; Culerrier, R.; Pratviel, G.; Van Damme, E.J.M.; Peumans, W.J.; Barre, A.; Rougé, P.; Benoist, H.; Pitié, M. Comparative Study of the Phototoxicity of Long-Wavelength Photosensitizers Targeted by the MornigaG Lectin. Bioconj. Chem., 2011, $22,1337-1344$ 
[94] Král, V.; Králová, J.; Kaplánek, R.; Bříza, T.; Martásek,P. Quo vadis porphyrin chemistry? Physiol. Res., 2006, 55, S3-S26.

[95] Walker, D.B.; Joshi, G.; Davis, A.P. Progress in biomimetic carbohydrate recognition. Cell. Mol. Life Sci., 2009, 66, 3177-3191.

[96] Murakami, H.; Nagasaki, T.; Hamachi, I., Shinkai, S. Sugar sensing utilizing aggregation properties of a boronic-acid-appended porphyrin. Tetrahedron Lett., 1993, 34, 6273-6276.

[97] Murakami, H.; Nagasaki, T.; Hamachi, I.; Shinkai, S. Sugar sensing utilizing aggregation properties of boronic-acid-appended porphyrins and metalloporphyrins. J. Chem. Soc., Perkin Trans. 2, 1994, 975-981.

[98] Imada, T.; Murakami, H.; Shinkai, S. Sugar-induced chiral orientation of a boronic-acid-appended porphyrin stack. Correlation between the absolute configuration and the CD (circular dichroism) sign. J. Chem. Soc., Chem. Commun., 1994, 1557-1558.

[99] Mizutani, T.; Murakami, T.; Matsumi, N.; Kurahashi, T.; Ogoshi, H. Molecular recognition of carbohydrates by functionalized zinc porphyrins. J. Chem. Soc., Chem. Commun., 1995, 1257-1258.

[100] Arimori, S.; Murakami, H.; Takeuchi, M.; Shinkai, S. Sugarcontrolled association and photoinduced electron transfer in boronic-acidappended porphyrins. J. Chem. Soc., Chem. Commun., 1995, 961-962.

[101] Arimori, S.; Takeuchi, M.; Shinkai, S. Sugar-controlled aggregate formation in boronic acid-appended porphyrin amphiphiles. J. Am. Chem. Soc., 1996, 118, 245-246.

[102] Wang, W.; Gao, X.; Wang, B. Boronic Acid-Based Sensors. Curr. Org. Chem., 2002, 6, 1285-1317.

[103] Hirata, O.; Yamamoto, M.; Sugiyasu, K.; Kubo, Y.; Ikeda, M.; Takeuchi, M.; Shinkai, S. Allosteric saccharide sensing by a phenylboronicacids-appended 5,15-bis(triarylethynyl) porphyrin. J. Supramol. Chem., 2003, 2, 133-142.

[104] Zhang, C.; Suslick, K.S. Syntheses of boronic-acid-appended metalloporphyrins as potential colorimetric sensors for sugars and carbohydrates. J. Porphyrins Phthalocyanines, 2005, 9, 659-666.

[105] James, T.D.; Sandanayake, K.R.A.S.; Shinkai, S. Saccharide Sensing with Molecular Receptors Based on Boronic Acid. Angew. Chem. Int. Ed Engl., 1996, 35, 1910-1922.

[106] Arimori, S.; Takeuchi, M.; Shinkai, S. Sugar-sensing by chiral orientation of dimeric boronic-acid-appended porphyrins which show selectivity for glucose and xylose. Chem. Lett., 1996, 77-78.

[107] Mizutani, T.; Kurahashi, T.; Murakami, T.; Matsumi, N.; Ogoshi, H. Molecular recognition of carbohydrates by zinc porphyrins: Lewis acid/Lewis base combinations as a dominant factor for their selectivity. $J$. Am. Chem. Soc., 1997, 119, 8991-9001.

[108] Suenaga, H.; Arimori, S.; Shinkai, S. Sugar-controlled associationdissociation equilibria between DNA and boronic acid-appended porphyrin. J. Chem. Soc., Perkin Trans. 2, 1996, 607-612.

[109] Hargrove, A.E.; Reyes, R.N.; Riddington, I.; Anslyn, E.V.; Sessler, J.L. Boronic Acid Porphyrin Receptor for Ginsenoside Sensing. Org. Lett., 2010, 12, 4804-4807.

[110] Zhao, W.-W.; Zhang, L.; Xu, J.-J.; Chen, H.-Y. Cell surface carbohydrates evaluation via a photoelectrochemical approach. Chem. Commun., 2012, 48, 9456-9458.

[111] Rusin, O.; Kral, V. Novel macrocycles with 1,1'-binaphthyl substituents for the recognition of saccharides. Chem. Commun., 1999, 2367-2368.

[112] Rusin, O.; Lang, K.; Kral, V. 1,1'-Binaphtyl-Substituted Macrocycles as Receptors for Saccharide Recognition. Chem. Eur. J., 2002, 8, 655-663.

[113] Wada, K.; Mizutani, T.; Kitagawa, S. Synthesis of functionalised porphyrins as oxygen ligand receptors. J. Org. Chem., 2003, 68, 5123-5131. [114] Dukh, M.; Šaman, D.; Lang, K.; Pouzar, V.; Černý, I.; Drašar, P.; Král, V. Steroid-porphyrin conjugate for saccharide sensing in protic media. Org. Biomol. Chem., 2003, 1, 3458-3463.

[115] Roger, L.; Majer, F.; Sergeeva, N.N.; Paszko, E.; Gilmer, J.F.; Senge, M.O.; Synthesis and biological evaluation of Foscan ${ }^{\mathbb{B}}$ bile acid conjugates to target esophageal cancer cells, Bioorg. Med. Chem. Lett., 2013, 23, 24952499.

[116] Bonar-Law, R.P.; Sanders, J.K.M. Polyol Recognition by a SteroidCapped Porphyrin. Enhancement and Modulation of Misfit Guest Binding by Added Water or Methanol. J. Am. Chem. Soc., 1995, 117, 259-271.

[117] Zelenka, K.; Trnka, T.; Tišlerová, I.; Král, V.; Dukh, M.; Drašar, P. Synthesis of Porphyrin Receptors Modified by Glycosylated Steroids. Collect. Czech. Chem. Commun. 2004, 69, 1149-1160.
[118] Ikeda, M.; Shinkai, S.; Osuka, A. Meso-meso-linked porphyrin dimer as a novel scaffold for the selective binding of oligosaccharides. Chem. Commun., 2000, 1047-1048.

[119] Sugasaki, A.; Sugiyasu, K.; Ikeda, M.; Takeuchi, M.; Shinkai, S. First Successful Molecular Design of an Artificial Lewis Oligosaccharide Binding System Utilising Positive Homotropic Allosterism. J. Am. Chem. Soc., 2001, 123, 10239-10244.

[120] Sugasaki, A.; Ikeda, M.; Takeuchi, M.; Koumoto, K.; Shinkai, S. The first example of positive allosterism in an aqueous saccharide- binding system designed on a $\mathrm{Ce}(\mathrm{IV})$ bis(porphyrinate) double decker scaffold Tetrahedron, 2000, 56, 4717-4723.

[121] Lu, W.B.; Zhang, L.H.; Ye, X.S. Porphyrin dimers as receptors for the selective binding of oligosaccharides. Sens. Actuators, B, 2006, 113, 354 360 .

[122] Kral, V.; Rusin, O.; Schmidtchen, F.P. Novel Porphyrin-Cryptand Cyclic Systems: Receptors for Saccharide Recognition in Water. Org. Lett., 2001, 3, 873-876.

[123] Kim, Y.H.; Hong, J.I. Molecular Recognition of Carbohydrates through Directional Hydrogen Bonds by Urea-Appended Porphyrins in Organic Media. Angew. Chem. Int. Ed., 2002, 41, 2947-2950.

[124] Ladomenou, K.; Bonar-Law, R.P. Urea porphyrins as simple receptors for sugars. Chem. Commun., 2002, 2108-2109.

[125] Lee, J.-D.; Greene, N.T.; Rushton, G.T.; Shimizu, K.D.; Hong, J.-I. Carbohydrate Recognition by Porphyrin-Based Molecularly Imprinted Polymers. Org. Lett., 2005, 7, 963-966.

[126] White, B.J.; Harmon, H.J. Optical Determination of Bacterial Exosporium Sugars Using Immobilized Porphyrins. IEEE Sensors J., 2005, $5,726-731$

[127] Lee, J.-D.; Kim, Y.-H.; Hong, J.-I. Carbohydrate Recognition through $\mathrm{H}-$ Bonding and $\mathrm{CH}-\pi$ Interactions by Porphyrin-Based Receptors. J. Org. Chem., 2010, 75, 7588-7595.

[128] Lee, J.-D.; Jang, D.; Hong, J.-I. Molecular Recognition of Amino Sugars by a Porphyrin-based Receptor in Aqueous Media. Bull. Korean Chem. Soc., 2010, 31, 2685-2688.

[129] Králová, J.; Koivukorpi, J.; Kejík, Z.; Poučková, P.; Sievänen, E.; Kolehmainen, E.; Král, V. Porphyrin-bile acid conjugates: from saccharide recognition in the solution to the selective cancer cell fluorescence detection. Org. Biomol. Chem., 2008, 6, 1548-1552

[130] Koivukorpi, J.; Sievänen, E.; Kolehmainen, E.; Král, V. Synthesis, Characterization, and Saccharide Binding Studies of Bile Acid - Porphyrin Conjugates. Molecules, 2007, 12, 13-24.

[131] Kalenius, E.; Koivukorpi, J.; Kolehmainen, E.; Vainiotalo, P. Noncovalent Saccharide Recognition by Means of a Tetrakis(bile acid)Porphyrin Conjugate: Selectivity, Cooperation, and Stability. Eur. J. Org. Chem., 2010, 1052-1058.

[132] Ferro, S.; Jori, G.; Sortino, S.; Stancanelli, R.; Nikolov, P.; Tognon, G.; Ricchelli, F.; Mazzaglia, A. Inclusion of 5-[4-(1Dodecanoylpyridinium)]-10,15,20-triphenylporphine in Supramolecular Aggregates of Cationic Amphiphilic Cyclodextrins: Physicochemical Characterization of the Complexes and Strengthening of the Antimicrobial Photosensitizing Activity. Biomacromolecules, 2009, 10, 2592-2600.

[133] Morisue, M.; Ueda, S.; Kurasawa, M.; Naito, M.; Kuroda, Y. Highly Fluorescent Slipped-Cofacial Phthalocyanine Dimer as a Shallow Inclusion Complex with $\alpha$-Cyclodextrin. J. Phys. Chem. A, 2012, 116, 5139-5144.

[134] Lee, S.J.; Park, K.; Oh, Y.-K.; Kwon, S.-H.; Her, S.; Kim, I.-S.; Choi, K.; Lee, S.J.; Kim, H.; Lee, S.G.; Kim, K.; Kwon, I.C. Tumor specificity and therapeutic efficacy of photosensitizer-encapsulated glycol chitosanbased nanoparticles in tumor bearing mice. Biomaterials, 2009, 30, 29292939.

[135] Senge., M.O. Excercises in molecular gymnastics-bending, stretching and twisting porphyrins. Chem. Commun., 2006, 243-256.

[136] Rothemund, P.; Menotti, A.R. Porphyin studies. IV. The synthesis of $\alpha, \beta, \gamma, \delta$-tetraphenylporphyrine. J. Am. Chem. Soc., 1941, 63, 267-270.

[137] Lindsey, J.S.; Schreiman, I.C.; Hsu, H.C.; Kearney, P.C.; Marguerettaz, A.M. Rothemund and Adler-Longo reactions revisited: Synthesis of tetraphenylporphyrins under equilibrium conditions. J. Org. Chem., 1987, 52, 827-836.

[138] Arsenault, G.P.; Bullock, E.; MacDonald, S.F. Pyrromethanes and porphyrins therefrom. J. Am. Chem. Soc., 1960, 82, 4384-4389.

[139] Lindsey, J.S. Synthetic Routes to meso-Patterened Porphyrins. Acc. Chem. Res., 2010, 43, 300-311.

[140] Senge, M.O. Stirring the porphyrin alphabet soup - functionalization reaction for porphyrins. Chem. Commun., 2011, 47, 1943-1960. 
[141] Wallace, D.M.; Leung, S.H.; Senge, M.O.; Smith, K.M. Rational tetraarylporphyrin syntheses: tetraarylporphyrins from the MacDonald route. J. Org. Chem., 1993, 58, 7245-7257.

[142] Lindsey, J.S. In: The Porphyrin Handbook; Kadish, K.M.; Smith, K.M.; Guilard, R., Eds.; Academic Press: San Diego, USA, 2000, Vol. 1, pp. 45-119.

[143] Sergeeva, N.N.; Senge, M.O.; Ryan, A. In: Handbook of Porphyrin Science. Kadish, K. M.; Smith, K.M.; Guilard, R., Eds.; World Scientific Publishing Co.: Singapore, 2010, Vol. 3, pp. 325-366.

[144] Senge, M.O. Nucleophilic Substitution as a Tool for the Synthesis of Unsymmetrical Porphyrins, Acc. Chem. Res., 2005, 38, 733-743.

[145] Senge, M.O.; Kalisch, W.W.; Bischoff, I. The reaction of porphyrins with organolithium reagents. Chem. Eur. J., 2000, 6, 2721-2738.

[146] Vicente, M.G.H. In: The Porphyrin Handbook. Kadish, K.M.; Smith, K. M.; Guilard, R., Eds.; Academic Press: San Diego, USA, 2000; Vol. 1, 149-199.

[147] Senge, M.O.; Shaker, Y.M.; Pintea, M.; Ryppa, C.; Hatscher, S.S.; Ryan, A.; Sergeeva, Y. Synthesis of meso-Substituted ABCD-type Porphyrins via Functionalization Reactions. Eur. J. Org. Chem., 2010, 237258.

[148] Ryan A.; Gehrold, A.; Perusitti, R.; Pintea, M.; Fazekas, M.; Locos, O. B.; Blaikie, F.; Senge, M.O. Porphyrin Dimers and Arrays. Eur. J. Org. Chem., 2011, 5817-5844.

[149] Aksenova, A.A.; Sebyakin, Y.L.; Mironov, A.F. Conjugates of Porphyrin with Carbohydrates. Russ. J. Bioorg. Chem., 2003, 29, 201-219; Bioorg. Khim., 2003, 29, 227-246.

[150] Pandey, R.K.; Zheng, G. In: The Porphyrin Handbook. Kadish, K.M.; Smith, K.M.; Guilard, R., Eds.; Academic Press: Boston, USA, 2000, Vol. 6, 157-230.

[151] Smith, C.D.; Prinsep, M.R.; Caplan, F.R.; Moore, R.E.; Patterson, G.M.L. Reversal of multiple drug resistance by tolyporphyrin, a novel cyanobacterial natural product. Oncol. Res., 1994, 6, 211-218.

[152] Minehan, T.G.; Kishi, Y. $\beta$-Selective $C$-Glycosidations: Lewis-acid mediated reactions of carbohydrates with silyl ketene acetals. Tetrahedron Lett., 1997, 38, 6815-6818.

[153] Minehan, T.G.; Kishi, Y. Extension of the Eschenmoser sulfide contraction/iminoester cyclisation method to the synthesis of Tolyporphrin chromatophores. Tetrahedron Lett., 1997, 38, 6811-6814.

[154] Schwesinger, R.; Waditschatka, R.; Rigby, J.; Nordmann, R.; Schweizer, W.B.; Zass, E.; Eschenmoser, A. Das PyrrocorphinLigandsystem: Synthese des 2,2,7,7,12,12,17-Heptamethyl-2,3,7,8,12,13hexahydroporphyrins. Helv. Chim. Acta, 1982, 65, 600-610.

[155] Minehan, T.G.; Kishi, Y. Total synthesis of the proposed structure of (+)-Tolyporphyrin A O,O-Diacetate. Angew. Chem. Int. Ed., 1999, 38, 923 925 .

[156] Minehan, T.G.; Cook-Blumberg, L.; Kishi, Y.; Prinsep, M.R.; Moore, R.E. Revised structure of Tolyporphyrin A. Angew. Chem. Int. Ed., 1999, 38, 926-928.

[157] Wang, W.; Kishi, Y. Synthesis and structure of tolyporphyrin A O,ODiacetate. Org. Lett., 1999, 1, 1129-1132.

[158] Hu, B.C.; Zhou, W.Y.; Liu, Z.L.; Cai, C.J.; Xu, S.C. Efficient synthesis of an A-B-C-tricycle fragment for a structural model of tolyporphin. J. Porphyrins Phthalocyanines, 2010, 14, 89-100.

[159] Ono, N.; Bougauchi, M.; Maruyama, K. Water-Soluble Porphyrins with Four Sugar Molecules. Tetrahedron Lett., 1992, 33, 1629-1632.

[160] Maillard, P.; Guerquin-Kern, J.-L.; Huel, C.; Momenteau, M. Glycoconjugated Porphyrins. 2. Synthesis of sterically constrained polyglycosylated compounds derived from tetraphenylporphyrins. J. Org. Chem., 1993, 58, 2774-2780.

[161] Maillard, Ph.; Vilain, S.; Huel, C.; Momenteau, M. Efficient Preparation of the $\alpha, \alpha, \alpha, \alpha$-Atropoisomer of meso-Tetrakis[2-(2,3,4,6tetraacetyl-O-B-glucosyl) phenyl] porphyrin. J. Org. Chem., 1994, 59, 2887 2890.

[162] Oulmi, D.; Maillard, P.; Guerquin-Kern, J.-L.; Huel, C.; Momenteau, M. Glycosylated Porphyrins. 3. Synthesis of Flat Amphiphilic Mixed meso(Glycosylated aryl)arylporphyrins and Mixed meso-(Glycosylated aryl)alkylporphyrins Bearing Some Mono- and Disaccharide Groups. $J$. Org. Chem., 1995, 60, 1554-1564.

[163] Griesbeck, A.G.; Miranda, M.A.; Uhlig, J. Sweet chiral porphyrins as singlet oxygen sensitizers for asymmetric Type II Photooxygenation. Photochem. Photobiol. Sci., 2011, 10, 1431-1435.

[164] Kohata, K.; Higashio, H.; Yamaguchi, Y.; Koketsu, M.; Odashima, T. Synthesis and characterization of new style of water-soluble glycosylated porphyrins as a spectrophotometric reagent for metal ions. Bull. Chem. Soc. Jpn., 1994, 67, 668-679.

[165] Hirohara, S.; Obata, M.; Saito, A.; Ogata, S.; Ohtsuki, C.; Higashida, S.; Ogura, S;. Okura, I.; Sugai, Y.; Mikata, Y.; Tanihara, M.; Yano, S. Cellular uptake and photocytotoxicity of glycoconjugated porphyrins in HeLa cells. Photochem. Photobiol., 2004, 80, 301-308.

[166] Hirohara, S.; Obata, M.; Ogura, S.; Okura, I.; Higashida, S.; Ohtsuki, C.; Ogata, S.; Nishikawa, Y.; Takenaka, M.; Ono, H.; Mikata, Y.; Yano, S. Hydrophobicity parameters $(\log P$ ) of glycoconjugated porphyrins for photodynamic therapy evaluated by reversed phase HPLC. J. Porphyrins Phthalocyanines, 2004, 8, 1289-1292.

[167] Mikata, Y.; Onchi, Y.; Tabata, K.; Ogura, S.-i.; Okura, I.; Ono, H.; Yano, S. Sugar-dependent photocytotoxic property of tetra- and octaglycoconjugated tetraphenylporphyrins. Tetrahedron Lett., 1998, 39, 45054508

[168] Maillard, Ph.; Huel, C.; Momenteau M. Synthesis of New MesoTetrakis (Glycosylated) Porphyrins. Tetrahedron Lett., 1992, 33, 80818084

[169] Cornia, M.; Casiraghi, G.; Binacchi, S.; Zanardi, F.; Rassu, G. Facile entry to 5,10,15,20-tetra-C-glycosylporphyrins. J. Org. Chem., 1994, 59, 1226-1230.

[170] Štěpánek, P.; Dukh, M.; Šaman, D.; Moravcová, J.; Kniežo, L.; Monti, D.; Venanzi, M.; Mancini, G.; Drašar, P. Synthesis and solvent driven selfaggregation studies of meso-"C $\mathrm{C}$-glycoside"-porphyrin derivatives. Org. Biomol. Chem., 2007, 5, 960-970.

[171] Cornia, M.; Menozzi, M.; Ragg, E.; Mazzini, S.; Scarafoni, A.; Zanardi, F.; Casiraghi, G. Synthesis and Utility of Novel C-mesoGlycosylated Metalloporphyrins. Tetrahedron, 2000, 56, 3977-3983.

[172] Pasetto, P.; Chen, X.; Drain, C.M.; Franck, R.W. Synthesis of hydrolytically stable porphyrin $C$ - and $S$-glycoconjugates in high yields. Chem. Commun., 2001, 81-82.

[173] Okada, M.; Kishibe, Y.; Ide, K.; Takahashi, T.; Hasegawa, T. Convenient Approach to Access Octa-Glycosylated Porphyrins via "Click Chemistry". Int. J. Carb. Chem., 2009, Article ID 305276.

[174] Gomes, M.C.; Woranovicz-Barreira, S.; Faustino, M.A.F.; Neves, M.G.P.M.S.; Tomé, A.C.; Almeida, A.; Cavaleiro, J.A.S.; Cunha, Â.; Tomé, J.P.C. Photodynamic Inactivation of Conidia of Penicillium chrysogenum by Cationic Porphyrins. Photochem. Photobiol. Sci., 2011, 10, 1735-1743.

[175] Fujimoto, K.; Miyata, T.; Aoyama Y. Saccharide-Directed Cell Recognition and Molecular Delivery Using Macrocyclic Saccharide Clusters: Masking of Hydrophobicity to Enhance the Saccharide Specificity. J. Am. Chem. Soc., 2000, 122, 3558-3559.

[176] Asayama, S.; Mori, T.; Nagaoka, S.; Kawakami, H. Chemical Modification of Manganese Porphyrins with Biomolecules for New Functional Antioxidants. J. Biomater. Sci. Polymer Edn., 2003, 14, 1169 1179.

[177] Hirohara, S.; Obata, M.; Alitomo, H.; Sharyo, K.; Ando, T.; Yano, S.; Tanihara, M. Synthesis and photocytotoxicity of S-glucosylated 5,10,15,20tetrakis(tetrafluorophenyl) porphyrin metal complexes as efficient ${ }^{1} \mathrm{O}_{2}$ generating glycoconjugates. Bioconj. Chem., 2009, 20, 944-952.

[178] Hirohara, S.; Obata, M.; Alitomo, H.; Sharyo, K.; Ando, T.; Tanihara, M.; Yano, S. Synthesis, photophysical properties and sugar-dependent in vitro photocytotoxicity of pyrrolidine-fused chlorins bearing $S$-glycosides. $J$. Photochem. Photobiol. B: Biol., 2009, 97, 22-33.

[179] Breslow, R.; Zhang, X.; Xu, R.; Maletic, M.; Merger, R. Selective Catalytic Oxidation of Substrates that Bind to Metalloporphyrin Enzyme Mimics Carrying Two or Four Cyclodextrin Groups and Related Metallosalens. J. Am. Chem. Soc., 1996, 118, 11678-11679.

[180] Breslow, R.; Gabriele, B.; Yang, J. Geometrically Directed Selective Steroid Hydroxylation with High Turnover by a Fluorinated Artificial Cytochrome P-450. Tetrahedron Lett., 1998, 39, 2887-2890.

[181] Rusin, O.; Kral, V.; Escobedo, J.O.; Strongin, R.M. A supramolecular approach to protein labeling. A novel fluorescent bioassay for concanavalin A activity. Org. Lett., 2004, 6, 1373-1376.

[182] Vilain-Deshayes, S.; Robert, A.; Maillard, Ph.; Meunier, B.; Momenteau, M. Enantioselective epoxidation of olefins by single-oxygen atom donors catalyzed by manganese-glycoconjugated porphyrins. J. Mol. Catal. A, 1996, 113, 23-34.

[183] Gaud, O.; Granet, R.; Kaouadij, M.; Kransz, P.; Blaise J.C.; Bolbach, G. Synthèse et analyse structurale de nouvelles méso-arylporphyrines glycosylées en vue de l'application en photothéraphie des cancers. Can. J. Chem., 1996, 74, 481-499. 
[184] Ménard, F.; Sol, V.; Ringot, C.; Granet, R.; Alves, S.; Le Morvan, C.; Queneau, Y.; Ono, N.; Krausz, P. Synthesis of tetraglucosyl- and tetrapolyamine-tetrabenzoporphyrin conjugates for an application in PDT. Bioorg. Med. Chem., 2009, 17, 7647-7657.

[185] Hamazawa, A.; Kinoshita, I.; Breedlove, B.; Isobe, K.; Shibata, M.; Kakuchi, T.; Hirohara, S.; Obata, M.; Mikata, Y.; Yano, S. Mesotetraphenylporphyrin having hexa-maltosyl and decyl chain as an amphiphilic photosensitizer toward photodynamic therapy. Chem. Lett., 2002, 388-389.

[186] Lee, C.H.; Li, F.; Iwamoto, K.; Dadok, J.; Bothner-By, A.A.; Lindsey, J.S. Synthetic approaches to regioisomerically pure porphyrins bearing four different meso-substituents. Tetrahedron, 1995, 51, 11645-11672.

[187] Oulmi, D.; Maillard, Ph.; Vever-Bizet, C.; Momenteau, M., Braultt, D. Glycosylated Porphyrins: Characterization of Association in Aqueous Solutions by Absorption and Fluorescence Spectroscopies and Determination of Singlet Oxygen Yield in Organic Media. Photochem. Photobiol., 1998, 67, 511-518.

[188] Bourhim, A.; Czernecki, S.; Krausz, P.; Viari, A.; Vigny, P. Glycosylated hematoporphyrins: A new approach in cancer phototherapy? $J$. Carbohydr. Chem., 1990, 9, 761-765.

[189] Driaf, K.; Krausz, P.; Verneuil, B.; Spiro, M.; Blais, J.C.; Bolbach, G. Glycosylated Cationic Porphyrins as Potential Agents in Cancer Phototherapy. Tetrahedron Lett., 1993, 34, 1027-1030.

[190] Driaf, K.; Granet, R.; Krausz, P.; Kaouadji, M.; Thomasson, F.; Chulia, A. J.; Verneuil, B.; Spiro, M.; Blais, J.C.; Bolbach, G. Synthesis of glycosylated cationic porphyrins as potential agents in photodynamic therapy. Can. J. Chem., 1996, 74, 1550-1563.

[191] Little, R.G. The mixed-aldehyde synthesis of difunctional tetraarylporphyrins. J. Heterocycl. Chem., 1981, 18, 129-133.

[192] Kaldapa, C.; Blais, J.C.; Carre, V.; Granet, R.; Sol, V.; Guilloton, M.; Spiro, M.; Krausz, P. Synthesis of new glycosylated neutral and cationic porphyrins dimers. Tetrahedron Lett., 2000, 41, 331-335.

[193] Fleischer, E.B.; Shachter, A.M. Linked Porphyrin Systems. J. Heterocycl. Chem., 1991, 28, 1693-1699.

[194] Sol, V.; Chaleix, V.; Champavier, Y.; Granet, R.; Huang, Y.-M.; Krausz, P. Glycosyl bis-porphyrin conjugates: Synthesis and potential application in PDT. Bioorg. Med. Chem., 2006, 14, 7745-7760.

[195] Chaleix, V.; Sol, V.; Huang, Y.-M.; Guilloton, M.; Granet, R.; Blais, J.C.; Krausz, P. RGD-Porphyrin Conjugates: Synthesis and Potential Application in Photodynamic Therapy. Eur. J. Org. Chem., 2003, 14861493.

[196] Casiraghi, G.; Cornia, M.; Rassu, G.; Del Sante, C.; Spanu, P. Synthesis and Transformation of Pyrrole $C$-Glycoconjugates. Tetrahedron, 1992, 48, 5619-5628.

[197] Hombrecher, H.K.; Ohm, S.; Koll, D. Synthesis of Galactopyranosyl Substituted Porphyrins. Tetrahedron, 1996, 52, 5441-5448.

[198] Laville, I.; Pigaglio, S.; Blais, J.-C.; Doz, F.; Loock, B.; Maillard, Ph.; Grierson, D.S.; Blais, J. Photodynamic efficiency of diethylene glycollinked glycoconjugated porphyrins in human retinoblastoma cells. J. Med. Chem., 2006, 49, 2558-2567.

[199] Sylvain, I.; Zerrouki, R.; Granet, R.; Huang, Y.M.; Lagorce, J.-F.; Guilloton, M.; Blais, J.-C.; Krausz, P. Synthesis and Biological Evaluation of Thioglycosylated Porphyrins for an Application in Photodynamic Therapy. Bioorg. Med. Chem., 2002, 10, 57-69.

[200] Hirohara, S.; Nishida, M.; Sharyo, K.; Obata, M.; Ando, T.; Tanihara, M. Synthesis, photophysical properties and photocytotoxicity of mono-, di-, tri- and tetra-glucosylated fluorophenylporphyrins. Bioorg. Med. Chem., 2010, $18,1526-1535$.

[201] Di Stasio, B.; Frochot, C.; Dumas, D.; Even, P.; Zwier, J.; Müller, A.; Didelon, J.; Guillemin, F.; Viriot, M.-L.; Barberi-Heyob M. The 2Aminoglucosamide Motif improves Cellular uptake and Photodynamic Activity of Tetraphenylporphyrin. Eur. J. Med. Chem., 2005, 40, 11111122.

[202] Wyrębek, P.; Osuch-Kwiatkowska, A.; Pakulski, Z.; Jarosz, S.; Ostrowski, S. The synthesis of sugar-decorated hydrophilic porphyrins. J. Porphyrins Phthalocyanines, 2013, 17, 384-391.

[202a] Mikata, Y.; Shibata, M.; Baba, Y.; Kakuchi, T.; Nakai, M.; Yano, S. Synthesis and photodynamic properties of maltohexaose-conjugated porphyrins. J. Porphyrins Phthalocyanines, 2012, 16, 1177-1185.

[203] Hao, E.; Jensen, T.J.; Vicente, M.G.H. Synthesis of porphyrincarbohydrate conjugates using "click" chemistry and their preliminary evaluation in human Hep2 cells. J. Porphyrins Phthalocyanines, 2009, 13, $51-59$
[204] Ballut, S.; Makky, A.; Loock, B.; Michel, J.-P.; Maillard, Ph.; Rosilio, V. New strategy for targeting of photosensitizers. Synthesis of glycodendrimeric phenylporphyrins, incorporation into a liposome membrane and interaction with a specific lectin. Chem. Commun., 2009, 224-226.

[205] Ballut, S.; Naud-Martin, D.; Loock, B.; Maillard, Ph. A Strategy for the Targeting of Photosensitizers. Synthesis, Characterization, and Photobiological Property of Porphyrins Bearing Glycodendrimeric Moieties. J. Org. Chem., 2011, 76, 2010-2028.

[206] McCarthy, J.R.; Bhaumik, J.; Merbouh, N.; Weissleder, R. Highyielding syntheses of hydrophilic conjugatable chlorins and bacteriochlorins. Org. Biomol. Chem., 2009, 7, 3430-3436.

[207] Sol, V.; Blais, J.C.; Carré, V.; Granet, R.; Guilloton, M.; Spiro, M.; Krausz, P. Synthesis, Spectroscopy, and Photocytotoxicity of Glycosylated Amino Acid Porphyrin Derivatives as Promising Molecules for Cancer Phototherapy. J. Org. Chem., 1999, 64, 4431-4444.

[208] Desroches, M.-C.; Kasselouri, A.; Meyniel, M.; Fontaine, P.; Goldmann, M.; Prognon, P.; Maillard, Ph.; Rosilio, V. Incorporation of Glycoconjugated Porphyrin Derivatives into Phospholipid Monolayers: A Screening Method for the Evaluation of Their Interaction with a Cell Membrane. Langmuir, 2004, 20, 11698-11705.

[209] Laville, I.; Pigaglio, S.; Blais, J.-C.; Loock, B.; Maillard, P.; Grierson, D.S.; Blais, J. A study of the stability of tri(glucosyloxyphenyl) chlorin, a sensitizer for photodynamic therapy, in human colon tumoral cells: a liquid chromatography and MALDI-TOF mass spectrometry analysis. Bioorg. Med. Chem., 2004, 12, 3673-3682.

[210] Maillard, Ph.; Loock, B.; Grierson, D.S.; Laville, I.; Blais, J.; Doz, F.; Desjardins, L.; Carrez, D.; Guerquin-Kern, J.-L.; Croisy, A. In vitro phototoxicity of glycoconjugated porphyrins and chlorins in colorectal adenocarcinoma (HT29) and retinoblastoma (Y79) cell lines. Photodiag. Photodyn. Ther., 2007, 4, 261-268.

[211] French, R.R.; Holzer, P.; Leuenberger, M.G.; Woggon, W.-D. A Supramolecular Enzyme Mimic That Catalyzes the 15,15' Double Bond Scission of $\beta, \beta$-Carotene. Angew. Chem. Int. Ed., 2000, 39, 1267-1269.

[212] Amessou, M.; Carrez, D.; Patin, D.; Sarr, M.; Grierson, D.S.; Croisy, A.; Tedesco, A.C.; Maillard, Ph.; Johannes, L. Retrograde delivery of photosensitizer (TPPp-O- $\beta-\mathrm{GluOH})_{3}$ selectively potentiates its photodynamic activity. Bioconjug. Chem., 2008, 19, 532-538.

[213] Dallagnol, J.C.C.; Ducatti, D.R.B.; Barreira, S.M.W.; Noseda, M.D. Duarte, M.E.R.; Gonçalves, A.G. Synthesis of porphyrin glycoconjugates bearing thiourea, thiocarbamate and carbamate connecting groups: influenceof the linker on chemical and photophysical properties. Dyes Pigm., 2014, 107, 69-80.

[214] Aicher, D.; Wiehe, A.; Stark, C.B.W. Synthesis of Glycoporphyrins Using Trichloroacetimidates as Glycosyl Donors. Synlett, 2010, 395-398.

[215] Samaroo, D.; Vinodu, M.; Chen, X.; Drain, C.M. mesoTetra(pentafluorophenyl)porphyrin as an Efficient Platform for Combinatorial Synthesis and the Selection of New Photodynamic Therapeutics using a Cancer Cell Line. J. Comb. Chem., 2007, 9, 998-1011. [216] Casiraghi, G.; Cornia, M.; Zanardi, F.; Rassu, G.; Ragg, E.; Bortolini, R. Synthesis and Characterization of Porphyrin-Sugar Carbon Conjugates. $J$. Org. Chem., 1994, 59, 1801-1808.

[217] Senge, M.O. A Conformational Study of 5,10,15,20-Tetraalkyl$22 \mathrm{H}^{+}, 24 \mathrm{H}^{+}$-porphyrindiium Salts (Dication Salts). Z. Naturforsch., 2000, $55 b, 336-344$

[218] Cornia, M.; Valenti, C.; Capacchi, S.; Cozzini, P. Synthesis, Characterisation and Conformational Studies of Lipophilic, Amphiphilic and Water-Soluble C-Glyco-Conjugated Porphyrins. Tetrahedron, 1998, 54, 8091-8106.

[219] Ló, S.M.S.; Cunico, J.C.; Ducatti, D.R.B.; Orsato, A.; Duarte, M.E.R.; Barreira, S.M.W.; Noseda, M.D.; Gonçalves, A.G. Synthesis of peracetylated $C$-1-deoxyalditol- and $C$-glycoside-dipyrranes via dithioacetal derivatives. Tetrahedron Lett., 2013, 54, 1137-1140.

[220] Davoust, E.; Granet, R.; Krausz, P.; Carré, V.; Guilloton, M. Synthesis of Glycosyl Strapped Porphyrins. Tetrahedron Lett., 1999, 40, 2513-2516.

[221] Feng, X.; Senge, M. O. An Efficient Synthesis of Highly Functionalized Asymmetric Porphyrins with Organolithium Reagents. $J$. Chem. Soc., Perkin Trans. 1, 2001, 1030-1038.

[222] Fülling, G.; Schröder, D.; Franck, B. Water soluble porphyrindiglycosides with photosensitizing properties. Angew. Chem. Int Ed. Engl., 1989, 28, 1519-1521. 
[223] Schell, C.; Hombrecher, H.K. Synthesis, Self-Assembling Properties and Incorporation of Carbohydrate-Substituted Porphyrins into Cell Membrane Models. Chem. Eur. J., 1999, 5, 587-598.

[224] da Silva, F. de C.; Ferreira, V.F.; de Souza, M.C.B.V.; Tomé, A.C.; Neves, M.G.P.M.S.; Silva, A.M.S.; Cavaleiro, J.A.S. Synthesis of Glycoporphyrins by Cross-Metathesis Reactions. Synlett, 2008, 1205-1207.

[225] Spiro, M.; Blais, J.C.; Bolbach, G.; Fournier, F.; Tabet, J.C.; Driaf, K.; Gaud, O.; Granet, R.; Krausz, P. Secondary ion mass spectrometry of glycosylated porphyrins. Int. J. Mass Spectrom. Ion Proc., 1994, 134, 229238

[226] Bourhim, A.; Gaud, O.; Granet, R.; Krausz, P.; Spiro, M. Synthesis of New Glycosylated Porphyrin Derivatives with a Hydrocarbon Spacer Arm. Synlett, 1993, 563-564.

[227] Domingues, M.R.M.; Domingues, P.; Reis, A.; Ferrer Correia, A.J.; Tome, J.P.C.; Tome, A.C.; Neves, M.G.P.M.S.; Cavaleiro, J.A.S. Structural characterization of glycoporphyrins by electrospray tandem mass spectrometry. J. Mass Spectrom., 2004, 39, 158-167.

[228] Domingues, M.R.M.; Domingues, P.; Neves, M.G.P.M.S.; Tomé, A.C.; Cavaleiro, J.A.S. Recent developments in the structural characterization of substituted meso-tetraarylporphyrins by electronspray tandem mass spectroscopy. J. Porphyrins Phthalocyanines, 2009, 13, 524527.

[229] Tomé, J.P.C.; Silva, E.M.P.; Pereira, A.M.V.M.; Alonso, C.M.A.; Faustino, M.A.F.; Neves, M.G.P.M.S.; Tomé, A.C.; Cavaleiro, J.A.S.; Tavares, S.A.P.; Duarte, R.R.; Caeiro, M.F.; Valdeira, M.L. Synthesis of neutral and cationic tripyridylporphyrin- $D$-galactose conjugates and the photoinactivation of HSV-1. Bioorg. Med. Chem., 2007, 15, 4705-4713. [230] Gomes, M.C.; Silva, S.; Faustino, M.A.F.; Neves, M.G.P.M.S.; Almeida, A.; Cavaleiro, J.A.S.; Tomé, J.P.C.; Cunha, Â. Cationic galactoporphyrin photosensitisers against UV-B resistant bacteria: oxidation of lipids and proteins by ${ }^{1} \mathrm{O}_{2}$. Photochem. Photobiol. Sci., 2013, 12, 262271.

[231] Achelle, S.; Couleaud, P.; Baldeck, P.; Teulade-Fichou, M.-P.; Maillard, Ph. Carbohydrate-Porphyrin Conjugates with Two-Photon Absorption Properties as Potential Photosensitizing Agents for Photodynamic Therapy. Eur. J. Org. Chem., 2011, 1271-1279.

[231a] Mikata, Y.; Sawaguchi, T.; Kakuchi, T.; Gottschaldt, M.; Schubert, U.S.; Ohi, H.; Yano, S. Control of the Aggregation Properties of Tris(maltohexaose)-Linked Porphyrins with an Alkyl Chain. Eur. J. Org. Chem., 2010, 663-671.

[232] Hammerer, F.; Achelle, S.; Baldeck, P.; Maillard, Ph.; TeuladeFichou, M.-P. Influence of Carbohydrate Biological Vectors on the TwoPhoton Resonance of Porphyrin Oligomers. J. Phys. Chem. A, 2011, 115, 6503-6508.

[233] Drobizhev, M.; Stepanenko, Y.; Dzenis, Y.; Karotki, A.; Rebane, A.; Taylor, P.N.; Anderson, H.L. Extremely Strong Near-IR Two-Photon Absorption in Conjugated Porphyrin Dimers:Quantitative Description with Three-Essential-States Model. J. Phys. Chem. B, 2005, 109, 7223-7236.

[234] Garcia, G.; Hammerer, F.; Poyer, F.; Achelle, S.; Teulade-Fichou, M.P.; Maillard, Ph. Carbohydrate-conjugated porphyrin dimers: Synthesis and photobiological evaluation for a potential application in one-photon and two-photon photodynamic therapy. Bioorg. Med. Chem., 2013, 21, 153-165. [235] Lupu, M.; Thomas, C.D.; Maillard, Ph.; Loock, B.; Chauvin, B.; Aerts, I.; Croisy, A.; Belloir, E.; Volk, A.; Mispelter, J. ${ }^{23} \mathrm{Na}$ MRI longitudinal follow-up of PDT in a xenograft model of human retinoblastoma, Photodiagn. Photodyn. Ther., 2009, 6, 214-220.

[236] Poyera, F.; Thomas, C.D.; Garcia, G.; Croisy, A.; Carrez, D.; Maillard, Ph.; Lupu, M.; Mispeltera, J. PDT induced bystander effect on human xenografted colorectal tumors as evidenced by sodium MRI, Photodiagn. Photodyn. Ther., 2012, 9, 303-309.

[237] Sol, V.; Charmot, A.; Krausz, P. Synthesis of New Glucosylated Porphyrins Bearing an $\alpha$-D-Linkage. J. Carbohydr. Chem., 2006, 25, 345360.

[238] Bonnett, R.; White, R.D.; Winfield, U.-J.; Berenbaum, M.C. Hydroporphyrins of the meso-tetra(hydroxyphenyl)porphyrin series as tumour photosensitizers. Biochem. J., 1989, 261, 277-280.

[239] Berenbaum, M.C.; Akande, S.L.; Bonnett, R.; Kaur, H.; Ioannou, S.; White, R.D.; Winfield, U.J. meso-Tetra(hydroxyphenyl)porphyrins, a new class of potent tumor photosensistizers with favorable selectivity. $B r . J$. Cancer, 1986, 54, 717-725.

[240] Zenkevich, E.; Sagun, E.; Knyukshto, V.; Shulga, A.; Mironov, A.; Efremova, O.; Bonnett, R.; Songca, S.P.; Kassem, M. Photophysical and photochemical properties of potential porphyrin and chlorin photosensitizers for PDT. J. Photochem. Photobiol. B: Biol., 1996, 33, 171-180.

[241] Bonnett, R.; Martínez, G. Photobleaching of Compounds of the 5,10,15,20-Tetrakis(m-hydroxyphenyl)-porphyrin Series (m-THPP, $m$ THPC, and $m$-THPBC). Org. Lett., 2002, 4, 2013-2016.

[242] Vargas, A.; Lange, N.; Arvinte, T.; Cerny, R.; Gurny, R.; Delie, F. Toward the understanding of the photodynamic activity of m-THPP encapsulated in PLGA nanoparticles: correlation between nanoparticle properties and in vivo activity. J. Drug Target., 2009, 17, 599-609.

[243] Sylvain, I.; Benhaddou, R.; Carre, V.; Cottaz, S.; Driguez, H.; Granet, R.; Guilloton, M.; Krausz, P. Synthesis and Biological Evaluation of Thioglycosylated meso-Arylporphyrins. J. Porphyrins Phthalocyanines, 1999, 3, 1-4.

[244] Bennett, S.; Von Itzstein, M.; Kiefel, M.J. A simple method for the preparation of thioglycosides of $\mathrm{N}$-acetylneuraminic acid. Carbohydr. Res., 1994, 259, 293-299.

[245] Snow, K.M.; Smith, K.M. Total syntheses of 8-formyl-8demethylprotoporphyrin IX, 8-(hydroxymethyl)-8-demethylprotoporphyrin IX, and 8-fluoromethyl analogs of protoporphyrin IX. J. Org. Chem., 1989, $54,3270-3281$

[246] Kenner, G.W.; McCombie, S.W.; Smith, K.M. Pyrroles and Related Compounds, XXI. Protection of Porphyrin Vinyl Groups. A Synthesis of Coproporphyrin-III from Protoporphyrin-IX. Liebigs Ann. Chem., 1973, 1329-1338.

[247] Chen, X.; Li Hui, L.; Foster, D.A.; Drain, C.M. Efficient Synthesis and Photodynamic Activity of Porphyrin-Saccharide Conjugates: Targeting and Incapacitating Cancer Cells. Biochemistry, 2004, 43, 10918-10929.

[248] Ahmed, S.; Davoust, E.; Savoie, H.; Boa, A.N.; Boyle, R.W. Thioglycosylated cationic porphyrins - convenient synthesis and photodynamic activity in vitro. Tetrahedron Lett., 2004, 45, 6045-6047.

[249] Silva, E.M.P.; Serra, V.V.; Ribeiro, A.O.; Tomé, J.P.C.; Domingues, P.; Faustino, M.A.F.; Neves, M.G.P.M.S.; Tomé, A.C.; Cavaleiro, J.A.S.; Ferrer-Correia, A.J.; Iamamoto, Y.; Domingues, M.R.M. Characterization of cationic glycoporphyrins by electrospray tandem mass spectrometry. Rap. Commun. Mass Spectrom., 2006, 20, 3605-3611.

[250] Hasegawa, T.; Umeda, M.; Numata, M.; Li, C.; Bae, A.-H.; Fujisawa, T.; Haraguchi, S.; Sakurai, K.; Shinkai, S. "Click chemistry" on polysaccharides: a convenient, general, and monitorable approach to develop $(1 \rightarrow 3)-\beta$ - $D$-glucans with various functional appendages. Carbohydr. Res, 2006, 341, 35-40.

[251] Garcia, G.; Naud-Martin, D.; Carrez, D.; Croisy, A.; Maillard, Ph. Microwave-mediated 'click-chemistry' synthesis of glycoporphyrin derivatives and in vitro photocytotoxicity for application in photodynamic therapy. Tetrahedron, 2011, 67, 4924-4932.

[252] Varamo, M.; Loock, B.; Maillard, P.; Grierson, D.S. Development of Strategies for the Regiocontrolled Synthesis of meso-5,10,20-Triaryl-2,3chlorins. Org. Lett., 2007, 9, 4689-4692.

[253] Kushwaha, D.; Tiwari, V.K. Click Chemistry Inspired Synthesis of Glycoporphyrin Dendrimers. J. Org. Chem., 2013, 78, 8184-8190.

[254] Ballardini, R.; Colonna, B.; Gandolfi, M.T.; Kalovidouris, S.A.; Orzel, L.; Raymo, F.M.; Stoddart, J.F. Porphyrin-Containing Glycodendrimers. Eur. J. Org. Chem., 2003, 288-294.

[255] Maillard, P.; Guerquin-Kern, J.-L.; Momenteau, M.; Gaspard, S. Glycoconjugated tetrapyrrolic macrocycles. J. Am. Chem. Soc., 1989, 111 9125-9127.

[256] Ballut, S.; Makky, A.; Chauvin, B.; Michel, J.-P.; Kasselouri, A.; Maillard, Ph.; Rosilio, V. Tumor targeting in photodynamic therapy. From glycoconjugated photosensitizers to glycodendrimeric one. Concept, design and properties. Org. Biomol. Chem., 2012, 10, 4485-4495.

[257] Pandey, R.K.; Isaac, M.; MacDonald, I.; Medforth, C.J.; Senge, M.O.; Dougherty, T.J.; Smith, K.M. Pinacol-Pinacolone rearrangement in vicDihydroxychlorins and bacteriachlorins: Effect of substituents at the peripheral position. J. Org. Chem., 1997, 62, 1463-1472.

[258] Mikata, Y.; Onchi, Y.; Shibata, M.; Kakuchi, T.; Ono, H.; Ogura, S.I.; Okura, I.; Yano, S. Synthesis and Phototoxic Property of Tetra- and OctaGlycoconjugated Tetraphenylchlorins. Bioorg. Med. Chem. Lett., 1998, 8, 3543-3548.

[259] Sol, V.; Blais, J.C.; Bolbach, G.; Carré, V.; Granet, R.; Guilloton, M.; Spiro, M.; Krausz, P. Toward Glycosylated Peptidic Porphyrins: a New Strategy for PDT? Tetrahedron Lett., 1997, 38, 6391-6393.

[260] Laville I., Figueiredo T., Loock B, Pigaglio, S., Maillard, Ph., Grierson, D.S., Carrez, D., Croisy, A.; Blais, J. Synthesis, cellular internalization and photodynamic activity of glucoconjugated derivatives of 
tri and tetra(meta-hydroxyphenyl)chlorins. Bioorg. Med. Chem., 2003, 11, 1643-1652.

[261] Hirohara, S.; Obata, M.; Ogata, S.-I.; Ohtsuki, C.; Higashida, S.; Ogura, S.-I.; Okura, I.; Takenaka, M.; Ono, H.; Sugai, Y.; Mikata, Y.; Tanihara, M.; Yano, S. Cellular uptake and photocytotoxicity of glycoconjugated chlorins in HeLa cells. J. Photochem. Photobiol. B: Biol., 2005, 78, 7-15.

[262] Hirohara, S.; Obata, M.; Ogata, S.-I.; Kajiwara, K.; Ohtsuki, C.; Tanihara, M.; Yano, S. Sugar-dependent aggregation of glycoconjugated chlorins and its effect on photocytotoxicity in HeLa cells. J. Photochem. Photobiol. B: Biol., 2006, 84, 56-63.

[263] Fischer, H.; Eckoldt, H. Überführung von Porphyrinen in Dioxychlorine durch Einwirkung von Osmiumtetroxyd. Liebigs Ann. Chem., 1940, 544, 138-162.

[264] Flitsch, W. Hydrogenated Porphyrin Derivatives: Hydroporphyrins. Adv. Heterocycl. Chem., 1988, 43, 73-126.

[265] Brückner, C.; Dolphin, D. 2,3-vic-Dihydroxy-mesotetraphenylchlorins from the osmium tetroxide oxidation of mesotetraphenylporphyrin. Tetrahedron Lett., 1995, 36, 3295-3298.

[266] Bonnett, R.; Nizhnik, A.N.; Berenbaum, M.C. Second generation tumour photosensitisers: the synthesis of octaalkyl chlorins and bacteriochlorins with graded amphiphilic character. J. Chem. Soc. Chem. Commum., 1989, 1822-1823.

[267] Tomé, A.C.; Neves, M.G.P.M.S.; Cavaleiro, J.A.S. Porphyrins and other pyrrolic macrocycles in cycloaddition reactions. J. Porphyrins Phthalocyanines, 2009, 13, 408-414.

[268] Silva, A.M.G.; Cavaleiro, J.A.S. In: Progress in Heterocyclic Chemistry; Gribble, G.W.; Joule, J.A. Ed.; Elsevier Science B. V: Amsterdam, 2008, Vol. 19, pp. 44-69.

[269] Silva, A.M.G.; Tome, A.C.; Neves, M.G.P.M.S.; Cavaleiro, J.A.S.; Perrone, D.; Dondoni, A. Porphyrins in 1,3-dipolar cycloadditions with sugar azomethine ylides. Synthesis of pyrrolidinoporphyrin glycoconjugates. Synlett, 2005, 857-859.

[270] Silva, A.M.G.; Tomé, A.C.; Neves, M.G.P.M.S.; Silva, A.M.S.; Cavaleiro, J.A.S.; Perrone, D.; Dondoni, A. Porphyrins in 1,3-dipolar cycloaddition reactions with sugar nitrones. Synthesis of glycoconjugated isoxazolidine-fused chlorins and bacteriochlorins. Tetrahedron Lett., 2002, 43, 603-605.

[271] Gomes, A.T.P.C.; Leão, R.A.C.; da Silva, F.C.; Neves, M.G.P.M.S.; Faustino, M.A.F; Tomé, A.C.; Silva, A.M.S.; Pinheiro, S.; de Souza, M.C.B.V.; Ferreira, V.; Cavleiro, J.A.S. Synthesis of new glycoporphyrins derivatives through carbohydrate-substituted $\alpha$-diazoacetates. J. Porphyrins Phthalocyanines, 2009, 13, 247-255.

[272] Obata, M.; Hirohara, S.; Sharyo, K.; Alitomo, H.; Kajiwara, K.; Ogata, S.-I.; Tanihara, M.; Ohtsuki, C.; Yano, S. Sugar-dependent photodynamic effect of glycoconjugated porphyrins: A study on photocytotoxicity, photophysical properties and binding behavior to bovine serum albumin (BSA). Biochim. Biophys. Acta, 2007, 1770, 1204-1211.

[273] Hirohara, S.; Obata, M.; Alitomo, H.; Sharyo, K.; Ogata, S.-I.; Ohtsuki, C.; Yano, S.; Ando, T.; Tanihara, M. Structure-photodynamic effect relationships of 24 glycoconjugated photosensitizers in HeLa cells. Biol. Pharm. Bull., 2008, 31, 2265-2272.

[274] Singh, S.; Aggarwal, A.; Thompson, S.; Tomé, J.P.C.; Zhu, X.; Samaroo, D.; Vinodu, M.; Gao, R.; Drain, C.M. Synthesis and Photophysical Properties of Thioglycosylated Chlorins, Isobacteriochlorins and Bacteriochlorins for Bioimaging and Diagnostics. Bioconj. Chem., 2010, 21, 2136-2146.

[275] Senge, M.O.; Vicente, M.G.H.; Parkin, S.R.; Hope, H.; Smith, K.M. Structural investigations on mono- and diacrolein-substituted nickel(II) porphyrins and nickel(II) benzochlorin. Model compounds for photosensitizers in photodynamic therapy. Z. Naturforsch., 1992, 47b, 11891202.

[276] Morgan, A.R.; Pangka, V.S.; Dolphin, D. Ready syntheses of benzoporphyrins via Diels-Alder reactions with protoporphyrin IX. $J$. Chem. Soc., Chem. Commun., 1984, 1047-1048.

[277] Maillard, Ph.; Hery, C.; Momenteau, M. Synthesis, Characterization and Photocytotoxicity of a Glycoconjugated meso-Monoarylbenzochlorin. Tetrahedron Lett., 1997, 38, 3731-3734.

[278] Zheng, G.; Graham, A.; Shibata, M.; Missert, J.R.; Oseroff, A.R.; Dougherty, T.J.; Pandey, R.K. Synthesis of $\beta$-Galactose-Conjugated Chlorins Derived by Enyne Metathesis as Galactin-Specific Photosensitizers for Photodynamic Therapy. J. Org. Chem., 2001, 66, 8709-8716.
[279] Li, G.; Pandey, R.K.; Graham, A.; Dobhal, M.O.; Mehta, R.; Chen, Y.; Gryshuk, A.; Rittenhouse-Olson, K.; Oseroff, A.; Pandey, R.K. Functionalization of OEP-Based Benzochlorins to Develop CarbohydrateConjugated Photosensitizers. Attempt to Target $\beta$-Galactoside-Recognized Proteins. J. Org. Chem., 2004, 69, 158-172.

[280] Graham, A.; Li, G.; Chen, Y.; Morgan, J.; Oseroff, A.; Dougherty, T.J.; Pandey, R.K. Structure-Activity Relationship of New Octaethylporphyrin-based Benzochlorins As Photosensitizers for Photodynamic Therapy. Photochem. Photobiol., 2003, 77, 561-566.

[281] Bertozzi, C.; Bednarski, M. C-glycosyl compounds bind to receptors on the surface of Escherichia coli and can target proteins to the organism. Carbohydr. Res., 1992, 223, 243-253.

[282] Nyman, E.S.; Hynninen, P.H. Research advances in the use of tetrapyrrolic photosensitizers for photodynamic therapy. J. Photochem. Photobiol. B: Biol., 2004, 73, 1-28.

[283] Senge, M.O.; Ryan, A.A.; Letchford, K.A.; MacGowan, S.A.; Mielke, T. Chlorophylls, Symmetry, Chirality, and Photosynthesis. Symmetry, 2014, 6, 781-843.

[284] Aksenova, A.A.; Sebyakin, Y.L.; Mironov, A.F. The Synthesis of Galactopyranosyl-Substituted Derivatives of Pheophorbide. Russ. J. Bioorg. Chem., 2000, 26, 111-114; Bioorg. Khim., 2000, 26, 126-129.

[285] Zhang, M.; Zhang, Z.; Blessington, D.; Li, H.; Busch, T.M.; Madrak, V.; Miles, J.; Chance, B.; Glickson, J.D.; Zheng, G. Pyropheophorbide 2Deoxyglucosamide: A New Photosensitizer Targeting Glucose Transporters. Bioconj. Chem., 2003, 14, 709-714.

[286] Bellnier, D.A.; Greco, W.R.; Loewen, G.M.; Nava H.; Oseroff, A.R. Pandey, R.K.; Tsuchida, T.; Dougherty, T.J. Population pharmacokinetics of the photodynamic therapy agent 2-[1-hexyloxyethyl]-2-devinyl pyropheophorbide-a in cancer patients. Cancer Res., 2003, 63, 1806-1813.

[287] Zheng, X.; Morgan, J.; Pandey, S.K.; Chen, Y.; Tracy, E.; Baumann, H.; Missert, J.R.; Batt, C.; Jackson, J.; Bellnier, D.A.; Henderson, B.W.; Pandey, R. K. Conjugation of 2-(1'-Hexyloxyethyl)-2devinylpyropheophorbidea (HPPH) to Carbohydrates Changes its Subcellular Distribution and Enhances Photodynamic Activity in Vivo. J. Med. Chem., 2009, 52, 4306-4318.

[288] Pandey, S.K.; Sajjad, M.; Chen, Y.; Yao, R.; Missert, J.R.; Batt, C.; Nabi, H.N.; Oseroff, A.R.; Pandey, R.K. Comparative positron-emission tomography (PET) imaging and phototherapeutic potential of I-labeled methyl-3-(10-iodonenxyloxyethyl) pyropheophorbide-a vs the corresponding glucose and galactose conjugates. J. Med. Chem., 2009, 52, 445-455.

[289] Hoober, J.K.; Sery, T.W.; Yamamoto, N. Photodynamic sensitizers from chlorophyll: purpurin-18 and chlorin p6. Photochem. Photobiol., 1988 48, 579-582.

[290] Pandey, S.K.; Zheng, X.; Morgan, J.; Missert, J.R.; Liu, T.-H.; Shibata, M.; Bellnier, D.A.; Oseroff, A.R.; Henderson, B.W.; Dougherty, T.J.; Pandey, R.K. Purpurinimide Carbohydrate Conjugates: Effect of the Position of the Carbohydrate Moiety in Photosensitizing Efficacy. Mol. Pharm., 2007, 4, 448-464.

[291] Grin, M.A.; Lonin, I.S.; Makarov, A.I.; Lakhina, A.A.; Toukach, F.V.; Kachala, V.V.; Orlova, A.V.; Mironov, A.F. Synthesis of chlorincarbohydrate conjugates by 'click chemistry'. Mendeleev Commun., 2008, 18, 135-137.

[292] Grin, M.A.; Lonin, I.S.; Lakhina, A.A.; Ol'shanskaya, E.S.; Makarov, A.I.; Sebyakin, Y.L.; Guryeva, L.Y.; Toukach, P.; Kononikhin, A.S.; Kuzmin, V.A.; Mironov, A.F. 1,3-Dipolar cycloaddition in the synthesis of glycoconjugates of natural chlorins and bacteriochlorins. J. Porphyrins Phthalocyanines, 2009, 13, 336-345.

[293] Mironov, A.F.; Grin, M.A. Synthesis of chlorin and bacteriochlorin conjugates for photodynamic and boron neutron capture therapy. $J$. Porphyrins Phthalocyanines, 2008, 12, 1163-1172.

[294] Li, F.; Bae, B.-C.; Na, K. Acetylated Hyaluronic Acid/Photosensitizer Conjugate for the Preparation of Nanogels with Controllable Phototoxicity: Synthesis, Characterization, Autophotoquenching Properties, and in vitro Phtotoxicity against HeLa Cells. Bioconjug. Chem., 2010, 21, 1312-1320. [295] Bae, B.-C.; Na, K. Self-quenching polysaccharide-based nano-gels of pullulan/folate-photosensitizer conjugates for photodynamic therapy. Biomaterials, 2010, 31, 6325-6335.

[296] Roitman, L.; Ehrenberg, B.; Nitzan, Y.; Kral, V.; Sessler, J.L. Spectroscopy and photosensitization of sapphyrins in solutions and biological membranes. Photochem. Photobiol., 1994, 60, 421-426. 
[297] Král, V.; Davis, J.; Andrievsky, A.; Kralová, J.; Synytsya, A.; Poucková, P.; Sessler, J.L. Synthesis and Biolocalization of Water-Soluble Sapphyrins. J. Med. Chem., 2002, 45, 1073-1078.

[298] Synytsya, A.; Král, V.; Synytsya, A.; Volka, K.; Sessler, J.L. In Vitro Interaction of Macrocyclic Photosensitizers with Intact Mitochrondria: a Spectroscopic Study. Biochim. Biophys. Acta, 2003, 1620, 85-96.

[299] Sessler, J.L.; Davis, J.M.; Král, V.; Kimbrough, T.; Lynch, V. Water soluble sapphyrins: potential fluorescent phosphate anion sensors. Org. Biomol. Chem, 2003, 1, 4113-4123.

[300] Štěpánek, P.; Šimák, O.; Nováková, Z.; Wimmer, Z.; Drašar, P. Asymmetrically substituted calix[4]pyrrole with chiral substituents. Org. Biomol. Chem., 2011, 9, 682-683.

[301] Dumoulina, F.; Durmuş, M.; Ahsen, V.; Nyokong, T. Synthetic pathways to water-soluble phthalocyanines and close analogs. Coord. Chem. Rev., 2010, 254, 2792-2847.

[302] Álvarez-Micó, X.; Calvete, M.J.F.; Hanack, M.; Ziegler, T. Expeditious Synthesis of Glycosylated Phthalocyanines. Synthesis, 2007, 14, 2186-2192.

[303] Iqbal, Z.; Lyubimtsev, A.; Hanack, M.; Thomas Ziegler, T. Anomerically glycosylated zinc(II) naphthalocyanines. Tetrahedron Lett., 2009, 50, 5681-5685.

[304] Ribeiro, A.O.; Tomé, J.P.C.; Neves, M.G.P.M.S.; Tomé, A.C.; Cavaleiro, J.A.S.; Iamamoto, Y.; Torres, T. [1,2,3,4-Tetrakis $(\alpha / \beta-D-$ galactopyranos-6-yl)-phthalocyaninato]zinc(II): a water-soluble phthalocyanine. Tetrahedron Lett., 2006, 47, 9177-9180.

[305] Zhang, P.; Zhang, S.; Han, G. Synthesis of Novel Asymmetric Zinc (II) Phthalocyanines Bearing Octadecyloxyl and Glucosyl Groups. Molecules, 2009, 14, 3688-3693.

[306] Álvarez-Micó, X.; Calvete, M.J.F.; Hanack, M.; Ziegler, T. A new glycosidation method through nitrite displacement on substituted nitrobenzenes. Carbohydr. Res., 2007, 342, 440-447.

[307] Alvarez-Mico, X.; Calvete, M.J.F.; Hanack, M.; Ziegler, T. The first example of anomeric glycoconjugation to phthalocyanines. Tetrahedron Lett., 2006, 47, 3283-3286.

[308] Zorlu, Y.; Ermeydan, M.A.; Dumoulin, F.; Ahsen, V.; Savoie, H.; Boyle, R.W. Glycerol and galactose substituted zinc phthalocyanines. Synthesis and photodynamic activity. Photochem. Photobiol. Sci., 2009, 8, 312-318.

[309] Soares, A.R.M.; Tomé, J.P.C.; Neves, M.G.P.M.S.; Tomé, A.C.; Cavaleiro, J.A.S.; Torres, T. Synthesis of water-soluble phthalocyanines bearing four or eight $D$-galactose units. Carbohydr. Res., 2009, 344, 507510 .

[310] Choi, C.-F.; Huang, J.-D.; Lo, P.-C.; Fong, W.-P.; Ng, D.K.P. Glycosylated zinc(II) phthalocyanines as efficient photosensitisers for photodynamic therapy. Synthesis, photophysical properties and in vitro photodynamic activity. Org. Biomol. Chem., 2008, 6, 2173-2181.

[311] Iqbal,Z.; Hanack, M.; Ziegler, T. Synthesis of an octasubstituted galactose zinc(II) phthalocyanine. Tetrahedron Lett., 2009, 50, 873-875.

[312] Silva, S.; Pereira, P.M.R.; Silva, P.; Paz, F.A.A.; Faustino M.A.F.; Cavaleiro, J.A.S.; Tomé, J.P.C. Porphyrin and phthalocyanine glycodendritic conjugates: synthesis, photophysical and photochemical properties. Chem. Commun., 2012, 48, 3608-3610.

[313] Reddy, M.R.; Shibata, N.; Yoshiyama, H.; Nakamura, S.; Toru, T. Synthesis and Spectral Properties of a Deoxyribose-Phthalocyanine Conjugate Using a Sonogashira Coupling Reaction. Synlett, 2007, 4, 628632.

[314] Lv, F.; He, X.; Lu, L.; Wu, L.; Liu, T. Synthesis, properties and nearinfrared imaging evaluation of glucose conjugated zinc phthalocyanine via Click reaction. J. Porphyrins Phthalocyanines, 2012, 16, 77-84.

[315] Lo, P.-C.; Chan, C.M.H.; Liu, J.-Y.; Fong, W.-P.; Ng, D.K.P. Highly Photocytotoxic Glucosylated Silicon(IV) Phthalocyanines. Effects of Peripheral Chloro Substitution on the Photophysical and Photodynamic Properties. J. Med. Chem., 2007, 50, 2100-2107.

[316] Lo, P.-C.; Fongm, W.-P.; Ng, D.K.P. Effects of Peripheral Chloro Substitution on the Photophysical Properties and in vitro Photodynamic Activities of Galactose-Conjugated Silicon(IV) Phthalocyanines. ChemMedChem, 2008, 3, 1110-1117.

[317] Lee, P.P.S.; Lo, P.-C.; Chan, E.Y.M.; Fong, W.-P.; Ko, W.-H.; Ng, D.K.P. Synthesis and in vitro photodynamic activity of novel galactosecontaining phthalocyanines. Tetrahedron Lett., 2005, 46, 1551-1554.

[318] Lo, P.-C.; Leung, S.C.H.; Chan, E.Y.M.; Fong, W.-P.; Ko, W.-H.; $\mathrm{Ng}$, D.K.P. Photodynamic effects of a novel series of silicon(IV) phthalocyanines against human colon adenocarcinoma cells. Photodiag. Photodyn. Ther., 2007, 4, 117-123.

[319] Ribeiro, A.O.; Tomé, J.P.C.; Neves, M.G.P.M.S.; Tomé, A.C.; Cavaleiro, J.A.S.; Serra, O.A.; Torres, T. First phthalocyanine- $\beta$ cyclodextrin dyads. Tetrahedron Lett., 2006, 47, 6129-6132.

[320] Liu, J.-Y.; Lo, P.-C.; Fong, W.-P.; Ng, D.K.P. Effects of the number and position of the substituents on the in vitro photodynamic activities of glucosylated zinc(II) phthalocyanines. Org. Biomol. Chem., 2009, 7, 15831591.

[321] Ermeydan, M.A.; Dumoulin, F.; Basova, T.V.; Bouchu, D.; Gürek, A.G.; Ahsen, V.; Lafont, D. Amphiphilic carbohydrate-phthalocyanine conjugates obtained by glycosylation or by azide-alkyne click reaction. New J. Chem., 2010, 34, 1153-1162.

[322] Kumru, U.; Ermeydan, M.A.; Dumoulin, F.; Ahsen, V. Amphiphilic galactosylated phthalocyanines. J. Porphyrins. Phthalocyanines, 2008, 12 1090-1095.

[323] Zorlu, Y.; Dumoulin, F.; Bouchu, D.; Ahsen, V.; Lafont, D. Monoglycoconjugated water-soluble phthalocyanines. Design and synthesis of potential selectively targeting PDT photosensitisers. Tetrahedron Lett., 2010, 51, 6615-6618.

[324] Lafont, D.; Zorlu, Y.; Savoie, H.; Albrieux, F.; Ahsen, V.; Boyle, R.W.; Dumoulin, F. Monoglycoconjugated phthalocyanines: Effect of sugar and linkage onphotodynamic activity. Photodiag. Photodyn. Ther., 2013 10, 252-259.

[325] Berthold, H.J.; Franke, S.; Thiem, J.; Schotten, T. Ex Pos Glycoconjugation of Phthalocyanines. J. Org. Chem., 2010, 75, 3859-3862.

[326] Bakar, M.B.; Oelgemöller, M.; Senge, M.O. Lead structures for applications in photodynamic therapy. Part 2: Synthetic studies for phototriggered release systems of bioconjugate porphyrin photosensitizers Tetrahedron, 2009, 65, 7064-7078.

[327] Rakestraw, S.L.; Tompkins, R.G.; Yarmush, M.L. Antibody-targeted photolysis: In vitro studies with Sn(IV) chlorin e6 covalently bound to monoclonal antibodies using a modified dextran carrier. Proc. Natl. Acad. Sci. USA, 1990, 87, 4217-4221.

[328] Peng, Q.; Moan, J.; Nesland, J.M. Correlation of subcellular and intratumoral photosensitizer localization with ultrastructural features after photodynamic therapy. Ultrastruct. Pathol., 1996, 20, 109-129.

[329] Moan, J.; Berg, K. The photodegradation of porphyrins in cells can be used to estimate the lifetime of singlet oxygen. Photochem. Photobiol., 1991, 53, 549-553.

[330] Foster, T.H.; Pearson, B.D.; Mitra, S.; Bigelow, C.E. Fluorescence anisotropy imagaing reveals localization of meso-tetrahydroylphenyl chlorin in the nuclear enevelope. Photochem. Photobiol., 2005, 81, 1544-1547.

[331] Mellish, K.J.; Cox, R.D.; Vernon, D.I.; Griffiths, J.; Brown, S.B. In vitro photodynamic activity of a series of methylene blue analogues, Photochem. Photobiol., 2002, 75, 392-397.

[332] Kessel, D. Porphyrin-lipoprotein association as a factor in porphyrin ocalization. Cancer Lett., 1986, 33, 183-188.

[333] Hatz, S.; Lambert, J.D.C.; Ogilby, P.R. Measuring the lifetime of singlet oxygen in a single cell: addressing the issue of cell viability. Photochem. Photobiol. Sci., 2007, 6, 1106-1116.

[334] Jori, G.; Fabris, C.; Soncin, M.; Ferro, S.; Coppellotti, O.; Dei, D.; Fantetti, L.; Chiti, G.; Roncucci, G. Photodynamic therapy in the treatment of microbial infections: Basic principles and perspective applications. Lasers Surg. Med., 2006, 38, 468-481.

[335] Sharman, W.M.; Allen, C.M.; Van Lier, J.E. Photodynamic therapeutics:basic principles and clinical applications. Drug Discov. Today, 1999, 4, 507-517

[336] Hatz, S.; Poulsen, L.; Ogilby, P. Time-resolved singlet oxygen phosphorescence measurements from photosensitized experiments in single cells: Effects of oxygen diffusion and oxygen concentration. Photochem. Photobiol., 2008, 84, 1284-1290.

[337] Sharman, W. M.; Allen, C. M.; Van Lier, J. E. Role of activated oxygen species in photodynamic therapy. Meth. Enzymol., 2000, 319, 376400 .

[338] Chen, Y.; Li, G.; Pandey, R.K. Synthesis of bacteriochlorins and their potential utility in photodynamic therapy (PDT). Curr. Org. Chem., 2004, 8 , $1105-1134$

[339] Boyle, R.W.; Dolphin, D. Structure and biodistribution relationship of photodynamic sensitizers. Photochem. Photobiol., 1996, 64, 469-485.

[340] Margaron, P.; Grégoire. M.-J.; Ŝĉasnár, V.; Ali, H.; Van Lier, J.E. Structure-Photodynamic activity relationships of a series of 4-substituted zinc phthalocyanines, Photochem. Photobiol., 1996, 63, 217-223. 
[341] Csík, G.; Balog, E.; Voszka, I.; Tölgyesi, F.; Oulmi, D.; Maillard, P.; Momenteau, M. Glycosylated derivatives of tetraphenyl porphyrin: photophysical characterization, self-aggregation and membrane binding. $J$. Photochem. Photobiol. B: Biol., 1998, 44, 216-224.

[342] Voszka, I.; Galántai, R.; Maillard, P.; Csík, G. Interaction of glycosylated tetraphenyl porphyrins with model lipid membranes of different compositions. J. Photochem. Photobiol. B: Biol., 1999, 52, 92-98. [343] Morlière, P.; Mazière, J.-C.; Santus, R.; Smith, C.D.; Prinsep, M.R.; Stobbe, C.C.; Fenning, M.C.; Golberg, J.L.; Chapman, J.D. Tolyporphrin: A natural product from cyanobacteria with potent photosensitizing activity against tumor cells in vitro and in vivo. Cancer Res., 1998, 58, 3571-3578.

[344] Berg, K., In: The fundamental bases of phototherapy. Young, A.R.; Yori, G.; Hönigsmann, H., Eds.; OEMF: Milan, 1996, pp. 181-207.

[345] Momenteau, M.; Oulmi, D.; Maillard, Ph.; Croisy, A. In vitro photobiological activity of a new series of photosensitizers: the glycoconjugated porphyrins. Proc. SPIE, 1994, 2325, 13-23.

[346] Thompson, S.; Chen, X.; Hui, L.; Toschi, A.; Foster, D.A. Drain, C.M. Low concentrations of a non-hydrolysable tetra-S-glycosylated porphyrin and low light induces apoptosis in human breast cancer cells via stress of the endoplasmic reticulum. Photochem. Photobiol. Sci., 2008, 7, 1415-1421.

[347] Giuntini, F.; Bryden, F.; Daly, R.; Scanlan, E.M.; Boyle, R.W. Huisgen-based conjugation of water-soluble porphyrins to deprotected sugars: towards mild strategies for the labelling of glycans. Org. Biomol. Chem., 2014, 12, 1203-1206.

[348] Vedachalam, S.; Choi, B.-H.; Pasunooti, K.K.; Ching, K.M.; Lee, K.; Yoon, H.S.; Liu, X.-W. Glycosylated porphyrin derivatives and their photodynamic activity in cancer cells. Med. Chem. Commun., 2011, 2, 371377.

[349] Kuroda,Y.; Hiroshige, T.; Sera, T.; Shiroiwa, T.; Tanaka, H.; Ogoshi, H. Cyclodextrin-Sandwiched Porphyrins. J. Am. Chem. Soc., 1989, 111, 1912-1913.

[350] Kuroda,Y.; Hiroshige, T.; Sera, H.; Ogoshi, H. Direct synthesis of a sandwich-type molecule involving porphyrin and two molecules of cycloheptaose. Carbohydr. Res., 1989, 192, 347-350.

[351] Kralova, J.; Synytsya, A.; Pouckova, P.; Koc, M.; Dvorak, M.; Král, V. Novel Porphyrin Conjugates with a Potent Photodynamic Antitumor Effect: Differential Efficacy of Mono- and Bis- $\beta$-cyclodextrin Derivatives In Vitro and In Vivo. Photochem. Photobiol., 2006, 82, 432-438.

[352] Králová, J.; Kejík, Z.; Bříza, T.; Poučková, P.; Král, A.; Martásek, P.; Král, V. Porphyrin-Cyclodextrin Conjugates as a Nanosystem for Versatile Drug Delivery and Multimodal Cancer Therapy. J. Med. Chem., 2010, 53, 128-138.

[353] Senge, M.O. $m$ THPC - A drug on its way from second generation to third generation photosensitizer? Photodiagn. Photodyn. Ther., 2012, 9, 170-179.

[354] Hirohara, S.; Kawasaki, Y.; Funasako, R.; Yasui, N.; Totani, M.; Alitomo, H.; Yuasa, J.; Kawai, T.; Oka, C,; Kawaichi, M.; Obata, M.; Tanihara, M. Sugar and Heavy Atom Effects of Glycoconjugated Palladium Complex on Phototoxicity. Bioconj. Chem., 2012, 23, 1881-1890.

[355] Ali, H.; Van Lier, J.E.; In: Handbook of Porphyrin Science. Kadish, K. M.; Smith, K.M.; Guilard, R., Eds.; World Scientific Publishing Co., Singapore, 2010, Vol. 4, pp. 1-119.

[356] Momenteau, M.; Maillard, Ph.; de Bélinay, M. A.; Carrez, D.; Croisy, A. Tetrapyrrolic glycosylated macrocycles for an application in PDT. $J$. Biomed. Optics, 1999, 4, 298-318.

[357] Morgan, J.; Jackson, J.D.; Zheng, X.; Pandey, S.K.; Pandey, R.K. Substrate affinity of photosensitizers derived from chlorophyll-a: The ABCG2 transporter affects the phototoxic response of side population stem cell-like cancer cells to photodynamic therapy. Mol. Pharm., 2010, 7, 17891804 .

[358] Hajri, A.; Wack, S.; Meyer, C.; Smith, M. K.; Leberquier, C.; Kedinger, M.; Aprahamian, M. In vitro and in vivo efficacy of Photofrin ${ }^{\circledR}$ and pheophorbide $a$, a bacteriochlorin, in photodynamic therapy of colonic cancer cells. Photochem. Photobiol., 2002, 75, 140-148.

[359] Soares, A.R.M.; Neves, M.G.P.M.S.; Tomé, A.C.; Iglesias-de la Cruz, M.C.; Zamarrón, A.; Carrasco, E.; González, S.; Cavaleiro, J.A.S.; Torres, T.; Guldi, D.M.; Juarranz, A. Glycophthalocyanines as Photosensitizers for Triggering Mitotic Catastrophe and Apoptosis in Cancer Cells. Chem. Res. Toxicol., 2012, 25, 940-951.

[360] Desroches, M.-C.; Bautista-Sanchez, A.; Lamotte, C.; Labeque, B.; Auchère, D.; Farinotti, R.; Maillard, P.; Grierson, D.S.; Prognon, P.; Kasselouri, A. Pharmacokinetics of a tri-glucoconjugated 5,10,15-(meta)- trihydroxyphenyl-20-phenyl porphyrin photosensitizer for PDT. A single dose study in the rat. J. Photochem. Photobiol. B: Biol., 2006, 85, 56-64. [361] Synytsya, A.; Král, V.; Blechová, M.; Volka, K. Biolocalisation and photochemical properties of two novel macrocyclic photosensitisers: a spectroscopic study. J. Photochem. Photobiol. B:Biol., 2004, 74, 73-84. [362] Synytsya, A.; Král, V.; Poučková, P.; Volka, K. Resonance Raman and UV-Visible Spectroscopic Studies of Water-Soluble Sapphyrin Derivative: Drug Localization in Tumor and Normal Mice Tissues. Appl. Spectrosc., 2001, 55, 142-148.

[363] Dai, T.; Fuchs, B.B.; Coleman, J.J.; Prates, R.A.; Astrakas, C.; Denis, T.G.St.; Ribeiro, M.S.; Mylonakis, E.; Hamblin, M.R.; Tegos, G.P. Concepts and Principles of Photodynamic Therapy as an Alternative Antifungal Discovery Platform. Front Microbiol., 2012, 3, 1-16.

[364] Calzavara-Pinton, P.; Rossi, M.T.; Sala, R.; Venturini, M. Photodynamic Antifungal Chemotherapy. Photochem. Photobiol., 2012, 88, 512-522.

[365] Carré, V.; Gaud, O.; Sylvain, I.; Bourdon, O.; Spiro, M.; Blais, J.; Granet, R.; Krausz, P.; Guilloton, M. Fungicidal properties of mesoarylglycosylporphyrins: influence of sugar substituents on photoinduced damage in the yeast Saccharomyces cerevisia. J. Photochem. Photobiol. B: Biol., 1999, 48, 57-62.

[366] Kanony, C.; Fabiano-Tixier, A.-S.; Ravanatz, J.-L.; Vicendo ,P.; Paillous, N. Photosensitization of DNA Damage by a New Cationic Pyropheophorbide Derivative: Sequence-Specific Formation of a Frank Scission. Photochem. Photobiol., 2003, 77, 659-667.

[367] Bernadou, J.; Pratviel, G.; Bennis, F.; Girardet M.; Meunier, B. Potassium monopersulfate and a water-soluble manganese porphyrin complex, $[\mathrm{Mn}(\mathrm{TMPyP})](\mathrm{OAc})_{5}$, as an efficient reagent for the oxidative cleavage of DNA. Biochemistry, 1989, 28, 7268-7275.

[368] Pratviel, G.; Pitié, M.; Bernadou, J.; Meunier, B. Mechanism of DNA cleavage by cationic manganese porphyrins: hydroxylations at the 1'-carbon and 5'-carbon atoms of deoxyriboses as initial damages. Nucleic Acids Res., 1991, 19, 6283-6298.

[369] Chaloin, L.; Bigey, P.; Loup, C.; Marin, M.; Galeotti, N.; Piechaczyk, M.; Heitz, F.; Meunie, B. Improvement of Porphyrin Cellular Delivery and Activity by Conjugation to a Carrier Peptide. Bioconj. Chem., 2001, 12, 691-700.

[370] Fiel, R.J.; Howard, J.C.; Mark, E.H.; Datta Gupta, N. Interaction of DNA with a porphyrin ligands: evidence of intercalation. Nucleic Acids Res., 1979, 5, 3093-3118.

[371] Far, S.; Kossanyi, A.; Verchère-Béaur, C.; Gresh, N.; Taillandier, E.; Perreé-Fauvet, M. Bis- and Tris-DNA Intercalating Porphyrins Designed to Target the Major Groove: Synthesis of Acridylbis-arginyl-porphyrins, Molecular Modelling of their DNA Complexes, and Experimental Tests. Eur. J. Org. Chem., 2004, 1781-1797.

[372] Biron, E.; Voyer, N. Towards sequence selective DNA binding: design, synthesis and DNA binding studies of novel bis-porphyrin peptidic nanostructures. Org. Biomol. Chem., 2008, 6, 2507-2515.

[373] Steenkeste, K.; Enescu, M.; Tfibel, F.; Pernot, P.; Far, S.; PerréeFauvet, M.; Fontaine-Aupart, M.-P. Structural dynamics and reactivity of a cationic mono(acridyl)bis(arginyl)porphyrin: A spectroscopic study down to femtoseconds. Phys. Chem. Chem. Phys., 2004, 6, 3299-3308.

[374] Mezö, G.; Herényi, L.; Habdas, J.; Majer, Z.; Myśliwa-Kurdziel, B.; Tóthf, K.; Csík, G. Syntheses and DNA binding of new cationic porphyrintetrapeptide conjugates. Biophys. Chem., 2011, 155, 36-44.

[375] Czuchajowski, L.; Habdas, J.; Niedbala, H.; Wandrekar, V. Porphyrinyl-uridines as the first water soluble porphyrinyl-nucleosides. Tetrahedron Lett., 1991, 32, 7511-7512.

[376] Reddy, M.R.; Shibata, N.; Kondo, Y.; Nakamura, S.; Toru, T. Design, Synthesis, and Spectroscopic Investigation of Zinc Dodecakis(trifluoroethoxy)-phthalocyanines Conjugated with Deoxyribonucleosides. Angew. Chem. Int. Ed., 2006, 45, 8163-8166.

[377] Hombrecher, H.K.; Schell, C. Carbohydrate Substituted Porphyrins. Synthesis, Characterization and Lipoprotein Binding Properties. Carbohydr. Polym., 1997, 34, 422-423.

[378] Maziere, J. C.; Morliere, P.; Santus, R. New trends in photobiology: The role of the low density lipoprotein receptor pathway in the delivery of lipophilic photosensitizers in the photodynamic therapy of tumours. $J$. Photochem. Photobiol. B: Biol., 1991, 8, 351-360.

[379] Mori, M.; Kuroda, T.; Obana, A.; Sakata, I.; Hirano, T.; Nakajima, S.; Hikida, M.; Kumagai, T. In vitro Plasma Protein Binding and Cellular Uptake of ATX-S10(Na), a Hydrophilic Chlorin Photosensitizer. Jpn. J. Cancer Res., 2000, 91, 845-852. 
[380] Makky, A.; Michel, J. P.; Ballut, S.; Kasselouri, A.; Maillard, Ph.; Rosilio, V. Effect of Cholesterol and Sugar on the Penetration of Glycodendrimeric Phenylporphyrins into Biomimetic Models of Retinoblastoma Cells Membranes. Langmuir, 2010, 26, 11145-11156.

[381] Arima, Y.; Akimoto, S.; Yamazaki, T.; Shibata, M.; Hirohara, S.; Yano, S.; Kakuchi, T.; Yamazaki, I. Excitation relaxation dynamics and molecular dispersion of maltohexaose-linked tetraphenylporphyrins in aqueous solution. Chem. Phys. Lett., 2002, 361, 152-158.

[382] Ibrahim, H.; Kasselouri, A.; You, C.; Maillard, Ph.; Rosilio, V.; Pansu, R.; Prognon, P. Meso-tetraphenyl porphyrin derivatives: The effect of structural modifications on binding to DMPC liposomes and albumin. $J$. Photochem. Photobiol. A: Chem., 2011, 217, 10-21.

[383] Matsuo, T.; Nagai, H.; Hisaeda, Y.; Hayashi, T. Construction of glycosylated myoglobin by reconstitutional method. Chem. Commun., 2006, 3131-3133.

[384] Makky, A.; Michel, J. P.; Kasselouri, A.; Briand, E.; Maillard, Ph.; Rosilio, V. Evaluation of the Specific Interactions between Glycodendrimeric Porphyrins, Free or Incorporated into Liposomes, and Concanavaline A by Fluorescence Spectroscopy, Surface Pressure, and QCM-D Measurements. Langmuir, 2010, 26, 12761-12768.

[385] Rosilio, V.; Makky, A.; Michel, J.-P.; Maillard, Ph. Interfacial behaviour of glycoconjugated tetraphenylporphyrins and their interaction with biomimetic models of the cell membrane. Ann. Pharm. Franc., 2012, 70, 219-226

[386] Makky, A.; Michel, J.P.; Maillard, Ph.; Rosilio, V. Biomimetic liposomes and planar supported bilayers for the assessment of glycodendrimeric porphyrins interaction with an immobilized lectin. Biophys. Biochim. Acta, 2011, 1808, 656-666.

[387] Makky, A.; Daghildjian, K.; Michel, J.-P.; Maillard, Ph.; Rosilio, V. Assessment of the relevance of supported planar bilayers for modeling specific interactions between glycodendrimeric porphyrins and retinoblastoma cells. Biochim. Biophys. Acta, 2012, 1818, 2831-2838.

[388] Bhanu, K.; Komath, S.S.; Maiya, B.G.; Swamy, M.J. Interaction of porphyrins with concavalin A and pea lection. Curr. Sci., 1997, 73, 598-602. [389] Komath, S.S.; Kenoth, R.; Giribabu, L.; Maiya B.G.; Swamy, M.J. Fluorescence and absorption spectroscopic studies on the interaction of porphyrins with snake gourd (Trichosanthes anguina) seed lectin. $J$. Photochem. Photobiol. B: Biol., 2000, 55, 49-55.

[390] Sultan, N.A.M.; Maiya, B.G.; Swamy, M.J. Thermodynamic analysis of porphyrin binding to Momordica charantia (bitter gourd) lectin. Eur. J. Biochem., 2004, 271, 3274-3282.

[391] Goel, M.; Damai, R.S.; Sethi, D.K.; Kaur, K.J.; Maiya, B.G.; Swamy, M.J.; Salunke, D.M. Crystal Structures of the PNA-Porphyrin Complex in the Presence and Absence of Lactose: Mapping the Conformational Changes on Lactose Binding, Interacting Surfaces, and Supramolecular Aggregations. Biochemistry, 2005, 44, 5588-5596.

[392] Komath, S.S.; Kavitha M.; Swamy, M.J. Beyond carbohydrate binding: new directions in plant lectin research. Org. Biomol. Chem., 2006, 4, 973-988.

[393] Kavitha, M.; Swamy, M.J. Thermodynamic Studies on the Interaction of Water-soluble Porphyrins with the Glucose/Mannose-specific Lectin from Garden Pea (Pisum sativum). IUBMB Life, 2006, 58, 720-730.

[394] D’Auria, S.; Petrova, L.; John, C.; Russev, G.; Varriale, A.; Bogoeva, V. Tumor-specific protein human galectin-1 interacts with anticancer agents. Mol. BioSyst., 2009, 5, 1331-1336.

[395] Bogoeva, V.P.; Varriale, A.; John, C.M.; D’Auria, S. Human galectin3 interacts with two anticancer drugs. Proteomics, 2010, 10, 1946-1953.

[396] Cecioni, S.; Matthews, S.E.; Blanchard, H.; Praly, J.-P.; Imberty, A.; Vidal, S. Synthesis of lactosylated glycoclusters and inhibition studies with plant and human lectins. Carbohydr. Res., 2012, 356, 132-141.

[397] Pandey, G.; Fatma, T.; Cowsik, S.M.; Komath, S.S. Specific interaction of jacalin with phycocyanin, a fluorescent phycobiliprotein. $J$. Photochem. Photobiol. B: Biol., 2009, 97, 87-93.

[398] Zhang, S.-P.; Zhao, J.-Q.; Jiang, L.-J. Photosensitized Formation of Singlet Oxygen by Phycobiliproteins in Neutral Aqueous Solutions. Free Rad. Res., 2000, 33, 489-496.

[399] Li, B.; Chu, X.; Gao, M.; Li, W. Apoptotic mechanism of MCF-7 breast cells in vivo and in vitro induced by photodynamic therapy with Cphycocyanin. Acta. Biochim. Biophys. Sin., 2010, 42, 80-89.

[400] Vedala, H.; Chen, Y.; Cecioni, S.; Imberty, A.; Vidal, S.; Star, A. Nanoelectronic Detection of Lectin-Carbohydrate Interactions Using Carbon Nanotubes. Nano Lett., 2011, 11, 170-176.
[401] Chen, Y.; Vedala, H.; Kotchey, G.P.; Audfray, A.; Cecioni, S.; Imberty, A.; Vidal, S.; Star, A. Electronic Detection of Lectins Using Carbohydrate-Functionalized Nanostructures: Graphene versus Carbon Nanotubes. ACS Nano, 2012, 6, 760-770.

[402] Rose, E.; Andrioletti, B.; Zrig, S.; Quelquejeu-Ethève, M. Enantioselective epoxidation of olefins with chiral metalloporphyrin catalysts. Chem. Soc. Rev., 2005, 34, 573-583.

[403] Che, C.-H.; Kar-Yan Lo, V.; Zhou, C.-Y.; Huang, J.-S. Selective functionalisation of saturated $\mathrm{C}-\mathrm{H}$ bonds with metalloporphyrin catalysts. Chem. Soc. Rev., 2011, 40, 1950-1975.

[404] Meunier, B. Metalloporphyrins as Versatile Catalysts for Oxidation Reactions and Oxidative DNA Cleavage. Chem. Rev., 1992, 92, 1411-1456. [405] Zhang, X.-B.; Guo, C.-C.; Xu, J.-B.; Ru, R.-Q. Synthesis of acetylglycosylated metalloporphyrins and their catalysis for cyclohexane oxidation with $\mathrm{PhIO}$ under mild conditions. J. Mol. Catal. A, 2000, 154, 31 38 .

[406] Maillard, Ph.; Guerquin-Kern, J.L.; Momenteau M. Catalytic Properties of Iron and Manganese Glycoconjugated Porphyrins.Tetrahedron Lett., 1991, 32, 4901-4904.

[407] Collman, J.P.; Zhang, X.; Lee, V.J.; Uffelman, E.S.; Brauman, J.I. Regioselective and Enantioselective Epoxidation Catalyzed by Metalloporphyrins. Science, 1993, 261, 1404-1411.

[408] Vilain, S.; Maillard, Ph.; Momenteau, M. Enantiomeric Epoxidation of 4-Chlorostyrene with $\mathrm{H}_{2} \mathrm{O}_{2}$ catalysed by Robust Chloro Manganese(III) meso-5,10,15,20-Tetra kis[2-chloro-6-(2,3,4,6-tetraacetyl-O- $\beta$-glucosyl)phenyl] Porphyrins. J. Chem. Soc., Chem. Commun., 1994, 1697-1698.

[409] Vilain-Deshayes, S.; Maillard, Ph.; Momenteau, M. Enantiomeric Epoxidation of 4-Chlorostyrene with $\mathrm{H}_{2} \mathrm{O}_{2}$ catalysed by Robust Chloro Manganese(III) meso-5,10,15,20-Tetra kis[2-chloro-6-(2,3,4,6-tetraacetylO- $\beta$-glucosyl)-phenyl] Porphyrins. J. Mol. Catal. A, 1996, 113, 201-208.

[410] Kuroda, Y.; Sera, T.; Ogoshi, H. Regioselectivities and Stereoselectivities of Singlet Oxygen Generated by Cyclodextrin Sandwiched Porphyrin Sensitization. Lipoxygenase-like Activity. J. Am. Chem. Soc., 1991, 113, 2793-2794.

[411] Kuroda, Y.; Hiroshige, T.; Ogoshi, H. Epoxidation Reaction Catalysed by Cyclodextrin Sandwich prophyrin in Aqueous Buffer Solution. J. Chem. Soc., Chem. Commun., 1990, 1594-1595.

[412] Kuroda, Y.; Ito, M.; Sera, T.; Ogoshi, H. Controlled Electron Transfer between Cyclodextrin-Sandwiched Porphyrin and Quinones. J. Am. Chem. Soc., 1993, 115, 7003-7004

[413] Yang, J.; Gabriele, B.; Belvedere, S.; Huang, Y.; Breslow, R. Catalytic Oxidations of Steroid Substrates by Artificial Cytochrome P-450 Enzymes. J. Org. Chem., 2002, 67, 5057-5067.

[414] Breslow, R.; Zhang, X.; Huang, Y. Selective Catalytic Hydroxylation of a Steroid by an Artificial Cytochrome P-450 Enzyme. J. Am. Chem. Soc., 1997, 119, 4535-4536.

[415] Breslow, R.; Huang, Y.; Zhang, X.; Yang, J. An artificial cytochrome P450 that hydroxylates unactivated carbons with regio- and stereoselectivity and useful catalytic turnovers. Proc. Natl. Acad. Sci. USA, 1997, 94, 1115611158 .

[416] Breslow, R.; Yan, J.; Belvedere, S. Catalytic hydroxylation of steroids by cytochrome P-450 mimics. Hydroxylation at C-9 with novel catalysts and steroid substrates. Tetrahedron Lett., 2002, 43, 363-365.

[417] Breslow, R.; Yang, J.; Yan, J. Biomimetic hydroxylation of saturated carbons with artificial cytochrome P-450 enzymes-liberating chemistry from the tyranny of functional groups. Tetrahedron Lett., 2002, 43, 653-659.

[418] Yang, G.; Breslow, R. Selective hydroxylation of a steroid at C-9 by an artificial cytochrome P-450. Angew. Chem., Int. Ed., 2000, 39, 2692 2695.

[419] Fang, Z.; Breslow, R. A thiolate ligand on a cytochrome P-450 mimic permits the use of simple environmentally benign oxidants for biomimetic steroid hydroxylation in water. Bioorg. Med. Chem. Lett., 2005, 15, 54635466.

[420] Yamazaki, S.-I.; Fujiwara, N.; Takeda, S; Yasuda, K. Electrochemical oxidation of sugars at moderate potentials catalysed by $\mathrm{Rh}$ porphyrins. Chem. Commun., 2010, 46, 3607-3609.

[421] Santos, L.M.; Baldwin, R.P. Liquid chromatography/electrochemical detection of carbohydrates at a cobalt phthalocyanine containing chemically modified electrode. Anal. Chem., 1987, 59, 1766-1770.

[422] Villagra, E.; Bedioui, F.; Nyokong, T.; Canales, J.C.; Sancy, M.; Páez, M.A.; Costamagna, J.; Zagal, J.H. Tuning the redox properties of Co-N4 macrocyclic complexes for the catalytic electrooxidation of glucose. Electrochim. Acta, 2008, 53, 4883-4888. 
[423] Berrera, C.; Zhukov, I.; Villagra, E.; Bedioui, F.; Páez, M.A.; Costamagna, J.; Zagal, J.H. Trends in reactivity of unsubstituted and substituted cobalt-phthalocyanines for the electrocatalysis of glucose oxidation. J. Electroanal. Chem., 2006, 589, 212-218.

[424] Sun, C.; Zhang, X.; Jiang, D.; Gao, Q.; Xu, H.; Sun, Y.; Zhang, X.; Shen, J. Electrocatalytic oxidation of carbohydrates at a molecular deposition film electrode based on water-soluble cobalt phthalocyanine and its application to flow-through detection. J. Electroanal. Chem., 1996, 411, 73-78.

[425] Takeuchi, M.; Yoda, S.; Chin, Y.; Shinkai, S. Sugar building-block as a fine-tunable link for electron-donor- electron-acceptor couples. Tetrahedron Lett., 1999, 40, 3745-3748.

[426] Lang, K.; Král, V.; Kapusta, P.; Kubát, P.; Vašek, P. Photoinduced electron transfer within porphyrin-cyclodextrin conjugates. Tetrahedron Lett., 2002, 43, 4919-4922.

[427] Záruba, K.; Vašek, P.; Král, V. Study of Molecular Recognition of $5,10,15,20$-tetrakis(pentafluorophenyl)porphyrin- $\beta$-cyclodextrin Conjugate Covalently Immobilized on a Silica Surface. Supramol. Chem., 2004, 16, 529-536.

[428] Sakakibara, K.; Nakatsubo, F.; French, A.D.; Rosenau, T. Chiroptical properties of an alternatingly functionalized cellotriose bearing two porphyrin groups. Chem. Commun., 2012, 48, 7672-7674.

[429] Wu, M.; Yu, Z.-W.; Liu, Y.; Feng, D.-F.; Yang, J.-J.; Yin, X.-B.;

Zhang, T.; Chen, D.-Y.; Liu, T.-J.; Feng, X.-Z. Glycosyl-Modified
Diporphyrins for in Vitro and in Vivo Fluorescence Imaging. ChemBioChem., 2013, 14, 979-986.

[430] Tamaru, S.; Nakamura, M.; Takeuchi, M.; Shinkai, S. Rational design of a sugar-appended porphyrin gelator that is forced to assemble into a onedimensional aggregate. Org. Lett., 2001, 3, 3631-3634.

[431] Kawano, S.-I.; Tamaru, S.-I.; Fujita, N.; Shinkai, S. Sol-Gel Polycondensation of Tetraethyl Orthosilicate (TEOS) in Sugar-Based Porphyrin Organogels: Inorganic Conversion of a Sugar-Directed Porphyrinic Fiber Library through Sol-Gel Transcription Processes. Chem. Eur. J., 2004, 10, 343-351.

[432] Batinić-Harerle, I.; Benov, L.; Spasojević, I.; Fridovich, I. The Ortho Effect Makes Manganese(III)Meso-Tetrakis( $N$-Methylpyridinium-2yl)Porphyrin a Powerful and Potentially Useful Superoxide Dismutase Mimic. J. Biol. Chem., 1998, 273, 24521-24528.

[433] Chiu, M.H.; Tamura, T.; Wadhwa, M.S.; Rice, K.G. In vivo targeting function of $N$-linked oligosaccharides with terminating galactose and $N$ acetylgalactosamine residues. J. Biol. Chem., 1994, 269, 16195-16202.

[434] Asayama, S.; Mizushima, K.; Nagaoka, S., Kawakami, H. Design of Metalloporphyrin-Carbohydrate Conjugates for a New Superoxide Dismutase Mimic with Cellular Recognition. Bioconj. Chem., 2004, 15 , 1360-1363. 
Curr. Med. Chem. 2015, 22, in press 